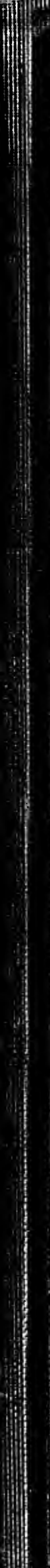











\section{DIARRHEAL, INFLAMMATORY,}

OBSTRUCTIVE, AND

\section{PARASITIC DISEASES}

OF THE GASTRO-INTESTINAL TRACT

BS:

SAMUEL GOODWIN GANT, M. D., LL. D.

PROFESSOR OF DISEASES OF THE COLON, SIGMOID FLEXURE, RECTLM, AND ANUS AT THE

NEW YORK POST-GRADUATE MEDIC.IL SCHOOL AND HOSPITAL

\section{ILLUSTRATED}

PHILADELPHIA AND LONDON

W. B. SAUNDERS COMPANY 
HII IC

$=11 d$

$1+1$

Copyright, 1915, by W. B. Saunders Company

PRINTED IN AMERICA

PRESS OF

W. B. SAUNDERS COMPANY

PHILADELPHIA 


\section{PREFACE}

The generous reception given the atuthor's recently published work on Constipation and Intestinal Obstruction (obstipation-Intestinal Stasis) has encomaged him in the belief that a volume along similar lines devoted to I darrheal, Inflammatory, (obstruetive, and l'arasitic Diseases of the Castro-intestinal Tract would be of ratue 10 the profession.

The dominant idea of the author in the preparation of this work has been to present to students and practitioners a complete yet practical treatise covering the etiology, pathology, symptoms, diagnosis, and treatment of acute and chronic diarther and allied affections, as well as diseases conseruent upon gastro-intestinal parasites.

To free the look of useless material and make it comsenient in size and easily comprehensilse, the author's discussions have been as briet as justice to the subject would permit, and technical torms, confusing nomenclature, profuse histologic data, complicated laboratory methols of examination, prolonged discussion of mooted points, wearying statistic tables, and obsolete views have been omitted. He has also endeavored to arrange the sul,jects in a logical and convenient form, so that the busy practitioner or student may quickly refer to whatever he desires relative to diarrheal and parasitic affections.

Many times the author has desired information concerning certain phases of diarrhea and has been unable to find it except hy culling an enormons amount of current literature, which repuired comsiderable time and labor, and repeated experiences of this kind helped to contvince him that a volume which would cover eliarthea in all its phates would prove useful alike to the internist, pediatrist, and surgeron. Ile has also been asked many times by physicians why he was devoting so

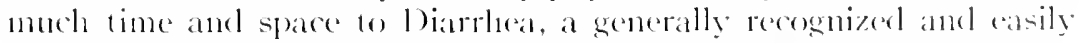
controllathe manifestation. In reply he would saly that thromgh dhe frequent questioning of students and pratelitioners will whom he has come in contact while lecturing in different citiess and teathing at the New York Post-Gradtate Merlical School atud llospital, atud at the University and Womens' Medical colleges (Kansas ("ily, Mo.), the author has become convinced that physicians generally do not turferstand the various types of diarthea, the modern methods of differ-

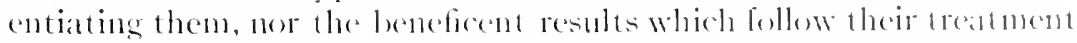
by directly irrigating the lesions responsible for the lexose morements or by surgical measures; and further, that a more comprehemive 
knowledge redative to diartheal, inflammatory, and parastic distases of the inte-tine in all their phase is deriralle.

Un account of the very - reat intere-t manifered concerning tropical and para-itic liacase frequent factor in diarrheal at home and in wur colonien. and the mumerous discoseries recently made per-

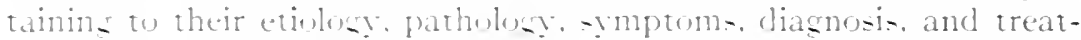
ment, the author ha-fully discused the relation of Purasitic Diseases (1) Diarrhea.

di-tinctive chapters have ahor been deveted to a "Fommlary" and the "Irrisating" and "Sureical Treament" of Diarrheal and Parasitic Affections of the Gastro-intestinal Tract.

So much prosere has been made during the past few years in the methods of examination and diagnostic technic relative to diarrhea, parastic and wher sa-tru-inte-tinal affectiuns. that it ha= leen deemed wise to diseds them in a seneral way in the special chapter entitled. "Examinution and Diconosis," and again in greater detail when considering their relation to individual forms of diarrhea in other sections of the work.

The author wishes to take this opportunity to thank Dr. Lamb. of the Army Medical Museum. for procuring photographs of specimens; Drs. S. Jurtimer Hill and F. Rolbin-for as-i-tance rendered during the preparation of the work: Mesrs. Howard I. Shannon and K. K. Bose for the excellent drawings seen throughout the book; E. F. Rinn for retouching numerou- photosraphs: and the II. R. Saunders Company for the many curresie- extended to the author.

With the hope that hi- work may stimulate interes in the class of diseases discureed, and prove helpful in the recognition and management of diarrheal. inflammatory, obstructive. and para-itic affections of the ca-tro-inte-tinal tract, the author offers this book to members of the profes-ion.

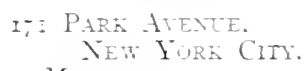




\section{CONTENTS}

('II.H'TER I PACE

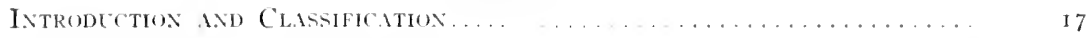

\section{(']AP'TK H}

Eximishon AND DugNosis

(II.APTER II]

ORG.ANIC DISFASES, MI.JRRHEA IN

Eye Diseases. 54-Mhuth Diseases, 54-Nasupharyngeal Diseases, 55-Thyroid

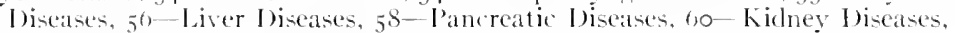
62-Diabetes Melitus, (1,3-Suprarenal Disease, (4- (ienital Diseases, 64Skin Diseases, 67-Bone Diseases, 68.

\section{(IIIPTER II}

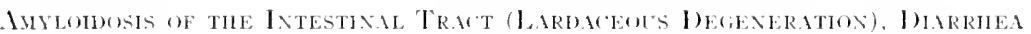
IN.

\section{CHISTIR I}

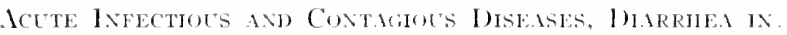

Measles, 7t Scarlet Fever, 7t - Varicellat, 7t - Variola, 75- Whomping-cough, 75 -Diphtheria, $75-\ln$ huenza, $75-$ Pneumonia, $70-$ Malaria, 78.

\section{CIIPTER VI}

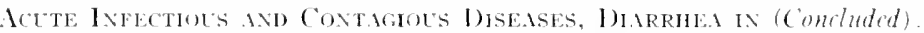

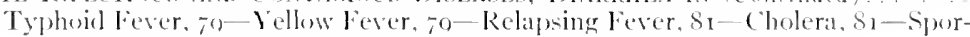

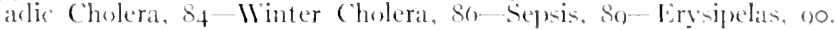

\section{CIJAPTIR VII}

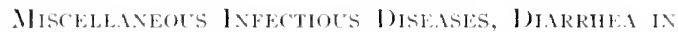

Cochin-China Diarrhea, o2-Sprue, o.3-Jill-(iarrhea, 04- Diarrhear Mlbat, o.t-

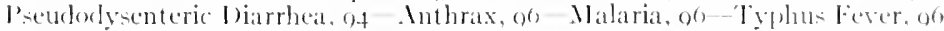

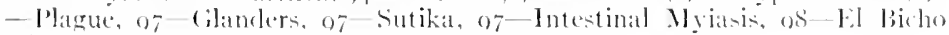

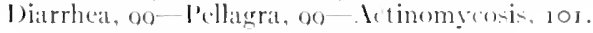

\section{('IIPYTE VII}

STMTRY DISEASES, IIIRRHIA IN

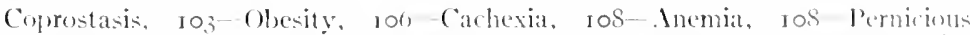

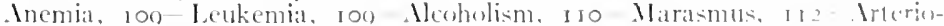

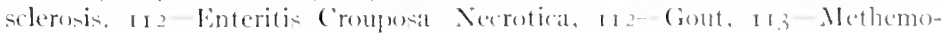
globinemia, 1 1.3-Sourvy, 1 13- Cerelorospinal Meningitis, 113. 
StNTRY DIARRHEAS.............. IIt

biarrhea from Irregularities in Livine. I 4-Diarrhea Cathartica. I $5-$ Diar-

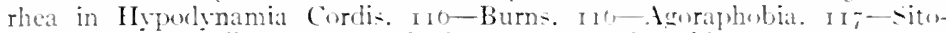

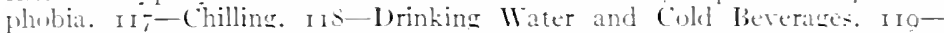
sun-strokes and Heat-strokes. I 20-Old Age. I I-Diarrhea Jocturna. I 2 IEosinophilic Diarrhea. Iz - Mechanic Diarrhea. I 22-Diarrhea irom Reflex Di-turbances, 122 .

\section{CHAPTER I}

Gastrogenic Imirrhes (Dyspeptic Diarrhes. Liexteric Diarrhea)

Achylia Gastrica. I 23-Hyperacidity. I 23-Malignancy. I 23-Atony. I $23-$ Motor Insufticiency: 123 .

\section{CHAPTER XI}

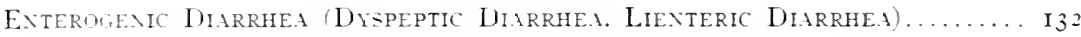
Unbalanced Suceus Entericus. 132-Duodenal Clcer. 132.

\section{CHAPTER XII}

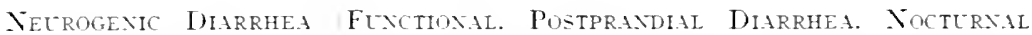

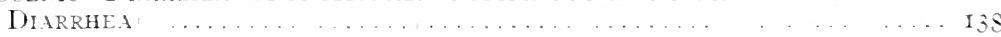

Psychic I liseases and Injuries of the Nervous system. I $35-R e f l e x$ Disturbances. 135 -Affections of the Thyroid Gland. I 35.

\section{CHAPTER XIII}

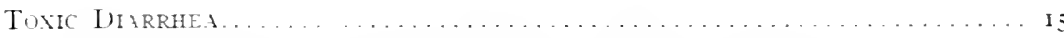

Meat-poisonine. I 50-Toxemia from Meat of Diseased Animals. 15 I-Toxemia from Decayed or Putrened Mcat. 152-Toxenia iron sausage-poisoning. I 52 - Puisonine irom Milk and It: Products. I 52-Fish and Shell-ish Poisoning.

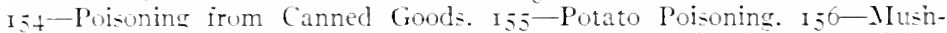
rom II suarin Poisoning. 15\%-Grain- or Seed-poisoning. I 5 -Other Fond Diartheas. I 5 S.

\section{CHAPTER NIV}

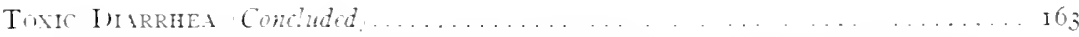

Arenic-poisoning. 165-Mercurial Poisoning. I65-Lead-poisoning. 166Phosphoru-pmisonine. Io6-- Acid-poisoning. 166-Alkali-poisoning. 16;Miriellaneous . Medicinal and Chemical Poisons. 160.

\section{CH.APTER XV}

COMPENSITORY DIARRHE $1 \ldots$

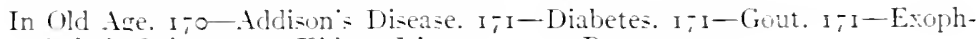

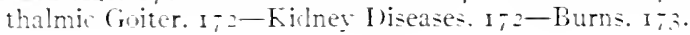

\section{CH.APTER XVI}

Exteritis. Colith. Exterocolits Nox-specific Intesinil Catirrh). IIARRHEA IN . . . . . . ITt

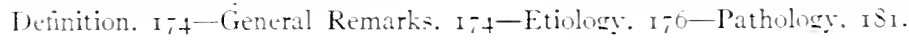

\section{CHAPTER MIII}

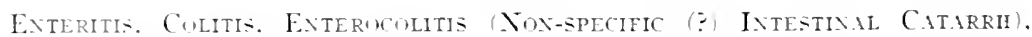

I)IRRIIEA IN Continut ....

symptoms. ist-Diannosis. roo.

\section{CH.APTER XIIII}

Exteritis. Colitis. Eyterocolitis Nux-spectfic ( InIRRIE I IX Concluded ................................. 200 Treatment, 200-Prugnosis. 200. 


\section{CII.IPTER XIX}

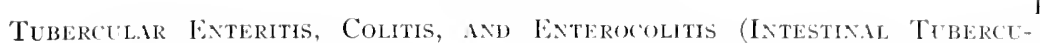

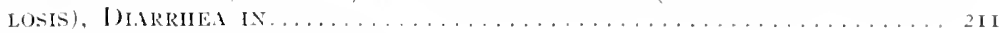
(ieneral liemarks, 21 I-Etiology, 2 I 3 .

\section{CHMPTER XX}

Tubercelar Exteritis, Colitis, and Exterocolttis (INTestinal Tíberce-

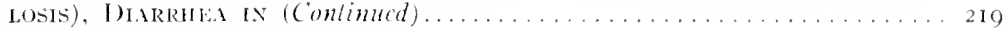
Classification, 219-Pathology, 220.

\section{CIIAPTER XXI}

Tubercular Exteritis, Colitis, avi) Exterocolitis (Ixtestinil Tebercu-

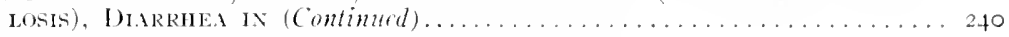
Symptoms, 240-Complications, 240-Serquelit. 240.

\section{CHAPTER XXII}

Tuberculir Enteritis, Colitis, and Enterocolitis (Intestinal Tuberce-

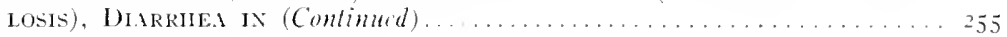

Diagnosis, 255 .

\section{CH.IPTER NXIII}

Teberclear Fateritis, Colitis, ANo Exterocolitis (Intestixil Tebercu-

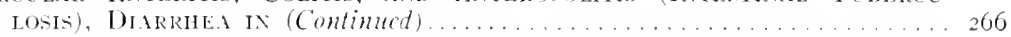

Treatment, 206-Prophylactic Measures, 267-bieting, 270-Medical Treatment, 271-Irrigating Treatment, 278-Serum Treatment, 282.

\section{CIIAPTER XXIV}

Tubercelar Exteritis, Colitis, axi) lixterocolitis (Intesticil, Tiberce-

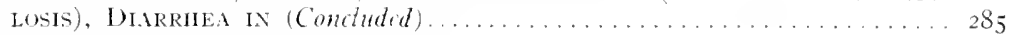
Surgical Treatment, 285 .

\section{CIIAPTER XXY}

Siphilitic Exteritis, Colitis, ind Exterocolitis (Intestixal Sypinlis), DiarRHEA IX. . . . . . . . . . . . . . . . . . . . . 294

General Remarks, 204-Etiology, 200-Pathology, 297.

\section{CHAPTER XXVI}

Syphlitic Exteritis, Colitis, ind Exterocolitis (Intestixil Syphilis), DiarRIEA IN (Continued).

Symptoms, 304-Diagnosis, 307-Prognosis, 31 I.

\section{CHAPTER XXVH}

Syphilitic Exteritis, Colitis, ind Exterocolitis (Intestindl Sypinls), DiarRHEA IX (Conduded).

Medicinal Treatment, 313-Salvarsan, 314-Surgical Treatment, 318.

\section{CIIAPTER XXVIII}

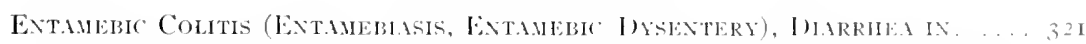

Definition, 32I-General Remarks, 32 I-Ilistory, 32,3-Etiology, 326-Geographic listribution. 3,30-Cultivation, 3,31-Classification, 332-Norpholagy, 3,36-Reproduction. 339 . 


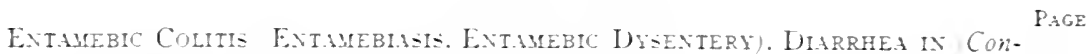
timet . . . . . . . . . . . . . . . 34 I Pathology Induding Tropical Liver . Bbece. . $3+1$.

\section{CH.APTER XXX}

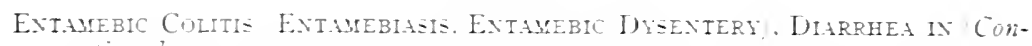

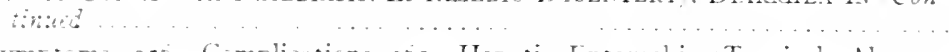
smptoms, si-Complicutions joj-Herti Entamelic Tropical Abscess,

\section{CHAPTER MYI}

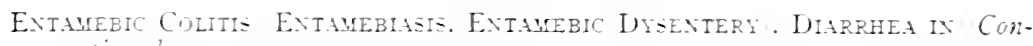
anied

Diagnosis 3:0-Prognosis. $3: \div$.

\section{CHAPTER XXYI}

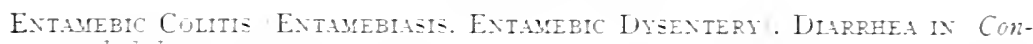

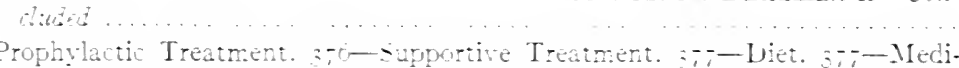

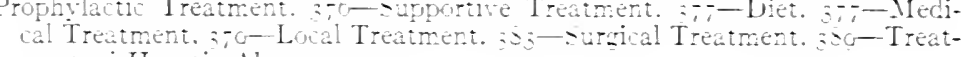
ment of Hepati Absces. sos.

\section{CH.APTER MXXIII}

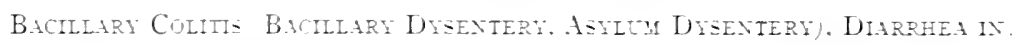

\section{CH.APTER MXYY}

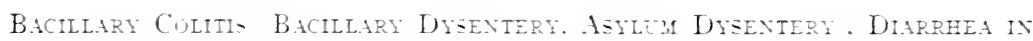
Constist Pathology :00. 


\section{CIIAPTER XXXIX}

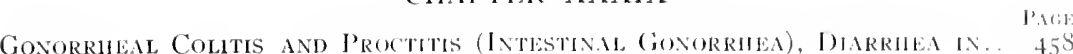

\section{CIIAPTER XL}

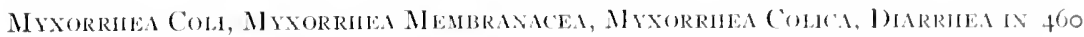
History, foo- litiology, foo-Pathology, to5-Symptoms, fo5-I iagnosis, foo -Treatment, fot-Prognosis, $4(0)$.

\section{CHAPTER XLI}

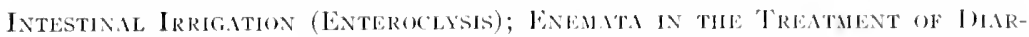

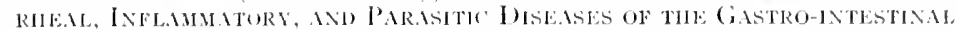

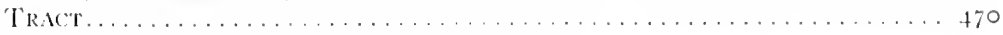

Irrigants, 473 - Technic of Bowcl Irrigation, 480.

\section{CIIAPTER XIII}

Obstructive (Mechanic, Surgicil) D)iarriea 488

General Remarks, $488-$ litiology, 490 .

\section{CHAPTER XIJI}

Obstructive (Mechinic, Surgical) Dharriea (Continued)............... 496 Symptoms, 406.

\section{CHAY'TER NLIV}

Obstructive (Mechanic, Surgical) Dharrien (Continued) 50.4

1)iagnosis, 504 .

\section{CHIPTER XIT}

Obstructive (Iechanic, Surgical) Diarrie. (Concluded).............. 510 Non-operative Treatment, $510-$ Suresical Treatment, 51 1.

\section{CHIPTER XLYI}

Post-oplerative Diarriea................................. 5 I0

Etiology and Pathology, 5 ro-Symptoms and Jiagnosis, 522-Treatment, $52,3$.

\section{CHAPTER NLITI}

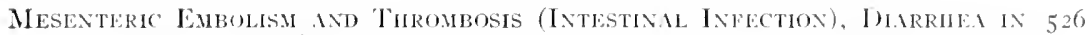
Etiolegy and Pathology, 520 -Symptoms and Diagnosis, 527 -l'rognosis, 528 Treatment, 528 .

\section{CIJAPTER XIVIII}

FORIILIRY

\section{CIIIPTER XIAX}

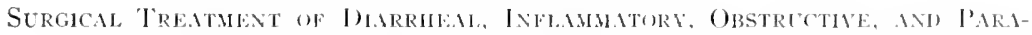

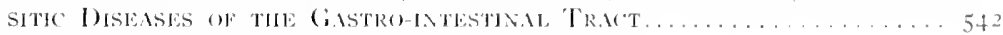
General kemarks, 542 - Prejaration of Pationt, 54.3.

\section{CIIPTER I}

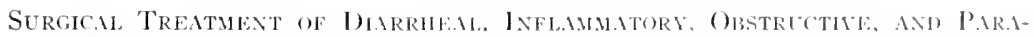

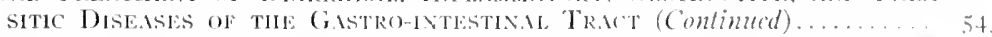

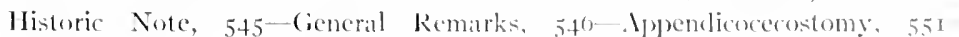
Enterostomy, 5.52-Colostomy, 552-Results of Tliromgh-and-through Irrigating Treatment following Appendicostomy and Cecostomy, 552- Technic of Cecostomy, 554 . 
Surgical Treatment of Diarrhede, Inflamutory, (Obstrlctive, and ParaSITIC MISEASES OF THE G.ASTRO-HNTESTIXAL TR.ACT (Continued) ........ 556

Technic of Gant's Enterocecustomy (Cecostomy), Appendicostomy, Appendicocecostomy, and Ippendico-enterocecostumy; 556 .

\section{CH.APTER LII}

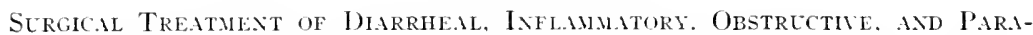

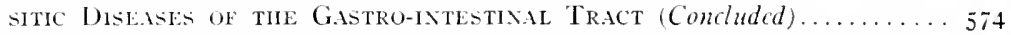

Technic of Enterostomy. Colostomy, Enterectomy. Cecectomy, Colectomy, Sigmuidectomy, Proctectomy, 57 .

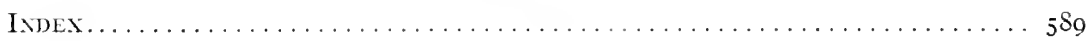




\section{CHAPTER I}

\section{INTRODUCTION AND CLASSIFICATION}

Introduction.-Diarrhea is a broad subject, and one which should interest alike physicians and surgeons, because the services of one or both are often required to relieve or permanently cure patients afflicted with it. Athough less frequently than constipation and intestinal stasis, diarrhea is met with quite of ten, and when persistent usually induces more suffering, digestive disturbances, loss of weight, and impairment to metabolism than constipation.

Diarrhea is a symptom characterized by frequent and fluid evacuations. This definition is not entirely satisfactory, because by some. fragmentary constipation is classed as a diarrhea because the patient has several daily movements; but this is a mistake, since the leces are semisolid or formed, and are cut off and ejected in smatl pieces in consecpuence of the irritable state of the levator ani and sphincter muscles. Again, some patients regularly have two or three daily evacuations, and the stools of others persistently remain mushy or semisolid, and yet health is maintained; consecfuently, such individuals do not suffer from diarrhea, though they or their physicians think they do, and believe something should be done to diminish the evacuations or cause them to becone formed. When, however, a patient has two or more morements daily containing mucus, pus, or bloot, a diagnosis of diarrhea is justifiable because evidently there is some pathologic change going on in the intestine which is causing abnormal peristalsis, the hypersecretion of mucus, or transuclation of fluid into the bowcl.

Owing to its many and varied types, it is necessary that practitioners who treat diarrhea should be adepts in the diagnosis and treatment of gastro-intestinal and allied diseases.

Diarrhea is a common topic in current literature and medieal societies, but the etiology, pathology, diagnosis, and especially the treatment of this condition are not understood; or, if they are, physicians generally do not take the pains or time to institute or "arry out a successful line of treatment. This hats been demonstrated many times in the author's private hospital and chinic work, where pattents who suffered from chronic chatrrhea for years in spite of constint medieal treatment and dieting have been speedify cured by the operative or other measures outlined in the following pages. Failure in many instances is due to the attentant in charge prescribing something which will quickly diminish or check the eracuations or lessen their fluidity, but does nothing to mitigate or remove the cause of the Irequent evacuations. 
While phy-icians are prone to look upon frequent loose movements as a manifestation of some other affection. the patient does not. and seeks treatment for the diarrhea which he con-iders responsible for his pain. gas disturbances. frequent evacuations. loss of appetite, diminished weight, detention from bu-ines-. inability to eat what he chones or participate in the pleasures of life because of the frequent vi-it- he must necesarily make to the toilet, and extra outlay for meelicines and doctors bills.

Prolonged diarrhea, through los of sleep, drain upon the system, worry, digestive di-turbances, and auto-intoxication which frequently accompanies it and tends to the production of other circulatory defects), lower the vitality and borlily re-isunce. and altogether makes the patient an eas prex to microbes. and is often the direct source of pathologic changes within the gut, in close proximity to it. and in distant parts becau-e the irritating fluid discharges serve to erode or break the continuity of the intestinal mucosa. permit specific and nonspecific bacteria to enter the circulation and be carried to remote parts. where they cauce trouble, or penetrate deeply into or through the inte-tinal wall with the resultant production of deep-seated mixed infection, ulcers, abscesses. fistule. pyemia, peritonitis, septic pneumonia, or other serious complications.

The profe-ion is usually prone to reaard diarrhea as being due to a single cause when its etiology is complex and embraces causes which are emotional. due to local disease in the gastro-intestinal tract. and those of a more ceneral character: but it matters not whether the disturling element belongs to the first. second, or third group, for it induces frequent evacuations by augmenting the gastro-intestinal secretions. stimulating frequent and prolonged peristalsis, diminishing or blocking the bowel lumen. so that there is only room for tluid feces to dribble past the obstructed point. all of which have received careful consideration in their proper place.

Loose morements. except those consequent upon intestinal obstruction, are as a rule. secondary to stimuli which directly or indirectly influence the bowel. Such stimuli may lead to diarrhea by causing hyperperistalsis, the transudation of fluid from the blood through the bowel wall into the intestinal lumen, and hypersecretion of mucus.

Stimuli responsible for diarrhea may be induced by psychic emotions, disease of the brain. cord, general or local nerve mechanism, inflammation. ulceration, neoplasms, strictures or foreign bodies in the intestine, or lesions affecting it from without, or anything which irritates the intestinal nerve-ganglia.

Occasionally, multiple or widely varying stimuli work simultaneously, so that a vicious circle is established. under which circumstances there is exaggerated intestinal motility, and the patient suffer cleptorally from diarrhea extremely difficult to relieve or cure.

Srometime the disturbing factors causing diarrhea are located in the stomach or small intestine, and the chyme is improperly digested 
or rushed through the gastro-intestinal tract before its watery constituent can be absorbed. Again, frepuent and fluid evacuations are secondary to inflammatory and ulcerative lesions which impair the absorptive power of the colonic mucosa, and, as a result, the thuid content of the bowel which should reath the circulation is evacuated.

Because of the many and varied types of diarthea it is impessible to formulate a routine treatment which will meet the comelitions in different cases, and diarrheat recpuires distinctive consideration and treatment in each case.

Those afflicted with this complaint who come to the author are asked the guestions, "Are you seeking temporary relief?" or "Do you desire that I shall institute a course of treatment which will have for its object a permanent cure?" hecause the therapeutic measures to be instituted hinge upon the patient s decision.

One can, by controlling the diet and administering medicines which contain astringents, antiseptics, opiates. ete. make the patient more comfortable and reeluce the frequency of the stools, but, when a cure is insisted upon, the symptomatic treatment (except in ursent cases) should be discarled in favor of surgical or other therapeutic measures which tend to eliminate one or all the causes-local, general, nervous, or psechic-responsible for the diarrhea.

Sufferers from chronic diarrhea are told, when beginning treatment, that it may require weeks or months to effect a cure, and that, to obtain the best results, it is necessary for them to make a business of getting well-carry out instructions and come for treatment as often and long as necessary.

The author speaks from experience when he says that a great deal can be accomplished in the handling of this class of cases by continuous treatment, and that the results are unsatisfactory when it is intertupted by patients who spasmodically apply for a prescription, irrigation, etc., or neglect the treatments because they are indifferent, busy, or wish to save a fee.

Frequent loose movements are regarted as a symptom of disease, and the physician is prone to concent rate his efforts exclusively against the latter, while he does little or nothing for the former, taking it for granted that the frepuent evacuations will cease when the affection responsible for diarrhea has heen cured, a procedure to be commented in some and condemned in other instances. Practitioners often fait in relieving diarrhea or curing the disease back of it becaluse they attribute the frequent evacuations 10 pathologic changen in the gastrointestinal tract, and fail of realize the frecuency with which diarrhea is caused by emotional and reflex disturbances. ontside toxemias (which act upon the intestine), nervous affections, abnormalities of the circulating media, discased organs or structures adjacent wo or remote from the bowel, and numerous other factors elsewhere discussed. Many physicians in the past have considered, and some at present regard, diarrhea as a medical disease, and treat it accordingly, 
when frequently the condition is surgical and camnot be corrected except through recourse to the operations described in the text. This is evidenced by the author s large experience. which has demonstrated that in many instance- diarrhea can be cured by surgical intervention after it has been unsuccesfully treated for years by medicine and other non-surgical measures.

Medicine can be relied upon to lesen pain. diminish frequency of eracuations. and, in rare instance, to affect a cure in acute and chronite diarrhea. but as a steneral rule. it is not dependable when it comes to permanently correcting or remoring the cause of the trouble. Ton often medicine is prescribed with the object of affording immediate relief. and not with the idea that it will permanently benefit the patient, ly busy, cartess. or ignorant practitioners, who do not try, or fail to find, the disturbing factors respon-ible for the diarrhea, and. as a result, thee sufferers are usually dowed for weeks, months, or vear: with morphin, opium. bismuth, tannic acid, chalk, salol, and like remedie: which lesen the morements while the patient is under their continued influence. but which otherwise do no good, since the diarrhea become equally or more marked as soon as drugs are withdrawn.

The indiscriminate prescribing of medicine for this affection is to be condemned. because they frequently dertroy the appetite. encourage insomnia. interfere with digetion. are nauseating. cause subsequent headaches, irritate the gastro-inte-tinal mucosa. lead to the formation of enterolith bismuth and salol. which cause obstruction, and patient-often lecome drus hobiats.

Recently sreat adrance has been made in the prophylactic. psichic, and dietetic treatment of diarrhea and allied conditions, alone or in conjunction with phrical therapeutic measures masage. electricity. vibration. hydrotherapy, concentrated light. etc. . irriqation, enteroclysis. where the fluid is introduced through the anu- or an artificial opening made in the small intestine enterostomy, appendix appendicustony . cecum cecostomy or colon (colostomy), or the surgical procedure = low where decribed.

Becaute of the surpri-ingly goorl permanent results obtained by the author in the survical treatment of diarrheal affections, and the frequent failure and complication. which follow the pernicious custom of univer-ally armini-tering medicine by mouth, he has, when feasible. recommended the former to the exclu-ion of the latter plan, and, as a rule. has not prescritud drues except when necessary to immediately relieve smptoms which have suddenly hecome annoying or dancerous.

Direct howel treatment is not employed a- frequently as it should be-certainly the author'- experience warrants him in the belief that more can be accomplished in the treatment of chronic loose movement soneequent upon non-specific and specific inflammatory diseate of the intestine by irrigation and topical applications than by havinc the patients diet and drug themselves. 
Inflamed and ulcerated areas in the intestinal mucosa readily respond to direct treatment after measures usually instituted for their cure have failed to heal them. Sometimes the colon can be treated by medicated irrigations introduced by way of the anus, but when, for any reason, the various segments of the bowel cannot be reached, or the procedure is not feasible, appendicostomy, cecostomy, or the author's cnterocecostomy are indicated, so that through-andthrough irrigation may be instituted.

Barring obstructiee, the vast majority of diartheas characterized by the presence of mucus, pus, and hlood in the stools can be quickly and permanently relieved in this way.

Mechanic, obstructioe. or suresical diarrhea is seldom mentioned in medical lectures or articles; yet it occurs frecuently, and if physicians appreciated its importance, and the ease with which it can he relieved or cured by operation, many patients would be saved who now die when treated in the ordinary way.

Obstructive diarrhea is fairly common, and may be produced by many pathologic lesions, foreign bodies, or fecal impatetion which block the bowel and retain solid, but allow the fluid feces to be frequently evacuated. This type of loose mesements is often overtooked, and opium and hismuth are prescriled to diminish the stools, remedies which tend to aggravate rather than relieve the patient's condition.

Surgical interention is indicated in the treatment of chronic diarthea as well as appendicitis when it is induced hy inflammatory, ulcerative, or olsstructing lesions which do not respond to ordinary measures.

Fragmentary constipation is sometimes mistaken and treated for diarrhea because the pationt goes to the toilet fretuently; but such errors would not be mate dicl the physician regularly examine the stools, for in these cases the excreta is ejected in short semisolid or firm pieces. Since the fecal bolus is divided into small sections through the scissors-like action of the external sphincter or levator ani muscle when irritated, or by o'Beirne's sphincter at the rectosigmoid juncture, it is obvious in this class of cases that the therapeutic anents usually employed in the treatment of diarrhea only make matters worse.

Classification.- At first one might think diarrheas easy to gromp, but on further sturly they iecome difficule or imposible to classify owing to their varied etiology, pathology, manifestations, and association with different local and general diseases. They may. however, be etiologically grouper as functional and organic, and clinically, into the acute and chronic types.

The atuthor has attempted to classify diarrheas etiologicully on pages 22 and 23, but he recognizes that this arrangement is not entirely satisfactory, since the canses of some affections responsible for diarrhea still remain undiscovered or in doubt. 


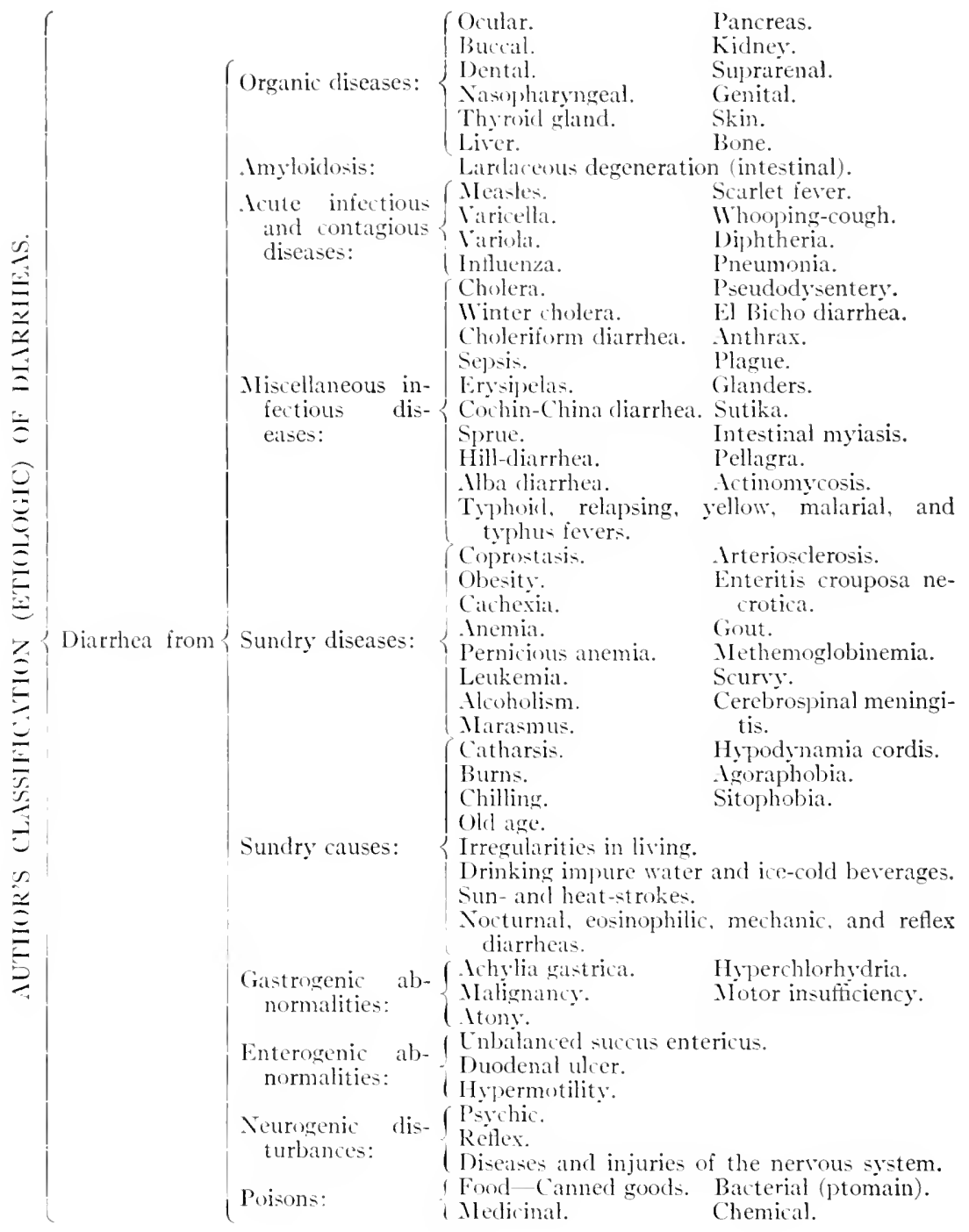

In his classification the author has endearored to get away from the term "dysentery," which is not a diseatse, but a symptom-complex, represented by abdominal pain, diarhea, tenesmus, mucus and blood in the stools, manifestations common to all inflammatory affections of the colon complicated by ulceration; and to this end he has employed the caption colitis plus the prefix of the etiologic lactor back of the intlammatory procesis to designate the disease-viz., non-specific (catarthal), entamebic, bacillary, helminthic, protozoal, tubercular. syphilitic, sonortheal, and malignant colitis.

IIith this arrangement the caption employed indicates the etio- 


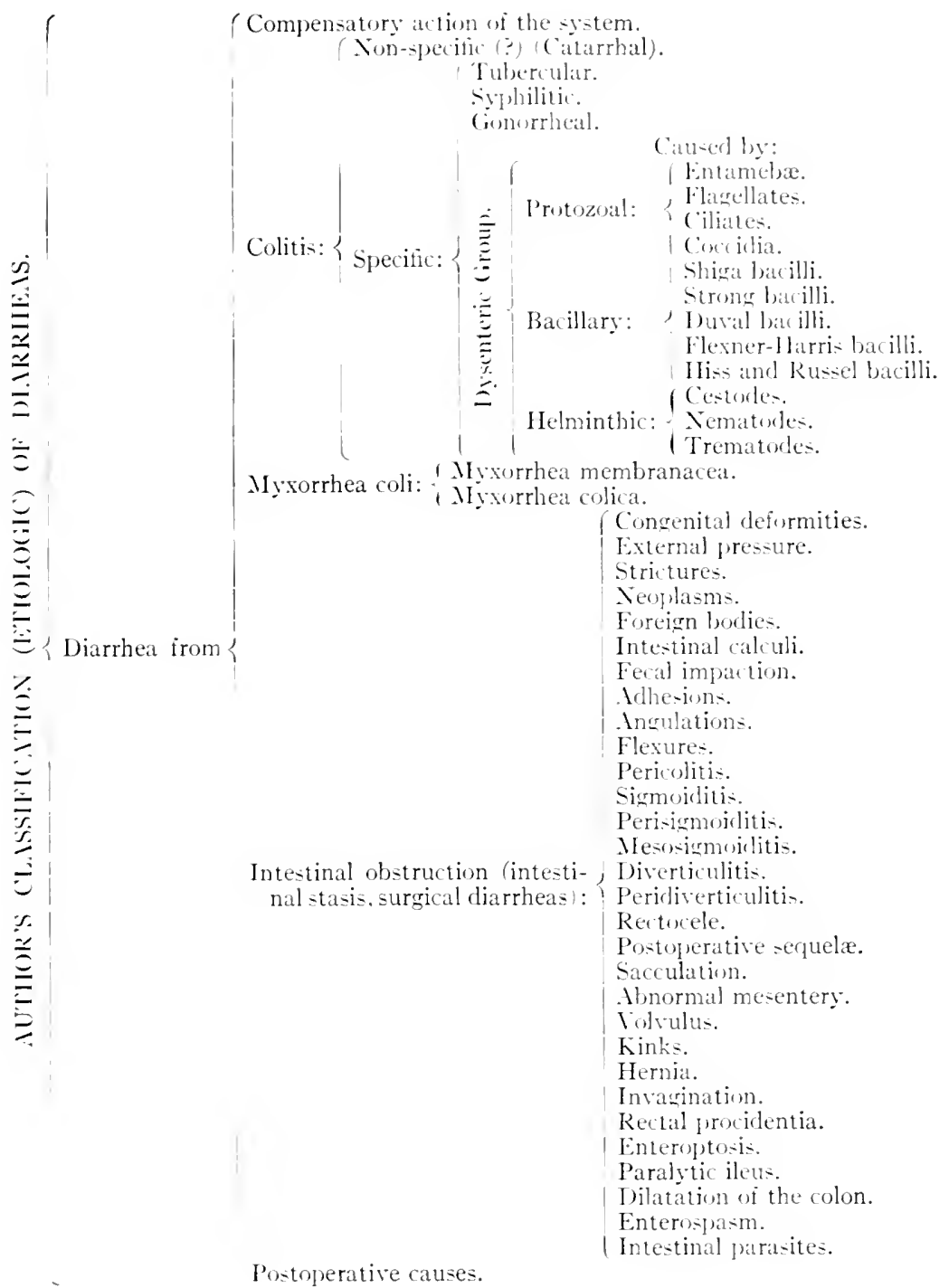

logic factor behind the inflammation, pathologic changes which characterized it, plan of treatment to be adopted, and leaves careless phy-sjcians without an excuse for diagnowing as dysentery and handling in a routine way all patients who complain of abdominal pain, tenesmus. diarrhea, and mucus and blood in the stools. 


\section{CHAPTER II}

\section{EXAMINATION AND DIAGNOSIS}

DIARRHEA is easy to diagnose because the patient will voluntarily or upon questioning say that the stools are more frequent and loose than they should be. It is, however, very difficult in many instances to ascertain what is the trouble. This is not to be wondered at when one sees the raried types of diarrhea and the almost innumerable functional and organic disturbances and other factors which may induce loose morements.

Usually there will be found a chief cause for the disturbance, but often several ctiologic factors are present, any one of which is sufficient to incite frequent or fluid evacuations, and must be considered when outlining the treatment. Occasionally diarrhea arises from defects in the bowel the result of diseases already cured, hut which have left their sequele to continue the loose movements until they are relieved or corrected. In order to make a rational diagnosis in this class of cases a routine method of examination should be followed. This consists in ubtaining a brief but intelligent history of the case from its beginning, along with the symptoms complained of, carefully inspectins the patient from every standpoint: palpating the spinal, abdominal, pelvic, and anorectal regions; percussing the liver, kidneys, pancreas, spleen, stomach, and intestines; practising succussion over the stomach and colon: making a chemic, macroscopic, and microscopic examination of the stomach and intestimal contents independently and following test-meals; examining the blood, urine, and secretions: distending the bowel with air, gas, or water, so that its segments may be outlined or obstructions located; inspecting the rectum and sigmoid Hexure through the proctosigmoidoscope; making a digital and specular cxamination of the vagina and anorectal region; taking radiographs of the viscera or studying them through the fluoroscope; and, in obscure cases, in examining the patient while under ether or following an exploratory incision.

History.-A complete, but not necessarily lengthy, history should he taken and recorded in each case, because in this way valuable information can often be obtained which would lead the attendant to suspect the nature of the trouble. One should draw the history from the patient by direct questioning instead of letting him tell his own story, as sone physicians do, and in this way ascertain how long he has leeen ill, if the cliarrhea was of sudelen onsed or came on gradually, is constant or intermittent, slight at one time and aggravated at ancther, alternates with constipation, if it was manifest from the start or secondary to another ailment, if the stools are foul smelling or 
contain blood, pus, or mucus, the feces are normal in shape, size, and consistence, the abdomen is tender on pressure, the stools are preceded, accompanied, or followed by tenesmus, straining or pain, and if the patient suffers from other manifestations indicative of disease that would cause diarrhea.

It is important to learn from the patient his age, oceupation, habits, character of food he eats, if he is dissipated or takes medicine which would increase the frequency and fluidity of the morements, and if he is afflicted with or has in the past suffered from digestive disturbances, gastritis, enteritis, appendicitis, abdominal or pelric disease, affections of the liver or pancreas, gall-stones, congenital deformities, operative sequele, or other local disease which would interfere with digestion or leave the mucosa irritable, inflaned, or ulcerated, diseases frequently complicated by tumefactions, adhesions, kinks, or stricture which may induce obstructive diarrhea.

One should also ascertain if the patient is paler than formerly. has lost weight, has nasal catarrh, bad teeth, furred tongue or foul

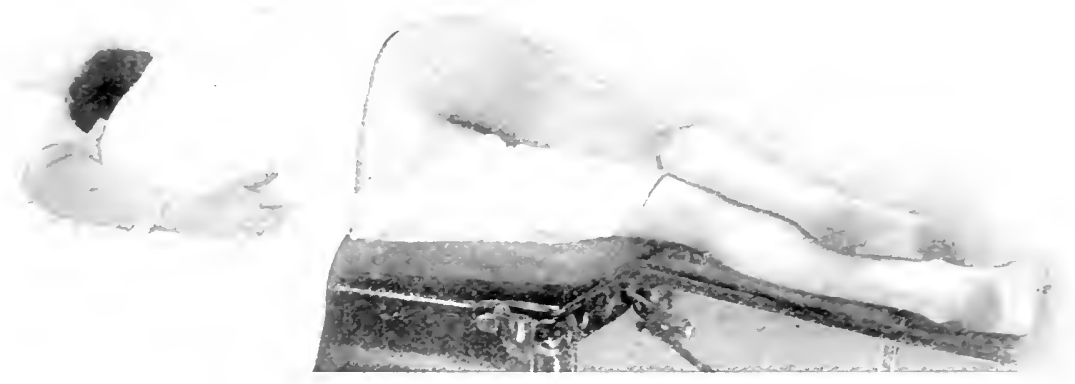

Fig. I.-Correct Sims' posture for rectal examination.

breath, disagreeable taste in the mouth, or suffers from loss of appetite, nausea, or vomiting. Finally, it is well to discover if the patient swallows with difficulty (esophageal stenosis or cardiospasm) or suffers from localized abdominal pain and tenderness on pressure, manifestations which indieate the presence of an ulcer, stricture, tumor, or foreign boty in the stomach or intestine, which might be responsilste for the frequent and loose evacuations. When the anmnesis is concluded the patient should be sistematically examined hy the diagnostic physical measures-inspection, succussion clapotement), auscultation, percussion, palpation, and inflation-and with esophagoscope, gastroscope, proctoscope, sigmoidosope, and rectal speculum, according to indications.

Position of the Patient:--Patients who suffer from obscure diarrhea should be examined whike standins. or on a table while resting upon the sides or back, with the limbs flexed and extended, during deep inspirations and expirations, and the posture should be changed during the examination, that any change in position of the hollow 
viscera, solid organ, or tumors may be noted. The Sims' knee-chest and inverted postures are rectuired for a complete digital, proctoscopic, and signoidoscopic examination of the rectum and sigmoid hexure (Figs. I and 5). By cxamining the patient while standing erect one can observe bulging from tumors, a pendulous belly due to enteroptosis, determine if the ablominal viscera, organs, or neoplasms previously felt lave descended to a lower level, and, by introducing the finger inte the rectum and having the patient strain, growths and other lesions not discoverable in other postures can be reached with the finger.

Inspection.- Ceneral inspection of the patient is a valuable aid in the diannosis of diarthea and allied ailments, because in this way one realily determines if the patient is cyanotic, jaundiced, anemic, pale, cachectic, has an wnhealthy color, a facial expression of anxiety, is debilitated, emaciated, has flabby muscles, hernia, congestion of the superficial veins of the abdomen, and if the abdominal wall is well developed, thin, flabby, or visibly protrudes.

A study of the abdomen in diarrheal subjects enables one to note if it is distended, bulges more prominently in one part than another, is depressed, pendulous, or overhanging. Irregularities which indicate contracting adhesions, an enlarged viscus, tumor, fecal impaction, enteroptosis or obstruction, and gas distention above the block induced by intestinal stenosis, angulation, volvulus or extra-intestinal pressure, conditions which frequently cause irritative or obstructive diarrheas, are noticeable.

A careful examination of the month will quickly show if the tongue is coated, the teeth are bad, or if ulcers are present, which would indicate syphilis or other types of infection, because one or all may at times induce gastro-intestinal disturbances, and inspection of the nose and pharyux will reveal if there is nasal catarrh or disease of the tonsils or throat which might have a bearing upon the case.

The rectum and sigmoid can be viewed through the proctosigmoidoscope, and ulcers, strictures, cancers, polypi, hemorrhoids, and other alfections can be quickly located.

Succussion or clapotement is useless, except in so far as it relates to the stomach and colon. When the abdomen is thin, and these organs are relaxed and contain a fair anount of fluid, a splashing sound may occasionally be induced by repeatedly and rapidly striking the aldelomen with the tips of the fingers and an idea obtained as to their position. From what has been said, it may be inferred that sucension is a cloubtful diagnostic aid, and is useful only in determining limitations of the stomach and large bowel.

Auscultation may be relied upon for detecting phthisis, bronchitis, pleurisy, leart murmurs, meteorism and ablominal gurgling sounde, and elelayed entrance of the fool into the stomach in esophageal stenceis, hut, except when these conditions in some way influence the novements, auscultation plays an unimportant part in the diagnosis of cliarrhea. 
Percussion is often helpful in locating tumors, fecal impaction, and other pathologic conditions which interfere with the fecal current and cause obstructive diarrhea. By percusing the abelomen one can sometimes determine if the intestine, erpectally the colon, and other abdominal and pelvic organs are diseatsed, enlarged, or displaced, and whether the bowel is empty or is partially or completely tilled with gas and feces. Percussion over an enlarged liver, tumor, or fecal mass gives a that note. while normally over the intestine the sound has a higher pitch. In cases of enteroptosis the degree of displacement and rlilatation can easily be ascertained ly carefully percussing along the course of the colon, first in the empty state, and then after it has been distenderl with gas or water. Again, this diagnostic adjurant is useful for locating twists, angulations, strictures, and other obstructive lesions of the intertine in conjunction with inflation. Following artificial distention with air or aas, the part of bowel below the block gives a very high note on percusion and makes the abdomen bulge outward. while the empty segment above gives a low note and the abdominal wall over it looks sunken in. Sometimes one obtains a hich and at others a low note over a tumor, a peculiarity accounted for by the presence of a gas-filled piece of intestine over it at one time. while at another nothing is interposed between the neoplasm and the abdominal wall. Ptosis and dilatation of the colon are easily diagnosed following inflation, because the outline, size, and location of the qut can be determined by percussion, palpation, and by separately inflating the stomach and large bowel, and the relation of the organs can be defined in coloptosis, gastroptosis, and splanchnoptosis.

Palpation is the most valuable of the measures employed in physical diagnosis. Important information can usually le obtained in obscure cases of diarrhea by first superficially and then deeply palpating the abdomen. with the patient first in one position and then in another, and with his legs extended or flexed. Superficial palpation enables one to detect uneren surfaces of the skin, enlarged reins, and character of the abdominal wall, and note if it $i=$ too thin, fat, rigid, or relased, for in the latter case it would not properly support the abdominal viscera. By means of firm, deep, single-handed or bimanual palpation one can locate tender and painful spots and gas collections, determine the thickness, size, location, and sensilsility of the hollow and solid viscera, and, in farorable cases, isolate neoplasms, angulations, invaginations, tumefactions, intestinal foreign bodies or hardened fecal accumulations (Fig. 2), and also (lifferentiate between fecal and benign and malignant tumors. Palpation will reveal that growths are hard, fixed, and non-indentable, and that fecal tumors are of a doughy consistence, movable, indentable, often multiple, and slip over the mucosa, in contradistinction to neeplatims. which are immovible.

In complicated cases the liver, spleen, pancreas, stomach, duoflenal region, colon, sigmoid thexure, and pelvic organs should le systematic- 
ally and separately palpated, superficially and deeply, and hernial openings and lymph-nodes should be carefully examined. Palpation is not reliable for determining the presence of pathologic lesions in the small intestine, except in stricture where gas accumulations and active peristalsis limited to certain segments of the bowel can be made out, but it renders valuable aid in the diagnosis of lesions affecting the appendix, colon, sigmoid flexure, rectum, and anal perineal regions.

When used in conjunction with inflation, palpation is decidedly useful in determining the presence of enterospasm, finding out if abdominal organs are abnormally large, hard or displaced, and for locating all varieties of obstruction within the colon by examining it before and after its distention with air, gas, or water. Lesions and tumors located in the sigmoid flexure and rectum can be palpated,

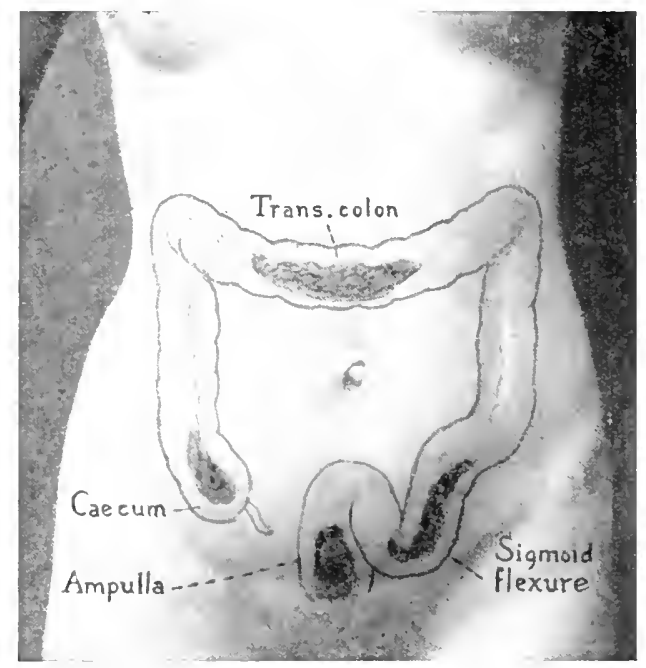

Fig. 2.- Showing most common locations of fecal impaction, named from below upward.

when high, through the sigmoidoscope, and, when low, with the finger. When examining the colon the hands should be placed at a right angle, and it should be rolled backward and forward or the bowel grasped between the fingers and thumb under leep pressure during expiration and inspiration, while the patient is in a recumbent posture with his legs flexed, or the intestine should be manipulated between the hands during bimanual palpation.

Examination of the colonic segments in this way frequently aids one in concluding if the gut is inflamed, ulcerated, displaced, firm and tule-like, spasmodically contracted, contains enteroliths or multiple pasty or nolular fecal impactions, under which circumstances the intestine feels like a string of sausage or is involved by a malignant srowth,

In (liarrheal subjects, sore spots, painful points, and isolated gas 
collections should be carefully noted, because they are indicative of ulcers, intlamed areas, and obstructive lesions which excite loose movements, and palpation of the hepatic, duodenal, and pancreatic regions shoukl not be overlooked, because of the correlation of these organs, which, when diseased, frepuently lead to diarrheat.

Spinal palpation should he practised as a routine measure, because in certain gastro-intestinal affections (ulcers, intlammation, and malignancy) the skin at the sicles of the spine possesses sensitive areas which make the patient wine when pressed upon. In persons who have gastric uleer the integument at the left of the tenth, elesenth, and twelfth dorsal vertebra is sensitive, while in those afflicted with cholelithiasis the same regions on the opposite side of the spine are involved, but in neurasthenia the skin on both sides of the vertelora (especially interseapular and sacral regions) are painful to the touch. In some instances the musculature adjacent to the spine is rigid in spots. and these, together with the painful points in the integument, can be determined by pressing with the finger-tips first on one side and then on the other, in successive stages, until the entire region of the rertebral column has been covered.

Inflation of the Stomach and Colon.-1)istention of the stomach and colon with air, gas, or water is a valuable diagnostic aid, particularly when it is employed in conjunction with inspection, percussion, and palpation, because it permits the examiner to determine with reasonable accuracy their position, size, and outline and differentiate them from other organs, neoplasms, and tumefactions.

Following gas distention one can obtain a clear tympanitic note over the stomach, which enables him to diagnose dilatation and ptosis of the organ, deteet conserfuent augmented peristaltic wares, locate lumors through change and position of the stomach as intlation takes place, in exceptional instances locate stenosis, and, when the gas rapilly ascapes into the bowel, to form an ideat as to whether or not pyloric resistance is below par.

Colonic inflation is extremely valuable for locating tender and painful spots, dearing up the diagnosis in olscure intestinal lesions, and should not le omitted lexause of its simplicity and the fact that it is kevoid of danger when intelligently practised. Following distention the intestine can be traced more accurately throughout its course; ptosis of colonic segments (congenital or accuired), conlargements, angulations, invagination, and points of olstruction (irrespective of cause) unrecognizalsle by other means can often be differentiated. Tumors in the gut wall are more easily mate out. neoplasms behind the peritoneum, tumors, and enlargement of other organs discernible before (particularly in persons having a thin atudemen) disappear as the intestine rises up with inllation, and gromths in the colon can occasionally be observed or felt to change their position along with the gut. Perforation should be suspected when air. gas, or water fail to distend the colom, thomgh it is possible that the distending median escapes past the ileocecal valve into the small 
bowet and not into the free peritoneal carity. Both the stomach and intestine should be emptied prior to inflation.

In supposed cases of large bowel obstruction the colon should be percused and palpated throughout its course, both before and following distention, because the gut will be found more tympanitic above the stenosis prior to inflation, owing to the collected intestinal gases, than below. while subserpent to the entrance of air into the bowet the reverse is the case because of the excessive amount of artificial air or ans in the terminal segment of intestine.

A rubber bag, distended with air or water, is frequenty employed by the author to ter the strength of the external and O'Beirne's sphincters, and measure the capacity of the rectum and locate angulations and strictures in the sigmoid flexure through which the bag has been introduced. In one instance following cecostomy, by inflating the colon above through the artificial opening and from below by way of the anus, a stricture was platinly defined in the central or dependent portion of the transverse colon. This patient suffered from extensive ulcerative entamelic colitis, for the relief of which cecostomy was performed, and a stenosis was suspected when regurgitation of the fluid through the cecum invariably followed its introduction. A eure follwwed resection of several inches of gut and subsequent medicated through-and-through irrigation. A definite idea may be formed in regard to the location of an obstruction consequent upon angulation. invagination. twists. strictures, or ptosis, and the size of the dilated colon can be accurately determined by measuring the amount of gas or water required to fill the bowel when dilated, or the sut below when blocked. Bowel inflation or distention with water or air is frequently impracticable because the pain incluced precludes the introduction of an amount sufficient to accomplish the desired result. Inflation with air or gas causes the patient more suffering than does filling the bowel with water, especially when the latter is emploved warm or hot (so $\mathrm{t}(\mathrm{)}) 10^{\circ} \mathrm{F}$.). lecause the heat soothes the intestinal musculature and nerves and minimizes enterospasm; warm oil is even more sedative than water.

The technic of gastric and colonic inflation and distention with air and water is comparatively simple. but when carelessly done is dangerous. because the healthy viscus may be, and the diseased (ulcerated) one is particularty apt to be, riptured.

tir and gas are preferable for the stomach, and may be introduced by means of an ordinary straight or stomach-tube, to which is attached a workable inflating bulh of fair size, or gases may be formed in the stomach by having the patient drink a half-glass of water containing a dram (4: gm.) of tartaric acid, and follow this shorty with a like amount of water in which has been dissolved a teaspoonful of sodium licartonate, a chemical combination which leads to the rapid formation of carbonic acid gas which inflates the stomach. As soon as posilde gas should be piped off to relieve the patient and lessen the danger of perforation should there be an ulcer or a stricture. In the 
presence of known ulecrs, strictures, cancers, allhesions, and heart alfections, inflation is contrat-indicated.

The simplest, guickest, and lest waty of intlating the colon is w introsluce at rectal or colon tube, and commere it with a compressed-atir apparatus, when the air is permitted to show lill the stut, while the themb is kept upon the valre connected with the rank to prevent the too rapicl escape of air or gats into the bowel, which would catuse consiclerable pain or posible rupture of the intestine. In the alserere of a lank or a bull), inverted siphons of lichy may les subtituled, or

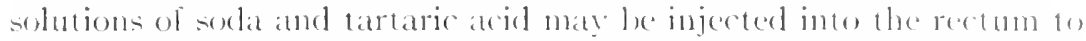
form gats whin the intestine. Sir or gats maly be retained ats bong ats

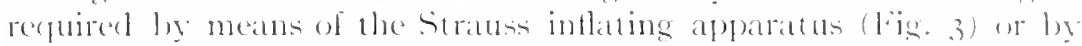
the introduction of a large-sized self-retaining anal dilator simulatuens with remosal of the inflating tube. Pain incident to colonic distention varies according to the amount of air, gas, or water employed,

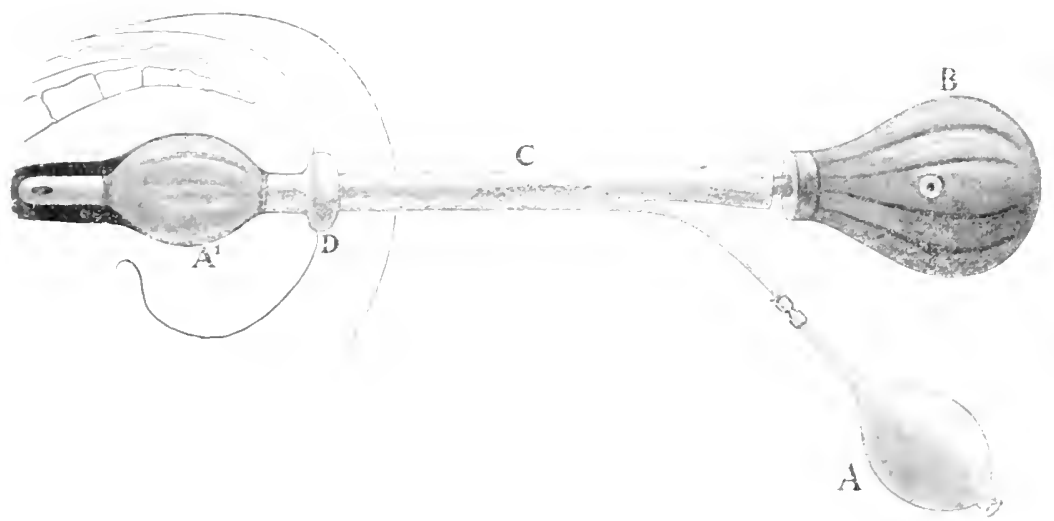

Fig. 3.--Inflating and irrigating rectal tube.

and the rapidity with which it is introduced, and instant relief usually follows passage of the proctuseope or colon tulbe and escape of the air, lout when the ristending agent is not permited we escape, the patient suffers several hours from colic or collapse. Nater is preferable to air or gas in cases of coloptosis and angulation, lecatuse, ou ing to its weight, sagging and kinks atre emphasizerl. Bỵ siphoning off the water following distention one can diagnose a colitis, ulecer or cancer by noting the contained hood, mucus, pus, or frasments of tissue as the fluid runs out, or, when this fails, by filtration or microscopic examination of the Huid. Inflation for therapentic and cliasnostic purposes can, if necessary, le limited to the rectum ly liseking the bowel at the anus and sigmoid with a water-or pnemmatic lowg; or the colon can be filled and the distending agent retaned within it as long as desired by means of the inflating apparatus shown in lig. 3.

Chemic, Macroscopic, and Microscopic Examination of the Stomach Contents. - When the size, shape, and position of the stom- 
ach have been determined through the aid of inspection, percussion, oscultation, clapotement, and palpation, with or without distention of the organ with gas or fluid, the contents of the stomach should be withdrawn at stated periods following normal and test-meals, and submitted to chemic, macroscopic, and microscopic examination, to determine whether the secretory, motor, and absorptive functions of the organ are deranged, and, if so, the cause, extent, and character of the disturbance. This precaution is most essential in obscure diarrhea of apparent alimentary origin, because, as will be seen in the special chapter devoted to the subject, there are many varieties of gastrogenic diarrheas, some of which cannot be diagnosed except by exclusion accomplished through the aid of a careful gastric analysis.

The etiologic factors. in diarrhea gastrica are at times difficult to ascertain, because in some instances the trouble is due solely to functional errors in the stomach; in others, to associated general disturbances, duodenal or pancreatic disease, or a catarthal inflammation of the small or large bowel, which tend to increase the number of evacuations. Gastrogenic diarrhea is, in most instances, traceable to subacidity or achylia gastrica, hyperacidity, atony, motor insufficency of the stomach, pyloric stenosis or malignancy, alone or combined, conditions which are fully discussed elsewhere. In these varieties of loose movements analytic study of the gastric. contents should be reinforced by a careful examination of the feces, for A. Smith has demonstrated by his test-meal that connective-tissue remnants are present in the stools of lienteric diarrhea (diarrhea gastrica); while Einhorn, Oppler, Schutz, and others have done much to establish the individuality of the above-named types of gastrogenic disturbances.

Test-meals.-Some patients who suffer from stomach trouble vomit frequently, and a portion of the contents can in this way be obtained for examination, but this is unsatisfactory, and it should be siphoned or suctioned out through a stomach-tube at a specified time following the administration of a test-meal, according to the plan of Riegel, Germain, Sévec, or breakfast of Ewald and Boas (the most universally used). Better results are obtained when the juice and admixed solids are withdrawn during the height of digestion, or one hour after the patient has eaten one or two rolls and drunk a cup of tea or water (Ewald-Boas meal), or from two to four hours following a mixed meal, including broth, meat, potatoes, and a roll (Riegel).

The test-meal should be taken upon an empty stomach, and no attempt should be made to remove it until digestion is well under way or completed, otherwise the tube will become clogged or the obtained juices will not be in the right ratio, which varies during the different stages of digestion.

Methods of Removing Stomach Contents.-Einhorn ${ }^{1}$ has designed a diminulive silver capsule-shaped stomach bucket, which he has the

${ }^{1}$ Diseases of the Stomach, $4_{\text {th }}$ ed., I 906, P. 8 I. 
patient swallow, and afterward withelraw by means of an attached silk cord after the bucket has remained in the stomach for a few moments and become partially or completely filled with gastric ingredients; but this apparatus is objectionalle becatuse it is eliffieult for solid matter to enter the bucket, and a sufficient amount of the contents is not always withdrawn to atmit a perfect eximination, and, further, because patients object to swallowing so large a metal boty, owing to the accompanying nausea and fear of its beeoming detached, and because its withdrawal induces considerable eliscomfort. Of the paraphernalia designed for extracting the gastric contents for analysis the stomach-tube is the most practical becaluse it is inexpensive, is easy to introduce, camnot become detached, does not injure the mucosa, is not complicated, and, when resorted to under proper conditions, accomplishes its purpose.

Tubes, to be of the greatest use, should be made of soft rubber, and sufficiently thick to give them a degree of stiffness, have a fair-sized opening in their conic distal end, and velvet eyes or slits scattered along the sides for an inch or more above the lower extremity, so that both fluids and soliels can readily enter it, and should be about 30 inches $(75 \mathrm{~cm}$.) in length. Small tubes should be employed for children. and the size of the larger variety should be varied to meet the conditions in alults of unusual stature, and those who suffer from spasmodic or organic stenosis of the esophatgus. An aspirating bulb, located alout the center of the pipe, aids in obtaining a free How of the gastric contents through its suction action when indicated, as, for instance, when the tube is libeked by mucus.

Stomach-tuhes are more useful for diagnostic than therapeutic purposes, and are rarely used in the treatment of gastric affections other than $t$, get rid of decomposing material in cancer cases and pyloric stenosis, and to wash out poisons which have been swallowed accidentaly or taken with suicidal intent.

Soft pipes are difficult to comptetely sterilize without injury, and it is adrisable for each patient to possess his own marked tube. Those used for clinic purposes should be carefully rinsed and kept in a bichlorid solution, but, even with this precaution, it is unwise to use on a heathy person a pipe which has been swallowed by an individual afflicted with syphilis, tuberculosis, or infectious diseases of the mouth or teeth.

The patient invariably objects to having the tube introduced the first tine, hut the fear can be dispellex in most instances by explaining the process, and informing him that the conserpuent discomfort is of short duration, and that removal of the tube will not cause pain or vomiting. The subject can stand or remain in the recumbent posture during introduction of the tube and extraction of the contents, but, as at rule, this can be accomplisheel with greater ease for the physician and less discomfort to the patient in the upright sitting position. with the head and shoulders slightly inclined forward, with the attendant standing to the right behind the chair, so that he may hold the 
tube in the patient's mouth with the left, while he aspirates or manipulates the outer end with the right hand (Fig. 4). In this way the contents can be certainly and slowly expressed into the receiving ressel.

In normal subjects depression of the tongue is unnecessary, but the patient should be told to close his mouth and swallow when the tube reaches the epiglottis, independently or guided with the finger. and, as he does so, it should be quickly and gently pushed downward through the esophagus into the stomach as the patient, under instruction, breathes deeply.

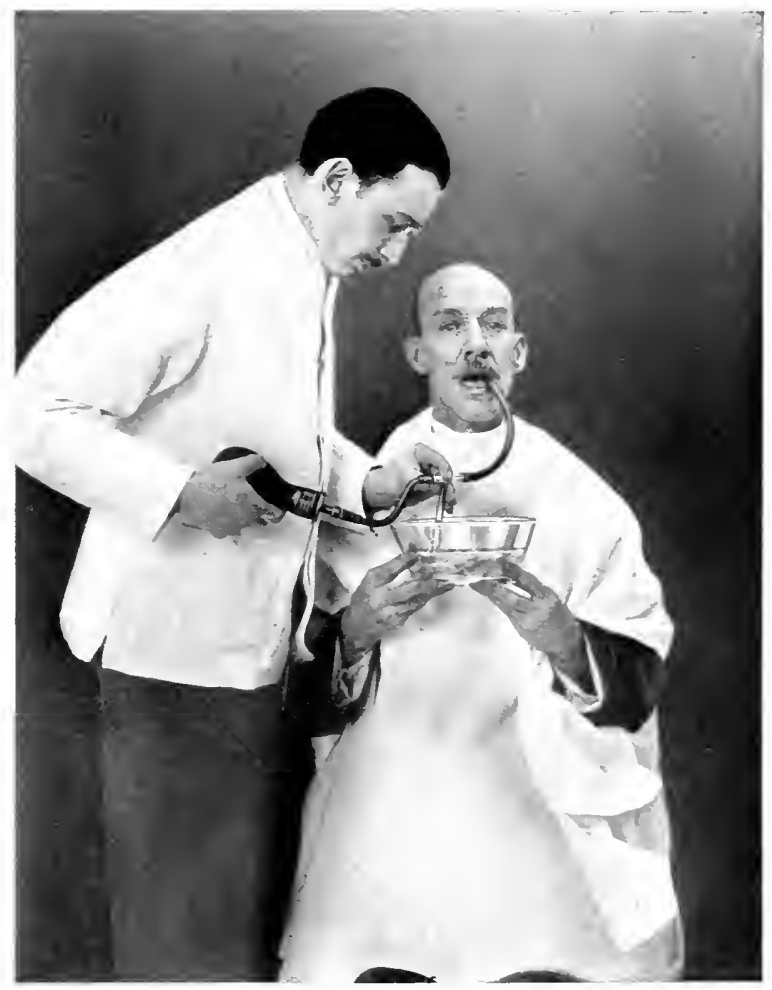

Fig. 7.-Removing the stomach contents for examination following a test-meal.

Before introduction of the tube false teeth should be removed. and it should be ascertained if there are contra-indications to its passage, such as angina pectoris, thoracic aneurysm, serious heart lesions. phthisis, bronchitis, gastric ulcer or carcinoma, old age, epilepsy, and other conditions wherein straining white romiting might prove dangerous.

Formerly the author required his office cusistants to examine chemically, macroscopically, and microscopically the gastric contents of patients suffering from diarrhea wherein it was thought that the 
source of the trouble was solely or partially located in the stomach, but in recent years he has referred the collected contents to the laboratory for examination, which, after all, is the best place, because the work is too laborious and eomplicated to be properly done in a private office.

It has been pointed out that subacidity, hyperacidity, atony, motor insufficiency of the stomach, pyloric stemosis or malignancy, singly or together, may incite sastrogenic diarrhea.

To describe the technic of the numerous tests and methods lecpuired for the identification of these abnormal conditions and the part played 1) them in the disturbance would recpuire more time, labor, and space than is warranted in a work of this character, and, becatse of this, the author refers the reater desiring such information to the stanctard works of Cohnheim, Finhorn, Boas, Reade, Nothnagel, Bassler. Ewald, and others who have dealt with the subject in a comprehensive and thoroughly practical manner.

Gastroscopy and Gastrodiaphany. - When the history, external examination, and analysis of the stomach contents fail to establish a diagnosis in diarrhea gastrica the stomach should be studied hy transillumination (gastrodiaphany) of its anterior wall, and its interior inspected and palpated through the aid of the gastroseope and artificial light, for, with the assistance of these diagnostic adjurants, the eolon and thickness of the mucosa can be determined, ulecrs located, and tumors outlined in a fair percentage of cases. The organ is rather eliffeult to examine with gastric illuminating instruments because of their size, great length, and skill reguired for their introduction and subsequent manipulation; consequently, at present gastroscopy is not as reliable a means of diagnosis as are cystoscopy or proctoseopy. Direct inspection of the stomach is more reliable for determining the character of lesions in the mucosa than stiagraphs, but the butter (discussed alsewhere in the chapter) are the most trustworthy for outlining the organ, locating strictures, tumors, displacements, and deformities, both following the administration of a carbonate of lismuth meal or filling the organ with a solution of the chemical mucilage of acacia and water, aud, in addition, one can with the $x$-rat and bimuth meal or injection observe the frequency and character of the gastric peristaltic waves, and estimate, with a fair degree of accuracy, the time reguired for the stomach to evacuate its contents, when healthy or diseased, by observation through the fluoroscope and radiographs mate at stated periods following antrance of the meal or injected solution.

The author's colleague, Dr. Max Finhorn, ${ }^{1}$ was a pionere in emphatsizing the diagnostic importance of gastrodiaphany and gastrosopy, and early designed instruments for hoth purposes. Recently, however, Dr. Chevallier Jackson' has improved upon his apparatus, and designed an instrument servicealsle both for esephagoseopy and

1 "Die Gastrodiaphanie." New Yorker med. Nonatsschrift. Now.. I s 8 .

"Medical Record, April 6, ino7: Jour. Imer. Med. Asoc. () tober 20, 1007. 
gastroscopy, that can also be used for drainage and suction of the secretions, and claims that with his appliance one can explore from one-half to three-quarters of the total gastric mucosa when the patient is profoundly anesthetized, and suggests that cocain is preferahle when the esophagus is to be examined. Better results are oldained when gastroscopy has been preceded by gastric lavage and morphin or atropin have been administered to minimize the trouble from the secretions, which freguently obscure or put out the light when the examination is made in a darkened room.

Examination of Feces.- The value of fecal examination has been greatly underestimated, owing to the fact that it is seldom practised by many physicians: (a) because of their repugnance to such work; $(b)$ the difficulty in keeping the stool warm for the necessary length of time; and $(c)$ their ignorance as to the technic of making the examination under varying conditions.

An analysis of the excreta should be made as a routine measure in the presence of diarrhea, because it would in nearly every instance give some idea as to the nature of the disturbance causing the increased fluid evacuations. A study of the gastric contents enables one to judge if the proportions of the stomach juices are relatively normal and ascertain if digestion within the organ is being properly carried out, while investigation of the feces will indicate if normal secretion, digestion, and absorption are taking place within the small intestine and colon, and whether or not there are disturbing factors in the bowel which would interfere with these functions or in other ways favor acute or chronic diarrhea.

Isually, normal and pathologic feces have a disgusting odlor, and because of this it is advisable to get them out of the office or hospital and to the laboratory as soon as possible. To this end the author, as soon as he has made a macroscopic study of the stool, places all or a portion of it in a Thermos bottle or other vehicle wherein it can be transported in a warm state to the laboratory, except in cases where an immediate diagnosis is imperative, or he wishes to demonstrate to the physician bringing the case entamebre, Balantidium coli, Shiga's or Flexner-Harris bacilli, parasites, their ova, or other micro-organisms supposedly responsible for the diarrhea.

Naturally in diarrhea the evacuations are changed from the normal in form, color, consistence, and frequency, but the extent to which such changes occur varies according to the cause of the disturbance, - (2) verity of the diarrhea, diet, amount of water consmed, and the character of the medicines atministered for its control, and they may be soft, mushy or fluid, and normal in amount or profuse.

It is advisable to consider the age of the patient in individuals supposedly affected with cliarrhea, and to bear in mind that in health an adult should have one, a child two, and an infant four or more stools datily, and that some individuals normally roid more than the ustkal number of evacuations in twenty-four hours and remain well.

ferfectly normal stomls have a slightly disagreeable odor, but when 
they contain indol, skatol, sulphureted hydrogen, menthane, and phosphin in abundance, as may occur in the presence of liver affections, wastro-enteritis, and somedimes constipation, they are particularly offensive. In dysenteric, tubercular, gonorrheal, spphilitic. and other forms of colitis complicated hy extensive ulceration, the dejecta possesses a disgusting odor, incident to the presence of an abundance of pus, bloot, and mucus which have been unduly retained.

Occasionally the movements of patients afflicted with cancer of the rectum or sigmoid are characteristically offensive, hut, as a rule. the elisagreeable odor does not appear until after the growth has degenerated, uleers have formed, and a discharge responsible for the oflor is produced. In choleriform gastro-intestinal disturbances, wherein the chyme and feces are rapidly rushed through the alingentary tract, the stools, which resemble rice-idater. have little, if any, orlor.

As a general rule, when the movements possess an ammoniacal odor it results from a decomposition of urine which has been voided along with the feces.

Fecal analysis, to be effective in obscure cases of diarrhea, shoukd embrace a careful macroscopic, chemic, and microscopic examination of the excreta made on different days following ordinary and test. meals.

Macroscopic Examination.-By means of macroscopic examination of the stools one can determine if they are slight or profuse, their color. form, and if they contain sections of or entire worms (tapeworms. Ascaris lumbricoides), tissue débris (polypi, degenerated cancer, etc.). intestinal sand, gall-stones, pancreatic calculi, enteroliths, foreign loodies. scybala, bismuth accumulations, mucus (gelatinous, casts or threads), pus, blood, or food remnants. Considerable information can be gained by studying the color of the dejecta, which is subject to marked changes in health and disease. Nilk (diet) colors the stools a light yellow; red wine, huchleberries, and salts of iron and magensia. brownish black; rhuharb, santonin, and senna, yellow' calomel. green; salol and beta-naphthol, aiolet; bismuth, black; methylene-hlue, bluish green; mucus in large quantities, yellowish gray; and cholera, strua: colored.

In aggravated types of diarrhea the stools have no more form than water: when less severe they flatten out in the chamber like a pancake; but when the patient does not have more than two or three movements daily the storls are semisolid. Some iclea may be obtained as to the cause of the trouble by studying their form. for when the sphincter is irritable and spasmodically contracts, or there is a stricture, polyp, or cancer located in the rectim, the feces may le evacuated grooved, like strings, indented or flat, and tape- or round and pencil-like. Obstructions focated in the small intestine and colon do not materially affect the form of the excreta because fluid feces escape by them to become solidified and le discharged in normal 
shape. Occasionally in subjects who suffer from diarrhea the feces are unduly retained, and large firm or small round hardened fecal maser scrhala collect and enemata are required to dislodge them.

Blood in the stouls is indicative of certain gastro-intestinal affections. When di-charged bright red it is usually incident to tumors. uleers. fissures or hemorrhoids located in the rectum or anal canal, and the bleteding may be copious or slight. depending upon the size of the verel involved. When the stools are dark brown or tar colored and re-tmble coffee-grounds. bleeding is from the stomach, small intestine, or upper colon, and the hlood has been retained sufficiently long for it to hecome clotted and undergo marked changes.

Mucus in one form or another is to be seen in the movements of nearly all individuals who suffer from diarrhea. and may vary greatly in character. for it may appear as tlakes or patches independently or upon impacted fecal masses. as casts in membranous enterocolitis. and may be gelatinous in jejunal diarrhea and tenacious in proctitis. ete. Formerly, sago-like bodies of regetable origin resembling frogspawn were frequently mistaken for coagulated mucus.

It is well to bear in mind that mueus is a normal product of the buwel, and that the amount secreted may be materially increased by catharsis, psychic emotions, and nervous derangements. independent of definte intestinal lesions. When mucus persistently shows in the stools it indicates a catarrhal state of the small intestine. colon, or both: hut when it is admixed with blood and pus it points to serious organic changes and indicates that the mucosa is eroded or affected by ulcerative stenotic or cancerous processes. When the mucus is very abundant and tenacious upon the feces, or when roided independently, the chief disturbance will he found in the lower sigmoid Hexure or rectum.

Membranous casts are usually formed in the colon, and when they closely resemble the bowel the probabilities are that they have formed in the descending colon, sigmoid flexure. or upper rectum. lt is extremely difficult to determine from what part of the intestine mucus comer. but when it is secreted in the small gut it is usually: not visible to the naked eye. but can be seen through the microscope. and gives to the stool a glistening appearance.

When there is a hypertrophic inflammation of the sigmoid flexure. rectum, or anus the inner lining of the bowel looks wet and is bathed with mucus. which slips out through the speculum when the patient is requested to strain. In membranous colitis, rope-like strings can - wmetime be seen projecting from the sigmoid into the rectum. and in catarthal intlammation of the colon. accompanied by the formation of tenacion mucus in large amounts. it can be seen through the prorto-cope winging downward from one rectal valve to another, and uncler reflected light looks as if the rectum was divided by a glass partition.

In acute proctitis the patient complains of tenesmus and frequently roid- mucu-alone or admixed with softened feces, but when 
the latter are firm the mucus covers the end or surrounds the entire fecal bolus when expelled.

Pus.-Thick, vellow pus is visible macroscopically only in the presence of spinal, abdominal, pelvic, and rectal al secesses or fistulate and when suppurating diverticula of the colon, sigmeid. or reetum discharge into the bowel. Brick-dust colored or pus andmixed with hlood and mucus is very much more common, ancl may lesencountered in the rarjous forms of ulcerative colitis, strictures, and cancers, and when abundant indicates that the lesions are numerous, or if single. that the mucosa is extensively involved; but when the amomnt is slight and streaks the feces or precerles their expulsion, it points to an ulcer, fissure, or blind internal fistula in the lower rectum or anal canal.

Indigestible or partially digested food remunts, such as peats. strings of beans, mushrooms, potatoes, and other vergetables, can be frequently seen with the naked eye, as can particles of meat, connective tissue, and fats, but less often and not so plainly, except when they are present in considerable amounts, which is indicative of impaired gastrogenic or in enterogenic digestion.

Finally, fatty stools the result of obstructive jaundice, overfat eating, and discase of the pancreas or its duct are macroscopically recognizable because of their oily or greasy appearance.

Chemic Examination of the Feces.-Ordinarily the feces are neutral or slightly alkaline in reaction, and sometimes there is a difference between the surface of and body of the feces. and both should be tested. Acidity of the excreta is augmented by a regetable diet and blocking of the bile-duct, and normally a prepared stool changes blue litmus-paper to red and the red to blue.

By Schmidt's bichlorid of mercury test healthy feces are colored red (showing hydrobilirubin), but when particles are given a greenish tint it indicates an abnormal state from unchanged bile. The reaction of the feces has slight if any diagnostic value in cletermining the etiology of diarrhea.

When, in spite of other diagnostic measures, the cause of the gatstric or intestinal disturbance still remains undiscovered, it is atvisable to test the feces for mucin, albumin, propeptone and peptone. starch, sugar, fat, blood. bile-pisment, biliary acids, urobilin, steatorrhea, trÿpsin. diastase, phenol, indol, skatol, biliary, intestinal, aud pancreatic concretions, according to the plan in standard work- devoted to the intertines and fecal examination.

Wicroscopic Examination of the Feces. In obscure cases macroscopic and chemic analysis inspection of the feces should be reinforced by a careful microscopic examination, for in this way important liasnostic signs can be deteeted which would otherwise go nurecognized. To be effective. fecal specimens should be examined dry, mostened with water, or diluted with lugol's solution or acetie acid, and compressed between cover-glasises until transparent, and them inspected through a low-power lens for the coarser clements, food remuants. tissue débris, parasites. and entanchat. etc., after which a higl-power 
lens should be substituted that bacterial flora, sarcince, clostridia, starch cells, etc., may be detected

The findings within the field vary considerably, dependent upon the diet and character of the gastro-intestinal disturbance, though some of the objects appear alike in healthy and pathologic dejecta, and it must be determined whether they are present in relatively too small or great amounts or numbers.

Microscopic inspection of the feces enables one to discover whether or not the paticnt is properly digesting his meat, fats, and connective tissue, and in a series of examinations the microscopic fields may show muscle-fibers, fat globules, connective-tissue fibers, vegetable elements, pus, blood, mucus, epithelia, tissue segments of worms, débris, eggs of intestinal parasites (tapeworms, ascarides, trichocephalus, etc.), colorless soaps, triple phosphates, oxalates, carbonates, calcium and Charcot-Leyden crystals, entamebe, Shiga's, FlexnerHarris or tubercle bacilli, Balantidium coli, infusoria, red and white blood-corpuscles, starch cells, clostridia (stain), plant cells, and numerous accidental and obligate micro-organisms, such as the coli, lactis aërogenes, bifidus, aërogenes capsulatus (gas forming), putrificus, and Boas-Oppler bacilli, etc.

Pathologic Significance of Microscopic Findings.-Yellow stain muscle-fibers in moderation are unimportant, but when numerous and massed, and their striations and nuclei are undisturbed, they indicate imperfect gastric or pancreatic digestion, and when they are greenish in color, catarrh of the ileum, fatty acid crystals, droplets, and diminutive pieces of $f a t$ in abundance point to blocking of the ductus cholidochus in liver and pancreatic diseases. Most crystals are of no diagnostic importance, but the rhomboid variety (hematoidin) occur following a hemorrhage, and Charcot-Leyden are found in the presence of helminthiasis, typhoid fever, dysentery, and phthisis. Epithelia in large numbers, together with augmentation of the white bloodcorpuscles, denote a chronic inflammatory state of the mucosa, and when the former are bile stained the small bowel is involved. Colorless signifies catarrhal colitis, and bile-stained mucous catarrh of the upper small intestine; fragments of tissue come from polypi, degenerating tumors, and ulcerative processes; numerous cholestridia and free starch cells are signs of fermentation and catarrhal and other derangements of the small gut, and sarcine complicate gastric dilatation. The coli, ärogenes capsulatus, and lactis ac̈rogenes bacilli in excessive numbers point to undue fermentative and putrefactive changes in the gastro-intestinal tract; the Boas-Oppler bacillus is commonly found in pyloric cancer; entamebre, the Shiga-FlexnerIIarris bacilli and Balantidium coli bacillus indicate a dysenteric colitis; typhoid and tubercle bacilli, with the usual manifestations, signify that the patient is suffering from typhoirl or tubercular inflammation of the intestine; ova prove the presence within the bowel of parasites. and the finding of Spirochetce pallida demonstrates that the patient hats syphilis. 
Urinary Examination in Diarrhea.-Frepuently a careful chemis, macroscopic, and microscopic examination of the urine is of sreat assistance in helping one to arrive at a correct diagnosis in the presence of olscure gastro-intestinal affections complicated lig acute or chronic diarrhea, and in detecting other causes which might augment the frequency and fluidity of the eracuations. for example, the amount of urine is increased in diabetic conditions, sclerosis, amyloielosis, and disturliances of the nervous system, and decreated in dedilitated individuals and those having a low blood-pressure, persons who partake sparingly of water, and in the presence of persistant hemorrhage, vomiting, or cliarrhea.

Indican, normally present in the urine, is greatly atugmenterd when intestinal putrefaction is active, when there is chronic obstruction of the small or large intestine, or the patient suffers from achylias getrical or hyperchlorhydria. Indicanuria usually complicates all disturbances that minimize peristaltic moxements, while the proportion of indican in the urine is lessened when the bowel is active and there is no fecal stasis.

Ethereal sulphuric acids are augmented by albuminoid putrefaction of the feces when retained from whatever cause, and infectious diseases, like diphtheria, scarlet fever, and erysipelas, and are diminished by typhoid, recurrent, and intermittent fevers.

In disturbances of the gastro-intestinal tract it is important that the amount of acetone in the urine be accurately determined because it is increased (also diacetic acid) in digestive disorders, catarrh of the stomach, gastro-enteritis, intestinal obstruction, parasitic diseases, fecal impaction, peritonitis, perityphlitis, cancer of the bowel, and diabetic conditions. Some investigators claim that albumoses in the urine indicate intestinal decomposition. typhoid or tubercular colitis, and gastric ulcers and cancer, while others hold they are unimportant as a diagnostic sign.

Markedly acid urine points to indigestion, intoxication, and nervous states; bile-pigments and acids may be detected in the urine of patients afflicted with certain hepatic diseases (congestion, cirrhosis. cancer, etc.), obstruction of the bile-ducts. or gastroduodenitis: blood in the urine is not an important sign of gastro-intestinal affections, though it has been noted in gastric cancer. Diminished nitrogen in the urine occasionally characterizes hepatic and nepluritic disturbances and gastric cancer; the output of urea is lessened when the liver improperly functionates or the flow of life is interfered with. while under these circumstances the amomt of uric acid is augmenterl: glucose denotes secondary digestive disturlanees from impaired metabolism rather than primary diseases of the gastro-intestinal tract; urinary sediments are unimportant as diagnosic signs in diarrhea conseguent upon disturbances within the alinentary tract.

Rectocolonic Injections (Lavage) in the Diagnosis of Intestinal Affections. - Water when injected into the bowel is uccasionally serviceable in locating the rarious types of intestinal obstruction. When 
only a few ounces can be injected it indicates that the block is in the rectum; when not more than a pint can be introckuced it points to obstruction in the sigmoid, but when two or more quarts flow readily into the colon the occlusion is located in the center of the transverse colon or higher up in the large bowel. Distention of the great intestine in this way, in connection with percussion and palpation, enables one to outline the gut and sometimes to quickly determine if it is ptotic, angulated, or twisted, and to isolate tumors when during the examination the patient is changed from one posture to another. When other intestinal lesions are present, colonic lavage is useful, because washed-out blood, mucus, pus, foreign bodies, food remnants, and tissue débris can be discovered by macroscopic, microscopic, or chemic examination.

Rectocolonic Transillumination.-The author has examined the abdomen along the course of the colon and sigmoid flexure following illumination of the gut in the empty state and when filled with water, but the results were so unsatisfactory that he has completely discarded transillumination, for at best it is useful only for isolating tumors located in the anterior intestinal walls, which can be more certainly identified by percussion and palpation.

Blood Examination.- An examination of the blood is of value in certain types of diarrhea, but a diagnosis upon the findings should not be conclusive unless they fit in with the history, clinical observations, and results obtained by other diagnostic measures. From the writer's experience he inclines to the belief that too much diagnostic importance is often placed upon the blood count and too little upon more simple and reliable methods of examination.

The make-up of the blood and its resistance to toxins vary widely in health and disease in different individuals, and this makes it extremely difficult to estimate the value of a blood examination in some of the more common and serious diseases affecting the stomach and intestine.

In cancer the blood often undergoes serious changes, which in some cases begin early and attack the red blood-corpuscles, which degenerate; while in other virulent types of malignancy composition of the blood is but slightly impaired.

In tuberculosis, blood disturbances are not always characteristic, except in miliary tuberculosis, where the leukocyte count is very low and the lymphocyte count is relatively augmented. Generally there is leukocytosis in tubercular subjects, a condition which becomes aggravated when the peritoneum is affected.

In sepsis, leukocytosis is rather characteristic, and the rise varies from 30,000 to 50,000 cells per cubic millimeter. No leukocytosis indicates that the infection is general, most virulent, and that it has seriously impaired the function of the bone-marrow, in which case there is an increased number of polynuclear cells, and in some instances an extreme degree of hemoglobinemia.

The blood in syphilis, when characteristically changed, shows a 
marked decrease in the number of red cells and a relatively low hemoglobin index almost as soon as the chancre appears, and a moderate leukocytosis during the eruptive stage.

In typhoid feerer considerable information of diagnostic inporlance is to be obtained through the blood examination by determining the relation of the leukocyte count to other cells, lecause usually hy the end of the second or third week the lymphoeyten increase ats the leukocytes. neutrophils, and eosinophils decrease, and since the latter multiply a little later as the fever subsides. As an example of the profound influence of typhoid fever upon the hlosed cells. Wiot gives his findings in a case as follows: The leukocyte count was 5000; polynuclear neutrophils were present in a proportion of 33 per cent.; large lymphocytes, 47 per cent.; small lymphocytes. 20 per cent.; no basophil or eosinophil cells were seen in the 500 leukocytecounted. The Widal reaction was positive at I to 200 within an hour.

In appendicitis, as in other active abdominal inflammatory conditions, there is both a leukocy tosis and a disturbed relation between the different forms of leukocytes. Here the polymorphonuclear cells are augmented, and in aggravated cases highly so, a change in the blook which indicates a gangrenous process in the appendix. Bacteria are frequently found in the blood, but under such circumstances they may or may not be a factor in the disease from which the patient sulfers.

Since the blood examinations and experiments made hy investigators indicate that micro-organisms can enter the cireulation when the continuity of the mucosa is broken, and that when it is not they can during digestion pass from the bile into the chyle, thence to the circulation, one authority maintains that the blood should be sterile or contain but a small number of bacteria during fasting, since the lungs and other organs act as a filter for the micro-organisms. It has also been claimed that in man bacteria pass from the intestine into the blood during a protracted death struggle, especially the colon bacillus, which is assumed to enter the circulation in large numbers before death, and to multiply preferably in the spleen, the bone-marrow, the liver, and the throid glant.

Proctoscopic and sigmoidoscopic examinations enable one to make a positive diagnosis of pathologic conditions situated in the upper rectum and sigmoid flexure which was inmosilde before the adrent of the proctoscope and sigmoidoscope. Force should never be used in the introduction of these instruments, otherwise there is danger of rupturing the bowel. The atuthor hat had one such areident, and similar cases have been reported. The rupture in most instances oecurs in the sigmosid flexure letween its a wo fixed peints. It recuires considerable practice to properly and painlens introduce the proctoscope or sigmoidoscope, and the correct knce-chest posture (Fig. 5) is essential to secure the desires amount of inflation. except when the instrument used has a glass-cap covering and a bull attach- 
ment for inflating, when the patient may be placed in the Sims position.

Isually the patient is placed in the genupectoral position (Fig. 5), the proctoscope or sigmoidoscope is oiled and introduced into

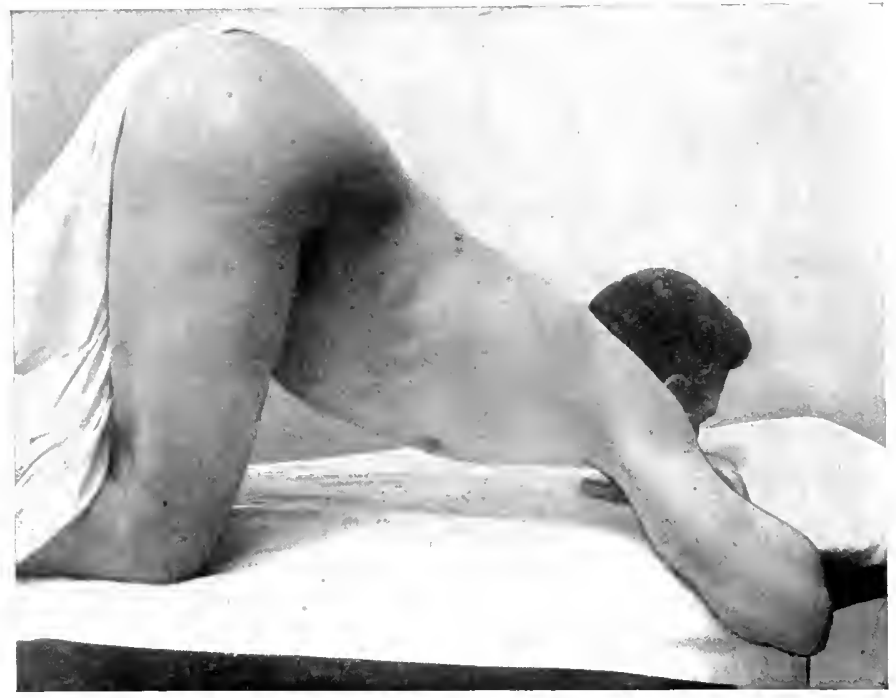

Fig. 5.-Correct genupectoral posture for proctoscopy.

the rectum, and directed downward and forward until it passes through the anal canal (Fig. 6). It is then pointed upward and

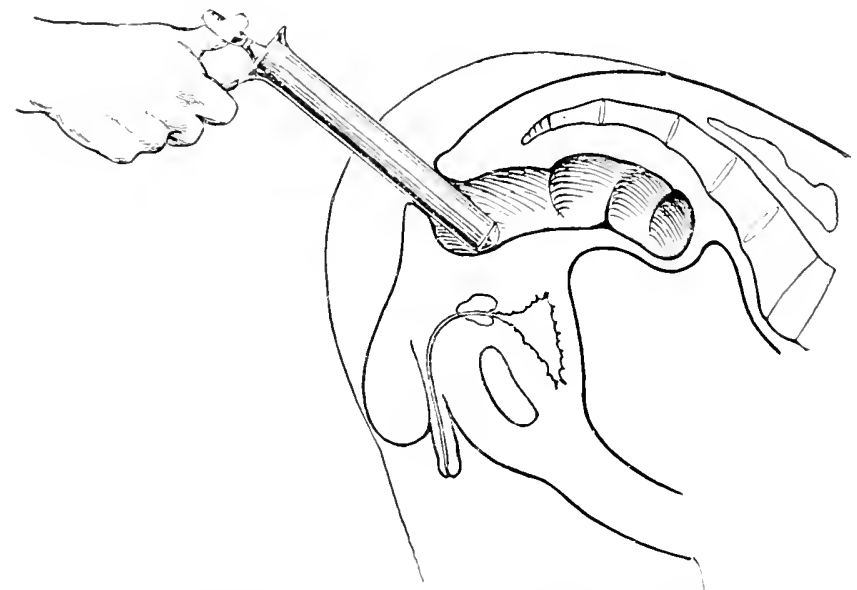

Fig. 6.- Method of introducing the proctoscope: First step

hackward until the promontory of the sacrum is reached (Fig. 7), when it is again directed downward and forward over the upper rectal 
value and into the sigmoid flextre (Fig. \&). The oluturator is then remored and the air permitted to rush in and dilate the bowed. When

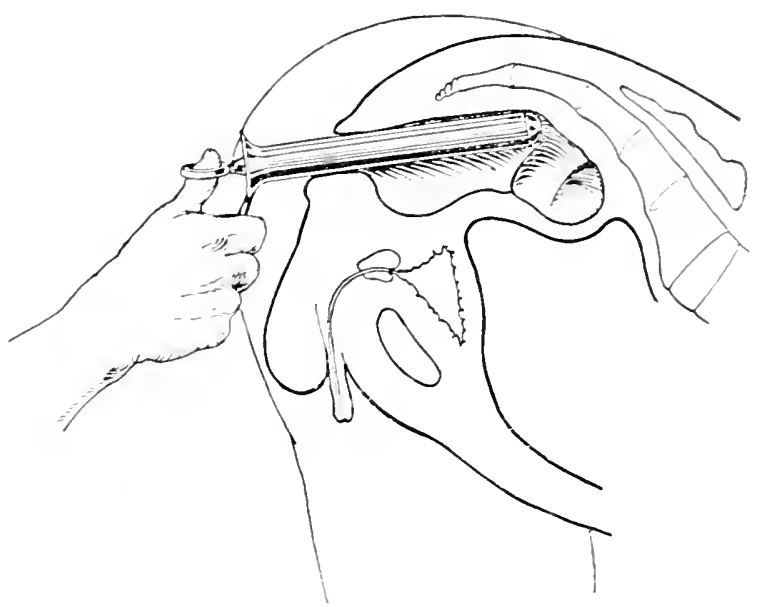

Fig. 7.-Method of introducing the prowasene: second step.

this has been accomplisherl. the sigmoid is examined. and at the instrment is slowly withdrawn a perfect view of every part of the

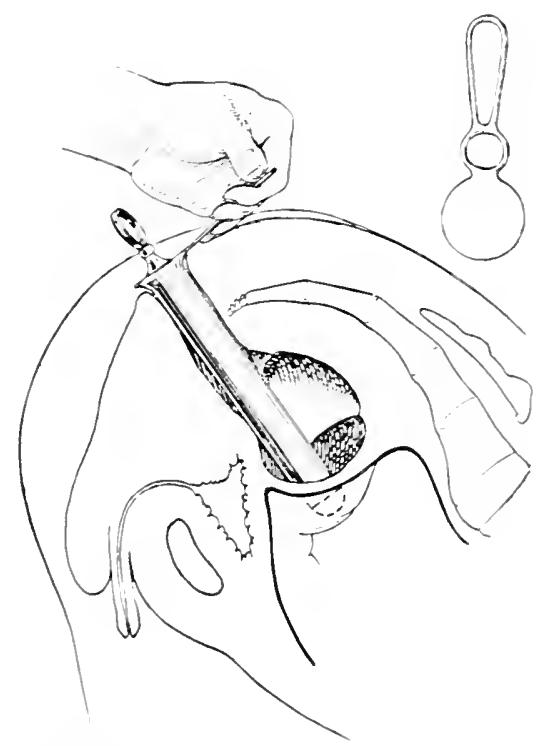

Fir. 8.- Methor of introducing the proctosope: Third step.

bowel can be obtained. In this way one can with accuracy disower any and all pathologic conditions within the lower lowel that interfere with the eracuations. 
When the air does not dilate the sigmoid flexure, a pneumatic sigmoidoscope should be introluced, with the obturator in place, until the middle valve has been passed. The obturator is then removed, and the rectum and bowel higher up are inflated and studied step by step, by pressing the bulb from time to time, as the instrument is passed higher up. In this way a splendid view is obtained, and any obstruction within the rectum or sigmoid may be easily and accurately located and examined. For this purpose Sims' position is the most comfortable one for the patient. With ordinary proctoscopes reflected light is employed, but with the pneumatic a small lamp is placed inside or outside, at the end of the tube, to pro-

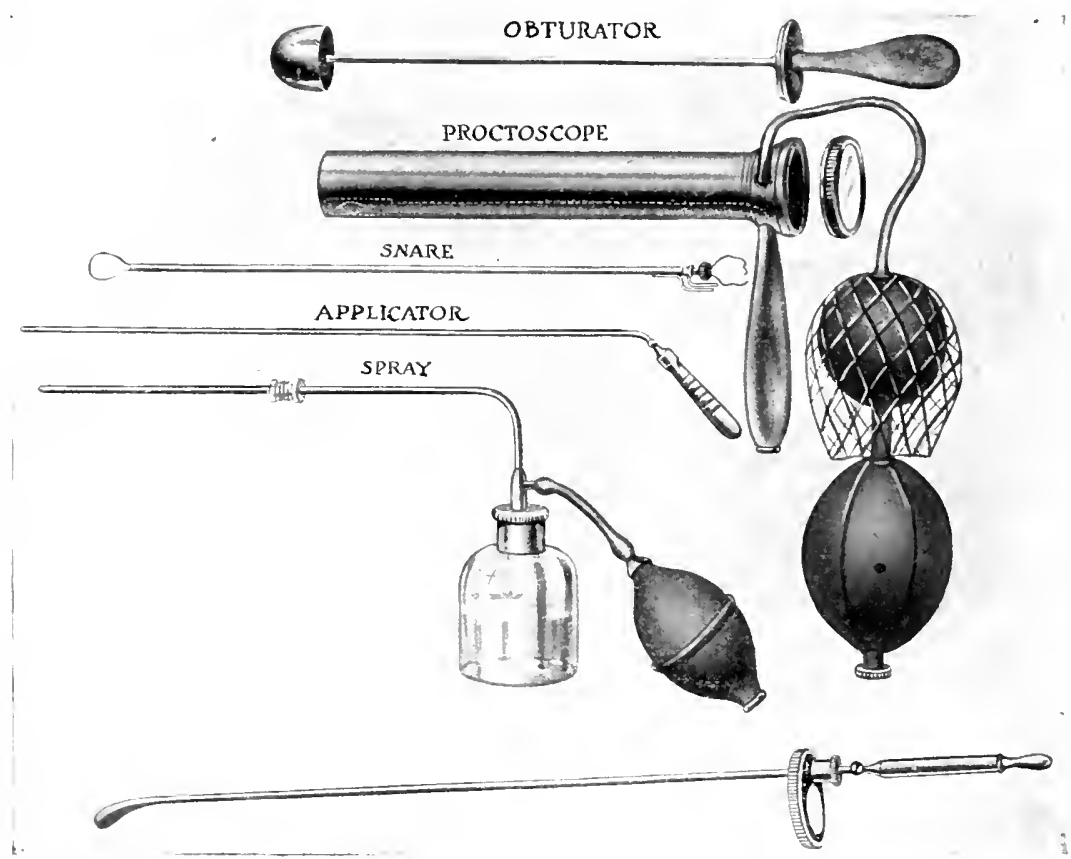

Fig. 9.-Laws' pneumatic proctosigmoidoscope.

vide the necessary illumination (Fig. 9). These instruments come in various sizes and lengths. Those most generally employed are 4, 8, and $\mathrm{I}+$ inches (IO, 20, and $35 \mathrm{~cm}$.) in length and a little less than $\mathrm{I}$ inch $(2.5 \mathrm{~cm}$.) in diameter.

Digital examination is the most reliable method of detecting disease in the lower howel, and much valuable information can be obtained by means of the educated finger (Fig. IO). In this way one (an easily and quickly diagnose fissures, ulcers, polypi, cancers, strictures, fecal impaction, foreign bodies, hemorrhoids, hypertrophy of the rectal valves and of the levator ani and sphincter muscles, thickening and rigidity of the bowel wall, diverticula, enlargement of the prostate, retroflexion of the uterus, deviated cocesx, and other 
conditions, which alone or together impede the feces in their downward course or cause diarrhea.

Digital examination, properly executed, induces but little discomfort, but when the finger is hurriedly and carelenty introduced

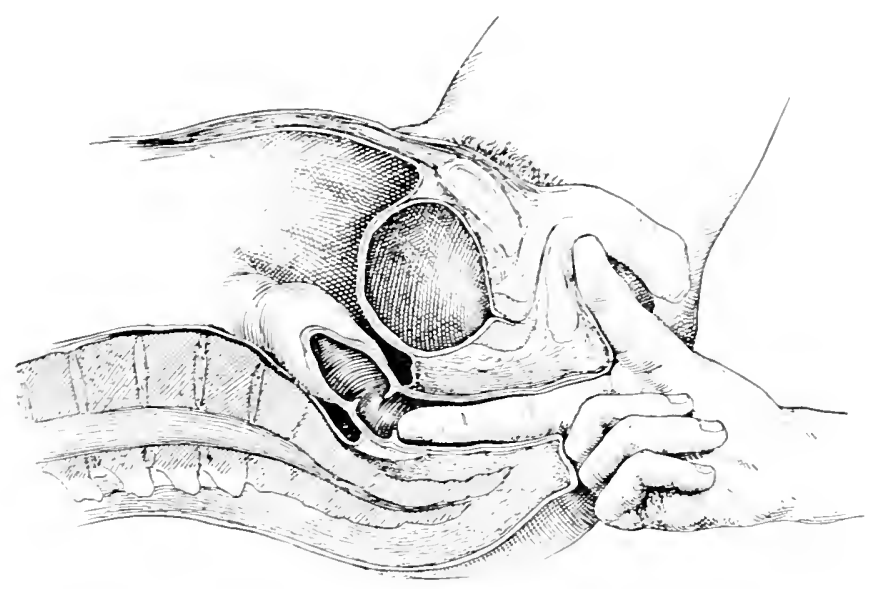

Fig. 10.-Correct method of digital examination with the patient in the lithotomy post ure.

it causes much unnecessary suffering. The nail should be pared. the finger oiled with some stiff lubricant, such as raselin, and passed slowly through the anus with a gentle boring motion. When the sphincter contracts, a few seconds should be allowed for it to relax; the examination may then be continued by sweeping the finger around the bowel. first in one direction and then in another. The condition of the sphincter, surface of the mucosa. prostate gland. uterus. bladder, raginal septum, sacrum, and coccyx may in this way be determined.

Rectal Specula.- Since the advent of the proctoscope, specula (Fig. I I) are rarely used for rectal ex-

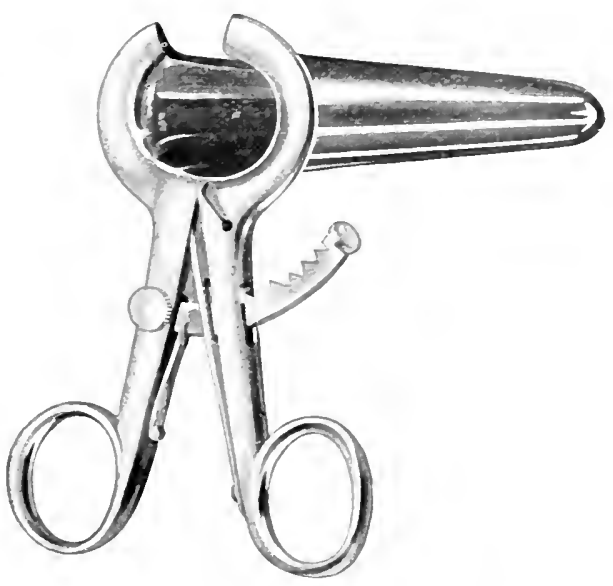

Fig. I1.-Author's examining sperulum. aminations, because they induce more suffering and do inot give as good a view of the parts as tubular instruments (proctoscopes?.

Röntgen Rays (r-rays).-C'ntil recently radiograms and fluoroscopic examinations were regarded as diagnostic noweltice in so far 
as they related to affections of the gastro-inte-tinal tract, but have gradually gained in faror. until now they are considered valuable diagnostic aids and are resorted to daily, and practical men do not venture a positive diagnosis in diarrhea or other obscure conditions of the stomach. small intestine, or colon until they have had röntgrams made. or inspected the workings of the stomach or bowel through the fluoroscope following the administration of bismuth, test-meals. or injections.

I-rays in the hands of the novice reveal little or nothing of diagnostic value, and are extremely dangerous to physician and patient unles they are properly protected. which is practically impos-ible with the equipment found in most offices. Formerly the author made radiograms and fluoroscopic examinations in his office,

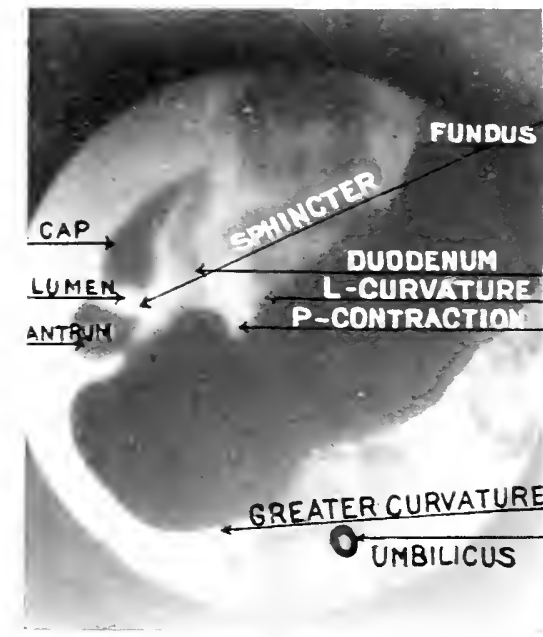

Fic. 12.-Radiogram showing gastric -urvatures. sphincter, fundus. and position of the duodenum erect posture.

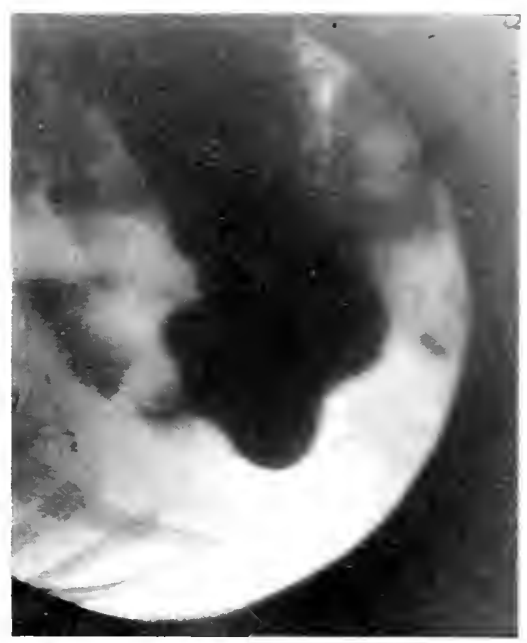

Fie. I3.-Radiogram showing relation of the stomach and adjacent small intestine prone posture. Taken by Cole).

lut now refers his patients for $x$-ray diagnosis to röntgenologists who maintain a fully equipped $x$-ray laboratory, and the change has proved eminenty satisfactory. hecause in this way more accurate radiogram - and Aluoroscopic riews have been obtained with less cost and annoyance to the patient than occurred under the older arrangement.

Phrsicians who do not employ the $x$-ray in connection with gastro-inte-tinal work are oblivious to its value, often fail to make a diasnosis. and miss many interesting and practical discoveries. Forrification of the diagnosis by means of $x$-ray pictures in cases where one has almost arrived at definite conclusions is often most gratifyince and encourages the physician. who can at once proceed intelliwntly with hyienic medical, or physical therapeutic measures. or urate when necessary with a greater degree of conidence. The 
author has had numerous radiograms made of supposedly normal individuals and those suspected of having gastro-intestinal derangements, and these pictures indicate that the size, contour, and position of the esoplatgus, stomach, duodemm, small intestine, colon, signoid thexure, rectum, and the solid viscera are abmormal or displaced very much more frepuently than the profession bedieves. Viewed from the standpoint of anatomy, it is the exception rather than the rule when one obtains in healthy or sick inclividuals racliosgrams which show all the viscera in position and without defects.

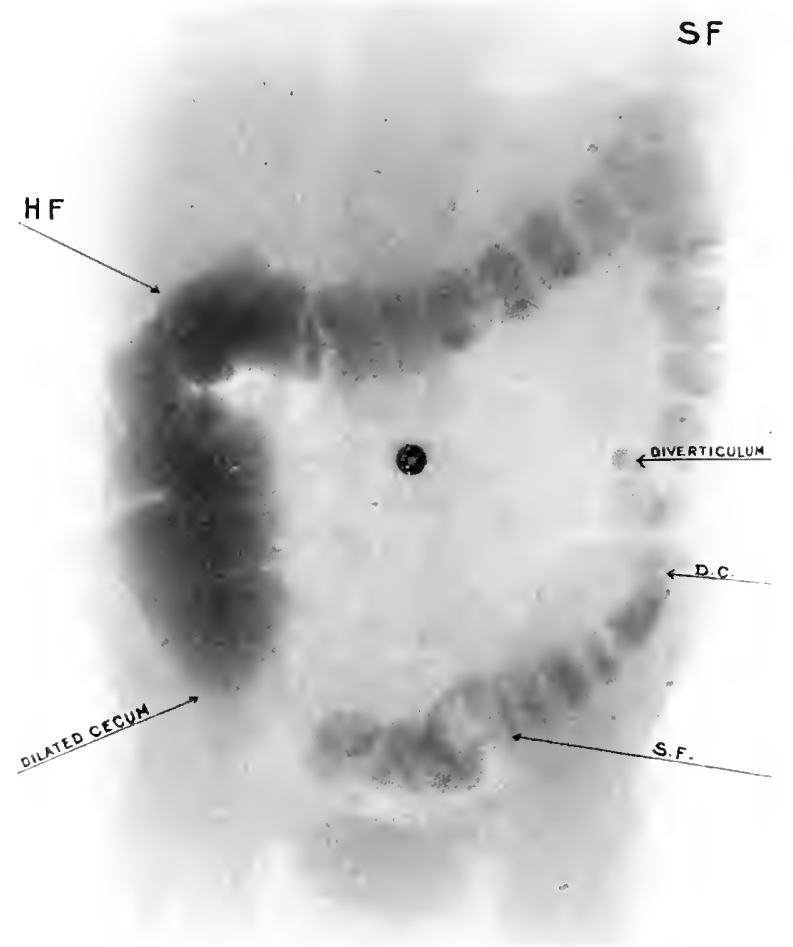

Fig. I t.- Radiogram of a diverticulum located in the descending colon and a dilaterl cecum.

Consequently, radiograms indicate that anatomists have incorrectly described the contour and position of the organs, that they frepuently change their position in healthy individuals, or they are often alinormally small or large, distorted or ptotic.

The esophagus can be slightly, and the stomach farty well, defined and studied with the aid of radiograms and fluoroseopic inspection (Fig. 12). F-ray investigation of the small intestine and, nuore particularly, its upper part, the duexlemum (lig. 1.3), is difficult and unsatisfactory because the lismuthized food passes through it rapidly, but when carefully taken excellent photographs can be wh- 
tained of the lower ileum, individual segments of the colon, and the sigmoid flexure and rectum, following a two days bismuth diet or filling of the large bowel with a bismuth solution. It is generally believed that water injected into the colon camnot be made to enter the small intestine, but elsewhere the author has given instances of where this has been accomplished, and more recently has been able to demonstrate by röntgrams that copious colonic bismuth injections do pass the ileocecal valve and enter the small bowel, and his pictures indicate that this not infrequently occurs, because in several radiograms the lower ileum has been made platinly visible

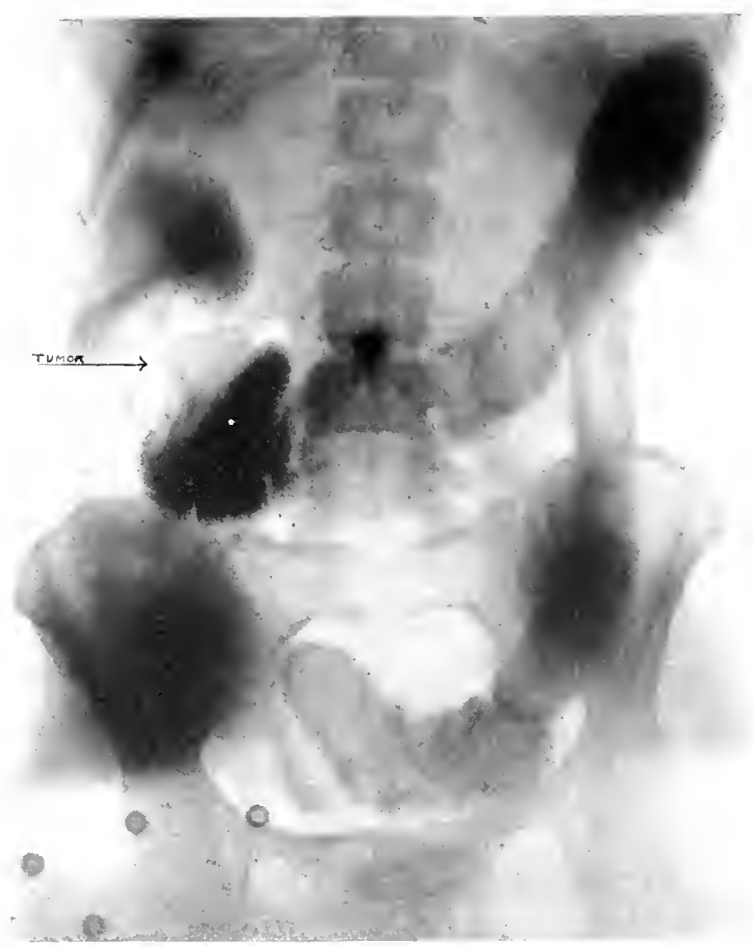

Fig. 15.-Radiogram of early carcinoma of the transverse colon without metastases. successfully removed by operation (Cole).

through bismuthization in this way in cases where none of the drug had been administered by way of the mouth (Figs. I4, 15). In one case lane's kinking of the ileum in proximity to the ileocecal juncture was very well shown. Burke? has been able, through radiograms, to demonstrate an hour-glass contraction of the duodenum incited by wo circular uleers, and others have in a few instances been able to photograph duodenal distortions. The x-ray and radiograms are

1 Cant, Constipation and Intestinal Obstruction, 1010, JP. 230, 231.

"Surgery, Gynecology, and Obstetrics, 1911. 
perhaps the most reliable diagnostic measures we have for locating metallic foreign bodies, bismuth accumulations, enteroliths, and calculi in the alimentary tract; defining esophageal strictures and cardiospasm; demonstrating the size, outline, and position of the stomach when abnormal, and the presence of strictures, tumors, or hourglass contraction within and athesions which pull upon it; showing the normal in healthy, and abnormal motility of the bowel in affections of the gastro-intestinal tract, which enables the observer wo determine the rapidity with which the food passes through the stomach and varions segments of the intestine and the time it takes for the colon to empty. which varies from wenty-four to forty-eight hours. This diagnostic adjuvant is most valuable in clearing up olscure lesions afferting the colon, signoid, or rectum, since following the administration of a bismuth meal or injection it can be photographerf

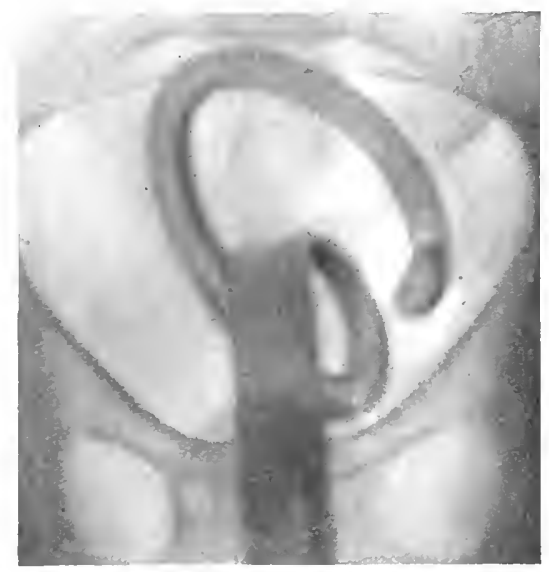

Fir. 16.-This radiogram demonstrates that it is sometimes impossible to introduce a tube into the colon, even though it is projected through a sigmoidoscope already in the lower simmoid hexure.

to advantage, and existing abnormalities. such as ptosis of one or all the colonic segments, angulations, twists, invaginations, binding adhesions, foreign bodies, diverticula, tumors, etc., located within or which involve them can be shown. In this way one can alio demonstrate the position of an introduced colon tube, and determine its usefulness for high colonic thushing, which is doubtful, since ratiograms indicate that the tube generally curls up in the rectum or lower sigmoid (Fig. I6) except in cases of colonic congenital or acpuirecl dilatation.

The fluoroscope is useful in studying gastro-intestinal peristaltic waves and following a bismuth meal through the stomach. small intestine, downward to the colon, and, to sonte extent, for noting displacement of the viscera and locating segments of gut wherein abnormal peristalsis takes place in the presence of strictures: hut for other purposes it is inferior to radiograms as a dianostic neasure, 
and should be discarded for them. because of this and the danger of burning the patient. owing to the lengthy exposure required. Considerable care and patience are required in obtaining radiograms of the viscera, and several pictures should be taken with the patient lying upon the abdomen, sides, back, and in the erect posture, so that changes in the location and character of the lesions and organs can be determined under varying conditions. Radiograms of the stomach. and particularly the colon, are often unsatisfactory in fat individuals, and such patients should be placed in the position which will bring the organ to be photographed nearest the plate.

Phrsicians should not hesitate to have patients $x$-raved by a competent röntgenologist. because burning now rarely occurs since the subject can be well protected from the rays and the pictures are taken with new and powerful machines in from one to ten seconds.

The technic of preparing the patient for radiography and fluoroscopic examination is not elaborate, but must be systematically. carried out, because when it is not, fluoroscopic examinations are unsutisfactory, will show nothing, and the pictures will be imperfect. Better results are obtained when the stomach and bowel are cleared of their content- before the bismuth is administered by mouth or is injected into the colon, and to this end it has been the author's practice to have the patient fast before the drug is consumed by mouth. and take a dose of castor oil the night before the colon is to be bismuthized from below. After the patient has been stripped to the hips. and guiding markers have been placed at the ensiform and umbilicus. he should be covered with a sheet. placed in the proper posture against the plate, and instructed to keep perfectly quiet. Of the various media administered hy mouth or injected into the colon to make the gastro-intestinal tract visible for fluorosopic examination and $x$-ray photograph, bismuth in one form or another has proved the least dangerous and expensive, the most effective. and is generally employed by expert röntgenologists. At first bismuth subnitrate was largely used, but in a few instances, where it was impure and contained arsenic, unpleasant or dangerous manifestations followed its administration, and because of this, and the fact that it contains more of the bismuth clement and gives equally good results. the subcarhonate has been substituted for it.

As a means of bismuthizing the stomach, small intestine, and colon from above the author employ - from I to 6 drams (3-23 gm.) for children and I to $3 \mathrm{oz} .(30-90 \mathrm{gm}$.) of bismuth subcarbonate for adults. admini-tered in meat, grary, butter, milk, cream, beaten egg, gruels, upple sauce, koumis- zoölak. menstruum of acacia, or other media that will retain the chemical in suspension for the necesary length of time. When the drug is used in the form of an injection for colonic work it may be succesfully emplosed suepended in acacia or admixed with fullers earth and water in the following propertions, viz: Bismuth sulucarb.. sij: fullers" earth. siv: water. (oij. Almosi any amount of hismuth subcarbonate may be used in the solution without doing 
harm, since absorption in the large bowel is not active; lout to andid danger from this souree the autbor makes a practice of washing out the bowel through the sigmoieloseope or a return-thow colon tube when fluoroseopic examinations have been completed and pietures laken. A quart (liter) of the solution is suffecent when the sigundeid is to le investigated; 2 guarts (liters) are necessiry for the transverse colon, and 3 quarts (liters) are repuired when the entire colon is to be inspected or photographed, and when it is to be eletermined whether or not the bismuth enters the lower ileum. The atvisability of photographing the patient while in different postures, to determine if the viscera or lesions change their position, has already been mentioneel. 'This practice is also interesting, because the level to which the bismuth settles in the hollow viseera is plainly shown when the picture is mate with the patient standing against the plate.

Radiograms and Huoroseopic examinations are rather impractical for general use because of the expense they incur, the time and clifficulty encountered in properly preparing the patient, and because the pictures are not always sufficiently distinct to enable the physician to complete the diagnosis. Radiographic plates and prints from them should be studied closely because they are often difficult to interpret, and rereading them frequently helps to define lesions unobserved at the first inspection. The novice should early learn to analyze the lights and shadows and to closely distinguish clear spots, which indicate accumulations of air or gas, from darker areas, which show that the viscera has been bismuthized at such points. In a few instances the author has obtained radiograms following inflation of the colon with gas and air independent of bismuthization, wherein certain segments of the healthy and diseased bowel were distinctly visible or the position, size, and the entire colon could be defined. Slight or partial inflation is sometimes useful in conjunction with bismuth because it distends the gut, lifts it upward, straightens out angulations or kinks, and brings intestinal neoplasms nearer the surface or displaces those pressing upon the gut; but, for general radiographic purposes, filling the gut with air or gas is not to be compared with either of the methoxls of bismuthizing the large bowel mentioned above.

Einhorn's test beads, food-carrving cups, duodenal tubes, obturators, and other diagnostic instruments are frecuently serviceable in helping one to arrive at a correct diagnosis in confusing cases of gastrointestinal affection, and in determining which segment of the alimentary tract is not functionating properly. 


\section{CHAPTER III}

\section{ORGANIC DISEASES, DIARRHEA IN}

\section{OCULAR, BUCCAL, DENTAL, NASOPHARYNGEAL, THYROID GLAND, IIVER, PANCREAS, KIDNEY, SUPRARENAL, GENITAL, URINARY, SKIN, BONE}

Eye Diseases, Diarrhea in.-Eye abnormalities, such as muscle strain, errors in refraction, and the disturbances caused by overwork from study, typewriting, bookkeeping, and the like, requiring close application of the eves. have been known to lead to diarrhea through making the sufferer extremely nervous, a manifestation of ten complicated by frequent watery movements containing neither pus, blood, nor mucus. Nore often profuse diarrhea, through its depleting effect and loss of the body fluid, leads, owing to weakness and lowered resistance, to certain serious ocular disturbances. Zimmermann has reported an interesting case of blindness and atrophy of the optic nerve following severe diarrhea, and Knies has pointed out that a profuse diarrhea may give rise to xerosis of the conjunctiva and cornea in children precisely as cholera in adults through desiccation and subsequent infection, and say's that the micro-organisms do not seem to develop very rapidly in the dry and cold cornea, and the process is not so rapidly progressive as in other forms of infectious keratitis at that age. Generally, but not invariably, the onset of xerotic keratitis in cholera infantum is the precursor of death. which, as a rule, occurs prior to perforation of the cornea, which otherwise follows. For obvious reasons the disease is almost invariably bilateral, although the two eves are often not affected to the same degree. Here the intestinal disease, as such, exerts a beneficent effect upon the development of the trouble in the eve. because the enormous loss of fluid in profuse diarrhea facilitates the drying out of unsheltered regions. Evidently there exists at the same time a sort of ptomain anesthesia, which causes the lack of sensibility in the cornea and the imperfect winking of the eyelids.

Treatment of diarrhea dependent upon strain or other eve affections which excite a neurotic condition consists, first, in correcting the ocular disturbance, and then treating the loose movements after the plan outlined in the chapter devoted to Neuropathic Diarrheas. (On the contrary, when the ocular manifestation is a sequel of profuse diarrhea, the frequent evacuations, irrespective of the cause, must be stopped: otherwise the therapeutic measures directed against the eye disease will fail.

Mouth Diseases, Diarrhea in.-This type of diarrhea may be incited by the swallowing of pathogenic bacteria in certain diseases involving the teeth or buccal mucous membrane. 
Patients whose molar teeth are tender, decayed, or absent rarely divicle the food as fincly as it should be, because the grinding is accomplished with teeth unsuitable for the purpose, and in consecpuence it is eleposited in the stomach in lumps which are not readily digested because of their size and the difficulty of the secretions saturating them.

Diarrhea from buccal causes most often results from the swallowing of germs which have accumulated upon the back of the tongue, between, or in carities of, the teeth, and on artificial plates and bridges where the patient does not properly cleanse them. Again, the loose movements may be attributed to unclean syphilitic or other ulcers of the mouth, the discharge coming from dental abscesses (owing to the action of the bacteria contained in the unhealthy secretions) and the swallowing of considerable saliva, which may cause dyspepsia.

The diagnosis can usually be readily made by examining the mouth for and detecting the causative factors already mentioned.

The treatment, which is obvious, consists in frequent cleansing of the roughened portion of the tongue, the teeth, cavities within them, plates, and bridges, as well as ulcers and abscess pockets. Teeth which are decayed beyond saving, and those which are detached from the gums or cause frequent irritation, should be drawn and the wound treated until healed, and operative interference is indicated when there is an acute abscess. chronic discharge from necrosed bone or root of a tooth. When remoring the source of the trouble does not cure indigestion and diarrhea, they should be treated symptomatically.

Nasopharyngeal Diseases, Diarrhea in.--Sufficient cases have been recorded in the literature to demonstrate that under favorable conditions nasopharyngeal affections are capable, directly or reflexly. of producing gastro-intestinal disturbance and diarrhea, and that disease of the stomach and intestine may at times affect the nose and throat.

In discussing these cases one authority says that the interdependence of the respiratory apparatus and gastro-intestinal tract is a vicious circle, and that nose and throat troubles can react upon the gastro-intestinal sỹstem or i'ice iersâ.

Nearly all practitioners have observed romiting and, frequently. loose movements to follow acute conditions of the nasopharynx. accompanied by a rise of temperature. edema of the mucosa, and prolonged spasms of coughing excited by a tickling in the throat or reflex disturbance. In other cases, as in coryza and chronic catarrhal affections, the same or more marked stomach and bowel symptoms may result from the almost constant hawking and swallowing of large quantities of mucus which is often virulently infectious. Again, the accessory sinuses draining into the nasopharyx are frequently the seat of fetid abscesses, as are also the tonsils and pharym. which spontaneously or following operation discharge into the throat; pus seething in pathogenic hacteria is swallowed in considerable amounts, setting up an irritative or infective gastro-enteriti- 
with increased eracuations. Evidence to this end has been obtained in finding of the same bacteria in the secretions taken from the nasopharyns. stomach, and intestines.

Children afflicted with adenoids greatly improve as regards appetite. digestion, intestinal ailments, and general metabolism following the removal of adenoids. and this, as White has suggested, is probably due as much to the stopping of swallowing unhealthy secretions as it is to benefit of the breathing apparatus. Arguing from this. there is reason to believe the other above-mentioned conditions can produce like disturbances or worse when virulent secretions reach the gastro-intestinal tract.

It is interesting to note. in this connection, the case of Aronsohn. where a patient had an immediate desire to evacuate the bowel following cocain applications to the nose in the treatment of asthma from which she had suffered six rears.

To show the reserse state. Coffin has called attention to the following ways in which disease of the upper air-passages may be produced or affected by those of the gastro-intestinal tract:

I Mechanically. by constipation. which causes slowed circulation. congested condition of the parts. and a consequently changed -ecretion.

(2) By Reflex Action.-Many interesting conditions are seen in the upper air-passages due to vasomotor changes and glandular activities. which are apparently due to reflex actions having their stimuli in the disordered condition of the gastro-intestinal tract.

(3) Toxemia. meaning those conditions found in the upper airpassages which are caused by toxic substances found in the blood as the result of imperfect digestion and disturbed metabolism.

The treatment of nasopharyngeal diarrhea depends upon the nature of the disease or lesion causing local irritation. reflex disturbance. and the formation of mucus or pus. the indication for the handling of which the reader is referred for a detailed description to the standard works on the nose and throat.

It is not necessary to do more here than briefly state that acute and chronic ab-ceses should be reached, curetted, and drained at the earliest posible moment. Hypersensitive areas. ulcers, and inflamed mucosa -hould be relieved by sprays. topical applications. and internal medication, the latter being particularly serviceable when the patient is neurotic. The most important features of the treatment, however, is to see that the nasopharyn is frequently cleansed, the paticnt educated to cough up and expectorate and not to swallow the obnoxiou- secretions, and to remore the adenoids.

Thyroid Diseases (Exophthalmic Goiter), Diarrhea in.-Diarrhea constitutes one of the most frequent and annoying manifestations of expphthalmic goiter, and it may usher in the disease or appear at any time and be transitory periodic, or remain permanentlyand add creatly to the suffering of the patient, and sometimes the stools escape involuntarily. Again, the loose morements may be in- 
dependent and of sudden onset or appear at the crises. when tachycardia, oevlar and other manifestations are most pronomeed, both in the incipient and later stages of the disease.

The evacuations are fluid, bile tinted, and contain whole peas. grains of corn, pieces of tomato, and other food remnants a few hours after they have been eaten, showing that digestion is impared and peristalsis accelerated. Here, as in neurotic diarrhea, the patient suffers trom nocturnal diarrhea; that is, has a number of passages in the early morning which rapidly follow each other.

Exophthalmic goiter subjects frecuently have a ravenous appetite, eat much more than they can digest, and this sooner or later leads to a true enterocolitis, which is sidenced by the presence of mucu, in the stools. This type of loose movements is similar to that induced by diminished pancreatic secretion described elsewhere. Balint and Molnar have studied this condition carefully, and conclude that instead of there being an insufficiency there is an increase of pancreatic secretion and perhaps of other juices. They say that it cannot be altogether denied that this generally increased secretion in possibly parallel to the overproduction of a peristaltic "hormone": this would serve to explain the azotorrhea and steatorrhea in the presence of abundant ferment. The specific action of the thyroid gland in these conditions seems to be supported ly the observation that myxedematous patients usually suffer from rebellious obstipation. In a case under the author's observation, concerning a woman with myxedema, the otherwise obstinate obstipation was at once replaced by regular stools, even by diarrhea. when the patient was given thyroid tablets; ice iersî. the olstipation returned as soon as the thyroid tablets were omitted.

It is furthermore noteworthy that the determination of the diastase in the feces may render very useful service in the diagnosis of the loss of pancreatic secretion.

In acute Basedow's disease the patient also complains of nausea and incessant romiting in addition to the diarrhea and other typical symptoms.

The diagnosis of exophthalmic goiter diarrhea is difficult or impossible where this condition ushers in the disease, but later. when the patient, in addition, begins to suffer from tachycardia, muscular trembling. exophthalmos, and enlargement of the gland, there is no excuse for a mistaken diagnosis.

The treatment should he non-operative or surgical, according to the condition of the patient when seen. In the incipient stages he can usually be benefited by changing his environment and sending him to the mountains, because in this way he gets rid of unpleasant companions, arduous indoor occupations and worries, and, in addition. has the benefit of plenty of fresh air and the soothing effect of eleration (3500 feet) upon the nerves and circulatory nstem. Patients who do not do well and those that are further advanced shoulel be given the benefit of the rest cure and a course of treatment, inclur- 
ing hydrotherapy, massage, electricity, exercise, and vibration to improve their general condition and quiet the nerves.

Generally, medical agents are useful in the symptomatic treatment, but have very little curative effect, though many remedies have been sugrested for the purpose. Anders reports + cases cured, 2 by the use of sodium salicylate (gr. $\mathrm{x}-0.60$ - four times a day), and 2 by the following combination:

R. Ext. digitalis

Ext, ergotie

Strychninæ sulph

Ferri arsenias

Ft. capsulas No. xxis

Sig.-One t. i. d. aiter meals. gr. iv $(0.25)$;

$\overline{5} \leqslant(2.0)$;

gr. ss $(0.03)$;

gr. ij $(0.12)$. -11 .

Improvement has also been known to follow the bromate of quinin, thyroidin, veratrum viride, aconite, belladonna, the glycerophosphate of sodium, and lecithin in conjunction with a milk diet. Farorable results have also followed the administration of the extract of the suprarenal gland, the cytotoxic serum of Rogers when the patient suffers from toxemia and the $x$-ray.

The operative treatment of exophthalmic goiter is more satisfactory than formerly, owing to improved technic, but the mortality and permanent results are still disappointing. In fact, the value of surgery and the best procedure to employ in these cases is difficult to decide, because a considerable number of cures and failures have followed partial thyroidectomy, resection of the sympathetic nerve, and tying of the nutrient blood-ressels.

For those especially interested in the treatment of exophthalmic goiter, comprehensive statistics are now to be had in the current literature covering the medicinal. serum, and surgical treatment of this affection.

The diarrhea usually ceases with the reduction of the gland, but may persist for a time thereafter, owing to the irritable state of the bowel or catarrhal condition which has been brought about. When during or following the treatment directed against the thyroidism the movements become very frequent, painful, and exhausting, opium in $\frac{1}{2}$-gr. ( 0.03 ) doses or morphin in smaller amounts. combined with an astringent or an antiseptic, should be administered sufficiently often to reduce the number of evacuations and secure for the patient the necessary comfort and rest.

Liver Diseases, Diarrhea in.-Diarrheal disturbances are encountered with comparative frequency in certain disorders of the liver, gall-bladder, and bile-ducts, particularly in nervous individuals so afflicted, and for some unaccountable reason the increased movements wsially take place shortly after eating and are aggravated by dark meat. This type of diarrhea is not so difficult to diagnose when there is marked derangement of the liver or its passages are blocked, but in less severe cases it is frequently impossible to differentiate between it and the prandial cliarrhea of Linossier, which, because of the prom- 
inence of the nervous manifestations associatled with it, is chescribed by the writer in the chapter devoted to Nervous Diarrheas.

This form of hepatic cliarrhea results from the considerable atecumulation and retention of bile which is eventually empticel into the bowel, and makes up all or a considerable proportion of the evacuattions when admixed with the feces. Both patient and intestine are already in a state of nervous irritatility, and the sudden discharge of so much bile into the sensitive bowel serves directly to stimulate the mucous glands to activity and the gut to abnormal peristalsis through rellex action.

Malignant and non-malignant growths, gall-stones, catarrhal inflammation, or other disease which leads to an obstruction of the gall-bladder, biliary passage, or both, usually induces constipation, but in aggravated cases persistent diarrhea results, and is due to the toxic effect of the absorbed bile upon the nervous apparatus and constitution, and prevention of the bile from reaching the intestince to participate in the fermentation and putrefaction going on within it. Owing to the absence of bile and its stimulating effect upon pancreatic secretion, intestinal indigestion prevails, and this, together with the abnormal increase of pathogenic bacteria and their oxins. resulting from a lack of the bactericidal influence of the bile, leads to the accumulation of irritants within the gut and diarrhea because of the attempts on the part of the bowel to expel them.

The writer has known gall-stones to incite diarrhea, both through their irritative effect upon the mucosa in their downward course and by blocking the intestine and setting up an obstructive diarrhea.

Diarrliea due to inflammatory or ulcerative lesions of the intestine may precede or complicate hepatic disease, but under all circumstances is aggravated when the bile pours over the lesions in abnormal amounts or is deficient and indigestion prevails.

Loose movements may be induced by icterus (jaundice), however produced, but is not an important manifestation except under the above-named conditions.

The various forms of cirrhosis at times cause diarrhea, and it is occasionally very aggravating in alcoholic subjects, due largely to the markedly congested state of the gastro-intestinal mucosa in these cases.

The disturbed portal circulation in this disease manifests itself by the congestive hyperemia of the digestive tract, which in the later stages of atrophic hepatic cirrhosis may lead to increatsed digestive disturbances, vomiting, diarrhea, and sometimes blood in the stools.

Symptoms and Diagnosis. - The symptom-complex varies in accordance with the nature of the hepatic disturbance. In the obstructive ypes there is jaundice and sensitiveness of the gastro-intestinal tract because of its congested state, the stools contain lumps of fat or glistening globules, and they, with expelled flatus, are very offensive, and. owing to the lack of bile, are ash or clay colored, and are of a dark- 
brown or greenish color when examined shortly after relief of the obstruction and emptying into the bowel of bile in large quantities.

The movements in this type of diarrhea do not occur with great frequency (four to five times claily), are more apt to be mushy or pasty than of a watery consistence, and are usually preceded by discomfort or severe pain, fulness, and heaviness in the epigastrium.

The nature of the loose movements may be suspected both in the absence of and when the evacuations are composed completely or almost entirely of bile.

Treatment.-Since the majority of hepatic diarrheas follow blocking of the biliary flow, the most rational method of procedure, particularly in urgent cases, is to explore the suspected region and remove the tumor, calculi, or the obstruction causing the trouble, except when due to a marked catarrhal state of the bile-passages, when temporary drainage may in suitable cases be instituted. In the latter, and in cases where there is no occasion to hurry, an attempt should be made to correct the difficultr with the aid of medicinal and hỵgienic measures.

It requires a considerable time to overcome a catarrhal inflammation which has attained headway enough to partially or completely. obstruct the flow of bile, but in lavorable instances relief is to be had from outdoor exercise, bland, nourishing diet, copious water drinking, hyclrotherapy, gentle or friction massage, nightly application of a cold girdle, or, in acute attacks, hot applications, free drinking of Vichy and other alkaline mineral waters, and by having the patient take antiseptics in liberal doses along with several ounces of olive oil twice daily.

When the liver is cirrhotic the diet should be regulated, and consists largely of bland, non-irritating foods, medicants, such as bismuth, charcoal, magnesia, and other remedies known to have a soothing effect upon an irritable mucosa, should be administered, and when the bowel involvement extends low down this treatment should be reinforced by intestinal irrigations, using mineral oil and bismuth or weak solutions of boric acid, ichthyol, or permanganate of potassium.

In all forms of hepatic diarrhea where the movements are excessive, frefuent, and irritating, or the patient suffers severely from pain inciclent to cramps or the passage of biliary calculi, morphin or, preferably, opium, gr. $\frac{1}{2}(0.03)$, and belladonna, gr. $\frac{1}{4}$ (0.0I5), should be prescribed to control the movements, relieve suffering and enterospasm, and cause a relaxation of the abdominal musculature.

Pancreatic Diseases (Diarrhea Adiposa), Diarrhea in.- This type of loose movements may result from duodenal disease, obstruction to the bile-passages, pancreatic affections (pancreatitis and cancer). crsts, or obstruction to the ducts, as above indicated; it occurs in felorile diseases of children; ingestion of an overabundance of oil, butter, cream, or other fatty medicaments or foods; from digestive disturbances which interfere or prevent the splitting up or absorption 
of the fatty elements of the chyme, or inereased peristalsis which hurries them through the intestine; and, finally, l, obliteration of the chrte vessels.

In adelition to diabetes mellitus, the characer and frepuency of the evacuations are materially changed in nearly all other panereatic affections, and are attributed by some to the diminished alkalinity of the chyme, but Rathery belicves that the food is improperly digested and excites peristalsis both by the quantity and puality of the intestinal contents, and that the suppression of the pancreatic juice catuses an increase in the watery and a diminution in the weight of the dry food substances referable to exaggerated motility of the intestine.

Physologists have for a long time taught that the lile and pancreatic thuids largely control the digestion and absorption of fats, and recenty an abundance of evidence has been adduced, through operation, experiments, and examination of the feces, to show that any obstructive or other disease which extensively involves the duodenum. gall-bladeler, pancreas, or the ducts leading to the small burel may cause fatly diarrhea.

Ehrstroem has reported a most interesting case of fat diarthea which resulted from obliteration of the chyle resseds at the mesenteric root.

()catsionally children afflicted with chronic intestinal catarrh suffer from an unusual amount of fat in the evacuations, under which circumstances the condition is designated as diarrheo adiposa, a manifestation which has received attention at the hands of Demme and Eichhorst. Here the stools have a fetid orlor, arising from the fatty acicls, an oily look, whitish or yellowish color, a pultaceous consistence, and which, according to Biedert, when mixed with water, microscopically show fat droplets heside and above each other, whereas normally they are few and isolated. The fat contents determined by extraction of dried feces with ether in healthy infants showed 3.8 to 20.3 per cent., while in those afflicted with fatty diarrhea the percentage varied from 4.17 to 67 per cent. of fats.

lixamination of the stools is most important in these cases because there is steatorrhea; they contain an abnormal amount of fat. which is sometimes macrosenpically visible in the form of glistening (Joplets, which, together with the pasty, clay-like appearance of the feces, is characteristic of the condition.

Treatment of Pancreatic and Fatty Diarrhea.-Since pancreatic diarrhea results from disease within the organ, or more frecpently to occlusion of its duct, nothing short of surgical intervention offers any hope of a permanent cure, and the operation must be selected acoording to intications, but preceding and following correction or improvement of the trouble, and also in fatty diarrheas from other sourese the diet should be regulated, fats being restricted and the patient given a supportive treatment. Surgery gives the best results in fatly diarrhea dependent upon affections of the gall-bladeler, bile-ducts. or duoclenal olstruction. 
When due to a catarrhal duodenitis, less radical measures, such as a non-irritative and non-fatty diet, the administration of saline laxatives, antiopetic and antidiarrheal remedies, together with colonic flushings, ustally suffice to diminish the oily constituents of the feces and lessen congestion of the intestinal mucosa. In some instances patients suffering from fatty diarrhea require symptomatic treatment, and when, in children, it is due to febrile disturbances, crrors in diet, or imperfect digestion, routine treatment is impracticable, and therapeutic measures should be instituted which will correct or remove the direct or indirect factor responsible for the diarrhea, afterward treating the bowel locally when necessary.

Kidney Diseases, Diarrhea in.-1)iarrhea not infrequently complicates organic and functional disturbances of the kidneys. The frequency of the movements may be slightly accelerated in one case or manifold in another, and the diarrhea may occur but once and be of short duration, periodic or persistent, and continue until the kidney trouble has ended fatally or heen reliered.

Chronic Nephritis, Diarrhea in.-Mackey has reported 2 cases of chronic nephritis, in both of which the bowel was found extensively ulcerated and the patient suffered severely from intestinal hemorrhages. Perforation occurred in one, and Mackey suggests that increased blood-pressure in this class of cases leads to hemorrhagic points which later become ulcers through the influence of bacteria. Such lesions, in turn, increase peristalsis and cause diarrhea.

In some instances diarrhea is probably the result of a co-existing gastrogenic disturbance or an enterocolitis, under which circumstances the movements bear a direct relation to the number and extent of the lesions, and contain mucus, pus, or blood alone or admixed. Diarrhea caused ly affections of the kidney is probably due to secondary derangement of the central nervous system or to the excretion into the bowel of irritating toxins, the latter being the most important etiologic factor, as evidenced by the fact that usually the frequent evacuations occur periodically and simultaneously with the exacerbations of the nephritic condition. At such times the movements are copious, very fluid, have an ammoniac odor, and may or may not be discolored, accordingly as they contain bile or blood.

Stiller ${ }^{1}$ says diarrheal albuminuria is due to an autotoxic action of the renal epithelium, and says the injurious action of the loss of water upon the heart and kidneys is farored by the infectious poison of the bacteria in cholera as well as in acute diarrhea.

Lremic ulcers have been repeatedly observed in the duodenum and other parts of the small and large lowel in subjects who had for a long time been afflicted with chronic nephritis. Other types of intestinal ulcers invariably excite peristalsis and augment the secretions and peristalsis and cause diarrhea, and there is every reason for beliesing that these ulcers act likewise. Considerable difference of opinion exists as regards the etiology of the lesions, and different

1 Ungarische Mediz. Presse. Nos. 3 and 4 , r 900. 
authorities have attributed them $10(a)$ irritation ley the carbonate of ammonia; (b) henorrhagic infarets into the sulmucosa; and (c) excretion of irritating toxins into the bowel.

Slightly increased evacuations are unimportant from a prognostic standpoint, but when exhatusting diarrhea is associated with natusea, vomiting, and migraine the patient is usually very ill, on the verge of serious uremic poisoning, and is likely to the unless the acute attack subsicles rapidly. Strange as it may seems, frefuent profuse serous evacuations are at times apparently helpful, because marked edema of the skin has been observed to disappear simultaneously with the attacks, probably owing to drainage of the parts through the circulation and by way of the intestine.

Bartels maintains blood, purulent masses, or mucus may occur in the stools in the absence of ulcers, and has shown hy autopsy that where transudation has taken place the intestinal mucosat is edematous.

Treatment.-Every effort should be made to limit the progress of - or heal-lesions within the kichey or correct any abnormality which interferes with the functionating power of the organ, to lessen the formation of the toxins and waste products which so profoundly affeet the general system, and, in addition, much can be done towarel making the patient comfortable and freeing the intestine of its irritating and poisonous contents hy flushing the colon with hot normal saline or a mildly medicated solution.

No special effort should be made, except in extreme cases, to control the cliarrhea, because the patient's condition frequently becomes worse when the number of evacuations are greatly diminished, and. further, because ordinary anticliarrheal remedies fail except in unctesirably large closes.

Diabetes Mellitus, Diarrhea in.-In rare instances this manifestation complicates diabetes mellitus as the result of a coexistent enteritis, the diet, or pancreatic disease. In the first, the slightly increased evacuations are semiliquid, contain mucus, and continue more or less regularly. In the second, the patient complains of distention, central abdominal pains, which are followed by a number of watery evacuations that bring immediate relief. While in the last, or pancreatic type of diabetes mellitus, the evacuations contain an excess of fat and have an offensive odor.

In some instances, where nawsea, vomiting, hiccup, and severe pain precete the moxements, a serious turn in the disease is incticated, and the patient may at any time pass into diabetic coma.

Treatment.-In handling this type of diarrhea much can be done toward relieving the patient by having him live an out-door life. cat at regular hours, and discard food known to cause indigestion or irritate the gastro-intestinal tract.

In addition, when there is an existing enteritis or colitis, bowel irrigation, medication, and the measures recommended elsewhere for the treatment of these conditions are indicated. 
When diabetes mellitus becomes fully developed it is necessary to limit the manufacture of sugar and amount of urine by improving the hygienic surroundings of the patient, restricting his diet as regards carbohydrates and farinaceous food, and by resorting to hydrotherapy, massage, and exercise to eliminate sugar. At the same time suitable medication and foods are preseribed to maintain his strength and increase his power of resistance. Opiates are peculiarly suitable in the treatment of diabetic diarrhea, because they not only limit or control the evacuations, but greatly assist metabolism, bring rest and sleep, and have a pronounced tendency toward limiting polyuria and the formation of sugar.

Suprarenal Disease (Addison's), Diarrhea in.-In this affection the suprarenal capsules become enlarged or degenerate, the skin assumes a bronze-like hue, the patient becomes feeble in body and mind, and is troubled with circulatory disturbances, pigmented spots, headache, dizziness, fatigue, roaring in the head, weak pulse, clammy skin, polyuria, indicanuria, and gastro-intestinal disturbances, which are evidenced by loss of appetite, nausea, and vomiting, pain in any or all of the abdominal regions, and persistent diarrhea.

The diagnosis is based principally upon the previously enumerated symptoms, discoloration of the skin, and the enlarged glands must be differentiated from carcinoma and tuberculosis, with which they are frequently associated locally and when they involve distant parts. While pigmentation of the skin is rather characteristic, it is well to bear in mind that cancer, hepatic disturbances, tuberculosis, and argyra affect the integument in a somewhat similar manner.

The prognosis is invariably bad, the patient dies of weakness, coma, delirium, or convulsions in about a year or eighteen months, but in some instances, where the disease is rapid, death ensues in six months, but in others he may live five or more years.

Treatment.- The treatment of Addison's disease is usually hopeless in so far as a cure is concerned, but much can be done to extend the patient's life and make him more comfortable by supportive and symptomatic remedies. They consist in keeping him quiet and restricting his diet to concentrated and easily digested foods which contain a liberal percentage of proteins. Iron and arsenic are the best representatives of the tonic medicines, particularly when employed in combination with nucleins or phosphorus. Bismuth, tannalbin, and other agents of the astringent variety in 5 -gr. (0.30) or larger doses, three or four times daily, frequently control diarrhea if moderate, but when it is severe opium should be used in conjunction with them or salol or beta-naphthol when an antiseptic is indicated. Nausea and romiting are minimized or relieved by cerium oxalate, champagne, or albumen-water. Extract of the suprarenal capsules has in some cases given very good results, but has not been tested a sufficient number of times to warrant the belief that it will prove universally. successful in this class of cases.

Genital Diseases, Diarrhea in.-Of the bowel disturbances, con- 
stipation and diarrhea, which frequently accompany abnormaliticand disease of the sexual organs in women, the latter at time-ardsmaterially to the patient's discomfort. Most often thi- -rmptom occurs during the menopause. and may result from the engorsed and highly sensitive state of the parts or to the extremely nerrou-condition of the patient. This type of frequent morement= is encountered most frequenty in hysteric and neurasthenic women. and particularly those suffering from uterine catarrh, tumors, displacements. or extensive cervical ulceration.

Not infrequently diseater of the female pelvic organs or gunital apparatus results in exten-ion of the inflammatory process to the sigmoid flexure or rectum. causing proctitis and acceleration of the eracuation - which contain considerable mucus, or an abscess forms. opens into the bowel. and sets up an irritative diarrhea accompanied by tenesmus. Abnormally frequent passages often occur where there is a complete laceration of the perineum. but here the troulble in not due to diarrhea. but to incontinence and the patient $=$ inability to retain liquid and semisolid feces until aborsption takes place and they become firm. This is easily demonstrable by correcting the deformity, when the stools arain become normal. A frequent desire to eracuate the brwel may alos accompany raginismus and the paseage of the child" - hearl during labor.

Persistent diarrhea, complicated by colonic distention. colic, and the discharge of an abnormal amount of mucus. has been ob-erved in the presence of ovarian tumors with axial rotation, the cau-e of which is not known. but the di-turbance is probalsy due to retlex impulse originating in the traumatized nertes. local concestion. pressure uponand irritation to-the bowel by the tumor mass. retention within the gut of feces and toxins from the same cause or rupture of the cyst into-or direct involvement of-the bowel by a disinteratintumor.

The author has also treated severe cases of diarrhea in women which apparently resuled from nervous and operative sequelit.

Treatment.- it is obvious when diarrhea is induced by direare or displacement of a part of the sexual apparatus that such an excitinfactor must be corrected before the frequent evacuations can be satisfactorily controlled. except where the bowel disurbance is the result of prechic impulses or nerrousness.

When diarrhea is traceable to nervederangement. strychnin. ar-enic. iron. or other tonics should be prescribed along with at non-irriating nourishing diet. moderate exercise in the fresh air and sunshine tw strengthen the patient: but the most important thing is tre encourase the sufferer and take her out of unhycienic and dismal surroundinsand better her environment.

When frequent movements are induced by reflex disturbance. pressure upon the bowel by a retroverted uterus, or the discharce into $i t$ of an abscess. surgical intervention is imperativt, and the diarthes quichly ceases following remoral of these conditions. 
When frequent evacuations dominate the symptom-complex, it may be necessary to prescribe opium and belladomna or other reliable antidiarrheal remedies to control the movements until the disease causing the trouble can be removed by operative or other measures.

Diarrhea which complicates abnormalities or disease of the male genital organs may be caused by luetic or gonorrheal infection; the discharge into the rectum of pus from prostatic abscesses, or urine through a rectovesical fistula; extension of inflammation; malignant or other diseases of the bladder, prostate, or urethra; vesical or urethral calculi; blocking of the rectum by an enlarged prostate, and reflex disturbances.

The source of irritation responsible for diarrhea can usually be ascertained by cystoscopy, sounding the bladder and urethra for calculi and strictures, palpating and percussing the resical region, investigating the rectum with the finger or through the proctoscope, and by examining the urine and feces to find out if they contain pus, gonococci, or anything which would assist in clearing up the diagnosis.

Treatment.-Diarrhea consequent upon disease of the male genital organs can be modified by medication, but a cure will not occur until the source of irritation or blocking has been removed by operation or relieved by treatment. To insure permanent results in this class of cases rectovesical fistule should be closed by plastic surgery, abscesses should be opened, curetted, and drained, resical tumors, calculi, and enlarged prostates should be removed, urethral strictures and sensitive spots causing reflex disturbances should be corrected, and topical applications should be made to the rectum, or it should be irrigated with antiseptic and stimulating solutions. when inflamed or ulcerated.

Urinary Diarrhea.-This type of diarrhea is most frequently associated with the retention and absorption of urine, but occasionallycomplicates septic and other disease processes of the urinary tract. The diarrheic manifestation may vary from a slight acceleration of the movements to manifold watery stools not unlike those designated as dysenteriform. The frequency and severity of the attacks depend upon the degree of toxemia, disturbance to the nervous system, and local irritation. When the first is very marked the patient also complains of nausea, romiting, and other evidences of severe gastro-intestinal involvement. The author has more often observed diarrhea in connection with impermeable stricture and purulent cystitis than in other ailments of the urinary apparatus, but in two instances has treated patients suffering from obstructive diarrhea caused by prostatic enlargement in one instance, and carcinomatous degeneration of the gland in another.

Raskai has recorded several cases where obstinate diarrhea was complicated by bacteruria, with and without cystitis, some of which were due to the colon bacillus.

Treatment.-This consists in correcting the local trouble, bowel irrigation, and the administration of drugs to control the frequent movements when feasible. 
Skin Diseases, Diarrhea in.-Diarrhea has been occasionally observed in connection with certain skin diseases, the attacks being of short duration in some and longer in others.

In urticarie (which is marked by pinkish, flat elevations of the skin accompanied by itching, stinging, or pricking sensations) frepuent evacuations have been known to oceur. both when no exciting cause for the ermption was discoverable and when it was caused ly the eating of shell-fish or due to ptomain-poisoning. In this class of calses, in addition to the itching and swollen skin patches in argrarattet cases, there may be dyspepsia, nausea, romiting, and colic, accompanied by frepuent offensive diarrheic movements. Some writers attribute the intestinal manifestations to the urticarial disturbance, but the author believes that the skin affection is secondary to. and caused by, gastro-intestinal ailments, particularly auto-intoxication (or ptomain toxins), since urticaria can be relieved most quickly by the administration of hydragogue cathartics in successive doses to cleanse the bowel.

Acrodynia (erythema endemicum), uncommon in this country, but frequently observed among the soldiers and prisoners of Paris and of far eastern countries, is accompanied by disturbance of the gastrointestinal tract, and is usually ushered in by anorexia, nausea, vomiting and diarrhea, and is supposed to be the result of the effect of an unknown toxin upon the central nervous system. In exceptional instances the movements may run as high as thirty or more daily and contain considerable blood. It resembles urticaria, in that there are pricking and stinging sensations and flattened wheals which are pigmented. This condition also resembles arsenic- and ergot-poisoning and pellagra. Patients complain of pain in the extremities and sometimes of edema and muscular spasm. The attack in favorable cases runs its course in from two to four weeks, but convalescence is longer in clebilitated and aged individuals.

In erythema nodosum the bowel ordinarily is not disturbed, but when it is, the paticnt complains of colic and diarrhea.

The infectious variety of erythema exudatium multiforme may in very severe cases be accompanied by buccal disturbances, ulcerattion or gangrene of the pharyngeal mucosa, indigestion, loss of appetite, vomiting, annoying diarrhea, and the evacuation of a considerable amount of blood both by way of the stomach and the bowel.

Both acute eczema and pemphigus iulgaris when in an aggravated form may, through the fever, nervous phenomena, or tyspeptic manifestations which accompany them, induce gastric and intestinal irritation and diarrhea. Pemphisus acutus is particularly inclined to bring about this condition. In many instances, where there are both gastro-intestinal and skin manifestations, it is frequenty imposible to determine which is the exciting factor.

Pityriasis rubra, the terminal stage of which is at times accompanied with loss of appetite, dyspepsia, diarrhea, and marasmus, and sclerema neonatorum occasionally greatly disturb the gastro-intestinal tract and cause diarrheat. 
Treatment.-For the treatment of the various dermatologic lesions with which diarrhea is associated the reader is referred to standard work: on skin diseases. because their due consideration here would be unnecesary in a work of this character.

Measures employed to reduce the number of eracuations in this clasis of cases must be changed according to the cause exciting the lowel disturbance. When the gastro-intestinal mucosa is irritable often much relief can be obtained by restricting the diet to fluid and semisolid or non-irritating foods that are easily digested and leave a small, in preference to a coarse, bulky residue. Naturally, condiments, ice-cold drinks. carbonated bererages, and foods which disagree with the patient should be interdicted.

Owing to the annosance caused ly the frequent eracuations, the patient desires a medicine which will afford him immediate relief. but in the majority of instances it is had practice to at once prescribe an opiate or other anticliarrheal remedy until after calomel or a hydragogue has been administered to correct hiliousness and dislodge fecal accumulations: the toxins which are formed locally or reach the intestines through the blood should be washed out or neutralized, because, while the diarrhea mas be temporarily checked, it will recur until the inte-tine has been thoroughly cleansed. Cathartics and laxatives internally administered do not alway- accomplish the desired results. and when they fail the colon should be repeatedly irrigated with a -aline or one of the antiseptic or mild astringent solutions mentioned in the chapter on Intestinal Irrigation until all sources of local irritation are removed. If. in spite of this and the treatment directed against the skin affection, the diarrhea is not controlled, opium and belladonna alone. or in combination with antiseptic or astringent agents. should be prescribed in suitable closes and repeated as often as nectsary.

Bone Diseases, Diarrhea in.--The author has on several occasions observed children who suffered from Pott' - or hip-joint disease or tubercular lesions of the bones in other parts who were also afflicted with diarthea, and who did not have ear diseases. degeneration of the intestines, or other organs. In some instances the intestinal disturbance was due to unhygienic surroundings and unsuitable food, but most often it was traceable to catarrhal inflammation of the bowel, which is so common in tubercular and syphilitic subjects.

In these cases improvement followed correction of the bone lesions, a suitable diet. supportive treatment, and bowel irrigation. Koll has reported + severe cases of fetid diarrhea associated with epiphyseal derelopment of the bone. in some of which cases there was hyperplastic and in others nutritional disturbances and atrophic changes in the osieous tissue. In two instances the bone disease was reliered by curing the intestinal disease, but the method of treatment and result in the other cases he did not reveal.

'Deulsch. Archiv, i. Klin. Med., rgro, vol. c, p. + sg. 


\section{CHAPTER IV}

\section{AMYLOIDOSIS OF THE INTESTINAL TRACT (LARDACEOUS DEGENERATION), DIARRHEA IN}

Amlomosis is not so common as formerly becaune it is largely. due to pus accumulations, cachexias, tuberculosis, and syphilis, all of which conditions are at present being treated earlier and more effectively, which in a measure tends to prevent complications and the development of this affection. Next to the kidney, liver, and spleen, the intestine is the most frepuent organ to undergo lardaceous degeneration, and the disease here is induced by the same causes which produce it elsewhere.

To show the comparative frequency of lardaceous degeneration of the bowel to that of other organs, Nothnagel has assembled the statistics of Loomis, Dickinson, and Goodhart, which total 468 cases, wherein amyloidosis affected the kidneys in 302; spleen, 273; liver, 20I: and the intestine in 163 cases.

Amyloid degeneration may involve all or any part of the digestive tract from the esophageal beginning to the anus. Ordinarily the entire bowel is involved, hut when it is not, the colon is most often the seat of the disease, and when the small intestine is attacked, only the ileum is the part affected.

The etiology and general character of intestinal amyloidosis are the same as in other parts, and it is not necessary to do more than discuss its gross appearance and state that amyloidosis may originate primarily (rarely) in the bowel or secondarily to degeneration of the kidney, liver, or spleen.

Mirchow ( 1855 ) was the first authority to give a classic anatomic description of intestinal amyloidosis and call attention to the manner in which it could be chemically diagnosticated.

In this affection the lymphoid follicles rarely undergo degeneration, but in the later stages of the disease the villi often disappear through atrophy or necrosis, and the intestine presents a waxy, gray, translucent, glistening appearance, and the mucosa appears somewhat swollen (Fig. I7). Ilecration nearly always characterizes lardaceous degeneration of the lowel, and the ulcers may be single, but are usually multiple. penetrating, and may be diminutive, large or small. extensive in size, particularly when there is an active mixed infection. Isually they are not very large and present a punched-out appearance, have smooth, raised and thickened edges, pale base, and generally show little tendency to heal. ()wing to the peculiar appearance given to the mucosa by the amyloid changes and the ulcers which 
penetrate it, it frequently resembles cheese (Fig. $I^{-1}$ ), and has been

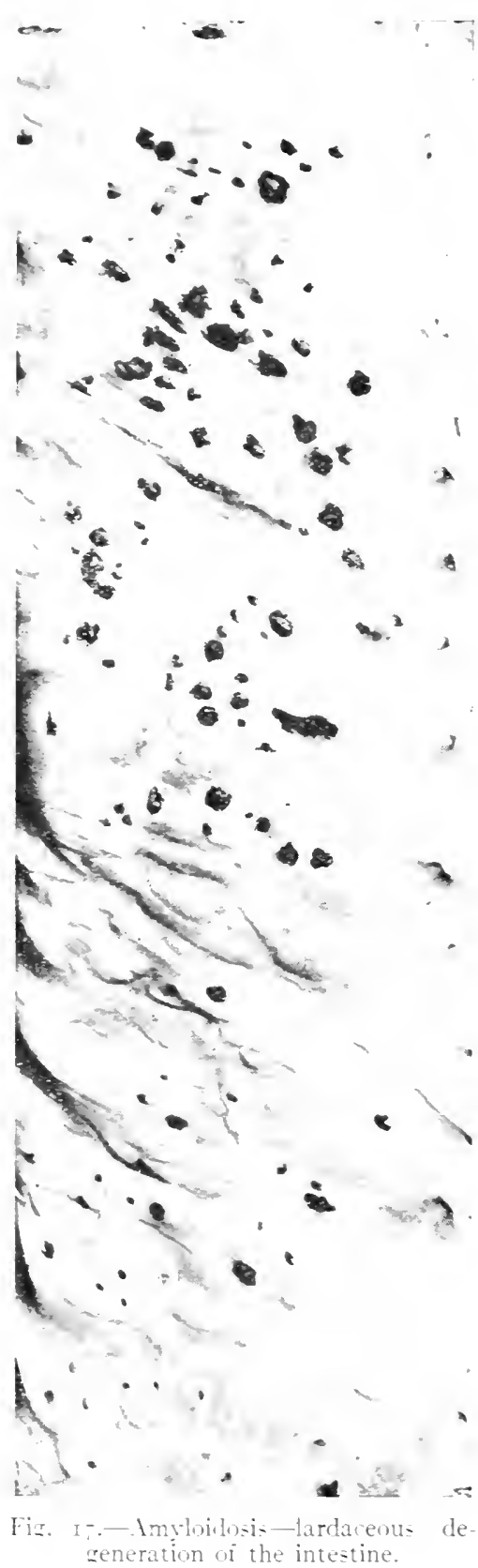
de-isnated by some "cheesy degeneration of the intestine."

The peculiar thing about intestinal amyloidosis is that the degeneration attacks principally the blood-ressels (particularly the arterial. involving the capillaries in the mucosa, arterial twigs in the muscularis, or the larger vessels of the intertinal wall alone or together. and shows a predilection for the media or muscular coat, though all the structures making up the intestinal wall may undergo a change. Owing to the rigidity and otherwise impaired condition of the rascular supply of the bowel. anemic spots result. which in time undergo necrosis and furm ulcers, but the authorities agree that all of the ulcerative areas seen are not attributable to this cause, and Colberg asserts that mechanic or chemic irritation must in some way play a part in the etiology of ulcerative amyloid degeneration of the intestine, perhaps through the breaking off of the fragile villi by the ingesta in its duwnward passage, or through irritation of the urinary products in the feces when the kidneys are lardaceous.

The symptoms of intestinal amyloidosis are indefinite in mild. but in typical cases they are fairly characteristic, and diarrhea constitutes the chief manifestation of the disease. The exciting cause of the frequent movements here differs from that of other forms of intestinal ulceration, in that they are due less to increased peristalsis and secretion of mucus than they are to a lack of absorption, owing to the condition of the mucos and impairment to the local circula1. Army Med. Museum. 
tion. The evacuations are frequent and watery, contain neither pus, blood, nor mucus, which one would expect to encounter in lesions of the same number and size from other causes, are not preceded l,y abdominal discomfort, nor is defecation accompanied log pain or tenesmus. This form of diarthea is comtinuous. olstinate, exhaustive, and is largely responsible for death in many cases.

The diagnosis of amyloid degeneration of the bowel is exceedingly clifficult, lunt can be arrived at in a fair proportion of the cases. once diarrhea has developed, ly (a) learning from the history whether or not the patient has had iuberculosis, bone suppuration, or syphilis. which might leat to it; (b) examining the kidneys, liver, and spleen to see if they are enlarged, rounded, bogeg, and lardaceous: (o) searching the urine for amyloid casts; and $(d)$ noting whether there is mucur. pus, or blood in the stools, the absence of which would indicate the amyloid cause of the frequent morements.

Again, in these cases there is less disturbance from gas, cramps, abdominal pain, and defecation causes but little if any annoyance; but when the kidney is seriously involved the patient shows dropsical tendencies of the lexs, the urine is pale, clear, increased in quantity. generally contains a considerable amount of albumin, and has a low specifie gravity.

Finally, Virchow has shown that the lardaceous intestine exhibits a brownish-red color when treated with iodin, turns bluse or violet when sulphuric acid is used, and a brilliant rose color when methylviolet is employed.

The treatment of intestinal amylodosis is guided largely according to its etiology, being antitubercular, syphilitic, or malarial when one. of these diseases is the inciting factor, or surgical when it results from pus accumulation or diseased bone. When the process is general, the patient should be treated symptomatically, since little, if anything. can be aceomplished toward a cure, and the same holds true in a large measure concerning amyloidosis of the entire intestinal tract.

When the degeneration is limited to a part of the ileum, a segment of the colon, or entire large bowel, more favorable results can be of tained in many instances when heroic treatment is instituted. Inder these circumstances (the condition of the patient permitting) the diseased portion of intestine should be resected, short circuited, or excluded, according to the plan described in the chapters levoted on the Surgical Freatment of Diarrhea.

When the patient refuses or is toos ill for surgical intervention, he should be given a supportise treatment, including a nutritive. non-irritating diet, improving his hyeituic surroundings, prescribing tonics of iron, arsenic, quinin or strychnin, arlminiscering opium in combination with bismuth, tanuigen, tannoform, lamnallin, or ichthalbin to diminish the exacuations, and in frequently washing out the colon with soothing, antiseptic, or healing atgents io improxe the condition of the mucosa and lessen the langer from mixed infection. 


\section{CHAPTER V}

\section{ACUTE INFECTIOUS AND CONTAGIOUS DISEASES, DIARRHEA IN}

\section{IIEASLES, SCARIET FEVER, VARICEILA, VARIOLA, WHOOPING-COUGH PERTUSSIS, DIPHTHERIA, INFLUENZA, PNEUMIONIA, IIALARIA}

Diarrhea of Acute Infectious Diseases.-The regularity of the movements and their consistence are interfered with at one time or another in nearly all seneral infectious and contagious diseases. In some instances obstinate constipation prevails, but frequently there is a diarrheal tendency and the evacuations are increased from three to fifteen or twenty daily, according to the sererity of the case, causing great discomfort and weakness.

Diarrhea may occur during the course of acute infectious diseases in adults, but is encountered most often in infants and children. and frequently proves a serious complication.

It is conceded that in this class of cases the direct or indirect cause of the sastro-intestinal disturbance and diarrhea under varying conditions may be of nervous. muscular, chemical, bacterial, or toxic orisin. Sometimes the bowel remains intact. while in other instances the mucosa becomes inflamed or ulcerated. owing to local infection. and the lesions increase the evacuations by a exposing the nerveendings to trauma and irritating discharges which lead to frequent and marked peristalsis: $b$ increasing the secretion of mucus and transudation of fluid into the bowel: $(c)$ interfering with absorption which leaves a greater amount of fluid to be evacuated. Acute infectious diseases particularly in early life exert a baneful influence because of the intestinal catarrh which accompanies them and their tendency to lower the patient's re-istance. which afford the colon hacillus and other pathogenic bacteria normal inhabitants of the bowel an opportunity to multiply and increase their toxic products. which in turn augments the gastro-inte-tinal disturbance by causing frequent movements, colic, tympanites, enterospasm, and constitutional manifetations. viz.: increased temperature, pulse-rate, and nervous symptoms.

Again, diarrhea in acute infectious diseases may without local lesions cause frequent morements through the action of toxins upon the intestinal nerses brain, or general nerrous syotem. Internists have directed attention to diarrhea with an infections basis without inte-tinal lesions, and point to the fact that in incipient typhoid. croupou- pneumonia. erysipelas. influenza. meases. etc.. diarrhea often ensues. In different cases this can be accounted for by the 
nervous state of the patient (psychical disturlance incited by the disease), effect of the toxins upon the nerves, or irritant action of the poison upon the intestinal mucosa. The author on various occasions has examined the rectum and sigmoid tlexure through the proctoscope and sigmoidoscope during and following attacks of atcute infectious rliseases wherein diarrhea was a complication. In every instance there were evidences of catarth (mucus, pus, or blood, alone or admixed), or erosions or ulcers were found in numbers sufficiont to account for the loose movements. In some instances the loral lesions were simple in character, and had existed previous to the infection, or were a manifestation of the disease; while in others, examination of the discharge, scrapings from the ulcers, or an analysis of the stools demonstrated that the lesions were specific and due to the infections disease.

In conjunction with his colleague, Dr. Pisck, a necropsy was performed by the author upon an infant who died from diphtheria wherein diarrhea was a serious complication. In this case the rectum and sigmoid flexure were extensively ulcerated, and the gross and microscopic examinations of the removed bowel showed that the lesions were diphtheric. From his examinations and experience with this and other acute constitutional infectious diseases the author believes that in the vast majority of instances bowel lesions are present and can be found where diarrhea is pronounced, and that in this class of cases more attention should be paid to bowel irrigation and topical applications than is at present being done to overcome complicating diarrhea.

Cases have been observed where involvement of the bowel was pronounced, and others where the local manifestations were less marked, but had not gotten well because the patient had been subjected to one acute infectious disease after another, which gave the bowel no opportunity to becone functionally normal. Lnder such circumstances and where the patient is left in a hysteric condition, responds actively to excitement or other emotion, or is neurasthenic, diarrhea may be continuous or occur periodically upon excitement for a long time after the infectious disease causing it has disappeared, thus making it necessary to care for the mental and nervous condition of the patient and avoid errors in diet which would exacerbate the diarrhea. In this connection it may be said that constipation prevails or the morements are but slightly increased when infection is mild, and diarrhea is frepuent and serious in virulent aggravated infections diseases.

Naturally, the consistence and composition of the stools vary in different cases, depending upon the nature of the infection, its effect upon the bowel, and presence of a pre-existing pathologic condition of the intestine. When diarrhea exists as a complication the evacuattions are usually watery, and fungi, protozoa, or specific bacteria (according to the nature of the infection) are present in the feces. but when there is a coexisting irritative or ulcerating enterocolitis, mucus, pus, and blood are also present. 
With these general remarks upon the relation of diarrheal manifestations to acute infectious diseases, the writer will now discuss the inclividual diseases.

Measles, Diarrhea in.-Generally the bowel is not greatly disturbed in measles, but occasionally its function is temporarily impaired, and the patient suffers from diarrhea or constipation. In rare instances the stools may become frequent, annoying, or even dangerous; in fact, next to bronchopneumonia, this symptom constitutes the most serious complication in infants and young children, but is seldom alarming in adults.

The small or large intestine, or both, may be affected, and ordinarily regularity of the stools is not greatly interfered with in the prodromal stage of measles, but when it is, the evacuations are profuse, occur in quick succession, rapidly exhaust the patient, and render the prognosis extremely rare. On the contrary, when the loose movements do not begin until after the eruptive stage, there is much less danger from this source, even though the evacuations are frequent and profuse. When diarrhea and bronchopneumonia both complicate measles, one may expect a rapid and fatal termination. In cases where accelerated evacuations occur simultaneously with coryza, or when absent, with nausea and romiting, they are excited by an intestinal exanthem, and the presence of bile in the feces inclicates that the trouble in part is attributable to biliousness. In the later stages, however, the evacuations frequently take on a choleriform or dysenteriform character-a most grave manifestation, particularly in young children.

I'sually the stools are watery, but may contain mucus, pure or in shreds, some blood, pus, or an admixture of all. In one case the cliarrhea may be limited to two or three movements and last but a day or two, while in another there may be ten to twenty morements daily, and the trouble may continue throughout the disease or remain indefinitely when the mucosa has undergone organic changes, the nerves become irritable, or digestion has been impaired. When enterocolitis is a complication the patient suffers considerably from cramps, tender spots along the colon, and voids a large amount of mucus, pus, and blood.

Scarlet Fever, Diarrhea in.-Scarlet ferer is complicated more frequently by constipation than diarrhea, but when there is sepsis the evacuations become frequent, foul, of a greenish color, and contain blood, an indication that the patient is or will become dangerously ill.

Varicella (Chicken-pox), Diarrhea in.-When diarrhea is a manifestation of chicken-pox it is usually due to errors in diet or incidental disease. This symptom most often occurs at the onset, is dangerous during this stage, and usually ceases with appearance of the eruption. The stools are profuse, obnoxious, contain blood (in the hemorrhagic form), and are very difficult to control with antidiarrheal remedies and colonic irrigation. 
Variola (Small-pox), Diarrhea in.-Frequency of the morements is often considerably increased in small-pox patients, but the diarrhea is encountered early, continues until the eruption appears, when it ceases and the patient feels greatly relieved. In exceptional cases, where this stmptom prevails during the later stages of the disease, the evacuations are offensive, exhausting. and indicate, in the absence or presence of intestinal hemorrhage, an unfarrorable prognosis.

Whooping-cough (Pertussis), Diarrhea in.-This is rarely accompanied by tiarrhea during the violent coughing stage, but during convalescence and later, owing to lowered resistance, the patient frequently develops simple gastro-intestinal catarrh or specific colitis when the subject is tubercular.

Diphtheria, Diarrhea in.-As has been shown by the case preriously referred to (in the general remarks upon Constitutional Infectious Diseases), diphtheria is sometimes accompanied ly severe specific or catarrhal intestinal disturbances and diarrhea. When the former prevails, the patient is profoundly poisoned, the intestine is congested, the mucosa is extensively ulcerated. and considerable sized sloughs may be expelled. Owing to these lesions. effects of toxins upon the nervous system and irritation to the glands and nerveendings at ulcerated spots caused by trauma of the feces and acrid discharges, persistent diarrhea is incited and the patient voids in rapid succession many offensive evacuation containing a large amount of pus, blood, and mucus, and unless relief comes promptly patients soon die from exhaustion or sepsis. This condition is aroidable when diphtheria is discovered early, antitoxin administered. and a proper course of treatment instituted.

In cases where frequent movements are due to the presence of an already existing enterocolitis, or one which is temporary and a part of the disease, the evacuations are less frequent and offensive. contain an abnormal amount of mucus, but little, if any, blood or pus.

Influenza, Diarrhea in.-Influenza is usually characterized by an inflamed condition of the mucous membranes, and very often the gastro-intestinal tract is involved sufficiently to cause nausea, romiting, and abdominal discomfort or pain, with an increased frequency of the eracuations, which contain considerable mucus. In some instances fliarrhea is persistent. in which case the inciting cause of the frequent morements mar be attributed to catarrh of the bowel, toxins which finel their way into it, irritable and nerrous state of the patient. or errors in diet. The diarrhea may last a flay or two throughout the attack, or continue regularly or periodically (when the patient rakes cold, eats indigestible food, or becomes nerious) for a long time after all signs of influenza have disappeared. Grip suljects have often a greatly lowered resistance and usually a congested. sensitive or eroded intestinal mucosa: all of which conditions predispose them to tubercular and other types of bowel infection which they withstand badly. 
Pneumonia, Diarrhea in.-Diarrhea often seriously complicates pneumonia in the earlier stages. The irritable condition of the bowel in some cases is attributable to aggravation by the disease of a preriously existing enterocolitis or to emigration of the pneumococci (Weichselbaum). Evidently the effect of the generated toxins upon the mucosa and the local and general nervous mechanism has considerable to do with causing and prolonging the diarrhea.

Treatment of Diarrhea Consequent Upon Acute Infectious Diseases.- In the handling of this class of affections the first and most important thing is to take the necessary precautions to prevent spreading of the disease (prophylaxis) by isolating the patient and carrying out other measures for this purpose. According to the nature of the ailment. the treatment should be symptomatic or constitutional.

In most instances the patient should be kept quietly in bed, and the diet restricted largely to fluids or semisolids which are nutritious and non-irritating.

When there are indications of biliousness or of a fecal accumulation or collection of retained putrefying food remnants in the colon, causing frequent morements, calomel in broken doses, 2 to $3 \mathrm{gr}$., followed in the morning by a saline laxative, is indicated before remedies are prescribed to control the diarrhea or other manifestations.

Ordinarily, in the treatment of diarrhea. fluids are interdicted or limited, but in the handling of acute infectious diseases where it is a complication a'ater should be freely given, except when it greatly accelerates frequency of the movements. This is necessary because in these affections the secretions are often partially or completely suppressed, toxins accumulate and become concentrated, making it important that the skin, liver, kidneys, and emunctories be stimulated to greater activity by hot baths, medication (diuretics, etc.), and increasing the body fluids.

Owing to a depleted constitution, lowered resistance incident to a complicating disease, or undue virulence of the toxemia, cardiac complications, weak heart, nerious depression, and prostration become troublesome manifestations. Lnder such circumstances much can be done to sustain the patient during the crisis by the administration of whisky, strychnin, digitalis, and nitroglycerin by mouth or hypotermically, but due care shoukd be taken to avoid overstimulation.

Feier plars in some a slight and in others an important part in the infectious diseases, and measures instituted for its control must be varied to meet the indications. Slight fever is controllable by sponging, intestinal lavage, and mild diuretics (such as potassium citrate, sweet spirits of niter, and aconite), but when it does not respond to these measures, good results are to be had from aspirin, acetanilid, phenacetin, or quinin, gr. $v(0.30)$, given as often as required, but the coal-tar preparations are not desirable because of their effect upon the heart, and should not be prescribed in large doses or continued 
for a lengthy period. When the temperature is regular and high, hydrotherapy (sponging, cold packs, baths, and bowel irrigation) should take precedence over drugs. because it is effective and is folllowed by a beneficent reaction upon the circulation, which strengthens and soothes the patient.

In some of these infectious diseases general or local sepsis is a serious complication. and it is necessary to keep the emunctories active, increase the fluiels of the body, support the patient with nourishing food and stimulants, administer constitutional remerlic(chlorid of mercury, creosote, etc.) and local antiseptics (salol, betanaphthol, salicylate of bismuth, etc.) in large and frepuent doses, ancl flush the colon two or three times daily with a normal saline or ichthyol, 2 per cent.; balsam of Peru, 2 per cent.; boric acid, 3 per cent.: potassium permanganate. I per cent. solution.

Controlling diarrhea complicating acute infections diseasc- in the main consists in treating these affections in the usual way. and in treating the diarrhea symptomatically.

When the moxements are influenced by emotions, precautions should be taken to prevent the patient from being frightened over his condition or excited in other ways. and when he is depreseded. strychnin, arsenic, and warm baths should le prescribed to culiet the nerves and court sleep.

When the bowel is clisturbed by local infection the treatment depends upon the nature and extent of the lesions.

Often there is simply a catarrhal inflammation of the mucosa. or it may be irritated by ulcers, bacteria or their toxin-. under which circumstances frequent and copious colonic irrigations with a normal warm saline. Carlshad salt. $\overline{3}$ ij to Oij $(60.0-1000.0)$, or a weak nncelicated solution such as boric acid, 2 per cent.; permanganate of potassium. I per cent.: balsam of Peru, ichthỵol. I per cent.: tannic acicl. 0.5 per cent.; or salicylate of soda. I per cent.. will prove effective in controlling diarrbea through their soothing and stimulating action upon the bowel, but when there are numerous erosion- or ulcers of considerable size the strength of the solution should le doubled or tripled. Large ulcers located in the sigmoid flexure and rectum. not otherwise curable, can usually be made to promptly heal by topical applications or cauterization made through the proctoseope.

Owing to the fact that frequent movements in this class of caseare attributable largely to nervous influence or lesions within the gut, astringent antidiarrheal remedies are contra-indicated in mo-t instances, frequently prove ineffective, and induce con-iderable irritation because they disturb digestion and fail to relieve the diarrhea.

Morphin or, preferably, opium, gr. $\frac{1}{4}(0.015)$, and the extract of belladonna, gr. $\frac{1}{8}(0.008)$, administered three or four times daily as long as necessary, are the remedies par excellence, because they limit or arrest the evacuations, relieve pain, and induce leep. When cramps are a complication and an opiate is undesirable, much relief 
is to be had from belladonna, gr. $\frac{1}{4}(0.015)$, and bot abdominal fomentations, which allay pain and muscular irritability.

Owing to the coryza and other evidences of a catarrhal inflammation of the air-passages in measles, influenza, and other acute constitutional infectious diseases, precautions should lee taken to protect the patient from drafts and breathing cold air to aroid severe ear and lung complications. In addition, in scarlet fever and diphtheria it is necesiary to frequently spray and cleanse the nose and throat with mild antiseptic solutions, because during the acute stages these parts are apt to become infected and the patient becomes septic, particularly in diphtheria cases where antitoxin is not administered early. When catarrhal pneumonia or bronchopneumonia complicate infectious diseases the patient should be protected from exposure and the inhalation of chilled air, and a supporting treatment, including nutritive fluid and semisolid foods, tonics, stimulants, and medicines which will relieve the cough, diarrhea, and other distressing symptoms, should be prescribed.

Malaria, Diarrhea in.-Diarrhea of malaria requires the same treatment as loose movements from the other infectious diseases, with the exception that quinin should be prescribed in liberal doses as of ten and as long as is necessary to destroy the malarial parasite.

Fairly good results have recently been reported from the use of antistreptococcic serum in the treatment of scarlet fever and inoperable cases of septicemia, and this would seen to justify the employment of this agent in the treatment of exhausting diarrheas originating from those and other septic sources.

Nearly all subjects of acute constitutional infectious diseases are left in a weakened condition, and have a lowered resistance and a tendency toward gastro-intestinal catarrh. Consequently, it is adrisable, as soon as possible, to change their surroundings and place them in the country, where they can get plenty of suitable exercise, nourishing food, and fresh air free from moisture.

From what has been said it is easy to understand why the treatment of diarrhea accompanying constitutional infectious diseases should be modified to suit the individual case. 


\section{CHAPTER VI}

\section{ACUTE INFECTIOUS AND CONTAGIOUS DISEASES, DIARRHEA IN (Concluded)}

\section{TYPHOID, RELAPSING, AND YELLOW FEVERS, CHOLERA, WINTER CHOLERA, CHOLERIFORM DIARRHEAS, SEPSIS, ERYSIPELAS}

Typhoid (Enteric) Fever.-Diarrhea may be encountered in any and all stages of typhoid fever, but usually follows constipation near the end of the second week or about the time decisive lesions are forming within the small bowel. No doubt the increased frecpuency of the evacuations are partially due to irritation, but they cannot be attributed solely to the swollen placques or ulcers (Figs. I $8-2$ I), ${ }^{1}$ because many autopsies have been performed where such lesions were both numerous and large and diarrhea was conspicuously absent. The diarrhea in these cases is partially due to local irritant effects of toxins generated within the intestine, and also to the general toxic condition of the patient, since it is known that nearly all septicemias induce frequency of the movements at one stage or another. It is also quite probable that the bile which would not unduly stimulate normal mucosa does irritate the inflamed gut here and exaggerates peristalsis.

In enteric fever the movements may vary from two or three to a dozen or more daily, according to the severity of the case; the stools are yellow and mushy at first and later thinner, resembling in color and consistency purée of peas (due to bile-pigment), alkaline in reaction, having a characteristically disagreeable odor, and, when left to stand, the solid matter settles. During the critical stage the evacuations may contain sloughing tissue particles and blood in small amounts when coming from capillaries, and in considerable amounts when from larger vessels. In other respects the macroscopic and microscopic appearance of the stool resembles that of cararrhal or infectious enterocolitis, ulcerative infection of the bowel, and general sepsis.

Yellow Fever, Diarrhea in.-This is an acute infectious disease (endemic and epidemic) which formerly destroyed many lives in the Southern States, Cuba, etc. but which has been almost eradicated by improved sanitation and isolating patients having the fever.

The lisease is characterized by a janndiced skin, anemic liver, friable pancreas, nephritis, dark blood, glandnlar enlargement, and acute catarrh of the gastro-intestinal mucosa (Fig. 22) complicated by mimute hemorrhages.

Symptoms.--Incubation (two to five days), anorexia, headache, etc First stage, chill, high fever, headache, aching in limbs, delirium, fast 
pulse, swollen gums, burning in stomach, nausea, and black romitus. 'Then weakness, jaundice, collapse, hemorrhages (gastric), fast pulse, blatck romitus, dry tongue, suppression of or deficient urine albumin), uremic convulsions or coma, and in fatal cases terminates in one week.

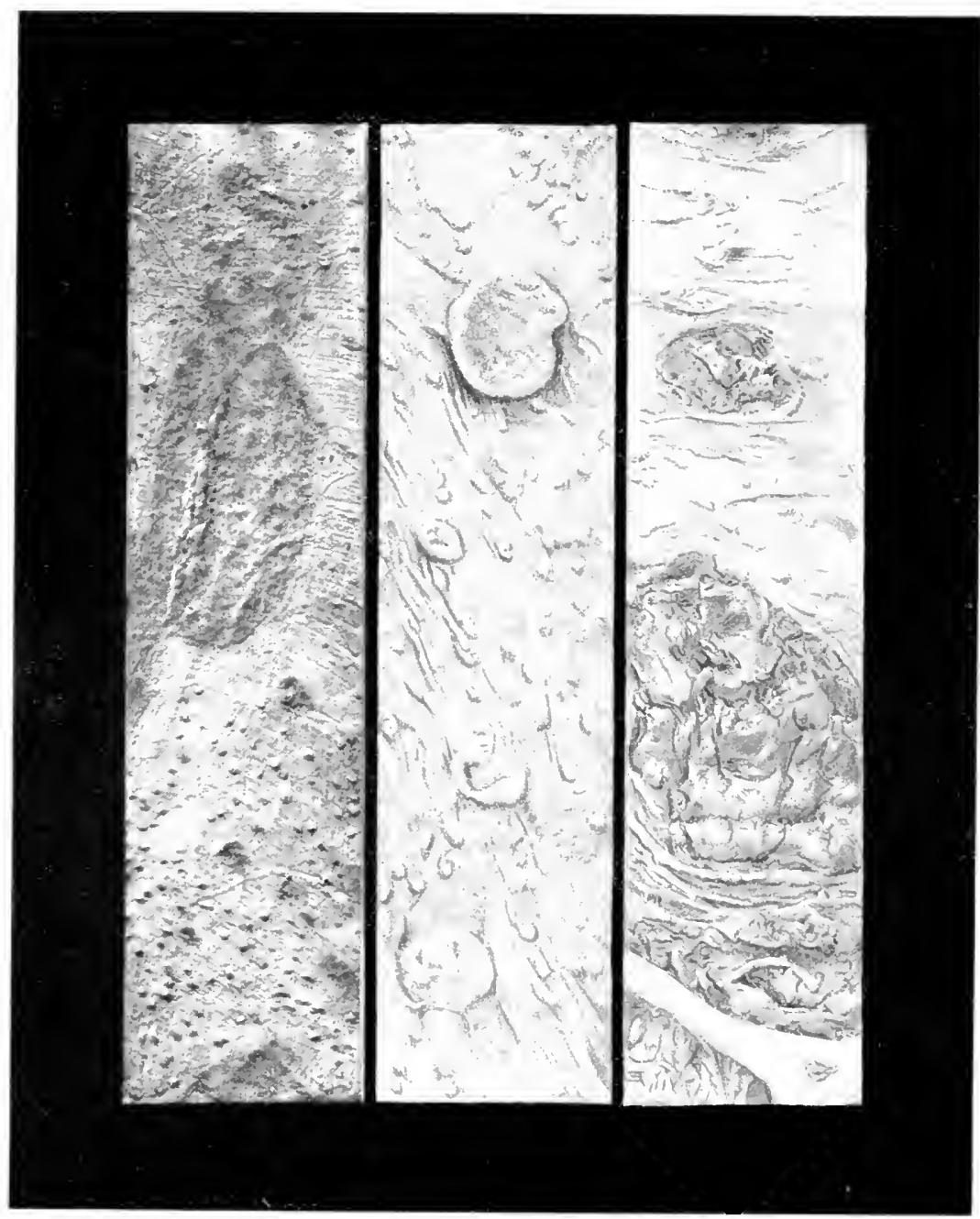

Firm. 18 .

Fig. IS.-Typhoid fever. Ippearance of mucosa, first week.
Fig. IO.

Fig. I - - Typhoid fever. Appearance of mucosa, second week.
Fing, 20

Fig. 20.-Tyzhoid fever. Appearance of mucosa, third week.

Diagnosis.-By symptoms, exchusion, and Wiclal's reaction.

Prosnosis. - The leath-rate may vary from I per cent. in light to Io per cent. in mild and 30 to 50 per cent. in severe forms of the lisease. 
Treatment consists in prophylaxis, guarantining, prevention against and destruction of the Stegomyia fasciata, having the pationt rest in bed or remain upon a flud or light diet, stimulating the emunctories, applying ice to the stomach, and administering sodium bicarlonate, gr. $\mathrm{x}$ to $\mathrm{xx}\left(0.65^{-1} \cdot 30\right)$, in Apollinaris water to eliminish acidity of the secretions.

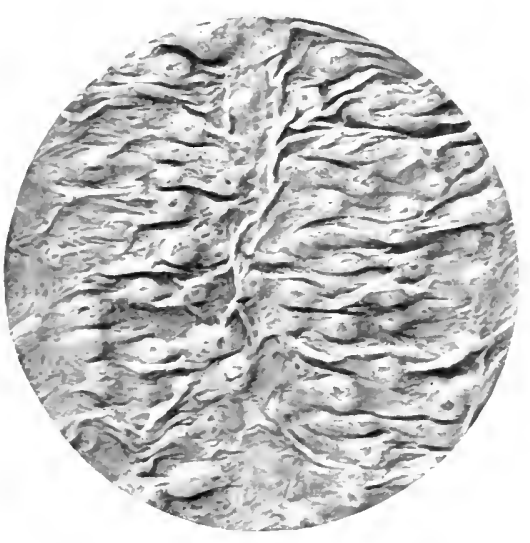

Fir. 21.-Typhoid fever. Section of transverse colon with enlarged glands ulcerated at their apices.

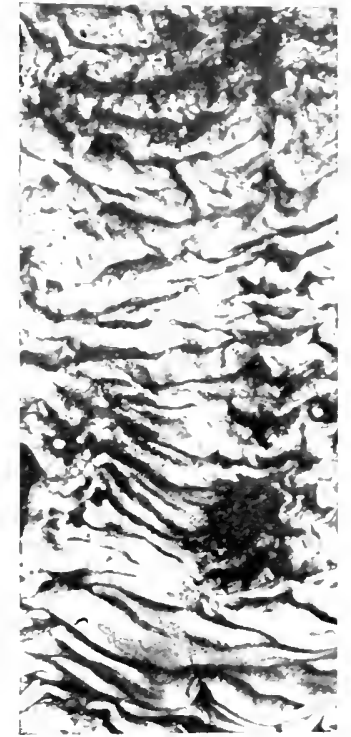

Fin. 22.- Yellow fever of the small intestine, showing capillary hemorrhages.

Relapsing Fever (Febris Recurrens), Diarrhea in.-This affection is caused by spirochetes, characterized by attacks of fever which last for about a week, and then subside to again recur in about the same time.

Relapsing fever is sometimes complicated by gastric disturbances. but diarrhea is a rare complication of the affection.

The treatment for it consists mainly in improving the hygienic condition of the patient, having him remain quiet while his temperature is high, regulating the diet, and giving an opiate when pain in the limbs cannot be otherwise relieved.

Cholera, Diarrhea in.-Epiclemic Asiatic cholera is greatly dreaded. because once it gets a hold it spreads rapidly and in a large proportion of the cases ends fatally. Fortumately, ravages from it are not nearly so disheartening as formerly, because national and local boards of health have learned how to arrest or provide against it. Recently it made considerable progress in Russia, southern Italy, and the Balkan States, but, in so far as out possessions are concerned, it has leen confined to the Philippines. except in 1873 and 1002 , when chelera obtained a strong footing in the Inited States.

The cause of this acute infectious disease is the commu bacillus of Koch or the Vibrio cholere Asiatice. This organism derive it 
name from its resemblance to a comma 1.$)$, looks somewhat like bacilli of chicken and pigeon cholera. is thicker and shorter than the tubercle hacillus, and is always deteetable in the discharge from persons infected. frequently with the colon bacillus, which aggrarates the condition.

Occasionally there is a short prodrome of ordinary diarrhea in Asiatic cholera, but in the vast majority of instances the disease is of a sudden onset. severe from the start. and terminates quickly and fatally in a goodly percentage of cases.

In severe cases in the beginning the patient complains of nausea and abdominal discomfort, and a little later of active peristalsis, with

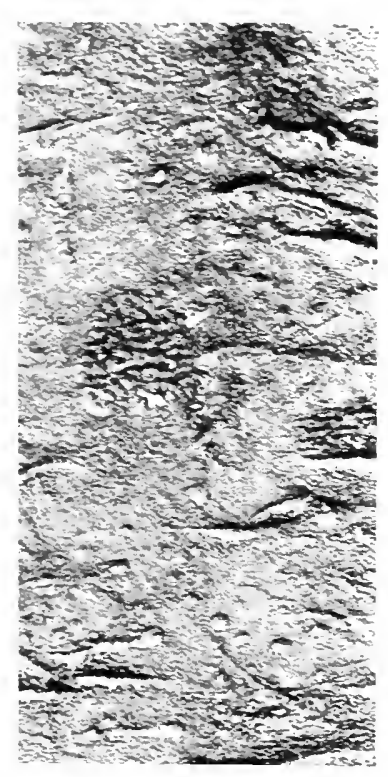

Fir. 23.-Appearance of the mucosa in Asiatic cholera. tenderness over the small intestine. which is soon followed by diarrhea and softened stools containing fecal matter. Shorty. however. the evacuations hecome characteristically- frequent, profuse, odorless, colorless, and painless, resemble whey or rice-water. are neutral or alkaline in reaction, contain no fecal matter (I to 2 per cent. solids). perhaps blood. and the patient romits food and transudated fluid, suffers from dizziness. cramps. complete loss of appetite, thirst. dryness of the tongue, mouth, and throat. sensations of heat and pressure from the Huid within the abdomen, gradually increasing weakened heart action, eridences of collapse. coldness, impaired circulation. livid skin, subnormal surface, muscular contraction in the extremities and elevated rectal temperature, pinched and exhausted facial expression, difficult respiration, partial suppression of urine. indifference to his surroundings. and. in fatal cases, rapidy suc. cumbs to general prostration.

When he survives the above manifestations, gradual improvement takes place. nausea becomes less marked, the appetite returns. the movements are less frequent, and the feces gradually resume their normal solidity, except where the bowel continues irritable or there are lesions which lead to periodical attacks of diarrhea.

As would be expected in a riolent intestinal disturbance of this character, the mucosa is acutely inflamed. swollen. edematous, and irritable. The follicles are enlarged (Fig. $23^{1}$ ) and. in the smail intestine occasionally strings of mucu- are found adherent to the mucous membrane, and the bowel is filled with the transudated rice-water discharge, especially in cases where there is a paretic tendency. Exceptionally, fairly well-dereloped ulcers are olserved.

The diagnosis of cholera is usually apparent when it is epidemic 
in the vicinity, but when there is doubt as to whether the patient suffers from Asiatic cholera, cholera morbus, or choleriform diarrhea. the diagmosis can be quickly cleared up by examining the stomb and finding the comma hacillus (veriffed ly gelatin or agar plate cultures), and by aseertaining if the patient has taken arenic, mercury, or other drug which would catuse evacuations simulating thone of cholera.

The prognosis of Asiatic cholera is grave, becallese a large percentage of the eases die within two days or later, during the so-called typhoid stage; oceasionally, however, the infection is not virulent and the patient recovers in a comparatively short time.

Treatment.-The prophylactic measures consist in isolating the patient and disinfecting the stools and everything that has leeen in contaet with him. Treatment of the disease proper ennists chiefly in withelrawing foods likely to irritate the stomach or intestine, administering calonel in large or broken doses when the patient is lilious, in prescribing morphin, gr. $\frac{1}{4}(0.015)$, or powdered opium, yr. $\frac{1}{2}(0.03)$, to relieve intestinal pain and cramps; water in abundance, 10 take the place of that lost by the constant transudation and rapicl evacuations.

During the comparatively recent epidemic in the Philippines benefit was derived from a normal salt solution (enteroclysis and hypodermoclysis). The more of the solution used, the better, and in order to get the quickest and best results the patient should be inverted, the sigmoidoscope introduced, and from a half to a gallon of the hot saline should be poured directly into the bowel, which is easily accomplished within five or ten minutes. From numerous experiments mate the author is certain that a portion of the solution thus introduced reaches the small intestine.

This treatment is soothing owing to the heat. strengthening because the fluid enters the circulation, and generally beneficial because it limits the multiplication of bacteria and washes out the toxins. The irrigations should be repeated as often as the patient will permit.

Kausch highly recommends the subcutaneous or, preferably, intravenous infusion of grape-sugar in the treatment of cholera. Beginning with 1.000 c.c. of a 5 per cent. solution, he gradually increases the concentration and quantity of fluid with each injection, which is made two or more times daily. Practically all the sugar is taken up by the organism and helps to sustain the system.

Gastric lavage, in combination with the treatment, is of great value in these cases. The author has never treated a case of Asiatic cholera, but from his experience in the handling of cholera nostris and choleriform diarrheas. dyentery, etc. he believes that many patients treated by the older plan could be saverl if. as soon as the nature of the disease was discovered. his cecostomy (see Figs. 1 +2, it5), which provides a guick and easy method of irrigaling both the small and large intestine, was performed, lecanse with the aid of this procedure complete intestinal irrigation of the entire intestinal tract could be carried ont as often as indicated with water or saline or medicated solutions. 
It has been demonstrated that cholera germs may be swallowed and do little or no damage in one case. while in another death shortly ensues, accordingly as they rapidly multiply or generate toxins. If this is so. then the line of treatment suggested by the author ought to greatly minimize the attack or affect a cure, becatuse the increase of hacteria and their toxins could be prevented by frequent gastrointertinal lavage.

Choleriform and Cholerine Diarrhea.-The expressions choleriform and cholerine have been employed by some authorities to indicate exceedingly mild Asiatic cholera, but they are confusing because they have aloo been used for other purposes. Consequently, from the author's view-point. all cases of cliarrhea incited by the comma bacillus should be designated as cholera. and the captions choleriform and cholerine should be reserved to indicate the profuse watery diarrhea in other conditions where the Tibrio cholerce Asiatice is absent. There terms are also frequently confused with cholera morbus of adults and cholera infantum of infants and children, conditions having practically the same etiology and pathology but which prevail during warm or hot periods. and because of this should be considered as seasonal ailments.

Choleriform and cholerine diarthea have no entity, and on account of this these terms are not emplosed by the author to indicate a particular type of disease, but to designate the intensity of the diarrheal condition in any ailment where the stools are frequent, thin, and composed of transudated fluid and otherwise resemble the evacuations in Asiatic cholera.

Cholera Nostras (Sporadic Cholera), Diarrhea in.-Cholera nostras may be encountered at any time of the year and at any age, but it unquestionably occurs very much more frequently in the warm months (June to September) than at other times.

Cholera nostras is generally believed to be of specific origin. and the colon bacillus is thought to play a prominent part in its production, and it may be that streptococci work in harmony with this organism, because virulent specimens of both have frequently been simultaneously discovered, but sufficient proof is not yet forthcoming to convince one that they are the only causes of this condition. In fact, the spirillum of Finkler and Prior is often discovered in evacuations coming from persons afflicted with cholera nostras, and some authorities considered this agent the specific factor in the disease. Again, the bacillus of Shiga and the Flexner-Harris and like organisms have also been detected, and some believe that ther are the principal causes of the troulle. Ordinarily, when either of the lastnamed micro-organisms are present and the disease is fully dereloped. definite lesions of the mucosa are discoverable through the sigmoidoscope, and the discharges contain more or less blood and some pus. while under other circumstances these manifestations are entirely absent or much less marked. Because of this, it has been the custom of the author to classify diarrheas, where the stools contained the Shiga 
or Flexner-Harris bacillus, as dysenteric, but in the absence of blood, where other organisms are present, as cholera nostras (morbus), this arrangement is not always satisfactory because of varying severity of the attacks and complications which arise.

In support of the theory that cholera nostrats is not of bacterial origin, some authorities site the well-known fact that an attack may be brought on by sudden overheating or chilling, the drinking of icewater ancl other cold beverages, and the eating of certain shellfish, ingestion of poisons, irritating foods and fruits, and impure water.

The symptoms are of sudden onset, and begin with nausea, which is followed shortly by vomiting, abdominal pain and tenesmus, and a violent or exhausting diarrhea. At first the stomach expels food, but later nothing but mucus and bile, and the evacuations, which in the beginning are composed of fecal matter, later rapidly follow each other and become soft, watery, and greatly resemble the ricewater discharges of Asiatic cholera. Within a few hours the patient shows the rapid progress of the disease in his thirst, pinched expression, clammy perspiration, cold skin, small weak pulse, impoverished circulation, indications of collapse, and often unconsciousness.

Exceptionally, persons afflicted with chotera nostras die, but, as a rule, the distressing symptoms gradually abate, partially or completely, unless sequele are left, in which case diarrhea may continue upon slight provocation.

In making the diasnosis it is necessary to differentiate between cholera morbus, amebic and bacillary dysentery, ptomain food poisoning, and Asiatic cholera (during epidemics), which when present are manifested by amebe or bacilli of Koch (comma bacillus). Shiga, or Flexner-Harris in the evacuations. When cultures show the colon bacilli alone or in conjuncture with streptococci to be of a virulent type, one is justified in attributing the cholera nostras to this organism, but when they are lacking, and there is evidence that the patient has taken considerable arsenic, mercury, been indiscreet in his diet or has eaten unhealthy food, the cause can be ascribed to mechanic irritation, putrefaction, or ptomain-poisoning.

The treatment of cholera nostras must be varied to suit the individual case at the time when seen, hecause it may be necessary to substitute one therapeutic measure for another, according as the symptoms vary from time to time, and the treatment effective in an adult is not always suitable for infants and children.

Ender all circumstances it is wise to temporarily restrict the diet to fluids consisting of predigested milk. soups. nourishing broths. strained gruels, and interdict cold drinks and carbonated beverages until the acute crisis is over, when raw or soft-boiled exgs, beef-juice extract or chopped beef, custards, milk pudding, milk toast, boiled rice, and similar articles of diet may be allowed. Later, as the patient gains strength and the movements have hecome nearly normal in frepuency and consistence, more solid foods agreeable to the patient 
can be gradually permitted. These patients suffer greatly from intestinal toxemia and los of the hody fluids. Consequently, it is advisahle to immediately prescribe copious bowel flushings until the patient ${ }^{\circ}$ condition is markedly improved, when the injections are given less often until inflammation of the mucosa subsides. A normal saline or weak solutions of magnesium sulphate. boric acid, ichthyol, tannic acid. or permanganate of potash ( I to 2 per cent.) may he enployed to eleanse the bowel. but the salt preparation is preferable hecause it accomplishes this purpose and is absorbed. partly replacing the fluid loss by the transudation.

Gastric lavage frequently affords the patient much relief, particularly in cases where chemic poisons or ptomains are the exciting cause. but when this simple measure does not arrest nausea and romiting. a mustard plaster applied to the stomach often brings the desired relief. The author has succeeded best in relieving gastric irritability and abdominal cramps by having the patient drink abundanty of hot water. use continuous hot moist abdominal applications, and by the administration of a pill or powder containing opium. gr. $\frac{1}{4} 0.015$, and belladonna. gr. $\frac{1}{9}(0.008)$. every two or three hours until the pain is relieved. except in urgent cases. where morphin hypodermically was administered.

In cases where the patient is bilious, has taken poison, there is fecal impaction or putrefying food remnants in the bowel, the above quieting medicines should be preceded by calomel. castor oil, or a saline laxative to stimulate the liver and free the bowel of its irritating contents.

Bland and antiseptic remedies. such as nagnesia, charcoal, bismuth subnitrate. subcarbonate or salicylate. salol or beta-naphthol, gr. $\quad 0.30$. three times a day, alone or in combination, do much toward restoring the bowel to its normal condition after acute manifestations have subsided.

Future attack are provided against by watching the patient's diet. preventing him from becoming overheated. taking violent exercise, exposing himself to inclement weather, and improving his general condition.

Winter Cholera.-In recent vears there have been a number of epidemics of intestinal thux, or choleriform diarrhea, which have broken out during cold weather. The manifestations of the condition are variable. and may resemble those of cholera. dysenteric colitis. typhoid fever or milder intestinal affections. The term "winter cholera" was first employed in Is I Michigan Board of Health). but the affection did not attract atuention until $\mathrm{s} s 9 \mathrm{~s}$, when sporadic cholera (winter diarrhea broke out at Escanaba, Mich., and caused a number of deaths. Epidemic of winter cholera occurred in Michigan City in I00I-02, and the disease has been encountered in other sections w the Middle Mest with more or less frequency since then.

The etiology of winter cholera is not understood. Some investisator: attribute it to cold weather. since it is met with only during the 
winter, but others maintain that such epidemics are traceable to polluted water, which is probably correct, since the causative agents of typhoid fever and bacillary colitis have been encountered in the stools of patients afflicted with winter cholera, as have also the colon and Bacillus enteritidis sporogenes, and other pathogenic organisms. No doubt in some instances there is a mixed infection. This type of diarrhea has frecpuently been associated with influenza, which some regard as the cause of the loose movements, but in the Escanaha epidemics (1907-08) Pfeiffer's bacillus was not demonstrable, and there was no reason for attributing the diarrhea to this cause.

Winter cholera has an uncertain pathology, and in different cases the changes in the intestinal mucosa incielent to it resemble those of non-specific enterocolitis, cholera, bacillary (dysenteric) colitis, and typhoid fever.

The symptoms are materially different in a series of cases, and Breitenbach. ${ }^{1}$ who has extensively investigated winter cholera, groups the manifestations of the affection into the following types, viz.:

Type I: Individual enjoying good health is suddenly taken with severe colicky pains, the bowel passages are increased from two to four a day. The stools are feculent, very offensive, at first semisolid, finally watery. These attacks are of short duration, seldom extending over a period of more than forty-eight hours, at the end of which time the patient may assume his former well-being. Because of the mildness of this type of disease it seldom comes to the notice of the physician.

Type 2: Individual enjoying good health is suddenly taken with feeling of malaise, nausea, and bilious vomiting. With or without these acute stomach symptoms at varying intervals, sharp colicky pains and a severe diarrhea appear. The stools are feculent, but very thin and offensive, and may number from one to twenty a day. Ol,jective symptoms are practically negative. Temperature is normal, pulse normal or slightly accelerated. The diagnosis of this condition is based on the acute onset, the characteristic gastro-intestinal disturbance, and the usual course of the disease. Patient is never really incapacitated for work, resorts to home remedies, or is subjected to routine treatment of castor oil in large doses. followed by astringents and bowel antiseptics, administered by his medical adviser, and is well in from twenty-four hours to three days.

Type 3: This type presents a most unusual array of symptoms. The cases in the history of their onset simulate, yet in their course and mortality differ materially from typhoid. Bowel symptoms vary in intensity, assuming in some few cases a dyenteric character, while in others there is constipation. Temperature in this class of cases varies from $98.6^{\circ}$ to $104^{\circ} \mathrm{F}$., is continuous but irregular. and, therefore, in this respect, entirely at variance with the remittent character of typhoid. The tongue is heavily furred, showing a

\footnotetext{
1 "Choleriform IDiarrhea of Cold Weather, Winter Cholera," Jour. Imer. Med. Assoc., October 3i, 1908.
} 
bright tip and margins. Delirium in these cases is of frequent occurrence. The 11 idal and diazo reactions are negative.

The disease is diagnosed from its occurrence during the winter months, the above symptoms, and finding of infecting agents in the stools, food, or water being consumed by the patient or community.

The mortality of winter cholera is rather high, and some idea of the seriousness of the affection may be sained by a study of the accompanying table compiled by Breitenbach:

\begin{tabular}{|c|c|c|c|}
\hline leut & $\begin{array}{l}\text { Estimated } \\
\text { population. }\end{array}$ & Deaths. & $\begin{array}{l}\text { Death-rate } \\
\text { per 100.000. }\end{array}$ \\
\hline Isos & 10.500 & 10 & 90 \\
\hline Isoa. & 10.500 & 8 & 70 \\
\hline 1000 & 10.003 & 25 & 247 \\
\hline 1001 & $10.4+4$ & I 5 & $1+3$ \\
\hline 1002 & $10 ., 05$ & 22 & 203 \\
\hline 100.3 & II.I t 6 & is & IOI \\
\hline 1004 & II .500 & 37 & 321 \\
\hline 1005 & I I . 500 & 49 & 426 \\
\hline 1005 & II .500 & 28 & $2+3$ \\
\hline $\mathrm{I} 0 \mathrm{O}^{-}$ & 11.500 & 8 & 69 \\
\hline
\end{tabular}

The treatment of winter cholera consists chiefly in protecting the community from extension of the disease by purifying the drinkingwater, isolating the patient, and destrosing his excreta, and, when the affection assumes a typhoid or dysenteric character, treating the sufferer as if he were afflicted with these diseases. In exceptional instances symptomatic must be substituted for curative measures.

Breitenbach's conclusions regarding winter cholera are as follows:

I) Winter cholera has no specific bacteriologic pathology, and is a synonym for the more common forms of bowel disturbances, choleriform in nature, occurring in cold weather.

2) The use of this term interchangeably with the nomenclature of other gastro-intestinal diseases shows the great need of more exact phra-erology, based on correct diagnosis.

3) Meteorologic conditions do not prove to be the exciting cause in these epidemic of winter cholera, but only as the factors instrumental in polluting water or milk are present do these epidemics occur.

4) Winter cholera, as typified by these choleriform manifestation- of diarrhea in cold weather, has no exciting cause in the activity of Pfeiffer: bacillus, and does not, therefore, exemplify gastrointestinal influenza.

5 Influenza, with a symptomatology centering itself in the wastro-intestinal tract, was present during epidemics of winter cholera. lout these isolated cases occurred during epidemic influenza. Diagnosis in these cases is established by proving the presence of the asocriated micro-oreanism.

6) To the end of -timulating a greater enthusiasm in demanding intelligent and adequate sanitary reform. instruction in sanitary 
science should demand a major consideration in the medical curriculum.

(7) Legislation controlling the watersheds and preventing contamination of public waters is imperative.

Sepsis, Diarrhea in.- This type of diarrhea is uncommon, lout when present it is difficult and often impossible to control, because it represents only one of the manifestations of a local or general sepsis which often terminates fatally. The loose movements may occur as a result of local infection within the bowel directly through excretion into the bowel of toxins, or reflexly through their effect upon the nervous apparatus, where, as the result of single or multiple septic foci located in different parts, pathogenic and pyogenic micro-organisms and their toxins have become disseminated throughout the body.

Acute sepsis may interfere with digestion. produce an irritable state of the stomach and intestine, and in this way, together with the toxins, cause moderate diarrhea in one instance, or temporary paralysis of the bowel and constipation in another. While hemorrhagic changes have occasionally been observed, proctoscopic and sigmoidoscopic examinations of the bowel at autopsies have shown that in these cases the nucosa usually remains intact, which would indicate that the disturbance responsible for the frequent movements is mainly of toxic origin. Some authorities have reported cases where diarrhea was a serious or fatal complication in women suffering from puerperal sepsis, but Jurgensen has never observed massive diarrheas under such circumstances.

In this class of cases septicemia and pyemia are generally caused by streptococci, staphylococci, preumococci, colon bacilli, gonococci, etc. Of these inciting factors, streptococci are encountered in about 75 per cent., and pneumococci and staphylococci in so per cent., of the cases. Other micro-organisms are met with much less frecuently.

The manifestations reflected wy gastro-intestinal disturlances consequent upon sepsis are loss of appetite, nausea, romiting, in certain cases diarrhea with simy evacuations containing some blool, mild or severe tenesmus, occasionally icterus or severe bleeding, and, in rare instances, peritonitis, through the formation of a septic embolism in one of the large ressels of the bowel or mesentery.

Rolleston, in summarizing his conclusions relative to purpura in infections complicated by diarrhea, says:

(1) Symptomatic purpura in infective diarrhea mainly occurs on the ablomen and chest of infants under the age of one year.

(2) It is usually a terminal phenomenon in prolonged cases.

(3) The prognosis in these cases is extremely grave.

The treatment of septic diarrhea consists primarily in removal of the foci ley operation and drainage when feasilsle, hut, when the process has become general, supportive measures, stimulation of the emunctories, abundant intake of fluids, administration of calomel, creosote, and other antiseptics and hỵdragogues to disinfect and cleanse the bowel are indicated. In the meanwhile the patient 
strength should be sustained by a nutritive diet, composed principally of liquids, such as milk, whites of eggs, strong broths, and meat juice, and hy stimulating the heart with whisky, strychnin, or ammonia. Quinin, gr. $x(0.60)$, lhree or four times datily influences the temperature when high, but, in the author's opinion, has no curative effect.

It is generally agreed that the most reliable agent to employ when eliminating bacteria and their toxins is a normal saline solution in large amounts, administered in the form of both hypodermoclysis and high enemata, which serve the double purpose of diluting the blood and cleansing the bowel of its irritating contents. The solution should be used as frequently and in as large amounts as the patient will stand.

When the patient is in a critical condition and surgical intervention for any reason is impracticable, antistreptococcic serum should be injected, although the results from it have thus far been disappointing. Anders recommends the employment at first of rooo units of Marmorek's serum daily or at longer intervals later, and says that Pearce prefers polyalent serum, which seemingly gives better results.

In pyemia. in addition to the above treatment, the exhausting sweats are best controlled by belladonna, gr. $\frac{1}{4}(0.008)$; atropin, gr. $\frac{1}{1}=(0.0005)$; or agaricin, gr. $\frac{1}{3}$ to $\frac{1}{4}(0.008-0.016)$, administered at night or oftener if indicated.

Erysipelas, Diarrhea in.-In this affection diarrhea is very often an annoying symptom, and the evacuation contains more or less blood, owing to the fact that the bowel at times becomes congested and ulcerated. Tillmann holds that the appearance of the bowel resembles that sometimes seen in cases of extensive burns. 


\section{MISCELLANEOUS INFECTIOUS DISEASES, DIARRHEA IN}

TROPICAL DIARRHEAS: COCHIN-CHINA DIARRHEA, SPRUE, HILL-DIARRHEA, DIARRHEA ALBA, PSEUDODYSENTERY, EL BICHO DIARRHEA, ANTHRAX, MALARIA, TYPHUS FEVER, PLAGUE, GLANDERS, SUTIKA, INTESTINAL MYIASIS, PELLAGRA, ACTINOMYCOSIS

Tropical Diarrheas: Cochin-China Diarrhea, Sprue, Hill-Diarrhea, Diarrhea Alba, Pseudodysentery, and El Bicho Diarrhea.- Inder the above captions the author will cliscuss certain types of diarrhea common to tropical countries, which are probably caused by entamebic or bacillary infection, but which are still considered as distinct affections by some of our best authorities.

Entamebic and bacillary colitis will not be discussed here because they have received full consideration in other chapters set apart for the purpose.

Diarrhea is prevalent in all tropical countries, and a number of reasons can be advanced to explain why this is so. In many instances the majority of the inhabitants of tropical lands are rendered amenable to infection because of their lowered resistance, brought about through unhealthy unhygienic surroundings, insufficient, poor, or improperly prepared food, and the intense heat and high humidity of such countries. Tropical diarrhea may result from infection of the individual, his food or water, by flies, gnats, fleas, mosquitos, and other carriers of infection which thrive in warm countries where the air contains considerable moisture. The chief cause of the loose movements in this class of cases is often traceable to water pollution. because the people are ignorant or careless as to hygienic conditions. and take but few if any precautions toward keeping their watersupply from becoming infected by decayed regetable and animal products, the excreta, etc.; nor do they appreciate the importance of or take precautionary measures to prevent, extension of the infection from afflicted to healthy individuals through proper disposition of their refuse.

The enormous benefits to be derived by preventive measures in diarrhea and other tropical diseases has been demonstrated in Cula, Porto Rico, and the Panama Canal Zone since they were taken over by our government.

Again, a number of infective and parasitic diseases which induce diarrhea are endemic in hot countries, but are seldom encountered in the northern part of the United States and in cold countries, except where infected individuals come from tropical countries, and persons become infected by contact with them. 
Dysentery and eertain parasitic affections have for a long time been encountered in the Southern States, particularly in hot and swampy districts, but it is only in recent years, since our soldiers, sailors, and citizens began returning from Cuba, Porto Rico. Panama, and the Philippines, that these diseases made their appearance in the north.

While diarrhea consequent upon simple enteritis or enterocolitis may be encountered in hot as in other countries, in most instances the loose movements are traceable to an intestinal infection of some kind or to atmospheric conditions.

Tropical diarrhea has been described by different authorities under the captions of Sprue, Psilosis Lingua et Mucose Intestini, Aphthre Tropica, Cochinchinitis, Hill-diarrhea, Pseudodysentery, and White Flux (Diarrhea Alba), but, from the author's study of the subject, it would appear that sprue (psilosis), pseudodysentery, Cochin-China diarrhea, and hill-diarrhea have individual characteristics, and that other so-called types of tropical diarrhea are variations of these affections.

Cochin-China Diarrhea.-This disease is so named because of its great prevalence in Cochin-China, where it has been responsible for a great many deaths, particularly during such epidemics as occurred in I 868 and I872. Cochinchinitis, or an affection with the same symptom-complex, has also been encountered with considerable frequency in India, southern China, Borneo, the Antilles, the Philippines, and South America. In these countries the whites are more susceptible to the disease than the yellow race, and children are more frequently attacked than adults, among whom the mortality is said to be 15 to 30 per cent.

The disease is considered to be infections, and is supposed to follow the ingestion of unhealthy water. which causes an enterocolitis.

The Strongyloides intestinalis are often found in great numbers in the stools of this class of patients, and were believed to be the cause of the disease until recent investigations demonstrated that they have but little if any pathologic significance in this connection. The author believes that Cochin-China diarrhea is due in some instances to entamebic (Entameba histolytica) and in others to bacillary (Shiga, Flexner, Strong bacilli, etc.) infection, and that the Strongyloides intestinalis simply aggravate the condition.

In two blood examinations by Lemoine small protoplasmic crowns with a refractive capsule, vesicular in shape, containing granules and an indistinct nucleus, were found. They were larger than red bloodcells, of constantly changing shape (the ameboid movements being observable for several hours), and he regarded them as the cause of Cochinchinitis. Owing to the absence of cultures and transmission, their etiologic connection, if any, was not established. The disease has also been attributed to other micro-organisms and parasites, but authors differ as to the organisms responsible for it. Lemoine, with reason, says that "doubtlessy" the diarrhea of hot climates is merely a symptom-complex, and it is probable that certain enteric 
affections grouped uncter this heading are due to various parasiten. such as protozoa, lamblia, megastoma of Cohnheim, trichomonas, and even all sorts of bacilli."

The symptom-complex of Cochin-China diarrhea is similar to that of catarrhal enterocolitis except that profound ancmia characterizes the disease. The movements are frequent and fluid, but do not contain pus or blood, and because of this and the abeenee of abdominal tenderness some authors hold that Cochinchiniti-should not be confused with tropical dysentery.

But little is known concerning the pathology of this conclition further than that there is congestion of the mucosa. swelling of the intestinal glands. which are covered with mucus, and a resemblance to catarrhal enteritis.

Sprue, Psilosis Linguæ et Mucosæ Intestini.-Thi- form of tropical diarrhea is particularly common to lndia and China, and differs principally from others in that the mucosa of the tongue and mouth early become exceedingly sensitive and ulcerated, from which circumstance the disease takes the name of "sprue," and the patient suffers from indigestion, diarrhea, emaciation, anemia, and may die from general weakness.

The etiology of psilosis, or sprue, is unknown, but the authorities who have studied it most concede its infective nature, although the inciting specific organism has not been discovered.

The attack is acute, frequently follows overeating; shortly the mouth and tongue become dry, swollen, glistening, sore. frequently furrowed, and the saliva acid; manifestations which are quickly followed by melancholia, indigestion, distress, tympanites, vomiting. and diarrhea with copious, foamy, offensive, pale-colored evacuations, which at the outset are greenish. The disturbances of the mouth and bowel usually occur and disappear about the same time. but the suffering from the former is much more distressing than the latter because of the pain incident to eating, talking, and swallowing.

Temporary improvement follows treatment, but the patient has frequent relapses, the disease wsually becomes chronic, the sufferer develops a deplorable anemia, and becomes emaciated through the great loss of weight ( 25 to 50 pounds in some instances).

The diarrhea is not severe in the incipient stages, since the patient rarely has more than three evacuations daily, but these are soft. exceedingly copious, and occur early in the morning. Later, as the ulcerated condition of the buccal cavity becomes more serious, their frecuency is increased, the patient rapidly becomes exhatsied. and assumes an anemic, pasty, characteristic appearance.

A diagnosis is comparatively easy, although sprue has been confused with entamelic and hacillary (o)litis (dyentery), cholera, and other severe types of inte-sinal infection, hut this is diffeult to understand because a comparion of the manifestations of it with such diseases readily shows their disimilarity, and in sprue the ulcerated condition of the mouth is pathognomonic. Lemoine says there in 
of ten a marked analogy between this condition and mucomembranous enterocolitis, but such a relation seems improbable to the author.

Bacteriologic examination of the feces is useful, since the finding of the specific micro-organisms of other diarrheal diseases would exchude sprue, and their absence woukl indicate this condition.

Hill-diarrhea. - This form of loose movements is frequently encountered in India and South America, or in Europeans who have lived in these countries. It is met with in persons who at one time or another have resided upon hills or mountains at an elevation of 6000 leet or more, and is supposed to result from a sudden change of temperature and eliminished atmospheric pressure (no specinc cause having been discovered, though it is probably a parasitic or a bacterial disease), which leads to chronic intestinal catarrh and diarrhea, but Cantlic thinks the condition arises from fine particles of gravel contained in the water. This affection may occur at any age and in either sex, but is more common in men than women, is rarely fatal, and often becomes chronic unless the pationt returns to a lower altitucle.

Persons afflicted with hill-diarrhea suffer from anorexia, malaise, Hatulency, dyspepsia, and a diarrhea wherein the movements are abundant, colorless, occur most often in the early morning hours, and have a frothy covering resembling that of sprue.

Diarrhea Alba (Diarrhea Chylosa, Celiac Disease).-This type of diarrhea, which has been described and clesignated ccliac discase by Gee, may occur at any age, but is encountered most frequently in children under five, and apparently results from a sort of combination between intestinal indigestion and catarrh, although parasites (Filaria sanguinis hominis) have been observed in connection with and may profluce it. This condition may be met with in any country, and because of its close resemblance has been confused with sprue and the hill-diarrhea of India. Diarrhea alba comes on slowly, and is characterized by emaciation, weakness, pallor, dropsy, tired feeling, boggy sensation on abdominal palpation, a moderately distended ablomen, and intestinal ulcers (unexplainable) have been observed. The mortality is high in these cases, but when the patient recovers he shows a manifest weakness in the legs and is often unable to junp.

The stools here differ from miscellaneous diarrheas, in that they are very bulky and are more abundant than the amount of food consumed would warrant, are soft, light colored (owing to the absence of bile), frothy, have an offensive odor, and closely resemble gruel or oatneal porridge.

The diagnosis is based upon the white and foamy character of the evacuations and finding of the filaria.

The prognosis in tropical diarrheas, excepting entanebic and bacillary, is good in midelle-aged and in youthful individuals, but grave in infancy and old age.

Treatment of Tropical Diarrheas.-The therapeutic measures em- 
ployed in the handling of patients afflicted with Cochin-China, sprue, hill-, and pseudodysenteric diarrhea are about the same, except in hill-diarthea, where it is advisable at the earliest opportunity to remove the patient from a high to a lower altitule. The most essential thing in the treatment is to protect him from the hot sun, keep him cool, resting in bed, and to see that he neither eats nor drinks contaninated water or food. On account of the ulcerated condition of the mouth and irritalle state of the intestine, coarse fords are contratindicated, and the patient should be sustained for a short or long time, according to indications, on milk, eggs, broths, and meatjuices, but warm milk in abundance causes the least discomfort and affords the necessary nourishment when taken in small amounts at short intervals. When after a few weeks the buccal condition improves, and the stools become normal or nearly so, the diet may be gradually increased until a fair amount of solids are taken. It is necessary for the patient to be cautious as regards his diet for a considerable time after an attack, as relapses are prone to oceur when the food is improperly cooked or he eats indiscriminately of beef, solids, raw vegetables, pickles, or drinks abundantly of alcoholic stimulants. When milk disagrees, this can often be corrected by mixing it with lime- or seltzer water.

The majority of patients recover under a strict milk diet, while medical agents avail but little, further than to control manifestations which suddenly become pronounced. At the beginning a laxative or calomel may be prescribed to advantage when biliousness or intestinal putrefaction are present, and Manson advocates intramuscular injections of arsenic and iron to relieve the anemia.

Much can be done to add to the patient's comfort by spraying the highly sensitive, inflamed, and ulcerated areas of the mouth and pharynx with a 4 per cent. solution of eucain to anesthetize them, and then painting, spraying, or bathing them with silver nitrate, 5 per cent., or other mild astringent or antiseptic solutions.

Stimulation and nutrient rectal enemata are indicated when food cannot be taken by mouth and the patient is rapidly being exhausted. A fruit diet has been recommended, but, reasoning from analogy in other diarrheas, this would seem to be had practice.

Diarrhea is best controlled by flushing the colon with soothing. antiseptic, and stimulating solutions, and by the administration of opium in small doses sufficiently often to reduce the evacuations. solidify the feces, relieve pain, and secure needed rest and sleep. But when these measures fail, appendicostomy or cecostomy and through-and-through irrigation are indicated.

The treatment of diarrhea alba is symptomatic in so far as the diarrhea is concerned, and consists in the administration of opiates, astringents, and antiseptics; but when there is reason to believe that the trouble is due to filariasis, thrmol, gr. ij to v $(0.12-0.30)$, (laily, has proved most efficacious, although as yed no effective specific has been discovered. 
Pseudodysenteric diarrhea is a type of toose movements encountered in hot countries, particularly Sumatra, wherein the evacuations have a dysenteriform appearance, but upon examination are found to contain neither entameba nor Shiga bacillus, though bacilli are present which become agglutinated through the patient's serum. The cultures resemble but are differentiated from Shiga bacillus by their mannite, and are not rendered immune by the Shiga serum.

Barmann-Schüffner have observed 6 cases in Sumatra, and state that on the basis of these findings it is justifiable to interpret these isolated bacteria as psendodysentery bacilli. Probably the disease, if not due to Shiga bacillus, is caused by bacilli of the Flexner, Hiss, or Harris types.

The symptoms, diagnosis, and treatment of this condition so closely resemble those of other tropical diarrheas as to make their further discussion superfluous.

Anthrax, Diarrhea in.-Anthrax may be accompanied by diarrheal manifestations, but this is exceptional, and when there are accelerated movements they are few in number, not of an exhausting type, rarely contain pus and blood, and are of slight importance as regards the prognosis, but are valuable from a diagnostic standpoint. When the bacilli in the form of immotile sporiferous rods are detected a diagnosis secondary to anthrax is justifiable.

Iwaschenzow has called attention to an epidemic of intestinal anthrax ( 7 cases) studied by him in Russia in the summer of 1909 , when all of the patients died. Three of the cases had been misinterpreted as ileus, on account of collapse, obstipation, and meteorism in the lower abdominal region. The observer directs attention to one symptom that is practically constant ( 5 of 7 cases), namely, the presence of free fluid in the lower abdominal cavity. The diagnosis was rendered during life in only $\mathrm{I}$ of 7 cases, and in this instance it was based on the history.

The treatment of external anthrax consists in excising the pustule, and when this is not feasible, in applying to or injecting into it weak solutions of carbolic acid; but in the internal variety, where the disease attacks the intestine and causes diarrhea, there is no hope for the patient, and he should be treated symptomatically and made comfortable while he lives.

Malaria, Diarrhea in.-Malaria generally runs its course without any serious bowel disturbances, but in exceptional instances there are evidences of a catarrhal inflammation of the intestine, when diarrhea may prevail to a slight degree, or in cases where the disease is malignant and anemia is prominent the evacuations may be very frequent, watery, and resemble those of cholera.

Typhus Fever, Diarrhea in.-Typhus fever is most often complicated by constipation, but moderate diarrhea may occur in the eruptive stages.

Plague (Black Death), Diarrhea in.-Plague is an infectious and often fatal disease caused by the Bacillus pestis bubonica; it is quite 
common in the far East, occasionally encountered in epidemic form in European countries, and cases have been olserved in the Inited States, particularly in San Francisco, where 31 cases were sturlied in

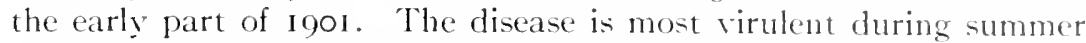
and fall, and is met with in unhygienic communities, and, aceorling to its severity, may be characterized by dermatologic manifestations, glandular enlargements, pneumonia, septicemia, or serere intestinal involvement. In the last type there are frefuent homorrhages, severe abdominal pain, and the patient suffers from what has heen designated "bloody diarrhea."

Glanders, Diarrhea in.-Human glanders is characterized ly nasal ulceration, but sometimes the lesions reach the mucosa of the stomach and intestines and cause bloody evacuations and diarrhea.

Sutika (Puerperal Diarrhea of Bengal), Diarrhea in.-Sutika is a complication of the plague, fully discussed in Pearse's report of the epidemic in Calcutta in the years $1904-05$. This condition appears with fever and very frequent movements in connection with childbirth, and the extent to which it prevails and the dangers from it are indicated by the following statistics (Pearse):

During a single year (1906-07) there occurred in Calcutta 228 deaths from sutika, as compared to 196 deaths from puerperal fever, and 80 deaths in the course of labor. The total number of deliveries registered in Calcutta is about 17,000 per year, and of this number over 1.3 per cent. of the puerperal women succumb to sutika.

So little is known of this remarkable disease that the author will rely chiefly upon Pearse for a description of it :

Sutika is characterized by the following symptoms: In the course of the first two weeks after delivery, sometimes later, a diarrhea makes its appearance which is usually not associated with pain. The number of stools, which are watery, sometimes fermenting, but contain neither mucus nor blood, varies between five and fifteen daily. In a certain number of the cases symptoms of dyspepsia are noted, and anorexia is present in practically all. On the other hand, there is neither vomiting, painful coughing, or anything to suggest disturbance in the pelvic region. Parallel with the diarrhea there develops fever, with an irregular course. The patients rapitly lose flesh, suffer from general prostration, and death takes place from exhaustion. Toward the terminal period there often develops edema of the lower extremities.

The course of the disease is somewhat variable: sometimes it is rather rapid, the patient succumbing within a few months; in other cases the condition drags along for a year or longer. The arerage duration of the diarrhea is from five to eight months. The condition, which has also been noted in other Bengal cities. attacks women of all ages, and is observed with the same freepuency among the Hindor population as among Mohammedans. On the other hand, it has not apparently been demonstrated in Europeans. The diannosis is not difficult, but the pathogenesis of the disease remains entirely 
obscure. It is certain, however, that we are not dealing with ordinary puerperal fever nor with dysentery, and there is no apparent reason for referring the trouble to tuberculosis.

Intestinal Myiasis, Diarrhea in.-Occasionally maggots (screwworms) find their way into the colon, causing extensive ulceration of the mucosa through their boring into it at various points, a condition usually accompanied by diarrhea, pus, and blood in the stools. The disease is common to tropical countries, but has seldom been encountered here. Einhorn has reported (personally to the author) a most interesting case of colonic myiasis (Fig. 24), where the bowel

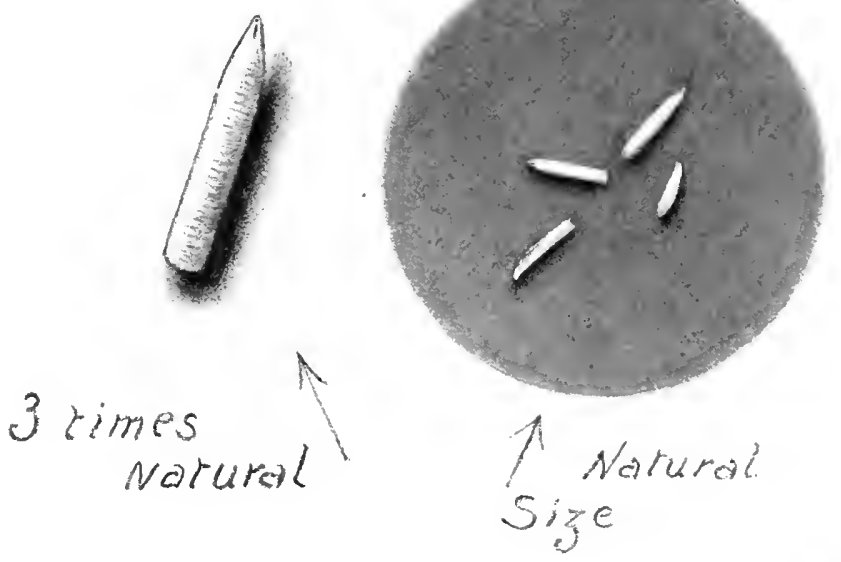

Fig. 24.-Intestinal myiasis. Note normal size of maggots on the right, and one magnified three times on the left. Detected in the feces of a patient suffering from intestinal indigestion.

apparently contained thousands of maggots, and Schlesinger and Weichselbaum ${ }^{1}$ studied a case of extensive colonic ulceration associated with colonic atony and recurring fecal impaction, which they regarded as due to intestinal myiasis.

The maggots, or larve, which are formed from eggs laid upon wounds or the edges of orifices by flies (Dermatobia cyaniventris) are "white, nore than $\frac{1}{2}$ inch in length (Fig. 24), and formed of twelve segments, carrying circles of minute spirally arranged spines, which give the creature a screw-like appearance" (Manson²). They penetrate deeply into whatever tissue they attack, and cause excruciating pain or even death when permitted an uninterrupted course.

McCampbell and Cooper ${ }^{3}$ reported a unique case of intestinal

\footnotetext{
1 Wien. Klin. Wochensehr., January 9, г90г.

"Tropical Diseases, th ed., ro07.

${ }^{3}$ Jour. Amer. Aled. Assoc, (Aclober o, 1900.
} 
myiasis due to infection from three species of dipterous laref. Which in the only instance where the disease was caused by a mixed infection.

The symptoms of colonic myiasis resemble throe of other typeof utceration in this region, but are not likely to be diagnosed unlene the maggots are microseopically seem in the feces or atre accielentally discovered when the excreta is being examineei for other infeetive agents.

The treatment consists in keeping the insects flies) from the patient, and in irrigating the colon with as strong a solution of carbolic acid, chloroform, or turpentine as the patient can stand.

El Bicho Diarrhea.-A severe and common type of diarrhea. known as El Bicho, is encountered in Brazil (particularly in the tropical marshy districts), and is thought to be a form of dyentery. The Indians of the hills suffer from it as soon as they descend to the low sections of the Republic. In these cases the rectum and colon exhale a putrid odor, the patient complains of fever. sometimes -loughing of the bowel is extensive, and diarrhea in extremely depresing.

Pellagra, Diarrhea in.-Nany theories have teen adrancerl tw explain the etiology of pellagra, but its cause is -till unknown. Any and all parts of the gastro-intestinal tract may le disturbed in pellagra, and manifestations, as gastralgia. prosi-. nausea, vomiting, san distention, diarrhea, coated tongue, and lack of appetite, are quite common, but the chief symptoms in a typic case are a sore mouth. indigestion, and diarrhea.

The part played by loose movements in pellagra has been concisely given by Roberts riz.: "The diarrhea usually precerter the dermatitis, but it may occur simultaneously, and Fritz has noticed that it is common for the two to appear together in those whose work keeps them in the sun. It alos shows that the diarrhea in the -rmptom of a systemic morbid proces. The diarrhea. stomatitis. and dermatitis reach their culmination together during the outbreak. The diarrhea comes gradually. lasts about a month all told, chi-appearing gradually as it came. In Tucker's 55 collected cases, diarrhea was present in 54, with remissions in the diarrhea in 36 cases. and diarrhea alternating with constipation in 30 cases. All ny cases except one had diarrhea, and, without exception, the more serere the diarrhea, the greater the prostration and exhaustion and the nuore apparently severe the pellagra. In some pellagrins the flux is severe as to merit the title of 'diarrheic pellagra.' The Egyptian case of Sandwith seem to have less diarrhea than either the Italian or American. Out of 166 cases. the bowets in 10.3 were normal. o had slight constipation, th with slight diarrhea. and s with exce-sive diarrhea.

"In the height of the spring attack the number of sools in the twenty-four hours varies from six to thirty, ten on wenty beine an average. In my own experience the number of stools is influenced neither by rest nor food, and the number is as great in the nisht as 
in the day, and often worse from 3 to 9 o'clock in the morning. In the early part of the attack and in the initial stage of the disease the diarrhea is more spasmolic in character and with far more peristaltic activity, so that the patient complains of abdominal pain and griping like a colic from indigestion. The stools at this time are thicker, contain more mucus and endothelial cells, the pellagrous odor is not so pervasive, and the stools do not come so freely as in the latter stages. At this time they may be tinged with blood, though not so commonly as in acute dysentery. They vary from gray and light brown to green in color.

"In the later stages of the disease the diarrhea assumes a more serous character, is more persistent, and far less amenable to treatment. It is almost a pure watery stool, usually of a light-green color, occasionally almost clear. At this time the acute phase of the disease may develop, and the diarrhea precede the delirium, and foreshadows marasmus and the approach of death. As the serous discharges increase, distention develops and paresis of the intestinal walls occurs. Rectitis, hemorrhoids, and anal fissures add to the cachexia and distress. As a rule, the mild cases do not develop a severe diarrhea, and the diarrhea ceases as the attack recedes. The diarrhea may be the only symptom of the fall exacerbation and may last for only a few days; in other cases, after the first spring attack, the bowels are always relaxed, and two to four stools a day are common. In the cases with constipation the attack is mild and short, and the disease progresses slowly. The life of the pellagrin is prolonged in inverse proportion to the severity and the persistence of the diarrhea.

"As the disease adrances the entire alimentary tract becomes inllamed; gastritis, enteritis, colitis, and rectitis are the foundations for gastric and intestinal ulceration, with blood, mucus, pus, and increased putrefaction and fermentation. At this stage indicanuria is common. Absorption is interfered with, and there is an increase in undigested food materials, especially fats, starch granules, plant cells, and muscle-fibers. The stoots are acid, as a rule, and gaseous, looking as if they hat been whipped, so numerous are the air-bubbles. Uncler the microscope there is an increase in the fat-globules, due probably to a decrease in the bile and pancreatic juice. If the stool in pellagrous diarrhea is put in a bottle or graduate and allowed to stand for several hours it separates into three layers-(1) above is the aqueous portion, serous in character, often colored a light vellow; (2) betow this a thick gray layer, composed of mucus, pus, and occasionally blood-cells; (3) a heary layer below, dark-brown or green in color, and composed chichy of waste matter from the food or ordinary fecal matter, in which is found clinging mucus that hat not separated. J. 1). Long, in his admirable sturlies, found ammonium and magnesim phosphate crystals, fatty acid crystals, calcium oxalate, cholesterin plates, and lungi."

The pathology of intestiual pellagra is not thoroughly understood, 
but it is known that the changes here are variathe and that momalls the bowel is thin. related. and atrophied, if not pienented.

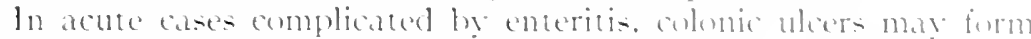
when the mucosa is onnested and not anemic, or under ofluer oir-

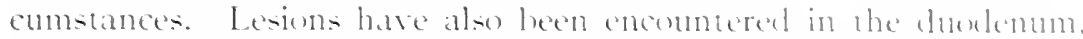
jejuntme and iletum, lut the mouth amel rectum are the part- of the

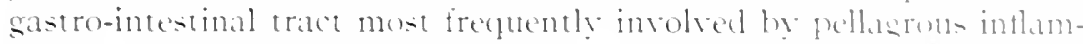

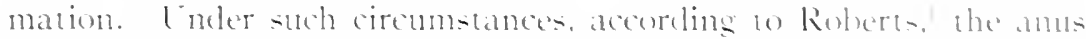
is often swollen, discolored, and finsures and hemerthoids aro acossionally present.

Treatment.-Pellagra is not always curable, hut a great thent can be done to retieve and cure the discase when the patient folluminstructions: though it tends to recur. Sometimes curative agents shoulel be discarded for smptomatic remedies.

Arsente preparations. Fowler"s solution, me $y$ to $x$ x $(0.30-1.30)$.

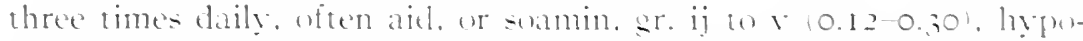

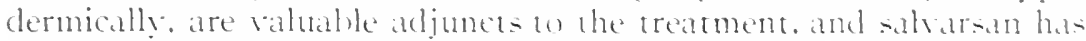
also proved helptul.

When the patient is weat. supportive remedies are indicated. and the diet should be as senerous as the subjects ondition will permit.

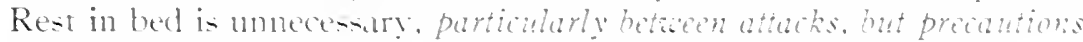
should be taten to see that the patient does not herome futigued.

Treatment of the diarthe requires special enmideration, and the plan followed hy Roberts appears to meet the resuliremento of the ordinary eased viz: "The diarthea is 10 a degree dependene on the decrease in the hydrochloric acte pepsin, and remin of antric juice. During the height of the attack. hewaver. When the diarrhea is apt to be worse. the stomatitis and the esophagitis make the mouth and esophagus so sensitive that the actel an le given only in smatl ghantities. if at all. At this time a prescription contaning lineture of nux romica, hismuth, and the elixir of lactated popsin in freantenty

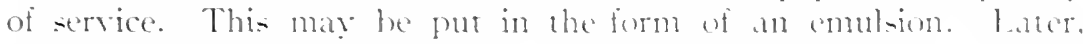
another prescription, containing dilute hyelrochlorie wid, mas he given when the condition of the mouth and throut permits. The

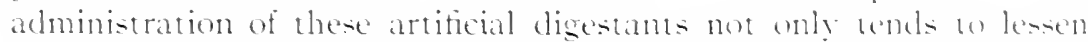
the diarrhea, but also atde digestion. farors ahsorption. increas s the stimulation, and promotes the metabolic activity, with as sain in weight. When the diarrhes is rery hate and drains the pationt wo a seriots degree. one should not hesitate to atminister opium. Berter a few hypodermies of morphin and rest thatn ner rest and the loss of many pounds. Lnstead of morphin. an oceasional den of paregorie.

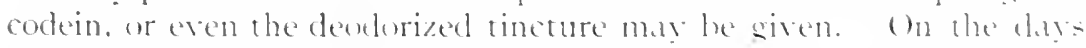
when the diarrhea is hald the patient should be quiet, hut not necessarily remain in herl. and the diet should he exther liepuicl or very lishe."

Actinomycosis (Big Jaw), Diarrhea in.-Thin affection is cintud by the ray fungus Streptothrix actinemyes. Aetinomyonsis is lese common in the Linted states and Britain than in ciermany, and the

$$
{ }^{1} \text { Pelligra, ios?. }
$$


infecting agent is probably taken in with the food. since "big jaw" frequently attacks cattle and hogs, and it has been encountered in persons who consumed raw wheat and rye.

Abdominal actinomycosis originates in the intestines, and is not confined to anr segment of the bowel, though the cecum and appendix are most frequently involved. The disease rarely, if ever, attacks children, and in the beginning often causes symptoms similar to those of perituphilitis.

The fungus maỹ produce superficial deposits upon or penetrate the mucosa ion its way to other parts of the body') and cause more or less infiltration of the intestinal tunics.

Perityphlitis actinomycotica is fairly common. and may be characterized by the manifestations of a local inflammation, tumor formation, or abscess and fistula. Cases of anorectal (secondary) actinomycotica have been reported where the lesions were low down or high up in the rectum, and caused firm, infiltrated masses or abscess and fistula. and stricture is a frequent sequel of the disease here.

Symptoms.-Diarrhea is a common manifestation of primary and secondary intestinal actinomýcosis, and may be induced by a localized inflammation (catarrh) in the colon or an obstruction (from a tumor formation. adhesion, or kink), which retains solid and permits the fluid feces to escape. In most cases the stomach is slightly disturbed, and diarrhea is irregular. with recurring attacks, and occasionally the actinomyces can be detected in the stools. Secondary growths, abscess, and fistula may form in other organs.

Diannosis. - The disease in the cecum. colon. and rectum should be suspected when a large painless. hard tumor is present which cannot be explained in other way's. particularly when it is connected with involvement of the abdominal wall or a fistula. With such a condition a diagnosis is made positive by finding actinomyces in the discharge or feces.

The prognosis of actinomycosis is often grave because of the extensive inroads made by the fungus and the tendency toward secondary infection of other segments of the bowel or organs. Thus far only about 20 per cent. of the cases of intestinal actinomycotica have been cured.

Treatment.-Potassium iodid, tuberculin, and the intravenous injection of silver preparations have been tried with unsatisfactory re-ult - Actinomycosis is a surgical disease. and a cure is not to be expected except in cases where all diseased foci are destroyed or remored. Sometimes remoral of the mas is adrisable. and at other times a number of small incisions is preferable, through which the fungus escapes. 


\section{CHAPTER VIII}

\section{SUNDRY DISEASES, DIARRHEA IN}

\section{COPROSTASIS, OBESITY, CACHEXIA, ANEMIA, PERNICIOUS ANEMIA, LEUKEMIA, ALCOHOLISM, MARASMUS, ARTERIOSCLEROSIS, ENTERITIS CROUPOSA NECROTICA, GOUT, METHEMOGLOBIN- EMIA, SCURVY, CEREBROSPINAL MENINGITIS}

Coprostasis, Diarrhea in.-Stercoral or coprostatic diarrhea is usually an acute process. It occurs in individuals who are accustomed to normal bowel movements, but who suddenly become markedly costive, those who suffer from chronic constipation and recurring impaction. and may complicate either atonic, spastic, or mechanic constipation (obstipation). Chronic stercoral diarrhea usually complicates olsstipation where there is an obstructing intestinal lesion-stricture. angulation, adhesion, diverticulum, pericolitis, external pressure. foreign body, twist, ptosis, chronic invagination, or tumor that partially blocks the bowel and causes slight or complete fecal retention. Occasionally it is encountered in functional or organic diseases which diminish the gastric or intestinal secretions, and where the intestine is inflamed, hypersensitive, or ulcerated, causing enterospasm or the simultaneous contraction of the circular and longitudinal intestinal muscular fibers (occlusion), or the bowel is partially or completely paralyzed as the result of a cord or brain lesion.

The loose movements here are induced by $(a)$ direct trauma to the mucosa by large putty-like fecal masses or numerous hard, irregular, nodular scrbala which collect in considerable numbers and clistend the bowel; (b) local irritation and auto-intoxication incident to the retention, multiplication, and absorption of the intestinal bacteria or their toxins; $(c)$ stercoral ulcers which expose the nerve-endings to the intestinal stimuli, which lead to almormal peristalsis; (d) the effect of psychic impulses upon the bowel brought about by worry; (e) the formation of irritating gases (marsh, $\mathrm{CH}_{4}$ : hyclrogen sulphid, $\mathrm{H}_{2} \mathrm{~S}$ ); and $(f)$ cathartics prescribed to soften the exacuations and prevent obstruction.

The author has elsewhere reported t5 cases of coprostasis, in the majority of which diarrhea was a complication. A stmmary of these cases has been given below, viz.:

Sex and Age.-Of the 45 cases, 22 were men and 2,3 were women. Their ages ranged from eighteen months to serenty-six years. 'Twentysix were thirty-five years or more, while ig were under that age.

\footnotetext{
${ }^{1}$ Gant, Lisealies of the Rectum and Anus, 1003.
} 
Location.-The impaction was located in the rectum 30 times; sigmoid and rectum, 5; sigmoid, 6; colon, sigmoid, and rectum, I; descending colon, I; cecum, I; transverse colon, I.

Weight.--The fecal accumulations ranged in weight from + ounces in a child to 2 pounds in an adult. The length of time these patients went without stool varied from two days to three months.

The causes of impaction directly and indirectly were as follows:

Stricture, 4; carcinoma, 4; pregnancy. I ; careless habits, 5; congenital malformation of the anus, I ; traumatic stricture, I ; paresis, 2 ; parched corn, 2; fruit- and berry-stones, 2; adhesions, 2; fibrous bands in rectum, $\mathrm{I}$; chronic constipation, 2 ; fissure, 2 ; loss of intestinal tonicity, 5; retroverted uterus, I; unknown, I; inability to evacuate the bowel after hemorrhoidal operation, I ; hypertrophied sphincter, I ; gall-stones, I ; sarcoma, I ; hypertrophied "rectal valve," I ; green corn with portion of cob, I; hypertrophied levator ani muscle, 1 ; disseminated polypi, I; enterolith, $\mathbf{~}$.

The author has recorded these cases with a view of pointing out the frequency of impaction, or coprostasis; the necessity of its prompt surgical treatment; and, further, to show the varied affections and conditions which may induce it.

The symptoms of stercoral or coprostatic diarrhea depend upon the nature of the trouble behind the constipation or obstruction, the number, location, and size of the impacted masses, and the duration and degree of obstruction caused by them.

In acute coprostasis the movements are not very frequent or exhausting, but the patient complains of gas distention, peristalic activity, gurgling sounds, and cramps. Relief quickly follows the evacuation of scrbalie or putty-like masses, semisolid and fluid feces, and pent-up foul gases $\left(\mathrm{CH}_{4} \mathrm{H}_{2} \mathrm{~S}\right)$, bacteria, toxins, and putrefying material.

In persons long afflicted with constipation or obstipation, where the bowel never completely empties itself, and there is chronic coprostatic or stercoral diarrhea, the symptom-complex differs and the additional following manifestations may at one time or another be observed: The evacuations continue abnormally frequent week after week, varying from four to ten daily, have a vile odor, are soft or fluid, contain mucus, pus, blood, and diminutive scybala, and induce considerable tenesmus and pain when evacuated, and the patient complains now and then of colicky pains, tympanites, and localized abdominal tenderness on pressure due to distention of the bowel, the ulcers, and a circumscribed peritonitis common in this conclition.

There are nearly always manifestations of profound intestinal autointoxication, viz.: restlessness, melancholia, muddy complexion, offensive breath, indigestion, loss of appetite, dizziness, night-sweats, albuminuria, nausea, vomiting, etc.

When impaction is in the rectum the movements are very frequent, the patient suffers from sensations of fulness, bearing-down pain, 
incessant desire to evacuate the bowel, tenesmus, and sacrocoecyeal discomfort.

The diagnosis of stercoral diarrhea is comparatively easy when a history has been taken; the above characteristic manifestations are exhibited and large hard, smooth, or nodular masiose can lee located along the colon, sigmoid, or rectum by palpation, instrumental or digital examination. Fecal tumors causing stercoral tharrhea are often confused with carcinomata and other neoplasms. but this is unnecessary, since fecal masses are indentable, movalile, and the bowel can be made to slide over them in contradistinction to growths. and there is no cachexia or loss of weight.

Differextial Diagnosis Betweex Fecal Iapaction and Carcinoma of the Large. INTestine AND Rectum

FlCCAL THMOR: (IMPACTION).

I. Single, large, firm, and globular in shape; or numerous, =mall, hard, and nodular.

2. Nou covered by mucous membrane.

3. Occupy lumen of the bowel.

4. Of doughy consistence and indentable.

5. Not attacherl.

6. Novable.

7. Occur at any age.

8. No cachexia.

9. Usually odorless.

io. Come on suddenly.

1. No previous history of pain or hemorrhages.

12. Not accompanierl by discharge of mucus or jelly-like stools.
CARETIOMA.

Two or more dense. rounded tumors.

Covered by mucosa except when ulcerated.

Project into the caliber of the intestine.

Hard and non-indentable.

Attached.

Xon-movable or slightly so.

In middle life and old age.

Cachexia.

Offen-ive odor.

Slowly:

Pain always, hemorrhages irequently:

Free discharce of mucus and sometimes of jelly-like evacuations.

The prognosis of stercoral diarrhea complicating atonic and spastic constipation is good, but when consequent upon chronic intestinal obstruction it will continue or occur periodically until the bluck is corrected by physical or surgical measures.

The treatment of coprostatic or stercoral diarrhea is simple and devoid of danger when it arises through carclessness in attending to the calls of nature, or the bowel has been tied up by medication. but in cases where fecal retention and diarrhea result from ohstipation induced by a mechanic obstruction, much ingenuity is required to keep the bowel free and control the diarrhea. In aggrarated cases, where masses and scybala cannot be entircly evacuated or quickly recur, an operation, such as intestinal exclusion, enterostomy, colostomy, cecostomy, appendicostomy, or resection, is usually imperative, but surgical intervention should not be resorted to until less radical measures have failed to relieve the patient.

In the presence of an olstruction, sweet, cotton-secei. mineral or castor oil, or licquid paraffin should be prescribed daily or periodically to soften and cause the retained fecal accumulations in be exacuated. but cathartics are contra-indicated in these cases becaluse they incite cramps, active peristalsis, and the transudation of fluid ints the 
bowel above the block, which adds to the patient's discomfort and does nothing toward getting rid of the fecal mass. In the absence of local pain and tenderness, careful massage may be employed to break up and dislodge the tumor, but when manipulation causes pain it indicates the presence of ulcers or peritonitis and should be discontinued.

Fecal masses responsible for stercoral diarrhea are located in the colon, sigmoid flexure, or rectum, and are amenable to treatment through the anus. When above the sigmoid flexure they can be eliminated by the administration of oil employed in conjunction with frequent colonic irrigation or high enemata. The most satisfactory media to employ for this purpose are soapsuds, slippery-elm water, and flaxseed tea because of their emollient and lubricating qualities. Two or three quarts (liters) are introduced and permitted to remain in the bowel as long as they can be retained, and the treatments should be repeated several times daily until relief is obtained. When the impactions become hard, are coated with mucus, and water will neither permeate nor dislodge them, large oil enemata, $\mathrm{Oj}$ to $\mathrm{ij}$ (500-I000), and turpentine, $5 \mathrm{j}$ (4.0), should be administered every five or six hours until the bowel is cleared. When soapsuds and oil enemata fail, a 25 per cent. solution of hydrogen peroxid, Oij (rooo), should be injected into the colon, for it will disintegrate the mass into smaller parts so that it can be washed out. Fecal impactions located in the sigmoid flexure and rectum can be broken up with the finger or gouge through the sigmoidoscope, and then evacuated by continuous soapsuds irrigation. After correcting the predisposing cause, dislodging and removing the offending feces, the bowel should be treated with antiseptic and soothing remedies internally and locally to heal the ulcers and soothe the inflamed mucosa.

Obesity, Diarrhea in.-A considerable number of obese individuals are gourmands, eat frequently and at irregular hours, partake freely of highly seasoned foods, and not infrequently imbibe alcoholic stimulants to excess, with the result that they take on still more fat, overtax the gastro-intestinal tract, impair the circulation, cause engorgement of the liver, and in many other ways disturb the body equilibrium. The writer has treated a number of fat persons who in this way have developed an irritable form of gastro-intestinal catarrh, and who still continued to gratify their appetite at the expense of their physical comfort, with the result that after partaking of large amounts of food, often in connection with several glasses of ice-water, they suffered from pain and cramps in the lower abdomen, followed by two or three watery movements and the expulsion of considerable offensive gas. Relief usually followed such movements, and comparative comfort prevailed until their next overindulgence. In some instances dietetic indiscretions induced a chronic gastro-intestinal catarrh and loose movements, varied from two or three to eight or ten watery evacuations daily containing considerable mucus, from which time there was often a gradual improvement, owing to 
the fact that less food was consumed, either because the patient lost his appetite or became frightened, ate less, and restricted himself to a more select diet.

Very severe diarrhea may also occur in the obese as the result of constipation and the accumulation of feces, which, when retained, form hard scybalous masses and excite frequent movements through their local action upon the gut or the formation of stercoral ulcers and consequent reflexes; but, of all the diarrheas encountered in very fat persons, the most intractable is that induced ly fatty degeneration of the liver, to which particular attention has been called by lisch.

Treatment.- Whether or not much is accomplished in the treatment of diarrhea in the olese individual depends principally upon his temperament and will power, because little or nothing can be done to better his condition if he pursues his usual faulty method of living; but much progress can be made toward relieving and curing the diarrhea and correcting other manifestations consequent upon the fat accumulation if he will place himself in the hands of one who understands his condition, and will rigidly follow instructions as long as may be repuired to reduce his weight and stop the diarrhea.

There is a wide variation in the diet lists which have been submitted by the different authorities for preventing the formationand getting rid-of surplus fat, as will be seen by sudy of the itinerary arranged by Banting, Ebstein, Oertel, and others.

Briefly stated, the best results are obtained by having these patients eat at regular hours, consume less food and water, abstain largely from alcoholic beverages, condiments, and appetizers, exercise in the open air, and refrain from eating sweets, farinaceous foods, fats, and fluids, except in very limited quantities.

Thus far no medical agent has been discovered which is harmless yet useful in this class of cases. The thyroid extract (desiccated), gr. j (0.06), three times daily, or thyroid (active principle) has been satisfactorily employed to reduce weight rapidly, but should be discarded or limited as soon as symptoms of thyroidism appear.

When diarrhea is consequent upon gastro-cnterocolitis this can be controlled by regulating the diet, flushing the bowel (with mild saline antiseptic and astringent solutions), and administering opiates, antiseptics, and astringents alone or in combination to diminish the number of stools.

Increased evacuations, which result from the accumulation of toxins or fecal impactions (stercoral (liarhea), are most quickly controlled by high colonic irrigations of water, saline, soap, or medicated solutions.

After all, most is accomplished by having the patient take care of himself, eat a reasonable amount, and at the same time partake of foods which are suitable and agree with him.

Leven observed several patients having chronic diarthea whose weight increased in sprite of the loose movements, and in whom 
cessation of the diarrhea, through suitable treatment, was followed by a diminution in the weight. These apparently paradoxic observation are explained by the author as due to the existence of a nerous realating apparatus for the weight of the body. the mechanisn of which may be disturbed by a pathologic process of any kind. He points out the necessity for a gradual emaciation of the obese, as a rapid loss of thesh depends rather upon a dangerous loss of water in-tead of upon a loss of fat.

Cachexia, Diarrhea in.-Diarrhea is often associated with cachexia. but the frequent movements should be considered as a manifestation of cancer, tuberculosis, pus accumulations. kidney lesions. or other serious affection where cachexia is a symptom.

Consequently. the treatment of diarrhea in calchexia should consist in relieving or correcting the pathologic condition responsible for it, along with measures directed against the intestine when there are local lesions which are in part responsible for the frequent eracuations.

Anemia, Diarrhea in.-Frequent eracuations in anemic subjects are encountered less often than obstipation, and when present the intestinal mucosa is rery sensitive, and there is an acute or chronic catarrhal inflammation of the bowel which is responsible for the increased eracuations and mucus. In this condition the anemia may be the result of general causes or local lesions of the intestine, accompanied by a few severe or many slight recurrent hemorrhages. In the latter case it is more than likely that the erosions or ulcers are the exciting cause of the diarrhea.

In pernicions anemia there may be constipation at one time or diarrhea at another, and while the latter prevails there is usually more or less gastric irritation.

Hertz claims that the peculiarities of this type of diarrhea consist in vellowish-green evacuations resembling those of trphoid fever at most six times daily). Which are not quite watery and rarely contain blood. The eracuations are independent of dyspeptic disturbances and are sometimes seen in the early-but usually occur in the later-stages of the disease.

Pernicious anemia may also be confused with ordinary anemia consequent upon intestinal hemorrhages from the ulcerative types of colitis, but can ordinarily be differentiated from it by getting a history of the case, inspecting the bowel through the sigmoidoscope, and hy examining the blood and stools.

Treatment. - IThen the mucosa is intact and the gastro-intestinal disturbances are only a part of the general anemic process, supportive measures. such as fresh air. nutritive food, regular hours, moderate exercise, and. if necessary, change of occupation or surroundings, are indicated in combination with iron, arsenic, strychnin, quinin, and other medicines which improve the condition of the hlood, tone up the nerrous srstem, and increase the bodily strength. When anemia or chlorosis is the result of intestinal lesions which 
bleed freely, the above plan of treatment is still indicated, but, in addition to this, liberal closes of bismuth subnitrate and salicylate, beta-naphthol, satol, guaiacol, tannin, tanniform, tamalbin, ichthoform, or other antiseptic and astringent remedies should be prescribed because they favor healing and lessen the formation of intestinal toxins.

Beneficial results are nearly always attainable in this class of cases by daily flushing the bowel from above through an appendiceal or cecal opening, or from below through the anus, with either normal saline, boric acid (2 per cent.), ichthyol (2 per cent.), permanganate of potassium (1 per cent.), or tannic acid ( per cent.) solutions, care being taken to see that the fluid reaches all parts of the colon.

When uleers are within reach of the sigmoiloscope, they can be made to heal more rapilly by topical applications of silver nitrate, 6 per cent.; ichthyol, 20 per cent.; balsam of Peru, 20 per cent., or, when unheathy, by stimulating them with full strength silver nitrate or copper sulphate.

The author has obtained some remarkable results with throughand-through irrigation in the treatment of chlorosis, ordinary and pernicious anemia following ordinary cecostomy (see Fig. 95), appendicostomy (see Fig. I 48 ), and his cecostomy, which provides a means of irrigating both the small and large intestine at the same time (see Fig. I +3 ), and employment of the above-mentioned or other irrigating solutions. The results demonstrate dearly that auto-intoxication is an important factor in the production of the anemic condition, or that there is some other close pathologic relation between the bowel and these ailments.

Improvement has been olserved in these cases where irrigation was restricted to the colon, but still beter results have been obtained when the author's method of freguently irrigating both the small and large intestine was practised. His enterocolonic irrigator (see Fig. I +2) (nables the attendant to wash out either the small intestine or colon, or both, at will, and also to cause the medicated solution to be retained as long as may be desired, which in some instances is a great achantage.

Pernicious Anemia, Diarrhea in.-Gastralgia, ablominal pain, and diarrhea are frepuent complications of pernicious anemia, and pyorrhea and an eroded tongue are often in evidence. The diarrhea in such cases can be attributed in part to the hypochlorhydria or achylia gastrica that prevails.

Diarrhea from this source is leest controlled by opiates during crises and rest in bed, fresh air, nourishing food, and Fowler's solution, miij to $v(0.200 .30)$ or more, three times didily. In acklition, other remedies known to improse the condition shonld be prescribed. The author has obtained remarkably good results in this class of casen from frepuent medicated colonic irrigations.

Leukemia, Diarrhea in.-In this affection the lowel, like other organs, freeguently becomes prinarily or secondarily involved (lig. 25. Hillman), and the patient may suffer from costiveness, diarrhea alone. 
or alternately. The evacuations when abnormally frequent are usually light colored and pasty, but when they contain a considerable amount of bile-pigment or blood they are brownish or even black, but in those cases where diarrhea results from enterocolitis the stools are more normal in color, contain mucus, and occasionally pus and blood. Frequently gas forms and causes meteorism, abdominal

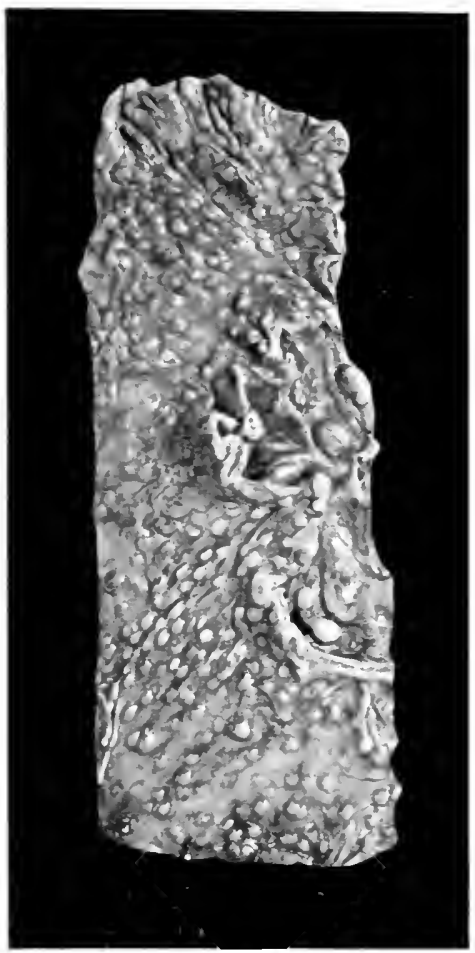

Fig. 25.-Limphatic leukemia inrolving the colon. Tote multiple subcutaneous nodules, and also enlarged lymph-nodes of mesocolon. pain, and a sensation of fulness and tenesmus may ensue when it and the feces are expelled. In rare instances ulcers of considerable size have been observed, and enlarged lymph-nodes have been found which were inflamed or necrosed.

In pseudoleukemia (Hodgkin's disease) diarrhea is seldom an early-but is frequently a late-complication in severe cases, and is very difficult to control throughout. The stools contain mucus, and occasionally mild or severe bleeding from hemorrhoids or lesions higher up takes place, and the glands adjacent to the intestine become markedly enlarged, but seldom cause intestinal manifestations.

The treatment of leukemia and pseudoleukemia is extremely unsatisfactory, though some authorities claim that much benefit can be obtained by exposing the enlarged glands to the Röntgen rays. Chief reliance, however, should be placed upon the supportive and symptomatic treatment of the patient.

Iron and arsenic, in conjunction with intestinal antiseptics, are serviceable in controlling diarrhea, but when these agents fail, an opiate should be given. In case there are lesions in the colon, irrigations and topical applications are indicated.

Surgery has been resorted to in this class of cases, but the results have been disappointing.

Alcoholism, Diarrhea in.-Individuals who consume in a few hours large amounts of whisky, brandy, gin, and other beverages containing from 30 to 60 per cent. or more of alcohol, or who regularly partake of alcohol in liberal amounts, are apt to develop an acute or chronic catarrhal gastro-enteritis and diarrhea.

In acute alcoholism the disturbance is of short duration, and the patient complains of nausea, vomiting, loss of muscular control, incoördination of speech, stertorous breathing, occasionally inconti- 
nence of urine and feces and, in ageravated cases, felirium tremens or coma, all of which manifestations abate or cease altogether shortly following a cessation of drinking.

Chronic alcoholism is now regarded as a clisease produced hy constant saturation of the body with alcohol, which in time profoundly. affects the general system, stomach, intestines, liver, and other orcans, and gradually produces the symptom-complex: anorexia, obnoxious breath, morning vomiting, gastric discomfort after eating, insomnia, headache, restlessness. clepression, dementia and delirium tremens. impaired metabolism, and constipation alone or alternating with diarrhea, or the latter may prevail.

Diarrhea in these cases results from the thickened, inflamed. eongested and often ulcerated intestinal mucosa, imperfect gastro-inte- tinal indigestion, interference with colonic absorption, disturbance of the biliary circulation, and the effect of alcohol upon the local and cerebrospinal nerve-centers controlling intestinal nutrition, secretion. and motility.

The treatment of diarrhea in acute alcoholism consists in witholrawing the drug, keeping the patient in bed on a liquid diet. washing out the stomach to abate nausea and romiting. flushing the bowel with soothing agents to relieve irritation and removing retained feces and toxins. prescribing remedies that will relieve headache and insure sleep until the patient has somewhat recoserect from the effects of his debauch, and in taking precautions to prevent further drinking.

In chronic alcoholism the above measures will suffice during exacerbations, but permanent results depend principally on the succe-s met with in limiting the amonnt of liquor consumed, regulating the diet. improving the nervous state, and in instituting therapeutic measures to minimize or cure the catarrhal gastro-enterocolitis responsilble for the frequent morements.

The diarrheal condition improves in farorable cases in proportion as the amount of alcohol consumed is eliminished and congestion of the mucosa becomes less; but frequently in chronic inebriates who have been cured there persists a chronic enterocolitis that requires further treatment. The occasional administration of saline laxatives is very serviceable because they diminish intestinal congestion. irete the bowel of imperfectly digested food, and have a favorable influence upon the eatarrh.

When enterocolitis and diarrhea dominate the symptom-complex of chronic alcoholism, the bowel deserves first consideration, and opium, gr. ${ }_{2}^{1}(0.03)$, and the extract of belladonna, gr. 10.000$)$, can be relied upon to control the movements, relieve pain and intestinal irritability, as can also morphin, laudanum, or other opiates, alone or in combination with bismuth, gr. $\mathrm{x}$ to $\mathrm{xx}(0.60-1.30)$, tannoform, ichthalbin, anel gallic acid, gr. v to $\mathrm{x}(0.30-0.60)$; but opiates should be prescribed with caution, otherwise the patient beromes habituated to them. 
When fermentation and putrefaction are marked, calomel, salol, beta-naph thol, and reliable antiseptics may be added to the treatment, but when there is a tendency to stagnation, gastric and intestinal lavage is indicated.

The treatment of alcoholic intestinal catarrh should not be quite so vigorous as other forms, since here the bowel is extremely irritable and often reacts against injected solutions having a strong stimulating tendency. On account of this peculiarity the writer has been accustomed to employ warm flaxseed tea, starch, or slippery-elm water, and very mild infusions of black or white oak bark, agents which soothe the bowel, reduce inflammation, and tend to heal erosions of the mucosa. In severe cases, where diarrhea is persistent and the stools, because of the presence of ulcers, contain considerable pus, blood, and mucus, stronger irrigants, such as boric acid, 2 to 4 per cent.; ichthyol, I to 2 per cent.; permanganate of potassium and balsam of Peru, I per cent., containing laudanum, w $\mathrm{xx}$ (I.30), and tincture of belladonna, mo $\mathrm{x}(0.60)$, are preferable.

With the patient in the proper position, and with the aid of the proctoscope, colon tube, and the irrigating container at the right height, a fluid can usually be made to reach all parts of the colon, but when this is not feasible, appendicostomy or cecostomy should be performed to insure through-and-through irrigation.

Marasmus, Diarrhea in.-Frequent evacuations are sometimes observed in chronic marasmus, but diarrhea is encountered in this affection more frequently and severely when it develops rapidly. The stools may resemble those of simple enterocolitis, or be watery and serous-like when transudation into the intestine is marked. In the latter, diarrhea is probably attributable to disturbance of the intestinal nervous mechanism, lack of absorption, or to impairment of the local circulatory apparatus.

Treatment.-Naturally, this type of loose movements occurs very much more often in infancy than in older subjects, due largely to improper feeding and malnutrition. From what has been said it may be inferred that the treatment is based upon improved feeding, nerve and systemic tonics, fresh air, sunshine. remedies to alleviate intestinal catarrh, and local irrigations to heal an inflamed and, in exceptional instances, an ulcerated mucosa, thereby reclucing irritation so that the nutrient media may remain in the intestine sufficiently long for absorption to take place.

Arteriosclerosis, Diarrhea in.-Arteriosclerosis of the mesenteric vessels often produces manifestations resembling gall-stone colic, and gastro-intestinal symptoms varied in character and cholera-like diarrhea from this source has been observed.

In such cases the treatment does not differ materially from that indicated in general arteriosclerosis.

Enteritis Crouposa Necrotica, Diarrhea in.-This condition is characterized by extensive sloughing, and then ulceration of the intestine, accompanied by diarrhea, irrespective of the causes of the 
condition, of which there are many. In the majority of instances the sloughing and loose movements are secondary to nephritis and uremia, mercurial poisoning, bacillary, balantidic, and entamebic colitis (alyentery). intestinal obstruction (complicated by interference with the circulation of the intestinal tunics), final stages of wasting diseases (syphilis, tuberculosis, septicemia, etc.), diphtheria, and occasionally preumonia.

The local symptoms of this form of diarrhea are the same as for other forms of ulcerative colitis.

The diagnosis is easily made with the aid of the sigmoidoscope and fecal examination.

The treatment consists in keeping the patient quiet, restricting the diet. administering opiates and bismuth to comfort the patient and diminish the number of stools; in irrigating the colon from betow or through an appendiceal or cecal opening and in resecting the diseased gut when less radical measures fail.

Gout, Diarrhea in.-Gout is more often complicated by constipation than diarrhea, but when loose movements prevail they are usually associated with flatulence, dyspepsia, pyrosis, and abdomina! pains.

The treatment is symptomatic as far as the gastro-intestinal manifestations are concerned, but the usual treatment for gout should be instituted to prevent a recurrence of the diarrhea.

Methemoglobinemia, Diarrhea in.-Chronic diarrhea has been frequently observed in this condition, and Gibson and Douglas suggested the name microbic cyanosis for the colon organism obtained from the blood of their patient, and Cärtner's bacillus has caused the disease in rats.

Scurvy (Scorbutus), Diarrhea in.-Scurvy is common among sailors who take long rovages, but its etiology is not known, and it is supposed to be induced by lack of fresh regetables, toxic substances in the food, or an unknown specific organism.

It comes on insidiously and is characterized by a loss in weight. diseased and bleeding gums, loose teeth, foul broth, swollen tongue, dry skin, ecchymosis, mental depression, and either constipation or diarrhea.

Treatment.-It can be cured by lemon-juice daily, together with wholesome meat and fresh vegetables. Much can be added to the patient's comfort by frequent mouth-washing with a weak permanganate or carbolic solution.

Cerebrospinal Meningitis, Diarrhea in.-Gastro-intestinal complications are usually not annoying in this affection, but when they are, diarrhea occurs less often than constipation. 


\section{CHAPTER IX \\ SUNDRY DIARRHEAS}

\section{DIARRHEAS FROM IRREGULARITIES IN LIVING, CATHARSIS, HYPO- DYNAMIA CORDIS, BURNS, AGORAPHOBIA, SITOPHOBIA, CHILL- ING, DRINKING IMPURE WATER AND ICE-COLD BEVERAGES, SUN- AND HEAT-STROKES, OLD AGE, NOCTURNAL, EOSINO- PHILIC, MECHANIC, AND REFLEX DIARRHEAS}

Irregularities in Living, Diarrhea from. ${ }^{1}-$ Meals, to be properly digested, should be taken at regular hours and eaten slowly and amidst pleasant surroundings. It is not surprising that individuals who eat much more than they need at all times of the day or night, and highly seasoned or indigestible foods, suffer from gastro-intestinal disturbances and diarrhea, because the organs are overworked and are being constantly irritated, particularly when the meals are hurriedly eaten and the food is improperly masticated, or is consumed very hot or ice cold.

Digestion is frequently interfered with because of the large amounts of whisky, beer, tea, coffee, wine, or ice-cold water consumed with the food, which abnormally dilute it, set up fermentation, or irritate the mucosa.

Indiscretions in diet are frequently responsible for diarrhea, although it must be remembered that some individuals can take care of certain foods better than others. Vegetables, like radishes, cabbage, turnips, celery, parsnips, and onions, which leave a coarse, irritating residue, and raw fruits, particularly when improperly masticated, are prone to induce indigestion and diarrhea, as does also an over amount of fat which may split up in the stomach, forming irritating fatty acids. Beef and pork are more difficult for the digestive fluids to break up than mutton, and the latter than chicken or fish; consequently, when it is found that one is indigestible, another should be substituted. Patients with an irritable gastro-intestinal tract should be warned against eating salted or smoked meat or fish, and the flesh of killed animals while in rigor mortis, because they are always tough, and salmon and herring, because of the contained oil. Meats are also more digestible when they are from young than old animals, and boiled instead of roasted, and the skin and ligamentous portions shoukd be discarded, because the digestive fluids act upon them slowly if at all. The consumption of fresh bread of ten interferes with digestion, while stale or toasted bread does not, because it is easily broken ui) and digested.

${ }^{1}$ See Gastrogenic and Enterogenic (Lienteric) Diarrhea. 
The symptoms in this type of diarrhea are about the same as those accompanying enteritis, elsewhere describerl.

The diagnosis is easy in some and difficult in other cases. The nature of the trouble. however, can ustally be determined hy getting the history and learning if the pationt is a gourmand, leads an irregular life, eats indigestible food, and by making one or more examinations of the gastric contents and feces following Schmidt s test-meal.

The treatment consists principally in having the patient alstain from alcohol, establish regular hours for sleeping, eating, and attending to the calls of nature, limiting the amount of food consumed, and restricting it to articles of diet known to agree with him. In most instances, gastric and intestinal lavage afford considerable relief by washing the stomach and bowel free from their irritating contents and quieting the irritable and inflamed mucosa, particularly when medicaments are added to the solution. When diarrhea persists in spite of this treatment, an opiate, sedative, antiseptic, or an astringent alone, or in combination, should be prescribed and administered as often as may be necessary to control the cracuations.

Diarrhea Cathartica.-Diarrhea is frequently a sefuel of chronic, atonic, spastic, and mechanic constipation, and may result from frequently recurring fecal impaction, which, through trama or the effects of retained toxins, causes a local inflammation, nlecration, or both, which may incite loose movements. Most often, however, diarrhea is produced by the long-continued emplosment in everincreasing dosage of laxatives, cathartics, and purgatives, which, through their general action and local irritation, continually cause an abnormal transudation of fluid into the intestines, glandular activity, and inflammation, with erosions or ulcerations of the mucosa.

Gastric or enterocolonic catarrh thus ensues similar to that which results from gastro-intestinal dyspepsia or which necessarily follows when unprepared fool is rushed through the gastro-intestinal tract. day after day, month after month, and year after year by the frequent and pernicious peristaltic moxements incited by the laking of drugs to secure the coveted daily evacuations.

Again, prolonged obnoxious medication in this way tenels to weaken the patient and lower his resistance, subjects him upon slight provocation to catarrhal infections and other diseases, and eventually produces an alnormal condition of the colon that leads to ulecration or prevents absorption. The fluidity of the movements in time cause an irritable state of the rectum characterized by tenesmus and an inability on the part of the patient to retain the fecen when they reach the lower bowel.

Drastic cathartics, such as colocyth, polophyllum, jalap, gamboge. elaterium, castor oil, and croton oil, act by enlivening peristalsis. IIydragogues or saline cathartics. such as solium sulphate and tartrate, magnesium sulphate and citrate, and potassium hitartrate, produce large watery movements through their liberal witholawal of water from the intestinal walls. Cholagogues, like mercury, aloes. 
and leptandra, both excite active peristalsis and an abnormal secretion of bile: the laxatices-riz., cascara sagrada, manna, tamarind, sulphur. olive oil, and hyoscymus - do the least harm, because they but mildy stimulate peristalsis and glandular activity, while mineral waters increase the frequency and fluidity of the evacuations through their saline constituents, thermic and mechanical action, and by increasing the amount of water in the bowel.

The treatment of diarrhea cathartica consists in discontinuing the medical agents responsible for the trouble, or, if they must be prescribed. using them in smaller amounts and through agents which will produce the minimum amount of gastro-intestinal disturbance. When urgent, antidiarrheal remedies may be judiciously prescribed until such time as the catarrhal condition of the bowel can be corrected.

Hypodynamia Cordis, Diarrhea in.--Occasionally, elderly patients complain of heart fatigue and suffer from diarrhea or spastic obstipation that does not respond to cathartics. According to Jaworski, the majority of these patients suffer from latent or manifest inflammatory or nutritive changes of the myocardium (chronic myocarditis, degeneration of the heart muscle, fatty heart, and arteriosclerosis). In this class of sufferers the author has been accustomed to regard the diarrhea as coprostatic, consecpuent upon enterospasm. In these cases he discards remedies ordinarily employed to empty the bowel (laxatives and cathartics) for heat applied to the abdomen in conjunction with belladonna. which tend to relax the intestinal musculature and liberate the accumulated feces. In some cases dislodgment and eracuation of the impacted masses can be quickly and satisfactorily obtained through the administration of olive or mineral oils and by copious hot soapsuds or oil enemata, agents which soften the feces and lubricate the bowel.

Burns, Diarrhea from.- One of the most remarkable and interesting manifestations connected with very extensive burns of the skin is the frequent formation of duodenal and, rarely, gastric ulcers, which sometimes cause perforation and peritonitis. Some authorities betieve this condition is due to thrombosis within the duodenal circulation. caused by the setting free of fibrin ferment, but this explanation has not met with universal approval, although no better has been offered. The hyperemic intlammatory and ulcerated condition is limited principally to the superior horizontal portion of the duodenum, and is very rarely encountered in the inferior extremity of this segment of gut or further along the intestinal tract. The lesions may be single or multiple and conglomerated, and show various stages of development with superficial hemorrhagic infarcts at one point, or deep uleers of consideralde size at another, about which the mucosid is congested. The lesions may develop rapidly and attain considerable size within forty-eight hours, but, as a rule, they manifest them-elves at the end of one or two weeks, and are so characteristic that, with the history, there is no reason for confusing them with other types of duodenal ulcers. 
In fatal cases death occurs in from three to five rays, sometimes from erosion of and hemorrhage from the pancreaticoluodenal artery, but most often from perforation and peritonitis. In more favorable instances diarrhed may be a complication and resule from impairment to the fligestive apparatus or irritation to the exposed nerveendings at the site of the lesions.

The treatment of diarrhea complicating extensive hurns is symptomatie. The chief thing is to relieve the terrible suffering induced loy the wound, which consists in protecting it from the air, puncturing the vesicles, kecping the parts submerged in water or covered with oil, applying antiseptic, soothing, and stimulating remodies, and later, skin-grafting, according to the stage and character of the lesion.

1)iarrhea here is treated by restricting the diet, administration of opiates, antiseptic and astringent remedies, as in other types of acute enteritis, together, when necessary, with flushing the bowel, but when perforation occurs the usual measures practised for the relief of peritonitis should be at once instituted, though operative interference is rarely permissible on accomnt of the patient's general condition.

Agoraphobia, Diarrhea in.-This form of loose movements occurs in certain individuals when in large audiences from the fear that they will have to go to the toilet, and the impulse thus generated of ten excites excessive peristalsis and even the transudation of fluid into the intestine, causing several fluid evacuations in rapid succession. Patients who have once hat this experience have it constantly before them, and are almost universally subjected 10 similar attacks while at receptions, in the theater, or restaurant, and more particularly when they are in doubt whether in case of need they can escape to the toilet. The author has known of cases where for the time being the patient lost control of the movements, they being involuntarily discharged, and Oppenheim calls attention to the fact that through this autosuggestion sphincteric tenesmus ensues, and then the intestinal contents are voided.

The treatment of this and other forms of psychic loose movements is fully discusioel in the chapter devoted to Nourogenic Diarrheats. and needs no further discussion here.

Sitophobia, Diarrhea in.-Paticnts who suffer from sitophobia. or the fear of eating hecause of the ill effects which they betieve will ensue, occasionally suffer from diarthea engendered ly gastro-intestinal dyspepsia. These sufferers restrict their diet to such a degree that the intestinal secretions are not sufficienty stimulaterl, matnutrition results, and the bowel becomes irritable do such an extent that it throws off the improperly digested food lefore it has an opportunity to be absorbed. Onee this type of loose movements is established, the diarrhea is continually aggravated thromgh psychic impulses consequent upon wory because of the frecpuent aracuations and the fear of them.

The treatment of sitophobic diarrhea is largely psychic, and con- 
sists in encouraging the patient to believe that his condition is not serious, that the eating of more food will not hurt him, and that in a short time he will recover, but the desired results can be most quickly and effectively accomplished by gradation of the amount of the food consumed until a normal diet has become established. In addition. rery neurotic individuals require remedies to correct this condition and enable them to obtain the necessary amount of rest and sleep.

Chilling, Diarrhea from.-Increased frequency of the evacuations is a common and distressing manifestation of "chilling" brought on through exposure to cold and dampness, as when the subject has been caught in a roaking rain. works under ground or in damp places, labors while he leans or sits upon moist earth or chilled stone, goes out improperly clothed when it is cold. exposes himself to drafts or while in a heated condition, goes into a cold-storage plant or other room having a low temperature.

This type of diarrhea may follow subjection of all or a part of the body, particularly the abdomen or feet. 10 chilling. and the number of the evacuations are as frequent after slight chilling as when the patient has a severe or congestive chill.

Fuw explanations have been offered as to the manner in which "taking cold" or chilling the body causes increased eracuations, and the-e are not convincing. There can be no doubt that sudden lowering of the surface temperature over a portion of or the entire body is accompanied by contraction of the cutaneous blood-ressels which leats to a congestion of the internal organs (particularly the intestine and visceral. Which favors an increased secretion of mucus similar to that ob-erved in the na-al passages during attacks of coryza. This explanation is not sati-factory to the author. hecause he has frequently studied the feces in these cases and the mucus contained in them has been small in amount and disproportionate to the numher of evacuations. Patients suffering from this type of diarrhea ordinarily have from five to eight evacuations daily, which indicates that peristalsis is markedly accelerated. a condition not likely to follow intestinal congestion alone. It is a question if this variety of loose morement- should not be grouped with neurogenic diarrheas. but this has not heen done because of custom. though the author consider that a nervou- element is the chicf factor in many of these cases. Elewhere it has heen pointed w that psichic emotions. disease in distant organs, and sudden shock can reflexty increase the frofuency and fluidity of the movements by causing hyperintal-in, excesive glandular activity and the transudation of fluid inter the bowel.

If the ontrolling nerve-centers and intestinal nerrous mechanism can be disturbed through the methods enumerated. there is reason to believe that diarrhea from chilling may arise from the same source, and result from the pronounced influence of cold upon the local nerves which carry the impulse to the centers from which it is re- 
flected to the intestinal nervous mechanism controlling the motor and secretory function of the bowel. It is possible that in some instances autosuggestion plays a part, the patient developing or affecting manifestations of a "cold" and intestinal disturlances simply hecause he has been eaught in a rain or has gotten his feet wet.

In aggravated cases true intestinal catarrh develops when the movements contain a greater amount of mucus, and diarrhea persists for a longer time, frectuently becoming chronic.

The treatment of diarrhea from chilling is mainly prophylactic, but, once contracted, symptomatic therapeutic measures are indicated. When seen early, the patient should be put to bed, given hot drinks, surrounded with blankets, and hot applications should be made to the abdomen to bring about a normal surface temperature and relieve congestion within the bowel, or this may be preceded by a hot hody or mustard foot-bath. When the chilly sensations continue, or the patient has a well-developed cold, a combination of Dover's powder, gr. $\vee(0.30)$, and quinin, gr. ij (0.12), administered every three or four hours, is effective because it arrests the chills, causes the patient to perspire, and diminishes the number of evacuations by lessening the secretions and peristalsis. When the patient suffers from cramps or aching pains, opium, gr. $\frac{1}{2}(0.03)$, and the extract of belladonna, gr. ? (0.008), or antikamnia, phenalgin, gr. x (0.60), every four hours, are serviceable. Warm, high, colonic water or medicated irrigations are also useful, because of the soothing effect of the heat upon the mucosa and its cleansing action.

Drinking Water and Cold Beverages, Diarrhea from.-The clrinking of pure water may cause soft or increaserl frecuency of the evacuations when it is consumed in large amounts or is taken ice cold under normal circumstances, but more particularly during hot weather or when the subject is overheated, and the same can be said of all cold beverages.

The drinking of large quantities of water induces diarrhea by mechanically stimulating peristaltic contractions and, possibly, glandular secretion, thereby increasing the amount and fluidity of the intestinal contents, and by diluting the gastro-intestinal secretions which interferes with digestion. The drinking of ice-cold water and beverages favors loose movements because they first induce a contraction of the superficial ressets in the mucosa, which is followed by a marked reaction and congestion of the membrane, which in turn temporarily leads to an abnormal secretion of the gastric and intestinal juices and eventually to intestinal catarrh.

Elsewhere the author has pointed out how chilling of the body surface acts upon the cutaneous nerres and causes loose movements, and here the same rules hold good, except that the cold comes directly in contact with the mucosa of the lowel instearl of the skin.

In certain communities, particularly Texas and New Mexico, the earth contains a considerable amonnt of alkali that contaminates the water of wells and springs, which, when drunk by a stranger or 
person unaccustomed to it, invariably induces a persistent and exhausting form of diarrhea. Drinking water containing other minerals, vegetable matter, parasites, refuse, or excreta is of ten responsible for this condition when it cannot be otherwise accounted for.

The treatment consists in having the patient drink less water and at a more moderate temperaure, and when the diarrhea is due to impure water it should be boiled or discarded for that which is pure. Then, in case the loose movements continue, they should be controlled or arrested by the usual antidiarrheal remedies, employed in conjunction with bowel irrigation when indicated.

Sun- and Heat-strokes, Diarrhea from.-Loose movements have been observed in these cases, both in patients who have recovered and those who died. but the evacuations were due more to rectal incontinence than to affections involving the mucosa of the gastro-intestinal tract. Nausea, vomiting. headache, vertigo, delirium, loss of consciousness, stercorous hreathing, and coma, one or all, may be present and, in addition, the patient not infrequently suffers from rectal and resical incontinence or suppression of urine, all of which manifestations may be accounted for through the effect of the sun or heat upon the local or general nervous mechanism.

These sufferers may be attacked while exposed to the sun, but there is a somewhat similar condition known as heat-stroke. prone to occur to workers who are enclosed in buildings exposed to high temperatures. such as obtain in glass-works, foundries, boiler-rooms, kitchens, etc. In the latter (heat-stroke) the attack may come on suddenly, gradually, or be preceded by loss of weight, weakness, dizziness, cramps, severe headache, disturbed vision, dry skin, rectal and resical irritability, but when it reaches a climax, there is hyperpyrexia, burning skin, delirim, convulsions, etc, along with diarrhea in some, or involuntary discharge of the feces in others.

The prognosis is more favorable in heat- than in sun-stroke.

The treatment of these conditions is largely prophylactic, and consists in having the patient avoid violent work when the sun is hottest or the temperature of the room is very high, using care as regards his tood, and avoiding iced drinks and excess of fluids. When an attack has occurred, efforts should he made to reduce the temperature by placing the patient in a cool room and applying cold packs. Stimulation by inhalations of ammonia or amyl nitrite should be resorted to, and when the patient has slightly recovered he should be placed upon a diet composed of skimmed or butter-milk, grape-juice, and boths until such times as he can consume semisolids. For control of the cramps and diarrhea, opium and belladonna or the ordinary diarrheal remedies are indicated, except when the loose movements result from incontinence, in which case they clo little or no good; and the freguent involuntary evacuations will continue until the patient dies or his general condition is improved and muscular tone has been restored to the rectoresical musculature. 
Old Age, Diarrhea in.--The digestion is always materially impared in the aged, and Schlesinger and Neumann, following Schmidt and Strassburger's plan of test-eliet and fecal examination practised upon 30 old people, found that they digested connertive tissue badly, but handled muscle-fiber, cereals, and fat very well. Elalerly individmals are of en afflicted with constipation becanse of imperfect digestion and the difficulty with which the intestinal content is propelled forward. In cases where feces are permited to collect and be retained to form single or multiple compart fecal masses, they soon excite a stercoral diarrhea which persists until the offending scybalous masses have been elislodged and evacuated.

Hence the treatment here consists in regulating the diet, giving an after-elinner pill to keep the bowel open, and administering castor oil and frefuent high colonic enemata of soapsuds or oil to soften the impacted accumulations so that they may be washed out. When the masses are large, hard, and locater in the sigmoid flexure or rectum, they are most quickly removed by introducing the sigmoidoscope, breaking them up with a gouge, and then they can easily be dislodged and washed out by continuous irrigation.

Diarrhea Nocturna.--(Sec Neurogenic Diarrhea.) The caption "diarrhea nocturna" is employed to indicate a type of loose movements wherein the stools are watery, rapidly succeed each other, and are voided in the carly morning between two and five o'clock, when the patient is resting quictly in bed, which ought to favor a diminution instead of an increase in the evacuations. This condition prevails in tuberculosis of the intestine and elsewhere, and ulceration of the colon may or may not cause the frequent and exhausting stools. The writer concedes tuberculosis may produce a type of diarrhea with similar symptoms, but on several occasions he has treated patients for diarrlea nocturna who hat no organic lesion of the intestine, lut suffered some organic or functional disease of the nervous system, where the evacuations were watery, frequent, and occurred in the early morning, and in whom the diarrheal state was aggravated by anxiety, sorrow, business worries, and psychic emotions of all kinds. Hence he holds that nocturnal should be grouped with the other neurogenic diarrheas discussed clsewhere.

Eosinophilic Diarrhea.-Neubauer and Stäubli ${ }^{1}$ have reported cases of this type of acute diarrhea with marked constitutional symptoms in which the mucosa was highly inflamed. During the height of the attack the eosinophils completely disappeared from the blood and returned when the symptoms abated. All cases occurred in young adults, and were accompanied ly sexere diarrhea, with hloxl, mucus. and Chareot-leyden crystals in the stools. Intestinal parasites, specifie bacteria, or gonococei were found. The rectal mucosa was congested and covered here and there with whitish exudates, but was not ulcerated.

These cases would indicate that the claim made ly many authors 'Munch. Med. Worhenschrift, 1906, p. 2380. 
to the effect that Charcot-Leyden crystals in the stools and an increased number of cosinophils in the blood are always indicative of intestinal parasites does not obtain.

The treatment is local and general.

Mechanic Diarrhea.-like others, mechanic stimuli irritate the mucosia and tend to augment the mucous glands to excessive activity and increase the frequency and strength of the peristaltic movements. Consequently, mechanic diarrhea may be induced by foreign bodies, enteroliths, gall-stones, intestinal sand, scybale, large impacted fecal masses, swallowing of metal-dust, drinking water contaminated by the feces of grasshoppers (Prout), decomposing granite (Hartigan), or lime-dust.

The treatment consists in eliminating the source of the irritation, and then treating the patient as if he had enterocolitis.

Reflex Disturbances, Diarrhea in.--Sometimes loose movements from this source are incident to disease or a growth involving neighboring organs (particularly sexual) distant from the gastro-intestinal tract which it indirectly disturbs. Castro-intestinal indigestion and diarrhea may result from an ulcer in the stomach or stricture in the rectum which inpairs the functions of the stomach; the small intestine and colon may, through reflex disturbances, he seriously interfered with when a small segment of the alimentary canal has become ptotic or diseased, or involved by adhesions, an angulation, invagination, twist, pericolic membrane, diverticulum, or blocked by a tumor, stricture, or foreign body.

Diseases or injury of the hrain, spinal cord, or nerves remote from the bowel may, under favorable circumstances, lead to watery evacuations or anal incontinence, and emotional disturbances, as excitement, fright, joy, and worry, frequently cause diarrhea. Many other causes of reflex diarrhea could be mentioned, but it is not necessary here to more than recall the fact that surgeons have in hundreds of instances succeeded in permanently curing chronic reflex diarrhea by operating and removing the cause of irritation to the local or general nerve mechanism which through reflex disturbances were responsible for the diarrhea.

Acute Embolic Enteritis and Colitis, Diarrhea in.-Like other organs the large and small intestine may become involved through hematologic emboli in the presence of ulcerative endocarditis, anthrax, septic angina, fibrinous pnemmonia, and other pyogenic processes.

Changes.- While the submucosa later is primarily attacked, the mucosa becomes softened, elematous, and characterized at first by punctiform hemorrhagic foci, or scattered nodules having hemorrhagic margins, and, later, by phlegmonous ulceration or abscess in the region of the vessels blocked by bacterial emboli.

The method of diagnosing and locally treating embolic enterocolitis resembles that of ulcerative and phlegmonous entamebic colitis, discussed elsewhere. 


\section{CHAPTER X}

\section{GASTROGENIC DIARRHEA (DYSPEPTIC DIARRHEA, LIENTERIC DIARRHEA)}

\section{ACHYLIA GASTRICA, HYPERACIDITY, MALIGNANCY, ATONY, MOTOR INSUFFICIENCY}

Comparatrely a few years ago nearly all rliarbeas were regarded as being due to functional or organic discase of the intestine, but today it is conceded that this condition is sometimes caused by disease of the stomach or interference with the gatstric secretions from nervous and other causes, and to Einhorn, Oppler, Schultz, and A. Schmidt belongs the chief credit for having pointed out this type of diarrhea, explained its etiology, outlined a rational method of diagnosis, and suggested meatsures for its correction. Except in lypical cases it is often extremely difficult to determine the exact part played by abnormalities of the stomach in diarrhea gastrica, because in some instances the stomach is solely responsible, in others there is associated disturbance of the duodemum and pancreas, or a catarthal condition of the small or large bowel, which help to increase frerpeney of the movements.

This type of diarthea may be induced by achylia sastrica (subacidity), hyperacidity, atony, motor insufficiency of the stomach or malignancy, either of which may temporarily or permanenty impair the functionating power of the intestine, particularly when it is alreatly affected or the patient has a lowered resistance.

In this affection there is usually but a slight discomfort in the stomach, the diarrheal manifestations leing the chicf source of trouble and from which the patient desires relief.

In achylia gastrica diarrhea, owing to the alsence of hychochloric aciel, that part of the digestion assigned to the stomach fails to take place, and the improperly prepared chyme is discharged into an ofttimes already irritable or nervous intestine which is incapable of completing digestion on account of the extra work thrown upon it. Consequently, it is not surprising that this almormal intestinal content. with its large boluses and abundant undigested comnective-tisste remnants, should irritate the intestinal mucosa to magnily its secretions and reflexly stimulate a more frecpuent and stronger peristalsis which invariably leads to increased fregueney of the evactations. Agam, the bactericidal quality of the chyme is materially rerluced through loss of the hychochloric acid, which allows the putrefictive and other pathogenic bacteria inhabiting the intestine 10 multiply and derelop virulent toxins with a tendency to aggravate the diarbeal condition, 
and it would appear that the connective-tissue food remnants afford the bacteria an excellent medium in which to derelop.

Hydrochloric acid is known to stimulate pancreatic activity and because of this lost influence the amount of pancreatic juice is materially diminished, which in itself is often sufficient to induce aggrarated diarrhea.

Hyperacidity (though less frequently than achylia) is an etiologic factor in gastrogenic diarrhea. In these cases there is a marked increase in the amount of hydrochloric acid present, while the organic acids show but slightly if at all. and there is a characteristic lessening in the amount of bile secretion. In discusing the manner by which hyperacidity diarhea is produced. Caultier maintains that in these cases the hypersecreted chyme sleceeds only very slow and imperceptibly in saturating its hydrochloric acid in the digestive passages, so that the fermentation acids which already exist in the stomach. increased by the fermentation acids which normally form in the intestine through the decomposition of the fatty substances, react upon the inte-tinal mucosa and lead to hypersecetion and peristaltic activity by irritation of the nerve-fibers, the peristalsis finally becoming strong enough to induce frequent and liquid stools.

Peculiarly enough. this condition produces. or at least is associated with. a considerable lessening of the flow of bile. a change which favors an increase of the diarrheic condition because the stimulating effect of the bile upon pancreatic secretion is minimized, the neutralizing effect of its alkaline qualities upon the upper intestinal acid content - is diminished. and modifies fat absorption, all of which interfere with the digestion and assimilation of food, thereby leaving an abnormal and larger residue which farors putrefaction, increased secretion of mucus, sets up marked peristaltic action. and brings about diarrhea. Caultier has called attention to the fact that acid diarrheas are associated with duodenal dystryp-ia, which alternates with constipation. and says that the former is the result of deficient pancreatic. and the latter to a les-ening of biliary, secretion.

In the atonic and deficient motility types of gastrogenic diarrheas the manner in which the frequent movements are excited have not been satisfactorily explained, but it is probable that the food and secretions remain until stagnant: in the meantime abnormal fermentation and putrefaction take place and the lacteria and toxins increase and cause tromble. Finally. when all or a part of the incompletely digested gastric contents. along with the irritative gases formed. are discharged into the mall intestine, they profoundly effect the delicate mucosa. directly lig contact and indirectly through action of the toxins upon the nerrous system. with the result that intestinal digestion is interfered with, assimilation is diminished, intestinal glands are excited to excesive activity, and the bowel is left in an irritable state and peristalsis readily responds to trauma induced by the food remnants.

Carcinoma of the stomach may also be classed among the causes 
of gastrogenic diarrhea. When located in the body of the cardiac end of the stomach the disturbance is due to fermentative changes and diminished hydrochloric acid, but malignancy usually attacks the pyloric end of the stomach, and under such circumstances there is impared digestion, achylia gastrica, and obstruction that caluses retention and stagnation of the food, which when discharged into the bowel sets up an irritative diarrhea.

Certain organisms, like yeast, fungi, proteus, hacilli, cocci, and streptococci of various kinds and forms, are usually found in large numbers in the feces of patients suffering from gastro-intestinal dyspeptic diarrhea, and it is reasonable to suppose that one or all play a greater or less part in exciting this condition, and the furst two are frequently encountered in enormous amomnts.

Symptoms.-Patients afflicted with gastrowenic diarrhea suffer but slightly from, and consecquently place but litle importance upon, gastric manifestation, but complain bitterly of the diarrhea, and many of them become hypochondriaes and think and talk of nothing but their intestinal ailment, much of the worry being due to the fact that it is thought incurable because of its chronicity. Sometimes this type of loose novements is continuous, hut occasionally after dietary incliscretions the attack may occur at shorter or longer periods or be continuous, according to the nature and gravity of the case, and those of an extremely nervous constitution suffer greatest and have more freguent passages than those who are not.

Early in diarrhea gastrica the intestine is not involved, but later become so, and an enteritis or enterocolitis gradually develops through trauma and constant irritation to the mucosa; in severe cases mucus. pus, and blood complicate the symptom-complex. The lienteric character of the stools is the chief manifestation in all forms of gastrogenic diarrhea, because, as A. Schmidt and others have repeatedly shown, the feces contain undigested connective-tissue remnants.

The fact has already leen mentioned that in the hyperacidity type of gastrogenic diarrhea there is a deficiency in both the biliary and pancreatic fluids. In such cases there is little or no anorexia, but the patient suffers from a coated tongue, waxy at first, and hater greenish complexion; gas distention, lenderness over the stomach and small intestine, weakness and losis of weight, restlessness, has little if any desire to attend to his social or business duties, and the acid, watery, frequent evacuations induce annoving tenesmus and irritate the skin, but when there is complete absence of the pancreat ic fluid the movements contain an abundance of fat: bright droplets are visible to the naked eye, as are remnants of starch and meat.

It has been demonstrated conclusively that diarrhea does not always occur in connection with the above-described gastric abnormalities, and because of this Schutz claims that when it does, the bowel has a lowered resistance.

Where the gastric changes lead to fermentative diarrbea the smomtoms are disseminated, the patient claming loss of appetite, malaise, 
central abdominal tenderness, pain, borborygmus, moderate diarrhea with light yellow, pasty, and foamy evacuations having an acid reaction and a foul odor, and containing starch and connective-tissue remnants.

In diarrhea gastrica consequent upon carcinoma, constipation is an early manifestation, and later alternates with diarrhea, but in the terminal stages frequent movements prevail in half the cases, and the number of stools is increased when the patient eats copiously of indigestible foods, and diminished under a restricted diet dominated by fluids.

Gastrogenic diarrhea may be suspected, but cannot be proved, when the gastric secretions vary slightly and examination of the stools demonstrate the absence of pus, blood, and mucus. Authorities best qualified to speak are inclined to consider diarrheas which are preceded by chronic gastric disturbances, those improved by the administration of hydrochloric acid or gastric lavage, where the stools contain pieces of meat or finer connective-tissue remmants, or large numbers of yeasts, sarcine, long bacilli, proteus, and other fermentative or putrefactive producing organisms, as being partially or entirely due to achylia gastrica, hyperacidity, atony, insufficient motility, or gastric malignancy.

Diagnosis.-To A. Schmidt more than anyone else belongs the credit for devising a rational method of differentiating gastrogenic (lienteric) from other diarrheas, for he has shown by his test-meal that connective-tissue remnants are found in all types of diarrhea gastrica, while Einhorn, Oppler, Schutz, and others have done much to isolate the different types of gastrogenic diarrhea by demonstrating that they are due respectively to achylia gastrica, hyperacidity, atony, and motor insufficiency, and that they are demonstrable by an examination of the stomach and its secretions.

The main reliance in the diagnosis consists in an analytic study of the feces following a test diet of two or three days' duration or such time as an evacuation from it can be obtained. A. Schmidt's itinerary is the best, and consists of milk, toast, oatmeal, eggs, beef, mashed potatoes, and butter. After such a diet the stool is placed in a ressel with water and macerated, and then, if connective-tissue shreds are visible on microscopic examination, it demonstrates the gastrogenic nature of the diarrhea, as in achylia gastrica, etc. The analysis also shows imperfectly digested vegetables, fats, and albuminoids, biliary deficiency, and when there is intestinal irritation, glairy mucus and discarded epithelial cells.

Schmidt has also pointed out that the abundant presence of micro-organisms which produce fermentation and decomposition, such as yeasts, sarcine, long bacilli, and others already alluded to, is adclitional evidence of gastric disturbance.

When diarrhea gastrica is due to hyperacidity or other abnormality, is complicated by deficient pancreatic secretion or achylia, the movements following the test-diet show an abundance of fat 
droplets, microscopic particles of meat. connective-tissue shreds, starch, and microscopically demonstrable connertive-tisane nuclei; the latter being digestible only by pancreatic secretion, conseguently Schmidt maintains that their presence always indicate pancreatic disturbance.

Inctependent or associated fermentative dyspepsia is roognnized by the simptom-complex alreaty emumerated and the coprologic findings, such as yellow, pasty. foamy, disagreeable aciel stosts, which contain large amounts of starch reminants and colostrum lutyricum (Zwieg). and their incubator test hows a marked carlohydrate fermentation (Kuttner). Small intestinal fermentation and putrefaction, according to Schmidt, may continue after the gastric error has been corrected.

Enteritis, or enterocolitis, may be present lefore or develop on the basis of a chronic gastrogenic diarrhea. under which circumstances abnormality of the gastric contents is manifest, connectivetissue remmants are present in the feces besides mucus, or in aggravated cases. pus and hlood, which indicate a catarrhal condition of the intestine, and, in addition to these diagnostic signs. the patient complains of cramps and frefuently suffers from auto-intoxication.

Biliary insufficiency. which often complicates hyperacidity and other forms of gastrogenic diarrhea. is indicated in the fecal analy is by poorly digested fats, muscle-fibers slightly stained with bile, and a weak Gmelin reaction.

Gastrogenic diarrhea, induced by an ulcer (peptic) in the stomach. is characterized by sitophobia inducing epigastric pain following eating. tenderness upon pressure in the epigastrium, ineligestion. vomiting. which affords relief, hemorrhage, pyloric spasm, collapse. anemia when bleeding has been copjous, and manifestations of shock and peritonitis following perforation.

Cancer of the stomach is usuatly located at the pylorus, though it may involve the leser curvature or cardia, and can be differentiated from other affections because it is prone to occur in midelle age or elderly persons. induces severe dragging pains in the epigastric region or back, vomiting of undigested ford. bleeding, metastasis of the abdominal lymph-nodes: the patient is cachectic, has indicanuria. there is frequently visible peristalsis. and when advanced a tumor can be made out by percusion or detecter by palpation: asain. in such cases a test-meal analysis shows that $\mathrm{HCl}$ and ferments are diminished, while lactic and fattr acids are abundant. Macroscopic and microscopic examination of the stomach contents show bright or clotted blood. pus. mueus. shreels of tissue. and the oppler-Buas bacillus and sarcinit. ( ancer of the protorus is complicated by stagnation of the stomach contents. and lactic acid fermentation or hydrochloric aciel is present when it results from degeneration of a priloric ulcer.

With a normal gastric content and no connective-ti-sue remnants, fat, mucus, pus, or blood in the stools, persistent foose movements 
simulating those of diarrhea gastrica should be diagnosed neurogenic diarrhea.

Treatment.-From what has been said about the etiology of gastrogenic diarrheas it is obvious that a routine treatment is impracticable. because in different cases the stomach is atonic, there is motor insufficiency, its contents are abnormal, there is biliary and pancreatic insufficiency. and enteritis may be a complication; all of which conditions require consideration when a plan of treatment is to be outlined. In this condition it is rery difficult to resist prescribing for the diarrhea and intestinal manifestations which dominate the clinical picture, but to be effective this must not be, and the treatment should be directed against the gastric abnormatity or true source of the trouble.

When diarrhea gastrica is consequent upon achylia or diminished acidity, liheral doses of hydrochloric acid or natural gastric juice should be administered three times daily at mealtime, and this agent is also the most effective remedy to prescribe in the absence of a changed secretion when there is atony or impaired motility of the stomach, but in these conditions schmidt recommends that a bitter tonic be combined with it.

Loose morements consequent upon hyperacidity are best controlled by alkaline and sedative preparations to neutralize excess of the acid, limit the secretion, and diminish intestinal irritability, such as the bicarbonate of soda, magnesia, charcoal, bismuth, cerium oxalate and calcium, either alone or in combination with belladonna, but when this condition is complicated by abnormal amounts of fats in the feces one of the reliable pancreatic digestants should be administered to overcome the deficiencr.

In the fermentatice type of gastrogenic diarrhea, magnesium peroxid has proved very satisfactory, and regarding its action Gaultier says, "This substance has the property of decomposing, in an acid medium, into a neutral salt of magnesium and oxrgenated water. The oxygenated water, in its turn, permits the escape of nascent oxygen into the intestine, which probably inhibits intestinal fermentations." Bismuth and charcoal are also useful agents to employ. in gastro-intestinal dyspepsia, but while waiting for medicines to accomplish their purpose much can be done to relieve diarrhea and other uncomfortable manifestations by keeping the patient in bed, applying hot applications to the abdomen, and restricting the diet.

While it is necessary to par attention to the diet, this part of the treatment of diarrhea gastrica is not nearly so important as it is in some other diseases of the stomach. Indigestible foods may aggravate the condition, but the evacuations are apt to occur with increased frecuency in this class of cases immediately after meals, irrespective of what has been eaten. It is important to see that the food is well preparecl and carefully masticated, to limit the amount of meat consmed (and interdict it altogether when raw or smoked), for otherwise the patient's condition will invariably be aggravated. A protein 
diet is most suitable in fermentative diarrheas, but where there is a hyperacidity, with pancreatic and biliary insufficiency, fats should be restricted and the bile flow stimulated by the atministration of roast meats and egess.

In gastrogenic diarrhea consectuent upon carcinoma, control of the diet is of great importance, and only foods which cause the least irritation and pain should be permitted, and they should be taken in small quantities and at short intervals.

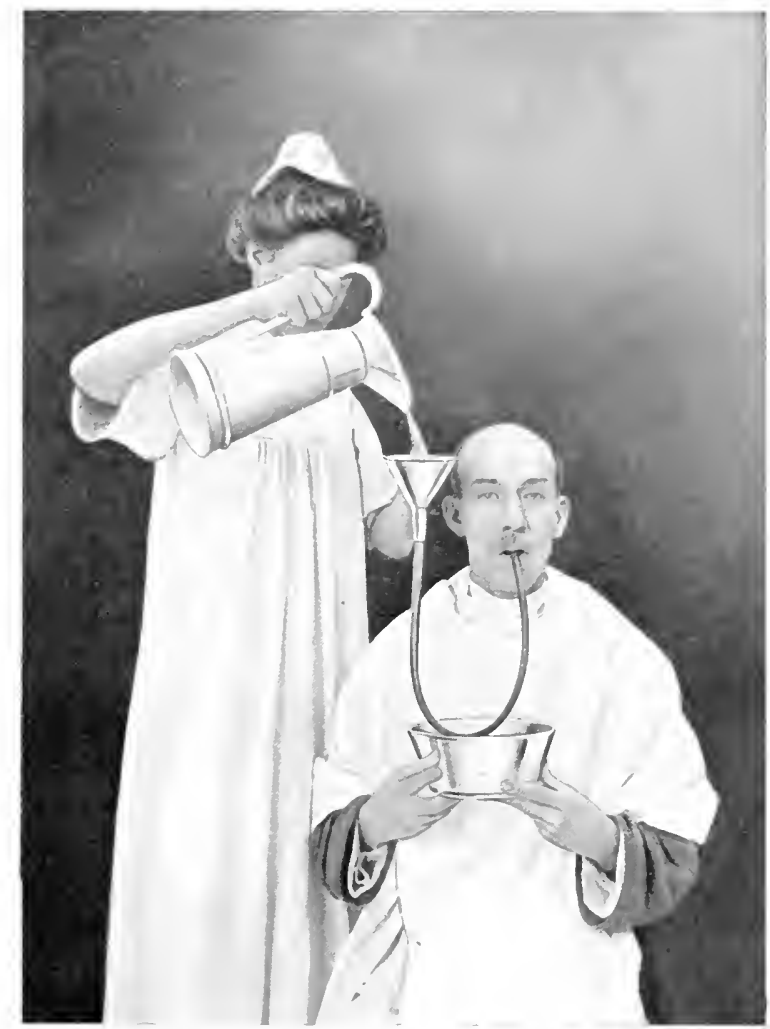

Fig. 26.-Washing out the stomach.

Where there is gastric atony or diminished motility, and the treatment alreaty outlined fails, open-air exercise, massage, dectric vibratory treatments, and hyllotherapy often render valuable asistance hy strengthening the organ. Patients afflicted with this type of diarrhea, who suffer greatly from gastric stagnation, fermentation, and putrefaction, resulting from imperfect secretions or malignant pyloric stenosis, are relieved hy nightly or more frecuent gastric lavage (Fig. 26). A normal saline or, in suitable cases, an alkaline solution is employed. When enteritis or colitis is present, as is eridenced by mucus, pus, or bloot in the stools, much can le done toward 
the patient's comfort by minimizing intestinal auto-intoxication, and healing the inflamed mucosa by colonic enteroclysis, using boric acid, 2 per cent.; ichthyol, balsam of Peru, I per cent., or permanganate of potassium, I per cent.; or permitting $\frac{1}{2}$ pint of olive oil containing I oz. of glycerin to flow into the bowel at night three times weekly or oltener.

Gastrogenic diarrhea is peculiar, in that the usual antidiarrheal remedies fail to control the movements and are contra-indicated, except opium extract, gr. ${ }_{4}^{1}(0.015)$, or this drug and belladonna extract, gr. $\frac{1}{8}(0.008)$, when the evacuations are exceedingly frequent and the patient suffers intensely from abdominal pains and cramps.

Patients who suffer from diarrhea incident to gastric ulcer should be put to bed for several weeks, and kept on a tluid diet reinforeed by nutritive enemata; the bowel should be kept open by small doses of Carlsbad or Epsom salts, administered daily; opium or morphin, gr. $\frac{1}{4}$ (0.0I5), employed to relieve pain, and astringents and antiseptics, as bismuth, gr. $\mathrm{xx}$ ( I.3O) ; silver nitrate, gr. $\frac{1}{6}$ (O.OI); or tannalbin, gr. $x$ (0.60), should be prescribed three times a day to diminish the stools and stimulate healing of the ulcer. When there is hyperacidity, alkaline remedies, such as sodium carbonate, gr. $\mathrm{xx}$ (I.30), should be administered in liberal doses in addition to the mineral waters already mentioned, and when the patient is anemic Fowler's solution of arsenic, niv (0.24), should be added to the treatment; when bleeding is profuse, extreme quiet is indicated, the ice-bag should be applied over the stomach, and ergot, ne xv (I.O), given hypodermically, and hypodermodysis administered if necessary.

In cases where rest, hygienic, dietetic, and medicinal measures fail to effect a cure, one should immediately resort to gastro-enterostomy, gastroduodenostomy, or pyloroplasty when the lesion is at the pylorus, and when there is perforation the opening in the stomach should be closed independently or in conjunction with enterostomy.

Carcinoma invariably terminates fatally, except when the growth is removed by a radical operation which necessitates resection of a small or considerable portion of the organ, and in some instances complete extirpation of the stomach (partial or complete gastrectomy).

Palliative treatment is indicated in the majority of cases because they reach the inoperable stage before the nature of the trouble has been determined. Such measures consist in restricting the patient to a selective fluid or non-irritating diet, preventing gastric fermentation, and administering opiates in liberal doses alone or in conjunction with antiseptics, astringents, and styptics to control pain, diminish frequency of the evacuations, and minimize the danger from hemorrhage. Cocain or orthoform added to the prescription affords the patient additional relief.

In the presence of stagnation, distention from gas, and excessive fermentation relief in some instances quickly follows gastric lavage. When well borne, hydrochloric acid may be prescribed to advantage to increase the efficiency of the gastric contents, and tonic remedies 
should be administered to stimulate the appetite and strengthen the patient.

When these measures fail, the patient grows steadily worse, and his suffering becones unbearable, palliative operations are justified. but the patient or his friends must be told that the oferation will diminish suffering and extend life, but that in no case will it effect a cure. When the cancer is located at the carliac extremity of the stomach, causes obstruction, and makes it extremely dificult for the patient to swallow thids. gastrostomy is indicated and afforts almost instantaneous relief. and is followed by improvement in the patient's general condition, because he can be comfortably fed at any time through the artificial opening.

Gastro-enterostomy is the operation of choice in inoperable cases where the tumor is located at or near the pylorus. irrespective of whether or not obstruction has occurred. This procedure prevents gastric stasis and undue fermentation, lessens the danger from bleeding, furthers digestion, relieves pain, and, to some extent, inhibits growth of the tumor by minimizing the trama to it. When the cancer is small and has not progressed too far, the operation is freguently. a valuable preliminary step to extirpation, because in the interval between the operations the debilitated state of the patient and irritable condition of the stomach can be improved, since disturbances consequent upon passage of the food through the strictured segment of the stomach are relieved. Except when the posterior wall is involved by the disease or adhesions. posterior is preferable to anterior gastro-enterostomy. In extreme cases, where the patient is sorely afflicted with pain and nourishment otherwise is impossible, and gastro-enterostomy is for any reason impracticable, jejunostomy is called for. Under other circumstances this operation is contra-inclicated. because it is impossible to introduce a sufficient amount of nourishment through the opening. and because the fluid chrme is being constanty discharged through the opening to annoy the patient and irritate the skin. 


\section{CHAPTER XI}

\section{ENTEROGENIC DIARRHEA (DYSPEPTIC DIARRHEA, LIEN- TERIC DIARRHEA)}

\section{UNBALANCED SUCCUS ENTERICUS, DUODENAL ULCER}

Dyspepsia intestinalis occurs more frequently than is generally supposed, because the diarrhea from this source is frequently attributed to gastrogenic disturbances or to various inflammatory and ulccrative lesions of the colon. Enterogenic dyspeptic loose movements may be marked in individuals when the mucosa is broken and when it is not, but usually it is more aggravated and difficult to control in the former than the latter.

This type of diarrhea may be primary when- $(a)$ it is due to a diminished secretion of the biliary, pancreatic, or true intestinal secretions; $(b)$ they are prevented from being discharged into the duodenum owing to obstruction within the ducts; $(c)$ there are pathogenic changes in the mucosa which interfere with or prevent intestinal digestion and assinilation; and (d) there are imperfect mastication and indiscretions in diet, and more food is consumed than the intestinal juices can take care of. Indigestible articles of diet (cabbage, sour-krout, cucumbers, and fresh fruit), after passing through the stomach, are mechanically irritative, or readily undergo fermentation and putrefaction.

Intestinal dyspepsia may be secondary to gastrogenic functional and organic discases, such as (a) hyperacidity, (b) achylia, (c) atony, (d) impaired motility, and (e) benign or malignant obstruction, which incluces intestinal catarrh or imperfect digestion, owing to the action of the abnormal gastric juice upon the food, or retention of the latter within the stomach until it is stagnant, and causes irritation after it passes the prlorus.

The etiologic factors of the secondary form of dyspepsia just enumerated require no further elucidation, since the effect of all in producing bowel disturbance has been fully discussed under the caption of Castrogenic Diarrheas. The part played in primary dyspepsia intestinalis by diseases and obstructions of the liver, pancreas, and their ducts, whereby too little or too much of the juices from these organs reach the intestine, has also received due consideration when outlining the etiology, symptomatology, diagnosis, and treatment of pancreatic, biliary, fatty, and alcoholic diarrheas and when discussing the physiology of digestion.

Functional and organic diseases which minimize or increase the gastric, pancreatic, or biliary fluids or ahter their relation to each other 
constitute the most frequent and perhaps persistent causes of enterogenic dyspeptic diarrhea. The lact should not be orerkoked that it may result from an alsdominal or decreased intestinal secretion resulting from nervous disturbance to the intestinal mechanism, impaired circulation, anemia, pathologic changes in the mucosid, or through a lowered resistance and undue multiplication of pathogenic bacteria and their toxins, normal inhalitants and products of the bowel, or those which reach it through the food.

Bacteria, through their augmentation of the fermentative and putrefactive processes, are sometimes responsil,le for intestinal dyspepsia and diarrhea through their abnormal multiplication and activity within the bowel, when there is a lowered resistance fparticularly in neurasthenic, anemic, and tubercular subjects) on the part of the patient, and when certain forms of pathogenic bacteria are introduced into the bowel by means of diseased water and foot.

The author will simply state here that this form of indigestion may occur where too much of one and too little of another kind of food is consumed; the fermentative juices are unequally balanced; or the subject has an idiosyncrasy to certain articles of diet.

Persons who consume an excessive amount of protein suffer less frequently and severely from enterogenic dyspeptic diarrhea than those who are indiscrete as to the quantity of fats and carbohydrates that they consume, because, after all, the loose movements in this class of cases are due principally to the irritating effect of the organic acids (lactic, acetic, butyric, and gases arising from the bacterial decomposition of the carbohydrates and fats) within the bowel, which are present in undue and harmful amounts when the last-named food products dominate the diet, or their digesting ferments are diminished in quantity, or are prevented by obstruction from reaching the intestinal canal.

Duodenal ulcers are occasionally causative factors in enterogenic diarrhea, because, through the irritation and reflex disturloances excited by them, peristalsis is augmented, the intestinal secretions are increased, and foorl is rushed through the small intestine into the colon before digestion has taken place, and, in consequence, remnants of the food elements appear macroscopically in the stools. The lesions occur most frequently in men, and may be secondary to extensive burns or mesenteric embolism with digestion of the infaret, or they may be induced by trauma or chronic hyperacidity (HCl). The characteristic manifestations of duodenal ulcers are indigestion and romiting after meals, severe cramp-like pains in the right hypochondriac region several hours after eating, and bloed in the stools.

The symptoms of enterogente dyspepsia vary and are modified in accordance with the degree of complicating gastric disturbance when present, type of intestinal incligestion inciting the troulule, the part played by gastro-intestinal catarrh, and, finally, by the general condition of the patient. 
When the stomach is involved, anorexia, sometimes acid eructations, nausea, and vomiting are present, and the patient complains of fulness and pain in the region of the epigastrium. The intestinal manifestations vary greatly in different cases. but loose movements, accompanied by distention, borborygmi, flatus, abdominal discomfort and pain, are always a pronounced feature, and it might not be inappropriate to designate this condition as al dyspeptic acid diarrhea, because in most instances the excessive irritation to the bowel, increased peristalsis, and secretion are largely due to abnormal acidity of the intestinal contents, on account of which the chyme is first hurried through the small intestine before digestion takes place, and then through the colon with such haste that absorption is impossible. The stools under these circumstances are acid, frequent, fluid, and cause considerable burning and tenesmus of the rectum and more or less irritation to the perianal shin once the attack is on, but such stools come from the upper intestine, and in the beginning are preceded by one or two semisolid or formed morements, composed of feces which had previously collected in the colon or rectum. The abnormal evacuations are frequently gelatinous in consistence owing to the presence of a large amount of mucus, under which circumstances it is difficult to determine whether it arises from dyspepsia or intestinal catarrh. In the latter, however, epithelial and isolated round cells are more noticeable, while in the former the mucus is apt to be bile stained.

In underfed children, with an idiosyncrasy to carbohydrates owing to the predominance of fat, the stools are of a light or gravish color, the Bacillus bifidus is present in considerable numbers, and the amount of indican and phenol in the urine is greatly exaggerated.

Schmidt and Strasburger have called attention to what they designate "intestinal fermentation dyspepsia." which closely resembles the jejunal type of diarrhea just discussed, which they believe is dependent upon insufficient digestion of starch as revealed by the fermentation test and stool analysis following a special diet.

Nothnagel has tersely summed up their views as follows: "The subjective symptoms are rather vague, but abdominal pains, chiefly in the umbilical region, fatigue, and discomfort are the most prominent. Abdomen frequently symmetrically distended, usually tender on palpation, either universally or limited to umbilical region or to the left of it. Examination of stomach rarely gives pathologic indications. Feces (after test-meal) evacuated somewhat more frequently, but no actual diarrhea. Stools are often foamy, light yellow, acid, with the odor of butyric acid. Other indications usually absent, particularly noticeable being the lack of mucus, and often of starch, but after ingestion of potato there occur plentiful empty potato cells." These authorities believe that cases may be of neurotic origin, the result of catarrh or purely functional in character.

The diagnosis of dyspeptic diarrhea is not always easy, and must be distinguished from catarrhal loose movements. In some instances 
the history will throw light upon the case, but ustually reliance is to be placed upon gastric and fecal analysis following test-meals to determine the part, if any, played by unbalanced gastric, biliary, or pancreatic juices, and also if there is imperfect protein, carbohydrate. or fat digestion. The presence of mucus, pus, or blond, when discosered, inclicates catarrhal organic changes.

From what has been said, acid and gelatinons evactations containing considerable bile-stained mucus without epithelial or round cells indicate the jejunal character of the diarrhea.

Anders groups the differentiating features between duofenal and gastric ulcers as follows; in other respects the manifestations of the two conelitions are about the same:

DITOI)ENAL LLLER.

Lsually occurs between twenty and forty years, except when rlue to external burns.

Males are more frequent sufferers than females in the proportion of 10 to 1 .

Onset markerl by intestinal hemorrhage. which may reur at intervals of rarying duration.

The metena may be preceder or accompanied by hematemesis, though not generally.

Blood in the discharges often is bright red, profuse, but not so marked as in gastrorrhagia; sometimes dark and tarry from the attion of acid chyme when slight.

Pain. due to acid, may come on late, two to four hours after meals; more often absent. It is localized a little abose and to the right of the umbilicus. Pain relievert by eating. owing to absorption of acid and closure of the pylorus.

Gastric crises occur without reference to time of taking fool.

Tomiting inconstant wilhout relation to ingestion of food and affords no relief.

Jaundice occasionally present irom occlusion of bile-duct.

No marked improvement after diet has been regulated.

Dorsal pain-point absent.
G.ASTRIC LLCER.

May occur at any age after childhord.

Females are the chict sufferers.

Gastric hemorrhage often occurs, preceded by other gastric symptoms. as a rule.

blood may appear in the stools, usually. after hematemesis.

The blood in the dejections is dark and tarry from the action of the crastric juices.

Pain, paroxysmal, greatly intluenced by taking food. Pain sharply localized in the epigastric region about 2 inches below the ensiform cartilage. I'sually. pain is ageravaterl by taking food.

Gastric crises come on soon after taking food.

Vomiting more common (during painful crisis) and afiords relief.

Jaundice absent.

Usually a marker! improvement follows regulation of diet.

Pain-point (tenth to twelfth dorsal vertebra on left side) usually present.

The treatment lere is largely dietary, and certain types of food should be interdicted or limited and others increased, according is the diet is improperly balanced and one variety is being partaken of in amounts larger than can be digested, and in rare instances, where solids cause undue irritation, it is actrisable to resort to a lifuid diet until later, when the patient can take care of solids.

In the presence of fermentative diarrhea and oft-repeated gadistention, regetables should be partaken of sparingly, and reliance should be placed upon eggs, soups, jellies, getatins, grueds, anel one of the lactic acid bacillus, sour milks, such as koumyss, matzoön,

\footnotetext{
${ }^{1}$ Practice of Medicine, 1910.
} 
bacillac, lactone, buttermilk, or lactoform. When there is marked distress from gas this can usually be relieved by having the patient take I or 2 drops of turpentine on lump sugar, three times daily, or ginger, peppermint, lime-water, or one of the carminative teas may answer the same purpose.

When the evacuations are foul, and result from putrefaction or albuminous decomposition, this is best corrected by gr. ij to iij (o.I2o.I8) of calomel in broken doses, administered weekly, until the stools are less frequent and offensive, using in the intervals satol, beta-naphthol, resorcin, guaiacol, ichthyol, we $\mathrm{v}(0.30)$, or salicylic acid, gr. v (0.30), four times daily, alone or in combination with charcoal, chalk, or bismuth, for their antiseptic action upon the putrid intestinal contents.

When enterogenic dyspeptic diarrhea cannot be controlled in the above way, the desired results are usually obtainable through the administration of opium or morphin, gr. $\frac{1}{4}$ (O.O15), with belladonna, gr. 10.008$)$, when there are cramps; or an astringent, as ichthyol, tanniform, tannalbin, or gallic acid, gr. $r(0.30)$, three or four times daily, which, because of their antidiarrheal (astringent) action decrease the number and fluidity of the movements.

When dyspepsia intestinalis is due to imperfect starch digestion. the author knows of no more effective remedies than taka diastase, gr. v (0.30), at mealtimes, but when the disturbance is due to the indigestion of meats and fats, pancreatin, gr. $v(0.30)$, is indicated.

Usually acute attacks of intestinal indigestion and diarrhea are controllable by the methods outlined, but when the condition becomes chronic it may be necessary to watch the diet carefully for a considerable time, to employ antidiarrheal remedies, but in smaller doses over a long period, and to direct treatment against catarrh of the intestine which arises sooner or later.

In aggravated cases the mucosa of the colon always becomes inflamed and sometimes crosions or ulcers form, and in neglected cases, owing to the part played by a mixed infection, they may rapidly increase in size, indications of which are to be seen in the presence of considerable pus, blood, and mucus in the stools. The astringent, sedative, and antiseptic remedies mentioned have a farorable action and are conducive to healing, but better and quicker results are obtained when the large intestine is irrigated daily with a normal saline, weak Carlsbad salt, ichthyol, boric acid or permanganate of potassium solution, or an infusion of flaxseed or oak bark.

The treatment outlined here relates principally to jejunal diarrhea, which represents the most common form of enterogenic loose movements. The therapeutic measures practised for the relief of this type of diarrhea, arising from gastric, hepatic, and pancreatic disturbances, have been omitted here since they have received separate and full consideration elsewhere.

Duodenal (peptic, otc.) ulcers require about the same treatment as gastric ulcers, the therapeutic measures for the relief of which have 
been fully discussed in the preceding chapter. The treatment indicated in these cases briefly summed up consist (a) in having the patient rest quietly in bed for several weeks; (b) restricting his nourishment to fluid foods and nutritive enemata; $(c)$ prescribing opiates, astringents, antiseptics, alkalies, and tonies to relieve pain, diminish the evacuations, minimize fermentation, heal the uleces, kesent the acidity of the chyne, and improve the patient's general condition: and $(d)$ when these and other therapeutic measures fail, sastro-enterostom $y^{\prime}$ resection, or a plastic operation should be resorted to to secure the necessary relief. 


\section{CHAPTER XII}

\section{NEUROGENIC DIARRHEA (FUNCTIONAL, POSTPRANDIAL DIARRHEA, NOCTURNAL DIARRHEA)}

\section{PSYCHIC DISEASES AND INJURIES OF THE NERVOUS SYSTEM, RE- FLEX DISTURBANCES, AFFECTIONS OF THE THYROID GLAND}

Neurotic individuals frequently suffer from irregular bowel movements. Most of them are troubled with constipation, but not a few complain bitterly of periodic attacks of diarrhea.

Nervous diarrhea may be encountered in persons suffering from organic nervous diseases, those who are nervous as the result of other ailments, and in normal high-strung or sensitive individuals, following emotional disturbances, frequent movements suddenly supervene.

Seldom in this class of individuals constipation or obstipation prevails for a considerable time, to give way alternately or continuously to diarrhea, when the nervous mechanism is interfered with by way of the brain, intestinal nerve-endings, or is affected through reflex impulses.

This type of increased evacuations occurs slightly more frequent in men than women and at any age, but is met with most often between twenty-five and fifty, or the time of life when the patient undergoes his greatest mental and physical strain, is apt to indulge in excesses or suffer from a seclentary occupation and confinement in poorly lighted and rentilated rooms, which cause suboxidation, a forertuner of nervous disturbances.

Nervous diarrhea is characterized by its sudden onset, periodicity, rapid succession of evacuations, extreme fluidity of the movements, with exception of the first, which may be semisolid or firm, and is most often encountered in emotional, neurasthenic, or hysteric subjects. It is generally conceded that the movements in these cases are caused by either hyperperistalsis, augmented glandular secretion, accelerated transudation of fluid into the bowel, or hypo-absorption, one or all resulting from abnormal impulses originating in the cerebrum, general nervous system, or more directly in the intestinal nerve plexuses.

Through reflex vasomotor phenomena originated by nervous influences, according to Lemoine, the intestinal ressels become dilated, which favors transudation of fluid into the intestine, causing the diarrheal crises.

It is customary to consider as nerious nearly all diarrheas which cannot be attributed to errors in diet or lesions of the gastro-intestinal tract, but undoubtedly many mistakes have followed this practice. 
The author believes, with Fleiner, that in the presence of true chronic diarrhea, which persists without interruption and merely varies in severity, that a diagnosis of nervous diarrhea should be made with great caution, and, while he does not doubt the existence of chronic diarrhea upon a nervous and psychopathic basis in exceptional cases, he is of the opinion that frepuent movements excited by other conditions are often ascribed to nervons diarrbea.

Indoubtedly, the intestinal nerve apparatus within the gut wall directly influences motility, secretion, and absorption, and the functionating power of the gut is stimulated or inhibted by discase within it affecting the nerve-endings, and constipation or diarrbea is the result in accordance as the impulses change.

It is efually true that the sole control of the various functionating powers of the intestine is not confined within it, because there is sufficient evidence to prove (as will be shown later) that certain organic diseases of the nerve mechanism in near and distant parts may lead to reflex disturbances which are capable of increasing or decreasing the evacuations, and that regularity of the morements is at times modified by psychic impulses.

Owing to the correlation which exists between the brain, cord, vagus nerve, the sympathetic system, and intestinal nervous mechanism, impulses are incapalele of transmission in either direction, for or against the welfare of the indiviclual, according to the location of the lesion or direction from which the disturbing or bealthful influence comes. With this knowledge, it is easy to understand that intestinal neurosis, such as diarrbea, may occur but once, periodically, or remain permanenty, in different cases, in accordance with the nature and duration of the pathologic lesion or exciting impulse back of it.

Psychic nerious diarrhea may be brought on by profound emotions of all kinds, such as sudken fright, prolonged terror, anxiety, anger, public speaking, trying a case before judge or jury, acting a play for the first time, taking an examination, being grilled upon the witness stand, excitement attending a journey or being aware of the fact that there will be no opportunity to evacuate the bowel for a given time.

The author bas treated patients from one or all of the above types of prychic diarrhea. One, a man fifty years of age, who had been a comic opera singer for thirly years, invariably suffered from griping pains, faintness, and diarrbea on each first night, during which he passed a number of watery evacuations, and suffered great mental agony while playing, because he did not know whether an accident would occur or not. Another case previously reported was that of a minister whose movements were normal in number and consistence except on Sunday, when they becane fluid and freepent, owing to the mental strain under which he labored in delivering his sermon and the knowledge that he conld not go to the toilet until after it was finished.

The author was informed by the foreman of a gang of Italian laborers that several of his men defecated in their clothing simul- 
taneously with the accidental explosion of an enormous amount of dynamite, and that the shock affected one of them to the extent that he suffered from watery movements for several days thereafter.

The author has known of other instances where diarrhea resulted from fright or worry, one of which was that of a lawyer who invariably suffered from diarrhea each time he tried a case. He has also treated a man, a highly sensitive individual, whose mucosa appeared to be sound, yet a diarrheic condition could be brought about by applying the electric current or the vibrator to the abdomen, by the introduction of the proctoscope, or by making topical application to the intestinal mucosa.

Cases of this kind have also been studied in individuals who were mursing friends or relatives fatally ill, whenever frequent movements were incidental to a depressed or fatigued mental state, anxiety, or fear that they would contract the disease; others have been observed where patients afflicted with constipation or other ailments became morried over their condition and diarrhea ensued, which increased their fear, and this, in turn, proportionately increased the frequency of the evacuations. When it was explained to them that their affliction was not serious, and that recovery would shortly follow, the movements became less frequent as their mental state improved, but in the case of one woman, whose fear could not be allayed, diarrhea became chronic, and proved to be more distressing than her original ailment. Numerous instances have been related or published where persons, particularly young women, have suddenly been attacked with nervous diarrhea when they were in the presence of company and in a restaurant, theater, or strange house, or taking a long automobile trip, and knew that there would be no opportunity to go to the toilet for a considerable time.

The author has also treated patients who suffered from colitis, cancer, etc., wherein the stools were made more frequent by suggesting to them that they needed an appendicostomy, cecostomy, colostomy, or intestinal resection or other operation, and Muszkats has reported a case of constipation with vasomotor instability where the patient voided from I to $6 \mathrm{oz}$. of thin mucus every time he became angry, the attack being devoid of pain, and the discharge contained neither shreds nor casts, in contradistinction to the evacuations of membranous colitis. Grasset, under the caption "psychosplanchnic" or cerebrovisceral neuropathy, describes what he considers a new form of gastro-intestinal neurosis, which is frequently accompanied by nervous dyspepsia, disturbance of intestinal secretions, olstipation often alternating with diarrhea, meteorism, and discharges of mucous collections containing shreds or casts, complicated by occasional heart and respiratory disturbances.

This condition may obtain in perfectly healthy individuals under the ahove and other forms of powerful psychic impression. but is most often encountered in persons who suffer from neurasthenia, hỵsteria, migraine, goiter, or tramatic neuroses. Ordinarily, con- 
stipation prevails in the presence of the more serious functional and organic diseases of the nervous system; sometimes, however, this condition alternates with diarrhea or gives way entirely to it, in connection with or independent of psychic impulses.

Frequent evacuations in neuropathic individuals are attributable mainly to accelerated peristalsis and transudation into the gut of fluid. Owing to peristaltic activity the stomach discharges improperly prepared food into the disfuieted small bowel, and the undigested food remnants are a souree of irritation and aggravate the conclition through their tendency to stimulate the glands to oversecretion and the sermicular movements to greater activity. This irritation continues in the large lowel where peristalsis is already marked, and transudation is active through the instigating nervous influence. Since the serous flud accumulates rupidly, and the segmental contractions of the colon are strone and frepuent, it necesarily follows that the evacuations must be watery and follow each other in fuick succession.

This condition is easily distinguishable from peristaltic unrest. where intestinal activity is limited principally to the small gut, and from membranous colitis, so frepuently encountered in nervous persons, becaluse of the small amount of mucus in the strols and the tolat absence of shreds and casts.

The frepuency, duration, and severity of the attacks are proportionate to the nervous state of the patient and the varying mental condition, the time recpuired to relieve his discuieted state, and the frepuency of his relapses. In neglected and porrly treated cases. where nervous diarrhea has existed for a considerable time, the patient is likely to contract chronic diarrhea as the result of the nervous disturbance, and the irritable and inflamed mucosa inducing the passage over it of undigested food remnants, acrid discharges, straining, and prolonged trauma to the intestine caused ly peristalsis.

Highly strung and nervous individuals who gormandize, eat hastily and generously of spiced foods, often at irregular hours or when fatigued, and consume an excess of alcoholic leverages, are prone to nersous diarrhea, because these habits lead to an irritalele state of the intestinal nerve-endings which abnormally respond to psychic and other stimuli.

There is a peculiar form of nervous diarrhea which occurs during or immerliately following the consumption of food. In some instances particular articles of dict will cause it, but in others frecuent evacuations are incited bs whatever is eaten. The attacks may follow any meal, but usually occur after lunch. Sharp pain is felt in the epigastrium almost as soon as the food has leen swallowed; it continues and then becomes more severe, and gradually works downward unt il it becomes agonizing. and is located in the lower and central part of the abdomen. By this time the patient is pale. weak. loreakisout in a colel perspiration, suffers from gas distention and borlorygmus and an imperative desire to empty the bowel, the straining incident to 
which increases his suffering, which continues until he has two or three evacuations that bring inexpressible relief, excepting intestinal soreness. The stools in this class of cases are watery, composed largely of bile and fluid feces. No doubt in some instances excessive biliary secretion is responsible for the trouble, but the author has treated patients where the crises were evidently influenced ly organic neurosis and powerful psychic emotions. This and the type of diarrhea which follows immediately after eating is termed "prandial" by Linossier.

Reflex disturbances are at times partially or solely responsible for attacks of nervous diarrhea, and the stimulating impulses may originate in the brain, intestine, or the peripheral parts. Occasionally, perfectly healthy individuals are attacked with diarrhea wherein the stools are frequent, watery, and occur in rapid succession following exposure to cold, sitting on damp steps or the ground, swimming in cold water, or getting wet feet, a type of disturbance which has been classified as nervous diarrhea, since the trouble evidently resulted from stimulation of the peripheral nerves.

Diarrhea is frequently associated with or caused by disease of the male and female genital organs, and results through reflex disturbance, because the crises occur at the times when the patient suffers most and is worried owing to aggravation of his local condition. This is particularly noticcable during the menstrual period in women, and especially those who suffer from dysmenorrhea, uterine catarrh, and displacements, extensive complete perineal tears, and cervical ulceration. The author has treated nervous men suffering from vesical, prostatic, and deep urethral trouble, who sought relief from diarrhea which accompanied exacerbation of their local condition, but in most instances they really suffered from tenesmus and not nervous diarrhea, as was evidenced by the fact that the stools were semisolid or firm, and not watery, as one would expect.

Certain diarrheas which accompany or follow the subsidence of perityphlitis, pericolitis, perisigmoiditis, and periproctitis, abdominal and pelvic inflammations, or peritonitis are classed as nervous, because they cannot be explained except through nerve irritation. The same may be said regarding pelvic and perirectal abscesses accompanied by frequent movements, with the possibility here that the excretion of toxins into the intestine is the exciting cause. The author has relieved patients who for a number of years had suffered from chronic diarrhea by the removal of a coccyx which was deriated anteriorly and irritated the rectum, or was fractured and caused pressure upon its nerves. He has known tumors to cause diarrhea by pressing upon the sympathetic plexuses in the petvis or compressing nerves at their exit from the cord.

He has also studied patients who suffered from a sensitive or so-called hysteric rectum, in whom an attack of diarrhea could be immediately brought on through the introduction of a proctoscope or application of topical medication to the mucosa. 
Both adults and children affected with extensive invagination and procidentia recti have been observed to suffer from diarrhea independent of the fact that the bowel was healthy. The frequent movements in these cases were probalny incited loy pulling upon the mesconteric nerves. At any rate, the diarrhea was cured simultanerms-ly with correction of the procidentia.

Large and small intlammatory and ulcerative lexions of all hinds located in the rectum and other part of the intertine are capalsle of inducing frequent evacuations through exposure of the terminal nerve-endings to the irritating intestinal bacteria, hard fecal mastes. and other contents of the colon.

Extremely nervous individuals and those who worry hecomes angered or are affected by other powerful psychic emotions, or who have intestinal disease. suffer more frequently and intensely from diarrhea than normal persons. In such cases the diarrhea should be attributed both to nervous influences and the pathologic state of the bowel.

Influensa is frefuently complicated by diarrhea. which in some instances is attributable to the catarrhal state of the bowet, and in others to influenza toxins which reach the inte-tinal nerves through the circulation. Diarrhea and loose or uncontrollable eracuations are at times associated with or complicate oreanic and functional diseases of the nerious system, although constipation is more often present under these circum-tances.

Diarrhea complicating epilepsy usually occurs during the cri-es. which are generally precerled by a rise in temperature and restles. ness on the part of the patient.

Intestinal auto-intoxication may profoundly influence frequency. of the evacuation, both in constipation. where the toxins are long retained, and in diarrhea, where the fluidity of the stools and erosions of the mucosa favor quick entrance of the poison into the circulation. The degree to which the patient is being poisoned under these circumstances is indicated through the effects of the toxins upon the general nervous system, as manifested by the character and frequency. of the attacks and by the amount of skatol and indican discorerable in the urine. Rodiet believes that the crises are the result of the action of these substances upon a predisposed organism.

In tabes dorsalis the usual constipated state may obtain throughout, alternate with diarrhea, or in exceptional instances the latter may prevail during intestinal crises, and be accompanied by abdominal pain, rectal tenesmus, and frerpuent discharge of exhausting, watery evacuations. Some of these attacks are traceable to psychic impulses, and others to constipation. fecal impaction, and auto-intoxication, but in many instances the etiology of the liarrhea remains obscure.

Tabetic diarrhea mas be intermittent or continuous. and the former, which begins in the preataxic stage, is severe and the movements occur involuntarily unless the bowel in instantaneously emptied. 
and while the attack lasts control of the evacuations is nearly if not entirely impossible.

Neurogenic diarrhea is also a common manifestation of neurasthenia and hysteria in the absence of intestinal lesions, attributable probably to an abnormal mental condition and irritability of the general nervous system.

Peculiarly enough, this complaint of ten complicates the terminal state of migraine, but no one as yet has offered a satisfactory explanation of the relationship unless it be accounted for in one of the above-mentioned ways.

The author has observed several patients who suffered from tenesmus and abnormally frequent semisolid or liquid movements caused by hypersensitice areas located in the sigmoid flexure and, rectum, which independently or when stimulated by the presence of feces incited an imperative desire to stool, a sensation which continued until several movements occurred in quick succession or relief was obtained through medication. He has also treated a number of nerrous children and occasionally adults who from infancy had been troubled with loose movements, which were inoluntarily discharged as was the urine, and in these cases the diarrhea or, rather, muscular paralysis which permitted the urine and feces to escape without warning, was traceable to injury or disease in the spinal nerve-centers which control the musculature of the rectum and bladder.

Symptoms.- The manifestations of neurogenic diarrhea differ from those of other causes principally in the following ways: It occurs in psychically disturbed or highly strung persons or those afflicted with functional or organic nervous disease. The attacks are periodic, of sudden onset, the movements are very thin and watery, occur in rapid succession without inducing tenesmus, take place usually in the early morning, may vary anywhere from five to twenty daily, and contain but little mucus and no pus or blood. The frequency, severity, and duration of the crises are governed by the type of emotion to which the patient is subjected, continued irritation to local nerves or general nerve mechanism, overactive exercise, fatigue, and other conditions which influence the nervous organism.

Diarrheal crises of nervous origin are frequently accompanied by dizziness, cerebral congestion, depressed feeling, difficult or rapid breathing, measiness about the heart, and sensations of heat and cold, loss of appetite, indigestion, abdominal weakness, intestinal unrest, borborygmus, occasionally tympanites, epigastric and fower abchominal pain, and in the type of nervous diarrhea which immediately follows the taking of food, an irresistible desire to stool.

In instances where gastric irritation predominates, vomiting is an annoying feature, and the substance evacuated may be undigested food or consist of mucus and bile. Some of the author's patients complained but slightly of intestinal disturbances, but in others, although there was no determinable lesion of the mucosa, passage of 
the food in some instances excited colic and enterospasm or pronounced peristalsis, which left the bowel sore.

When these patients suffer continually from pain and diarrhea persists regularly for a long time, there are localized tender syouts along the intestine, the stools erontain a eonsiderable amount of pus, blood, and mucus, and there is much pain preceding and tenesmus during defecation, the diarrhea may be attributed both to nervous disturbances and enteritis or enterocolitis.

Diagnosis.-In typic cases. where the above-mentioned characteristic symptoms are present, nervous diarrhea may be recognized with comparative ease, but in less marked instances. particularly in nervous individuals, it is exceedingly diffeult to differentiate between neurogenic and diarrhea induced by simple or specific lesions.

Some authorities, with reason, attach ansiderable importance to the absence of mucus from the stools, attributable to the transudation of fluid into the bowel in contradistinction to mucus, pus, and blook which appear in the feces where organic changes are present in the mucosa.

Of bowel diseases, membranous colitis is most often confused with neuropathic diarrhea, because patients thus afflicted are always very nervous once the ailment has fully developed, but this condition can ordinarily be distinguished from it by the frequent attacks of obstipation incilent 10 enterospasms, and the presence of mucous casts or shreds in the evacuations.

With the exception of myxorhea membranacea, practically all forms of colitis, because of their exhausting discharges. loss of blood, and accompanying toxemia, cause rapid emaciation and loss of weight. sallow complexion, anemia, anorexia, furred tongue, weakened and rapid pulse, marked gastro-intestinal disturbances, and other signs of greatly impaired health. all of which manifestations may be absent or minimized in nervous diarrhea, which usually occurs in comparatively healthy individuals, owing to the fact that nutrition is disturbed to a less degree.

In diagnosing neurogenic diarrhea the author relies chiefly upon the sudden onset and periodicity of the attacks, marked fluiclity of the stools, rapid sucession in which the evacuations follow each other in the early morning, the manifestation or presence of organic or functional nerve disease (hysteria, tabes, exophthalmic goiter, etc.), or the eridence of profound psychic emotion, together with a thorough investigation of the abdominal viscera, an analysis of the feces, and a careful digital proctoscopic and sigmoidoscopic examination of the rectum and sigmoid flexure.

Fecal analysis will show the evacuations to be composed largely of a serous fluid in nervous diarrhea, and soft fecal matter containing an abundance of mucus and some pus and blood in the more common types of diarrhea (catarthal and infective enterocolitis-).

Instrumental examination of the bowel materially helps to chear up the diagnosis, because in nervous diarrhea the mucosil shows pale 
and unchanged, while in the simple and infectious types of enterocolitis it appears red, swollen, or edematous, and there are crosions or ulcers variable in size and extent, and the lesions and intervening mucosa are smeared with an admixture of mucus, pus, and blood. Medication is belpful in the differential diagnosis for antidiarrheal remedies which are known to control the usual forms of loose movements, but do little if any good when diarrhea is caused by psychic impulses, functional or organic nervous diseases. Pariser regards tenderness on pressure of the abdominal ganglia as the characteristic stigma of nervous diarrhea, but this diagnostic sign has not proved reliable in the author's hands. A differentiation is difficult in cases where persons previously afflicted with nervous diarrhea contract enteritis.

Diarrhea should not be considered as neurogenic and attributed to tabes dorsalis, exoph thalmic goiter, neurasthenia, hysteria, migraine, or other organic or functional nervous ailments except when there are confirmative evidences of their existence and the bowel is found to be intact.

Treatment.-Before outlining the treatment it is necessary to determine whether the diarrhea is truly neurotic or is due to lesions of the intestine, or both, because the therapeutic measures indicated in the two conditions vary greatly. Again, when hysteria, neurasthenia, or other nervous disorder are directly or indirectly responsible for the frequent movements, these must be corrected, otherwise a cure cannot be accomplished.

Psychic emotions are the sole cause of neurogenic diarrhea in some instances and aggravate it in others, and because of this it is of the utmost importance that the patient's mentality should receive due consicleration when planning the treatment.

In the handling of this class of cases much more can be accomplished with psychotherapy and physical therapeutic measures for building up the general health than by the administration of drugs, which temporarily quiet the nervous mechanism and leave it irritable when their effects have worn off. Neurotic subjects who suffer from diarrhea consider their condition serious, and should not be told that it amounts to nothing, otherwise they will lose confidence and discontinue the treatment; nor should their trouble be magnified, which would cause them to worry, and lead to an increased number of evacuations. It is of the utmost importance for the physician to maintain a checrful disposition while with these patients, to tell them that they have a slight ailment, but one which can be easily corrected, and encourage them diaily by remarking that both their general and local conditions have improved. 'The author makes it a rule in these cases to treat the bowel with soothing oils, sprays, and innocent topical applications, both to diminish local irritation and relieve the patient's mind. This is the sine qua non of the treatment, for the reason that it eases the patient's mind, owing to the fact that it convinces him that something definite is being done to effect a permanent cure. 
Soothing electric currents and light friction masisge, when well borne, are helpful, because they are impresive, wothe the nerver, encourage sleep, and strengthen the mustes, and the same can le said of hydrotherapy judiciously employed, luat vibratory treatments and heary matsage are contra-indicated lecande the tend to irritate the sensitive nerves, induce insomnia, and increase the number of movements.

Some inclividual can remain at home. come to the office, ancl be successfully treated, but others cannot, because improvement is prevented by their uncongenial and depresing surroundings, family Wrangles, business worries, or other factors which aggravate their nervous state. Such persons shoutel be sent either to a sanitarium, to take the rest cure amicl quiet surroundings and where a -uitable diet can be prescribed along with other therapeutic measures or to the country, away from their ofd surroundings. business associates, friends, and relatives, where they can take sufficient exercise, have congenial companions, spend their time in the open air, and overcome the suboxidation and insomnia from which they suffer. At the same time they should be compelled to lead a regular life as regards the time for eating. sleeping, and attending to the calls of nature.

The diet should be non-irritating, bountiful, and nutritious, and the patient should be adviserl against the eating of foods difficult to digest or which irritate the stomach and intestine, and the drinking of alcoholic, iced, or carbonated beverages, which tend to increase the frequency and fluidity of the movements.

In some instances a cold girdle, applied to the abdomen in the intervals between attack-, makes the patient feel better and strengthens the abdominal and intestinal musculature, but during the crises, when there is griping and soreness of the intestine, cold should be displaced by hot applications, which diminish pain and irritability.

When heat does not prove effective, and the evacuations are rapidy exhausting, the patient's relief is oltainer by a hypodermic injection containing morphin. gr. $\frac{1}{4}(0.015)$, and atropin, gr. $\frac{1}{1} \overline{1}(0.0006)$, which mary be repeated in one hour if necessary, or the remedies may be employed in suppositories when rectal tenesmus and pain are annoying.

Astringent remedies are contra-indicated because they do litule or no good, and frequently aggravate the condition by interfering with the digestive secretions.

Except in the most distressing cases, patients afflicted with neurogenic diarthea feel perfectly well in the intervals between the attachs. and this respite should be taken advantage of to leeter the condition of their nervous system l,y prescribing tonirs to improve the circulation, strengthen the nerves, and tone up the digestive apparatus. but in toing this drugs should not he prescribed which would tend to upset the stomach or disturl, cligestion.

Of the various remedies employed for the purpose, arsenic, iron preparations, and nutrient tonic emulsions, such as Russell's. have 
proved most useful. (hareot has obtained good results with arsenic and bromid used as follows:

R. Sol. arsenic, liowleri Aq. amyolal. amar. $\bar{a} \bar{a} \bar{j} \mathrm{j}(4.0) .-\mathrm{M}$.

I)et. ad vitripat.

Sigr.-Berinning with + drops daily after meals, increase the dose $\mathrm{I}$ drop every second day until 20 drops are taken. then drop back to $t$, and repeat the process. Give at night the effervescent salt of sandow in milk or water.

The following combination is to be relied upon. both to quiet the mental state, encourage sleep, and to diminish the number of eracuations when the patient is extremely nervous:

R. Tinct belladonne

Kalii bromati

.àā gr. $\operatorname{xxx}(2.0)$;

Natrii bromati.

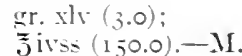

Aq. Font.

Sir. - I tablespoontul three or four times daily.

In some instances it has been found necessary to prescribe veronal, trional, or sulphonal, gr. xi (0.97), bromid of soda, gr. xxx (I.95), or, in extreme cases, the latter in combination with chloral, gr. $x(0.60)$, to quiet the nerves and secure much-needed sleep.

In the handling of this type of diarrhea it is well to bear in mind that there are irequent intervals when the regularity of the evacuations is not disturbed. During such periods, hygienic, dietetic, and tonic therapentic measures should be continued and antidiarrheal agents stopped, but during crises tonics should be side-tracked for remedies which can be depended upon to minimize or control the pain and exhausting diarrhea. For this purpose hypodermics of morphin, gr. \& to $\frac{1}{4}(0.008-0.015)$, the tincture of opium, ne $x(0.60)$, or powdered opium, gr. j to iss (0.06-0.09), administered two or three times daily until pain is arrested and the movements are diminished, are the most reliable remedies, but when there is considerable intestinal measiness, cramps, or enterospasm. the extract of belladonna, gr. ? (0.008), should be combined with an opiate.

little or nothing is to be expected from antiseptics or the ordinary remedies which are serviceable in other types of diarrhea.

Frequenty there coexists with neurogenic a diarrhea incident to gastritis, enteritis, or enterocolitis, and under such circumstances it is advisible to treat both conditions. In addition to the above, it is necessary to institute moderate or strict dietetic measures. Spices. greasy, sour, and foods which contain considerable cellulose and leave a large irritating residue should be prohibited, and those that are bland. Huid, or semisolid and digestible substituted (milk and limewater. buttermilk, broth, gruels, etc.). Ice-cream, cold carbonated drinks, and alcohol should be limited or discarded altogether, because they usually increase the evacuations.

When there is excesive putrefaction and enteritis, as is evidenced by regular frequent daily evacuations containing mucus, blood, and 
pus, antiseptic agents, such as bismulh subnitrate or salol, gr. $x$ (0.60); beta-naphthol, gr. ij to $\mathrm{v}(0.120 .30)$, and astringent remedies, such as tamalhin, ichthalbin, ichthoform, gr. $x(0.60)$, abministered three times daily, adel very much to the patient's comfort and frecpuently arrest or limit the number of morements.

A great deal can be done to alleviate the patient's condition when there is catarrle or enterocolitis by daily irrigating the bowel from helow by way of the anus, or from above through an artificial appendiceal or ceral opening.

In the presence of simple catarrh much benefit is to be deriver from a normal saline, (arblual salt, or sulphate of maguesia solution. Ĵs to $\mathrm{j}(1,5,30)$ to water $0 \mathrm{ij}(1000)$, lut when there are erosions or uleers. Warm mild antiseptics or astringent solutions, such as boric acid, permanganate of potassium, ichthyol, and salicylate of sola, 1 to 2 per cent., etc., should be employed to flush the bowel daily or three times weckly, according to the severity of the diarrhea. When the evacuations are very frepuent, and contain an abundance of pus and blood, a silver nitrate solution, gr. x to $0 \mathrm{j}(0.60-500)$, should be substituted for the above remedies; but when enterospasm is troublesome the latter should be alternated every other day with warm mineral, olive, cotton-seed, or crute oil, poured directly into the colon through a sigmoidoscope, with the patient in the inverted posture; this soothes the inflamed mucosa and muscular irritability of the howel. 


\section{CHAPTER XII}

\section{TOXIC DIARRHEA}

\section{FOOD, CANNED GOODS, BACTERIAL (PTOMAIN) POISONING}

ACUTE and chronic diarrheas, mild, severe, and fatal, are consequent upon bacteria, ptomains, contamination through metal containers, and other poisons accidentally eaten very much more frequently than is generally believed, irrespective of the fact that gastro-intestinal and nerve irritation from these sources are being detected more often than formerly, and physicians and the laity have been educated to the importance of properly selecting and protecting food.

Formerly it was customary to consider all toxemias induced by food products as resulting from ptomains, but more recent investigations have demonstrated beyond question that the poisoning is more frequently due to infection from ingested bacteria than to the toxins generated by them. In some quarters the chief source of poisoning was thought to be due to contamination of the food by metal when canned, or cooked in a brass ressel, but, while this is possible and occasionally occurs, we now know that the disturbance in the vast majority of instances is caused by bacteria or their toxins.

The unhealthy article of diet causing the trouble may have become contaminated by pathogenic germs through an animal haring been diseased, putrefying before being eaten, or, when healthy, being left exposed to the myriads of micro-organisms in the air. Naturally, when food is consumed raw, poisoning is more apt to follow than when the meat is boiled or roasted, because nothing is done to kill the bacteria, but, while this is true, it is well to bear in mind that their products, the ptomains, are frequently not destroyed in this way, and may remain to cause profound toxic manifestations after thorough cooking.

Poisoning has followed the eating of a large variety of foods, and of these, veal, beef, pork, sausage, chicken salad, potted tongue, lobsters, oysters, crabs, clams, fish, canned goods (all sorts), ice-cream, custard, puddings, potatoes, beans, and mushrooms have been most frecuently associated with and contained the toxin responsible for diarrhea from food-poisoning.

Meat-poisoning, Diarrhea from.-A perusal of the literature indicates that diarthea from food-poisoning is induced more frequently by meat than other articles of diet known to have caused tosemia. The disturbance does not always result from a particular kind of meat, cases having been observed where it followed the eating of beef, mut- 
ton, pork, veal, mince-meat, chicken salad, salmages, and various canned meats, often raw and less freepently when cooked.

Bollinger, who has made an elaborate study of meat-poisoning. believes that in four-fifths of the cases it results from eating lhe meat of animals already diseased when killed. Bacteria may fourish in raw or slightly cooked meat, and are destroyed by luorough losiling or roasting, but their ptomains may be rendered inere or modified by cooking or retain their toxic qualities thereafter. Most persons believe that smoking meat destroys bacteria, comteracts and renders the toxius inert, and prevents its luture infection, lut epielemics have often followed the eating of smoked meat and herring, and investigation of these cases has shown that this smoking does not alway's render meat proof against bacteria or their toxins.

Badly putrefied meat is readily retectable from its oclor, but when it is but slightly tainted it is likely to be eaten, since it looks, smells, and lastes normal. Chicken and game have been known to undergo bacterial changes while in cold storage, and they readily decay when remosed from the cold. That the consumption of putrefying food does not always make one ill is evidenced by the fart that ducks and partridges are often eaten when very "high" without causing any disturbance. Meat-poisoned stools are but slightly infectious, the writer having been able to fincl but a single case, that of a woman, who became infected from the discharges of her boy while nursing him for ptomain-poisoning.

A description of the pathologic findings in meat infection and ptomain-poisoning requires but brief consideration, because they are unimportant in comparison with the serious manifestations to be relieved.

In all types of meat-poisoning except botulism (sausage-poisoning) organic changes are confined chiefly to the digestive tract, as is shown by the gastro-enteritis which obtains, and results from congestion, slight edema, and sometimes hemorrhagic spots in the mucosa. Hemorrlagic areas may also appear in the serosa of the pleura, pericardium, and skin. In particularly virulent cases the follicles are swollen, undergo necrosis, ulcers are formed and blecting ensues, and sometimes enlargement of the mesenteric glands and spleen are manifest.

In sausage-poisoning the central nervous system is profoundly affected, leading to secretory disturbances and muscular paralysis.

Meat toxemias may be grouped into those induced by $(a)$ consuming meat of diseased animals, (b) eating that which is putrefied, and $(c)$ partaking of unhealthy satusages.

Toxemia from the Meat of Diseased Animals. - Numerous epidemics have occurred where soldiers in large mumbers or other persons congregated together (family parties, exc.) have suffered mildly, severely, or fatally from diarrhea and other distressing manifestations which followed the eating of meat derived from animals diseased when slaughtered.

Food toxemias have not only been traced to the eating of dis- 
eased veal, beef, etc., which was eaten, but also to the flesh of healthy animals which had become contaminated through contact with diseased meat. This form of poisoning has usually followed consumption of the meat of calves that had infected navels or other discase, cows having gastro-enteritis or unhealthy udders, and it has resulted from the eating of meat from diseated steers, hogs, horses (with abscesses), and sheep. The author formerly lived in Kansas City, where there are numerous slaughter-houses, and of his personal knowledge knows that it was often the practice of farmers and cattlemen in the Mest to rush diseased stock to market along with healthy, that they might aroid the loss which would otherwise follow from animal epidemics then killing their stock. This practice still obtains, but to a less degree, owing to the fact that animals to be slaughtered must first undergo government inspection.

The bacteriology of food-poisoning due to eating the meat of diseased animals is not well understood. hut the paratyphoid and Bacillus enteritidis are consiclered to be the chief etiologic factors because of the frequency with which they have been associated with this type of infection. The trouble, however, may lue due to either of these germs or to their products (ptomains) which have formed in the meat before or after it was ingested.

Toxemia from Decayed or Putrefied Meat.-Diarrhea from this source is encountered very much more frequently in the summer than in winter months. because heat farors the multiplication and activity of putrefactive bacteria (proteus group). This form of meat-poisoning may follow the ingestion of the flesh of both healthy and diseased animals, particularly when chopped up and exposed to the air for a considerable time in a warm place, and preservative mea-ures have not been taken to protect it.

While numerous kinds of putrefactive bacteria may be present, the poisoning from decayed meat is usually traceable to the proteus group alone, or associated with colon bacilli, although in one recorded instance the toxemia was due to the Bacillus subtilis.

Toxemia from Sausage-poisoning (Botulism).-Food-poisoning from this source has been observed more frequently in German-speaking countries than elsewhere, because Germans are large consumers of sausage, and frequently eat it raw or cold. which offers a greater chance for infection, since nothing is done to destroy the contained hacteria or neutralize their toxins. Toxemia from this source has been traced to diseased animals, those in poor condition, careless handling of the meat, its insufficient smoking, and to the time elapsed between slaughtering of the animal and manufacture of the sausage. The pathogenicity in sausage-poisoning has been attributed to the Bacillus botulinis, an anaërobic germ, which has also been associated with poisoning from the eating of beans and ham.

Poisoning from Milk and Its Products, Diarrhea in.-Infected milk is accasionally responsible for loose movements in adults. and is concerled to be the most frequent etiologic factor in the diarrhea of in- 
fants, in whom the mortality is very great, particularly among arificially (bottle) fed chilelen during the heated summer months.

irilk, ice-cream, custards, cream-puffs, sauces, puddings, and cheese have all caused poisoning which induced gastro-enteritio and tharrhea. In some instances their toxic effect produced nothing more than slight temporary annoyance, while in others distresing manifestations or death ensued. In some instances poisoning wats ernfined to an individual or family party, while in others a large number of persons (soldiers, ete.) were affected by the contaminated milk or its products.

Bacilli may contaminate the milk of diseased animats affected with tuberculosis, but most often it becomes infected through dust on the animal, filthy hands of the milker, unclean receptactes, or from exposure when left uncovered for a considerable period. Milk contains myriads of many different types of bacteria, but the colon and the Bacillus enteritidis of Gärtner are thought to be the most frepuent disturbing factors, while the Bacillus enteritidis sporosenes. staphylococci, streptococi, paratyphoid, and possibly typhoid bacillus have been frequently found.

In addition, Vamghan has pointed out that the non-pathogenic or seprophytic germs frepuently contaminate milk and its by-products and form ptomains, particulary tyrotoxicon, which is capalile of inciting a choleriform diarrhea.

Ice-cream has very frequently been responsible for diarrhea, hut not so often hecause of its low temperature as it is to the fact that it has become poisonous through flatoring extract or the container. Isually poisoning from this source has followed the eating of ranilla ice-cream, and the toxemia has been attributed to the flavoring extract, but more recent investigations, particularly those of Wassermann and Vaughan, have demonstrated the bacterial origin of the poison.

The symptoms of poisoning induced hy milk, ice-cream, custards, pudding, cheese, or other foorl having a milk basis may be slight or very grave, and are always more dangerous to infants and children than adults. Sometimes poisoning does nothing more than upset the stomach and slightly increase evacuations, but at others toxemia is marked and the patient suffers from chilly sensations, headache, diziness, disturbed vision, subnormal temperature, abdominal tenderness and pain, cramps, diarrhea, bloody stools, persistent rectal tenesmus, exhaustion, and, in very aggravated cases, unconsciousness and eollapse. Poisoning from cheese frepuenty does not manifest itself until from six to fifteen hours after it has been eaten. but the symptoms from ice-cream, custard, and milk poisoning are frecpuently noticcable immediately, or, at the latest, in from one to taio hours.

The diannosis is determined he ascortaining if the milk came from a healthy cow, if it has been contaminated by dirt in the handling or through exposure and by examining the milk, cheese, cuabard, or putding for bacteria and toxins which cause these forms of toxtmial.

Diarrhea from cheese-poisoning is fairly common, and the anthor 
recently treated a patient who almost died from this type of loose movements and the violent vomiting which accompanied them. In this and other instances there was nothing about the color, odor, or taste of the cheese which would learl one to suspect that it was poisonous. Vaughan attributes the toxic action of the cheese to the alkaloidal fyrotoxicon, but more recent investigators indicate that the disturbance is bacterial, and it is not unreasonable to suppose that the toxemia may be due to either or both.

At different times cheese-poisoning has been attributed to a bacillus of the colon type (Holst), anaërobic (Pflüger), similar to Bacillus botulinus, typhoid and paratyphoid bacillus, and to the proteus bacillus.

Fish and Shell-fish Poisoning, Diarrhea from.-Toxemia from the eating of fish or their roe is met with now and then, but does not occur as frequently as poisoning from oysters, crabs, clams, mussels, snails, and other shell-fish.

Fish toxemia may ensue from the eating of fish (sturgeon) which are naturally poisonous at all times, and others which are toxic only during the spawning season. One is more apt, however, to become poisoned from the eating of the roc of pike, German carp, and the Japanese fugu than from meat, and it is claimed that the liver and bile of certain fish have been known to produce ill effects.

In this country ptomain-poisoning has frequently occurred from eating oysters, lobsters, crabs, clams, mussels, and, rarely, snails. In some instances they were contaminated while in their beds through sewage, and became carriers of typhoid, dysenteric, and other types of infecting agents. Fish and shell-fish toxemias may be induced by the eating of them when already diseased before being caught, or where they were healthy in the beginning, but became infected through uncleanliness or carelessness, and were permitted to become infected before being cooked or served raw. Poisoning may ensue as a result of bacterial infection or their toxins, or both, but the former can be destroyed by boiling, while the latter cannot in every instance, and it is well to bear in mind that unconsumed cooked meat may easily become infected when left exposed, particularly in warm weather.

The manifestations are mild when putrefaction is just beginning, but the symptoms produced by eating fish and shell-fish which have undergone marked putrefaction (noticeable by its odor) are very much more pronounced, owing to the increased number and activity of contained bacilli and their toxic products.

To obviate danger from this source fish more than twenty-four hours old should not be eaten unless they have been properly iced, and the same applies to cooked fish and shell-fish; nor should fresh fish, oysters, lobsters, etc., be permitted to come in contact with those caught or cooked earlier or placed in a refrigerator before cleansing, otherwise they are likely to become infected; for the same reason, the eating of canned oysters, lobsters, or fish, which have been opened 
and allowed to stand is dangerous, and it is well to use only fresh dressings with them, because poisoning has resulted from this source.

Infection in this class of cases has been attributed to the proteus, colon, and paratyphoid bacillus, alone or collectively, in lifferent cases, and other organisms are supposed to participate, iut their identity thus far has not been establisherl.

Poisoning from Canned Goods, Diarrhea in.-During the past decade the amount of canned goods consumed has increased at an enormous rate, owing to their cheajoness, greater assortment, being readily and easily prepared for the table, because many thousands of persons of limited means are compelled to live in diminutive apartments having small kitchens or kitchenettes which are not suited for the preparation of more elaborate dishes; the limited time left for cooking by individuals who must work for a living; and, finally, they are used extensively because they can be easily carried and indefinitely kept by fishermen, hunters, explorers, soldiers, and others who are on the move or go to out-of-the-way places. In fact, with their aid one can within a few moments prepare a nice hot course dinner, including everything from soup to kessert.

A few vears ago, when canned goods first became popular, many cases of poisoning were rejorted from this source; and this led 10 an investigation by the authorities, who discosered that the trouble was due largely to the selertion of imperfect or spoiled foods, uncleanliness, their carcless canning, insufficient sterilization or undue exposure to pathogenic bacteria, which later results in their decomposition and the formation of poisonous toxins. Recently, however, poisoning from this source has not been frequent, because the authorities now maintain a supervision over the canning of food.

When toxemia results from bacterial poisoning it may be from the canning of decomposed products, sound food stuffs which contain germs not destroyed in the preserving process, or by virulent toxins in the food formed by bacteria and not always destroyed by heat. While meat-and fish-poisoning are very much more common, toxemia has been observed on a number of occisions to follow the eating of canned regetables, particularly string-beans, and, strange as it may seem, in the two most celchrated outhreaks from this source, those of Darmstadt, 1904, and leipzig, 1906, the tromble was attributed in the former to a germ identical with the Bacillus butyricus, and in the latter to colon and paratyphoid bacilli-bacteria responsible for most meat toxemias.

As a rule, when markerl hacterial decomposition has raken place it causes what is known as a "blown can," or one wherein the ends of the tin are rounded and bulging, which in itself evidences the poisonous nature of the contents and should warn the purchaser not to buy the goods. The laity, even at the present time, leelieve that the poisonings that follow the eating of canned food are practically always due to the metal in the can which contaminates its contents. but it hats been demonstrated by numerous investigators that toxemia rarely 
results from this source. This type of poisoning may be caused by either tin or lead, the latter being by far the most frequent. It is said that in Germany toxemia has not arisen from either of these sources on account of the law providing that the tin used shall not contain more than I per cent., nor the solder ro per cent., of lead.

Lehmann has pointed out that the amount of contained tin increases the longer the contents remain in the can; is greater in decomposed canned foods and in pickled fish or herring, where the acid favors the breaking up of the tin. Consequently, fruit and the like containing acid is less apt to eause trouble when preserved in glass or crockery. Poisoning from copper has been known to follow the boiling of vegetables and acid substances in copper-lined containers, but this must be extremely rare, since much of the cooking in the larger hotels is done in copper ressels and poisoning does not result. In these supposed cases the poisoning was probably due to the lead which reached the foot where the copper covering had become defective. Bolduan says that the presence of lead can be determined by boiling a + per cent. solution of acetic acid in the ressel for one-half hour, and then passing sulphuretted hydrogen through it, when a black discoloration or precipitate shows the presence of lead.

The symptoms of toxenia consequent upon the consumption of contaminated canned goods varies somewhat according to the character of the food and contained toxins. In the cases of Fischer, where 2 I persons were poisoned by bean salad and I I died, the manifestations of the toxemia were very severe, appeared one or two days later, and were similar to those described under sausage-poisoning. Autopsy showed congestion of the intestine with hemorrhagic spots beneath the mucosa. While in the Leipzig outbreak, che to stringbeans, all of the 250 persons affected recovered, and the dizziness and gastro-intestinal manifestations, including diarrhea, came on within a few hours, and lasted but two or three, or at most four days, leaving no ill effects, while the recovered Fischer cases hat a very prolonged convalescence. Because of the long time which elapsed in Fischer's cases, and the short period intervening between the eating of the beans and onset of the symptoms in the leipzig cases, it would seem that poisoning in the former was due to bacterial action, and the latter instances to their toxins.

The diannosis depends largely upon obtaining a history as to the nature of food which has been eaten, and in examining the container and a sample of the food to determine if bacteria, toxins, or chemicals are present which might cause trouble.

From what has already been said, it can be inferred that the prognosis may be serious in some and favorable in other cases, depending upon the virulency of the poison and resistance of the patient.

Potato-poisoning, Diarrhea of.-A few cases of poisoning from potato salad have been recorded. but in the vast majority of instances where the toxic element of potatoes has been sufficiently virulent to cause gastro-intestinal and other severe manifestations 
the poisoning has occurred among soldiers or other assemblages where large amounts of food have been poorly selected, handled by unclean hands, or improperly cooked. This disturbance may be brought on by eating green, diseatsed, shrunken-up, diseolored, or actirely spromting potatoes, and is in most instances supposed to be due to the contained solanin which is present at all times, hut in increased amounts under the above circumstanees.

Solanin is founcl in all parts of the potato, but is most abunclant in the peel, and gradually decreases from there toward the center. Authorities on this type of poisoning are inclined to the belief that toxemia results more frepuenty through bacterial action than through solanin. which must be consumed in large amounts to procluee aggravating or dangerous symptoms. (ne is led to this conclusion because in later years individual poisoning and outbreaks among soldiers have followed eating potatess, or the salad from them, which were peeled in large (juantities the night or even a longer time lefore their consumption. Since solanin is largely contained in the peel, and is not materially increased, if at all, by previous peeting of the potato, it would seem that the trouble might be clue to infection, either from their handling or the setting in of hacterial decomposition upon the uncovered potatoes. Bieudomne has pointed out that proteus bacilli are the inciting micro-organisms in the decomposition process.

In the Hammellourg instance, where I so soldiers were poisoned hy potato salad, the above micro-organisms were found in abundance, and mice fed upon the salad died within a day of severe gastro-intestinal svmptoms, and it was found that the poisoning resulted from a toxin formed upon the potato by the bacilli during warm weather.

Bolduan has suggested that other bacteria may be etiologic factors, and that sinee typhoid and paratyphoid hacilli grow well upon potatese, these organisms should be sought for in the microscopic examination.

The symptoms vary in potato-poisoning, but in typic cases they are fairly characteristic. Isually the patient first complains of headaches, which are quickly followed by gastro-enterospasms and a severe type of diarrhea which produces marked exhaustion. In aggravated cases there may be a rise in the temperature, fast pulse. dilated pupils, feeling of faintness, and occasionally the patient complains of chilling, lassitude, and may have nausea and romiting. but rarely if ever dies. I sllatly they recover in from two or three days to a week, but when the poisoning is serere, the gastro-intestinal tract may remain irritable for a longer time to continue the diarrhea.

Mushroom (Muscarin) Poisoning, Diarrhea of.-There are several rarieties of mushrooms which may be cultivated or found growing wild, some of which are harmless exlibles, while others are extremely toxic because of the alkaloid, muscarin, contained in them. Mushrooms should never be eaten mutil after they have been dried aud boiled, because in this way only can the muscarin escape through evaporation. 
Gastric and intestinal manifestations are invariably present in mushroom-poisoning. At first there is nausea, romiting. and pain in the stomach and cramps in the lower part of the abdomen, diarrhea, jaundice, and hemoglobinuric, but later the pulse-beat is retarded, there is imperfect rision, and in severe cases coma and conrulsion:

Grain- or Seed-poisoning, Diarrhea of.-Gastro-intestinal disturbances and diarrhea have been known to follow the eating of spoiled or contaminated grain foud stuff-. such as rye. corn, chichenpeas, and the symptom-complex arising from these forms of toxemia have heen derignated ergotismus, maidismus (pellagra), and lathyrismus. In all the truble has ensued from the eating of bread or other edibles prepared from the meal of the above-mentioned grains.

Ergotismus, which occurred more frequently among early settlers, is attributed to the fungoid parasite claiceps purpurea. which infests rye, wheat, and other food product- raised on new more frequently than those grown on old ground, or soil which has undergone cultiration for a considerable time. Ergotisnus may produce abdominal cramps, tingling of the extremities, convulsions, delirium, other evidences of a profoundly disturbed nervous system or dry gangrene, and in exceptional cases it produces diarrhea.

Maidismus, or pellagra, is a disturbance of metabolism frequently encountered in Lombardy. Spain, and our Southern States, where corn bread constitutes a regular and important part of the daily diet. The disease is thought to be due to bacteria or more likely: to their toxins. Which contaminate the meal from which bread is made. a food stuff easily infected when left exposed to the air, or when kept in a damp cellar or room. Repeated eating of bread made from spoiled meal may soner or later lead to pronounced chronic nutritional disturbancer, indicated by los of appetite, imperfect digestion, bodily weakness. tired feeling, and frequenty diarrhea. Here diarrhea is very profuce, and the trouble appears to be rather the effect of a constitutional toxemia working through the nervous apparatus than to local levions within the bowel, although occasionally gastroenteritis has been sbserved in the later stages. which are often complicated by mouth. throat, and skin affections, together with paresthesia, headache, and melancholia, and occasionally the fatty degeneration of the internal organs.

Lathyrismus, or chicken-pea poisoning, is almost unheard of in this country, and since this type of toxemia leads to paraplegia and other profound nerrou- disturhances, and seldom, if ever, produces diarrhea, nothing further need be said concerning it.

Other Food Diarrheas.-In thi- connection the author wishes to call attention to the fact that food diarrhea is not always consequent upon poison contained in the articles of diet above mentioned, or toxins formed within the body, becallse he has treated many patients wherein the diarrhecs resulted from gormandizing. improper mastication, eating of indigestible food stuffs, or those which left a 
large, rough, or otherwise irritating residue to excite peristalsis and glandular secretion.

The diagnosis of ptomain-poisoning may be easy, but is usually difficult, because it is necessary to exclude poisoning from other articles of diet before the trouble ean be attributerl to the meat. When this has been done, the greatest task remains, viz., that of finding out whether toxendia was catued by (a) the meat from diseased animals (bacilli-enteritidis or paratyphoid); (b) recared nuat (bacilli-proteus or colon), and (c) sallsage-poisoning (anaërobic Bacillus botulinus). Again, it is no easy matter to differentiate between poisoning resulting from bacteria and that following their toxins formed before or after the meat has entered the body. From what has already been said, it maty be inferred that some idea of the nature of the trouble (ptomain-poisoning) can be gained from a clear history of other cases and by observing the symptom-complex, but it is imposible to determine the exact nature of the disturbing elenent except by means of hacteriologic examination, although some have attempted to do so by sera. Conseduently, it is important to quickly select, prepare, and protect a portion of the suspected food and immediately send it to the laboratory for chemic, microscopic, and experimental examination. In so far as the blood is concerned, changes are unimportant, but in rare instances the causative agents are discoverable within it.

The symptoms of meat- or ptomain-poisoning vary greatly, according to the character and extent of the infection. In one instance the disturbance may be slight and of short duration, and in another profound, and remain as a secpuel to disturb the gastro-intestinal tract for a considerable time, or it may cause death anywhere in from four to ten days.

The phenomena following bacterial activity appear a few hours after ingestion, and while similar in some respects in all cases, necessarily vary according to the type, amount, and virulence of the toxin or inciting bacteria. The gastro-intestinal tract is usually slightly or profoundly disturbed in all cases, but in Bacillus botulinus, or sausage-poisoning, the nervous system is affected to a much greater extent than in the other types of meat toxemias. Lnimportant attacks are usually of short duration, and the patient recovers within a week or ten days, but when he is profoundly poisoned, acute manifestations may last a short time, be subacute for wecks, become chronic, or continue through life.

The mildest form of ptomain-poisoning is accompanied by nausca, vomiting, anorexia, dizziness, blanching of the skin, which frequently has a greenish hue, elevated or subnormal temperature, cold perspiration, marked muscular weakness, mild or severe abdominal pain, bursting occipital healache, restlessness, explosive diarrhea, and yellow and offensive stools.

Again, when toxemia is more pronounced, the attack closely resembles that of cholera, in so far as romiting, purging, and colorless, 
watery evacuations (fifteen to twenty the first day) are concerned, and the patient suffers from thirst, a subnormal temperature, livid skin, fast pulse. cramps in the abdomen and legs. depression, dimness of rision. difficult breathing. coated tongue. buccal soreness, lassitude, retention of urine, and sometimes icterus. convulsions, paralysis, or collapse.

The symptoms enumerated rather indicate that bacterial products (toxins) are responsible for the poisoning. but when the disturbance begins with a chill. followed by a rapid rise of temperature (which continues irregular or elevated for several days), and the condition of the patient assumes a typhoid-like character (pea-soup-like stools), bacterial infection is considered to be the exciting cause of the gastrointestinal and other manifestations of the poisoning.

The abdomen is usually tender or painful on pressure, generally distended. but in rare instances retracted, the liver is sometimes and the spleen frequenty enlarged and palpable after the second day, and albumin. which is occasionally abundant in the urine, gradually subsides with the attack. as does hematuria.

Huebener has called attention to the fact that the urine often contains the pathogenic agents at an early date (Bacillus enteritidis. Gärtner group, and paratyphoid bacillus groupl. In protracted cases long-continued bacteriuria and chronic cystitis may develop.

Erythema. urticaria. hemorrhagic spots. and other superficial cutaneous disturbances are frequent complications, and Leutz has pointed out that herpes labialis is a characteristic manifestation in meat-poisoning due to paratyphoid infection.

The symptons of botulism. or sausage-poisoning. require separate consideration. because here ob-tinate constipation prevails more often than diarrhea, and further, because they differ markedly in some way from those of other meat toxemias. Patients afflicted with botulism may suffer from minor gastro-intestinal disturbances, but their nerious and secretory stems are always greatly impaired. and the consequent manifestations resemble those of belladonnapoisoning focular disturbances. Aysphagia, aphonia, and dryness of the mouth and throat. due to deficient salivary secretion or retention of urine).

In the +12 cases of sausage-poisoning collected by Senkpiel there was a mortality of to per cent.

The author has treated a great many patients for chronic diarrhea who have formerly suffered from food- or ptomain-poisoning. The loose movements in this class of sufferers were traceable to an irritable or influmed gastro-intestinal mucosa in the majority of cases, but in some it was ascribed to the neurotic condition in which the patient was left. Owing to the nervous state of the patient and irritable condition of the stomach and bowel, attacks of indigestion and diarrhea were frequently brought on by psichic disturbances Ifright, grief. worry). indiscretions in diet. and exposure.

In addition to impaired digestion and loose movements, his 
patients, while undergoing a long convalescence (especially elderly and persons having little resistance), have often suffered from gencral and cardiac weakness, anorexia, headaches, anemia, and nervous phenomena which made them easy victims to infertious diseases.

Death most often occurs within twenty-four hours, but has been known to supervene three weeks or longer afterward, and the mortality from poisoning nay be high or low according to the type of and amount of poison consumed. In some epidemics a large number of individuals have died, while others have had narrow escapes.

The symptoms of tish-, oyster-, crals-, muscel-, and snail-poisoning resemble each other, but the manifestations of fish-poisoning come on usually several hours after eating, while those of ovisters and crabs appear in taio or three hours or sooner, but toxemia from mussels is indicated within from fifteen to thirty minutes.

In mild cases of fish- or shell-fish-poisoning the patient complains of nothing more than slight natusea, vomiting, diarrhea, dizziness, and headache; these simptoms gradually recede, and he entirely recovers within wo or three dass. In more atroravated cases, in addition, the patient complains of throat dryness or constriction, weakness, rapid pulse, difficult respiration and aggravated gastro-enteritis, evideneed by persistent nausea, romiting, erythema, urticaria, or swelling of the skin, and exhaustive freguent mosements, which cause rectal tenesmus and contain considerable blood and mucus. Besides these manifestations in extremely severe or fatal cases, the patient may also suffer from dyspnea, delirium, coma, and complete collapse.

Patients afflicted with crab- or mussel-poisoning may aho suffer from hematuria, convulsions, a mild form of paralysis, and severe diarrhea or obstinate constipation.

The treatment of the various forms of food-poisoning will be discussed together, because, in a large measure, the prophylactic, symptomatic, and curative therapeutic measures in all are very much the same.

Prophylaxis consists in selecting healthy meat, milk, and other fookls, in protecting them from the air and keeping them in a dry cold place, in partaking of cooked in preference to raw foods, and having all animals and meats thoroughly inspected, requiring that all vegetables and food products should be under supervision while being chosen and canned. prohibiting the eating of food stuff- which have been exposed to the air sufficienty long for putrefaction to begin, and, above all. to see that refrigerators in the home, hotels, and restaurants are thoroughly cleaned daily. and that ofd or undesirable fish, meat, shell-fish, and other artictes of diet which may lse diseared do not come in contact with fresh supplies.

The treatment is largely symptomatic, since specific antidotes to the varjous poisons have not been discovered. When urgent toxic symptoms manifest themselves while drinking milk or eating, or immediately thereafter, the stomach should be washed out, or ipecac. 
mustard, apomorphin, or other reliable emetic should be administered to evacuate the poison and relieve present and prevent future distress. When the patient is seen at a later time, after the toxin has passed into the bowel, gastric lavage should be instituted for the patient's comfort, and castor oil, calomel, or a saline cathartic should be administered to free the bowel of its irritant contents, this to be followed by copious high colonic irrigation. Slippery-elm water, flaxseed-tea, mucilaginous drinks, and sechatives like charcoal, magnesium, and bismuth should be administered in liberal amounts to relieve gastro-intestinal irritation after elimination of the poison. When the sufferer is exhausted from prolonged nausea, romiting, and purging, he should be strengthened by strychnin, digitalis, whisky or brandy by mouth or hypolermically, and if in great pain, hot applications should be applied over the abdomen to soothe the bowel and relieve cramps. It frequently becomes necessary to prescribe remedies to relieve the diarrhea, and for this there is nothing better than morphin or opium, gr. $\frac{1}{2}(0.03)$, combined with the extract of belladonna, gr. $\frac{1}{4}$ (O.oI5), adding calomel, salol, or beta-naphthol, gr. $x(0.60)$, three times daily, when an antiseptic is indicated to minimize putrefactive changes. When the patient is profoundly ill an ice-bag should be applied to the head and subcutaneous salt infusion practised. Where the condition takes on a typhoid character (as frequently in botulism) the treatment is about the same as for typhoid fever.

In aggravated cases, where the gastro-intestinal mucosa is left chronically inflamed, remedies used in the treatment of gastro-intestinal catarrh, described elsewhere, are indicated. Many individuals who have undergone meat- and other types of food-poisoning are frequently left in an extremely nervous state and otherwise run down, and it is advisable to have them change their environments, live a free, happy life in the open air, and take a tonic until such time as they are restored to their former health.

The treatment of mushroom (muscarin) poisoning is obviously symptomatic, and consists in washing out or emptying the stomach with emetics, prescribing a cathartic to free the intestine of the poison, the administration of stimulants, and giving atropin alone or in combination with morphin to counteract the toxic effect of the muscarin and to relieve colic and diminish the number of evacuations.

The treatment of maidismus (pellagra), or grain-poisoning, is largely prophylactic, and consists in seeing that precautions are taken that grain used for food purposes is pure, and that the meal from it is neither left exposed nor where it can accumulate moisture, which caluses it to ferment and putrefy. For those afflicted, supportive measures should be instituted, and antidiarrheal remedies, etc., prescribed to control loose movements and relieve other unpleasant symptoms. 


\section{CHAPTER XIV}

\section{TOXIC DIARRHEA (Concluded)}

\section{MEDICINAL-CHEMICAL POISONING}

THERE is a long list of medicines and chemical agents which, when introduced into the body $1, y$ mouth, through the anus. by inumetion. subcutaneously, or inhalation, incite acute or cluronic diarrhea through their effect upon the local or distant nerve-centers by entering the blood, and later being exereted into the intestine, and most frefuently because of their direct irritating action upon the mucosa, which set up a catarrh of the stomach. small intestine, or colon; or a gastroenterocolitis wherein the mucous membrane is congested. eroded, or ulcerated. Of these numerous etiologic factors, the writer will discuss only those which are most often responsible for the more chronic types of diarrhea, since the others are apt to terminate fatally, or when the patient recovers the intestinal manifestations are inclined to subside quickly.

In discussing the relation of inorganic poisons to diarrhea, nothing will be said concerning the diagnosis except what is stated here. because it depends largely upon getting the history of the case and determining if the patient has taken a drug. and. if so, the amount and kind. In addition, it is important to study the symptom-complex carefully, because certain poisons develop characteristic manifestations. Finally, it may be stated that information as to the nature of the poison can in some instances be obtained by inspecting the mouth for burns and discolorations, and by making chemic, macroscopic, and microscopic examinations of the stomach contents. feces, urine, and blood, but these diagnostic measures are of but comparatively little value.

Arsenic-poisoning, Diarrhea of.-Here the poison may gain entrance through being taken deliberately for suicidal purposes or through habit in neurotic individuals: accielentally hy inhalation or absorption, in arsenic miners and artificial flower, shot, glass, paper. and analine dye makers. taxidermists, and by women who take it internally or rub it in the skin to improse the complexion.

One of the most frequent and distressing manifestations of acute or subacute aranic-poisoning is disturbance to the gastro-intestinal tract, the nature of which depends upon the amount taken at one time and the accumulative quantity present in the system, where the drug has been taken in liberal amounts for a considerable times. P'orome suddenly becoming poisoned from this agent suffer dizziness, nallata, vomiting, headache, weakness, twitching, pulfy eyelicls, epigastric pain. 
distressing thirst, and frequent rice-water evacuations (resembling choteriform stools), manifestations which appear in from a half-hour to six hours following ingestion of the poison, gradually increase in severity, and often terminate fatally within one or two days in untreated or improperly handled cases. Other symptoms of this and the chronic form of arsenic-poisoning are irritation of the respiratory tract, skin eruption, hematuria, albuminuria, cardiac weakness, multiple neuritis, muscular spasm, and tenesmus following the frequent, exhausting, fluid evacuations.

The diffuse debilitating dysenteriform-like diarrhea encountered in arsenic-poisoning is attributable to the direct irritating action of the drug upon the mucosa (which results in active gastro-enterocolitis) and effect of the poison upon the general and local intestinal nervous mechanism, and, finally, in chronic aggravated cases, to fatty degeneration of the intestinal musculature.

Autopsy findings show the intestine relaxed, containing mucus and pasty or less often fluid feces, and the serosa inflamed and mucosa congested or excoriated. Hemorrhagic infarcts variable in number are at times observed in the edematous mucosa, with enlargement of the solitary and conglomerate follicles, and occasionally ulcers with a hemorrhagic base.

Treatment.-The essentials in the treatment of diarrhea consequent upon arsenic-poisoning consist in (a) stopping its administration and use and changing the vocation of patients who work in it; $(b)$ instituting measures which will eliminate it from the system; and, finally, (c) treating the gastro-intestinal catarrh induced by it.

In acute arsenic-poisoning, where the patient is seen shortly after taking the drug, repeated gastric and intestinal lavage with hot water or medicated solutions add greatly to the patient's comfort and minimizes toxic manifestations by dislodging and washing out the poison and soothing the irritated gastro-intestinal tract. When this is not feasible, or lavage fails to accomplish the desired result, emetics should be tried, but when marked toxic symptoms still prevail, an iron preparation is indicated, the most generally used of which is the compound of magnesia and iron hyclrate in tablespoonful doses every fifteen to forty minutes until the patient is retieved.

Here we are more particularly interested in chronic arsenic-poisoning, for the reason that it is most of ten the inciting factor in diarrhea. In this class of cases the treatment should be supportive, symptomatic, and directed against gastro-intestinal irritation and catarrh, along with preventing further entrance of the drug into the body and in eliminating it from the system as speedily as possible by the internal administration of potassium iodid, stimulating the emunctories, and subjecting the patient to hot baths and friction massage. Local treatment of the bowel consists in the internal administration of opium and astringent and antiseptic agents to relieve pain, quiet peristalsis and soothe the intlamed mucosa, and in frequently irrigating the bowel with mild solutions of boric acid, ichthyol, salts, or oils. 
Mercurial Poisoning, Diarrhea of.-Diarrhea from acute mercurial poisoning requires slight attention, except when the patient recovers and it is a serpuel, becalue it constitutes one of the mirus manifestations observalse after the swallowing of bichlorid tablets (or other forms of mercury) aecirtentally or with suiciulal intent. For the immediate relief of this class of sufferers the stomach should be washerl out, they should be surrounded with hot-water bottles, and given an opiate to relieve pain, stimulant - to hrace them up, and white of egess and other alluminous sulstances to combine with the mercurial salt and minimize its action. Later. becaune of the gastroenteritis, the patient should be kept for a short time upon at fluder or non-irritating diet.

The physician is mont frequenty called upon to treat gastro-intestinal manifestations arising from chronic mercurial poisoning that are the sequelce of atute poisoning, and mercurialism resulting from the unaroidable inhalation of the chemical loy miners. smelters, and persons engaged in making mirrors. thermometers, felt hats, and vermilion pigment, or its absorption through the skin by contact when prescribed in the form of medication. for at the proper temperature the agent is volatile and readily finds its way into the blood through the avenue mentioned. Consequently, when it regularly enters the system for a considerable time it disturbs the stomach and intestine locally, and through it effect upon the general and nervotl= - ystems causes an aggravated type of diarrhea, as is evidenced ly anemia. emaciation. foul breath. muscular weakness. falling out of the hair, brittleness of the nails. metallic taste, salivation, stomatitis, buccal ulcers, nausea and vomiting, inflammation of the stomach, and pronounced enterocolitio (with and without ulcers).

Almkist believes that in mercurial colitis and stomatitis the mucosa of the colon and of the buccal cavity hecomes loosened or erorled as the result of local putrefactive changes. and that the hydrogen sulphid gas formed by these proceses is in part reabsorbed. When the blood contains mercury a depo-it of sulphur and mercury appears in the superficial capillaries and fincls it way into the endrythelial cells of the vessel walls. and leads to disturbances in the circulation and nutrition. Theorganiom endeavors to remose the deposit of mercury from the vasular walls through the leukocyte- hence. in long-standing cases abundant granule of mercury are found in the leukrocytes. When the deposit is consideralste and cannot be removed by the leukecyten, necrosis of the tir-ue follow:

The treatment is evident, and consists in discontinuing mercurial preparations and in changing the occupation of the patient. so that he may not lee competlerl to inhale or aboest) the mineral. Mluch comfort is to be had from frequent rin-ing with a mouth-wash containing the chlorate of potassium and myrrh or antiseptic and astringeme solutions, occasional gastric lavage milel non-irritating diet, -ulphur baths, and liberal doses of potascium iodid. which help to eliminate the mercury. In addition to this, a supportive treatment should be 
instituted and the intestine should receive attention through the administration of bismuth, tannalbin, or ichthalbin in 5-gr. (0.30) doses four times daily, to lessen the secretions and heal the inflamed or ulcerated mucosa, along with beta-naphthol, salol, and other antiseptic remedies when there are pronounced fermentative or putrefactive disturbances. This treatment should be reinforced by daily or triweekly high colonic irrigations, using weak or strong solutions (according to indications) of silver nitrate, boric acid, formalin, ichthyol, krameria, and soda or salicylic acid, to remove the toxins, débris, mucus, and fecal accumulations and to clean and stimulate healing of the inflamed or ulcerated mucosa.

Lead-poisoning, Diarrhea of.-Lead-poisoning is fairly common among painters, plumbers, and persons who mine or make sheet-lead or shot. Lead, when consumed with the food, drinking water from lead-lined cisterns or pipes, and as lead chromate used to give the yellow color to certain articles of diet, such as butter, candy, etc., may produce plumbism. This mineral passes through the skin with difficulty, is readily absorled through the gastro-intestinal tract and lung, and is chiefly eliminated through the kidneys. It is not necessary to enumerate the usual familiar symptoms of lead-poisoning further than those which relate to the gastro-intestinal tract. These patients usually complain of lead-colic or pains radiating from the central abdomen, marked constipation, but in rare instances they suffer from an aggravating diarrhea alone or alternating with constipation. The diagnosis is usually easy, and the treatment is simple, and consists in forestalling the further consumption or absorption of lead, the administration of opium or morphin, gr. $\frac{1}{8}$ to $\frac{1}{4}(0.008-0.015)$, in combination with belladonna, gr. 1 (0.008), to relieve pain, cramps, hypersecretion, abnormal peristalsis, and in this way control the diarrhea, and, where constipation prevails or alternites with the frequent movements, a laxative, such as salts or castor oil, is indicated to procure the daily evacuations and prevent fecal impaction, a not infrequent complication of plumbism.

Phosphorus-poisoning, Diarrhea of.--Phosphorus-poisoning excites nausea, romiting, pain in the hepatic and epigastric regions, jaundice, hematuria, intestinal hemorrhage, and, in rare instances, diarrhea, and is characterized by ecchymoses in the serous and mucous membranes, kidners, and elsewhere.

Briefly stated, the treatment consists in gastro-intestinal lavage (with a weak potassium permanganate solution), employing emetics, administering mucilaginous drinks, and prescribing opiates to control pain and diarrhea, and laxatives when constipation is troublesome.

Acid Poisoning, Diarrhea of.-The manifestations of and the manner in which mineral and organic acicls-hydrochloric, nitric, nitrous, sulphuric, sulphurous, oxalic, tartaric, acetic, and carbolic -produce diarrhea so closely resemble each other that their separate discusion is unnecessary. The disturbance incident to them is usually acute, though occasionally chronic gastro-intestinal irrita- 
tion remains as a sequel to these poisons, which are taken accidentally, with suicidal intent, or administered in mistaken dosage or erroneously for some other drug. The most intense suffering here results from burns in the mouth, throat, esophagus, and stomach, which may remain superficial or lead to extensive necrosis and ulceration, followed by stricture. Ender such circumstances swallowing is painful, the patient complains of a choking sensation, profuse salivation, and distention of the abelomen, which is tender upon pressure. There is an increased frequency of the evacuations, which, because of their bloody character, resemble those of colitis and other inflammatory and ulcerative lesions of the small bowel or colon.

The symptoms of colonic calarrh and diarrhea supposedly result chiefly from a loss of alkali which is necessary to metabolism. Very soon after acid has been introduced into the stomach there begins an increased acid excretion through the kidneys, and a passage of acid fluid through the large intestine, giving rise to secondary diarrhea. It is not necessarily the ingested and absorbed acid itself, which is speedily excreted, for the body gives off large amounts of its stored alkali for the neutralization of the ingested acid, and the excretory organs endeavor to compensate the resulting loss of alkali by eliminating considerable quantities of compounds with an acid reaction from the body. The effect, of course, is identical.

When instituting the treatment, if the patient is seen immediately gastric lavage is indicated, but later, owing to the danger of perforation, distention of the stomach with water or neutralizing agents is extremely dangerous, because it is impossible to foretell the extent and depth of the eschars. The most desirable remedies to prescribe are magnesia (lime-water), soap in liberal doses, or a few drops of liculur sode in mucilage, to counteract the escharotic effect of the acid.

Carbolic-acid-poisoning constitutes the most frequent of the above group, and the treatment descrves separate consideration, since it differs somewhat from the above. Briefly summed, this consists in gastric lavage, using lime-water or alcohol (25 per cent.) liberally, which is an antidote to carbolic acil.

When the patient is out of danger from immediate effects of the poisoning he should be placed upon a fluid diet or, if necessary, rectal nutrient enemata, given oil, bismuth, magnesia, charcoal, and like sedatire and soothing agents alone, or in combination with antidiarrheal remedies to relieve gastro-intestinal pain and diminish diarrhea. In the meantime, stimulants and remedies which sustain the system should be administered. The subsequent treatment of enteritis and colitis from carbolic-aciel-poisoning is the same as that recommended elsewhere for the relief of these conditions from other causes.

Alkali-poisoning, Diarrhea of.-The milder alkalies-solium carbonate, potassium acetate, citrate, carbonate, etc.-when administered in large doses or for a long tine maty canse slight irritation to the intestinal mucosa and interfere with digestion, but strong or calustic 
alkalies-calcium (slaked lime), calcium oxid (quicklime), potassium hydrate (caustic potash), sodium hydrate (caustic soda), ammonia, and lye-accidentally or deliberately swallowed are frequently the cause of severe diarrhea. Of these, lye-poisoning is most common, particularly among children, because it is generally used for scrubbing, and is often left around, so that they can readily get hold of and swallow it.

The immediate manifestations of caustic alkali-poisoning is severe burning pain from the mouth to the stomach, difficult swallowing, esophageal spasms, loss of voice, salivation, irritative cough, severe vomiting, expectoration of bloody mucus and shreds of the mucosa, colic, which is closely followed by diarrhea, and there may be respiratory disturbances, muscular spasms, disturbed action of the liver and kidneys, and a severe gastro-enteritis may prevail after the patient has recovered from the poison.

The treatment, when the patient is seen immediately or soon after taking the caustic, consists in having him drink freely of a weak solution of vinegar, dilute acids or lemon-juice, or wash out the stomach with these agents to neutralize or evacuate the poison. In addlition, demulcents-milk, flour, water, and oils-should be administered to protect the raw mucosa, along with opium or morphin in combination with atropin, to relieve pain and visceral spasms.

In aggravated cases where, because of spasm or edema of the glottis, breathing is difficult or impossible, immediate tracheotomy is indicated.

Fluid and soft nourishment is permissible when the patient can swallow and digest them, but frequently, owing to the raw condition of the throat, esophagus, and stomach, this is impossible, and he must be sustained by frecpuent nutrient enemata. Later, bismuth, chalk, charcoal, tannalbin, ichthoform, and beta-naphthol, gr. $v$ to $\times(0.30-$ $0.60)$, should be prescribed three or four times daily because of their soothing, astringent, antiseptic, or healing action upon the stomach and bowel, and tendency to restrict the number of evacuations. Because of the necessity of continuing the treatment for a long time it is not advisable to permit the sufferers to take opiates, because they may become habitués to the drug, yet it is urgent that something should be done to allay the severe gastric and intestinal pains, and for this purpose the author knows of no more reliable local analgesics than aspirin, orthoform, and analgin, gr. v (0.30), administered according to indications.

When there is colitis, with and without ulceration, colonic irrigation with ichthyol, balsam of Peru, or horic acid ( 1 to 2 per cent.) to heal the bowel, alternating with oil, which has a soothing effect upon the mucosa, are to be recommended in connection with the above treatment, and continued unless the intestinal manifestations subside.

Miscellaneous Medicinal and Chemical Poisons, Diarrhea of.In addition to the above poisons, there are numerous medicinal and chemical agents which, when taken in large amounts, accidentally or 
purposely, gain entrance into the circulation in other ways, produce diarrheal manifestations, slight or grave, but in practically all cases the increased frequency of the evacuations results from an acute or chronic gastro-enterocolitis induced directly or indirectly by the poison. Because of their relatively slight importance to the subject their individual symptomatology and diagnostic features will be omited, and the author will simply name them and give their antidotes in parentheses. The poisons included in this list are:

Turpentine (magnesium sulphate and emulcents).

Cantharides (demulcents, saline cathartics, and opinm; oils and fats interdicted).

Physostigmin (fixed alkalies and atropin hypodermically).

Pilocarpin (atropin and morphin hypodermically).

Colchicum (opium and astringents).

Digitalis (aconite, nitroglycerin, and opium).

Ergot (stimulation and general application of heat).

Silier salts (table salt, demulcents, and milk).

Nicotin (restrict tobacco).

Conium (emetics, tannic acid).

Lobelia (tannic acid, lavase, and stimulants).

Iodin (starch or arrow-root).

Zinc salts (white of eas. demulcents, and sodimm carbonate).

Copper salts (demuleents and albumins).

In addition to the administration of the above antidotes, it is frequently necessary to resort to immediate lavage, prescribe stimulants and supportive remedies, administer an opiate to relieve pain, restrict the diet, and later, when sequele and diarrhea are present, to have the patient take internal remedies and irrigate the howel for the purpose of controlling the gastro-intestinal catarrh caused by the poison. 


\section{CHAPTER XV}

\section{COMPENSATORY DIARRHEA}

THIs rather unusual form of diarrhea is due to a disturbed metabolism and not to organic changes in the bowel or digestive derangements. ${ }^{1}$ It may be encountered in a number of diseases, the most frequent of which are thyroidism, gout, diabetes, Bright's and Addison's diseases, pellagra, severe injuries, extensive burns, climatic disturbances, and certain affections of the liver. It occurs when metabolism of an organ is materially interfered with through organic changes or nervous influence and there is an accumulation of excretory products (some toxic) which cannot escape normally, and hence find a means of exit through the bowel, to cause diarrhea by increasing the fluid, augmenting the secretions, and exciting peristalsis.

This form of diarrhea is symptomatic of arrest or perversion of function of the skin, lungs, kidneys, or organs of internal secretion, and is corrective in effect. The definition includes those cases where the intestinal tract vicariously assumes the excretory functions of other organs or eliminates products which are the result of defective metabolism, and does not embrace functional diarrhea when the intestine merely rids itself of accumulated foreign materials. Stern ${ }^{2}$ holds there are three types of compensatory cliarrhea, viz.: (i) Diarrhea concomitant with disturbed catabolism. (2) Diarrhea resulting from disease of excretory organs. (3) Diarrhea occurring during the period of physiologic decline.

In discussing compensatory diarrhea Niles has pointed out that it may be present in elderly persons undergoing physiologic decline, owing to incompletely catabolized products which are not excreted, and result from toxic states where the toxins are eliminated by the intestine as in the climacteric period.

In this type of loose movements the evacuations are exceedingly poisonous, and it is often questionable if the diarrhea should be arrested, because in stopping it the toxins from the disease could not escape and would accumulate, to seriously impair the health or cause death.

Compensatory diarrhea may lead to an inflamed or ulcerated state of the mucosa and the loose movements continue after the original cause of the diarrhea has been removed, under which circumstances local treatment of the bowel is necessary to effect a cure.

Old Age, Diarrhea in.- In old age the intestinal tract is the last organ to lose its tonicity. Since the rest of the body deteriorates,

1 The author is under obligations to Dr. Sidney Adler for assistance in looking up the literature of compensatory diarrhea.

2 Jour. Amer. Med. Assoc., August 8, i Sos. 
lessening the activity with which the toxic materials are excreterl, the intestine in a measure takes up the work of the kidneys and lungs. and assists in keeping up the systemic erpuilibrium.

In old age there is a change in the kidney and skin function, and there is also a general decline of all the organs of the boty. Women more than men are subject o diarrhea, and this is especially seen at or after the chimacteric period. Some of these women have never suffered from chronic diarthea before this period. but with the aptearance of the menopatuse retrogresive changes wecur, and the woxie prote uets in the blood which were in patrt thrown off hy the men-trual blood mus now find other means of egress. The osmotic presure in controlled in women by the menstrual lowe of blood. After this is lost at ibe menopante the hypersmotic hlowel seckis a chance to filter through the tiscue offering the least resistance, such as the intertine. and the filtrate is full of toxic material and cause- diarrhea. Sometimes as the fatient grows older the diarrhea increasen. though structural and functional changes in the intestinal wall may cause it. People who perspire freely are seldom subject to chronic diarrhea. Therenon ${ }^{1}$ says that the equilibriun is retained in elderly perons by increased intertinal elimination. which compensates for the lesened activity of the external integument.

Addison's Disease, Diarrhea in. - The most common form of diarrhea occurring in this condition arises at or toward the close of this affection, and comes on without any olvious cause. Retraction of the abdominal wall reembling peritonitis, colicky pains, with watery discharges, lead guickly to collapse or delirium and coma. There is another form, owever, which occurs in the earlier stages of the discase which is in no wy exhausting. but which tends to improve the general conclition. The disease is characterized by asthenia, pigmentation of the skin, general loss of appetite and muscular energy, conditions which are improved by the liarrhea.

Diabetes.-Diabetic coma is often ushered in by a gastro-intestinal attack. The diarrhea, however. may be of a compensatory nature and warl off coma. This condition would come earlier and more frequently if the intestine dicl not get rid of the toxins at the proper time. Diarrhea, as a complication of diabetes. may be due to the continued sugar-free diet causing an intestinal insufficiency or to the metabolic processes and rapid osmosis through the interinal wall of the toxic materials. It is through this means that sugar is excreted by the intestine, and the urine may berome entirely free from sugar. After one of these attach the urine is often free from sugar, acetone. and diacetic acid. Sugar is not always found in the feees during diarrheal attacks, nor is it found in the storkls of cliabetics whose bowel action is normal. In no other diarrleal condition is sugar found. Following the compensatory diarrhea the general nervous condition of the patient also improses.

Gout.-Gouty individual- (expecially oleere) are inclined to con1 "De la Diarrhée chez les Viellarst.." Thè-e de Parí. 1sos. 
stipation. In cases of irregular gout the paroxysms are ushered in by attacks of intestinal stasis. It is not an uncommon occurrence for these paroxysms to be entirely prevented by free bowel movements, ridding the body of the toxins. The urates and alloxur bodies often clog up the kidneys in the chronic gouty conditions, seriously interfering with their permeability; the intestinal function must then take on the extra work and act for the kidneys too. The feces in these cases contain considerable amounts of urates and alloxur bases. Clinically, we are able to demonstrate the compensatory action of the intestine by the decrease of urine secreted and the dark stool of nuclein substances, tenesmus, and pain felt in the bowel.

In retrocedent or suppressed gout there may be severe gastrointestinal symptoms, pain, vomiting, diarrhea and great depression, and death may occur during one of these attacks.

Exophthalmic Goiter, Diarrhea in.-Excessive sweating is sometimes seen in this condition, and has a corrective action. In some cases diarrhea alternates with the sweating. When these diarrheal attacks last longer than five to six days structural changes in the intestine will be encountered. Diarrhea in this condition lasts but a day or so, and is always of a compensatory nature.

Kidney Diseases, Diarrhea from.-In uremia, where the function of the kidney is seriously interrupted, and urea, ammonium carbonate, creatin, and creatinin get into the blood, diarrhea of ten ensues through cerebral stimulation, action of the toxins upon the intestinal mucosa, vasomotor disturbances, peristaltic activity, or transudation of toxic fluid from the blood into the intestine. Where there are no uremic ulcers in the bowel, diarrhea must, of necessity, be included in the body's compensation. When compensatory diarrhea has been in effect for some time and ulcers are present the loose movements may be caused by the lesions, efforts of nature to protect herself against toxins, or both.

Diarrhea after the formation of uremic ulcers is changed in effect and in regard to the character of the excreta. The occurrence of an attack of compensatory diarrhea is an indication that there is no immediate danger from uremic convulsions, dyspnea, or coma. It is often seen in a uremic condition that the compensatory diarrhea will occur more than once, and the general condition of the patient improves with each attack. The elimination of toxic materials by the intestine causes congestion and, in time, erosions of the mucosa, but the patient lives in comparative ease while the intestinal tract is caring for the elimination of the renal excreta.

Hirschler has shown experimentally that ammonium carbonate and creatin are not only strong central intestinal stimulants, but also peripheral irritants. Iremic vomiting is in the same class with an ulcerative process in the duodenum or other portion of the intestinal tract, due to the enzyme formed in the blood causing thrombi.

${ }^{1}$ Jubilar-Arbeiten der Schuler Korayi's Pester, Medichine-Chirug. Presse, I891, No. 30 . 
Such evacuations are not corrective but pathogenic, and tend to weaken an already exhausted borly.

Burns, Diarrhea from. When a consideralble portion of the lusly surface is hurned toxic alsorption symptoms arise through injury to the protoplasm, hood, loss of skin activity, toxins, and slock. Anong the phenomena sooner or later olserved are diarlacal, retention of urine, delirimu, coma, and sometimes a mantacal conclition.

In some cases of extensive lurns, where the patient is comatose, pulseless, and respiration is hardly noticeable, diarrleas sets in and the general condition inproses at once, for the urinary retention disap)pears and the pulse becomes more regular, the intestine taking care of catabolic dimination.

The treatment of compensatory diarrhea must necessarily vary in different cases, and should be directed against the affected part of the oreanism, with the olject of exualizing metabolism, so that the harmful products responsible for the diarrhea will not be profluced in abuormal amounts. Where the movements become very frequent, offensive, or exhausting, and the bowel is inflamed or ulcerated, the intestine should be cleansed with medicinal solutions and antidiarrleal remedies preseribed to relieve pain, diminish the movements, and enalse the patient to oldain rest and sleep. 


\section{CHAPTER XII}

\section{ENTERITIS, COLITIS, ENTEROCOLITIS (NON-SPECIFIC (?) INTESTINAL CATARRH), DIARRHEA IN}

\section{DEFINITION, GENERAL REMARKS, ETIOLOGY, PATHOLOGY}

Definition.-Inflammation (catarrhal?) of the mucosa of the small or large intestines, both or any one of their sections. which, according to the segment or part of the bowel involved, is designated enteritis, duodenitis. jejunitis, ileitis, colitis, appendicitis, typhlitis, sigmoiditis, proctitis, or enterocolitis when the entire intestine is affected.

General Remarks. - The condition commonly characterized as enteritis, colitis, or enterocolitis constitutes the most common of all intestinal disturbances. and while it is a disease that causes considerable annoyance, distress, and often renders the subject partially. or completely unfit for social or business duties, it rarely ends fatally except in the very young or old, and when it is complicated by mixed infection or some other disease.

Enterocolitis is encountered in all countries, climates, nationalities. walks of life, and in both sexes, though it is more prevalent among infants and children and in men, particularly those who are exposed, than in others.

The affection is encountered most often in persons between twentyfive and forty years of age, and may be acute or chronic. but is found in the latter form most frequently. because when acute it is either not sufficiently distressing to cause worry or the patient attempts to cure himself with home remedies or patent medicines, and further, owing to the fact that chronic enterocolitis is often accompanied by diarrhea, colic, digestive disturbances, or other manifestations that make it imperative for the one afflicted to seek relief. Owing to the similarity of the symptoms in the presence of gastric and enteric or colonic inflammation of the mucous membrane, sastritis, enteritis, and colitis are frequently confused, and the one mistaken and treated for the other, but it is well to bear in mind that sometimes the entire gastrointestinal tract may be involved in the catarrhal inflammation (gastroenterocolitis).

Any or all portions of the intestinal mucosa may be involved, but the duodenum, rectocolonic segment, and ileum are the sections most commonly affected. The first. owing to its shape and close relation to the stomach, the discharges from which frequently and quickly change the intestinal contents from an alkaline to an acid reaction. The second. because firm and nodular feets, gas, and toxins frequently 
collect in the rectum and remain to irritate the mucous membrane, and the third, owing to the fact that the maximum lacterial activity takes place within the ileum, which is conducive to the catarthal state.

Colitis, however, is very much more common than enteritis, and catarhal inflammation is observed very much more frepuently in the rectum and sigmoid (proctosigmoiditis) than in other portions of the large intestine. The author holds that catarhal enterocolitis is not nearly so common as is believed by the profession generally, because in numerous instances, by means of getting the history, going over the patient thoroughly, and making a careful macroscopic and microscopic examination of the feces and discharges, he has been able to demonstrate that patients previously treated for this affection were suffering from tubercular, syphilitic, entamebic, balantidic, helminthic, coccilic, or ciliate colitis; and in other instances he has been able to slow that the bowel disturbance was due to a gastrogenic, enterogenic, hepatogenic, pancreatogenic derangement, or other local or general ailment which incited diarrhea, colic, or the discharge of mucus in the absence of catarthal involvement of the gut.

Catarrhal enteritis or colitis may pave the way for or be a sequel of the above-named infections and disturbances, or may complicate them and make differentiation of the one from the other extremely difficult, hence the affection may be either primary or secondary. Enterocolitis has not infreguently been confused with intestinal cancer, benign growths, stricture, angulation, twisting, ptosis, enteroliths, foreign bodies, or other lesions or agents which cause irritation, obstruction, inflammation, or ulereration of the bowel.

Usually, when a patient complains of indigestion, unduly frequent and loose movements, colic or ablominal soreness and mucus in the dejecta, in the absence of obvious evidence to show that the trouble is due to another infection, a diagnosis of intestinal catarrh is often erroneously made.

Diarrhea is regarded as one of the pathognomonic inclications of catarrhal cnterocolitis, but many patients having the lisease do not exhibit this symptom, or, if so, not until late, but complain chiefly of costiveness or constipation alternating with loose movements, or suffer several days from the former and then the latter, under which circumstances the catarthal state is mistaken for chronic intestinal obstruction. Iniversal success in the treatment of intestinal catarth will not be reached until physicians realize that constipation and diurrhea often gohand and gloxe in this complaint, and must be rationally treated.

Each year demonstrates that a greater proportion of patients who were formerly thought to be afflicted with catarrhal enterocolitis suffer from inflammation of the mucosa, induced ly specifie agents, such as entameba, Balantidium coli, bacilli (Shiga, Flexner, Hiss, etc.), coccidia, flagellates, ciliates, etc., which emphasizes the importance of routine 
fecal examinations in all cases, so that ordinary catarrhal may be differentiated from the specific infections of the intestine.

Etiology of Enteritis, Colitis, and Enterocolitis.-The causes of enteritis, colitis, and enterocolitis are so numerous, varied, and complicated that to attempt a detailed explanation of each would be futile in a work of this scope; hence, the author will be content with having named the more frequent etiologic factors of the diseases and discussing only the causes of greatest importance.

No hard-and-fast line can be drawn which would clearly separate the causes of and the tissue changes which take place in acute and chronic inflammation of the intestine, and because of this the etiology and pathology of acute and chronic intestinal catarrh (enteritis, colitis, and enterocolitis) will both be considered together. The author will, however, endeavor to point out their chief etiologic factors, and discuss in logical sequence the pathologic changes which take place in the bowel during the acute and chronic stages of the disease.

In order to understand the etiology and pathology of this affection it is well to constantly bear in mind the fact that enterocolitis may be primary and hegin independently as such, or be secondary to and induced by one or more of the local and general ailments named below as causative factors.

Named in the order of their frequency and importance, the chief primary and secondary etiologic factors in the production of acute and chronic catarrh in the upper and lower intestines are:

(I) Dietary indiscretions (gormandizing, eating at irregular hours, too fast or too often, consuming indigestible foods or foods too hot or cold, that contain acid, are stimulating or leave a coarse irritating residte, and those which are unripe, infected, raw, improperly cooked or partially spoiled, and ice-cold drinks during meals).

(2) Unhygienic surroundings.

(3) Occupations which are arduous and lead to frequent exposure, etc.

(4) Age (very young, one to two years, and over sixty).

(5) Mahutrition (starvation, improper feeding, intestinal disturbances, etc.).

(6) Atmospheric influences (a sudden change from a high to a low temperature and vice versâ).

(7) Extreme heat (from the sun, violent exercise, working in foundries, etc.).

(8) Impure water (contaminated by minerals, sewage, helminths, entamelox, bacilli, etc.).

(9) Sitting in a chaft or upon cold steps, or keeping on wet clothing or shoes which would give one a cold.

(Io) Chronic irritants and poisons administered in the form of medicine, swallowed accidentally or taken with suicidal intent (mineral acids, mercury, arsenic, cantharides, copper, tartar emetic, spices, pepper, mustard, garlic, alcohol, chloroform, ether, alkalies, aromatic acids, volatile ethereal oils, etc., and laxatives and purga- 
tives, such as the hydragogues or salts and drastic purgatives like senna, jalap, croton oil, and colocynth, which irritate, inflame, or erode the mucosa).

(I I) Chronic irritants of the blood which act upon the mucosa in the presence of extensive cutaneous burns, and catarrh of uremic nephritis and mercurial poison.

(12) Mechanical irritants (gall-stones, enteroliths, intestinal sand, scybala, bismutl balls, foreign bodies, parched corm, seeds, fruit, stones, coins, and movable or ptotic neighboring or distant organs).

(13) Acute and chronic intestinal obstructions, abnormalities, and displacements (malignant and benign tumors, splanchnoptosis, ptosis of the intestine or other organs, adhesions, extra bowel pressure, stricture, angulations, volvulus, invagination, strangulation, divertieula, or the extension of inflammatory processes from other organs and structures to the gut).

(It) Local. general, and infectious diseases of the intestine (measles, whooping-cough, scarlet fever, diphtheria, chicken-pox, erysipelas, anthrax, grip, pneumonia, malaria, rheumatism, typhoid, ptomainpoisoning, cholera, sepsis, and tubercular, syphilitic, gonorrheal, entamebic, bacillary, balantidic, helminthic, flagellate, ciliate, or coccidic colitis (dysentery), and inflammatory states of the bowel incident to the Proteus vulgaris, colon bacillus, Bacillus enteritidis (Gärtner's), sporogenes. streptococci, Bacillus butyricus, paratyphoid bacilli, and others of the accidental and obligate pathogenic microorganisms of the intestinal canal).

(I5) Constipation and fecal impaction.

(16) Neuroses and psychic impressions which influence the motor and secretory functions of the intestine.

(I7) Extension of inflammation or disease from other organs to the gut (gastritis, appendicitis, peritonitis, ulcers. cancer, suppurative conditions, tuberculosis, etc.).

(i 8$)$ Disturbances (obstructive) of the heart, liver, and lungs or splanchnic circulation, which result from cachectic states (observed in cancer, malaria, chronic intestinal auto-intoxication, and Bright's and Addison's diseases).

(ig) Frequently drinking an abundance of ice-water and cold beverages when overheated or during meals.

(20) Swallowing infected sputum in dental and nasopharyngeal suppuration.

Dietary indiscretions of varions kinds often lead to a catarrhal state in the stomach, intestine, or both. Frequent overloading of the former is a factor, becalse it distends the stomach, trammatizes the mucosa, and sooner or later leads to impairment of the motor and secretory functions of the organ, with the result that the food is insufficiently salivated, broken up, and digested, and, in addition, the gastric juice abnormally mised is cliseharged into the intestine almost continuously, so that the digestive apparatus has but little if any time to recuperate between meals. Again, when heary and indigestilsle 
food accumulates in excessive amounts, it farors intestinal catarrh and loose movements through the abnormal stimulus which it gives to peristalsis and the secretory glands. Indue intestinal activity and congestion of the mucosa may also be incited by ice-cold drinks, eating very hot food, and the consumption of fruit, sausages, certain fish, and other articles of diet difficult to digest. Foods, when taken in large amounts and those which are improperly balanced, lead to undue fermentation, putrefaction, and the formation of lactic, butyric, acidic, succinic, and other acids and gases which pare the way for the catarrhal state. Raw, unhealthy, and improperly cooked regetables, fruits, and meats are frequently difficult to digest, and are, therefore, conducive to bowel disturbances, particularly in infants and children. Some individuals have an idiosyncrasy to certain articles of diet, which, when consumed even in very small amounts, excite a catarrhal discharge, viz.: milk may produce a diarrhea in one indiridual and constipation in another. Food infection and ptomain-poisoning, as, for instance, the eating of meat from diseased animals or that which has been exposed and infected beforehand, or other foods which contain pathogenic bacteria, their poisons or elemental toxins, like canned goods, fish, and shell-fish. impure or spoiled grain food stuffs, diseased potatoes, mushrooms, etc., often induce catarrhal colitis.

Intestinal inflammation is frequently secondary to gastrogenic derangements where there is subacidity, achylia gastrica, hyperacidity, atony, motor insufficiency, or malignancy. which, according to the methods elsewhere described (see Gastrogenic Diarrhea, Chapter X), lead to stagnation within the stomach, poor or no digestion, and the emptying into the gut of an abnormal juice and incompletely broken up and digested food masses that are beyond the ability of the intestine to handle. Such a condition interferes with the functionating powers of the liver and pancreas, owing to the abnormal stimuli which is brought about.

Hepatogenic and pancreatogenic disturbances resulting from reflex irritation and organic disease have also been known to aggravate or cause intestinal catarrh where the biliary and pancreatic secretions are augmented or reduced sufficiently to impair the intestinal juice.

Enterogenic dyspepsia (sce Enterogenic Diarrhea, Chapter XI) is also a common cause of catarrhal enteritis, colitis, and enterocolitis. This type of catarrh is primary when $(a)$ it is due to a diminished secretion of the biliary, pancreatic, or true intestinal secretions: (b) they are prevented from being discharged into the duodenum owing to olstruction within the ducts: $(c)$ there are pathogenic changes in the mucosa which would interfere with or prerent intestinal digestion and assimilation: and $(d)$ there are imperfect mastication and indiscretions in diet, where more food is consumed than the intestinal juices can take care of, it is indigestible (such as cabbage, sourcrout, cucumbers, and fresh fruit) after passing through the stomach, it is still mechanically irritative, or where the food readily undergoes fermentation and putrefaction; and secondary when it is consequent 
upon gastrogenic abmormalities, such as subacidity, achylia gastrica, hyperacidity, atony, motor insufficiency, or cancer.

The chemicals and medicines named in the aloove list, which gain entrance to the bowel accilentally or by administration, when very strong sometimes have a cauterizing effect or set up an active inflammation which terminates in acute or chronic catarrh, but remedies like mereury, arsenic, silver, and the enumerated drastic purgatives often lead to a subacute catarrhal inflammation either through repeated irritation of the mueosa, incident to their passage through the bowel, their effect upon the general or local nervous apparatus, tendency to augment peristalsis, or increase the exudation of fluids into the howel or stimulate the intestinal glands to secrete an oversupply of mucus.

Chemical irritants and toxins which reach the bowel through the circulation produce catarrhal inflammation of the mucous membrane solely through their irritating propensity, while mechanical irritants, such as gall-stones, enteroliths, foreign bodies, act in a similar manner, except that, in addition. they tranmatize the mucosa and may cause ulceration and suppuration when they become lodged or encysted.

Acute and chronic intestinal obstruction, however produced, invariably incluce a local catarrhal inflammation or enterocolitis when the block is high, because scybala accumulate and trammatize the gut, the pathogenic and pyogenic accidental and obligate intestinal microorganisms greatly multiply and generate poisons offensive to the bowel, and gases which form in an enormous amount as a result of putrefaction and irritation to the mesenteric nerves stretch and irritate the intestine along with retained mucus and fluid feces, and because the discharge from ulcers above the obstruction intensely irritate the mucosa and cause congestion, and later, when emptied into the bowel below the block. are so offensive that they produce a catarrhal inflammation which may be complicated ly well-marked erosions, ulcers, or both.

Children's diseases, such as measles, whooping-cough, scarlet fever, pneumonia, diphtheria, digestive disturbances, etc., not infrequently. accompany or induce intestinal catarrh, owing to the changes in the mucosa. Inder these circumstances sepsis may complicate them, or the effect of toxins may be manifest in the circulating media.

It is natural that a catarrhal intlammation of the intestine should be a forerunner of syphilitic, tubercular, gonorrheal, and the different types of dysenteric colitis because of its tendency to lower the patient's vitality and leave the mucous membrane uncovered, so that the specific agents of these infections may readily find suitable loelgment and a gateway to the circulation.

It is also easy to understand why catarthal enteritis, colitis, or enterocolitis should be a secpuel to such infectious diseases of the bowel, because the mucous membrane is repeatedly insulted for such a long time ly the specific organisms, lesions of the acompanying mixed infection, generated toxins, and offensive discharge. In not 
a few instances considerable ingenuity and patience are required to cure the intestinal catarrh after the original infection has completely disappearect. and in some cases. where there is a resultant stricture, this must be treated or extirpated before the patient can obtain permanent relief.

Constipation and coprostasis are nearly always complicated by colonic catarrh, and frequently by stercoral diarrhea, owing to the irritation procluced by retained offensive feces, toxins, gas and discharges. and bruising of the mucosa by scybake and the formation of alcers through the pressure of impacted masses which accumulate at the bowel flexures. Once lesions have formed. peristalsis is augmented through action of the intestinal content upon the terminal nerve filaments, and when the fecal collection assumes proportions sufficient to almost block the gut, a tendency to strain is constant, and evacuations are frequent and fluid because solicl excreta cannot get by:

Veurotic diarrheas hare been fully discussed elsewhere (see Neurogenic Diarrheas. Chapter XII. and it is not necessary to do more here than mention the fact that when disturbing neuroses continue for a long time they interfere with digestion. the formation of normal gastro-intestinal juices and cause undigested food to be rushed through the alimentary tract. which irritates the mucosa and sooner or later leack to catarrhal enterocolitis.

Lnquestionably. disturbance of the splanchnic circulation is conducive to enterocolitis. for stasis of the portal ressels caused by obstructive liver and heart affections induces congestion of the abdominal circulation, and engorgement of these ressels, however else produced. favors a hyperemic state of the mucosa. The conclusions of Türck. who attempted to demonstrate this experimentally. are as follows, riz.:

"Strong chemic irritation produces conge-tion of all the splanchnic or abdominal ressels.

"The rasomotor disturbance thus produced results in motor disturbances of the stomach and intestines.

"Prolonged distention of the stomach or intestines with air or gas result in splanchnic congestion. followed by collapse.

"Toxins formed in the stomach may produce splanchnic congestion as a local reflex irritation, or by absorption."

On a few occasions the author has treated patients afflicted with localized enteritis, coliti- igmoiditi-. or proctitis which were secondary. to inflammatory suppurative or malignant disease in other organs. viz.. the prostate, bladder. pelvis, upper abelomen. stomach. appendix. or other structure. Aumerous cases of catarrhal and other forms of colitis have been recorded where the distase could be traced to an unhealthy appendix which were cured by appendicostomy or appendectomy.

From what has been said, it may be inferred that in the rast majority of instances catarrhal enteritis. colitis, or enterocolitis is dut to pathogenie micro-organisms, toxins of whatever kind, foreign 
bodies, intestinal distortion, or, in fact, anything which irritates the mucosa continuously or repeatedly at shorter or longer intervals.

Pathology of Enteritis, Colitis, and Enterocolitis. - The changes which take place in the bowel in catarrh of the intestine vary greatly in different cases, dependent upon whether the disease is primary or secondary to another local or constitutional clisease, the nature of the catse or etiologic factors which produce $i t$, and the stage of the intlammatory process when seen.

The appearance of the mucous membrane in the presence of catarrhal enteritis, colitis, or enterocolitis is about the same as that observed in the gastric and mucous membranes elsewhere when similarly affected. A review of the above-named etiologic factors in the disease explains why patients may suffer from catarrh of the entire gastro-intestinal tract or any one of its several segments, viz.. sastroenteritis, enteritis, enterocolitis, appendicitis, typhlitis, colitis, sigmoiditis, or proctitis, though certain portions of the bowel (duodenum, ileum, colon, and rectum) are very much more frequently the seat of catarrhal inflammation than others. In other words, this affection may be distinctly circumscribed or extend through a considerable length of intestine, according to the virulence of the inflammation, extent of the toxins causing $i t$, or character and location of the irritation. Catarrhal inflammation of any or all parts of the small or large intestine is ever changing when a series of cases are studied, owing to its varying degrees, since the mucosa may be (a) simply congested or inflamed; (b) eroded; (c) ulcerated; $(d)$ marked by hemormagic infarcts; (e) intolice in a phlesmonous (gangrenous) process; or $(f)$ covered with a diphtheric membrane, though the two last-named conditions complicate infectious more often than catarrhal enterocolitis.

The manifestations of the disease are not always commensurate with changes in the mucosa and decper structures of the gut, for often patients suffer greatly when the bowel appears normal or slightly congested, and, again, those afflicted with enterocolitis, where the intestine is intensely inflamed, eroded, or ulcerated, may suffer but slightly from the discase.

Acute Catarrhal Enteritis.-In this affection the mucons nembrane is hyperemic, sensitive, swollen, and of ten edematous over small or large circumscribed areas or throughout a considerable length of the intestine, and occasionally the inflammatory process extends to the submucosa. In some instances, according to the degree of inflammation and character of the intestinal contents, the color of the mucosa varies from a pink to a dark purple, or presents a dull, choudy, gravish appearance. The inflammatory process concentrates at the summit of the valvulat conniventes about the lymph-vessels, villi, and solitary follicles, but seldom involves Peyer's patches, and oceat sionally punctate hemorrhagic areas are manifest.

Under the microscope the secreting cells show marked activity, and when the inflammation is active leukocytes collect about the tubular glands and the capillaries in the submucosa. "The bloot- 
ressels of the mucosa, and usually the submucosa, are more or less distended with blood. Occasionally small extravasations can be seen, chiefly between the crypts of Lieberkiihn. The interstices between these glands are frequently wider than normal and contain more or less abundant masses of round cells; the latter appearance may be considered characteristic of true inflammatory catarrh. Round cells are also abundant in the most superficial part of the submucosa, immediately under the muscularis mucose, and in the deeper layers of the submucosa, chiefly around the blood-vessels. The swelling of the solitary follicles-if they are swollen at all-and of Peyer's patches is probably due to proliferation of the essential cells of these glandular structures and in part to immigration of round cells. The epithelium of the mucosa in almost all cases is detached, particularly in the large intestine. This I regard as merely a postmortem phenomenon, and 1 have performed a number of control experiments in animals that strengthen this view" (Nothnagel).

The lining of the bowel may be dry and hot in the beginning of acute enterocolitis, but in a short time becomes abundantly smeared with mucus or a discharge composed of it, pus, blood, bile-pigment, epithelial cells, mucous shreds, liquid feces, and undigested food remnants, according to the virulence of the inflammation and changes in the gut. In aggravated cases the discharge, which is enormous, may be attributed to the exudation of fluid into the intestine, augmented gastro-intestinal juices, and to hypersecretion of mucus or goblet-cells.

The amount of pus and blood contained in the mucoid evacuations bears a direct relation to the number of crosions and ulcers present. Once the epithelium is destroyed, pathogenic and pyogenic bacteria of the bowel begin to play a part, mixed infection ensues, and the affection passes from a simple catarrhal to a suppurative inflammation of the mucosa, which may, in aggravated cases, be followed by extensive ulceration, submucous abscesses and fistula, or a diffused phlegmonous inflammation which destroys the mucous membrane over large areas, and sometimes causes death from exhaustion, toxemia, or perforation and peritonitis.

Hemorrhages have occurred in the different stages and types of intestinal catarth, uncler which circumstances the affection is ordinarily designated as hemorrhagic enteritis, colitis, or enterocolitis. Bleeding may be the first manifestation to suggest an inflammatory condition of the bowel or appear at any time during its progress. Hemorrhagic areas may appear as diminutive, dark, petechial-like spots, crosions, or over the whole or extensive sections of the bowel where the mucosa is soft, spongy, and presents an appearance typic of this complication.

Hemorrhagic enterocolitis resembles typhoid fover in its manifestations, but, according to "Mummery," it is due to a primary infection of the colon with the Diplococcus pnemmonia. The mortality 
of this condition is very much higher than that for ortinary catarrhal enterocolitis, but the process can usually be arrested by through-andthrough colonic irrigation.

When from unknown causes mucus collects upon the inner surface of the bowel, and is evacuated en masse in the form of tube-like moldings of the intestine, the inflammatory process is diagnosed as desquamative enteritis or colitis, according to the seandent of bowel involved. This condition, which has also been called pseudo-enteritis, enteritis crouposa endemica, enteritis diphtheritica. elc., may in some inexplainable way occur in the presence of both catarthal and sperific inflammations of the bowel as well as mercurial poisoning, nephritis. etc., both in infancy and in achult life. When the disease is accompanied by necrotic changes in the bowet it is characterized by a suclen onse, high temperature, toxemia, and dysenteric symptoms, which frequently and quickly lead to death through exhaustion. In case the exulation is superficial, tenacious membranes are visible, but when the deeper structures are involved the mucosa has a granular, raspberry-like appearance, dotted here and there with exudative deposits. but where the inflammation extends entirely through the intestine the bowel becomes at least six tines its normal thickness. [sually this variety of colitis is most marked in the sigmoid Hexure and rectum. It is essential that acute desquamative or membranous enteritis or colitis be differentiated from the chronic form known as myorrhea coli (colica mucosa, enteritis membranacea, etc.).

When the solitary follicles and agminated glands in catarrhal enteritis or colitis become involved, the process is designated as follicular enteritis or colitis, a condition more often caused by bacterial invasion in persons having a lowered resistance. As a rule, intestinal catarrh paves the way for the specific organisms of dysentery or bacteria of the intestine, which dominate the disease once they participate in it. In this form of inllammation the follicles are swollen, protrude beyond the mucous surface, and are manifest as anemic spots resting upon a highly colored basic membrane. Usually follicles within the colon do not project above the surface, but early ulcerate and form depressed lesions shortly following infection, though occasionally they have been observed as pinhead like elevations before breaking down. Owing to swelling which ensues in Peyer's patches the mucosa possesses a somewhat corrugated appearance. There is an accumulation of lymphoid and round cells in the follicular parenchyma, which, together with congestion of the diminutive blood-ressels, acount for the distention, swelling, and necrosis which follow. There is also cellular infiltration around the follicles and into the lymph-vessels. Then necrosisocenrs, dininutive abscesses may form and individual uleces coalesee to make larger raw surfaces corered with mucus containing some blood-cells. Moodward holds that in some instances infection first takes place, alscesses result, and the diminutive opening or lesion is caused by the escaping discharge. 
Allorecht, in a case of suppurative follicular enteritis, attributed it to a hacillu de-ionated hy him as Bacterim psendotuberculosis rodentum. becaute of it reemliance to the plagute bacillus found among rats. The pathologic findings also resembled those found in plague.

The pathology of chronic intestinal catarh is in many respects, -imilar to that of the ateute variety. because in many instances the tran-itional stage from the latter into the former is imperceptible. and the muco-a $\mathrm{i}$ - concested. swollen. and covered with mucus. but its coloring is paler. often heing of a gray or slate hue when there is an extravastion of pigment hetween the glands and at the summit of the villi.

The discharce contains considerable mucus and epithelium, the collection of round cell- is -maller. and connective-tis-ue proliferation is more marked. In chronic catarrh the intestine may undergo atrophie or. more frequenty, hupertrophic changes. In the hypertrophic form the gland- are long. irregular in their course, and tend toward the formation of pouches. and sometimes. where their mouth are abnormally closed. secretory cysts form cystic enteritisl. The author has known this condition to progress until the connectivetissue hyperplasia caused marked thickening and stenosis of the bowel, and in a number of cases treated the hypertrophic changes led to the formation of multiple small and larse polypi in the colon colitis polyposal. which in some instance- could be clearly seen through the sigmoidoscope. When such growths were present the evacuations were particularly offensive, and usually contained considerable mucus thoroughy mixed with pus and a trace or large amount of blood. When the -olitary and acminated gland-are involved, owing to the shape of their contour the condition has been designated as enteritis nodularis ly Orth. In the presence of atrophic changes the reverse attains, and the mucous membrane and other intestinal tunics diminish in thicknes. villi are less prominent or absent. and the krmph-nodules rupture or become necrotic, giving way to follicular ulceration. In chronic catarrh, ulceration may or may not he a complication. but when present the lesion - are much more numerous and extensive in the later than earlier sase of the disease. particularly in ignoranty treated or neglected cases. This is due in a larse measure to activity of the colon bacillus and other pathosenic micro-organisms which beconte troublesome as soon as the mucosa is eroded by the irritating discharge or straining. Once the ulcerative process has become extensive it is difficult to arrest with the best treatment. and when repair takes place the former location of ulcers is indicated by diminutive whiti-h sars and puckered depresions. which give the mucosa an uneren indentated appearance, or when a number of ulcers have persi-tently coalesced to form large encircling, raw areas the cicatricial ti-sue from them causes partial or complete stenoses.

Clcerative lesions may appear as superficial erosions. small ulcers, or occasionally lesions of considerable size, but except in the presence 
of a virmlent mixed infection the destruction of tissue is not so extensive or deep as that caused by the various types of infeetious colitin: consequently, severe hemorrhage, submucous absece-e- glandular involvement, perforation, peritonitis. and stricture selrlom complicate simple catarrhal enteritis, colitis. or enterocolitis.

Lsually, when a patient suffers regularly week after week from chronic diarrhea and has about the same number of evactation-daty. ulcerative colitis prevails. and the frequency of the eacuations is proportionate to the number of ulcers in the bowel, and the diarrhea will improve or get worse accordingly as the ulcerative proces is healed or permitted to extend. 


\section{CHAPTER ATII}

\section{ENTERITIS, COLITIS, ENTEROCOLITIS (NON-SPECIFIC (?) INTESTINAL CATARRH), DIARRHEA IN (Continued)}

\section{SYMPTOMS, DIAGNOSIS}

Symptoms.-The manifestation- that accompany non-specific enteritis and colitis are numerous and extremely variable in a series of cases dependent $(a)$ upon whether the affection is acute or chronic: (b) virulence of the inflammation: (c) cause of the disturbance: (d) disease complicating it: (e) condition of the patient's mind and nervous mechanism: ( $f$ ) part played by digestive disturbances: $(g)$ sequele; (h) location and extent of the catarrhal inflammation: and (i) other factors which at one time or another influence the symptomatology.

While the smptoms of acute and chronic enterocolitis are similar in some, they differ materially in other respects, but in both there is a variation in the accompanying manifestations when the inflammatory process is located in different segments of the small intestine and colon. Following a discussion of the usual symptoms of enterocolitis the disturbances which point to catarrh in the several segments of the gut (duodenitis, ileitis. colitis, sigmoiditis. and proctitis) will be pointed out. The author recognizes that intestinal catarrh may arise in and be confined to any and all parts of the small and large intestine, and he believes that in a goodly percentage of cases the inflammation involves at least a part of both the small gut and colon. and for this reason he considers it advisable to discuss the disturbances arising from the affections under the caption of enterocolitis. Lnquestionably, colitis frequently exists independently, but the probabilities are that enteritis does not exist for any great length of time, because the irritating discharges and poor digestion which accompany it must necessarily learl to an inflamed state of the colonic mucosa. owing to the fact that it is being constantly irritated by acrid discharges and coarse, undigested food remnants.

Acute enterocolitis occasionally comes on slowly, and the patient complains of anorexia, feeling of malaise. and abdominal discomfort, but generally there are no prodromata, and shortly following the onset of disease (which is sudden) the patient complains of severe sensations, of pressure and fulness in the abdomen, intestinal soreness and gurgling forborymus, loring, griping or colicky pains (which begin in the mesogastrium and radiate in all directions), diarrhea, sometimes natsea and pain, loss of appetite, and a temperature of $\mathrm{IOI} \mathrm{I}^{\circ} \mathrm{F}$. in mild and $103^{\circ}$ to $\mathrm{IO}^{\circ} \mathrm{F}$. in severe cases. The characteristic manifestations are the slightly griping or the colicky pains and diarrhea, but the former gradually or inmediately subside following 
the evacuations, which are frequently explosize in character. Often the movements closely follow one mpon the other, and then the patient has a temporary respite from diarrhea and pain until the next crisis. 'This is the case in catarrh of the small gut, but not in colitis, where the movements are not always preceded ly griping, occur at more regular intervals, and ustally are not of the explosive variety. When the upper is aflected and the lower intestine remains normal, the patient may void considerable mucus, but diarrhea is slight or absent, and the dejecta is semisolicl or firm, because the fluid feces are hurried through the small gut into the colon by almormal peristalsis, and remain there long enough for their watery constituent to be partially or completely absorbed. However, in the arerage case of acute enterocolitis, cliarthea is marked, and the number of evacuations vary from two or three to fifteen or lwenty daily, and in the beginning are composed of feculent masses, and later of a watery or highly irritating fluid (containing feces, mucus, and serum) which scalds the rectum and induces tenesmus.

Diarrhea here is attributable to $(a)$ peristalsis, which rushes the umprepared chyme and feces through the gut so quickly that the water cannot be taken up: (b) impairment of the absorptive mechanism of the colon: (c) serons transudation into the bowel; $(d)$ increased activity of the secretory glands: (e) mental impressions and neuroses which inlluence the intestinal motor and secretory nerves: $(f)$ gastrogenic, hepalogenic, pancreatogenic, and enterogenic disturbances which unbalance the gastro-intestinal juices; $(g)$ toxins which act constitutionally or upon the bowel; and $(h)$ anything which causes irritation of the intestinal mucosa. In these cases the quantity of the discharge is out of proportion to the food ingested, and the first stools have a brownish tint, but later are of a pale-yellow color and foul smelling. Ordinarily, diarrhea is more persistent, and the number of evacnations greater $i n$ colitis than enteritis. The odor of the dejecta may be normal, absent, or very offensive, and the reaction is acid or usually alkaline, according to the food consumed.

In some instances the fluid movements are preceded or accompanied by collections or evacuations of scybalat, the expulsion of which is followed by marked relief.

Macroscopic and microscopic examination of the dejecta in a series of cases will show that they are composed of fecal matter, mucus, pus, blood, epithelium, leukocytes, calcium phosphates, oxalates, fat, undigested food remnants (muscle-filers, albuminous particles, catsein, undigested fat, particles of starch, etc.), and living or dead micro-organisms, separately or together, according to the diet, cause of the inflammation, or changes taking place in the bowel.

Duodenal tubes and buckets are also useful diagnostic adjurants in this class of cases.

Although constipation and diarrhea are usually considered as opposite manifestations, both are frequenty symptoms of enterocolitis, and more particularly eatarrh of the large intestine. Sometimes one 
and sometimes the other dominates the condition, but more often there are alternating loose movements and costiveness, or diarrhea may supervene for several dars or weeks and then give way to constipation. In this class of cases constipation or obstipation may ensue when the intestine becomes blocked by an enterolith or foreign body, and when the bowel contains an irritant of any kind which induces hypertonus of the muscular tunic and causes enterospasm (spastic constipation) which partially or completely blocks the gut. The modus operandi by means of which frequent fluid stools are incited has already been given.

Catarrhal constipation is frequently a troublesome symptom in the presence of ileocecal catarrh, which renders the valve irritable and causes it to contract at irregular intervals or remain closed for shorter or longer periods, and in membranous enteritis characterized by dried shreds or sheets of mucus or tubular casts of the bowel, which obstruct the intestine or cause its musculature to contract and excite spastic constipation until this membrane has been expelled.

Enterospasm may involve the small intestine and induce delayed evacuations, but most frequently occurs in the colon, being induced occasionally by indigestible food remnants, such as cucumbers, peas, sweet potatoes, etc., which irritate the gut and give rise to tetanus, and bearing-down, labor-like pains.

Feier may not appear, be slight, or, in exceptional cases, where the inflammation is virulent, the temperature may rise several degrees, but fever is more regularly present, higher, and persistent in infectious than in catarrbal enterocolitis. When nausea and somiting are annoying there is usually some complicating gastrogenic disturbance; steatorrhed indicates that the pancreatic secretion is deficient or absent, and, when biliary secretion is marked, Gmelin's reaction for unchanged bile-pigment is positive. As has been previously inclicated, pain may be manifest as a discomfort, or be of the boring, colicky variety, accompanied by an intense desire to evacuate the bowel, and there is also intestinal tenderness upon pressure.

The amount and character of the mucus in the stools is dependent upon the extent and virulence of the inflammation and nature of the irritant causing it, but the quantity of accompanving pus and blood is slight when erosions and ulcers are few, and considerable when they are large and numerous. 1)ark, clotted blood points to lesions in the small intestine or upper colon; bright fresh blood indicates bleeding from the rectum (though hoth are rare).

In hemorrhagic colitis bleeding is unimportant when it comes from petechial spots, but is dangerous when the mucosa is spongy and raw over extensive areas.

When acute enteritis or enterocolitis is of short duration or moderately severe it does not impair the general health, but when the inflammation is virulent or long drawn out, metabolism is disturbed, malnutrition takes place, the patient becomes exhausted, melancholy, anemic, languid, indifferent to social and business duties, has no ap- 
petite, complains of headache, loses weight, and suffers from a poor circulation and muscular weakness.

Chronic Catarrhal Enteritis, Colitis, and Enterocolitis. (hronic intestinal catarrh nay he primary and a continuation of the acute form, or secondary to the infectious buwel di-eaten. constitutional

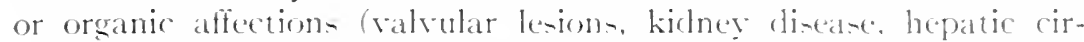
rhosis, cancer, stricture, etc.), or one of the many etiolugic factors in catarrh of the bowel; conseguently, the manifestations of the clisease vary greatly in different cases. As a rule, howerer, diurrhed is the dominating symptom. and frecplently it is the only one tos sugerest the nature of the trouble. By the time catarrhal inflammation has become chronic it is usually centered in the colson, the mucona of which has beeome irritable, intlamed, and occasionally eroded, or ulcerated lesion (from mixed infection, etc.). which tend to augment the frequeney and fludity of the morements, because they stimulate mucous glands to oversecrete and expose terminal nerve filament- to the irritants within the bowel. which excite stimuli that bring about frequent strong and prolonged peristaltic contractions and tead to expulsion of the feces and discharge. Those interented in a further study of the symptoms of diarrhea and the changes which take place in the stools uncler varying conditions in chronic inte-tinal catarrh. are referred to Nothnagel.' who has given a clear description of them.

When the inflammation is active at a given point, or some irritant or foreign body becomes lodged and excites simultaneous contraction of the circular and longitudinal muscular fibers, they cause spantic constipation (enterospasm!. Which may continue for several hours or days, or may alternate with diarrhea. While the destruction of tistue is very much les marked in chronic catarrhal than in infectious enterocolitis, it may take place, and, whing to the erosions and ulcer. intestinal auto-intoxication. with its train of smptoms. bleeding, and suppurative follicular colitis. may be present, and submucoss abscesese and fistula have been known to form in neglected cases.

Intestinal tenderness is characteristic, and aldominal pain and cramps may occur. but lese frecpuently and in a milder form thas in acute enterocolitis, and it is notable that suffering is create-t sereral hours after meals, particularly when the patient has been indi-creet in diex. Inder such circumstances, in ardelition to pain there are sensations of aludominal distention (b) hating and an imperative desire for an evacuation, which crmes shortly and relieses the patient. When gases collect in enormous quantities the patient become- uneary and suffers from lorloorymi, colicky pains, shortness of breath, palpitation. cereloral congestion, vertigo, or angina pectoris. which prevail until the gas is bekhed up or exapes through the anus.

When catarrhal inflammation is concentraterl in the sigmoid flexure or rectum (particularly') a con-tant de-ire for an evacuation. intense straining during the same, and hurning and dull aching pain in the lower bowel after stosl dominate other symptoms.

${ }^{1}$ Diseases of the Intertine and Peritoneum. American ed. I00; , 1\% I03. 
Chronic catarrh is a persistent affection, and requires months or vears to cure: though when properly treated the variety and intensity of the manifestations are subject to marked changes. Victims of it sometimes complain bitterly of eliarrhea and mucous discharges for a time, and then for a short or long interval these symptoms are quiescent, during which tme the patient leads a comfortable existence and is able to attend to his duties. The state of the bowel and depleted condition of the subject makes him an easy. victim to infections discases of the intestine and some organic and constitutional ailments. Among the more common complications are appendicitis, rheumatism, refiex phenomena, intestinal lithiasis, dyspepsia, indicanuria. and pneumonia. When catarrhal enterocolitis persints for a very long time and is cured. the functional disturbances dependent upon it ceast. but the bowel never becomes perfectly normal because the mucous membrane remains thickened, spongy, dotted over with excrescences or scars, and the glands are hypersensitive or atrophied, and the gut may be strictured, hypertrophied or contracted, and tube-like.

The objectize symptoms most frequently present in cases of enterocolitis are the diarrhea and constanty changing character of the stools, gas distention, tenderness on palpation, pot-belly when enteroptosis is a factor, and now and then splashing sounds after eating or drinking when the abdomen is tapped suddenly one or more times.

Diagnosis.-In suspected cases of enteritis or colitis it is advisable to ascertain if the condition started as such or followed some other disease - tubercular, syphilitic, helminthic, entamebic, or bacillary colitis: enterogenic, gastrogenic, hepatogenic, or pancreatogenic dyspnea; circulatory obstruction, neuroses. etc.-thoroughly examine and analyze the gastric juice and feces. for by so doing many doubtful points can be cleared up.

By studying the pathology and analyzing the symptomatology outlined above indicative of catarrhal inflammation. it is comparatively easy to diagnose simple enteritis, colitis, or enterocolitis, and to determine whether or not the affection is acute or chronic.

It is much more difficult, however. to define the limitations of the disease and to isolate the part or segment of the small or large intestine when the catarrhal inflammation does not extensively involve the upper or lower bowel, or both, and ret this is essential if the patient is to be rationally treated. Owing to the diagnostic importance of determining the exact location of the disease when it is circumscribed. the author will first outline the best methods of doing this, and will then call attention to some esential features useful in making a differential diagnosis between intestinal catarrh and other affections the manifestations of which often simulate those of enteritis and colitis.

Localization of Intestinal Catarrh (Enteritis, Colitis, Enterocolitis, Duodenitis, Jejunitis, Ileitis, Cecitis, Sigmoiditis, and Proctitis).-When there is a gastro-enteritis or colitis the diagnosis is fairly easy when the stomach and intestinal contents are examined 
following test-meals by a summarization of their chicf manifestations. In case the catarrh is of gastrosenic origin, and particularly when it results from achylia gastrica, periodic diarrhea ensnes. Ciastrogenic dyspepsia most freguently excites catarrl in the upper small intestine, and undigested food remnants appear in the dejecta shortly thereafter.

The localization of intestinal catarrh is of en very difficult because the symptoms induced by inflammation of the entire and individual sections of the gut are mainly the same, and because disturbances from the disease located in the same portion of the gut in different cases vary in accordance with the exciting cause, virulence of the inflammation, and the part, if any, played by complicating ailments. At times. When the history has been taken into consideration, the patient studied for a sufficient length of time, the stomach and bowel contents repeatedly inspected and analyzed, macroscopically, microscopically, before and following the administration of test-meals, and the sufferer subjected to a thorough physical examination, one can determine if a particular part or several segments of the intestine are involved in the catarrhal process.

Since the therapeutics of catarrhal duodenitis, jejunitis, ileitis, ileocecitis, typhlitis, colitis, sigmoiditis, and proctitis vary, the author will briefly call attention to their peculiarities, that they may be individually diagnosed, after he has mentioned the essential points of differentiation between enteritis and colitis.

Catarrh of the small intestine occurs very much less frequently than catarrhal colitis, and in the former the inflammation in most cases, sooner or later, extends to the lower bowel, but non-specific colonic catarrh seldom passes upward to the ilem or higher.

Enteritis (Catarrh of the Small Intestine).-One of the chief characteristics of enteritis is the rumbling heard and felt by palpation in the intestinal coils of the right iliac fossa. There is increased fermentation and a multiplication of the bacteria, and likewise an augmentation of the gases (carbonic, hydrogen sulphid, etc.) which stimulate peristalsis.

Catarrh of the small intestine does not necessarily influence either the stools or act of defecation, and in a series of cases the evacuations may be normal, slighty increased, or delayed, particularly when the inflammation is confined to the upper segment. Owing, howerer, to the participation of gastrogenic or local disturbances, dyspepsia, abdominal distention, soreness on pressure, belching of gases, and boring pains in the umbilical region are frequent manifestations. When the inflammatory process extensively involves the mucosa of the small gut, diarthea is the dominating symptom, because peristalsis is ahnormally stimulated, the secretory function of the bowel is enhanced, and chyme improperly prepared in the jejunum passes into, irritates, and produces undue activity of the colon. I inder such circumstances the evacuations frequenty have an acid reation, the odor of volatile acids, are light brown, yellowish or greenish in color 
(in presence of hydrobilirubin), foamy, mixed with gas, contain unchanged bile-pigment, muscle-fibers, fat globules, epithelia, etc., and when there is a chronic catarrh of the small gut, Rosenheim's testdiet will demonstrate the presence of well-preserved starch bodies without sheaths (which easily give the iodin reaction) or remnants of fat (flakes, crystals, drops). According to Rosenheim, these findings of disturbed assimilation point to enteritis only when they are demonstrable in formed stools, for neurogenic and other severe diarrheas may be associated with them. Mucus is often absent from the dejecta in catarrh of the upper small intestine, but is more frequently present when the inflammatory process involves the ileum.

Tenderness upon pressure in the region of the mesogastrium and colicky pains are more aggravated in enteritis than in colitis.

Circumscribed pain and tenderness in the region of the appendix, cecum, and rectum point to catarrh in these localities, but under other circumstances are unreliable as diagnostic signs of localized catarrh, because they are so disseminated that the patient cannot locate them, but pain at and centered about the umbilicus and tenderness upon pressure point to enteritis, and when the same manifestations are obtained in the right and left iliac regions the patient probably suffers from enterocolitis.

Nothnagel maintains that yellow mucous granules in the stools are characteristic of catarrh in the small bowel or upper colon, but this is denied by Boas and A. Schmidt.

Urinary examination is not a reliable diagnostic aid in cases of intestinal catarrh, though now and then albuminuria and cylindruria (Fischl) are complications, hyaline casts have appeared in the urine, and indican and the ethylsulphuric acids so common in many bowel ailments are frequently denonstrable in this condition. Where the stools give an acid reaction it indicates a catarrhal state of the small intestine.

Duodenitis.-Catarrh limited to the duodenum is characterized by icterus incident to swelling or blocking of the ductus choledochus, but in the absence of this manifestation a diagnosis of duodenitis must be made with reservation. In the presence of icterus, if localized pain and tenderness on pressure above the umbilicus, gastric disturbances, constipation alone or alternating with diarrhea, and extensive cutaneous burns are manifest, one is justified in concluding that he has a catarrhal duodenitis to deal with. This condition has been, but need not be, mistaken for typhlitis and appendicitis, because the pain consequent upon the latter is right sided. Microscopic and macroscopic mucus in the dejecta also point to this affection, as do undigested food remnants under the above circumstances.

Jejunitis and Ileitis.-A catarrhal inflammation of the jejunum is impossible, and catarrl of the ileum is extremely difficult to diagnose except by postmortem examination. Since there is no means of differentiating between catarhal inflammation in these segments of the 
gut, and ileitis is more frequent and troublesome, only the differentiating points of the latter will be discussed. In the jejunum or in catarrh of the ileum (particularly the upper part) bile-pigment is frequently present and changes the mucus to an orange or greenishyellow hue, and also discolors the epithelium, round cells, and fat. The stools have an acid reaction and contain more or less mucus mixed with feces. The amount and character of the former and whether or not the stools are softened, fluid, and freefuent depends upon the extent of the catarrhal process and the degree of peristalsis and glandular activity caused by it. When a major portion or the entire ileum and jejunmm are affected, borborysmi, colicky pains. intestinal distention, gurgling, occasional bloating, and soreness or tenderness on pressure over the central portion of the abdomen are troublesome manifestations. Chief reliance, however. should be placed upon a careful macroscopic and microscopic examination of the dejecta before and following test-meals. Frequently the feces remain firm or semisolid when the inflammation is confined to the ileum; most often, however, there is an enterocolitis, the dominant symptom of which is daily or periodic diarrhea. Diminutive and larger undigested food remnants, such as starch. Fat. and musclefibers, point to enterogenic dyspepsia and ileitis, as does undecomposed bile-pigment, demonstrable by Gmelin's test. Nothnagel lays great stress upon the bile-stained stools and very small, pigmented balls of mucus in this condition (ileitis). Here, as in catarrhal inflammation in other parts of the bowel, indicanuria is marked.

Colitis.-When attempting a diagnosis of catarrhal colitis it is well to bear in mind that the inflammatory process may be confined exclusively to the large bowel, in which case there are present welldefined manifestations and signs in the dejecta which greatly help one to identify it, or there may be an enterocolitis and accompanying manifestations that are confusing. In catarrhal colitis, pure and simple, gastrogenic disturbances are rarely present and undigested food remnants seldom appear in the stools. Slight. moderate. or exhausting diarrhea is the pathognomonic symptom of catarrhal colitis. and always prevails except when spastic constipation (enterospasm) is brought on by ulcers, inflamed areas, or collections of dried mucus which excite the simultancous contraction of the circular and longitudinal muscular layers of the bowel. This form of obstipation may alternate with diarrhea or dominate it for a period of several hours. days, or weeks, and is encountered more often in membranous than in catarrhal colitis. While these patients complain of soreness and tenderness on pressure, the discomfort is felt over the course of the colon and is not concentrated at the midabdominal region as in enteritis, and, though they suffer severely from colic, the pains are less frequent and intense than those which complicate catarrhat inflammation of the upper small intestine and enterocolitis. Borborygni and gurgling on palpation. common to enteritis of the duodenum, jejunum, and ileum, are rarely encountered when the inflammation is 
confines to the eolon. Patients afflicted with colitis often maintain a fuirly good appetite and digestion in contratistinction to enteritis, but they are often in a run-down condition because of the exhaustion consequent upon frequent and fluid movements and complicating mixed infection. Sufferers from colitis are often despondent and nervous on account of the persistent diarrhea, but depression and nervous phenomena are more frepuent and exaggerated in enterocolitis, because here, in addition to suffering from auto-intoxication, the patient worries because he knows if he eats it will aggravate his condition. Naturally, when the catarrhal process is diffused, and when the mucosa is eroded or ulcerated, the accompanying manifestations are augmented: the diarrhea, because of increased exudation, peristalsis, and secretion; soreness and pain, owing to the lesions; muscular spasms. from exposure of terminal nerve filaments to irritation, and the stools more watery; the absorption of fluids in the colon has been diminished or arrested. When the disease is influenced by neuroses or psychic impulses the daily evacuations are increased, are sanguineous in character, and are roided in the early morning (in rapid succession), and later in the day when the bowel is inflamed or ulcerated.

Mucus, in small or large amounts, may be seen, macroscopically, in the dejecta, in the form of jelly, threads, whitish balls, membranous casts, or as a glistening covering for scybalæ or lumpy feces, when the catarrhal process involves the transverse and descending colons, sigmoid flexure, and rectum. The quantity of mucus secreted in the upper colon and small intestine under the same conditions is considerably less, and usually cannot be seen with the naked eye, but appears as threads or brown or greenish grains under the microscope. Again, mucus intimately mixes with feces in upper intestinal catarrh, and appears free in the presence of sigmoiditis or proctitis. Pus, blood, burning pain, and tenesmus increase in amount or degree in proportion as the inflammatory process approaches the anus.

Percussion usually brings forth a distinctly tympanitic note, and by palpation the colon is often recognizable owing to its hyperplastic condition, distended state, or its knot-like feel when there is enterospasm. Deep palpation also elicits tenderness over the entire colon or an individual segment when the catarrh is circumscribed. When the transverse colon is filled with gas it raises upward and lessens hepatic dulness, and complete colonic distention interferes with respiration, disturbs the heart, and may cause angulation or twisting of the gut, localized pain, and obstruction at points where it is bound down.

Catarhal colitis is not accompanied by a material rise in temperature except when there is mixed infection, under which circumstances the temperature may be irregular, and vary from $100^{\circ}$ to $\mathrm{IOI}^{\circ} \mathrm{F}$. or rise to $103^{\circ}$ or $104^{\circ} \mathrm{F}$., when submucous or deeper abscesses form.

In concluding the discussion regarding the points of differentiation between enteritis and colitis it may be as well to mention that the 
latter runs a more regular course than the former, which is inclined to periodicity, so that the patient is fairly combortable and able to be about for a time, and then is confincel to his beel, owing to the intense suffering which prevalis doring the exacorbations or crisis.

When there is enterocolitis, diarrhea is a more marked srmptom than in catarrhal enteritis or colitis, and the majority of evacuations occur in the early morning, owing to fecal fermentation which takes place during the night.

Anders differentiates between enteric catarh and colic as follows:

EATERIC CATARRH.

Diarrhea is generally present.

Fever maty be slight or marked.

Pain is griping and followed by diarrheal stools.

Tenderness in the interval between pains.
COLIC.

Constipation is present.

Noferer.

Pain is colicky, nore severe, and is not followerl by diarrheal discharces.

No sensitiveness on palpation.

Ileocecal valve catarrh (catarth of Bauhin's valve) has been discussed as an entity hy some. but it is doubtful if it can be identified, for it is frequently associated with catarrhal inflammation of the ascending colon, the ceeum, or ileum.

The ileocecal valve is a favorite site for intestinal disease, particularly the infectious types (ameliasis, tuberculosis, typloid ferer, actinomycosis, etc.). The feces in ileocecal valve catarh are fluid and putrescent, mucus is present in the form of threads, and undigested food remnants do not appear. Patients thus afflicted complain of localized discomfort or pain and bloating, on account of which the affection has been mistaken for enteritis. Occasionally the evacuattions are irregular in character and either const ipation or diarrhea may prevail. Cecal gurgling is also indicative of catarrh at and about Batuhin's valie.

Typhlitis is accompanied by manifestations similar to those just mentioned, but constipation is a more frequent complication, and cecal gats distention is more marked and pressure over the head of the colon elicits decided tenderness or pain and slight gurgling.

Appendicitis (catarrhal) is accompanied hy harp, characteristic pain locaterl at McBurney's point, tenderness over the cecal region on pressure, rigidity of the aldominal muscles, and frequently coustipation, though in exceptional cases slight or severe diurthea may prevail.

As the inflammation beemes more marked, mucus collects within the appendix, the patient's suffering is intensifed, the local circulation is impaired, and soon perforation or gangrene ensues, conditions which leat to peritonitis, a rise in the temperature, and symptoms of collapse. In the chronic form the patient may recover from a severe altack and remain in comparatively good health for weeks, months, vears, or until another crisis sets in.

Sigmoiditis incluces the manifestations common 10 eatarr in other segments of the colon, but is distinguishable by the fulness 
apparent in the left iliac fossa, increased thickness of the gut, localized pain and soreness, and augmented evacuations.

Proctitis.-Catarhal inflammation of the rectum is typified by persistent diarrhea, incessant desire to empty the bowel, unrelieved feeling following the movements, occasional colicky pains in the sigmoid region, burning in the rectum, and numerous small evacuations, accompanied and followed by straining.

Small round, mucus-covered scybala are discharged from time to time, but usually mucus is not admixed with the feces, as in enteritis or colitis, but is evacuated free and in large amounts in the form of strings, casts, jelly-like masses, or a thin, glairy discharge. When constipation prevails, mucus may be evacuated, pure, ahead of the movement, or as a coating for the lowermost hardened end of the fecal mass. When the rectal mucosa is extensively inflamed, eroded, or ulcerated the levator ani and sphineter muscles spasmodically contract and greatly enhance the paticnt's suffering, under which circumstances either or both muscles clasp the finger like a strong rubber band when it is introduced into the rectum.

In these cases, when the inflammatory process is acute, the mucosa feels hot, swollen, and slippery. There is no difficulty in diagnosing acute and chronic sigmoiditis and proctitis, because the bowel can be accurately inspected through the sigmoidoscope or proctoscope.

A painstaking abdominal examination is essential in cases of enteritis and colitis, because in this way one can gain much information regarding the localization of painful and tender spots upon pressure, tumors, olstruction, the degree and location of gas accumulations, peristaltic wares, enterospasm, impacted fecal masses, splashing and gurgling sounds, and sometimes the limitation of the catarrhal inflammation or other abnormal condition which might cause, simulate, or complicate enterocolitis.

Mistaken diagnoses are common when this or infectious bowel diseases are located in the upper colon, cecum, or segments of the small intestine, but no one except the ignorant or careless should confuse enterocolitis, colonic infections, obstructive, or other lesions with each other when they are situated in the sigmoid flexure or rectum, because their nature, appearance, and exact location can be accurately determined by proctosigmoidoscopic inspection and digital examination of the lower bowel.

A close analytic, macroscopic, and microscopic study of the urine is useful, lut not always positively indicative of enterocolitis, though in characteristic cases it is hypertoxic and contains skatol, indol, indican, occasionally a slight amount of albumin, and the proportions of solids may vary when the temperature is high or the patient is excessively run down.

Blood examination is necessary, and will give some idea as to whether or not the patient is anemic, sulfering from a mixed infection, auto-intoxication, or peritoneal involvement. Aiter all, the chief diagnostic reliance in catarrh of the small in testine, colon, or individual 
segments of the gut is based upon repeated examinations of the stomach and bowel contents independently of and following Schmidt's or other test-meals, for in this way only can one form any idea as to whether the ingredients of the gastric juice and suceus entericus are properly balanced, if the feces contain entameba, bacilli, helmintles, or other infective agents, forcign bodlies, pus, blood, nucus, threats of tissue or undigested food remnants, the funding of which greatly aid in clearing up the diagnosis. The author will not further discusm the macroscopic and microscopic findings in the feces here becatuse this has been done in the symptomatology and more fully in (hapter II devoted to The Diagnosis of Diarrhea.

In this and simulative affections of the bowel Kuttuer lays considerable stress upon the color of the dejecta and summarizes his conclusions as follows, viz.:

\section{Ochcr-colored stools:}

In adults these stools suggest disturbances in the small bowel.

\section{Green stools:}

In adults these stools sugrest jejunal diarrhea.

In infants they are rlue to increase of alkali in the upper parts of the intestine. or to an oxyclizing ferment.

\section{Fermenting stools:}

These stools are usually light brown. foamy, and have an acid or cheesy odor. They generally indicate the milder intestinal disturbances.

\section{Putrefying stools:}

These stools are dark colored and indicate serious trouble, such as dysentery or carcinoma of the large intestine.

\section{Differential Diagnosis of Catarrhal Enteritis, Colitis, and Entero-} colitis.-Catarrh of the small and large intestine has been frequently confused with affections of the bowed which induce irregular or fluid movements and ailments of other organs which cause diarrhea. The following are the diseases most often mistaken for intestinal catarth, viz.: Ptomain-poisoning, typhoid fever, appendicitis, peritonitis, colic, infections diseases of the small intestine and colon, tuberctulosis. entamobiasis, hacillary dyentery, helminthiasis, lues, gonorrhea, amyloid degeneration, neirogenic diarrhea, membranous enterocolitis. intestinal nleers from burns, diseases of the pancreas and liver, gastrogenic and enterogenic dyspepsia, chronic coprostasis and cancer, benign tumors, stricture, and other forms of bowel olstruction.

Ptomain-poisoning can be differentiated from enteritis and colitis by the extreme suddentess of the attack. sererity of the simptoms (vomiting, pallor, diarrhea, tendency toward collapse, and monensciousness), taking a history, ascertaining if the patient has consumed unhealthy shell-fish, spoiled or infected meat, ice-cream, canned goots, or mushrooms, etc., and ly examining suspected food and the feces for bacilli and toxins that might canse the trouble.

Typhoid feer is distinguibhable by the rose-rash, dicrotic pulse, 
high and charateristic temperature-curve. stupor, enlarged spleen or liver. and Midal test.

Appendicitis comes on suddenly, is characterized by intense pain in the risht iliac region, rigidity of the adbominal musces. tenderness and pain upon presure over the cecal region, constipation, etc.

Peritonitis quickly lead= to general abdominal distention. pain and tendernes- upon presure, constipation. marked rise in temperature, fart and thread-like pulse, facial expresion indicative of suffering and anxiety. tympany, and mu-cular rigidity.

Colic is usually traceable to gall-biadder or duct disturbances, or more frequently wo the eating of indigestible food or backing up of gate- proximal to a fecal impaction.

Inferions diseases of the inte-tine. such as tubercular, syphilitic. gonorrheal. entamebic, helminthic, balantidic, hacillary and coccidic colitis. etc.. are cunfined chichly to the colon, and can be accurately diagnoed by means of fecal and blood examinations. which reveal the specific etiolusic agent-tubercle. Shiga, Flexner, strong. Hiss, or other hacilli, round-, tape-, or hook-worms or their eggs, coccidia, gonococci, spirochete- or other microscopic organism-responsible for the infection. Infections enteritis or colitis is further distinguishable by obstinate diarritea, sallow complexion, serious digestive disturbances, tenesmus. and the frequent abundant discharge of pus. blood. or mueus. and their lack of response to the usual dietary and medicinal treatment. When tuberculosis is suspected. examination of the lungs is imperative, because this ugpe of enteritis or colitis is nearly always secondary and the infection is caused by hacilli which have been coushed up and wallowed. Patients afflicted with intestinal infections are more weakened in mind and body than those afflicted with simple or catarrhal enterocolitis. and frequenty suffer from abscesses in local or distant parts wing to extensive ulceration and the mixed infection which ensue once continuity of the mucosa is broken.

Amyloid degeneration in the later stages is sometimes accompanied by manife-tations =imilar to those of an intertinal catarrh. but it affect- the patient more generally, and the history will indicate that other viscera were involved prior to the appearance of troublesome inte-tinal simptoms.

- Veurogenic diarhea is mistaken for this condition more often than the above affections, but it differentiation is not difficult because it occurs in nervous individuals easily influenced by psychic impulses. the stools are -anguineous, take place in the early morning, occur in rapidsuccesion, contain no blood or mucus. are passed without pain or tenesmu-, and the diarrhea can be improved or controlled hy psychotherapy and physical therapeutic measure better than by medication, dieting, and enteroclysis.

Mempanous colitis has been mistaken for catarrhal enteritis or coliti-, although there is little reason for it hecause spastic constipation is alway - a prominent symptom. diarrhea is seldom if ever a complication, and when it occurs in extremely nervous individuals or persons 
suffering from angulation, twisting, adhesions, or blocking of the gut, and, further, because the mucosa is less often congested and mucus visibly appears in the stools periodically in the form of long strips, twisted strings, large jelly-like matseses or casts of the lowel.

Enteritis consefuent upon cutaneous burns can be diagnosed hy obtaining a history of the injury.

Malignant and benign growths which obstruct the intestine and lead to specific or stercoral inflammation and ulceration frepuently catuse diarrbea, gas distention, digestive disturlanees, and mucus, pus, and blood in the dejecta; but their diagnosis is not difficule when high up, and is easy when the tumor is located in the sigmoid flexure or reetum, and can be inspected through the signoidoscope or felt with the finger. In the presence of malignancy the patient is cachectic, rapidly loses weight, and the evacuations are frequent and offensive.

Tumors and intestinal adhesions, angulation, twisting, iniagination, and foreign bodies of the colon can usually be diagnosed by locating tender and painful spots, noting the distended gut above and empty gut below the block, palpating for swellings and ascertaining if diarrhea, which may alternate with constipation, was preceded by dificulty in securing daily evacuations. Frequent stools, accompanied by marked straining and no relief to the patient, are indicative of olsstructive lesions situated in the sigmoid flexure or rectum or at the anus.

Pancreatic bowel disturbances are rare, and can usually be differentiated from catarrhal enterocolitis by fecal exanination, which shows an abmormal amount of fat in the dejecta.

Gastrogenic and enterogenic dyspepsia may simulate enterocolitis, cause or be associated with it, and often a correct diagnosis cannot be arrived at until the stomach and intestinal contents have been repeatedly analyzed and studied macroscopically, microscopically, and chemically to determine if there is subacidity or achylia gastrica, hyperacidity, atony or motor insufficiency, or abnormality in the ratio of the ingredients composing the succus entericus.

Finally, when attempting a differential diagnosis of catarrhal enteritis, colitis, or enterocolitis it is well to exclude organic diseases of the lungs, heart, and liver because in some instances, owing to the venous olstruction which accompanies them, the blood-serum exudes into the bowel and increases the fluidity of the feces and number of daily eracuations. 


\section{CHAPTER XVIII}

\section{ENTERITIS, COLITIS, ENTEROCOLITIS (NON-SPECIFIC (?) INTESTINAL CATARRH), DIARRHEA IN (Concluded)}

\section{TREATMENT, PROGNOSIS}

THE treatment of inflammatory conditions involving the small and large intestine, as well as their individual segments, requires considerable ingenuity and patience to obtain a satisfactory result. Recently, because of our better understanding of the etiology of this and allied affections, considerable progress has been made in the treatment of enteritis and colitis, but much remains to be done before the treatment can be relied upon to control or arrest catarrhal enterocolitis in a reasonable time without leaving sequelas.

It is conceded that acute catarrh of the small intestine, colon, or both can, in the majority of instances, be quickly and satisfactorily relieved; chronic enteritis and colitis cannot, because in this class of cases the inflammation has existed for a long time and organic changes have taken place in the mucosa and, perhaps, other of the intestinal tunics to such an extent that it is difficult or impossible to overcome them, even though the progress of the catarrhal inflammation has been checked. Owing to this abnormal state the intestine remains irritable after the troublesome manifestations of enterocolitis have been relieved, and the patient may be subjected to relapse in case he becomes indiscrete as to his diet, consumes alcoholic stimulants, becomes overactive, or subjects himself to exposure during inclement weather. To this extent the prognosis of chronic enteritis and colitis is bad, but it should be remembered that catarrhal symptoms are controllable and the disease is curable in many instances, and in others it can be regulated so that the patient remains comfortable and can be self-supporting if he will follow instructions.

Cases of acute and chronic enteritis and colitis require individual consideration from a therapentic standpoint, because the diet and remedial measures suitable for one are unfit for another on account of the varied etiology of the disease. The author will follow the usual custom of separately discussing the therapeutics of acute and chronic catarrhal inflammation of the intestine, because the methods of handling them differ and the treatment of the former is simple in comparison with the latter.

Acute Enteritis, Colitis, and Enterocolitis.-Medical treatment is not required in the handling of acute catarrhal inflammation of the bowel in many instances, because the attack is of short duration and subsides spontaneously, or the patient can be relieved by having him 
rest in bed and restrict his diet to thid and non-irritating nourishment, and by alleviating his discomfort and pain through the application of turpentine stupes to the alselomen.

Before instituting the tratmont in this class of cases it must first be ascertained whether or not the gut is infected, the liver or pancreas are abnormal, the bowel manifestations are modified by malaria or general affections, and if there is gastrogenic or enterogenic dyspepsia or other ailment which would cause or augment intestinal catarrh. When such complications arise they must either be corrected before or receive due consideration while the enteritis and colitis are being cared for.

In cases of enterocolitis, pure and simpte prophylactic measures, such as removing irritating toxins, fecal impactions, foreign bodies from the bowel, protecting the patient against exposure, rest in bed, and control of the diet, are indicated.

Rest in bed is of the greatest importance during acute crises, particularly when the temperature is high or diarrhea and cramps are distressing, but when these manifestations subside the patient may be permitted to be up and around during his convalescence.

Control of the diet must not be overlooked in acute enteritis and colitis, since indiscretions as regards the amount and character of the food eaten invariably aggravate the catarrhal intlammation. The preliminary dict should be sparing, simple, and non-irritating, and composed mainly of hot black peppermint or camomile tea, cognac, cocoa, strained gruels, and nourishing soups. Later, as the soreness, pain, and diarrhea become less annoying, koumiss, zoölak, the Metchnikoff sour milk, barley, rice, cereals, bread and butter, scraped meats, soft-boiled eggs, and baked or mashed potatoes may be permitted, and after a few days, if the patient continues to improve, pigeon, chicken, and afterward real and other meats may be eaten. During the attacks, meat, raw fruil, green vegetables, alcohol, cold drinks and ice-cream, cucumbers, fat, acids, and sweets are contra-indicated, and the patient should be cautioned against fasts and overeating.

Dietary indiscretions frequenty cause a relapse, and for this reason it is advisable for the patient to guard his diet for a few lays, weeks, or until he has completely recovered.

'The medical treatment of enterocolitis is largely symptomatic, and consists chietly in prescrilning remedies which will (a) free the bowel of toxins, scybala, and other irritating substances; (b) relieve enterospasm and pain, and (c) diminish the frequency and fluidity of the stools.

In the preliminary treatment calomel should be administered in divided doses, and in an amount varying from 3 to $10 \mathrm{gr}$. (0.2 0.6) because of its cathartic and antiseptic effect, but in the alsence of biliousness, castor oil, $5 \mathrm{j}$ to ij $(30-60)$, may be substituted for it. Where purging of the bowel is desirable, Epsom or Cilauber's salls, z $\mathrm{j}$ to $\mathrm{ij}(3060)$, or a saline mineral water (Carabana, Hunyadi, Apenta, etc.) should be prescribed as often as indicated. 
In the average case belladonna is preferable to opium and other drugs for the relief of discomfort and pain, because in enterocolitis they arise mainly from cramps and the soreness consequent upon them, a conclition for which the tincture of belladonna, np Io (0.60), or atropin, $\frac{1}{1} \bar{g}, \mathrm{gr}$. (0.0006), are ideal, because they produce complete relaxation of the gastro-intestinal musculature when administered three or four times daily. The benefit derived from these agents can be greatly enhanced by keeping the patient quiet in bed, applying hot abdominal stupes, and administering hot colonic saline enemata, adjuncts which should be discontinued as soon as relief is obtained. In the presence of intense pain or persistent diarrhea, when belladonna fails to meet the indications, tincture of opium, me Io (0.60), or codein, gr. $\frac{1}{3}$ to $\frac{1}{2}(0.02-0.03)$, every three hours, will bring prompt relief and enable the patient to sleep. Opiates are invariably contra-indicated in the beginning, because they arrest peristalsis and frequently prevent the expulsion of toxins or other substances which are irritating the intestinal mucosa.

In extremely aggravated cases, where the bowel is highly inflamed or eroded, and pain and diarrhea are troublesome, temporary relief may be secured through the agency of salol, guaiacol, bismuth, tannalbin, chalk, beta-naphthol, or other antiseptic, soothing, and astringent remedies in 5- or $10-g r$. doses $(0.30-0.60)$, three or four times daily, alone or in combination with belladonna or opium.

Enemata or enteroclysis are often beneficial, but occasionally they induce considerable pain and are promptly expelled. The irrigants which have proved most useful in the author's practice are normal saline and ichthyol, protargol, argyrol, and permanganate solutions (I: 2000), administered through a return-flow tube or a fountain-syringe when they are to be retained. The number and amount of the irrigations require to be varied in accordance with the extent and severity of the inflammation. Often slippery elm or oatmeal water and infusions of camomile or flaxseed tea are more soothing and give better results than the above irrigations.

Treatment of Chronic Enterocolitis (Enteritis, Colitis).- The therapentics required here depend upon the chronicity of the disease, dominant symptoms, infection, and the part played by other ailments. First of all, the hygienic condition of the sufferer should be improved as much as possible. Complete or temporary rest in bed is advisable, since it secures inactivity of the mind and body; and this class of patients should avoid violent exercise, particularly during the heat of the day, exposure to inclement weather, irregular hours, rapid eating or overfeeding, partaking of intoxicating drinks, attending to business or social duties which excite or make them nervous, and drinking unboiled water that might be infected with entamebx, dysenteric bacilli, or helminths, etc.

Individuals suffering from chronic enterocolitis should guard their diet closely and, so far as practicable, carry out the dietary suggestions made below. 
Dietetic Treatment.-The dietary in this class of cases requires frequent changing to meet the indieations, both in reference to the amount and articles of foos composing it. While it is arlvisable to closely restrict the diet during times when the intertine in extremely irritalse and the patient suffers sererely from diarbeat and pain. it is mot good reatment to sarve thene patient-or limit them to a particular variety of foud rlurine the interval hetwen the erises. because when this is clone the lon in ribality will oxererene the lenefits derived from other sources. Many inclividuat afflicterl with chronic colitis and enteroeolitis imprese fater when permited atmost at full diet, foods known to disagree with them are excluelect, and oils, medication, and irrientions are emplosed to minimize irritation and heal lesoms within the bowel. In no cale should a diet be indugurated until after the gastro-intertinal contents have been repeatedly examined following schmidt's test-meals, and, under all circumstance- when instituted it should be mate as bland and non-irritating ats po-ible. and contain a liberal amount of easily absorbable nutriment.

Permitted Foods and Drinks.-During seiere attacks the cliet thould be closely restricted as regards the amount and character of the fords. and only those which are not irritating and leave little residue. such as mourishing soups. peppermint and camomile tea, barley- and ricewater, cocoa, and strained gruels, should be consumed. Milk aterilizeel, boiled, or with lime-water) in suitable cases is an aclvisable adjunct to the treatment, but it is sometimesobjectionahle, beeause it may" agree with one and aggravate the condition in another patient.

In mild or subacute cases of catarrhal colitis, in addition to the above, eggs (soft boiled or scrambled). gluten preparatitns, gruets. rice, cereals, arrowront, custards, sweetbreads, hrains, strained meat soups, extracts or jellies. scraped or lean roast beef, selecterd fish. milk if it agrees, chicken, vegetable pureés. macaroni, sago. fresh butter, toasted and stale bread, houmis, matzoön. zoölak. sour milk made from Bacillus bulgaricus, weak tea, and cocoa may le taken in reasonable amounts.

In chronic colitis, where the patient suffers but slightly from cliarrhea or cramps except. during exacerbations, a more liberal cliet. including meats and most vexetables, maly be permitted.

Patients afflicted with catarrhal inflammation are better when they eat oftener and consume a. smaller amount at each meal, and less meat should be allowed when the patient suffers from indicanuria, and fewer vegetables when fermentation is active. Milk slould be prescribed if feasible hecaune it is nourishing. helps to control the intertinal flora. When cooked with salieylic acid creaty diminishes intertinal bacteria, and the fat from it is superior to that olstainerl from vegetables. Lactolucillin and sour milk probluced lex means of the Bacillus bulgaricus are frectuenty lut not always uneful in this clatss of cases, becante they tered to displace the colon hacillus. Schmidt holds that the latter can elecompose starch and allumin, and recommends pure carbohydrates when putrefaction predominater. anel 
albumin when fermentation is excessive. The value of the sour milk treatment can be augmented by employing sugar of milk with it.

A prolonged cereal diet is often oljectionable. since it may cause or ageravate diarrhea in patients having hyperchlorhydria.

Theat-juices, beef-tea, and allumin preparations favor the formation of alhumoses, and, according to schmidt, are indicated in pancreatic insufficiency, as evidenced by undigested meat-fibers in the stool. When vegetable remnant-, cellulose, etc., appear in the dejecta, they indicate enterogenic dyspepsia with fermentation; and starchy foods, barring wheat flour, sago, macaroni, toast, farina, and arrowroot. which are the least harmful, should be discontinued or selected with care.

In order that the inexperienced practitioner may obtain a practical idea as to the best manner of arranging the diet in cases of enterocolitis complicated by diarthea, the author has appended the list of Cohnheim, which is a very good one:

7.00 A. M.: Mineral water. hot, and taken in small doses of ( $2 \frac{1}{2}$ to $5 \mathrm{oz} .-75^{-}$ I 50 c.c.l. The choice of the water depends upon the state of the gastric seretions.

7.30 A. M.: Eichel cocoa (two teaspooniuls to a cup) in water. and toasted white bread and butter.

10.00 A. M.: A cereal soup with butter. toast with butter. eggs. and scraped ham. I.00 P. M.: Broth rith arits, noodles. macaroni. and white meat: in mild cases, regetable purées and one glassiul of blueberry wine.

4.00 P. 3.: Same as -.30 1. M.

6.00 P. 1.: Nineral water.

7.00 to 5.00 P. $3 .:$ Tea with red wine or blueberry wine, toast, butter, and cold white meat.

9.00 to ro.00 P. 3.: A cupiul oi hot peppermint-tea.

In mild cases. when the stool is of a pulpy consistency, or after improvement in severe cases, white bread, carrots. filet, and baked fish may be allowed.

Contra-indicated Foods and Drinks.-Always during acute crises and usually in subacute and chronic colitis the following foods should be interdicted or cautiously permitted, particularly when diarrhea and pain are persistent: Raw fruits foranges, pears, dates, figs, bananas, and apples): salads: vegetables (peas, beans, celery, cabbage, turnips, caulitlower, radishes, green corn, mushrooms, carrots, onions. cucumbers. potatoes, etc.), particularly those which are rich in cellulose or faror intestinal fermentation and putrefaction; spices, salt; pastries, rye, and newly haked bread containing yeast, sugar, organic acids. alcohol (beer, ale. whisky, and sour wines): strong tea and coffee, cold and carlonatert water, lemonade, ice-cream, fats, smoked and insufficiently cooked meat and fish, fried and hard-boiled eggs, shell-fi-h, erary, and starchen.

Massage and vibration are useful adjuvants in the treatment of chronic colitis complicated by atrophy or atony of the bowel, deficient intestinal motility and constipation, because they encourage glandular and muscular actirity, help to dislodge impactions, and propel the 
feces toward the anus. Iilectricity may be employed for similar purposes, using the faradic or, preferably, galvanic current (interrupted or continuous), and applying one electrode to the abdomen or spine and introducing the other into the bowel, which may or may not contain a normal saline solution to conduct the electricity to the upper colon.

Hydrotherapy is a more useful agent than the above physical measures, and the water may be used internally or in the form of showers, Scotch douches, or baths. Cold douches and baths produce a tonic effect upon the strong, owing to the remarkable cutaneous reaction which follows them, but are harmful to aeak subjects and debilitated patients because of the shock which follows their administration, and soothing and stimulating atam salt or needle-baths should be substituted for them. The Priessnitz wet-pack to the abdomen is universally recommended in the treatment of enterocolitis, because it strengthens the viscera and abdominal musculature, besides adding much to the patient's comfort. Cool water drinking is adrantageous in the presence of constipation, owing to its tonic effect upon the bowel, but the water should be drunk as hot as bearable when the intestine is irritable or the patient suffers from enterospasm or gaspains.

Mineral waters are frequently useful in the treatment of chronic colitis, but their employment must be modified to meet the indications in mild and severe types of colitis and in the presence of gastric derangements. They are often advertised as cures for intestinal catarrh by mineral springs companies when such is not the case. though often better results are obtained from them (in watering-places) because of the régime enforced upon the patient while there. When there is a deficiency of the ingredients composing the gastric juice, waters which contain sodium chlorid, such as Homburg. Miesbaden. Hawthorne, and Blue Lick Springs, are extremely helpful, particularly when employed with other therapeutic measures; but when the secretion of the stomach is normal, or there is hyperchlorhydria, Carlsbad, Marienbad, Vichy, or French Lick Springs water is indicated. These and similar mineral waters are more effective when taken upon an empty stomach and at the body or a warmer temperature, and should be administered in smaller amounts to depleted than to strong individuals. Waters of this class are contra-indicated in enterocolitis when diarrhea is persistent or the patient suffers from cramps, unless it is evident that the bowel contains some irritant (dried discharge, such as mucus, scybala, etc.) which they would expel; at other times they afford relief whether taken by mouth or used in the form of enemata or irrigations, but it is not known whether their beneficient action is due to their alkaline effect upon the mucosat or chemical changes which take place in the gastro-intestinal contents through their agency.

Medical Treatment. - Merlicines, except when intelligently used, in the treatment of chronic catarhal enterocolitis do a great deal more 
harm than good. Drugs should be employed for the relief of symptoms rather than cure of the disease, and always give better results when used in conjunction with the therapeutic measures previously outlined than when prescribed independently. When diarrhea and pain are persistent drugs are indicated when these symptoms cannot be overcome by rest, correcting the diet, and hot fomentations, but opiates and insoluble agents, like bismuth, should be cautiously administered, because the first may lead to the drug habit and the second to the formation of bismuth enteroliths, which sometimes remain in the bowel indefinitely and intensify the patient's suffering or cause obstruction, as has been witnessed by the author in a number of instances.

Where patients are very nervous or greatly depleted, Fowler's solution of arsenic, gtt. ij to $\mathrm{x}(0.12-0.60)$. Blaud's pills, carbonated guaiacol, gr. $x(0.60)$, or a reliable iron preparation should be included in the treatment to improve the patient's general condition.

Antiseptic, soothing, and astringent remedies which diminish the intestinal secretions, irritability of the mucosa, fermentation, putrefaction, and peristaltic activity, faror solidification of the feces and tend to minimize diarrhea and pain, such as bismuth preparations, gr. $\mathrm{x}$ to $\mathrm{xx}(0.6-1.3)$; uzara, gtt. $\mathrm{xxx}(2.0)$; creosote, gtt. $\mathrm{j}$ to $\mathrm{x}$ (0.06-0.60); beta-naphthol, gr. v (0.30); salol, gr. v to $\mathrm{x}(0.30-0.60)$; boric acid, gr. iv $(0.24)$; tannigen, tannalbin, or tamnopin, gr. $\mathrm{r}$ to $\mathrm{xv}$ (0.3-I.o); ichthalbin, gr. x $10 \mathrm{xx}(0.6-\mathbf{I} .3)$; fortoin, gr. iv (0.24), etc., administered three or more times daily, according to indications, do much toward relieving the patient's distress, quieting the inflammatory process and healing ulcers within the bowel. Methyleneblue (Io cg.) is a reliable remedy to employ in connection with lactose when diarrhea is persistent. When it is desirable to administer a remedy which will protect the inflamed mucosa, calcium carbonate or phosphate, chalk or charcoal, gr. xv (I.O), three or four times daily, alone or in conjunction with olive oil, nutralol, or petroleum, $\overline{3} j(30.0)$, give the best results.

When other measures fail to rclieve the patient's diarrhea and pain, opium is the remedy par excellence, independently or combined with the above-mentioned drugs. Quickest relief follows when it is administered in the form of morphin, gr. $\frac{1}{5}$ to $\frac{1}{4}(0.0008-0.015)$, but when there is no occasion for haste, opium extract, gr. $\frac{1}{2}(0.03)$, or tincture, gtt. $\mathrm{V}$ to $\mathrm{x}(0.30-0.60)$, codein, gr. $\frac{1}{4}(0.015)$, or heroin, gr. $\frac{1}{10}$ (0.006), adninistered according to indications, invariably relieve pain, minimize the diarrhea, and enable the patient to sleep.

When complications are present in addition to the above, other remedies are indicated, viz., in anorexia, nux romica; hyperchlorhydria, an alkali or dilute nitric acid; anemia, iron preparations; flatus, sulphocarbonate of zinc; hyperacidity with constipation, powdered magnesia or cooking-soda; enterospasm, belladonna, etc., and should be administered in suitable doses as often as required.

Calomel and soda, of each gr. $\frac{1}{2}(0.03)$, administered half-hourly until 3 gr. have been taken, is the most reliable agent to employ when 
the patient is bilious, suffers from intestinal toxemia, or there is an irritant in the bowel to be expelled, when, on the following morning, a saline is taken to carry it off.

Constipation can usually be controlled by the saline mineral waters, Juss to j ( I5-30), previotisly mentioned; ("arlsbad, gr. xxx (2.0); Epsom, Cilauber's, or Rochelle salts, jj to ij ( 4 - \& c.c.), two or three times daily, are preferable to other laxatives because of their benefieent action upon the intestinal mucosa. Enematia of olive, sweet, almond, or mineral oil when injected warm usually secure an immediate evacuation, or a ro per cent. solution of gelatin, $\bar{j} \mathrm{j}$ to $\mathrm{ij}$ (30-60 c.c.), which is particularly soothing to the inflamed bowel, may be substituted for the oil.

Irrigations. - Bowel tlushings with normal saline and other medicated solutions are beneficiai in the treatment of chronic catarrhal colitis and enterocolitis, because they riel the gut of irritating toxins, undigested food remnants, hardened fecal masses, foreign bodies and offensive bacteria, reduce inflammation, heal ulcers, and supply the bowel with additional water when there is exhaustive diarrhea. Occasionally cold irrigations $\left(65^{\circ} \mathrm{F}\right.$.) are agreeable, but, as a rule. enteroclysis is more effective when employed warm or hot ( $10^{\circ}$ to $120^{\circ} \mathrm{F}$.), because hot solutions are soothing, retained longer. allay nervousness, diminish pain, and make the bowel feel more comfortable. Once daily or three times weekly is sufficient in ordinary cases of catarrhal enterocolitis, but the bowel should be thoroughly flushed morning and night when the mucosa is ulcerated. there is a mixer! infection, and pus, blood, and mucus collect quickly.

There are no specific irrigants, and the usefulness of medicated bowel washings should be attributed more to the healing and mechanical actions of the solution, in getting rid of irritating déloris and curing ulcers, than to the specific action of the remedy. The amount of fluid employed should rary from a few ounces to + quarts, depending upon the extent of the catarrhal inflammation and accompanying changes, but more copious irrigation and injections are to be aroided. because they distend and cause the bowel to sag, induce pain, and may. cause perforation, except when used through a double-flow irrigating tube. It is essential that the position of the patient be frequently changed, so that all parts of the intestinal mucosa are reached during the irrigation.

Good results are obtainable in this class of cases from normal saline or boric acid (3 per cent.), ichthyol ( 2 per cent.), balsam of Peru ( per cent.), permanganate of potassium (I per cent.), protargol or argyrol (5 per cent.), or silver nitrate, gr, r to Oij (0.30 gm. to 1000 c.c.). solutions, introduced through the appendix, cecum, or anus.

Better results can sometimes be olbtained by alternating the irrigations with copious enemata $\mathrm{Oj}(500)$ of warm, olive. cotton-seed, or mineral oil alone or containing bismuth, aristol, salol, jij (8.o), etc., which soothe and protect the inflamed mucosa and augment healing. 
Surgical Treatment.-Surgical measures are less often called for in the treatment of enteritis and colitis than in the infectious types of colitis, but when the bowel is extremely irritable, croded or ulcerated, or the patient suffers from mixed infection and other therapeutic measures fail, it may be necessary to resort to appendicostomy, cecostomy, or Gant's enterocecostomy', procedures which enable one to put the bowel more or less at rest and to practice daily through-andthrough irrigation. For a further description of the indications and technic of these operations, the reader is referred to the chapters devoted to the surgical treatment of catarrhal, diarrheal, and parasitic affections of the gastro-intestinal tract.

Opotherapy and organotherapy have been employed with some success by others in the treatment of chronic catarrhal and infectious enterocolitis, but the author has not employed them sufficiently to warrant recommending them in this class of cases. Intestinal extracts and secretin $(0.05)$ have also proved helpful in the treatment of chronic catarrhal, tubercular, dysenteric, and mucomembranous enterocolitis, constipation, and biliousness, but sometimes they irritate the intestinal mucosa and must be stopped. Pancreatic opotherapy is said to be useful alone or in conjunction with secretin when diarrhea is consequent upon pancreatic affections, nervous phenomena, and colitis with intolerance to milk.

Vaccines.-The uscfulness of vaccines in the treatment of typhoid fever has been established beyond question, and sera have proved effective in cases of bacillary, but not in entamebic, colitis. In the two former the causative organisms liberate endotoxins following autolysis, enabling one to manufacture a reliable therapeutic agent which minimizes or arrests the infective process, but the remedy, owing to its toxicity, should be administered with caution.

Vaccines have been prepared from Bacillus coli communis and coliform bacilli by Hale White and Eyre, and used with some success in the treatment of the class of diseases under discussion.

The bolus or clay treatment introduced by Stumpf is highly recommended by Gäcrtner in gastro-intestinal affections complicated by diarrhea and meteorism, because of its inhibitive action upon bacterial growth, particularly when the clay (bolus alba) is given upon an empty stomach, and in doses varying from $\frac{3}{3}$ iss to iijss $(50-100)$ in $\frac{1}{2}$ pint (250 c.c.) of water. It has been found particularly useful in chronic catarrhal and tubercular bowel diseases, and for protecting patients exposed to typhoid and those about to be operated upon against infection.

The Rosenberg dry treatment has been successfully employed by the author in proctitis and sigmoiditis, but the method is useless in the treatment of colitis, because the powder cannot be applied to the entire large bowel except following the author's enterocecostomy. Rosenberg considers it superior to other methods of treatment, owing to the prolonged action of the remedy, which clings for hours following its application to the inflamed or ulcerated mucosa. The powder 
may be applied with swals, but the author prefers to place the patient in the knee-chest posture, insert the sismoideseope, intlate the bowed, and then introduce the remedy through a power-blower and with the aid of compressed air continuing the application nntil the mucosa is covered with the powder. Such a treatment minimizes pain, lesens tenesmus, and assists in healing the lesions. but the atuthor comsiders both topical applications amel medicated irrigation, as outlined abose. more effective than Rosenbers's remedy, which is composed of tannic acid and magnesium, though bismuth, xeroform, and zinc are sometimes emplosed.

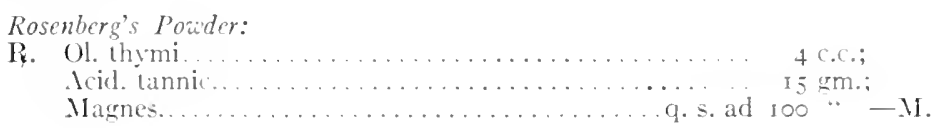

Clifton Springs Pack.-This abdominal pack, which is composed of equal parts of mustard, red pepper, and prickly ash bark, thoroughly mixed, is a valuable agent for relieving pain and discomfort and ruieting peristalsis during diarrheic crises on account of its moisture and counterirritant action. It is applied by putting a tablespoonful of the mixture in a quart of warm water, saturating flannel cloth in the solution, and placing them orer the abdomen. In the absence of the pack, hot turpentine stupes may be sulstituted.

The Fango and similar compreses are useful in some instances.

Inflation of the intestine with oxygen has, in the author's hands. proved a valuable adjunct to the treatment, because it immerliately braces the patient up, improves his anemic condition, augments peristalsis (when constipation prevails), increases intestinal disinfection, and augments the amount of oxygen in the blood. Oxygen can be easily introduced into the alimentary tract through tubes comnected with a tank pasied into the stomach and rectum. A. Schmidt combines agar (oxyagar) and insufflates 2 to + quarts (liter-) twice daily through Einhorn s duodenal tule, which is followed by an odorless flatus, and later by diminished intestinal fermentation and putrefaction. Carbonic acid gas, introduced through the rectum. has leen frequently employed in the treatment of intestinal catarh by A. Rose. who claims that its effects are stimulating, strengthening, and antiseptic. The author has employed the method in a few instances with disappointing results, and has abandoned it, owing to the distention pains which accompany and follow the treatment.

Prognosis of Enteritis, Colitis, and Enterocolitis. - Mrults uslally suffer considerable amnoyance and discomfort from acute catarhal enteritis, colitis, or enterocolitis, but recover in a lew days or weeks, except in complicated cases, where the disease becomes chronic. Infants, very young children, the aged, and enfeebled individuals often become greatly exhausted and freeguently succumls to it unless carefully handled.

Chronic intestinal catarrh is distressing, greatly delilitates the 
patient, and frequently incapacitates him for business and social duties, and, when neglected or ignoranty treated, death may ensue from mental and physical exhanstion. This affection rarely causes death directly, but occasionally it is responsible for the patient's end, because it sucks his vitality and gets the intestinal mucosa inflamed and eroded, conditions which pave the way for constitutional and local infectious and other diseases having much higher mortality than catarrhal enterocolitis. As a rule, under favorable conditions, acute intestinal catarrh subsides in a few days or weeks, and chronic abates in a few months, when the patient is protected against exposure, arduous labor and unhealthy surroundings, is properly clothed, fed, treated, and placed in the midst of cheerful surroundings to improve his despondency. 


\section{CHAPTER XIX}

\section{TUBERCULAR ENTERITIS, COLITIS, AND ENTEROCOLITIS (INTESTINAL TUBERCULOSIS), DIARRHEA IN}

\section{GENERAL REMARKS, ETIOLOGY}

General Remarks.-Clinicians at all familiar with intestinal affections concede that tuberculosis is an important factor in diarrhea because of its frequency as a cause, and the difficulty encountered in relieving or curing this type of loose movements.

The fregtent evacuations of tubercular subjects may be excited by tuberculosis of the bowcl or an intestinal catarrh which preceded or made its appearance simultaneously with plithisis or tubercular infection in the bowel or elsewhere. The comprehensive statistics hereafter quoted relative to postmortems held upon persons sulfering from this disease show that the bowel is usually affected by a simple enteritis (most common) or tubercular lesions of the intestine, either of which are sufficient to account for the loose movements so frequently complained of by tubercular subjects, irrespective of where the disease is located.

Relative Frequency of Tuberculosis in Different Parts of the Intestine.-All segments of the intestine are liable to tubercular infection, but some parts of the bowel are more frequently affected than others. Statistics bear out the author's personal observations that the lower half of the small bowel is very much more often attacked by tuberculosis than the upper, the cecum is, in the vast majority of cases, the farorite location, and, next to the ascending colon, the anorectal region is more frequently the seat of foci than other portions of the large bowel.

Nikoljski, in 120 operations and 70 autopsies performed upon persons suffering from tubercular intestinal stricture, rarely found the stenoses in the upper small intestine or colon, but encountered them frequently in the lower ileum or cecum, while (aird ( I cases) observed them at the ileocecal valve and cecum in 6 , in the small intestine alone in 4 , and the ascending colon in I casc. Eisenharclt's statistics show tuberculosis of the small intestine in 83 , and of the large howel in 135 cases, but does not indicate the segments of gut involved. In Eisenbach's collection of tubercular infections, the disease was located in the small bowel nine times, cectm twenty-seven, and the descending colon and appendix each once. Reach cites Strehl's unigue case of multiple strictures, fourteen of which were located in the ileum and the other in the hepatic flexure.

The most claborate and extensively quoted statisties rolating to this subject are those of fienwick and l)oelwell, who, in 2000 atutopsies performed upon tubercular subjects, found infection of the intestine 
in 883 cases. The lesions were located in the different parts of the bowel in conjunction with other segments or alone as follows: Duodenum, 3.t per cent.; alone, none; jejunum, 28-1.+ per cent.; ileum, 60.2-4.+ per cent.; ileocecal, 85-9.6 per cent.; appendix, 503.8 per cent.; ascending colon, $51.4-1.8$ per cent.; transverse colon, 30.6-1 per cent.; descending colon, 21.0 per cent.-none; sigmoid Hexure, 13.5 per cent.-none; rectum, I4.I per cent.-none.

The duodenum is seldom involved in the tubercular process, but when it is the stomach is frecuently diseased. The author has been unable to find many reported cases of duokenal tuberculosis, and of these Maylard mentions 3. In the first the duodenum and pancreas were massed together with tubercular caseous glands, in the second there was a large transverse ulcer, and in the third a small round ulcer with thickened edges, $\frac{1}{2}$ inch (I.25 c.c.) distant from the pylorus and the tubercles, involved the peritoneum. Hamilton has recorded a case in connection with gastric tubereulosis of a large ulcer at the pyloric extremity of the duodenum, the base of which was covered with tubercles. Mayo Robson has called attention to a case of duodenal tubercular stricture discorered while performing pyloroplasty upon a young girl. Reach observed but one instance of duodenal involvement in 21 collected cases of multiple intestinal tubercular stricture, and this patient suffered from the disease in other parts of the intestine.

The author has encountered tuberculosis of the duodenal mucosa in only two instances, but he has observed its peritoneal covering freely studded with tubercles and the muscular tunic appeared to be involved in other cases. In the first instance the tubercles were limited to the duodenum, but in the other they extended over the entire small intestine and covered the ceeum.

Jejunal tuberculosis is more frequent than duodenal, but the disease does not occur here so frequently as in the ileum. White gives two sets of statistics of bowel tulereulosis complicating phthisis - in the first series (57 cases) the jejunum was involved 7 and the ileum 32 times, while in the second (66 cases), tuberculosis occurred in the jejunum 20 (30.3 percent.) and in the ileum .36 times ( 54.5 per cent.), but in the remaining o cases its location was not given. These last figures do not differ materially from those of Fenwick and Dodwell, who state that the jejunum was involved in the tubercular process in 28 per cent. of the eases. Mikulicz has recorded 3 cases of jejunal stricture, including one of his own, and Reach has recorded another.

The upper ileum is not much more frepuently attacked by tuberculosis than the distal end of the jejumum, but its lower extremity very often becomes involved because of frequent extension of the disease upward from the cecum (19. 27). Hemmeter examined the intestines of 56 persons who died of phthisis, and in $1+$ instances tubercular ulcers were observed in the ileum and colon.

Alglave, in the 28 cases of granular ulcerative tubercular lesions of the bowel, found ro simultancous lesions of the ileum, cecum, and 
appendix, 6 simultaneous lesions of the ileum and cecum without lesion of the appendix, 7 lesions of the ileum withesut invelvement of the cecum or appendix, I calse of simultanerous lesion of the cecum and appendix without lesion of the ileum, i lesions of the cecum without involvement of ileum or appendix. I case of lesion of the appendix without lesion of cecum or ileum. In other words, in 2.3 of the 28 cases the ileum was exclusively affected in 7 eases, in 20 of 28 cases the cecum was diseased (3 times exclusively), and in I 2 of 28 cases the appendix was affected (once exclusively).

The cecum is very frecpuently involved in cases of pulmonary tulerculosis, and in intestinal tuberculosis the disease is located at the cecum very much more frequently than in the remainder of the bowel, and all forms of the disease are encountered in this region. Fenwick and I odwell found the cecum involved in 85 per cent. of all cares, and in 9.6 per cent. of the cases the direare was limited exclusively to this region.

Alglare says the enteroperitoneal form of intestinal tuberculosis is peculiar to the ileocecal region, and that when other parts of the intestine are involved the lesions centralize in and adjacent to the cecum. Regarding the hypertrophic or neoplastic type. he states that it may occur in any part of the intestine, but its seat of predilection is the ileocecal angle.

To indicate the frequency of the tubercular process in this region the author will cite the statistics of two authorities whose names are most prominently associated with cecal tuberculosis. Hartmann has collected 299

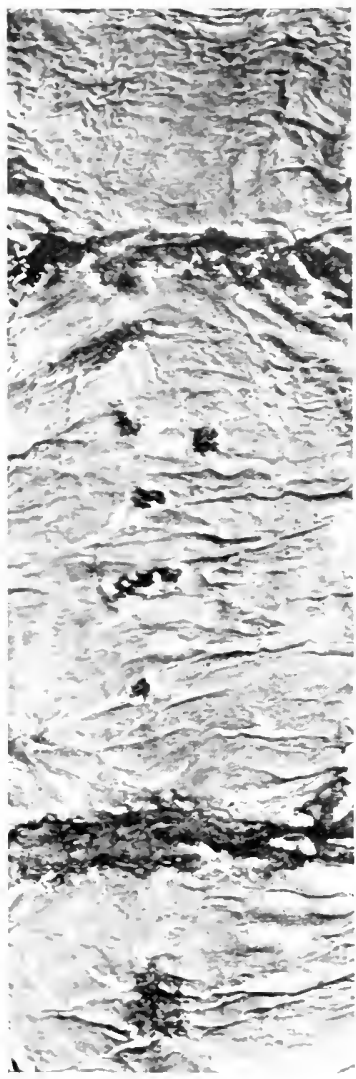

Fig. 27-Small and large enteric girdle cicatrizing tubercular ulcers of the ileum. operations for, and Campieche has recorded 379 cases of, ileocecal tuberculosis, and there are many other scattered cases. These statistics, with others which could be added. demonstrate conclusively that tuberculosis attacks the ileocecal angle more frequently than any other segment of the bowel.

\section{ETIOLOGY}

Predisposing Causes of Intestinal Tuberculosis. - Intestinal tuberculosis may occur in otherwise healthy inclividuals, but is most ofter encountered in persons who have a hereditary tendency to the disease, arduous occupations or those which subject them to exposure. poor food, or insuficienty ventilated rooms and unhygienic surround- 
ings, conditions which lower their resistance and render them liable to infeetion by tubercle bacilli and other inlectious agents.

Bowel tuberculosis has frequently been traced to affections of the gastro-intestinal tract accompanied by inflammation or ulceration of the mucosa and irritating discharges, and a sufficient number of cases of tuberculosis secondary to trama have been recoreled to warrant the belief that the tranmatism which ensues in consequence of surgical operations, constipation and recurring impaction, invagination. ptosis, angulation or twisting of the bowel, pressure upon or irritation to the gut induced by tumors or disease in neighboring organs, tend to interfere with the howel and pave the way for tubercular infection. Young hottle-fed children are predisposed to intestinal tuberculosis more frequently than adults because of the food consumed and the prevalence among them of cliarrhea, constipation, and other bowel affections.

Age.-Intestinal tuberculosis may occur at almost any time from infancy to old age. The author has treated a girl of three for enteric tuberculosis, a man of seventy for the neoplastic type, and other patients of various ages, young and old. Nevertheless, age is an important factor in intestinal tuberculosis, as is evidenced by the statistics upon the subject. Practically all authorities agree that this affection in its different forms occurs far more frequently in persons between twenty and forty years of age, and especially in the third decade, than at any other time, and the writer's observations corroborate these views.

Primary tuberculosis is encountered most of ten in young children, and the neoplastic type in older persons. The following table, representing the statistics of Crowder, illustrates very well the relative frequency with which tuberculosis of the intestine occurs in the various stages of life and the frequency with which both sexes are attacked.

\begin{tabular}{|c|c|c|c|}
\hline Age. & Male. & Female. & Total. \\
\hline I to ro........ & o & 0 & $\circ$ \\
\hline I I to $20 \ldots$ & 4 & 7 & II \\
\hline 2 I to $30 \ldots$ & 10 & It & 24 \\
\hline 3 i to $40 \ldots$ & I9 & 10 & 20 \\
\hline 4 to 50 . & 3 & 6 & 9 \\
\hline 51060. & 3 & 5 & s \\
\hline
\end{tabular}

Sex.-The sex is of no etiologic importance in intestinal tuberculosis, and the different types of the disease seem to affect men and women with about equal frequency, though in the author's experience the male sex has slightly predominated, as was the case in Nikojski's I 70 collected cases of tubercular intestinal stricture. In Esenbach's series of 27 cases of intestinal tuberculosis there were 1.3 men and it women, while in Hartmann's 217 collected operated cases of ileocecal hyperplastic tuberculosis there were 105 males and 1 I 2 females.

Method of Infection in Intestinal Tuberculosis.-Tulerculosis of the intestine invariably results from the invasion of its tunics by 
tubercle bacilli. Isually the tubercular process is due to human tubercle bacilli, but it may be caused by bovine bacilli (typus bovinus). A great deal of discussion was excited by Koch at the British Medical Congress, London, rgor, when he made the statement that bovine tubercle bacilli were different from the human, and that it was cloubtful if they are communicable to man. Investigations were starterl shorty thereafter to determine the nature of typus boainus and the extent to which it would cause tuberculosis in human beings, and the earlier experiments and observations tended to support the theory of Kech, for the bovine bacillus was rarely discovered in tubereular manifestations of adults or children, but when found it was encountered most frequently in tuberculosis of the intestines and mesenteric glands.

Gradually evidence has been accumulating which disproves the views of Koch, and demonstrates bevond question that tuberculosis of the intestine and elsewhere can be contracted from the milk and meat of tuberculous animals. While it is now generally agreed that the bovine bacillus can cause the disease in man, the best authorities concede that the tuberculous process is caused by human bacilli in the majority of cases.

In intestinal and other forms of tuberculosis, bacilli may gain access to the body through the inhalation of contaminated dusi, being carried to the mouth by the fingers, toys, walking sticks, pencils. and other objects, the ingestion of milk, cream, cheese, butter, meat, or other kinds of infected edibles, or scratching of the anus.

While food products are known to occasionally be the carrying agents of tubercle bacilli (primary tuberculosis), it is a fact that intestinal tuberculosis is secondary to and caused by the swallowing of tubercle bacilli from lesions in the lungs, larynx, nasopharynx, esophatgus, stomach, liver, or diseased gut higher up in the vast majority of cases, and it has been shown that tubercle bacilli can pass through the stomach and infect the intestine. Because of the infrequency with which tuberculosis is encountered in the stomach, jejunum, and upper ileum, it would appear that the gastric juice (hydrochloric acid) in some way attenuates the bacilli temporarily, and that they regain virulence after having reached the cecum and colon, where the disease is most common, and propulsion through the bowel is slow, or the stasis common to this region favors their pathogenic development.

The direction from which the bacilli come varies in different cases. Ordinarily they gain entrance to the gut, attack the mucosa, and the tubercular process extends outward, but in rare instances the peritoneal coat first becomes involved through extension of the disease from the mesenteric glands, ubercular organs, or infected lymph or blood. Astute observers hold that human and bovine tubercle bacilli may, under favorable conditions, penctrate the healthy or diseaserl intestine and infect neighboring lymph-nodes or other structures adjacent to or distant from the lowel.

While it is admited that tubercle hacilli may possibly pass through healthy bowel, all agree that such occurrences are rare, and that when 
they penetrate the gut or find their way into its circulation, the mucosa has already been denuded of its epithelial lining by erosions or ulcers. Fiaten and schottelius fed animals tubercular material; infection of the mesenteric glands and other parts ensued, which would indicate that tubercle bacilli do find their way through the bowel and produce tubercular foci in other parts.

Since boine bacilli apparenty can infect human beings, and find their wiy through the nornal and diseased intestine to other structures, it would seem that they might be responsible for the primary tuberculosis of the mesenteric lymph-nodes, intestine, and elsewhere.

Instances of primary intestinal tuberculosis have been reported without autopsy finclings, where the diagnosis was based upon the history, general and local examination, and absence of tuberculosis elsewhere, or, when present, where it occurred secondary to bowel involvement or was induced by extension of the intestinal infection by contiguity or otherwise. Such reports should be looked upon with skepticism, because primary intestinal tuberculosis can be diagnosed by autopsy only and where tubercular processes elsewhere have been excluded.

It is not definitely known whether bowel tuberculosis is ever inherited or not, but in 2 or 3 cases the disease has been observed in children varying in age from a few days to three weeks old, born of tubercular parents, and one is justified in assuming that the disease may be congenital.

AUTHOR'S TABLE SHOWING THE FREQUEACY WITH WHICH INTESTINAL TUBERCULOSIS COMPLICATES THE DISEASE ELSEWHERE

\begin{tabular}{|c|c|c|}
\hline Name. & $\begin{array}{l}\text { Number of tubercular } \\
\text { cases. }\end{array}$ & $\begin{array}{l}\text { Percentage complicated with } \\
\text { intestinal tuberculosis. }\end{array}$ \\
\hline White (Phipps Institute) ............... & 266 & +5.1 \\
\hline 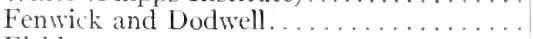 & $\mathrm{SS}_{3}$ & 56.6 \\
\hline Eichhorst.................... & 462 & 21.9 \\
\hline Heinze . . . . . . . . . . . . . . . & 1226 & 51.0 \\
\hline Eisenhardt (by Crowder).............. & 1000 & 56.6 \\
\hline 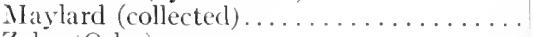 & 200 & 40.7 (children) \\
\hline 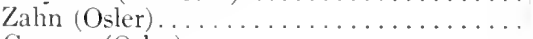 & Number not stated & 63.21 \\
\hline 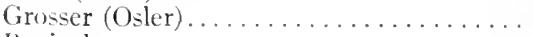 & " 64 & 47.0 \\
\hline Bagrinsky . . . . . . . . . . . . . . . & 063 & $\begin{array}{c}\text { All intestinal tubercu- } \\
\text { losis secondary }\end{array}$ \\
\hline Höning (Cornet) ........... & Number not stated & 70.0 \\
\hline Weigert and Orth (Cornet) ..... & 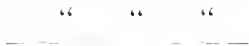 & 90.0 \\
\hline Total. & 5090 & 49.27 \\
\hline
\end{tabular}

Excluding the statistics of Höning and Veigert and Orth, the average percentage is +2.45 per cent. Herxheimer, quoted by Crowder, states that of 58 cases of intestinal tuberculosis, 98.2 per cent. were secondary. In 100 cases of intestinal tuberculosis (ulcers, catarrh, fistula, abscesses, etc.) observed by the author, tuberculosis developed in the bowel secondary to the disease elsewhere (lungs, 70 per cent.) in 75 per cent. of the cases. 
Primary and Secondary Tuberculosis. - I pertisal of the literature bearing upon tuberculosis demonstrates conclusively that intestinal tuberculosis may have its origin either primarily or secondarily in the bowel, but there is still a wide divereence of opinion ats to the relative frefuency of these types of infection, and comprelensive statisties have beem loroughe forward by atthorities to corroborate their respective clains. Even so great an atuthority ats koch made the statement shortly before his death that he hat observed hut few cases of primary infection. An analysis of the figures inflicates conclusively that in a large pereentage of ases bowel tuberculosis is secondary to the diseate in other parts. especially the lungs; that primary infection oecturs more frectuently than is sipposed, and when present is molatly caused by the bovine lacillus (typus bovinus) which has gained acess to the alimentary tract throtgh contaminated milk or food.

To show the frectuent association of intestinal tuberculosis with the disease elew where in adults and chiletren, and the relative frecpuency of primary and secondary infections, the author has collected and tabulated the following statistics.

Pertik's Statistics. - Primary intestinal tuberculosis is calculated by Zahn at 2.27 per cent. Grosier found only a single case among 1 to autopsies. Secondary intestinal tuberculosis, in the origin of which the condition of the stomach is evidently an important factor, was met with in 63: 2 I per cent. of all cases of pulmonary 1uberculosis, according to Zahn, and in 47 per cent., according to Crrosser.

ACTIOR'S TABLE SIOWING FREQLENCY OF PRIMARY INTESTIALL TLBERCLLOSIS

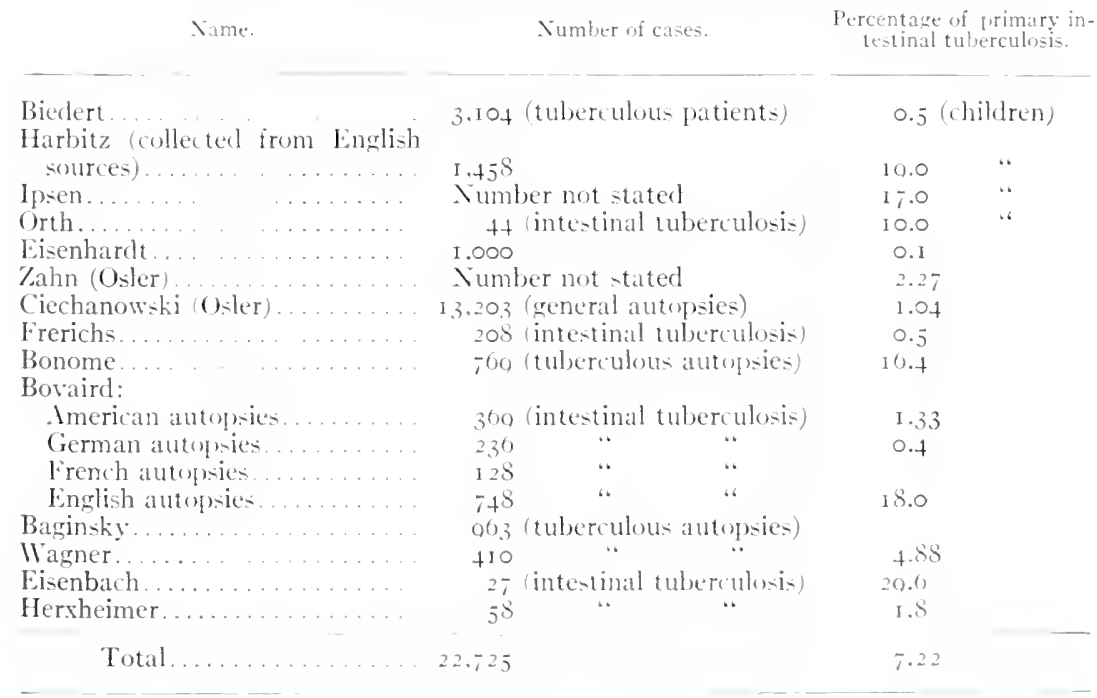

${ }^{1}$ Münch. Med. Wochschrít, No. 2, r902. "Inaugural Discertation. Tübingen, :200. 
The atuthor's comprehensive study of the subject and personal experience with intestinal tuberculosis warrants the statement that the discase is primary in less than ro per cent. of the cases, and when it is, the lesions usually originate in the anorectal region.

To show the apparent correlation between diphtheria and intestinal tuberculosis, and determine the proportion of primary infections in these cases, Osler collected the statistics of Heller, Councilman, Mallory, Pearce, and Baginsky as follows, which, when carefully studied, throws considerable light upon an obscure feature of tuberculosis:

\begin{tabular}{|c|c|c|c|c|}
\hline & $\begin{array}{l}\text { Deaths from } \\
\text { diphtheria. }\end{array}$ & $\begin{array}{l}\text { General and } \\
\text { other tuber- } \\
\text { culosis. }\end{array}$ & $\begin{array}{c}\text { Intestinal tuber- } \\
\text { culosis. }\end{array}$ & $\begin{array}{l}\text { Primary intestinal } \\
\text { tuberculosis. }\end{array}$ \\
\hline Heller ..... . . . . . . & 714 & I 40 & 53 & 7.4 per cent. \\
\hline Councilman, Mallory, Pearce & 220 & 35 & 13 & 5.9 \\
\hline Baginsky .............. & 806 & 144 & 6 & 0.7 \\
\hline
\end{tabular}

An analysis of these figures indicates that intestinal and general tuberculosis are predisposing causes of diphtheria.

Many theories have been advanced to explain why the disease attacks the cecum so very much more frequently than other parts of the intestine. A study of the statistics already given show conclusively that the cecum, the lower extremity of the ileum, and beginning of the ascending colon are the seat of the disease in 85 per cent. or a larger proportion of all cases of intestinal tubercular infections, and for this reason tuberculosis of the ileocecal region deserves separate consideration.

It is conceded that tubercle bacilli are able to pass through the stomach and infect the bowel, but it is difficult to understand why it is that the duodenum, jejunum, and upperileum, with which they first come in contact, escape, while the cecum is so frequently attacked. There are many reasons which can be advanced which would help to explain the predilection of tuberculosis for the cecum and adjacent ileum and colon, viz.:

(I) Because the fluidity of the feces and more pronounced peristalsis in the small intestine hurry the tubercle bacilli through the upper segments of the small bowel so quickly that they have no opportunity to cause infection.

(2) The gastric juice at tenuates the bacilli, and they do not recover sufficiently to set up a tubercular process until they have reached the cecum or colon, where they are retained long enough for them to regain their vitality and find a lodging-place.

(3) In the ileocecal region the feces have a strong alkaline reaction, while higher up they are acid owing to the gastric juice, which inlibits bacillary activity.

(4) In the cecum and ascending colon the feces are prone to collect and form large putty-like or hardened masses, which sometimes traumatize the mucosa, produce catarrh, erosions or ulcers, and a mucous discharge, conditions which favor a local infection by tubercle bacilli.

(5) The abundant lymphatic distribution here favors the development of tuberculosis in the ileocecal region. 


\section{CHAPTER XX}

\section{TUBERCULAR ENTERITIS, COLITIS, AND ENTEROCOLITIS (INTESTINAL TUBERCULOSIS), DIARRHEA IN (Continued) \\ CLASSIFICATION, PATHOLOGY}

Classification.-It is not so easy as it would at first appear to classify tubercular lesions of the intestinal tract which are capable of inciting diarrhea, because there are several types each of which presents a different picture when encountered in its various stages. and. further, because the authorities have evidently discused the same forms of the disease under different names.

It has been the custom of the author, following the plan of Alglave. to group the lesions of intestinal tuberculosis according to the part and manner in which the disease attacks the intestinal wall, lymph-nodes. and peritoneum, viz.:

(i) Enteric or superficial ulcerative type.

(2) Enteroperitoneal or deep ulceratice type.

(3) Hyperplastic (hypertrophic. neoplastic).

(4) Fibrosclerotic.

(5) Glandular.

(6) Peritoneal.

All varieties of tuberculosis. no matter whether they attack the intestine from within or without. produce slight or persistent diarrheal attacks at one stage or another. Enteric occurs far more frequently. than enteroperitoneal tuberculosis. which is encountered more often than the hyperplastic (neoplastic) form. The other ype- of the disease are of rare occurrence.

In some instances two varieties may be present in indepenctent parts of the gut, or they may be localized in a single segment. under which circumstances it is difficult to diagnose the true condition hy clinical and pathologic findings.

The relative frequency with which the different types occur is very well shown in the 52 specimens of intestinal tuberculosis mentioned by Alglave and tabulated by the author.

TABLE OF 22 SPECIMEXS OF INTESTIXAL TLBERCLLOSIS

\begin{tabular}{|c|c|c|c|}
\hline & Varieties. & Number of casen. & Percentage. \\
\hline Enteric.... & & 28 & $=3.0$ \\
\hline Enteroperitoneal. & $\ldots \ldots \ldots$ & 14 & 20.0 \\
\hline Hyperplastic... & $\ldots \ldots \ldots$ & 5 & 0.0 \\
\hline Peritoneal ........ & $\ldots \ldots \ldots \ldots$ & 4 & 7.7 \\
\hline Glanclular....... & & I & 1.0 \\
\hline Total.... & $\ldots \ldots \ldots \ldots \ldots \ldots \ldots \ldots$ & 52 & \\
\hline
\end{tabular}


The author has made a comprehensive study of the published statistic upon intestinal tuberculo-is. and from this analysis and his persmal experience he feel-ju-tified in =tating that the enteric or superficial uleerative type prevails in about no per cent. of the cases. He is aware that the peritoneum is involved in perhaps 20 per cent. of the cases, but believes that in many instance- the serosa becomes affected secondarily from extensions of the tubercular proces or mixed infection, which would explain the frequency with which the peritoneal coat is observed to be liseased during operations or at autopsies upon tubercular subjects. Again. considerable infiltration may take place in the crats of the intestine in enteric tuberculosis, and because of the thickness and firmness induced in the gut wall the process is occasionally mistaken for the hyperplastic variety. or. When the lymphnodes become secondarily involved. for the slandular type of the di-ease.

The enteric and enteroperitoneal forms. like hyperplastic tuberculosis of the gut, are comparatively rare in the upper small bowel and colon. although they are found considerably more frequenty than the hyperplastic. but the different varieties are encountered in the ileocecal region in about 85 per cent. of all cases.

The stati-tics of Hemmeter and other authorities upon the subject show that there is an enteritis or enterocolitis (catarrhal or tubercular in practically all person- who die of tuberculosis of the lungs or other orcans. If this is so, and there is no reason to believe otherwise. intestinal tuherculosis is not only secondary. but occurs frequenty.

\section{PATHOLOGY}

Enteric Supericial Llecrative Type) Tuberculosis.-Ilcerative is the most frequent type of intestinal tuberculosis. and when present excite- a persistent diarrhea. This condition is usually secondary to tubercular lesion = in the lung and elswhere. It is met with in all parts of the intestinal tract. but shows a predilection for the lower ileum. cecum, ileocecal valve, and ascending colon Fig. 28), regions bountifully supplied with lymphoid tissue. solitary follicles, and Peyer $=$ patches. In the 2 cases onteric tuberculosis mentioned in the preceding table the ileum was affected exclusively in 7 . in 20 the cecum was diseased 13 times exclusively while in 12 cases the appendix was intolved once exclu-irely).

In specimens examined by the author the ulcerated areas were numerou- and most marked opposite the mesenteric attachment, indicating involvement of Peyer's patches. though in places the solitary follicles were included $i$ the destructive process.

Formerly it was thought that the tubercular foci originated in the superficial mucosa independenty of these patches and glands. but the investigations of Orth and Baumgarten have conclusively shown that the reverte is true, and that the disease has its inception in these structures. and that tubercle hacilli and giant cells are to be found woth in the closed follicles and base of the ulcers. In catarrhal, ty- 
phoid, and other infections the entire Peyer's patch is affected, but in tuberculosis a single gland or several inclividual follicles or small aggregations of glands within the patch may le attacked, while others remain healthy. Shortly following infection the solitary glands or part of Peyer's patch affected become nore prominent, owing to distention of the follicles with tubercular tissue and hacillary intlanmation, and later, through impairment to nutrition or caseation, overlying structures give way and diminutive lesions form. As soon as the mucosa become ulcerated the disease progresses rapidly because of the irritating poisons emanating from the tubercular foci, formation and subsecuent cascation of new tubercles, mixed infection and necrosis from the endarteritis, and in time extensive ulcurated areas form through enlargement of a single lesion or the coatescing of several lesions.

Some authorities bolice that extension of the discase is due principally to the successive formation and caseation of the tubereles, but the author holds with Maylard that when the destructive process is markel, progression is principally due to mixed infection resulting from the tulicercular foci and lowered resistance of the patient, for the granulated mucosa, fecal stasis, and the pathogenic and saprophytic micro-organisms within the bowel all favor a seconclary or mixed infection. White in many instances pathogenic cocci predominate in the lesions, evidences of the tubercular process an be determined by careful study. In fact.

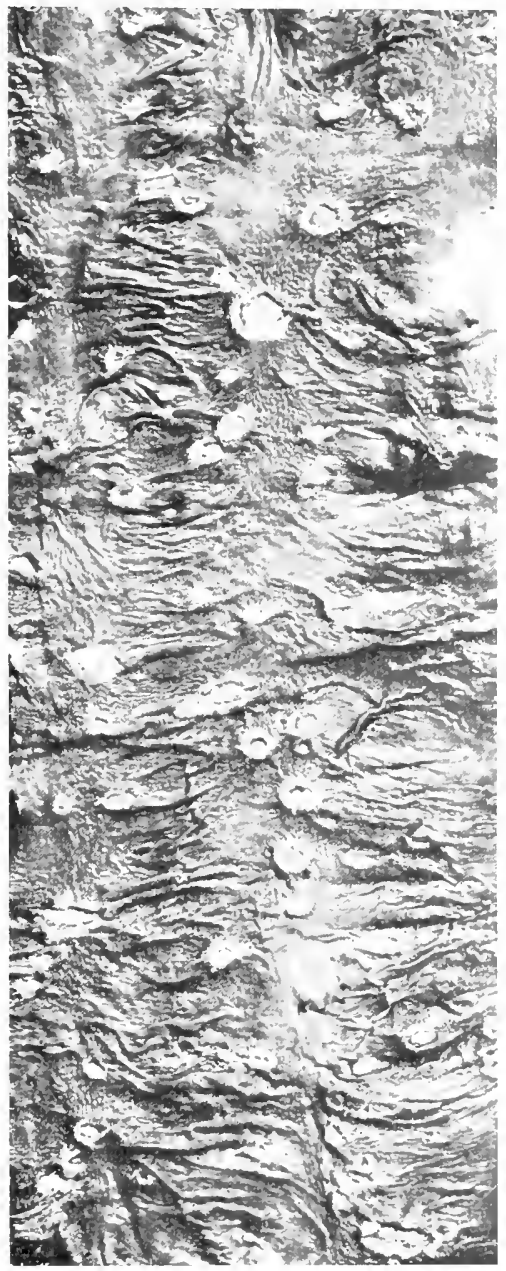

Fig. 28. - Tubercular ulceration (ascending colon). ${ }^{\mathrm{I}}$ where there are enormous ulcers with excavated edges or the mueosa is in shreds or completely destroyed over an area of several inches the destruction of tissues is tue more to the mixed than to the original or tubercular infection. IVhen other micro-organisms participate actively in the inflammatory process the mucosa suffers greatly and perforation as well is likely to take place. 
Enteric tulerentar ulcers rarely penetrate beyond the mucosa, and, except when mixed infection is a complication, they show a tendency to heal, an evidence of which is to be seen in the scar tissue at the balie.

In some instances the disease extends without apparent limitation, but in others healing takes place at one point while ulceration is progressing in another.

The characteristics of tubercular ulcers of the intestine are fairly well known. These lesions are usually gray in color, have a greasy appearance, a macerated base (composerl of degenerated and caseat-

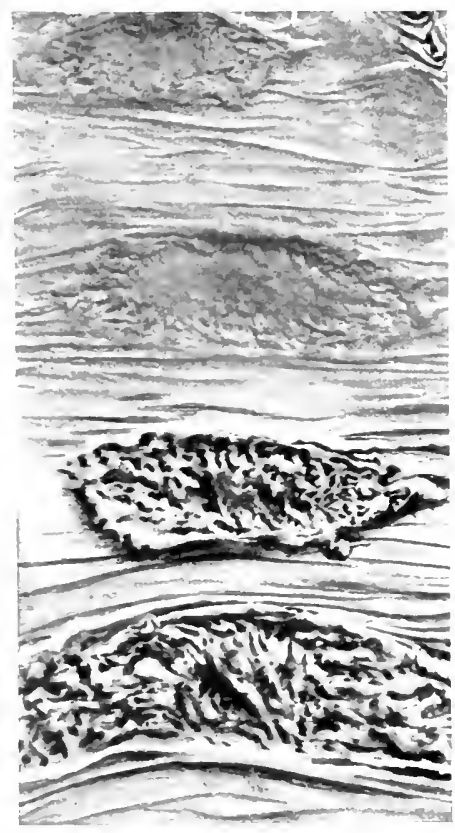

Fin. 20.-Tubercular ulcers (ileum). (Lower two touched up to look like specimen.)!

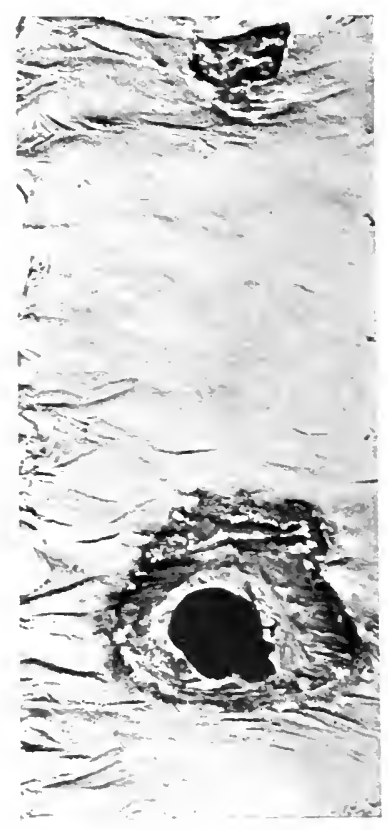

Fig. 30.-Perforating intestinal tuberculosis (adult with phthisis). ${ }^{1}$

ing tisste and fragments of mucosa), their edges may be smooth or irregular, soft or indurated, swollen or edematous, mindermined (Fig. 29) or bulging (rarely), and tubercles (often in a state of caseation) are frequently to be seen on the margins or near the lesions. In the enteric form of intestinal tuberculosis the ulcers are usually superficial. but may extend to the muscular tunjc, or, in rare instances, penetrate the bowel (Fig. 30) and cause peritonitis or abscess. They vary in shape and may be circular, elongated, or irregular: single or several lesions nay coalesce. Isually, however, the ulcers take the direction of the vessels and extent transversely to the long

1.Army Med. Nuseum. 
axis of the bowel, until they partiatly or completely encircle it, from whence they derive the name of girdle nleers (Figs. 29,31). On aceount of ensuing infittration, the cicatrices formed when the uleers heal, or enterospasm conseguent upon the irritation of the terminal nerve filaments, the bowel may be obstructed to a varying degres. Enteric tubercular ulcers show less tenclency to heal than those causert by catarrhat and other forms of utcerative colitis.

Enteric is sometimes described as granulating tuluerculosis, becaluse of the uneven surface of the mucosa produced by the prominence of the distended solitary glands and uneven surface of the mucosa after they have broken down.

The peritonewm shows evidences of the tubercular process in all varieties of the disease independently and when perforation has taken place. Sometimes, even in the mild cases of enteric tuberculosis, dark spots are to be seen upon the peritoneum over the base of ulcers; the serosa is slightly swollen and presents an uneven surface (Figs. 32, 33). When ulcers penetrate deeply into the intestinal wall. exulates are thrown out and the serosa becomes agghtinated to atjacent organs or structures and loops of intestine, and. in the presence of a perforation, local or general peritonitis ensues. which is of ten $\mathrm{com}$ plicated by a pyostercoral fistula. The intestinal wall varies in the ulcerative tubercutar colitis, and may be thickened by the exulates. thinned through the destructive processes, and, in either case, it may be resistant or fragile.

Lsually only diminutive ressels are eroded, but occasionally larger arteriotes are involved by the lesions, in which case profuse hemorrhages occur.

Enteroperitoneal (Deep Ulceratie) Tuberculosis. - The author regards enteroperitoneal (Fig. $3 \mathrm{I}$ ) as the most dangerous type of intestinal tuberculosis. because the destructive process progresses rapidly, is very extensive, always difficult and frequently impossible to arrest, and terminates fatally more often than other forms of the disease. It is serious because tubercular foci are present sooner or later in both the mucous membrane and serosa, and frequently the process starts simultaneously (hematogenously) in both. Again, in enteroperitoneal unberculosis caseation takes place early because of the virulence of the infection and activity of other pathogenic organisms, and the lesions resulting therefrom rapidly extend to and involve all the bowel tunics and bring about serions complications, viz.: constitutional manifestations, extensive ulceration, formation of scar-tissue and stenosis, toxemia, hemorrhages, papillomatous excrescences, and often perforation. adhesions, peritonitis, abseess, and pyostercoral fistula. which fint an outlet through neighboring organs or the abdominal wall, and with stricture where the patient recovers.

Very often the pathogenic bacteria which normally inhahit the bowel are active in these cases, and the destruction of tissue which follows is due more to them than the cascation of tubercles. When mixed infection is pronounced, elestruction of the dissue molles, and 
often the mucosa is destroyed over considerable areas or is ragged and marked by deep excavated ulcers. Here, as in other tubercular

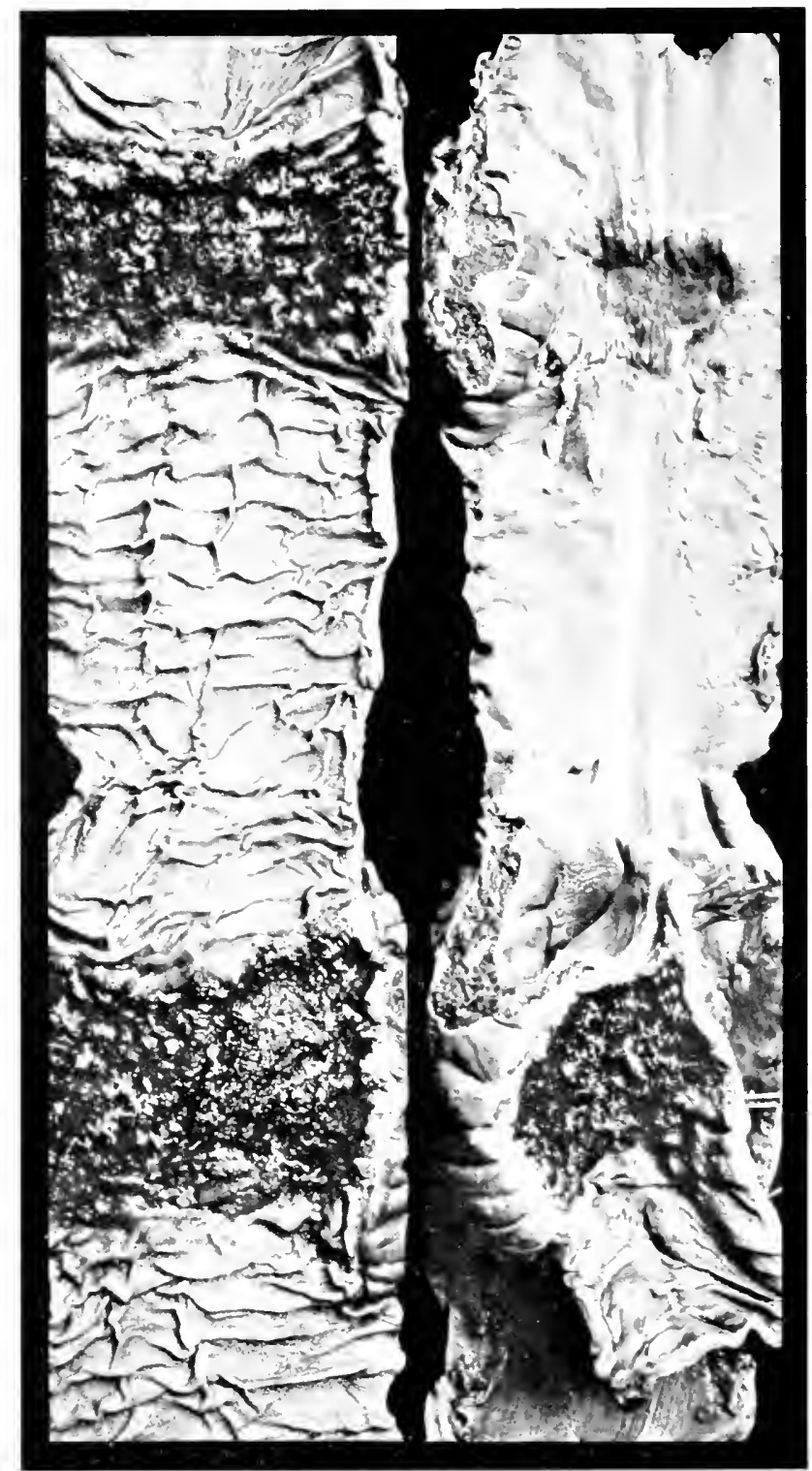

Fig. 31.-Extenşive enteroperitoneal tubercular lesions (ileum). Left: Nucosa. Right: peritoneal surface. ${ }^{1}$

ulcerative processes, the lesions most frequently take a direction at right angles to the long axis of the bowel.

s Army Med. Museum. 
A study of the author's personal together with pulplished cases indicates that the tubereulat process ustally originates in the mucosa and rapidly extends outwarel and involves the entire gut wall (lige. 32) and mesenterie glands ly contiguity, or through lympli-chanuets, while in a few instances it starts in neighboring glamels or the peritoneum and works inward or attacks the gut intrinsically and extrinsically at the same time, uncler which circumstatuers all the coats become involved with great raphelity. It is encountered witle regularity in the lower ileum, the cecum, ascending colon, and appendix, is not so frecpuently localized as the enteric type, but nore often con-

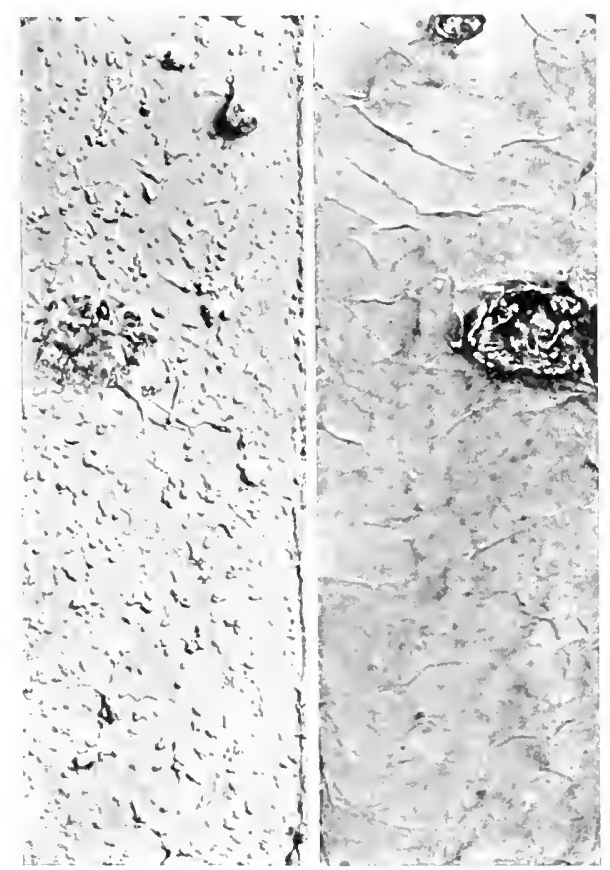

Fig. 32.-Enteroperitoneal tubercular ulcers and miliary deposits (ileum). I.cft: Peri toneal surface, Right: Mlucosa. ${ }^{1}$

tinuously involves sereral fect of the small and large intestine, lhough in some cases there may be intervening areas of healthy gert.

The author has olserved hut 3 cases whore the process appeated to be confined to segments of the small bowed, and in these it was more pronounced in the lower ilemm. The ileocecal region is usually diseased, and focimaty extend either to the ilem or fower colonic segments and rectum.

Because of peritoncal involvement and amount of infiltates thrown ont, the gut becomes glued to the appendix, coils of the smatl bowel, other organs, or parietes in the early stages of the discitse,

\section{Army Med. Museum.}


which interferes with peristalsis, incites enterospasm, obstipation, or alternating diarrhea and constipation.

The mesenteric glands are enlarged through inflammatory changes or. more often, tubercular foci. The tubercular process may be arrested hy calcification or the nodes may be partially or completely destroyed through caseation and suppuration, the discharge from which finds its way to the agglutinated bowel or abdominal covering to cause peritonitis or abseess.

Perforation occurs in only 6 to 8 per cent. of the cases of the enteric variety lecause the process is often arrested at the muscular tunic, but takes place more often in the enteroperitoneal type, owing to the fact that all of the intestinal coats are involved. The bowel in these forms of the disease has no fibrosclerotic covering like the neoplastic type to protect the abdominal cavity from infection in case of perforation, but the gut is usually encompassed by adhesions and infiltrates sufficiently to limit the infective process and prevent peritonitis. The barriers act as a shield against the formation of abscesses in the majority of instances, but are not sufficiently strong to restrain the pus and feces at all times. Consequently, abdominal pyostercoral fistulae are encountered in this far more frequently than the other types of intestinal tuberculosis. In the anorectal region this form of tuberculosis is a frequent cause of abscesses and fistulae, because there are neither a peritoneum nor adhesions to protect the perirectal tissues and limit the suppurating process.

Intrinsically, enteroperitoneal tuberculosis extends in the same way and has the same characteristics as the enteric type, with the exception that the ulcers rarely cicatrize and form stenoses, but may lead to more or less obstruction through the presence of complicating large or small papillomatous or polypoid growths which macroscopically and microscopically resemble benign adenomata.

Lsually in this form of the disease miliary tubercles are to be seen in patches scattered throughout the peritoneal covering of the inrolved gut (Fig. 32), some as smooth nodules and others as excoriated diminutive swellings when caseation has taken place. The serosa is always highly congested, and all of the bowel tunics are extremely fragile and readily tear when the slightest tension is made upon the gut or an attempt is made to separate one intestinal coil from another. In this class of cases the gut has a doughy feel and ulcers are not as readily discernible from without as in the enteric form, owing to the thickened condition of the howel. In hoth types of the disease caseation may take place and minute intermural abscesses may form which open through the peritoneum into the gut lumen.

Finally. enteroperitoneal tuberculosis more rapidly goes to a fatal termination through exhaustion of the patient or other complications. because there is but little tendency on the part of the tubercles to undergo calcification or of the ulcers to cicatrize. In other respects the changes which take place in the enteroperitoneal are similar to those of the ulcerative enteric type and require no further elucidation. 
Hyperplastic (Hypertrophic Neoplastic) Tuberculosis. - II istory and General Remarks. - Cecal (hyperplastic) tuberculosis as an entity was but little known until the present decade, and comparatively nothing is to be found in text-books on medicine and surgery concerning it. The current literature prior to i 890 contained but a few scattered articles upon this interesting condition, hut in the last few years several valuable contributions have been made to the subject, so that at present the etiology, pathology, symptoms, chagnosis, and treatment of ileocecal neoplastic tubereulosis is fairly well understood.

The excellent contribution of Hartmann and Pilliet (I fogr) served to excite great interest in this subject, and their publication was followed shortly afterward by other articles along similar lines. It remained for Copuet (1894) to call attention to and apply the term of hyperplastic to a frecuent type of cecal tuberculosis associated with tumor formation. Among those who have done most to increase our knowledge concerning ileocecal neoplastic tuberculosis the names of Durante, Alglave, Tissier, Tiery, Eisenhardt, Conrath, Crowder, Epstein, Mayo Robson, Keetley, Kidd, Fenwick and Dodwell, Lartigan, Campiche, Wagner, and Cumston stand out most prominently.

Hyperplastic, from a surgical standpoint, constitutes the most interesting type of tuberculosis, because here, as the result of infection, a tubercular mass forms which may attain considerable dimensions, yet it is one which can be removed with comparatively little danger and generally with the assurance that the patient will be cured. Because of their size and clense character these neoplasms have often been mistaken for fecal impactions, malignant growths, and other tumor formations, and have been designated as pseudoneoplasms and neoplastic u ubercular tumors.

The author has treated wo patients afflicted with this condition wherein a mistaken diagnosis of carcinoma was made by himsell and other diagnosticians, and the true condition was not discovered until operation. The first case was that of an old man who had been sick for months, was emaciated, and suffered from chronic obstruction, but whose case presented several features not characteristic of a cancerous process, and where at operation the non-malignancy of the trouble was confirmed by the picture presented and the examination of a section of the tumor. In the second case the tumor was assunced to be cancer by one physician and a fecal impaction incident to stricture by another, but the author held otherwise, on account of the patient's age (thirty years), the chronicity of the disease and unusual history. and advised an exploratory examination, which revealed the tubercular nature of the tumor.

Age appears to have a prectisposing influence in the production of neoplastic tuberculosis, and individuals between the ages of twenty and forty (those most actively engaged in life's work) are most frequently afflicted. This affection has been encountered in young children and the aged, but it occurs most often in the third decale. 
Guinon and Pater have recorded a case, that of a girl of four years, who suffered from this condition, and the author has operated for the relief of a large neoplastic tumor involving the cecum in a man sixty-eight years of age.

In Hofmeister's series (35 cases) there were 18 women and 17 men, and in Hartmann's 219 operated cases there were 112 females and ioz males.

Neoplastic tuberculosis has been detected in different segments of the small and large intestine, but show's a decided predilection for the cecal and ileocecal regions. In fact, it diminishes rapidly in frequency the further one gets away from the cecum, both as regards the small and large bowel, but it has been encountered in the rectum fairly often.

The appended table shows the distribution of the neoplasm in the 100 cases collected by Mummery:

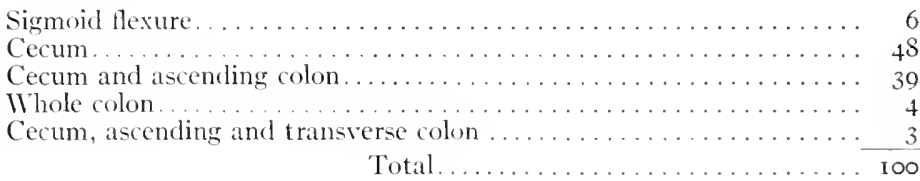

Conrath holds that the cecum is the seat of the disease in 85 per cent. of all intestinal tubercular infections, and the statistics of Hofmeister (9I cases) show that in 60 per cent. there was ileocecal involvement. To emphasize the frequency and surgical importance of ileocecal hyperplastic tuberculosis it is only necessary to mention that Hartmann, as far back as 1906, collected 219 cases operated upon for the relief of this condition, and that current literature demonstrates that this affection is being diagnosed and treated very much more often now than formerly.

IIyperplastic occurs less frequently than either enteric or enteroperitoneal types, as is shown hy Bernay's statistics of tubercular intestinal stricture, where the stenosis was induced by this condition in but 8 out of 70 cases.

Neoplastic is usually independent of the other forms of tuberculosis. but instances have been recorded where it and the enteric or enteroperitoneal form of the disease were found in the same and different segments of the gut.

Hyperplastic tuberculosis may involve a single segment of the small intestine alone, but is usually concomitant with ileocecal tuberculosis, and takes its origin at or in close proximity to the ileocecal valve, when the swelling may be felt in the small gut, but the enlargement is smaller and less resistant than neoplastic tubercular growths of the cecum, colon, or rectum.

Neoplastic tubercular tumors are usually single, but may be multiple, with sometimes healthy gut intervening, as occurred in the cases of Trendelenburg, where there were five, and of Borsch, where there were four, enlargements; or extensive segments of the bowel 
may undergo hyperplastic degeneration and leave the gut rigid and tube-like (sas-pipe intestine), similar to the cases reporet] by Lartigau and others. The writer possesses a specimen of a woman thirty-five years of age who suffered from chronic olstruction of this type. where the rectum and sigmoid flexure had completely undergone fibrosis and nothing short of excision relieved her.

Hyperplastic tuberculosis differs from other types of muberculosis in that it is chronic, and the disease progresses so slowly that dangerous manifestations may not appear for many months or years. It has been observed where this type of intestinal tuberculosis is associated with tubercular infection chewhere that the later is always less active than when it is a complication of the enteric or enteroperitoneal types. From what has been said it may be inferred that a person suffering from neoplastic uberculosis, alone or concomitant with the disease elsewhere, has a greater resistance and lives longer than individuals under similar circumstances who are afflicted with the more virulent forms of bowel tuberculosis. This power of resistance stands the patient well in case of operation, and recovery is quicker and more apt to be permanent following surgical intervention for the relief of neoplastic than other types of intestinal tuberculosis.

Neoplastic, like other tubercular processes, may be primary or secondary, and some contend that the disease is always primary. Certainly, statistics concerning tubercular lesions of the intestine prove that the hyperplastic form is secondary to involvement of the lungs, bowel, or other organs very much less often than either the enteric or enteroperitoneal types.

The following statistics of Mummery indicate the infrequency with which hyperplastic tuberculosis of the intestine is associated with tubercular disease elsewhere, viz.:

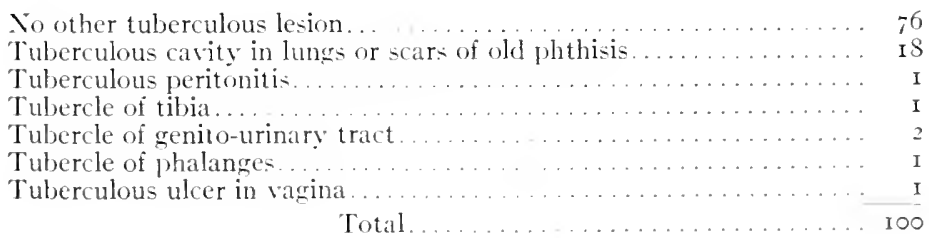

Hyperplastic tuberculosis of the intestine may be caused hy human or bosine bacilli, and the infection may be direct (from milk and food) or secondary to the disease in the lungs. In the boly, bovine (typus bovinus) are less active than human tubercle bacilli, and hecause of this and the face that the hyperplastic is more chronic than other formof tuberculosis, and the frequency with which these hacilli are swallowed in milk, cream, butter, cheese, and meat, it is not unreasonable to assume that primary infection is fairly common and induced by bovine bacilli; but when neoplastic uberculosis is secondary to lung involvement it is probably caused ly hmman tubercle hacilli.

Hyperplastic (neoplastic) tubercular swellings are usually present 
as sharply defined, fixed or slightly movable, firm, smooth, oval, or irregular tumor formations. Usually the hypertrophic process inrolves the entire circumference of the cecum uniformly, but occasionally the infiltration is more marked and the cecal watl thicker posteriorly. In a few instances the disease has been limited to the side of the bowel, producing an inward projection which caused obstruction and a similar enlargement externally. In the vast majority of cases the infiltration involves all the tunics of the gut (Fig. 33), but in a few necropsies it has been limited to one or more coats, under

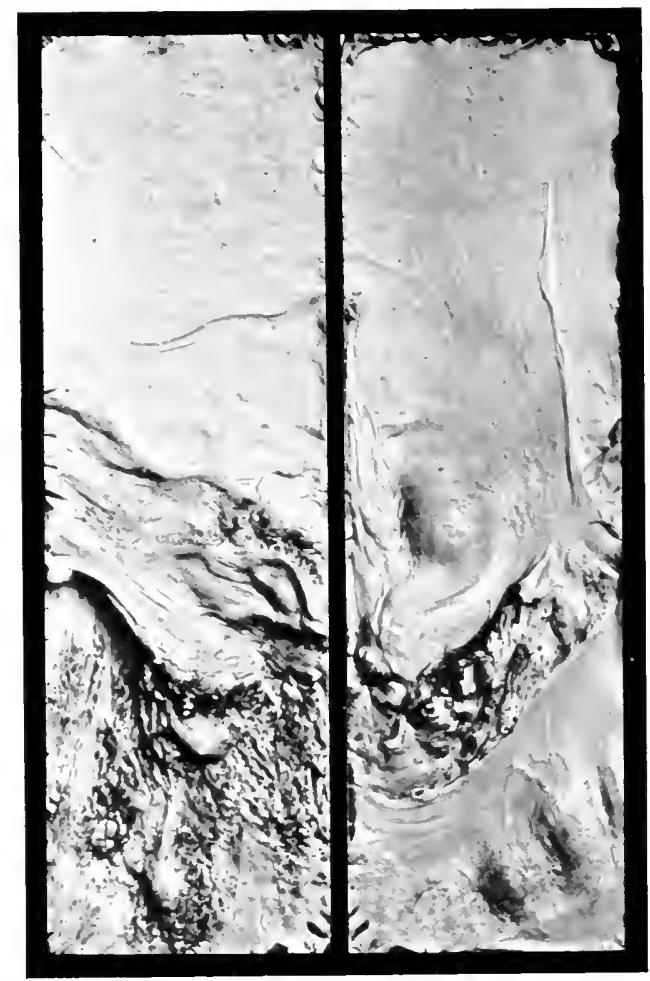

Fig. 33.-Left: Tubercular ulceration and tumor of cecum. Right: Tubercular tumor of peritoneum.

which circumstances the infiltrates are located principally in the subserosa and submucosa. The enlarged cecum, inchuding a part of the ileum and ascending colon when involved, are not entirely responsible for the large size of these tumors, since they are invariabiy encapsulated by a fibro-adipose sheath, varying in thickness from $\frac{3}{4}$ to $I_{4}^{\frac{1}{4}} \mathrm{in}$. $(2-+\mathrm{cm}$.), which, together with surrounding adhesions, help to make up the swelling. This pseudocapsule can be separated from the enlarged bowel by careful dissection during operation or autopsy. In exceptional cases where the neoplasm is of very considerable size

1 Army Med. Museum. 
the mass is made up of the hyperplastic cecum, its capsule, appendix, athesions, intestinal coils, and enlarged lymph-nodes glued together. The cecum is usually adherent laterally and posteriorly, but is rarely attached to the anterior abelominal wall by the adhesions.

The covering resembles that found about tubercular kidneys, and is macle up largely of normal adipose tissue and fat which has undergone fibrous changes. In the author's case, where perforation had taken place and a pericecal abscess was discovered, the fibrous capsule hat evidently limited the infection and prevented general peritonitis.

Upon section, hyperplastic tubercular tumors are resistant to the knife, grayish-white in color, and resemble cartilage, owing to the presence of an abundance of fibrous tissue. The gut wall varies in thickness from $\frac{1}{2}$ to $\mathrm{I}$ inch $(\mathrm{I}-3 \mathrm{~cm}$.) or more, a condition which explatins the size of the swelling and manifestations of olstruction. In the earlier stages the lumen may be but slightly occluded, but later it is often almost or quite obliterated, specimens having been examined where it was difficult or impossible to introduce a probe through the stenosis. In these cases the gut is rigid, non-collapsible, and the blocking results from the infiltration and thickening of the intestinal tunics, and not from the contraction of cicatricial tissue, as occurs in ulecrative intestinal tubereulosis. The stricture may be annular or tubular, the inner surface being smooth when the mucosa is intact, or irregular when ulcers or papillomatous growths are present, and the gut proximal to the stenosis is often dilated. The ofstruction is not always produced solely by hyperplasia, as there may be a concomitant tubercular enteritis or colitis, which results in the formation of cicatricial tissue, and that, in turn, helps to diminish the lumen of the gut through contraction.

In exceptional instances the smooth cartilaginous appearance of the thickened intestinal wall may show a carity the result of caseattion, under which circumstances the bowel has less resistance when compressed between the fingers.

In this class of cases the ensuing stricture never presents as a crescentic fold or membranous partition. Through the formation of hyperplastic tissue and shortening which follows, the ceemm and ascending colon are frepuently displaced upward. The circulation is considerably impaired in these neoplastic swellings, but occasionally one portion is found quite vaseular.

The mucous membrane in hyperplastic tulerculosis may remain intact, but more often it is involved in the tubercular process, and by mixed infection, fecal impaction, or other non-tubercular types of colitis is inllamed, eroded, or ulcerated. Because the continuity of the mucous membrane is occasionally broken some authorities contend that fibrosis in these cases is secondary to the ulcerative process, but this riew is refuted by the fact that many cases of neoplastic tuberculosis have been reported wherein the mucosa remained normal and presented no evidences of scar-tissue. Sometimes both the hyper- 
plastic and ulcerative types of tuherculosis are present in the same case, under which circumstances the fibrous change in the gut is attributed to both. In this affection the mucosia is usually thrown into transverse folds through atrophy of the submucosa, and is considerably thickened owing to the tubercular colitis. Tuhercles are occasionally to be seen and may undergo caseation, but in aggravated cases, where considerable sized areas of the mucous membrane slough. it is due to impairment of its cireulation or breaking down of tubercular fuci in or beneath it. When there are a number of such large ulcers the surface of the mucosa may be mammillated or the membrane may be undermined.

The most characteristic feature of hypertrophic tuberculosis is the presence of numerous polypoid or papillomatous growths having pedunculated or sesile attachments which project into the gut lumen. These excrescences vary in number, size, and shape, but are usually from birdshot to hazel-nut size. clubbed at their extremities, and are sometimes seen projecting from either side through the ileocecal ralve. In one case they may be isolated and few in number, or in another numerous and segregated. They are not of tubercular, but inflammatory origin, although caseating foci have, in rare instances, been found in then. They originate in the submucosa and consist mainly of connective-tissue and round-cell infiltration, and have an epithelial covering similar to that of the mucosa. Lartigau and others found neither tubercles. necrosis, nor giant cells within them, but tubercles have been observed upon their mucous covering and elsewhere.

Then the mucosa is inrolved in the tubular process, tubercles containing giant and an abundance of round cells. and sometimes necrotic or caseating areas. are observed, but in simple catarrhal inflammation only round-celled infiltration is discernible.

Hyperplastic tuberculosis originates in or attacks the submucosa early. The proces is more active here and in the subserosa than elswhere, and. as a result, active round-celled infiltration ensues and the submucosi becomes considerably thichened five to eight times normal. Whitish in color. and resembles cartilage in its hardness and translucency. Tubercles. associated with or without caseation, giant, spindle, and small round cells are encountered here with more or les resularity: polymorphonuclear and granular eosinophil cells have been seen in this laver near the mucosa, and tubercle bacilli in small numbers may be found when a sufficient number of sections are examined. It $\mathrm{i}$ here and in the suberosa that the conflict takes place hetween the tuhercular invasion and the inflammatory reaction inaugurated br the sistem to combat extension of the disease, and in consequence there is a piling up of hyperplastic tissue in these tunics.

( Wing to the fact that the mucusa and it-vestel- are so markedly involved in this form of tuberculosis, some authorities maintain that it is of hematogenoms origin.

The muscular tumic is also thickened in hyperplastic tuberculosis owing to round-celled infiltration, and not to any increase in the num- 
ber and size of the muscle-fibers. When there is a true bypertrophy in these cases it is compensatory, and takes plate in the dilated sedyments of bowel proximal to the ofstructing mass. In neoplastic tuberculosis the muscle bundles appear paler than wormal and the fibers are often separated by connective-tissue formations, fut the changes in the musculature is much less marked than in the tunics on either side.

The subserosa ordinarily is extensively involved in the hyperplastic process and is greatly thickemed by round-celled inliltration. The cellular elements here are the same as in the submocosa, with the exception of the accumulation of fat cells in the vacuolated spaces. Tubercles are rarely found in this tumic, but when seen present occasionally minute absesses formed through the breaking down of cascous deposits, and when perforation takes place a localized or general peritonitis ensues. The peri- and endarteritis always present in tubercular manifestations are pronouneed here on account of the vaseularity of the subserosa. Conrath holels there are two ypes of neoplastic tuberculosis, viz., subserous and submucons, and that the former is more frequent, is causerl by infected ileocecal glands, and involves other coats of the bowel through lymphatic extensions.

The large amount of hyperplastic tissue, together with the filorous bands existing in the subserosa, are largely responsilile for the hard, tumor-like formation noticeable in neoplastic tuberculosis.

The peritoneal coat is the least changed of the intestinal tunics, is slightly increased in thickness, and may at times become dotted over with tuberctes or involved by athesions which distort the bowel.

In neglected cases of hypertrophic tuberculosis, where roundcelled infiltration is extensive and the gut lumen diminished, the intestinal coats proximal to the stenosis are thinned and may be ruptured or perforated. while the bowel is distended, or when tubercles undergo caseation. Under such circumstances local or general infection takes place, and pyostercoral alscesses form and empty into other organs or through the abdominal wall.

Appendiced ineliement nearly always occurs in hyperplastic ileocecal tuberculosis. Tsually the involvement is confuned to adhesions which bind it to the tumor mass and angulate or displace it. When the hyperplasia attacks the appendix the process is found more frecuently at the base than the lip or other parts, owing to extension of the disease from the ileocecal region. The author recently attempted appendicostony for the relief of ulcerative colitis, lut this procedure was abandoned for cecostomy because of the discased condition of the appenelix, which called for an appendectomy. The appendix was more han four times its normal size, indurated, studeled here and there with tubereles, and when opened the mucosa and other tunics were found greatly thickened and compesed largedy of fibrous tissue: upon section it showerl the usual hyperplastic changes common to neoplastic tuberculosis. Lese frepuenty the infection occurs primarily in the appendix and extends to and involves the cecum and adjacent struetures, and, when the disease is virulent, per- 
foration of or, in rare instances, complete destruction of the appendix ensues.

Glandular Inioliement in Neoplastic Tuberculosis.-1leocecal adenopathy in one form or another invariably occurs in hyperplastic intestinal tuberculosis, manifests itself early in the disease, and may be limited to the ileocecal angle, where it is always marked or extends to lymph-nodes at a considerable distance from active tubercular foci. In fact, enlargements of the mesenteric, retroperitoneal, and sometimes omental glands have been observed in these cases, but most often the nodes in the vicinity of the cecum and along the ileocecal ressels are chiefly affected. Occasionally one finds single or multiple small and large glands bound up in the inflammatory mass. The enlargement of the lymph-nodes may result from a simple or tubercular colitis or mixed infection in hyperplastic ileocecal disease, or the

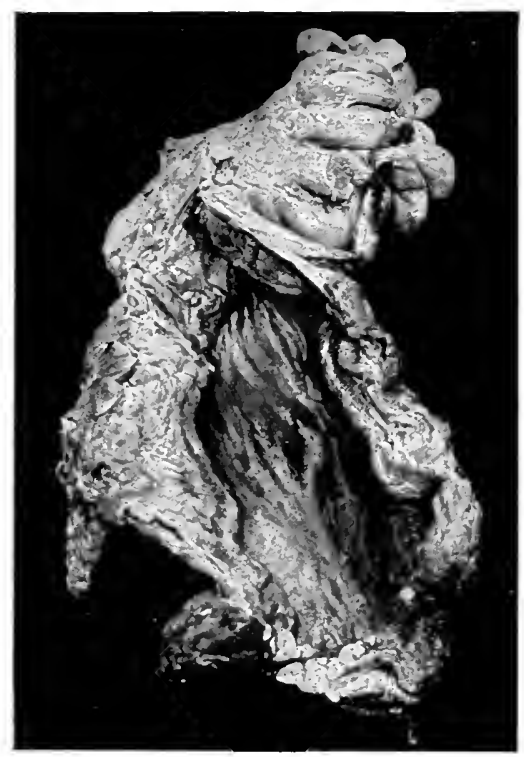

Fig. 34.-Stricture of the rectum due to chronic proliferating stenosing tubercular proctitis (fibrosclerotic tuberculosis). Note thickening of rectal wall. (Author's case.) the blood-vessels to undergo connective-tissue changes independent of the formation of cicatricial tissue, which converts the pliable intestine into a short or long fibrous tube.

When olstruction results from this condition the mucosa is rarely ulcerated, and when it is, the lesions are secondary. In cases operated upon by the author and specimens examined by him presenting the picture of fibrosclerotic tuberculosis the mucous membrane was poorly nourished, of a pearlish-gray color, and thrown into longitudinal 
or concentric rings through contraction of the outer unics, and there were no evidences of healed ulcers. In one case a section was examined and pronounced by the pathologist hibrosclerotic ubereulosis, from the presence of tubercular tissue and extensive connective-tissue changes in the submucosa.

It has been customary to incluele this variety when grouping tubercular manifestations of the intestine, and for lhat reason the writer has separately discussed fibrosclerotic or atrophic tuberculosis, though, according to his view, its pathology does not differ suffeicntly from that of the hyperplastic ype to entitle it to individual consiteration. It is true that sometimes the so-called tubercular gas-pipe intestine is encountered, and the changes within the gut cannot be attributed to the cicatricial tissue of healed ulcers, nor is the bowel encapsulated by the fibro-adipose covering present in true neoplastic tuberculosis, but otherwise the condition closely resembles hyperplastic tuberculosis, and it is probably a variation of it.

Again, it is likely that many strictures ascribed to atrophy or sclerotic changes have, in reality, been induced by scar-tissue following the healing of ulcers in the mucosa. Busse records 3 cases bearing out this argument, and say's that, upon the basis of his fundings, "there is no proof of a non-ulcerating submucous tuberculosis of the intestine," and, further, that "the previous existence of ulcers must be assumed as the cause of stricture," a view maintained by many modern authorities upon this subject.

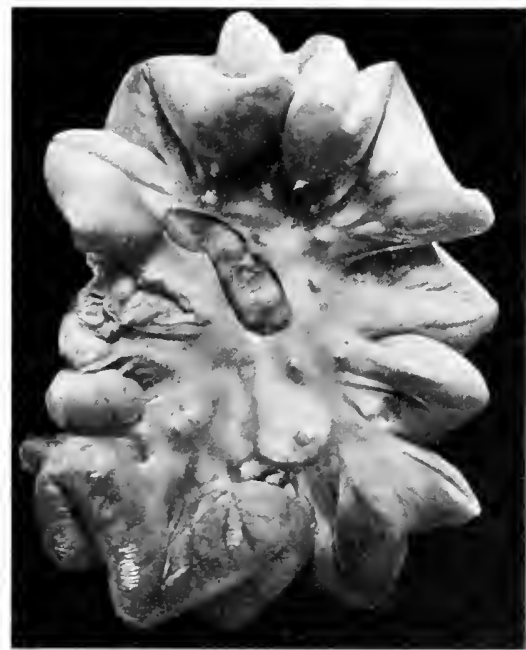

Fig. 35.-Tuberculosis of the mesentery and lymph-nodes. From the evidence at hand it

would appear that the type of tuberculosis under discussion has not an entity, but it is a condition with peculiar features, which may be induced either ly enteric (ulcerating) or hyperplastic tuberculosis.

Glandular Tuberculosis. - This type of tuberculosis will receive but brief attention because of its rarity, its independence of intestinal involvement, and the infrequency with which it causes diarrhea.

The mesenteric glands adjacent to and lymph-nodes situated at a considerable distance from the bowel may become secondarily involved in intestinal tuberculosis, or, less frequently, they may become infected primarily and the disease extend to the bowel (Fig. 35).

Primary infection of the mesenteric glands may occur at any age, but is most frepuent in females between the ages of three and ten. and 
the group of glands most commonly attacked is situated in the ileocecal region (ileocecal adenopathy). Vautrin has recorded 3 cases of primary involvement of these glands, and maintains that the abundant follicles and rich network of lymphatics favor infection of glands of this region.

It has been shown elsewhere that tubercle bacilli can penetrate the intestinal wall when it is healthy or diseased, and under such circumstances it is easy for the bacilli to pass from the bowel (by way of the blood or lymph-channels) to and infect the mesenteric, retroperitoneal, and omental glands primarily or secondarily, both with and without ulceration of the mucosa. Hemmeter, in 56 necropsies upon persons who died of phthisis, observed tuberculosis of the mesenteric, omental, or retroperitoneal glands in 12 instances, in 6 of which the entire intestinal mucosa was intact and in + there was enteritis or colitis.

The mesenteric glands, like the intestine, are involved secondarily very much more frequently than primarily, and when the disease originates in the lymph-nodes it is probably caused by bovine bacilli which have gained entrance to the bowel.

Concerning primary and secondary glandular infection in children, Carrière says that the mesenteric glands may become secondarily infected, and this is the more common type of the disease. Primary tuberculosis of these nodes was found by him in only 2 of 200 cases. Among 2000 examined patients it conld be demonstrated in only 9 per cent. of the cases.

Secondary tuberculosis of the mesenteric glands is common in children, and Carrière observed it in connection with pulmonary tuberculosis in 30 per cent.; tuberculous peritonitis, to per cent.; tuberculous enteritis, 20 per cent.; tuberculous glandular affections, 5 per cent., and bone-and-joint tuberculosis in 5 per cent. of the cases.

One or many of the lymph-nodes may be diseased, and, according to circumstances, the enlargement may present as a minute or large nodule, or single or multiple conglomerate masses, which may be mistaken for impactions, cancer, and other tumor formations. Such swellings are exceptionally palpable, but, as a rule, they are not detected except during operation or at autopsy. It is well to remember, however, that the mesenteric lymph-nodes may become swollen as a result of other types of infection, and that they are not necessarily tubercular even when such a process is present in the lungs, intestines, or elsewhere.

Tubercular glands behind the peritoneum and in the omentum or mesenteric folds may caseate and suppurate, or, as is usually the case, undergo calcareous degeneration.

In the author's cases of intestinal tuberculosis extensive secondary glandular involvement has generally been observed.

When the diseased glands are few and small, meteorism may ensue through interference to the mesenteric nerves and vessels, but when they are large and numerous, intestinal stenosis may result from pressure or the formation of contracting adhesions and connective 
tissue. The author has observed one case where the mesenteric gland were enlarged and the arljacent bowel had undersone fibrosis and presented a slistening appearance and leathery feel, and there is a unique specimen of ilencecal adenopathy in the pathologic laberatory of Columbia University wherein the slandular mass is hen s-eres size and caused intestinal olstruction.

The enlarged gland- may reduce in size spontaneously or, when tubercular, undereso calcification and feel like bullets within the mesentery, or they may suppurate and discharge into the browel or the peritoneal cavity, causing a local or general peritoniti-

When the noxes break down or a localized peritonitis re-ults from other causes, they may become agglutinated to adjacent coils of

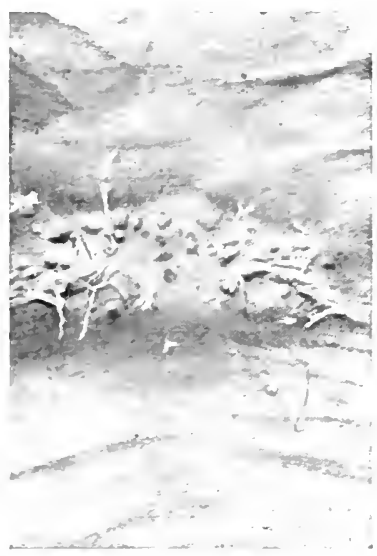

Fin. 36.-Tubercular deposits in the peritoneum opposite ulcers in the small intestine.

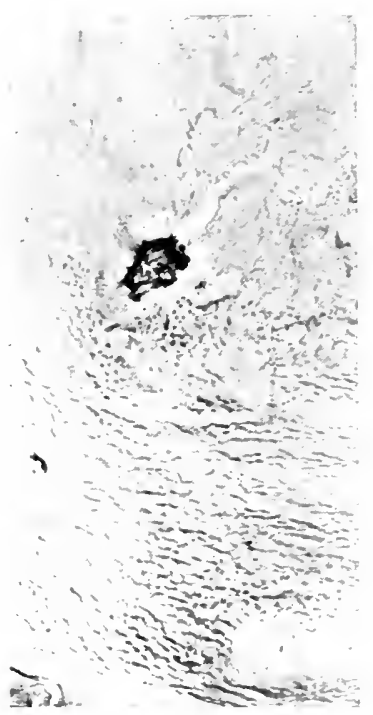

Fir. - - Ciantric tubercular ulcer. Moman twenty-three. phthisis. 1

intestine and form a tumor mass discernible by palpation and inspec(ion.

In -tudying intestinal tuberculosis it is well tr) bear in mind that the glands associated with and those located at a con-ideratsle ditance from the tuberculous bowel may become affected primarily or secondarily in the various types of intertinal tuberculosi-.

Peritoneal Tuberculosis Fig. 36).-The peritronem is nearly alwars involved in intestinal tuberculosis incirlent to the presence of tubercle bacilli or mixed infection or exteneion of the inflammators process from disease within the bowed, and nearly alway- complicaterl by diarrhea.

Peritoneal tuberculosi- is variable and exceedingly difficult to

$$
\text { 'Army Med. Museum. }
$$


classily from either a clinical or pathologic standpoint, but Murphy's arrangement of this type has been generally accepted, viz.:

(i) Disseminated, exuclative, miliary, non-confluent, or serous (ascitic) variety.

(2) Nodular, ulcerative, or perforative (the least frequent) variety.

(3) Aclhesive, fibroplastic, eystic, or obliterative variety.

(4) Suppurative, circumscribed, or general mixed infection.

One may gain some idea of the frequency of tubercular peritoneal involvement in tubercular subjects by studying the postmortem statistics of Cummins, who found this condition in 11 per cent. of 3405 autopsies, and the Io7 cases investigated by Philips, where peritoneal involvement was complicated by tuberculosis of the lung in 99, intestine in 80 , and pleura in 60 instances.

Tuberculosis of the Stomach.-Gastric tuberculosis (Fig. 37) requires mentioning here because when the stomach is involved independently or with the bowel, indigestion and diarrhea are annoying complications. The infection is encountered here less of ten than in the upper small bowel, where it is rare, and the museums contain but few specimens of gastric tuberculosis and not many cases have been reported.

Gastric tuberculosis may be primary or secondary and the accompanying lesions unimportant or extensive, and it is usually mistaken for some other disease or the diagnosis is not made until it has become serious.

Maylard has collected several interesting cases of gastric tuberculosis; Ellis has reported 2 instances where the disease was secondary to pulmonary tuberculosis, and Clayton and Wilkinson collected 173 and reported 43 cases of their own, discovered during autopsies upon tubercular subjects.

The pathology, symptoms, diagnosis, and treatment of gastric tuberculosis are practically similar to those of intestinal tuberculosis, and do not call for further consideration here.

Tuberculosis of the Appendix.-Appendiceal tuberculosis occurs more often than is generally supposed, and the appendix may be primarily (rare) or secondarily involved, in which case the disease may be limited to it (Fig. 38), or extend to the cecum and from thence to the small intestine or colon. Tuberculosis shows a predilection for the appendix on account of its structure, liability to catarrhal inflammation, richness in adenoid tissue, lymph-follickes, and pathogenic bacteria, imperfect drainage, and its attachment to the cecum, the most common site of intestinal tuberculosis. Crandiani says that ileocecal tuberculosis originates secondarily in most cases following tubercular lesions of the appendix, but the concensus of opinion is that the appendix usually becomes involved through extension of the disease from the cecum.

Tuibercular foci here are frequent in cases of disseminated intestinal tuberculosis, and the appendix is tubercular in from $\mathrm{I}$ to 2 per cent. of alt cases of appendicitis. The hyperplastic type involves the appendix 
less frequently than other forms of intestinal tuberculosis, and when it does, in most instances the infection occurs through contiguity with the cecum. Frequently, where the appendix is massed with ileocecal tubercular tumors, it is not diseased, and because of this some authorities hold that tuberculosis of the appendix is rare.

Of 27 cases of bowel tuberculosis recorded by Eisenhach, the appendix was involved in 4 . In $14+$ antopsies on patients having tubercular appendicitis Leseur found the appendix was solely affected in 8.3 per cent; Fenwick and Dodwell, in 2000 autopsies upon tubercular subjects, observed that the appendix was involved solely in the tubercular process in 17 cases, and was associated with lesions in other parts of the bowel in 50 per cent. of the cases.

Gandiani has recorded an interesting case of tubercular appendicitis in a girl twenty years of age, where the disease was in the initial stage and more fully developed than in the cecum, from which he argued that it originated in the appendix and involved the cecum secondarily.

Tuberculosis of the Colon and Sigmoid Flexure.-The discase is encountered in the ileocecal region in about 85 per cent. of the cases, but in the colon and sigmoid it usually occurs at the points most often subjected to

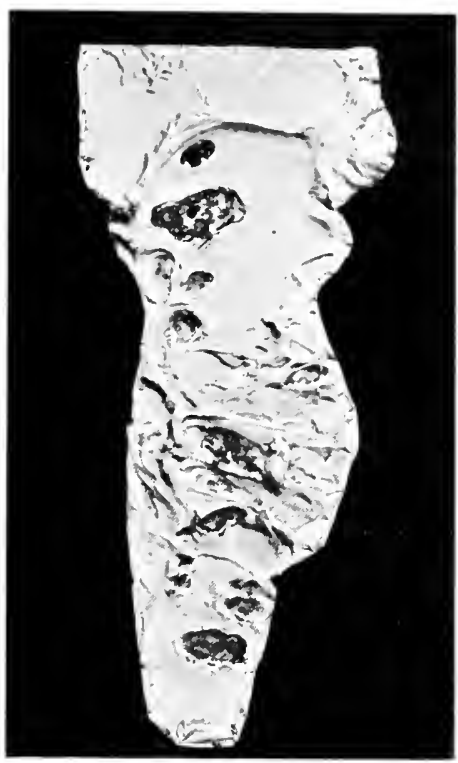

Fig. 38.--Tubercular ulceration of the appendix. ${ }^{1}$ trauma by the feces, viz., hepatic, splenic, and sigmoid flexures. When the lesions are in the sigmoid they are more numerous and larger than elsewhere in the colon.

Tuberculosis of the Rectum.-Anorectal tuberculosis is quite common, and the infection is more difficult to control here than when it is in the small or large intestine, and is frequently complicated by abscess and fistula. Mixed infection plays an important part in rectosigmoidal tuberculosis, and persistent diarrhea, with an abundance of pus, hlood, and mucus in the stools, invariably complicates the disease here.

\footnotetext{
${ }^{1}$ Army Med. Museum.
} 


\section{CHAPTER XXI}

\section{TUBERCULAR ENTERITIS, COLITIS, AND ENTEROCOLITIS (INTESTINAL TUBERCULOSIS), DIARRHEA IN (Continued)}

\section{SYMPTOMS, COMPLICATIONS, SEQUELE}

General Remarks.-In many respects the symptoms and complications of bowel tuberculosis are the same as those produced by other inflammatory and ulcerative affections of the intestine, the most prominent of which are diarrhea. intermittent attacks of abdominal tenderness, pain, cramps, and the presence of mucus, pus, and blood in the stools. What has been said applies more particularly to the disease in its incipient stage, because, when it is more fully dereloped. the patient presents a sallow complexion and the usual characteristic appearance of the tubereular subject, but these peculiarities may be mistaken for other forms of enteritis and colitis accompanied by mixed infection and auto-intoxication, where the patient presents an unhealthy appearance and other manifestations which closely resemble those of bowel tuberculosis.

The differentiating symptoms between intestinal tuberculosis and other ailments likely to be mistaken for it have been outlined in the following chapter.

The manifestations induced by bowel tuberculosis are similar in some and differ in other cases. hecause of the varying types of the infection. They also vary in all stages of the disease and when the lesions are located in different segments of the bowel. The disturbances which accompany tuberculosis of the small intestine are less severe than those induced by the disease at the ileocecal region or colon, and ulcerative and neoplastic lesions situated in the lower sigmoid and rectum excite a more intense type of diarrhea, pain, and tenesmus, and the stools contain a greater amount of pus. blood, and mucus than when the foci are in the upper colon.

In all forms of intestinal tuberculosis the symptons are fewer and les severe in the earlier stages and when the infection is mild than when the disease is virulent or has fully developed. Tuberculosis would not induce such distressing manifestations nor terminate fatally so often were it not for the mised infection incited by the pathogenic intestinal micro-organisms as sonn as the continuity of the mucosi has heen broken, a complication which oecurs in all forms of this disease, but is more marked in enteroperitoneal and ulcerative types. where the denuded areas are numerous and large.

While it is true that bowel tuherculosis may originate primarily in the gut, the lact remains that in most instances it is secondary to disedee in the lungs and other organs, conseguently, in making a diagnosis it in necessiry to bear in mind that very often the symptom- 
complex is made up of the manifestations arising from tubercular foci in both the lungs and bowel; hence they are more apt to be confused than when either the one or the other is involved. Knowing this, it is easy to understand why the destructive process causes less suffering and progresses more stowly in primary than seondary intestinal tubereulosis, where the patient is already in a deplorable condition from phthisis before the sputum infects the bowel, following which rapid decline and death ensue muless the discase is promply arrested.

In so far as the intestine is concerned the origin of the discase is unimportant, because the clinical picture presented by the intestinal lesions in the various forms of tulerculosis is the same in leoth primary and secondary.

Before considering the symptomatology of the individual forms of intestinal tuberculosis, the author would recall that, when the lungs are involvel, the patient suffers from an evening temperature, nightsweats, emaciation, impaired digestion, discomfort in the chest, cough, hemorrhages, and the usual indications of phthisis in acklition to charrhea and other gastro-intestinal disturbances.

Enteric (Ulcerative) and Enteroperitoneal Tuberculosis.-Because of the similarity of the clinical pictures produced hy ulcerative (granular) and enteroperitoneal tuberculosis of the intestine, particularly in their earlier stages, it would be impracticable to consicler their symptomatology separately. In fact, one encounters great difficulty in distinguishing between the manifestations induced by them and other types of enterocolit is.

To avoid repetition the author in his general remarks upon intestinal tuberculosis pointed out the manifestations in these cases when the lungs are intolved, and nothing more is necessary here than to empluasize the symptoms which arise from foci in the lowel and other organs.

In the incipient stages the unimportant symptoms, such as diminisher appetite, imperfect digestion, softening of the evacuations or occasional slight diarrheal attacks and discomfort in the region of the navel, are not sufficiently characteristic to leat one to suspect the tubercular nature of the disease, and they are usmally ascribed to gastro-intestinal catarrh or colitis from other causes. When the tubercular process makes rapid stricles, characteristic manifestations quickly become obvious, but this is exceptional and, as a rule, the symptom-complex gradually becomes exaggerated until, at the end of a few weeks or (at most) months, the clinical picture is sufficiently clear to cause one to suspeet intestinal tulerentosis, particularly when the patient already has phthisis. By this tine he looks very ill, has lost considerable weight and strength, and suffers from malaise. hats anemia, a sullow complexion, poor appetite, concentrated urine, furred tonsue. foul breath, indigestion, a slight elezation of temperature, incredsed pulse-rate, occasional night-sceuts, is restless and worried over his condition, and suffers from diarrliea, a discharge of pus, blesel, and mucus in the stools, abdominal pain, tenderness, and gas distention, which reguire separate consideration on account of their importance. 
Diarrhea is the chief srmptom of bowel tuberculosis, varies in different cases accordine to the stage, location, and extent of the disease, and the presence or absence of other complicating intestinal affections.

In the incipient stage. where the inflammation dominates the ulcerative process, the morements become softer. contain a small amount of mucus, and may take place with normal or increased frequency, but later, through the formation of new and extension of old lesions and the part played by mixed infection, an aggrarated type of diarrhea develops. and the patient mav have anywere from eight to fifteen movements within twenty-four hours, principally at night. on account of which this condition has been designated by some authorities as diarrhea nocturna.

In some instances diarrhea may continue over a considerable period, but usually it is intermittent, during which time the patient may not have more than two or three stools daily. and then, through action of the food or other inexplicable cause, there is an acute crisis accompanied by cramping pain, sensation of weakness, anorexia, and abdominal distention, which is followed by the escape of the gas and several Huid movements having a penetrating. fetid odor, and containing remnants of undigested food and fats. Freeing the bowel of retained foul gases and feces brings immediate relief. When the disease is not arrested the crises gradually increase in number and severity until diarrhea is persistent and the griping pains are almost continuous. Under such circumstances. opiates, bismuth, and other therapeutic remedies. administered to alleviate suffering and diminish frequency of the evacuations, frequently fail to relieve the patient. The movements are semisolid, at first mushy or lumpy and light colored, and finally fluid, and contain mucus. blood, and pus in amounts corresponding with the number and size of the lesions.

When a ressel of considerable size and high up is involved the feces frequently resmble coffee-grounds (black diarrhea), owing to free bleeding and contained clotted blood. Stools of fresh blood indicate one or more bleeding ulcers in the rectum. but when clear blood precedes the movements, it is suggestive of hemorrhoids, an ulcer, or fissure at the anal margin. Pus and blood are found less often and in smaller quantities in tubercular than other types of colitis, excepting catarrhal. Frequenty the blood in the evacuations of persons suffering from intestinal tuberculosis is occult. and is found only by the microscope as hematin crystals and blood-cells.

The author has often observed that when tubercular lesions are situated in the sigmoid flexure or rectum they cause a much greater frequency and fluidity of the stools than when located in the colon or small bowel, and that ulcerative affections are always more pronounced in the colon than in the small intestine. the ulcers being larger and more numerous. The frequency and solidity of the stools are modified in two ways, viz., through impairment to or destruction of large areas of mucosa, which limits the absorption of water and causes 
irritation to the exposed nerves; this reflexly leads to abnormal peristalsis, glandular secretion, and the pouring of mucus into the bowel, which softens the fecal content and increases the number of evacuations. When the small intestine is involved the mosements are fewer in number except when the colon is also ulecrated.

In these cases there may be as much irritation and the contents are rapidly rushed through the small bowel on account of the increased peristalsis, but, owing to their longer retention in the healthy colon, the watery content of the stool is absorbed and the feces may be evacuated soft or firm in consistence. In most instances, however, the large intestine, through infection or irritation induced by the discharges from above, becomes diseased when the stools are materially increased.

Apart from the influence of location, the number of daily evacuations in intestinal tuberculosis, the degree of digestive disturbance, the amount of tenclerness and pain, and the quantity and character of the discharges, pus, mucus, and débris, bear a direct ratio to the extent of the lesions, viz., they are more aggravated when the ulcers become numerous, deep, and extensive, and less annoying when the lesions are few, small, and superficial.

While diarrhea is the pathognomonic simptom of bowel tuberculosis, it is well to bear in mind that occasionally this class of patients do not suffer from frequent movements, but are troubled with obstipation or constipation alternating with diarrhea, induced by enterospasm, excited through nerve irritation in the ulcerative and enteroperitoneal types where the mucosa is denuded or obstruction caused by the tumor in hyperplastic tuberculosis; or possibly, through mixed infection, a virulent toxin is produced in the gut which reaches the nervecenters and produces a paralytic or inhibitory influence upon the nerves controlling the motor apparatus of the gut, resulting in irregular and infrequent evacuations. In several cases where constipation was marked the inner coats of the intestine were studeled with small lesions or extensive denucled areas of several coalesced uleers, or by an obstruction induced by a tuluercular neoplastic swelling.

Pain and Tenderness.-Pain in one form or another is encountered in all types and stages of tuberculosis, but its character and severity depend largely upon the following conditions, viz.: (a) the degree of digestive disturbances and $(b)$ the number, extent, and location of the gut lesions. The most intense suffering (so-called acute crises with griping pains) is usually incited by indigestible food, which causes the formation of irritating gases and enterospasm accompanied by agonizing, sickening, disseminated pain, which continues until the putrefying food and gases are expelled. Between attacks the patient usually feels uncomfortable or occasionally suffers pain in the region of the navel. During crises, peristalsis is active, and the patient complains of loud intestinal noises, and, when enterospasm is a complication, the bowel is rigid and a'ermicular movements in the proximal intestine can of ten be seen or felt through the parietes. 
Extensive ulcers have been revealed at autopsies that caused little if any pain during life, but, as a rule, it will be found that the patient's suffering bears a direct ratio to the extent of the destructive process. Much of the discomfort complained of is caused by the irritating discharge which remain in contact with the ulcers or passes over them. Occasionally some lexion = are sluggish. while others are extremely sensitive to the discharge and bowel contents. which explains why it is that the patients sometimes complain of intense pain at a definite point along the bowel preceding, during, and following an evacuation. Naturally. when ulcers are numerous and scattered throughout long segments of gut. suffering is proportionately greater, and it is in this type of cases that one observes tenderness upon pressure throughout the length of the colon and often over the entire abdomen, but in some instances the sorenes in attributable to backed-up gases or enterospasm. Pain and tenderness are usually emphasized in the lower colon. sigmoid, and rectum. because by the time the feces reach these part they are firm or nodular. difficult to propel, and cause trauma to the ulcer in their downward passage. Lesions situated in the lower rectum and at the anus invariably induce greater pain than when higher up because here the bowel is more bountifully supplied with sensory nerve. it is very narrow, and surrounded by the muscles which contract and produce sphincteralgia when the ulcers are traumatized during defecation.

The author has observed that in ulcerative (enteric) and enteroperitoneal intestinal tuberculosis crises occur more frequently, last longer. and cause greater suffering when there is an enterocolitis than when the lesions are confined to the large bowel. and that comparative slight gas accumulations within the gut of ten induce intense pain, while filling of the bowel with water or an irrigating solution does not, which would indicate that the pain was not due to the distention, but to the irritating quality of the gas. The rectum is nearly always dilated in diarrheal subjects and feels like a gas-filled cavity, while in con-tipation the reverse is true, and the finger comes in contact with the rectal wall as soon as introduced.

Owing to repeated or continuous gas distention many sufferers from intestinal tuberculosis present a bulging abdomen not unlike the pot-belly of the enteroptotic subject. but exceptionally the abdominal wall is contracted. This class of sufferers have a habit of rubling the belly, probalily because through the massage they are alite to work the ga- downward, to be expelled. while others obtain relief by leaning over and pre-ning the abdomen against the legs during the cries. When the tulercular proces is extensive and inflammation i- acute the abdomen is often firm or rigid through muscular contraction incited hy reflex impulses. and not a a result of accumulated gas.

Malsh lays-tres- upon this manifestation. and has compiled statistic = to show the relative frequency with which diarmea abdominal pain. tenderness upon pressure. and rigidity of the abdominal muscles 
occur in tuberculosis. He found that of 76 patients who had tubercular ulcers of the intestine, 30.6 per cent. had tenderness, 25 per cent. abdominal rigidity, and 6.58 per cent. all four symptoms, while in the remaining 24 cases of intestinal tuberculosis, where there were no ulcers, tenderness was manifest in 29.2 per cent., rigidity in 25 per cent., and diarrhea, abdominal pain, tenderness, and rigidity $i n+$ pere cent.

The author has oftein observel the tightness of the aliclominal wall, but has not encomtered muscular rigidity with anything like the frequency with which it occurred in Malsh's eases.

Iecrative and enteroperitoneal tuberculosis, like the neoplastic form, occurs most frequently at the ileocecal angle, and when the process is acute or involves the appendix, ileocecal tuberculosis in the earlier stages has been mistaken for appendicitis.

When intestinal uberculosis is permitted to reach the final stages through extension of the caseating and ulcerative process, it extends deep into the intestinal tunics or perforation takes place, circumscribed or general peritonitis, abscess and fistula, opening through the abdomen in the right iliac region are complications, or the patient may die from sepsis, exhaustion consequent upon the diarrhea, or from phthisis, where intestinal infection is secondary 10 phthisis.

Enteroperitoneal extends very rapidly, is extremely difficult to control, and is complicated by annoying or dangerous sequelie more frequently than enteric or meeratize intestinal tuberculosis. In both types the deplorable state of the bowel in the later stages is attributable mainly to mixed infection induced by pathogenic bacteria (especially the Bacillus coli communis) normal inhabitants of the gut. Incomplicated tubercular lesions extend slowly and are inclined to remain limited, but progress rapidly and produce ugly, deep, irregular, excarated, ulcerated areas when mixed dominates tubercular infection.

Enteric (granular, tilcerative) tuberculosis is rarely accompanied by the formation of abdominal or ischiorectal abscess and fistula, because, as a rule, it is limited to the mucosa, but enteroperitoneal is, because all the intestinal tunics are involved. They are comparatively rare complications of colonic tuberculosis because the gut is of ten protected by peritoneal adhesions, but are common in the rectal region owing to the absence of the serous covering.

Walsh has compiled a table to show the frecpuency with which tuberculosis in the different segments of the intestine is complicated by abscess, viz.:

Ulceration of small intestine

Ulceration of large intestine.

Ulceration of both large and small intestine.

Ulceration of rectum.

Absence of ulceration.

ISCHIORECTAL ABSCESS.

\begin{tabular}{c|cc} 
Present. & Absent. & Nist recorded. \\
0 & 13 & 0 \\
0 & 12 & 2 \\
8 & $4 \mathrm{r}$ & 0 \\
0 & 1 & 0 \\
1 & 22 & 0
\end{tabular}


When periproctitis takes place and there is an abscess forming, the patients complain of chilly sensations, sharp rise in temperature, feeling of malaise, and continuous throbling pain, symptoms which disappear as soon as the pus has been evacuated or finds an outlet through the bowel or ischiorectal fossa.

Rarely enteric and enteroperitoneal lesions have been known to improve under treatment or heal spontaneously, but, as a rule, partial or complete stenosis ensues, owing to the formation and contraction of scar-tissue at the site of the ulcers. which induces the ordinary manifestations of chronic intestinal obstruction.

The condition of patients afflicted with intestinal tuberculosis is frequently aggravated by the presence of papillomatous excrescences and cutaneous hemorrioids where irritating discharges constantly pour over the mucosa and anal skin.

While the mesenteric almost invariably, and the retroperitoneal and omental lymph-nodes occasionally, become enlarged, they produce no symptoms except tenderness upon pressure and palpable swellings unless they caseate and break down, under which circumstances there is a rise in the temperature, often followed by circumscribed peritonitis and the formation of an abscess which opens into the abdominal cavity or drains into the bowel or through the abdominal wall.

The author has observed cases where there was general miliary tuberculosis that simultaneously involved the intestine and other parts, accompanied by a marked elevation of temperature, emaciation, and profound gastro-intestinal disturbances, which, in spite of treatment, soon terminated fatally, and he has treated other patients where the disease was apparently primary in the bowel, but later extended to other organs, all of whom died.

Symptoms of Hyperplastic (Neoplastic) Tuberculosis.-The symptoms of hyperplastic (neoplastic) or ileocecal tuberculosis in the small bowel resemble those of other types of the disease, but when the colon, sigmoid, or rectum are involved, neoplastic tuberculosis presents manifestations which are fairly characteristic, but they vary in the different stages of the disease.

In cases treated by the author definite symptoms were not manifest until eight months, two and five vears after the intestinal disturbance started. When studying the symptomatology of this affection it is well to bear in mind that it is tumor forming, and causes a varying degree of obstruction, reflex phenomena, and in the later stages constitutional manifestations, dyspepsia, loss of weight, and irregular evacuations, which contain mucus except when there is ulceration or the tumor has broken down, when the feces are admixed with mucus, pus, blood and tissue débris, and have a disgusting odor.

At the onset of the trouble, which is insidious, there is an inexplicable slight disturbance in the alimentary tract that cannot be located in the stomach or intestine, and which does not interfere with 
the appetite or bowel movements. At the end of a few weeks or months progress of the disease is indicated by a failing appetite, irritable stomach and intestine, slight discomfort, sinking sensation in the abelomen, and occasional slight attacks of diarrhea. Later, when the swelling has assumed proportions sufficient to interfere with the fecal current and the circulation, lymphatics, and nervous apparatus of the gut, local and gencral manifestations of the disease become more apparent. Either through direct or reflex disturbances to the digestive tract mild crises occur, during which the patient has severe indigestion, becomes nauseated and romits, suffers from cramps, intestinal gas distention, frequent fluid or lumpy evactations containing considerable mucus, a feeling of discomfort and tenderness in the right iliac fossa, and rigidity of the abdominal muscles. Nonths later. or from one to three years from the time the patient became ill, the manifestations of the disease become sufficiently characteristic to enable one to group them and complete the diagnosis. By this time the subject has a tubercular complexion, has lost considerable weight, runs an occasional temperature, has intermittent attachs of colic, diarrhea, the stools containing undigested food remnants, fat, a large amount of mucus, and sometimes pus and blood when there are ulcers, and the patient worries over his condition, believing it to be cancerous. In addition, he complains of a sensation of blockage in the right side, and usually a fair-sized, firm, fixed, oval tumor can be seen or felt in the ileocecal region.

Examination reveals a firm, oval, tender tumor in the right iliac fossa, with sometimes palpable retroperitoneal or mesenteric lymphnodes in the ileocecal angle, or a large irregular swelling when the appendix, cecum, ileum, and adjoining structures become enmassed. The neoplasms frequently can be easily felt or seen bulging the abdominal wall. Owing to the partial obstruction induced by the growth the patient suffers from obstipation or constipation, alternating with diarrhea, between the crises.

In the final stages of the disease there is almost continuous diarrhea because only fluid feces can pass the obstruction, and the patient suffers intensely from griping pains, fecal impactions, putrefaction, and retained gases; all of which conditions favor a general septic condition, pyrexia, rapid pulse, and other evidences of auto-intoxication, and he becomes rapidly weaker and emaciated through the frequent evacuations and discharges, which often contain mucus, pus, and blood in consequence of the breaking down of the mesenteric glands or bowel wall through caseation, or the formation of deep ulcers resulting from combined tubercular and mixed infection present.

Occasionally the destructive process extends until a perforation occurs which results in peritonitis or the formation of simple or pyostercoral abscesses, which may remain circumscribed or form fistulous sinuses which open externally in the iliae fossa or at the navel in children. Even yet the prognosis is fairly good when the involved gut is excised and the sinus is dissected out, but if the disease is not now 
arrested the patient will soon die from exhaustion as a result of malnutrition, infection, or diarrhea.

In addition to the manifestations already enumerated, when acute attacks are excited by the administration of purgatives or other causes, meteorism, gurgling. and splashing sounds can be detected in the dilated proximal end of the ileum and pain is severe throughout the abdomen, which is usually protuberant.

The symptoms of hyperplastic and other types. of tuberculosis in the colon and sigmoid flexure are the same as when the ileocecal region is attacked, but when the rectum is involved all symptoms are worse, and the patient complains bitterly of sensations of fulness, weight and bearing-down in the bowel, and tenesmus at stool.

The symptoms of peritoneal tuberculosis may be insidious, under which circumstances they are characterized by emaciation, sallow complexion, anorexia, coated tongue, abdominal discomfort, obstinate diarrhea or constipation, tenderness upon pressure, slightly elevated evening temperature and a gradually quickening pulse, or the attack may come on suddenly as the result of an acute exacerbation or perforation when the symptoms become urgent, the patient has a high temperature. night-sweats, and other evidences of pus formation, riz., fast pulse, generalized abdominal pain and distention, and fluid in the abdomen, which has a protuberant appearance and doughy feel.

In fibrosclerotic tuberculosis the symptoms are the same as in stricture of a corresponding segment of the bowel from other causes, viz., constipation alternating with diarrhea, recurring fecal impaction and gas distention, occasional colic, malnutrition, muddy or sallow complexion, frequent attacks of indigestion, rumbling within the gut, exaggerated peristalsis, and, when the block is in the lower sigmoid or rectum, an incessant desire to stool, bearing-down pains, and an unrelieved sensation following stool. In this form of bowel tuberculosis, diarrhea persists because fluids only can pass the involved segment, but blood, pus, and mucus in the evacuations are not so abundant because stricture formation here is not preceded by extensive ulceration. The general condition of patients suffering from fibrosclerotic tuberculosis is fairly good because the disease is not nearly so exhausting as enteric (ulcerative) and enteroperitoneal tuberculosis.

The symptoms of intestinal peritoneal tuberculosis are indefinite until peritonitis ensues. Usually it causes more or less bowel distortion, and is not suspected until the abdomen is opened; hence it is usually mistaken for other types of intestinal tuberculosis.

The symptoms of glandular tuberculosis are about the same as those caused by tubercular infection of the colon, except that gas distentions are more frequent and pronounced, the glands are occasionally palpable, and diarrhea, accompanied by mucus, pus, and blood in the stools, is less annoying, owing to the fact that the mucosa is not ulcerated. 
Complications and Sequelæ of Intestinal Tuberculosis.-Ilaving outlined the general and local symptoms as encountered in the various stages of the different types of howel tuberculosis, the atuthor will now briefly discuss the more common and serious complications and secuelie which may result from intestinal tuberculosis, viz:

(I) Stricture.

(2) Peritonitis with and without perforation.

(3) Adhesions, kinks, and twists.

(4) Abdominal and anorectal abscesses and fistulic.

(5) Cencralization of the infection (miliary tuberculosis).

(6) Carcinomatous grafting upon the tubercular process.

Stricture.-The most frequent, annoying, and difficult to cure of the resultant disturbances of intestinal tuberculosis is stenosis, a condition sometimes encountered in all parts of the intestine in the different types of the discase. This type of stricture may be met with in any country, at all ages, in both sexes, and remains through life to cause partial or complete obstruction unless. cured by surgical intervention.

Like other intestinal tubercular affections, stricture is encountered most often in persons between twenty and forty years of age, although one case has been observed in a child three and one-half and another in a man sixty-eight years old.

This type of stenosis is located fairly often. in the small intestine, an evidence of which is to he found in the statistics of Berney, who, as far back as 1899 , collected 70 cases; since then numerous cases have been reported. The obstruction is rarely encountered in the jejunum or upper ileum, and shows a decided predilection for the cecum. rectum, sigmoid, ileocecal valve, and the lower ileum, and has been encountered less often in other segments of the small and large intestine, viz.: Caird (I I cases), small intestine alone, $t$; ileocecal region, 6 ; ascending colon, I ; Fuch (9 cases), ileum, 2 ; cecum, 7 ; Erdheim, ileum, 4; Trinkler, jejunum, I: Mikulicz, jejunum, 2; Balrous, jejunum, I : V'öhtz, ileum. I ; Reach, duodenum, I ; ileum, 8; Geosz, ascending colon, multiple. I ; transverse colon, I; Streh! (cited by Reach), ileum, It; hepatic flexure, I; Mummery, Ioo collected cases (hyperplastic tuberculosis), cecum, 48 ; cecum and ascending colon, 39; cecum, ascending and transverse colon, 3 ; colon, 4 ; sigmoid flexure, 6 .

In the author's cases (It) of intestinal tubercular stenosis the obstruction occurred as follows: ileum, 2; ileocecal region, 2; transverse colon, I; descending colon, I; sigmoid flexure, I; rectum, 6: anus, $\mathbf{I}$.

Tubercular strictures may be single or multiple, but the former are most common in hyperplastic tuberculosis of the large bowel, and the latter in the enteric and enteroperitoneal (ulcerative) types involving the small gut. The author has had the good fortune to observe both forms of tubercular stricture, and in one of his cases the bowel was obstructed at four points.

Busse has recorded + cases of multiple tubercular stenoses, in 1 of 
which there were ten occlusions, but Maylard has gone him one better and records a case where there were eleven. In Vöhtz's case the bowel was narrowed 12 inches $(30 \mathrm{~cm}$.) above the ileocecal valve and again $+\frac{1}{3}$ feet $(130 \mathrm{~cm}$.) higher up; in Reach's, the first stricture was in the duodenum and the last of several in the lower ileum, while in Geosz's multiple stenoses were found in the ascending and transverse colons.

Strictures may result from either the enteric (ulcerative), enteroperitoneal, hyperplastic, or fibrosclerotic types of tuberculosis, and the character of the occlusion varies greatly in different cases, for the stenosis is annular and involves but a short segment of gut in the first two varieties and tube-like in the last named, and may obstruct anywhere from 1 to 10 inches $(25 \mathrm{~cm}$.) or more of the gut. In the former the occlusion is produced through the contraction of cicatricial tissue from healed ulcers, and upon digital examination the rigid ring-like annular constriction feels like a syphilitic stricture. In the fibrosclerotic or stenosing variety the intestinal tunics (especially the submucosa and subserosa) undergo fibrosis, and contract to convert a considerable length of the bowel into a firm, narrow, resisting tube (the so-called gas-pipe intestine), so that it is frequently impossible to introduce the finger or instrument through the stricture for diagnostic purposes. Stenoses induced by hyperplastic tuberculosis occur at the ileocecal region in about 85 per cent., and in the rectum in the majority of other cases, and the occlusion does not result from the contraction of cicatrices of healed ulcers or conversion of the bowel coats into fibrous tissue, but is caused by an encroachment upon the gut lumen by enormous round-celled proliferation in the gut wall and peri-intestinal structures, which lead to the formation of a tumor which projects into and outside the cecum or involved segment of intestine. Sometimes the stenosing process or cell proliferation may be restricted to a halfcircle of the bowel or extend along one side and parallel with the gut, under which circumstances the obstructions are present as crescentic-shaped spurs or long indurated ridges.

Usually the mucosa remains intact below a tubercular stricture, but is partially or completely destroyed at and above the constriction, owing to impaired circulation, trauma, mixed infection, and collections of scybala, conditions accompanied by the discharge of blood, pus, and mucus in large amounts. Often fecoliths or infected material become embedded in the sores, resulting in the formation of submucous or perirectal abscesses and fistulæ above the stenotic level, the pus from which drains downward to form abscesses or fistulæ at the anal margin, ischiorectal fossa, and elsewhere, that may be simple (streptococcic or colon bacilli) or tubercular.

The bowel proximal to the obstruction is usually dilated, owing to the tendency of gas and feces to collect there, and its walls may be either thinned or thickened. In the small bowel compensatory muscular liypertrophy sometimes takes place, but in the lower bowel the increased thickness of the gut lumen is attributable to the inflammatory changes and not to muscular development. 
The caliber of the stricture may be moderate in size or so small it is difficult to see or pass with an instrument. The symptom-complex induced by the intestinal tubercular strieture depends mainly upon the degree of obstruction present. When the block is slight the patient complains of tenesmus and constipation, alternating with cliarrhea. If the obstruction is moterate, there is a loaking-up of gas, fecal impaction, semsation of weight and fulness in the lowel, and a more frequent desire to stool, which is accompanied by straining and gives no relief, but where ocelusion is almost complete, the abromen remains distended, there is an incesiant desire to empty the bowel and an inability to do so, except when, through the aid of medication or enemata, the foces are renclered fluid and expelled in small quantities and at freguent intervals without freeing the bowed of the scybalid. Finally, when the stenosis is complete the usual sympoms of acute intestinal olstruetion are present, and will continute antil the stricture is enlargerl, excised, or an artificial anus is marle abose it.

Strictures of the lower lowel are encomered most frequently in the rectum, and their location and character can be determined by digital examination, but when situated higher in the rectum and sigmoid, they are diagnosed ly carrying the proctoscope up and inspecting them. No attempt should be made to force the instrument or a bougie through a stricture more than 3 inches abore the anus the peritoneal attachment), because, owing to the ulecrated condition of the gut, it is liable to be ruptured.

Peritonitis IIth ond ITithout Perforation.-In extensive tubercular as in other chronic inflammatory and ulcerative lesions of the intestine, the peritoneum may be specifically or otherwise involved. In such cases it is swollen, reddened, cdematous, and dark spots appear upon its surface at the sites of corresponding lesions in the mucosa. As a result the serosa becomes agglutinated to the abdominal parietes, loops of intestine, omentum, and other organs or adjacent structures having a peritoneal covering, but further than soreness and interference with peristalsis the general and local manifestations consequent upon peritoneal involvement are unimportant.

When peritonitic inflammation results from the caseation of diseased mesenteric or retroperitoneal glands, perforation takes place from a tubercular or mixed infection ulcer, or the passage of tubercle bacilli and other pathogenic micro-organisms through the diseased intestine, the infection is virulent, widespread, and terminates in general or eircumseribed peritonitis often complicated by abseess and fistula, and the patient suffers from abdominal pain, discention, tenderness and muscular rigidity, high or subnormal temperature, nausea, romiting, restlessness, cold extremities, impared circulation. chilly sensations, blueness of the skin, constipation or aggravated diarrhea, has a septic skin, leukocytosis, and the usual constitutional manifestations of sepsis.

In extreme cases, where the peritoneal involvement is a part of general miliary tuberculosis, the omentum and gut are thickly studeded 
over with whitish or yellowish caseating tubercles. As a rule, miliary peritoneal tuberculosis does not respond to surgical or other treatment because it is part of a general infection which sooner or later terminates fatally.

Adhesions, Kinks, and Tiists.--Intestinal tuberculosis, particularly the severer ypes, owing to peritoneal inflammation or the complicating abscess, is invariably accompanied by the formation of exudates or organized string, fan, or band-like massive adhesions which bind the gut to adjacent structures, having a peritoneal covering which materially interferes with the functionating power of the bowel. Where peritonitis has been general, a number of intestinal loops may be found glued together or with neighboring or distant organs, and form narrow, broad, short, or long adhesions.

The phenomena induced by adhesions depend upon their number, size, and relation to the gut, riz.: bandular obstruct the bowel by pressing upon or entwining themselves about it, or fan-shaped (multiple) angulate or twist it by pulling in opposite directions, or when one end of an adhesion is connected to one, and the other to another segment gut, and membranous adhesions by compressing a lengthy piece of the bowel and causing intestinal stasis.

Kinking of the bowel sometimes follows the healing of extensive tubercular ulcers and the formation of cicatricial tissue.

The clinical manifestations induced by adhesions, kinks, and twists depend upon the extent to which the gut is affected, and when occlusion is slight they interfere with peristalsis, cause slight gastrointestinal distortions, constipation or loose movements, localized tenderness, and occasional enterospasm, but when the gut lumen is almost or quite obliterated by them the abdomen is distended with gas, sensitive and rigid, and the patient suffers from obstipation and recurring fecal impactions or persistent diarrhea, severe abdominal pain from gas accumulations or enterospasm. nausea, romiting, and the usual manifestations of acute intestinal obstruction, or necrosis when pressure sloughing ensues.

Abdominal and anorectal abscesses and fistulce are infrequent complications of bowel tuberculosis, and may be caused by tubercle bacilli or mixed infection which reaches the peritoneal cavity or perirectal spaces through perforation or escape of pathogenic micro-organisms through the non-resistant, diseased gut.

Mixed infection abscesses of the abdomen induce acute and profound pus manifestations, and terminate fatally if not prompty evacuated, while tubercular abscesses are inclined to chronicity and the pus may accumulate in enomous quantities without creating acute symptoms. The discharge in both types of abscess may or may not contain fecal matter. and burrow its way into the gut, hollow organs, or follow the bowel downward to open into or near the rectum. In children abdominal abscesses usually open in the median line near the navel or weakest point in the parietes. In adults afflicted with ulcerative or enteroperitoneal tuberculosis the abscesses may discharge 
upon the abdominal wall at any point, depending upon the location of intestinal lesions, but in the hyperplastic sype the discharge finds an ontlet in the right iliac fossat.

Enteroperitoncal tuberculesis is frectently arcompanied by small or considerable sized inter-and intramural abseesses, which may penetrate the gut, lift the mucosa upward, and open into the intestine below; a condition exidenced hy the finding of yellowi pus in the stools.

Abscesses and fistulit are encountered more frepuently in the anorectal regions than higher up, hecause here the protecting inlluence of the peritoneum and peri-intestinal athesions are absent, the lesions are more extensive, and the bowed is constantly trammatized by the feces.

Abscesses here are designated pelivectel when they originate in the pelvis, perirectal when located at the side of the gut, ischiorectal when situated in the ischiorectal fossa, marginal when at the mucocutaneous border, follicular when the hair-follicles become infected, and submucous when pus collects beneath the mucosit.

Fistula resulting from such alscesses are named accorling to the number, location, and relation of their openings, viz., complete, when there is one opening in the rectum and one upon the surlace or skin: complete internal, when loth are in the rectum: complete external. when both are upon the surface; blind external, when the outlet is through the skin; blind internal, when in the rectum; rectoraginal, when the sinus communicates with both bowel and vagina; rectourethral or aesical, when it extends between the gut and the urethra or blatkler; horseshoe, when the tract encircles the rectum and opens into it and upon the buttock upon either side of the anus; and complex, when there are numerous openings in the bowel, upon the surface, and communications betwen the rectum, labia, vagina, urethra, bladder, or pelvis.

Complete fistula is the most common type, and in this and other forms, when the tract connects with the rectum, the internal opening is located in the posterior median line $\frac{1}{2}$ inch above the anus in 90 per cent. of the cases.

From the standpoint of prognosis there are threc types of anorectal fistula- the tubercular, difficult to heal when operated; ordinary, occurring in individuals devitalized by phthisis which resplute a considerable time; and simple, which are easy to cure because there are no constitutional disturbances to devitalize the patient.

Generalisation of the Infection (.Miliary Tuberculosis). - Ceneralization of the process occasionally occurs secondary to intestinal tuberculosis elsewhere, under which circumstances miliary 1 ulereles are distributed throughout the various organs. In such cases the peritoneum (Fig. 32) and omentum present a speckled appearance produced by the projecting, smooth or caseating. white or yellowish tinted tubercles, which upon the mucous and skin surfaces of the anorectal region appear in patches and soon break down (owing to the trauma 
to which they are subjected), to form superficial cup-shaped ulcers. These lesions may extend over a considerable area individually or by coalescence, but are not inclined to attack the deeper structures as are other forms of tuberculosis.

The prognosis of intestinal miliary tuberculosis is unfarorable because it is secondary to and rarely develops until after the discase has become well established elsewhere, and the patient is debilitated and unable to withstand the ravages of the tuberculous process.

Carcinomatous Grafting Upon the Tubercular Process.-Carcinoma frequently complicates intestinal tuberculosis or follows it when the ulcers heal. The writer believes that the disease here, either through its traumatic influence induced through intestinal activity or irritation to the mucosa consequent upon the constant passage over it of the discharges, is a predisposing cause of intestinal cancer. He has been led to this conclusion through observations made upon 2 patients colostomized for cancer, in whom the malignant process had rapidly developed. These patients gave a history of having had chronic colitis and diarrhea, one for eighteen months and the other for two years prior to cancerous obstruction in the sigmoid and rectum. Both individuals had been emaciated, and manifested evidences of tuberculosis for a long time preceding the crisis incited by the growth. In the rectal case, tuberculous appearing ulcers were observable only above and below the neoplasm, and tubercle bacilli were found in scrapings from them. In the other, when the gut was withdrawn for the purpose of making an artificial anus to relieve the cancerous obstruction in the sigmoid, it was found studded with tubercles, and the peritoneum was found inflamed and thickened in the vicinity of the ulcers; on the mucous membrane in the rectal case tubercular lesions were plentiful throughout, and there was a large raw area upon the perineal skin, on the surface of which were numerous fresh and caseating tubercles. This patient was also suffering from phthisis.

On another occasion, where a number of rectal and abdominal glands were removed with a carcinoma at the rectosigmoidal juncture, some were inflamed, others malignant, and the remainder tuberculous and in a state of caseation. In this connection it is well to remember that tuberculosis shows a predilection for the cecum, sigmoid, and rectum, the most common sites of carcinomatous degeneration. Crowder has also reported 2 cases of carcinoma associated with intestinal tuberculosis. 


\section{CHAPTER XXII}

\section{TUBERCULAR ENTERITIS, COLITIS, AND ENTEROCOLITIS (INTESTINAL TUBERCULOSIS), DIARRHEA IN (Continued)}

\section{DIAGNOSIS}

General Remarks.-It is frequently difficult or impossible (except by operation or autopsy) to distinguish tubercular lesions from those caused by catarrhal and infectious diseases of the bowel. because the intestinal and other manifestations induced by them are similar, and, further, because tubercular infection may complicate a simple or infectious enterocolitis. It may at times require prolonged observation and astute diagnostic ability to determine the type of tuberculous lesions one has to deal with, irrespective of whether they insolve the small or large intestine, or both, owing to the fact that in all varieties there may be complicating foci in other organs, the patient shows constitutional characteristics of the disease, gastric and intestinal disturbances are present; there is no difference in so far as a tuberculin or diazo urinary reaction is concerned, and further. because the patient may be affected simultaneously with different types of intestinal tuberculosis in the same or different segments of the bowel, which would blend the manifestations and confuse the diagnosis.

In the majority of advanced cases, however, the symptoms of the different types of infection are sufficiently typic to enable the clinician, with the aid of the microscope, to distinguish between them, and determine if another affection is associated with the tuberculosis:

The author carefully examines for intestinal tuberculosis all persons, roung or old, coming to him who are emaciated, have a sallow complexion, suffer from digestive disturbances or diarrhea, pass pus, bloot, or mucus, singly or together, and have a cough or give a family history of phthisis.

Diarrhea is the pathognomonic symptom of bowel tuberculosis, but occasionally this class of patients suffer from con-tipation or ob-tipation, alternating with diarrhea, when the bowel is ulcerated or blocked by a tubercular tumor.

I'sually', tubercular lesions of all kinds and wherever located induce a slight or aggravated diarrhea, but, as a rule. the morements are more frequent and fluid when the lesions are located in the lower sigmoid and rectum than when situated in the small intestine and colon, because the disease is always more aggravated in the-e regions.

Diarrhea, in the main, results from irritation to exposed nervefilaments, which cause hyperglandular secretion, frequent and prolonged peristaltic contractions and deficient colonic absorption com- 
sequent upon the inflammatory and ulcerative state of the mucosa, factors which, in working together, increase the watery constituent of the intestinal contents and cause frequent evacuations.

The number of morements, amount of pus, blood, and mucus discharged and degree of suffering induced by intestinal tuberculosis corresponds closely with the extent to which the bowel is denuded of its mucosa.

The author will now outline the chief points of differentiation between the different types of tuberculosis, and this disease and other affections which mimic it, following which he will discuss the complications of intestinal tuberculosis.

\section{Diagnosis of Enteric (Ulcerative) and Enteroperitoneal Tubercu-} losis.-In most instances it is comparatively easy to differentiate between hyperplastic and other forms of intestinal tuberculosis, but between the ulcerative and enteroperitoneal types of the disease it is always difficult, because in both constitutional manifestations are present, gastro-intestinal disturbances are about the same, and both are accompanied by discharge of mucus, blood, and pus. The most significant differential feature between them is that the symptoms of enteroperitoneal become aggravated very quickly. are more difficult to control, and the disease terminates fatally sooner and more often than enteric tuberculosis. In the former (enteroperitoneal) the entire wall becomes involved early by the inflammatory and ulcerative processes and the disease extends rapidly within and without the bowel, owing to caseation and necrotic changes and extension of the disease through the coalescing of formed ulcers and mixed infection. Outwardly, eridences of the disease are shown in the uneven, congested peritoneum and exudates and adhesions which are largely responsible for the pain and tenderness elicited by pressure. Sometimes through the lymphatics perforation or infection takes place, resulting in circumscribed peritonitis, matting together of the intestines and adjacent organs, and the formation of an ordinary or pyostercoral abscess, which becomes encysted or discharges through the gut, or finds an outlet through the abdominal wall.

The general manifestations of enteroperitoneal tuberculosis are more profound lecause of extensive miliary deposits observable in the peritoneum and bowel, and its frequent association with tubercular lesions in other organs. particularly the lungs, from whence autoinoculation takes place by way of the blood when the phthisic lesions involve a ressel, or by swallowing infected sputum.

Owing to the through-and-through diseased condition of the gut wall, it is very tender upon pressure. and attacks of enterospasm or cramps occur more frequently and severely in enteroperitoneal than ulcerative tuberculosis where the mucosa is chiefly involved. Again, obstructive manifestations are observable less often in the former than the latter because the disease early terminates fatally, or the ulcers do not heal and form strictures, as the therapeutic measures directed against them do not reach the diseased midlle and outer tunics. 
Pus, blood, and mucus are present in slight or considerable anount in both types of tuberculosis, and in catarrhal, syphilitic, dyenteric, gonorrheal, or diphtheric colitis. Consequently, it is impossilsle 10 differentiate between ulerative or enteroperitoneal tulereulosis hy examining the discharges, but a careful microsopic examination of the stools enables one to diagnose tulereular from other types of colitis when the bacilli are found. 'Tubercle bacilli are rarely present, or, if so, they are setdom found ly examining the stools, particularly when the lesions are located in the small lowel, but the auther has occasionally been able to detect them in tuberculosis of the colon and rectum, but more often in the scrapings of ulcers than in the clischarges or feces.

The lest way to oldain material for examination when the disease involves the rectum or sigmoid is to introcluce a proctosigmoidoseope, expose raw areas and curet their bases and edges, reserving the remored débris for examination, for bacilli are found more frequently and with less trouble in infected tissue in the discharges or feces than in the usual method where the evacuated discharges are examined.

A certain amount of information can be obtained by examining the fragments of tissue found in the stools of tuberculous patients to see whether they result from the necrotic tissue or undigested meat remnants.

Too much importance is placed upon the finding of tuhercle bacilli, even when present, because they may be found in the feces of individuals who have phthisis alone or both lung and intestinal infection. Klose was able to demonstrate tubercle bacilli in the evacuations of 47 out of 60 consumptives in whom there was no evidence of intestinal involvement.

Chrostek and Stromayer place considerable importance upon a urinary examination in persons suspected of having intestinal tuberculosis, because, after arministering peptone, they have been able to demonstrate albuminuria. From the author's experience he is inclined to place more reliance upon Ehrlich's diazo-reaction than the finding of albumin under the circumstances enumerated.

Nuch more importance is 10 te placed upon a positive tuberculin reaction test (particularly in children), as indicated by a marked rise in temperature, natsea, restlessness, muscular pains, and other subjective symptoms, which, when obtaned, points strongly toward the tubercular nature of the disease. Tuluerculin may be sulisfactorily employed in the form of an intramuscular injection, gr. $\frac{1}{15}$ to (0.004-0.006), when lut a single dose is given, or as practised by Löwenstrin and Kaufmann, who administer it in doses of gr. is (0.002), repeating about every four days until three or four injections have been made, maintaining that each dose prepares the pationt for a more favorable reaction. When the alose is ineffective, the losage, beginning at gr. $\frac{1}{315}(0.002)$, is increased to gr. $\frac{1}{1}$ - $(0.005)$, or exen gr. $\frac{1}{6}$ $(0.0 \mathrm{I})$, until the reaction is obtained. 
Preferably the injections should be given in the evening, and the temperature should be taken every few hours, and when it rises $\mathbf{5}$ degrees Koch considers the reaction positive.

The part played by the opsonic index is not so valuable in diagnosis as it is in treatment, ret it deserves to be mentioned. It is unreliable here because the same indications are present in tuberculosis of the lung as of the intestines, and when the disease attacks several organs the opsonic index does not help one in finding out which particular organ is involved in the tuberculous process.

French, mentioned by Maylard, says: "In the case of tubercle bacilli the opsonic indices of a series of healthy individuals may vary from 0.8 to $\mathrm{I} .2$ or thereabouts, but in tuberculous patients the index may be as low as 0.3 or as high as $\mathbf{I} .8$, or even higher. The estimation, therefore, may be of considerable diagnostic value in cases where there is doubt as to whether the lesion is tuberculous or not. If the index is below 0.7 or above 1.3 the argument will be in favor of tubercle, the resisting power of the patient being low in the first case, high in the second. . . It has been found that after injecting $\frac{1}{5000} \mathrm{mg}$. of tuberculin R. into a healthy man the opsonic index falls slightly for about two days, then rises to slightly above normal, and then returns to what it was originally. A similar injection into a tuberculous patient is followed by a considerable fall in the opsonic index, the latter remaining below what it originally was for a week or more, by which time it has begun to rise again above what it was before. The initial fall after the injection is called the negative phase. This negative phase is quite short in healthy people, long in tuberculous subjects, so that we have here an additional means of diagnosis."

An examination of the blood is sometimes useful because leukocytosis is high in malignant ulceration of the intestine, and slight or absent in tuberculosis of the bowel. The author does not underestimate the value of tuberculin, peptonitic and diaso reactions, and blood analysis, but places greater reliance upon the subjective and objective symptoms of intestinal tuberculosis, particularly when tubercle bacilli are found in the stools.

Thus far the differential diagnosis of ulcerative and enteroperitoneal tuberculosis has been discussed in a general way, and it now remains to call attention to the principal points of differentiation between them and neoplastic tuberculosis, and a few of the more common affections which have been confused with them.

The manifestations of enteric, enteroperitoneal, and neoplastic tuberculosis closely resemble each other in the incipient stage because of the accompanying gastro-intestinal disturbances, but when the disease has become fairly well developed the diagnostic picture of hyperplastic tuberculosis varies greatly from that of the other forms. Briefly stated, neoplastic differs from other types of intestinal tuberculosis in that it occurs in early adult life, develops more slowly, causes less emaciation, diarrhea is less aggravated, obstruction more marked, and there is a characteristic right-sided tenderness, fulness, 
pain, and tumor, comparatively little or no pus, blood, and mucus, and postoperative results are very much better.

So-called fibrosclerotic nuberculosis (which the author believes to be a variety of the neoplastic type) often causes diarthea and other manifestations resembling ulcerative tuberculosis, but is distinguished from them by its chronicity, hard, firm, tule-like feel of the intestine, the slight amount of pus, blood, and mucus in the stools, and absence of ulceration prior to the stricture formation.

Amyloid degeneration sometimes resembles tuberculosis of the intestine, and the diagnosis must he arrived at by exclusion. This form of degeneration is encountered in the small, and tuberculosis occurs in the large, intestine; the mucosa of the former is pale and waxy and villi are missing, while in the latter it is often red, swollen and edematous, and the villi are distinguishable except at ulcerated points. In amyloiclosis the diagnosis is based principally upon the iodin reaction, which, when applied to diseased arcas, turns them a brownish-red color, the hue of which is converted to violet by the addition of sulphuric acid, and a pink color when methyl-violet is employed, hut the diagnosis is not always possible during the patient's lifetime.

Actinomycosis in many particulars resembles hyperplastic, but has been mistaken for both enteric (ulcerative) and enteroperitoneal, tuberculosis. This condition is accompanied by gastralgia and enteralgia, and more frequently induces constipation than diarrhea, at least during the earlier stages. The mucosa presents an uneven surface because of nodular swellings which in no way resemble tubercles, but when these granulomatous deposits break down they form lesions which look like tubercular ulcers, especially when chronic, owing to the part played by mixed infection in both. In the later stages of the disease there is no excuse for confusing actinomycosis with ulecrative tuberculosis because it is accompanied in rapid succession by the formation of a tumor in the cecal region, painful infiltration of the abdominal wall, and the opening of sinuses through it (especially low down), from which exudes a viscid discharge containing débris and typic yellow sulphur-like granules, which, moler the microscope, show the fungus. In the absence of sinuses a positive diagnosis is impossible, except when the fungus is revealed by urinary or fecal examination.

Syphilitic can be differentiated from tubercular discase of the intestine by obtaining a history of chancre, olserving specific lesions elsewhere, funding the spirochetes, obtaining a positive Wassermann reaction, improving the pationt's condition by the administration of mercury and potassium, arsenic preparations, or salvarsan in the absence of a tuberculin reaction.

Typhoid ferer is easily differentiated because of variance in the symptom-complex and positive 1 idial reaction.

Gastric and duodenal ulcers are occasionally mistaken for tubercular lesions of the intestine, but this is not likely to occur when due 
care is taken, because the constitutional manifestations of tuberculosis are absent, and when the lesions are in the stomach or duodenum the patient suffers more acutely from pain in these regions, romiting, inability to retain food, hematemesis and agony shortly following eating because of the pouring out of the hydrochloric acid; and, further, for the reason that the stools contain no pus, blood, and little mucus, all of which are found in intestinal tuberculosis.

Dysentery is more often confused with intestinal ulcerative tuberculosis than any other affection. The former very rarely attacks the small intestine, being confined principally to the colon and, more particularly, the sigmoid flexure and upper rectum, where characteristic stellate ulcers can be observed through the sigmoidoscope. Diarrhea, mucus, and pus occur with equal frequency in both affections, and vary according to the duration and extent of the disease, but bleeding and tenesmus are more pronounced in dysenteric ulceration, and the pain which accompanies this condition is sharper and more centrally located than in tuberculosis. The finding of entamebre or bacilli of Shiga, Flexner, or Hiss is necessary to complete the diagnosis.

Dyspeptic enteritis, which may be mistaken for or complicate all types of intestinal tuberculosis, is caused by gastrogenic clisturbances such as hypo- and byperchlorhydria, under which circumstances there is usually interference with the biliary and pancreatic secretions. Here the stools are not increased in number, but contain glairy mucus and induce more or less tenesmus. Later, when both the stomach and intestine are involved and the secretions are augmented, the morements are more numerous, soft, offensive, and foamy, but differ from ulcerative tubercular enteritis in that the diarrhea alternates with constipation.

Chronic enteritis resulting from ptomain-poisoning somewhat resembles intestinal tuberculosis, but the history of a sudden and violent onset, very great pain, nausea, vomiting, collapse, and exhausting diarrhea go far toward differentiating this condition. In these violently toxic diarrheas the number of polymuclear cells is considerably increased (Loeper), and the absence of amebre and tubercle bacilli in the stools helps to confirm the diagnosis of ptomain-poisoning.

Diagnosis of Hyperplastic (Ileocecal) Tuberculosis.-Hyperplastic tuberculosis, irrespective of whether it is located in the small or large intestine, is never diagnosed in the carlier stages except by chance, because the gastro-intestinal clerangements excited by the infiltrating process, which is unimportant at this time, are but slight, and differ but little if at all from other and more common intestinal ailments. Nor does the author believe it possible to make a diagnosis of hyperplastic involvement of the small intestine in the vast majority of cases in any stage of the discase, becausc the neoplastic thickening is less marked here than in the colon, which makes it almost or quite impossible for the clinician to detect the swelling by palpation or other means, and, further, since if the tumor could be discovered it could not be differentiated with certainty from other neoplasms causing obstruc- 
tion. Even when the disease has progressed to a considerable degree, and is located at its ustal site in the ileoceal region, neoplastic tuberculosis is rarely suspected. because of the infrefuency with which it occurs and the fact that the consepuent disturlance to the digestive apparatus, right-sided swelling, tenderness and pain, loss of weight, integumentary manifestations of anto-intoxication, and the occasional presence of mueus in the stonk, may all or in part be produced by disturbances of the stomach and howel, appendicitis, circumseriberl peritonitis, tumors, or disease within the ablomen or pelvis.

It is not so difficult, however, to recognize neoplastic tulercular tumors of the large bowel and, particularly, of the cecum, the seat of their predilection, when the disease has fully derefoperl, and one considers the history of the case, the symptoms as outlined abore, makes a careful examination of the abdomen, and hears in mind the diagnostic peculiarities of the disease included in the following description:

One is justified in suspecting hyperplastic tuluerculosis in individwals between twenty and forty who give a history of or suffer from phthisis, are chronically ill, have a poor appetite, indigestion, become emaciated slowly, run an occasional degree of temperature, complain of diarrhea or frepuent moxements alternating with constipatiom, tenderness upon pressure, pain and fulness in the right iliac fossa. Later, when the tumor has issumed fair proportions, if cancer lee excluded according to the methor of differentiation outlined below, there is no reason why a correct diagnosis should not be made in the vast majority of instances. The neoplasm is recognized by its predilection for the cerum, limitation to the ileocecal region, slow growth, bulging appearance, firmness. large size, oval shape, fixation, fibroadipose capsule, sensitiveness on pressure, dulness on percussion, extensive involvement of the mesenteric and retroperitoneal slands. presence of polypoid and papillomatous growth in the affected sut, partial or complete obstruction induced. crater-like ulcers when the neoplasm degenerates, pericecal adhesions, circumscribed peritonitis, and occasionally pyostercoral abscess and listula when perforation takes place.

The papillomatous vegetations mentioned are pathognomonic of the discase, and are encountered very much more often in this than other types of the disease. Peristalitic moxements of the intestinc can occasionally be felt and seen during the attacks and when the patient suffers from cramps, but this symptom is less frepuent and severe in neoplastic than it is in either the ulcerative or enteroperiuneal type of tuberculosis. The manifestations of olstruction are always present, naturally vary aceording to the degree of stenosis. Jut distention, gurgling, and splashing souncls are at intervals present in nearly all cases, owing to the backing up of gers and fuid feces, and, as a result, a tympanitic is substituted for a dull percussion note.

In those exceptional cases where ulceration early complicates tumor formation, and the process reaches the peritonemm, hyperplastic closely resembles enteroperitoneal tuberculosis until the true state of 
affairs is revealed by the manifestation of a tumor in the right iliac fossa.

In this form of the disease the mucosa is frequently not disturbed, and when it is, ulceration occurs in the later stages of the disease after the circulation has become impaired by the neoplasm, when, through the necrotic processes, extensive deep ulcers are formed which in no way resemble the superficial types seen in the other varieties of intestinal tuberculosis. This goes far to explain why it is that individuals afflicted with this form of tuberculosis do not suffer so early, frequently, or so severely as persons having the ulcerative or enteroperitoneal forms of tuberculosis, where numerous lesions are found in the mucosa almost from the incipiency of the disease. Consequently, pus, blood, and mucus in the stool occur less often and in smaller amount in the neoplastic than in the varieties of the disease just mentioned, and the odor of the stools is less fetid, owing to the fact that the discharge, mixed infection, and the accompanying putrefaction is correspondingly less.

Differential Diagnosis of Hyperplastic (Ileocecal) Tuberculosis.It is always extremely difficult if not impossible to make a diagnosis of hyperplastic tuberculosis when the discase is situated in the small intestine, except by exploratory laparotomy or autopsy, and then, in many instances, not without the aid of the microscope, since here the symptoms resemble enteritis or obstruction, and the pathologic changes in the gut cannot be made out owing to the smallness of the tumor.

It cannot be denied that ileocecal tubercular neoplasms have often been mistaken for other diseases and growths, particularly cancer, but in the light of our present knowledge concerning this affection mistaken diagnoses should be extremely rare, because these enlargements have the peculiarities and symptoms previously noted, which, when carefully studied, should distinguish them from other ailments.

Hyperplastic tubercular formations of the intestine have been most frequently mistaken for carcinoma, appendicitis, perityphlitis, chronic adenitis, actinomycosis, fecal impaction, gummata, psoas abscess, and massive adhesions. Naturally, it is confused most frequently with carcinoma because of its firm, tumor-like consistency, and the fact that cancer is so frequently encountered at the cecum and in the rectum, the most common sites of hyperplastic tuberculosis, and, further, because the profession has not been well acquainted with this disease nor sufficiently watchful for the neoplastic type of the trouble. Many patients are unnecessarily permitted to die yearly by general surgeons who mistake neoplastic intestinal tumors for inoperable cancers, and refuse them a raclical operation when the growth could be enucleated with comparative safety and without leaving annoying sequeliz.

In order to avoid unnecessary detail in describing the characteristics of carcinoma and neoplastic tuberculosis the author has condensed the differential data in the accompanying table, which contains the diagnostic characteristics of these ailments: 
DIFFERENTIAL DIAGNOSIS BETWEEN ILEOCECAL HIPERPLASTIC TUBERCULOSIS AND CANCER

IHPERPLASTIC TUBERCLLOSIS OF THE CECUN.

C.ARCHNOMA UF THE RECTLW.

Age

Twenty to forty (principally third deciule). Forty to sixty. (hildhoud very rare.

Childhood not uncommon.

Onsel and Progress

Slow.

Ratpid.

Duration

Two to three years.

Four to ten months.

Usually slight.

$$
\text { Pain }
$$

More frequent and markel.

Absent.

$$
\text { Cachexia }
$$

P'resent.

Slow and slight.

\section{Emaciation}

Rapid and pronouncerl.

Indigestion

Early and marked.

Late manifestation.

Ferer

Common.

Uncommon.

Obstruction

Late and partial.

Garly and marked.

Mdominal Tenderness

More or less.

U’nusual.

Present.

Gurgling and Splashing

Rarely so.

\section{Peristalsis}

Marked.

Slight.

Position

Cecum normal position.

\section{Locution}

Inrwhere in the colon, particularly at the flexures.

Mobilit

Fixed.

Slightly movable.

Intestine Palpable

Rareks.

\section{Abscess and Fistula}

Vers rarely.

\section{Hemorrhige}

Very rare.

More frequent.

Blood and Pus in Slools

Uccasionally. 
DIFFERENTI.AL DIAGNOSIS BETIEEN ILEOCECAL HYPERPLASTIC TUBERCULOSIS INI) C.INCER (Continued)

HYPERPLASTIC TLBERCLLOSIS OF TIE CECUM.

CIRCINOMA OF THE RECTUM.

Tubercular occasionally.

\section{Bacilli in Stools}

Absent.

Fetid Odor to Stools

Slight.

Charateristically foul.

Practically always.

\section{Peritonitis (Circumseribed)}

Occasionally.

Tubcrulin Reaction

Usually positive.

Absent.

Lcukocytosis

Leukopenia frequent.

Moderate.

Frequently present.

Ehrlich's Diaso-raction

Fre

Frequent (lungs, etc.).

Thickened.

Absent.

Intotement of Other Organs

Infrequent (liver).

.tdjacent Gut

Normal.

Mescntery and Periloncum

Always extensively involved.

Sometimes and less extensively.

\section{Titmor Characteristics}

Broad, oval, and smooth.

More nodular and firm.

\section{Bowel IV'all}

Subserosa especially affected.

Submucosa involved.

Rarely breaks down.

\section{Retrogressive Changes}

Degenerates and forms crater-like ulcers.

Giant cells, tubercles, bacilli.

\section{Microscopically}

Characteristic tumor cells.

Hyperplastic ileocecal tuberculosis, more particularly in its earlier stages, has been mistaken for chronic appendicitis because of its location and the corresponding tenderness, pain, and infiltration which, to a varying degree, are encountered in both. Later, however, when the tubercular process has become neoplastic in character, one is not liable to be deceived and mistake it for appendiceal or perityphlitic inflammation because the tumor induces obstruction, can be seen or felt, the more acute manifestations of the latter are not noticeable, and since the attacks of appendicitis and perityphlitis are more intermittent.

Neoplastic tuberculosis has been diagnosed in a few instances as enlarged masserl glands where the nodes have become inflamed or specifically diseased, surrounded and fixed by exudates and adhesions, or degenerated, forming abscesses and fistula. Such a mis- 
take is rarely made by the careful observer who is familiar with this class of intestinal tuberculosis, and is on the outlook for pulmomary lesions, obstruction, and other signs of the disease, but where the glands are tulerentar and there is a tumor formation a diannosis is impossible, becanse the tuberculin and Ehrlich's diazo-reaction are positive in both.

In actinomycosis, which shows a predilection for the cecum, the neoplasms resemble that of hyperplast ir tubereulosis, but the former induces greater pain, more frepuently breaks down, forming chronic sinuses, the scrapings and discharge from which contain the fungus (sulphur-like granules) of atetinomycosis, while in that from tulsercular fistule may frepuently be found tuberde bacilli.

The incidence of simple impaction in the young and old and frequency of neoplastic tuberculosis between twenty and forty is a valuable aid in the differentiation of the two conditions. Again, in coprostasis the tumors form more quickly, are apt to be nodulated, are multiple, inclentable, can be manipulated, and may atso be present in other segments of the large bowel. They also excite a more aggravated type of diarrlea and toxemia, both of which, together with the tumor masses, disappear when the bowel has heen cleared by massage, medication, or enemata.

Right-sided massive adhesions from whatever cause, neoplasms of the ovary, tube, and the uterus, osteoma, nephroptosis (movabte kidney), and sarcoma have probably been mistaken for tubercular neoplasms in the ileocecal region, but, as a rule, they can be easily exchuted by obtaining a history of the case, making a thorough examination, and by comparing the symptoms and diagnostic foatures of these diseases with those of hyperplastic tulerculosis.

Gummata, as has been pointed out in the chapter on Syphilis, are encountered very rarely in the colon, but when found in the cerum and of fair size they somewhat resemble hyperplastic tubercular sweltings, but can be differentiated from them by the history of a previous chancre, the discovery of mucocutancous syphilids, spirochetes, a positive Wassermann and negative tubereulin reaction, and by the administration of mercury and potassimm iodid or Elntich's "606," which, if followerl hy a diminution of the size of the growth. points to a gummin.

Psoas abscesses may be differentiated by reflex pain, absence of tenderness on presisure, presence of fluctuation, sof tness and changeableness in position by manipulation, and its association with spinat necrosis, as indicated by the cusuing deformity.

Peritoneal Tuberculosis. The diagnosis is at times confusing in slow-cleveloping peritoneal tuberculosis, except when the patient gives a history of phthisis and suffers from diarrhea, and the stools contain pus, blood, and mucus. In uncomplicated cases, where the attack comes on suddenly, it is impossible to differentiate tubercular from other varieties of peritonitis except by laparotomy. 


\section{CHAPTER XXIII}

\section{TUBERCULAR ENTERITIS, COLIITIS, AND ENTEROCOLITIS (INTESTINAL TUBERCULOSIS), DIARRHEA IN (Continued)}

\section{TREATMENT}

PROPHYLACTIC, DIETARY, MEDICINAL, IRRIGATING, SERUM

General Remarks.-The treatment of diarrhea consequent upon tubercular lesions of the intestine (colitis) can occasionally be carried to a successful issue. In the most farorable cases it requires a long course of treatment to arrest the process, and in more aggravated it is often impossible to effect a cure, and when a good result is obtained, annoying or dangerous sequela often prevail.

Routine treatment cannot be followed in this class of cases because of the varying types and virulence of the infection, different phenomena induced by them in their constantly changing stages, uncertain part played by mixed infection, varying location and extent of the disease, and because in one instance there is a local condition to deal with, while in another foci are located, both in the lung and the bowel; the manifestations of either may predominate or their combined disturbances be of such a nature as to require special consideration. Again, diarrhea, pain, hemorrhage, or other symptoms may become suddenly so severe that it is necessary to suspend other therapeutic measures and treat the patient symptomatically to relieve his suffering or, when there is obstruction, save his life.

While it is true that in a few instances tubercular foci originate in the bowel or perianal region, remain fairly circumscribed, induce but moderate general manifestations, and can be cured by local treatment, it cannot be clenied that in most instances bowel tuberculosis is secondary to or complicated by lung involvement, under which circumstances serious general and local manifestations are induced and the patient requires both constitutional and local treatment.

From what has been said it may be inferred that the treatment is variable, hence the author will discuss the therapeutic measures useful in the treatment of intestinal tuberculosis under the following headings:

(I) Prophylactic measures.

(2) Measures for improving the general health.

(3) Medical treatment.

(4) Serum treatment.

(5) Irrigating treatment.

(6) Topical application treatment.

( 7 ) Surgical treatment. 
Prophylactic Measures.-The prophylactic treatment of phthisis and diarrhea of intestinal tuberculosis in persons who have inherited a tendency toward these conditions, have a weakencel constitution, or suffer from some acute or chronic inflammatory or ulcerative disease of the intestine consists in improving their hygienic surroundings by having them sleep in well-rentilated rooms, exercise in the fresh air, change a sedentary for a more active occupation, partake of nourishing food, aroid the dairy products of tuberculous coms, exposure, rooms or sleeping cars which have been oceupied hy consumptives, irregular habits, and association with persons afflicted with tuberculosis.

It is not known what percentage of persons vaccinated with tuberculin are prevented from contracting tuberculosis, nor has it been determined to what degree the effect of the disease is minimized in those who become infected. However, since some favorable results have been recorded and the inoculation is not dangerous, it would seem that one is justified in advising vaccination for persons who show a wakened constitution through their inherited tendency to tuberculosis.

Individuals already afflicted with phthisis should be compelled to expectorate into an air-tight receptacle, and destroy the sputum to prevent the drying and scattering of the infected material which might prove destructive to healthy individuals. They should be instructed not to swallow their sputum, to irrigate the cup and free it of hacilli. and, finally, in country districts where privies are used to destroy the excrement.

Measures for Improving the General Health.- Some individuals inherit a weakened constitution, and persons afflicted with tuberculosis of the bowel, lungs, or both suffer from debility and a lowered resistance as the result of the tuberculous process and mixed infection.

In proportion as we increase the nutrition and resistance of the patient we can prevent infection, forestall auto-inoculation, and arrest extension of existing foci.

When the patient is run down and his general condition needs building up, this is best accomplished by (a) improving his mental state; (b) recpuring him to take tonics and tissue builders; (c) rest during crises; $(d)$ live in the open air or with the windows of his room open; (e) indulge in moderate exercise except when weakened by fever or diarrhea; $(f)$ clothe himself coolly in summer and warmly in winter; and $(g)$ consume plenty of nourishing foods (nilk, cream, eggs, etc.).

Improing the Wental Condition of the Patient.- Nearly all tubercular subjects are in a replorable state mentally, either because they think they have an incurable disease or they worry orer their inability to support their family. This anxiety is enough to deplete the system, for all have observed strong, healthy, phlegmatic individuals lose in weight and health, become extremely nervous, and suffer from digestive disturbances through worrying, and if such a condition ob- 
tains in healthy individuals, it emphasizes the importance of eliminating the mental factor in tuhercular subjects. The condition of the distorted mind can often be quickly improved when the physician and relative of the patient assume a spirit of cheerfulness. encourage him by minimizing the seriousness of his condition. citing instances of others similarly afflicted who have been cured, preventing him from becoming melancholy by means of innocent amusements or taking him out for a change of surroundings, and by not constantly reminding him of his ailment through the frequent administration of disagreeable drugs.

Rest -in hed when the patient is quite ill, or in a comfortable chair when he feels better-does much toward relieving his mind, improving digestion, lowering the temperature, preventing fatigue, and overcoming insomnia. conditions made worse by physical activity. Wright claims to minimize the effects of the tubercular process by rest, and has shown that massage and mobility of tuberculous foci induces the formation of a larger amount of tuberculin than would otherwise ensue. which is distributed through the body to induce toxic phenomena. Evidence of the injurious effect of active exercise in this class of cases may be observed in the lessening of the opsonic index.

The author has frequently noticed that tubercular subjects in a sanitarium. where rest constitutes a compulsory part of the treatment, do better than office or dispensary patients who receive ambulatory treatment and are not permitted sufficient quiet.

Exercise.-Both overactivity and inactivity are harmful, and, because of this, it is necessary for these patients to partake of a reasonable amount of rest and of the right kind of exercise to improve the circulation, oxygenation, open the skin pores, stimulate the appetite, prevent muscular atrophy, and cause the various organs to functionate properly. Violent and gymnastic or indoor exercises are contraindicated because they exhaust him and lead to his breathing dusty and impure air. Walking or driving in the park or woods constitute the most suitable forms of exercise. lecause they afford entertainment, fill the lungs with fresh air, stimulate the emunctories, give the patient a greater relish for food, improve digestion, lessen insomnia, and give him something to do. It becomes necessary to change the mode of exercise. graduate or suspend it temporarily or altogether when it tends to make the patient restless, causes al rise in the temperature, or fatigue. In suitable cases shower or cool baths, followed by friction with a rough towel or massage. improve the condition, but hot baths. electricity, and vibration do little if any good, because they make the patient nervous and increase the number of the exacuations.

Fresh Air.-1t has been frequently observed that both men and animals who are cooper up in small, poorly ventilated quarters develop tuberculosis more frequently than when they live a free life in the open air and are not required to comply with the exacting demands of closely populated civilized communities. 
Oliver Wendell Holmes many years ago expressed his view as to the value of light and air lo man as Eollows:

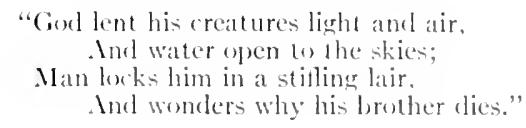

In fact, fresh air constitutes the essential feature in the treatment of tubercular subjects, irrespective of whether they rematin at home, go to a sanitarium, or live in the wilds of a higher altitule. Certainly. all farr-minded persons must admit that the results oldained at Troudeatis, the loomis, and other santaria elsewhere, where the fresh-air treatment predominates, are far superior to the older methot, where the patient was compelled to remain at home amid poor hygienic surroundings and was constantly dosed with cod-liver oil and other remedies which impared his digestion without doing him any good. Inder the present résime aggravated cases with extensive lung destruction live much longer than formerly, and many persons in the incipient stage of the disease recoter. The cure is of ten permanent, but reinfection may take place in persons benefited in a high altiude who suddenly return to a lower one and live in croweled districts andicl their former muhealthy surroundings. The fresh-air treatment consists in having the patient sleep in a spacious room with all the windows wide open, or live and sleep in the open, care being taken to see that he is kept dry, warm, and out of drafts.

The writer has often observed marked improsement and a rapid gain in weight in persons who suffered from phthisis, intestinal or anorectal tuberculosis, treated at home and in santaria, when they obtain the necessary air in conjunction with the treatment, but it has seemed to him that the results are more farorable when the patient goes to at higher altitude ( 800101000 feet) and spents most of his time resting or loitering about in the fiekls.

Clothing the patient properly is important, that he may spend his time in the fresh air during the winter without getting his leet, abdomen, or body chilled. which invariably aggravates the ubercular process; conseguently, it is advisable for him to he dotherl in woolen underwear, protect the dese and abdomen from cold hy a pad or binder, and the feet and outer clothing from getting wet by means of rubber shoes and a rancoit. In summer the clothing should be of a lighter material so he will not perspire freety, which would tend to exhatust and catuse him to take cold.

Change of Occupetion. -Frequently the infection is attributable to, or the condition of individuals already afflicted with tuberculosis is greatly aggravated by, the paticnt's occupation, which refuires him to live a seelentary life, or a more active one in the midst of small, poorly ventilated rooms, in factories, mines, ete., where the lungs are frepuently irritated by the inhalation of ritiated gases and dust, and which necessitates his suldenly changing from one temperature 
to another (as is the case in iron foundries and cold-storage plants), or keeps him exposed to all kinds of weather. If this is the case, it is the duty of the physician to advise tubercular subjects to change their abode, and, when necessary, the occupation, if it is responsible for the condition, because improvenent will not take place under the prevailing conditions, and when a cure has been accomplished through a change of location, fresh air, and good food they should be prevented from returning to the surroundings which brought on their illness.

Dieting.--Proper control of the diet constitutes a valuable feature in all inflammatory ulcerative and stenotic lesions of the intestine which induce diarrhea, particularly of tubercular origin. Dieting is rarely curative in itself, but is an aid in the symptomatic treatment of intestinal tuberculosis, a fact easily demonstrable by having the patient change from suitable to foods which disagree with him and vice versâ. It plays a much more important part in the treatment of tuberculosis located in the small than in the large bowel and rectum, which are not so sensitive to a change in the secretions or irritation from food remnants. The patient should have two diet lists-one containing food he can eat and the other that which he cannot. This practice works well in some instances, but in other cases the results are not good, because the edibles suitable to one individual frequently aggravate the condition of another, and the amount of food that can be digested varies with different persons.

When outlining a diet it is well to take into consideration the type of infection and its progress, because some forms and stages of a tubercular process appear to be more sensitive to the action of food than others-viz., cramps, gas distention, and diarrhea are less pronounced in enteric than in enteroperitoneal tuberculosis where the lesions are extensive and involve the entire gut wall.

While the author acknowledges the value of controlling the diet, he believes dieting is often overdone to the patient's detriment, because a sufficient amount of suitable nourishing food is not prescribed to sustain metabolism.

The author will now list the foods which should, and those which should not, be permitted in the average case of tubercular diarrhea, viz.:

Foods Permitted.- When the eracuations are frequent, solid foods which leave a coarse, bulky, irritating residue should be prohibited and the patient placed upon a liquid or semisolid diet, consisting of milk, koumiss, zoölak, or buttermilk (when well borne), strained gruels, milk-toast, cocoa, ordinary and beef-tea, nourishing soups; and later, cereals, raw or soft-boiled eggs, rennet and custard, until the attack has subsided, when he may be permitted to eat butter, cream, vegetable purées, sparingly of squab, chicken or quail, certain well-cooked varieties of fish, scraped beef, and other foods which experience has shown he can digest. When the lesions are confined exclusively to the lower bowel, a chop or small piece of steak may be allowerl once daily during the intervals of attacks. 
Foods Prohibited. - As a rule the following articles of diet should be excluded in acute and chronic ubercular diarrhea: raw fruits. cold drinks, ice-cream, colel carbonated beverages, eider, honey, berries, fruit juices, dates, cabbage, beets, roots, or vegetables rich in cellubse, coffee, cheese, fresh or hot bread, potatoes, meat in large quantities, shell-fish or other edibles which resist the digestive juices. chill the intestine, or irritate the inflamed and ulcerated mucosa.

From what has been said it will be seen that a routine diet in this affection is impossible, and that it is necessary to change the ford from time to time.

Medical Treatment.- Csually at one time or another drugs are necessary in the treatment of the various forms of intestinal tuberculosis, and particularly when diarrhea is the dominant symptom. The author knows of no drug which is a specific in bowel tuberculosis. In fact, he believes that more good comes from medicine srmptomatically prescribed than when it is administered with the object of curing the patient. He does not question but that, with the aid of the remedies hereafter described, the degree of pain, amount of gas distention, and freffuency of the stools and other manifestations consequent upon the diseased bowel can be diminished or temporarily alleviated, but does doubt whether they do any permanent good through actual healing of the inflamed or ulcerated mucosa responsible for the diarrhea. Many times he has seen diarrhea partially or completely checked for days, weeks, and exen months with drugs only to start up again as soon as they had been stopped. If medicines administered by mouth cannot be relied upon in this class of cases, they should be discarded or seldom prescribed, because drugs disturb digestion, impair the appetite. diminish the secretions and upset the nervous apparatus. and cause the subject to become habituated to them, and when insoluble often collect and form enteroliths which constantly irritate the intestine or obstruct the bowel. Therefore. more reliance should be placed on the therapeutic measures elsewhere recommended in the treatment.

On several occasions the writer has removed black, putty-like. bismuth accumulations which raried in amount from a few ounces to as much as 3 pound in one instance. and others have removed similar accumulations of bismuth and salol. Bismuth impactions, because of their insolubility, cannot be disolved or dislodged by catharsis or enemata, anel must be evacuated with a spoon or the fingers when near the anus, and through the sigmoidoscrope. With a narrow, loner-handled scoop, when higher up. Such arcumulationsare liack, pulty-like, and result from the continued daily administration of large amounts of bismuth to control chronic diarrhea.

In one case the author removerl altogether four bismuth tumors (orange size) from a patient who had taken the drug for three years, the first of which was rlelivered six months, the second nine months. and the third a year and a half after she had -topped taking the drug, and the fourth four years following the last. At the time the first mass 
was evacuated others could be felt in the cecum and transierse colon, but evidently the bowel had become tolerant to the chemical, since it caused no further discomfort than sensations of fulness and weight in the bowel.

In another patient (a young man of twenty) who suffered from rectal carcinoma and frequent movements incident to the obstruction, an enormous tumor was located by digital examination and abdominal palpation, the lower extremity of which was hard, the middle soft, and the upper part fairly firm and nodulated, and a later examination and exploratory incision proved the lower rectal portion to be carcinomatous, the middle bismuth putty. and the upper a fecal impaction. The growth. which at first appeared inoperable, was easily removed by perianal excision following dislodgment of the feces and bismuth with a gouge, and enemata introduced through a proctoscope inserted berond the cancerous obstruction.

For purposes of convenience the author will now discuss the medicines and sera which have been most successully employed in the treatment of tubercular diarrhea under the following headings:
(I) Tonics.
(2) Antiseptic and soothing agents.
(3) Srmptomatic remedies.
(4) Irrigating solutions.
(5) Topical remedies.
16. Serum treatment.

Tonic Remedies.--Tonics are prescribed with the object of improving the digestive apparatus, increasing metabolism and building up the general condition of the patient, but they are unreliable except when emploved in combination with the other and better therapeutic measures elsewhere reommended in the treatment of persons afficted with phthisis, intestinal tuberculosis, or both.

There can be no question that this class of patients need fats, and that they should be prescribed liberally in the form of milk, cream. butter, cream cheese, thinly sliced and properly cooked bacon. and in the incipient stages non-irritating oils (olive and almond) may. be given when tolerated, because they soothe the inflamed bowel and supply a much-needed food. The author has often observed marked improvement follow the administration of Russell's emulsion of mixed fat $=$ and regetable products $(\overline{5}=-$ - I 5$)$ three times. daily, in connection with the open-air treatment. His patients have not been improved through giving them an emulsion or other cod-liver oil preparation, but he has known these remedies to make them worse by destroving the appetite and interfering with digestion. Better results are usually obtained from forced feeding and kecping the patients' bowels open by laxatives than from the medicinal tonic treatment.

In suitable cases. where the patient is anemic. run down, nervous, and has an irritable cough. considerable relief is to be had from the administration of iron reduced iron). gr. I to $2(0.06-0.12)$; the sulphate, gr. $\frac{1}{2}$ to I $(0.0,-0.06)$; Blaud's pills, I to 2 ; arsenic (Fowler's 
solution), ne 2 to $10(0.12-0.60)$; crensote, no I to $3(0.06-0.18)$; guaiacol, mo $8(0.50)$; guaiacol carlonate, gr. Io to i $5(0.60-$ I.0), individually or simultaneously.

Sergent has been treating pulmonary tuberculosis according to Ferrier's recaleification lime treatment to resupply lime, the lons of which, he believes, prepares the soil for tuberculosis and promote it development, and eonsiders it the lest method of treating tuberculosis because it prevents the undue hoss of lime and favors recalcification. He has employed the method in $157+$ cases. and states that 306 patients have been unter observation for from six months to fre years, and general improvement was marked in 0 f per cent.: general and local. in to per eent.; It per cent. were apparently cured, while in + per cent. the disease was ummodified. Ferrier assumes that the principal cause of the losis of lime is acid fermentation in the disestive tract. which must be prevented and combated by lime salts given in substance and in mineral waters.

The treatment of pulmonary tuberculosis by the hyportermic injections of succinimid of mercury (gr. $\frac{1}{3} \cdot[0.013]$ in wh $10[0.60]$ of water) into the deep muscles of the buttock has proved beneficial in several cases treated by Dr. Barton L. Wright ( $\mathrm{L}^{+}$. S. Army), who originated the method, and others. He made thirty injections on atternate days, and then substituted potassium iodid tablets. Io gr. (0.60), in a wineglass of water three times daily during the following two weeks, after which merlication was stopper for a week, and then the mercurial injections were again started and the method continued as long as necessary: In most case- the physical signs and number of bacilli rapidly decreased frollowing the injections, and in some instances the improvement was permanent. but in others relapses occurred.

Brown recommends iodin injections in phthisis and tuberculo-is of the boivel-riz.:

R. Iodoform

Acacia powder.

Glycerin

Carbolic acid

Boiled distilled water

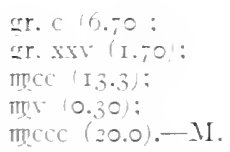

Dose.-I2 to It gr. $10.70-0.85$, for non-pulmonary cazes: 5 to I 2 gr. $10.50-0.70$ ior pulmonary cases.

The injection is followed by a rise of temperature for a day or 1 wo. and is repeated every two or three weeks. Several cases are reported in which marked improiement or a cure resulted.

Antiseptic and Soothing Remedies.-The internal admini-tration of so-called gastro-inte-tinal antiseptic has been extensively practised by the author among his private and dispensary patients for a number of years, and the different remeelies recommended for the purpese have been prescribed in small and larese doses in the various eypes of inflammatory and ulcerative lesions of the intestine. inclutine tulserculosis, with the result that he has found antiseptics unrelialsle in so far as their germicidal powers are concerned. 
In sone cases microbic activity scemed to be temporarily counteracted by antiseptic drugs taken by the mouth, but no permanent benefit followed their administration, probably because if the dosage had been sufficient to accomplish the purpose the patient would have died from the effects of the medicine.

Recent authorities on gastro-enterology have but little to say in favor of the antiseptic treatment of bowel diseases in comparison to what was said in their favor a few years ago, shortly following the publication of Bouchard's work on "Auto-intoxication in Disease."

Antiseptics are frequently prescribed with the idea of lessening intestinal fermentation and putrefaction, neutralizing toxins of retained discharges and feces, and preventing the multiplication of pathogenic bacteria, but, in so far as the author's experience is concerned, the administration of these drugs with the object of arresting diarrhea incited by tubercular foci through destruction of the bacilli is utterly useless, because, in his opinion, they are not destroyed, and it is doubtful if they are even attenuated.

It is evident that tubercle bacilli have considerable resistance against these remedies and acid media because they successfully pass through antiseptic juices (acid) of the stomach and the digestive secretions of the small intestine, which are supposed to have certain bactericidal powers. The chances are, that if a chug were discovered which would successfully destroy these and other pathogenic bacteria it would interfere with the digestive process, poison the patient, or destroy the mucosa. A serum is more likely to be discovered which, acting through the blood, would prevent and cure tubercular lesions than a chemical which would produce a cure through its local effect upon bacteria.

The following are the medicines which have accomplished the best results in the antiseptic treatment of tubercular and other specific lesions in the small intestine and colon, used alone or in combination with other drugs-viz., calomel, gr. $\frac{1}{2}$ to 2 (0.03-0.12); bismuth subnitrate, gr. Io to $30(0.60-2.0)$; carbonate, gr. Io to 20 (0.60-I.30); subsalicylate, gr. I to 3 (0.06-0.I 8$)$; beta-naphthol, gr. I to 3 (0.06-0.I 8); creosote, wr I to 2 (0.06-0.12); formol, salol, gr. 3 to IO (0.I 8-0.60); tannoform, gr. + to $8(0.2+-0.50)$; dilute solutions of hydrochloric (me Io to I5 [0.60-I.o]) and sulphuric acid (ne Io to 20 [0.60-I.30]); boric acid, gr. 5 to Io $(0.30-0.60)$; benzoate of soda, gr. 10 to $15(0.60-\mathbf{I} .0)$; fortoin (cotoin-formaldehyd, recommended by Rotschild in treatment of tubercular diarrhea because of its favorable influence upon the circulation), gr. + to $8\left(0.24^{-0.50)}\right.$, three times daily; catechu, gr. 8 (0.50); and fortoin, gr. $+(0.24)$, one powder three times daily.

Renon and Grundel have employed methylene-blue in $5+$ cases wherein the stools rapidly diminished from fifteen to twenty to normal within three days. The methylene-blue was administered in I $\frac{1}{2}$-gr. (0.09) doses combined with lactose to make it more tolerant. The improvement noted outweighs the disadvantages of the drug. 
which discolors the urine and produces an unpleasant taste. The writer has for years employed this remedy in the local treatment of tubercular and other intestinal lesions, and the remarkably good results following its employment have been attributed to its antiseptic, soothing, and healing qualities.

Calomel in divided doses ( $\mathrm{gr} .11$ [0.0I5] repeated), where the patient has bilious attacks. helps in getting rid of surplus bile and diminishing fermentation and putrefaction, particularly when followed next morning by a moderate dose of citrate or sulphate of magnesia, mineral draft, or Seillitz poweler.

Of the antiseptics named, beta-naphthol, subnitrate of bismuth, and salol alone or in combination have given the best results in the author's practice, but more through their soothing and protective action upon the inflamed and ulcerated mucosa than by their antiseptic action upon the intestinal contents.

Others of the more reliable soothing and protective remedies are the carbonate and phosphate of calcium, gr. Io to $30(0.60-1.30)$; pulverized chalk, gr. I 5 to 60 ( I.0-4.o); charcoal, gr. I 5 to 30 (1.0-2.0), alone or in combination with olive oil, neutratol, etc., in tablespoonful or larger doses twice daily, when the oil does not upset the stomach.

Bismuth subnitrate and salicylate are useful because they are constipating, have a sedative and styptic action upon the inflamed mucosa, stimulate the healing of lesions, have a decomposing eflect upon sulphuretted hyctrogen, and can be administered for days, months, or years in Io-gr. (0.60) or larger doses from three to five times daily without disturbing digestion. The principal objection to their continued administration is that they may accumulate and form enteroliths that cause partial or complete obstruction.

Routine dosage is impossible with these remedies in the treatment of diarrhea of tubercular origin, because a much greater amount is required in one case than another on account of tolerance on the part of the patient or virulence of the infection. The accompanying formule can be relied upon to relieve pain and limit the stools in emergency cases:

R. Ext. opii. gr. $\frac{1}{6}(0.01)$ : Bismuth salicylatis.................... iij (0.18).-M. Ft. pilulas No. i.

Sig.-One three times daily.

R. Syrr. zingib. Tr. opii $\operatorname{mix}(2.60)$ : Mist. creta miv $(0.24)$; $\tilde{J}=2(15.0)$. - 11 .

Sig.-Tablespoonful every four hours.

R. Pulv, cretx compos.. gr. xr (1.0): Bismuth subgallate.. Irr. $r(0.30)$;

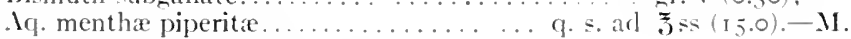

Sig.-Tablespoonful every four hours.

R. Tr. krameria Tr. opii. $\operatorname{mpxxx}(2.0) ;$ Mist. cret $x$....... miा $(0.30)$;

Sig. - Tablespoonful every four hours. $3=s(15.0)-11$. 
Antiseptics administered by mouth often accomplish very little in the treatment of intestinal tuberculosis. but when these same renedies are applied directly to the diseased mucosa in the form of a powder by inflation or irrigating solutions improvement in the patient's condition rapidly follows.

Symptomatic Remedies.-It irequenty becomes necessary to temporarily suspend the curative treatment of intestinal tuberculosis and direct our efforts against a particular symptom which distresses the patient greatly.

The most frequent and pronounced symptom of tubercular lesions of the bowel is intermittent or continuous diarhea. In mild cases the morement can be controlled fairly well with antiseptics and astringents. but where the lesions are extensive and there is marked infection, the number of stools can be diminished but not arrested. When disease of the stomach or small intestine complicates tubercular foci in the colon. it must be treated also; otherwise the diarrhea will not be entirely relieved.

of the antidiarheal medicines. opim constitutes the remedy par excllenee, and invariably partially or completely controls the evacuations. This useful adjuvant in the treatment of tubercular diarrhea and accompanying cramps and pain may be used alone or in combination in the following forms and dosage viz., pulveris, gr. I to $\mathrm{I}_{2}$ (0.06-0.09: extract, gr. $\frac{1}{2}(0.03)$ : tincture, wh 5 to $10(0.30-0.60)$; camphorated tincture of opium, 5 i to ij (4.0-8.0): Dorer's powder, gr. 5 to Io $(0.30-0.60)$ : morphin sulphate. gr. $\frac{1}{4}$ to $\frac{1}{4}(0.008-0.015)$; codein sulphate, gr. $\frac{1}{4}$ to I $(0.015-0.06)$ : heroin, gr. $\frac{1}{10}$ to $\frac{1}{20}(0.006-$ 0.0031 .

The drus may be admini-tered by mouth. hypodermically, or in the form of suppo-itories. according to the urgency of the case or inclination of the patient. A few decades ago opium was almost universally prescribed in the form of a powder or pill. but many physicians tolay prefer morphin, heroin, or codein. The latter are more convenient and are taken with better grace, but powdered or gum opium control- the movements and accompanying discomforts better because it-action i- clue to the contained resin. The beneficient effect of opium in tulercular diarrhea is due to its power of inhibiting peristal-is, favoring absorption and nutrition (by quieting the bowel), relieving muscular spasm, quieting the pain from gas accumulations, and checking the -ecretions.

From a curative-tandpoint opium serves to tie up the bowel. so that it acts as a splint and heeps the intertinal le-ions within it quiet and give them an opportunity to heal. When enterospasm is frequent or serere. good result are obtainable by combining gr. 1 to $\frac{1}{4}(0.008-$ 0.015 of the extract or Huidextrat of belladonna with it.

When there is hyerfermentation or putrefaction, or the bowel is irritalle, an antiseptic, astringent. or sedative remedy may be comlined with opium to adrantage. The frequent stools of tubercular diarrhea can genterally le contrulled by the adninistration of Dover's 
powder, gr. Io (0.60), and bismuth subnitrate or carbonate, gr. 30 (2.0), three or more times daily.

Where the stomach is easily upset or the patient suffers from rectal tenesmus and pain, suppositories containing cocain or morphin, gr. $\frac{1}{4}$ (0.0I5), in combination with bellarlonna, gr. $\frac{1}{4}$ (0.008), inserted two or three times daily, do much toward relieving the patient's suffering and reducing diarrluca.

Styptic remedies alone, or in combination with opium or bismuth, are fargely relied upon by some physicians to control diarrhea and encourage intestinal ulcers to heal. (of the astringents, lead acetate, gr. I (0.06); alum, gr. 5 (0.30); tamnic acirl, gr. 5 10 \& (0.30-0.50); tincture of cocoa, gtt. 5 to $10(0.30-0.60)$; gallic acid, gr. 10 to $15(0.60-$ 1.0); tannalbin, gr. 15 to $60(1.0-4.0)$; tamnigen, gr. 4 to 8 ( $0.24-0.50)$; tannocol, gr. Io to I5 (0.60-I.O); ichthoform, gr. I5 to 30 (I.O-2.0); ichthalbin, gr. I5 to 30 ( I.O-2.0); subgallate of bismuth, gr. 5 to 20 (0.30-I.30); and silver nitrate, gr. $\frac{1}{60}$ to $\frac{1}{30}(0.00 \mathrm{I}-0.002)$, in $5 \mathrm{ij}(8.0)$ of water every two hours (Jacoby), have been most frecuently employed.

Silver nitrate is very effective, but sometimes when long continued gives to the skin a darkened and phosphoreseent hue (argyria).

Acetate of lead, in combination with opium, is serviceable for checking diarrhea and in alleviating cramps and abdominal soreness, and, when used in connection with hot moist applications to the abelominal wall, reduces muscular rigidity.

Connterindications to antidiarrheal remedies in the treatment of intestinal tuberculosis are stricture, fecal impaction, enteroliths, retained discharges, and putrefying foods.

It is advisable to preseribe appetizers, tonics, and other remedies which will aid digestion, so that the patient can digest a reasonable amount of ford and keep up metabolism. The gastric and intestinal contents should be examined as to their alkalinity or acidity, so that in case either is deficient the trouble can be corrected by having the patient take dilute hychochloric or sulphuric acid in small doses where there is hypo-acidity; or magnesia, bicarbonate of soda, or lime-water when there is hyperacidity.

Pejosin may lec combined with hydrochloric aciol to arlwantage in gastric atonic dyspepsia, hut when the dyspepsia is of intestinal origin pancreatin is useful, and $11 \mathrm{x}$ vomica and gentian can be relied upon to tone up the gastric glands and clear up hilions attacks.

Cohnheim recommends the tincture of creosote, gtt. \& $(0.50)$ in a spoonful of red wine three times daily, in the treatment of tubercular dyspepsia, the amount being gradually raised to gtt. 30 ( 1.3 ). which is continued for three months.

Fever which cannot be controlled by rest, fresh air, tepid water, or alcohol sponge baths should be kept within bounds by antipyretic agents-aspirin, antipyrin, etc., gr. 5 to ro $(0.30-0.60)$, three times daily-remedies to be avoided if possible, hecause of their usually harmful effects upon the heart. 
Night-siveats can be speedily overcome by forced feeding and the fresh-air treatment, but when they cannot a few drops of the tincture of helladonna or the injection of atropin, gr. 1100 (0.0006), usually accomplish the desired results.

In this connection it might le of interest to note that Flemer has obtained some rery good result w wh gelatin in the treatment of intestinal tuberculosis, and that Heiser has found that where tuberculosis and hookworm coexist the former is much easier placed under control when the latter has been eliminated.

Irrigating Treatment.- The irrigating treatment of intestinal tuberculosis is always beneficial, but the results from it are much better in some than other forms.

It is more effective in the enteric or superficial ulcerative than in either the enteroperitoneal (Fig. 39), hyperplastic, fibrosclerotic,

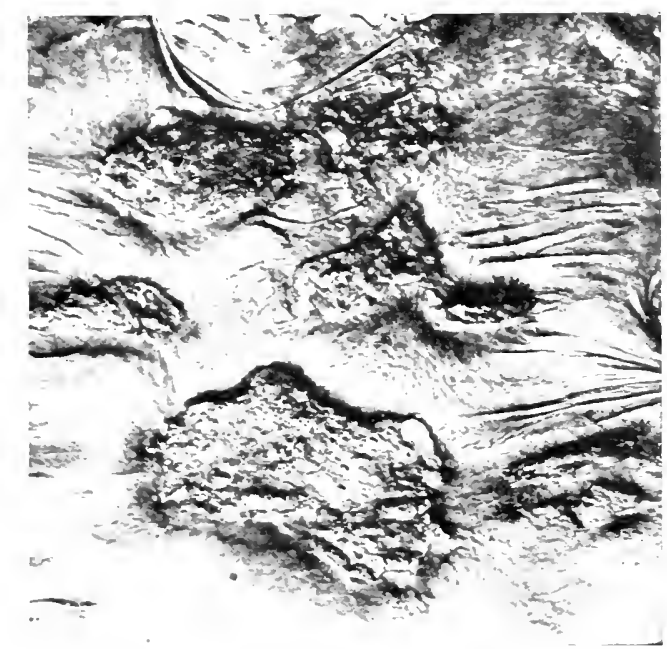

Fig. 30.-Tubercular ulcers of the colon. Note their number, large size, and lack of tendency toward girdle formation. The rounded edges of the lesions, the scar tissue, and puckered condition of the mucosa indicate that the tubercular process has been arrested. ${ }^{1}$

glandular, or peritoneal varieties. lecause the lesions here are limited to the superficial layers of the mucosa, which are easily reached, reansed, and stimulated by the medicated fluid.

In the enteroperitoneal or deep ulcerative type, where the mucosa, serosia, or entire bowel wall are involved, enteroclysis helps directly and indirectly to improve the patient's condition by dislodging irritating feces, débris, and discharges, neutralizing, destroying, or evacuating toxins, attenuating. killing, or flushing out tubercle bacilli and other pathogenic organisms, healing ulcers and limiting extension of the disease by improving the bowel resistance, purifying the circulating fluids. and minimizing the danger from specific and mixed infection.

Except in the abote-mentioned ways these measures are of little 1. Army Ned. Nuscum. 
conseguence in the treatment of this type of tuberculosis once the foct have become established in the serosa, muscularis, and mesenteric or retroperitoneal glands.

In hyperplastic (tumor-forming) tuberculosis the neoplasm is frequently located in the cecal region or rectum, induces a marked intlammatory reation in the intestines, but does not uleerate unt il late when it has assumed considerable size. Conseduenty, enteroclysis accomplishes very little in the earlier stages of neoplastic tuberculosis. but later. when tubercular mixed infection and stercoral ulcers are present, it does considerable toward preventing extension of the ulcerated areas, formation of abscesses, and auto-intoxication, arresting hemorrhage, neutralizing poisons, ridding the bowel of irritating discharges, toxins, and hacteria, evacuating gas accumulations, and relieving soreness, cramps, muscular rigidity, and pain when emplored hot $\left(100^{\circ}\right.$ to $110^{\circ} \mathrm{F}$.).

This type of tuberculosis is not curable by enteroclysis or medicated irrigations because the disease extends intrinsically and extrinsically by cell proliferation, forming tubercular masses of such size that the solution could not be expected to cause its obliteration since it does not reach all parts of the growth. The statistics given in the surgical treatment show that numerous cases bave heen reported where tubercular neoplasms have heen diminished or mate to disappear by short-circuiting the involved gut. probably through affording rest to the affected part. If hyperplastic tuberculosis is amenable to this procedure, it should he still more so to proper intestinal irrigationswhich diminish peristalsis, soothe the intlamed bowel, heal ulcers. and cleanse the infected area of bacteria. toxins. discharges, and other irritants. Quicker and better results are obtained when through-andthrough irrigation, described elsewhere. is provided fur at the time of short-circuiting. to prevent backing up of the feces and permit bowel Hushing. This combined procedure is devoid of the dingers of resection. and is indicated in all cases where the patient teclines extirpation of the neoplasm or its remoral is impratcticable and as a try-out with a riew to resection in case it fails.

In the fibrosclerotic (stricture-forming) tulerculosis the irrigating treatment alone or in connection with entero-indstomosis has the same indications as in the hyperplastic type.

Glandular and peritoneal tuberculosis are lenefited by enterenclyos as independent affections, and also when they are anociated with bowel tuberculosis. The author has shown hy statistics that there is alwars a simple or specific catarrbal intlammation of the muensa in persons who suffer from phthisis, infection of other organs. or intertinal tuberculosis, therefore irrigation is beneficial in these caser because it attennates or prevents the lokgment of swallowed hacilli, soothes and heals the bowel. minimizes auto-intoxication. improver the circulation, and increases nutrition when oils or nutrient solutions are emplovert.

${ }^{1}$ Gant, Constipalion and Inteslinat Obstruction, pr. $+15 \cdot+10$. 
The irrigants used in the treatment of tubercular are practically the same as for other types of colitis. The chief benefit derived from irrigating solutions is attributable to their mechanic effect in cleansing the inflamed mucosa and ulcers. areas of bacteria, toxins, acrid discharges. putrefying fond remnants. and feete.

Cold solution $-55^{\circ}$ to $75^{\circ} \mathrm{F}$. induce diecomfort and enterospasm. while hot ones $100^{\circ}$ to $115^{\circ} \mathrm{F}$. soothe the sut diminish soreness, and relieve this condition.

When the storls are numerous. bleeding is profuse. and the patient is being exhauted by the diarrhea. irrigations of silver nitrate. gr. xxx to Oij 2-I000), are administered three times weekly, after which the bowel is washed out with a normal salt solution to remove any exces of silver. When the eracuations have been considerably reduced and the patient feet- better. one of the following milder solutions should be substituted for the silver. viz.: boric acid. 3 per cent.; ichthyol. I to 2 per cent.: balam of Peru. I per cent.: permanganate of pota-ium. I per cent.: siker nitrate, gr. I to Oij (0.30-1000): protarsol or argyrol. 5 per cent.-irrigants the strength of which may be - lishtly increased or decreased according to the severity of the ulceration and consequent diarrhea.

Very often the treatment: are best alternated with warm olive. cotton-seed, or mineral oil injections, alone or containing bismuth, aristol. salol. salicylate of soda. morphin. etc. which soothe the mucosa. protect and heal ulcers, lesen putrefation, or partially disinfect the bowel.

A description of the different way of irrigating the colon and small inte-tine and admini-tering enemata ha- been given in detail in Chapter XlI devoted to the purpose. and a repetition would be out of place here.

Topical Applications. - Formerly, topical applications were made throush a speculum and limited to the lower rectum, but with the aid of the simmoidoscope. applieator- and reflected light one can locate and treat lesions located in the lwwer sigmoid flexure and all parts of the rectum Fiss to. $4 \mathrm{I}$ ).

In ascravated case- of intestinal tuberculosis different types) where there are large single or multiple sluggish ulcers which do not re-pond to medicated irrigations. much can often be done to hasten the patient's recovery hy dieting and measure- recommended for upbuildins the seneral health, and topical applications made to ulcers. the inflamed mucrea. or both.

Persi-tent le-ion- heal faster when cauterized with an electric or Paquelin cautery, but some patient ofject to them. in which case a -ilver nitrate or copper sulphate solution full strength) should be -ub-tituted and applied by means of cotton on an applicator introduced through a ismoidoscope. while care in being taken to keep the olution from coming in contact with the mucosa between the lesions.

When the ulcers take on a healthier appearance and for less 
obstinate lesions, ichthyol and glycerin or the balsam of Peru, 50 per cent., are preferable to strong silver and copper solutions. Nitric and other acids accomplish less and should not be employed, heause

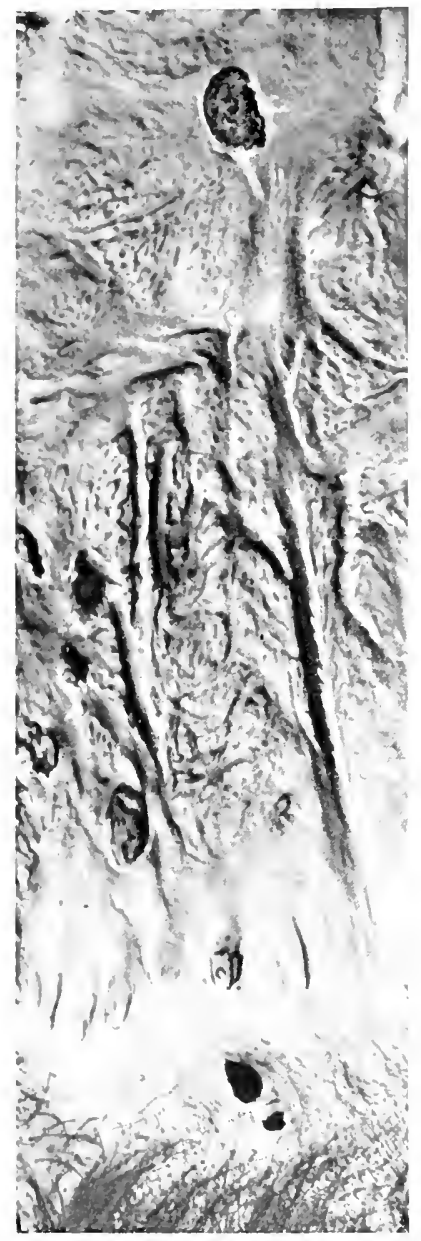

Fig. 40.-Chronic tuberculosis of the anorectal region complicated by ulceration, alberess, fistula, perforation, and the formation of varying shaped and sized ridges of scar-tissue from healed lesions.! it is difficult to prevent them from sprearling from disceased to healthy tissues, where they cause sloughing.

In the average case, or where one has to deal with superficial lesions or those having a tendency to heat, silver nitrate, 6 per cent., protargol, 20 per cent., balsam of Peru, 25 per cent, or ichthyol, 15 per cent., give satisfactory results when applied to the lesions three times weekly and the bowel is irrigated on alternate days. Now and then stimulatingapplications make the patient worse, in which case the lesions and ad-

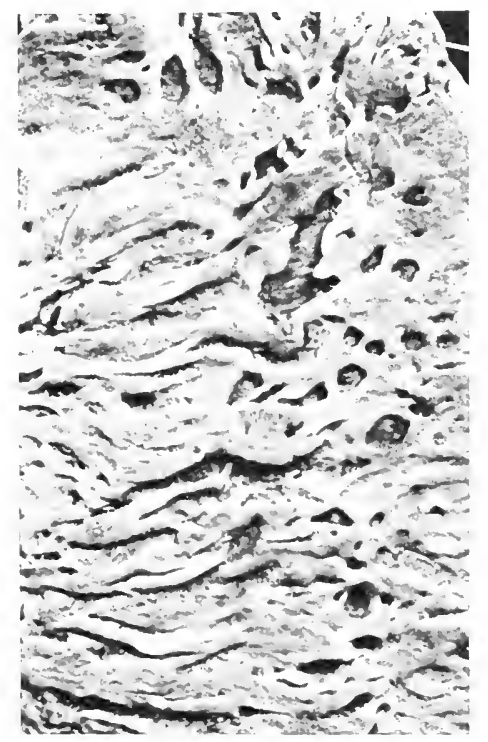

Figr. +r.-Tubercular ulceration of rectum.

jacent mucosa should be treated with methylene-lulue, 10 per cent.. a remedy of the highest value in the treatment of all chronic ulcers. Where the mucosa is inflamed and aroled from the discharge, lurning pain and tendernesis can be diminished by mopping the ulcerated rectal mucous membrane with cocainized oil or that containing hismuth 
or aristol, which form a coating over and protect it from irritation by the feces.

Where there are no ulcers and the patient suffers from a simple catarrhal or a tubercular sigmoidoproctitis, his condition can be greatly improved by introducing the sigmoidoscope and inflating the bowel with boric acid or a powder composed of the following:

R. Tamnic acid ........................

Bismuth subnitrate..................... gr. $\mathrm{xx}(\mathrm{I} \cdot 3)$;

l'ulv. alum.....................

I'ulv. talcum................................

Sig.-Insufflate the bowel daily.

In case the intestine is inllamed, sensitive, and marked by pinpoint ulcers, the author has found the accompanying formula very uselul:

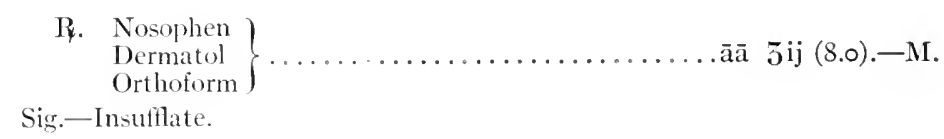

Where the patient is nervous and complains of extreme pain from topical or other treatment, his suffering can be reduced by spraying or swabbing the mucosa with cocain, 8 per cent., but the solution should not be injected, as a dangerous amount will be quickly absorbed.

Having given the medical, the author will now discuss the serum and vaccine treatment of intestinal tuberculosis, alone and when complicated by lung involvement.

Serum Treatment.-Various sera, including Koch's old and new tuberculins and bacillary emulsions and vaccine preparations, have been employed in the prophylactic treatment of general and tuberculosis of the intestine, but the results have not been particularly flattering. Thus far they have not proved useful in the treatment of foci in the bowel which causes acute or chronic diarrhea, and injections of them have occasionally been followed by serious toxic manifestations and abscess.

Maragliano has produced a serum (cows') for which he claims much when employed as a vaccine to immunize the patient and as a therapeutic measure to cure tuberculosis. Of the many cases treated with his serum in Italy there were 250 incipient cases where the lesions were circumscribed and there was no fever, of whom 38 per cent. were cured and 50 per cent. greatly improved, but at the Phipps Institute this serum was given a thorough trial and proved unreliable in old, and of no more value in incipient, cases than if the patient had been subjected to the Trodeaus or sanitarium treatment. Marmorek's horse serum has exhibited a tendency to immunize and improve the condition of tubercular animals, but in man the good results have not been sulficient to offset its toxic effects. 
It has been shown repeatedly that the index is lowered in tulercular subjects when regions of the foci are traumatizerl. Conseguently, the administration of Koch's new tuberculin R. (which is made of disintegrated bacilli) and similar preparations are valuable aids in the diagnosis of intestinal tuberculosis, since their employment is indicated by a rise of the opsonic index and the treatment is accompanied by increase in the opsonins which are antagonistic to tubercle bacilli.

The hygienic, dietetic, and medicinal treatment of peritoneal tuberculosis is the same as when the gut is affected. but non-surgical and operative therapeutic measures must be selected according to indications. Some patients do well under colonic irrigation when they are kept quietly in bed, given a light nutritious diet, cold applicationare made to the abdomen, and the rapid pulse, high temperature. constipation, diarrhea, and other manifestations are symptomatically treaterl.

The surgical measures most of ten resorted to in tubercular peritonitis are aspiration. laparotomy, and excision of abdominal tubercular foci. The first is applicable when ascites is marked, but is often unsatisfactory because the speration must be repeated and there is danger of injuring the intestine with aspirating needle or trocar. I aparotomy is not dangerous and gives the best results, but no satisfactory explanation has been advanced as to why a cure sometimes follows thin procedure. It may be due to relieving distention, pernitting the escape of retained toxins, admitting light or air to the abdominal carity, mechanical irritation to the peritoneum, effect upon the nerses thromeh psichic impulses, excitement brought about by the preparation for operation and after-treatment which encourages the patient to believe that he will be cured, or some other unknown cause. While the abdomen is opened, complicating adhesions should be broken up, rents in the viscera should be repaired, and tubercular deposits exeised when feasible. According to Treves and others, about one-third of the patients who suffer from tubercular peritonitis are curable and many others are improved by laparotomy. No doubt many of these patients remain well, but the author's experience would lead him to expect a relapse in a goodly percentage of the cases.

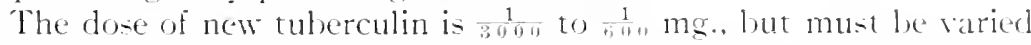
and arlministered at shorter or longer intervals, according to the opsonic indices. It is not advisable to prescribe a large amount and make the injections frequently. In fact, one treatment should not follow another until the simptoms of the former have subsided, otherwise the remedy instearl of helping may have a contrary effect upon the patient. The administration should be discontinued when the toxic effect of the tuberculin becomes annoying or dangerous, and discontinued when the patient becomes tolerant of it.

Naturally, the sera act more farorably in incipient than in ageravated cases of tuberculosis where intestinal or other lemions are extensive and there are suppurating areas. Concerning the tuberculin 
treatment. Koehler and Lenzmann say: "In a general waỹ it may be said that the initial stages of tuberculosis are preferably subjected to treatment with old tuberculin. whereas the adranced and especially the very chronic cases are more amenable to the tubercle bacilli emulsion. Certain precarious cases, which do not readily tolerate old tuberculin, and react to every injection with persistent and high fever, should be treated by means of tuberculin T. R. or the mild preparation- from germ-free tubercle bacilli bouillon."

From the writer's experience and his perusal of the literature concerning sera and vaccine therapy, he believes them worthy of a trial when the process is in the incipient stage, and as an immunizing agent in persons of an inherited predisposition to tuberculosis, but considers them of but very little value in the treatment of fully developed foci in the intestine, and particularly when complicated by lung involvement, and advises against their use because of their tendency to produce unpleasant or toxic symptoms and abscess which may result fatally.

From what has been attained in the prevention and treatment of diphtheria, typhoid fever. syphilis, small-pox, and other infectious diseases, and favorable cases of tuberculosis by sera, vaccines, etc., the chances are that before long an agent will be discovered that will prevent or cure tuberculosis of the lungs, intestine, and elsewhere. 


\section{CHAPTER XXIV}

\section{TUBERCULAR ENTERITIS, COLITIS, AND ENTEROCOLITIS INTESTINAL TUBERCULOSIS, DIARRHEA IN (Concluded)}

\section{SURGICAL TREATMENT}

StraICAL intervention is indicated in bowel tuberculosis (colitis when the various measures already recommended for the relief and cure of this alfection fail. when the type of infection is virulent, and the local lesions progress rapidly or profoundly influence the patient's general health. when dangerous bleeding takes place and the patient suffers frequently and greatly from cramps, or his strength is being rapidly exhausted because of the frequent morements. and in chronically aggravated cases complicated by stricture, ahscess. fistula, and peritonitis from perforation.

Surgical intervention is not always satisfactory in the treatment of tubercular colitis and diarrhea beeause the ulcers often heal slowly or extend, owing to the subject's lowered resistance conserfuent upon his lack of appetite, poor digestion, bat assimilation, and debilitated condition.

Except in urgent cases the author never suggests an operation in this class of cases until he has first made a thorough general and local examination, endearored to build the patient up by a proper diet, having him spend his time in the fresh air, giving him tonics and colonic irrigations.

One should operate as quickly for the relief of one type of intertinal tuberculosis as another, and for any dangerou- complication which might arise if the patient has sufficient strength. hut when he is debilitated, or going to die shortly from foci in the lungs or elsewhere. nothing can be accomplished by operation. Most patients suffering from bowel tuberculosis have an active or latent infection in the lung. a condition which under favorable circum-tances may be arerarated by the other and terminate fatally. Becaute of thi- the author operates under local anesthesia, but uses gas or gas-oxyen when general narcosis is reepuired.

Surgical profedures designed for the treatment of howel tuberculosis have for their object one of three things-removal of the disease. putting the gut at rest, or proxiding for through-ankl-through irrigation.

Extirpation of tubercular foci is accomplished ly enterectomy. cecectomy. sigmoidectomy. or proctectom $\mathrm{y}$ aceording to the location of the disease. 
Exclusion (short-circuiting) of the liseased intestine is accomplished by lateral entero-anastomosis (Maisonneuve) or unilateral or bilateral exclusion, and bout flushing is achieved by means of enterostomy (duodenostomy. jejunostomy. ileostomy), appendicostomy. cecostomy, appendiceal cecostomy. cccostomy aith an arrangement for irrigating large and small intestines (Gant's), and colostomy.

When the patient's condition permits. resection is the operation of choice, because it offers the patient the best chance for a quick and permanent recovery. While it cannot be denied that this class of patients withstand operation as well as those afflicted with other intestinal affections. it must be admitted that the procedure is always franght with some danger because of the time required, intestinal trauma, and injury to adjacent organs incident to the destroying of adhesions and freeing the gut. The experience of the author is in harmony with that of other operators, in that the danger is about the same whether a small or large piece of intestine is excised. He has treated patients where several inches or feet of the large or small intestine were removed who suffered but little from shock and made an uninterrupted recovery. Multiple resections are necessary where widely separated segments of gut are diseased and when tubercular foci develop subsequent to operation. Instances of such cases will be found in the statistics given below.

The author has at times found it adrantageous to short-circuit and put the diseased bowel at rest. or perform appendicostomy or cecostomy as a preliminary step to resection when the patient's condition would not warrant a more radical operation.

Nest to resection, short-circuiting is the favored procedure in this class of cases. Intestinal exclusion consists in diverting the fecal current from a large or small part of the bowel, so that the lesions within it mav obtain rest and heal. The author has resorted to exclusion only six times in treating intestinal tuberculosis. but has practised it with satisfaction many times for the relief of other conditions. ${ }^{1}$ and the operation, with few exceptions, has not proved tedious, difficult, or dangerous.

A segment of the intestine may be excluded (a) by simple enteroanastomosis with a Murphy button or suture (Fig. 176$) ;(b)$ by closing the howel on one side of the lesion and anastomosing the proximal end with healthy bowel below-unilateral exclusion (Fig. I $8 \mathrm{I}$ ); and (c) by dividing the intestine on both sides of the diseased area and joining or closing the proximal and distal ends-bilateral exclusion (Fig. I $T$ )

Of the three procedures, the former is usually practised in bowel tuberculosis. When doing either of these operations the writer usually provides a way (appendicostomy or cecostomy, etc.) of flushing the diseased bowel to relieve the excluded gut of backed-up feces and rlischarges, and thus treat the lesions. It is really necessary to shortcircuit the small intestine because in the majority of cases tubercular

\footnotetext{
${ }^{1}$ Gant. Constipation and Intestinal Obstruction. p. 4 ro.
} 
lesions are located in the cecal region or the recum, and when called for it is advisable to connect the proximal end of the small with the distal end of the large gut. Ender such cireumstances the morements are thuid and frepuent at first, but in a short time the ileum gradually takes upon itself the functions of the colon, and the stools beeome fewer and more solid week by week, until hy the end of two or three months they usually become normal in number, form, and onsistence.

Entero-anatsomosis may also be called for as a preliminary nueasure to excision in ageravated cases, and following this operation when there is a resultant stricture. The llattering results which follow thene methods of exchding the diseased bowel have been verified by the author, and are shown in the appended statistical tables.

These procedures (entero-anastomosis and unilateral and bilateral exclusion) are indicated when there are complications recpuiring immediate attention in the presence of serious lung involvement, when the patient is greatly debilitated or exhausted, the bowel is extensively diseased, or a neoplastic tubercular tumor is large and adherent everywhere, numerous athesions which may bind the aflected fragile segment to the intestinal or other organs, when there are chronic pyostercoral fistula, extensive glandular involvement, and when, for any reason, extirpation is out of the question, or it has been performed and the disease has returned or stenosis has followed.

The unfarorable features of intestinal exclusion are: it is more often palliative than curative, healing takes place slower than following extirpation, the discharge and bacilli continue to pour down over healthy gut, the secretions and feces sometimes back up and become stagnated, no provision is made for the direct treatment of the lesions, the disease may attack the anastomotic opening and require a second operation. no attempt is made to correct or remove peri-intestinal adhesions, abscesses, fistuke, and diseased glands causing the patient discomfort, foci are left from which the disease may extend or become generalized, and he must necessarily suffer for several weeks from diarrhea until the feces once more become firm.

Through-and-through irrigation (by way of a cecal or appendiceal opening) is always indicated in persons who have intestinal tuberculosis and suffer severely from exhausting diarrhea. locterial and chemical toxins. bleeding, irritation and tenesmus incited by acrid discharges, gas accumulations or cramps incident to fermentation or putrefying food within the intestine. when enterodysis from bedow does not accomplish the desired results. In some instances all the infected foci are located in the lower sigmoid flexure and rectum, and under these conditions flushing the bowel through the anus usually proves effective because the fluid reaches them: but, on the contrary when the inflammation or uleers extend throughout the large howe irrigation from below often fails lecause the solution etres not reach all the segments of the diseased colon on aceount of the patient position, type of irrigator employed, or the irrigating ube curls up in the rectum or sigmoid flexure and prevents the solution from flowing inte 
the upper colon. Inder such circumstances an artificial inlet should be established ly appendicostomy (Fig. +2), cecostomy (Fig. 159), appendiceal cecostomy, the author's cecostomy, by means of which the large and small bowel can be separately or simultaneously flushed, or colostomy. These procedures, with the author's cecostomy, may be employed in the treatment of tuberculosis involving all or a part of the colon, but they (with the exception of the author's cecostomy, Fig. I59) are not satisfactory when there is enterocolit is because the solution cannot be made to pass the ileocecal valve, and his operation should be performed, since it provieles for thorough irrigation of both the large and small intestine.

While through-and-through irrigation (Fig. 42) is beneficial in all varieties of intestinal tuberculosis, its palliative and curative effects are elecidedly more marked in the enteric form of tuberculosis which

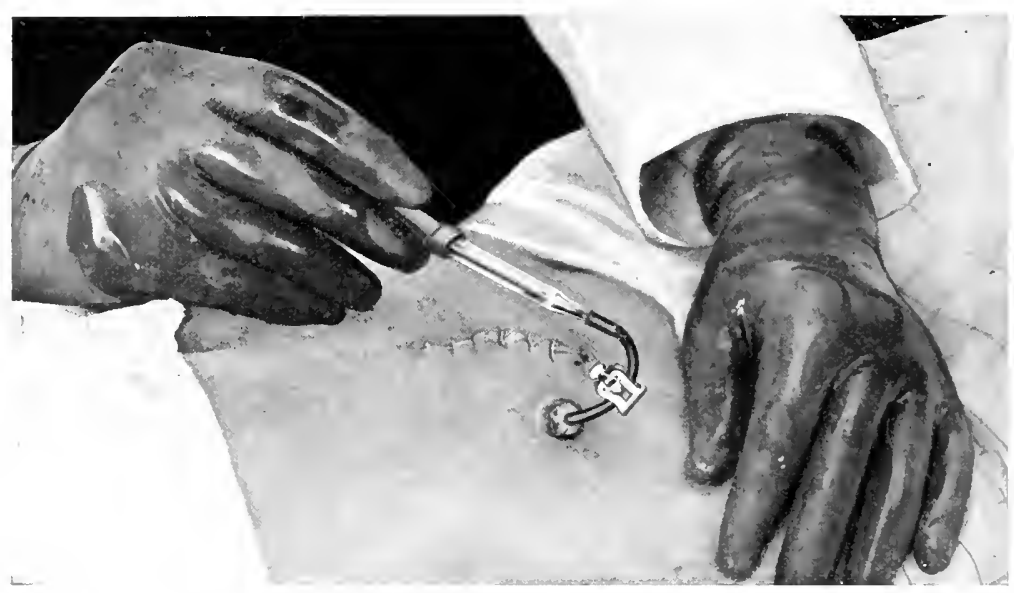

Fig. 42.- Through-and-through irrigation following the author's appendicostomy.

involves the mucosa superficially than in either the enteroperitoneal, which attacks all the intestinal coats from within and without; the hyperplastic, wherein there is extensive tumor formation and ulceration in the later stages; the stenotic, where the tunics are converted into fibrous tissue; the glandular, with inflaned and caseating lymph-nodes; or the peritoneal types.

There is a catarthal or tubercular inflammation, erosion, or ulceration of the mucosa in all forms at one stage or another of the disease, conditions invariably improved or cured when the bowel is properly irrigated daily or three times weekly with one of the irrigants already montioned.

Through-and-through irrigation is preferable to the introduction of a solution from below because larger amounts and stronger solutions can be used, owing to the fact that they pass rapidly through the gut, particularly when a small proctoscope has been introduced 
through which they may escape. less pain accompanies this procedure than the former, for the reason that the fluid flows in and out in a continuous stream. lout when it is injected throush the anus there is consiglerable distention pain by the time the neetesary amount of fluid has been introelueerl.

The above-mamed operations have occasionally leen employed as a preliminary treatment to heal ulcers and improve the patient's general conclition prior to extirpation or resection, and in conjunction with intestinal exclusion to prevent stagnation and faror a more speedy convalescence through cleansing of the lesions.

The irrigants employed in this nuse complete method of enteroclysis have already been given or will be discussed in the chapter which deals with intestinal irrigation. The author has not pointed out the special features and methods of performing the operationof excision, exclusion, appendicostomy, cecostony. and colostomy here, because their indications and technic have been given in the chapters set apart for the surgical treatment of diarrhea.

In concluding the treatment of intestinal tuberculssis nothing more is necessary than to summarize the statistical result of the above-mentioned operations and point out their relative value under varying circumstances.

STATISTICS CONCERNING ENTERECTOMY, CECECTOMY, COLECTOMY, AND PROCTECTOMY

Cecectomy and Ileocecectomy.-Lnder this heading are grouped the statistics relative to extirpation of the cecum, lower ileum, proximal end of the ascending colon, and the appendix, alone or together.

\section{ILEOCOLECTOMY}

\begin{tabular}{|c|c|c|c|}
\hline Author. & $\begin{array}{l}\text { Personal and } \\
\text { collected cases. }\end{array}$ & Nortality. & Cures. \\
\hline & & Per cent. & Per cent. \\
\hline 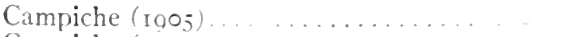 & I 54 & I 2.0 & +1.0 \\
\hline 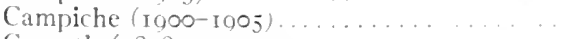 & 50 & & 30.0 \\
\hline 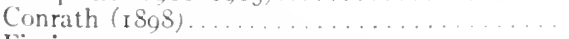 & 60 & & $3 \mathrm{I} .0$ \\
\hline Fiori. .... & 7 & I +.2 & $7 \mathrm{r} . \mathrm{O}(2 \mathrm{grs})$ \\
\hline Frague Surcical Clinic (Rubesch) .......... & I 2 & 8.3 & 53.3 \\
\hline 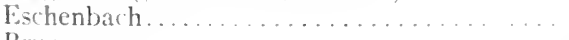 & 24 & 29.1 & \\
\hline 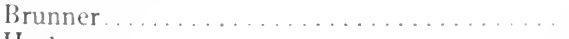 & 125 & 25.0 & \\
\hline Harlmann . . . . . . . . . . . . . . & I 28 & $2 \mathrm{I} . \mathrm{S}$ & \\
\hline 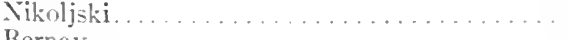 & & 31.0 & \\
\hline Bernay . . . . . . . . . . . . . . . . . . . . & 37 & I $3 \cdot 5$ & \\
\hline Lotheissen . . . . . . . . . . . . . . . . & 17 & 17.6 & \\
\hline Nicoloysen. . . . . . . . . . . . . . . . . . . . & $4 i$ & I O. I & \\
\hline 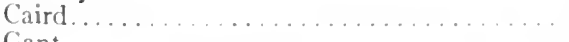 & I I & 30.3 & \\
\hline 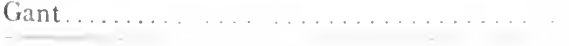 & 3 & $.33 \cdot 3$ & \\
\hline Total $\ldots \ldots \ldots \ldots \ldots \ldots$ & 675 & & \\
\hline Average mortality .............. & $5^{6} 5$ & $2 I . S$ & \\
\hline Average cures............... & $28_{3}$ & $4^{0.4}$ & \\
\hline
\end{tabular}




\section{INTESTINAL EXCLUSION}

ENTERO-ANASTOMUSIS, UNLLATERAL AND BILATERAL EACLUSION

\begin{tabular}{|c|c|c|c|}
\hline Author. & $\begin{array}{l}\text { Personal and } \\
\text { collected cases. }\end{array}$ & Mortality. & Results. \\
\hline & & Percent. & \\
\hline Kubesch................ & I 3 & 7.60 & \\
\hline Hartmann. . . . . . . . . . & $3 \mathbf{I}$ & 9.67 & \\
\hline 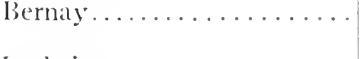 & 4 & 25.00 & $\begin{array}{l}3 \text { patients recovered; } 1 \text { well } \\
\text { two years later. }\end{array}$ \\
\hline Lotheissen.............. & I 5 & 20.60 & \\
\hline Nikoljski.............. & 40 & 20.40 & $\begin{array}{l}65.3 \text { per cent. lived one year } \\
\text { or more. }\end{array}$ \\
\hline 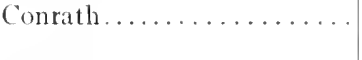 & IO & $\ldots$ & $\begin{array}{l}\text { Immediate and remote re- } \\
\text { sults excellent. }\end{array}$ \\
\hline Halberer.............. & I 3 & 38.4 & $\begin{array}{l}8 \text { patients lived eleven months } \\
\text { to five years. }\end{array}$ \\
\hline 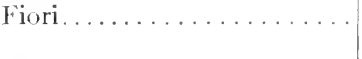 & 4 & $\cdots$ & $\begin{array}{l}75 \text { per cent. survived after } \\
\text { three years. }\end{array}$ \\
\hline Brunner........... & 36 & 8.33 & \\
\hline Giant..... & 3 & $\cdots$ & $\begin{array}{l}\text { Still living, eleven months, } \\
\text { two years, and three years. }\end{array}$ \\
\hline Total......... & 178 & 13.60 & \\
\hline
\end{tabular}

OPERATIVE TREATMENT OF INTESTINAL TUBERCULOSIS

\begin{tabular}{|c|c|c|c|c|}
\hline Autlor. & Nature of operation. & $\begin{array}{l}\text { Number of } \\
\text { cases. }\end{array}$ & Deaths. & $\begin{array}{l}\text { Parcentage of } \\
\text { mortality. }\end{array}$ \\
\hline \multirow{10}{*}{$\begin{array}{l}\text { Ilartmann } \\
\text { (Groves): }\end{array}$} & Partial resection of cecum..... & 9 & I & I I \\
\hline & $\begin{array}{l}\text { Resection with end-to-end an- } \\
\text { astomosis....................... }\end{array}$ & 78 & I9 & 24 \\
\hline & $\begin{array}{l}\text { Resection with side-to-side an- } \\
\text { astomosis. }\end{array}$ & $3 \mathbf{I}$ & 5 & I 6 \\
\hline & Resection a Deux temps........ & 10 & 3 & 33 \\
\hline & Ileocolostomyr . . . . . . . . . . & 20 & 4 & 14 \\
\hline & Unilateral exclusion.... . . & 0 & I & I I \\
\hline & Bilateral exclusion. . . . . . . & 22 & 2 & 9 \\
\hline & End-to-side (anastomosis)..... & IO & 3 & 15 \\
\hline & Multiple operations..... ... . & 22 & 8 & 36 \\
\hline & Total and average percentage. & 220 & 40 & 20 \\
\hline \multirow[t]{6}{*}{ Conrath: } & Resection............... & 58 & I I & I9 \\
\hline & Resection of intestinal wall ... & 6 & & \\
\hline & Entero-anastomosis. . . . . . . . & IO & & \\
\hline & Enucleations. . . . . . . . . & 8 & 2 & 25 \\
\hline & Exploratory laparotomy....... & 4 & & \\
\hline & Total and average percentage. & 86 & 13 & I5 \\
\hline
\end{tabular}


OPLR.ITIVE TREATMENT OF INTESTINAL STENOSES

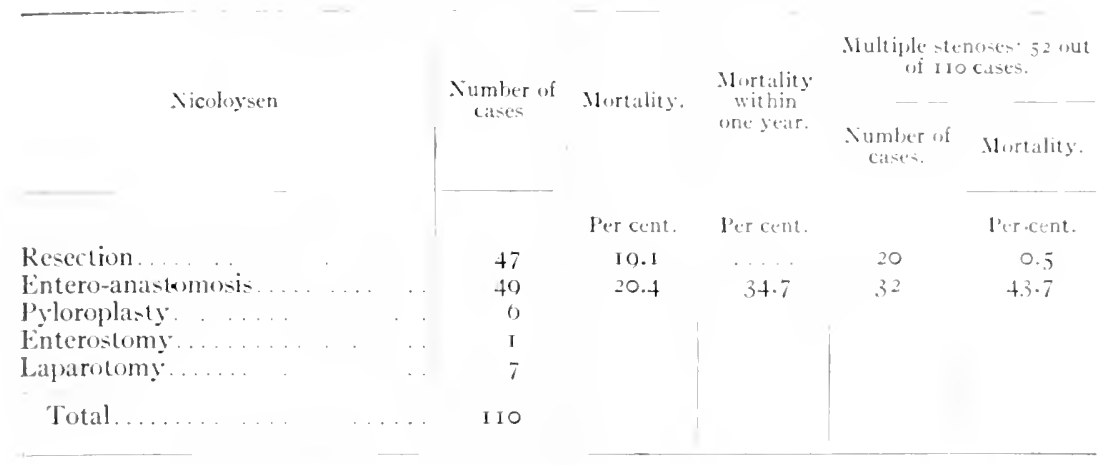

In the above tables, together with those of Conrath and Hartmann quoted by Groves, and Brumer's statistics, the mortality and percentage of recoveries are bairly well shown. From the author's personal experience, the statistics given, and an analysis of the opinions of prominent surgeons concerning the operative treatment of tuberculosis and the relative values of the different procedures, he has drawn the following conclusions:

(I) The mortality of operations performed for the relief of intestinal tuberculosis appears high and the results not very good, but, when one takes into consideration the condition of the average tubercular subject, the fact that until within a few years all of these patients died. and that the mortality from them is but slightly if any greater than for the same procedures done for non-tubercular lesions, he realizes that considerable progress has been made in the surgical treatment of intestinal tuberculosis.

(2) Neoplastic tuberculosis is practically always encountered at the ileocecal region, and in the operative statistics it was found but once elsewhere above the rectum, and that was at the juncture of the transverse and descending colons.

(3) Hyperplastic or ileocecal tuberculosis is a purefy surgical affection, and the results following resection of the diseased gut are splendid and oftained with comparatively little danger.

(4) The mortality following resection of tubercular tumors is much less than after other types of tuberculosis.

(5) The high mortality (30 per cent.) which formerly prevaled in this type of resection should, with our present knowledge and technic, be about io per cent.

(6) Exclusion (usually entero-anastomosis) is inclicated in hyperplastic tuberculosis when there is extensive lung involvement, the patient is greatly debilitated or exhausted from diarrhea, when the tumor is very large and bound down by extensive adhesions, or complicated by abscess or fistula-conditions which make resection extremely dangerous. 
(7) Unilateral and bilateral exclusion usually temporarily or completely relieve the most distressing manifestations of the disease, and cause the neoplasm to shrink up or effect its permanent disappearance.

(8) From the frequency with which ileocecal tubercular tumors are encountered and cured it is quite evident that they were formerly mistaken for carcinoma, and surgeons declined operation because of their size and connections. Certainly the records of a decade ago show but comparatively few cecal excisions for the relief of tubercular tumor formations.

(9) There is less danger from lung complications in this than other types of tuberculosis, because the infection is primary and less virulent.

(Io) Results are very much better when the swelling or ulcerated areas are diagnosed and promptly removed than after the infection has been permitted to extend to other segments of the intestine.

(I I) Entero-anastomosis (Maisonneuve), unilateral and bilateral exclusion, were formerly regarded as palliative measures, but today they are considered a curative power, and some operators say they are preferable to resection in most instances.

(I 2) Entero-anastomosis is the procedure of choice when the tubercular process extensively involves the small and large bowel and excision of the diseased segment is impracticable, and should be substituted for resection when the patient is unable to withstand a prolonged operation.

$(I, 3)$ Convalescence following exclusion is longer than after successful extirpation of the tubercular foci.

(I I) In hyperplastic or ileocecal tuberculosis, where the disease is localized and possibly primary, excision is preferable to anastomosis except under the above-named conditions.

(I5) Complications are fewer and the results better when short-circuiting is reinforced by enterostomy, appendicostomy, cecostomy, or temporary colostomy, so that the cliseased bowel can be cleansed of feces and discharges.

(16) Entero-anastomosis of the proximal with the distal gut puts the affected area at rest and favors healing of the lesions, while excision eliminates it altogether.

(17) Short-circuiting, when the ileum is joined to the sigmoid flexure or rectum (ileorectostomy), is followed by frequent movements for several weeks, or until the lower ileum begins to take upon itself the work of the colon, when the stools become normal in number and consistency.

(18) The low mortality following bilateral exclusion is partially offset by the annoying fistula left to drain the diseased intestine.

(i9) The chief objection to entero-anastomosis and unilateral exclusion in the treatment of tuberculosis is that the diseased area is left, and nothing is done to prevent the hacilli and irritating discharges from pouring into the healthy bowel below.

(20) Bilateral exclusion in inoperable cases and where fistula is 
present is superior to the other forms, because the gut is closed above and below the foci and the intestinal eontents cannot reach them, nor can infective material enter the healthy intestine.

(21) Partial or eomplete exelusion may loe reorted to as a preliminary step to excision in cases where, at the first operation, remesal of the diseased bowel gut is found inatrisable becalue of the patient's condition.

(22) It may aloo be necessary to short-cireuit the bowel to relieve obstruetion from stricture at the point of union.

(23) Exclusion operations, particularly entero-anastomosis, are less clangerous than extirpation because they recuire less time and cause little or slight fleterling.

(24) Stricture demands practically the same operative treatment as other tubercular intestinal lesions; consefuently, the statistic of operations for stenoses have been given with proceclures employed in the treating of other forms of bowel tuberculosis. but, fror the sake of comparison, a summary of Nicoloyen's statistic has leeen appended.

(25) Strictures occur rarely in the jejunum and duotenum, but are found fairly often in the lower ileum and cecal regions, and are usually single, but may le multiple.

(26) Broken-down glands easy to extirpate should be removerl. but when glands are simply swollen and difficult to reach they are best left alone, since many cures have followed where enlarged nodes were found at operation that were not removed.

(27) It makes very little difference whether one removes a short or long piece of the gut in so far as the severity of the operation is concerned. for on many occasions either a few inches or several feet of the intestine have been successfully resected.

(28) The suture-alone method is preferable to the Murphy button or other mechanical appliance, reinforced by Lembert stitches. and side-to-side should take precerlence over end-to-side or end-to-end anastomosis in this class of cases.

(29) The frequency with which enormous tubercular tumors and single or multiple strictures have been encountered indicate that operative measures are often postponed longer than they should be in bowel tuberculosis.

(30) Laparotomy may be of service in tubercular peritonitis. but is useless when the disease involves the intestine. 


\section{CHAPTER XYV}

\section{SYPHILITIC ENTERITIS, COLITIS, AND ENTEROCOLITIS INTESTINAL SYPHILIS', DIARRHEA IN}

\section{GENERAL REMIARKS, ETIOLOGY. PATHOLOGY}

THERE is a paucity of literature relative to siphilitic enterocolitis, but a study of it, together with his personal experience. has convinced the author that while luetic intestinal lesions sometimes canse chronic diarrhea, they do so les often than older writers would have us believe. and this is surprising when one takes into consideration the large number of people who suffer from syphilis and the frequency with which the diseare attacks other organs or parts of the body. The -tatistic of two decades ago indicate that intestinal lues, particularly of the rectum, was exceedingly common. and no doubt many cases of chronic diarrhea were attributed to this disease.

With modern diagnostic methods one can readily ascertain whether the subject is syphilitic or not. which can be accomplished by discovering spirochetes, obtaining a positive Wassermann reaction, and administering salvarsan. which eliminates luetic manifestations.

Proctologists and other who frequently study the lower bowel through a proctosigmoidoscope agree that siphilitic lesions here are comparatively rare. and that strictures common to the rectum, which formerly were attributed to lues, are caused by catarrhal and other form of colitis where mixed infection plays an important part.

Old text-hook- devoted to diseates of the rectum attribute from to to 60 per cent. of all rectal strictures to syphilis. but modern works on proctology do not asign more than Io per cent. to this cause.

In the past. clinicians and investigators often mistook the lesions of other diseases for those of syphilim, because they did not understand the etiology of lue- were not familiar with proctoscopic and sigmoidoscopic examinations, did not have the Wassermann reaction to aid them, and were not accustomed to studying the feces through the microsope, which would have enabled them to find entameber dysenteric baciliti. Balantidium coli. helminths (or ova), and thus differentiate between the different types of infections colitis.

Oceasionally the author has observed a catarrhal inflammation, erusion-s superficial, then deep extensive ulcers, and. finally, stricture follow each other in moderate or quick succesion. that were incident to entamebic, bacillary, tuberculons, or gonorrheal infection. More often he has encountered catarrhal coloproctitis, which when neglected led to the formation of the above-numerated lesions and caused per-istent diarrhea, a condition which in many instances had been 
previously diagnosed as syphilis of the rectum. The author does not helieve that lues or other types of infections colitis cause extensive destruction of tissue, but that they break the continuity of the mucosa, and that thereafter mixed infection, participated in by accidental and obligate pathogenic micro-organisms, takes place, as a result of which numerous large excoriated ulcers form that are responsible for the persistent diarrhea and stools contaning pus, blood, and mucus which characterize aggravated cases of colitis.

From what has been said it may be inferred that strictures of the lower bowel may result from any inflammatory disease of the colon accompanied by ulceration, and that such a condition is seldom brought about by luetic involvement of the intestine.

The author has treated 35 patients suffering from diarrhea who gatre a history of syphilis, exhibited stigmata or typic luetic manifestations. wherein the intlaned ulcerative or stenotic rectal lesions were responsible for the trouble, characteristic and diagnosed as luetic. He can recall only 7 cases of chronic diarrhea which were attributed to syphilitic lesions located exclusively in the small intestine or colon. Two of these patients, one six (boy) and the other eighteen (girl), at the age of one month had congenital syphilis which was exhibited by Hutchinson's teeth, enlarged fontanels, mucous patches of the mouth and throat, and fissures about the anus or vulva. In each case one or both parents admitted having syphilis, and the diarrhea and other manifestations improved while under specific treatment and relapsed when it was discontinued, as was shown in one instance by a positive Wassermann reaction obtained later. In both cases the rectum and sigmoid appeared healthy, and because of this and the fact that these little patients suffered frequently from abdominal cramps, distention, and soreness, a diagnosis of enteritis was made.

Four other cases, two men and wo women, whose ages ranged from nineteen to thirty-seven, suffered from acquired syphilis; each admitted having had a chancre, and in 3 this evidence was verified by the presence of typic initial cicatrices. All + cases gave a clear history of having suffered from the usual manifestations of lues-ferer. skin eruption, erosions of the mouth and throat, enlarged glands, and falling out of the hair. In I case, a man twenty-three rears of age. the clinical diagnosis of lues was confurmed by finding of the spirochata pallida and obtaining a positive Wassermann reaction, lute in the other 3 cases the latter methods of diagnosis were not employed. since the patients were treated before their discovery.

The diagnosis of syphilitic diarrhea in these cases was hasecl upon these findings: the patients had syphilis, the fluid movements were controlled by antiluetic treatment, the negative findings of the fecal examinations, and no other infection (dyentery, tuberculosis, efe.) which would account for the diarrhea could be discovered.

The seventh case was a bookmaker, fifty years of age. riddled with syphilis, who suffered from auto-intoxication and extensive uleeration of the entire colon which excited a most persistent diarrhea. This 
being before the advent of appendicostomy and cecostomy, colostomy was advised, and when the author attempted to lift the bowel upward it tore in two, and it was with great difficulty that the hard, brittle, and ragged ends were brought out and sutured to the skin to form the desired anus. The entire colon was involved, and many deep ulcers could be seen through the intestinal serosa. After a painstaking examination in this case the author became convinced that the frequent and fluid movements were due to syphilitic degeneration of the colon, for the rectum and sigmoid, as high as they could be inspected through the sigmoidoscope. were extensively involved in the luetic process.

In obscure cases of chronic diarrhea, where syphilis is suspected, the author considers macro- and microscopic examination of the feces exceedingly important, because in lues the findings are negative, but where the trouble is induced by dysentery, tuberculosis, gonorrhea, or helminths, the stools contain the specific organisms responsible for the infection, which, when found, render the diagnosis positive.

The etiology of intestinal syphilis inducing diarrhea is the same (viz., spirochetes) as that for lues affecting other parts of the body. This dreaded disease may be contracted through heredity from either or both parents, or by direct infection during sexual intercourse. Physicians have been known to become inoculated with syphilitic virus while operating upon or treating syphilitic subjects, and others have been infected through vaccination, nursing, kissing, drinking from unclean vessels, or by using a syringe, rectal tube, or toilet articles which had been previously employed by an individual suffering from lues.

Syphilis originally manifests itself in the form of a chancre upon the penis, labia, lip, tongue, skin, rectum, or at the anus. Persons suffering from acquired syphilitic diarrhea show or give a history of having had the initial, secondary, and tertiary stages of the disease, while children with congenital syphilis may be affected with gummata and late lesions of lues without having had the symptoms common to the earlier stages of acquired syphilis.

The theory of Lustgarten that lues is caused by a micro-organism resembling tubercle and smegma bacilli has been rejected in favor of that of Schaudinn and Hoffmann, who since I905 have maintained that the Spirochata pallida (Treponema pallidum) are the etiologic factors in syphilis. Spirochetes have been so frequently demonstrated in the initial sore, lesions of the skin, mucosa, and involved lymphatics by laboratory workers at home and abroad that one feets compelled to admit that it is the chief or sole cause of syphilis.

It is unnecessary here to give a detailed description of Spirochata pallida, since standard works on syphilology and pathology give the necessary information concerning it, and, further, because its presence cannot he demonstrated in the later stages of lues, and more especially in persons who have taken antisyphilitic treatment. Spirochetes 
have been demonstrated in consiclerable numbers in the intestinal mucosa of a luetic fetus (Sinmonds), and olserved in strand-like formation about the glands in an infant who died at the age of four days from purulent peritonitis (Fränkel). When discoverable, they are present in considerable numbers on and in the ulcerated areas, in the mucosa and glands of Licherkühn, or the part of the gut abundantly supplied with lymphatics. As a rule, they are not demonstrable in the lesions or discharges of patients suffering from syphilitic colitis causing chronic diarrhea.

Pathology.-Chronic diarrhea may be induced by syphilis involving the small intestine, colon, or rectum, individually or collectively. Congenital and accuired luctic lesions which cause diarthea are encountered very much more frepuently in the rectum than in either the small intestine, colon, or sigmoid flexure. It is an interesting and established fact that when syphilis attacks the bowel above the rectum it shows a predilection for and involves the jejunum more frequently than the stomach, duodenum, ileum, or colon.

No one has offered a satisfactory explanation as to why lues at tacks the jejunum and rectum so much more frequently than other parts of the bowel, nor do we know why a purely syphilitic stricture of the rectum should be met with so much more often (seren to one) in women than in men. There must be some reason why this is so, because other ulcerative lesions of the parts of the bowel do not show the same tendency to involve these particular segments of the gut so frecuently, and attack women and men in about the same ratio.

The rectum and anus are usually involved in both hereditary and acquired intestinal syphilis, but when the small intestine, especially the jejunum, is affected, lues is usually congenital, and a careful examination will reveal that the patient has Hutchinson's teeth, anal fissures, enlarge fontanels, or other stigmata of inherited syphilis.

The luetic lesions which cause diarrhea vary greatly as regards their location and appearance, and pathologic changes may involve the superficial mucosa, extend completely through the gut and attack the mesentery, or cause perforation and peritonitis.

More is known of the clinical aspect and the gross and minute pathology of the luetic rectum than of the symptoms and the anatomic changes which take place in syphilis of the small intestine and colon because syphilis is more common in this region, and the disease has been more closely stuclied here, owing to the fact that the rectum is accessible to digital and proctoscopic examination, removal of diseased tissue for microscopic examination is easily accomplished, and, moreover, the feces and discharges are readily obtained for inspection and analysis. In acquired, the destruction of tissue is greater than in congenital intestinal syphilis because the disease has existed longer and progressed farther. Adults and childen who have inherited syphilis and suffer from chronic diarrhea show about the same intestinal lesions as those who suffer from acpuired lues, though, as a rule, they are not so extensive, and there is no appreciable difference between the 
changes which take place in the tissues in the two forms in the presence of syplailitic colitis.

There is a difference of opinion as to whether the lymply or blood is the carrier of the infection to the intestine, lut from our present kmowledge it would appear that the blood is mainly responsible, though there is considerable evidence to support the claim that hoth lymph and blood plis their respective parts in producing intestinal syphilis. Once the bowel is involved, the blood-resseds become the chief factors in furthering the disease lecaluse the lumen of the arteries is gratually diminished by progressive endarteritis and connectivetissue thickening of the adrentitial, and, in consepuence, the tissues are cleprived of blook, necrosis takes place, and uleers form, which, when healed, sometimes caluse strictures.

Chronic diarrhea of syphilitic origin may be induced by a variety of intestinal lesions: (a) catarrhal inflammation (cuteritis and colitis syphilitica); (b) ulcers; (c) gummata; and $(d)$ strictures. The degree of the diarthea varies in different cases depending upon the duration

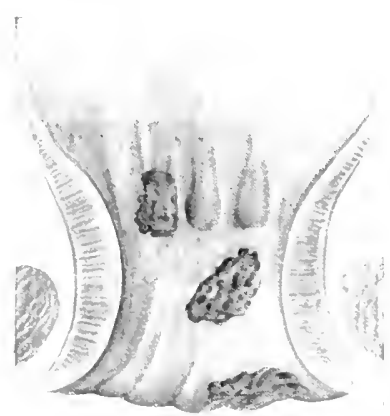

Fig. 43.-Syphilinic uheration of the anal canal and rectum (alter auther's case). of the discase catusing it, the part played by mixed infection, and extent and character of the lesion.

Enteritis and Colitis Syphilitica-Catarrhal inflammation of the small intestine and colon incident to syphilis is encountered more frequently in the earlier than the later stages of intestinal lues, but the manner in which the inflammatory condition is produced is not known unless, as Jullien believes, the infection takes place through the lymphatics, though some one has suggested it is due to toxins emanating from the spirocherta pallicla which have, through the excretory process, been deposited mpon the mucosa.

Syphilitic Lleers of the Intestine.-Intestinal ulcers (Fig. 4.3) of syphilitic origin which incite chronic diarthea may be single or multiple, in pairs or groups, closely assembled or widely scattered throughout the bowel, superficial or deep, variable in color, and their edges may. be rageed, sharply defined, or rounded. In enteritis syphilitica there is nothing more than slight erosions of the mucosa, but when the luetic process attacks the follicles of lieberkühn or Peyer's patches the ulcers are larger and leeper. The most destructive syphilitic ulcers are those formed by the breaking down of lesions, which vary in size, aie crater-like in character, and cause the most persistent type of luetic colitis.

In contradistinetion to other ulcerative intestinal lesions, luetic uleers usually have a rounded, raised, haid border instead of undermined edges. Syphifitic-like tuberenlar ulcers are inclined to follow the hlood-ressels and encircle the bowel, and as healing takes place 
and cicatricial tissue accummlates, the sears are prone to contract and form strictures. Multiple stenoses complicate syphilis more frepuently than other infections discases of the intestine, of which diarthea is a manifestation.

Syphilitic ulceration, in the form of clancres, mucous patehes, and gummata, atlack the rectum in dillerent stages of lues, but uleers of the small intestine and colon oceme early in the secomelary and tertiary stages of the disease. Mixed infection is more active when the lesions are in the colon and rectum, and diarrhea is more persistent than when they are located in the small intestine. In either case, when ulecrs are numerous or extensive and leep, the evaluations are

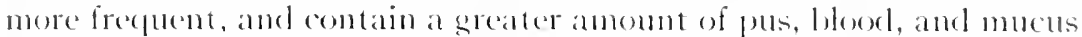
than when the ulcerative process is insignificant. In some instinces, in congental and acpured syphilis involving the upper segments of the bowed, from twenty af fifty uleers have feen observed. Isually. syphilitic diarrhea results most frecpuently from involvement of the rectum, and the uleers causing the loose morements under such circumstances will be described under a separate hearling.

Pathology of Gummata and Strictures.-Cimmmatous infiltration is generally conceded to be the most common manilestation of intestinal syphilis attacking the small intestine and colon. In the rectum, howerer, gummata are exceedingly rare, and a perusal of the literature and the stanclard works on proxtology show that lust few authenticated calses of gummatous infiltration of this part of the intestine have been reported. In a large experience covering twenty years the author has encombered this condition but hree times.

Gummata imvolving the small intestine and colon may be single or multiple and appear as dinninutive miliary oved swellings, large sancershaped devations, or, more frecpuently, as raised or ridge-like infiltrattions which encirele the gut at a right angle, usually following the direction of the blooxt-ressels. There has been considerable discussion by writers upon syphilologry, and experiments have leen marle to determine in just what layer of the gut wall gummata originate. All authorities agree, howerer, that in certain cases during its progress the infiltration may include singly or collectively the mucosa, sulmucosa, muscularis, and serosa, or extend to and involve the mesentery. Amost conchusive evidence is at hand to support the theory that this type of intestinal syphilis originates in the comnective tisste of the submucosa, and may, when untreated, extend in either or both directions, according to the duration of the disease and its virulence.

Gummata may remain intact or profuce diarhea through obstruction where they encircle the bowel of project inte its homen. These infiltrating tumors may also muclerge retrogresive changes where one or many superlicial or penetrating ulcers expese the ferminal nerrelibments to the intestinal contents which serve to irritate the nerous mechanism, and through reflex action exoite frepuent and prolonged peristaltic contraction and an increase in the number of the evacuations. 
These ulcers may amount to nothing more than superficial erosions of the mucous membrane, or they may be progressive, and in successive stages involve one or all of the intestinal tunics and cause perforation during fetal life or subsequently, a complication that has been observed a number of times. The author has treated but one patient, and in this instance death took place from peritonit is within forty-eight hours. Unfortunately, he was unable to secure a postmortem to determine whether or not the perforation was caused by a degenerating gumma or syphilitic colitis. The patient was a woman forty years of age, who had all the manifestations of lues, but no cachexia or other symptoms which would lead one to suspect cancer, tuberculosis, or dysentery. A diagnosis of gummatous infiltration was based upon the clinical history, conspicuous evidence of syphilis, and the peculiar saucershaped swelling located in the central part of the ascending colon which caused partial obstruction. These negative symptoms, together with the fact that the ahdomen became rapidly distended and peritonitis followed shortly the feeling of sharp pain at the site of the tumor, led the author to believe that perforation had taken place incident to a penetrating ulcer and not as the result of obstruction. This patient had been placed in the hospital and was being prepared for intestinal resection, but perforation occurred and she died before the operation. Non-degenerating and ulcerating gummatous infiltrations invariably lead to peritoneal involvement and the formation of false membranes or adhesions which surround or bind the bowel to other organs, or cause the massing together of several loops of intestine by exudates. When, as a result of the destructive process, infection or perforation takes place, peritonitis ensues or abscess and a fecal fistula form. In deplorable cases the mesentery, through extension of the inflammation and infiltration, becomes swollen, hard, and tends to produce contraction, narrowing, or angulation of the gut.

There is very little if any difference between the character of gastric and intestinal gummata. In both, the mucosa, submucosa, and muscularis may be involved by the infiltrating process, which is of the round-celled variety. Brunner, Kleinschmidt, and other close students of intestinal syphilis have recently confirmed the views of Mracek, who maintains that the infiltration begins in the adrentitia of the arterial walls and gradually progresses until it greatly narrows or completely occludes the lumen of the ressels, and that the veins do not become involved until later. Frequently, the narrowing or obliterating process which occurs in the arterioles as the adventitia becomes involved is accelerated by changes which take place simultaneously in the intima as a result of an obliterating endarteritis. If the blood-ressels become partially or completely incapacitated in this manner, it makes plain the reason why ulceration is a common manifestation of gummatous tumors, for impairment to the circulation necessarily brings about fatty degeneration and necrosis of the involved structures. As the necrotic tissue gives way, small or large, superficial or deep ulcers form and add to the gravity of the situation. Ulcera- 
tion usually begins in the center of the tumor mass, and individual ulcers have a greenish-colored base and a raised sclerotic border. When retrogressive changes have progressed to a considerable extent a number of ulcerated areas, variable in size, shape, and depth, are to be seen.

Under such circumstances the surface of the gumma presents certain peculiar characteristics. for at one point a rapidly extending ulcer may be observed, at another one marked by heahthy granulations and partly healed, and at still another a chepression covered with a glistening white scar, which indicates the location of a healed or completely cicatrized ulcer. If the destructive process is not now arrested, the ulcerated areas rapidly extend, become deeper, encircle the bowel, and when healed cause partial or complete intestinal occlusion (stricture). In some instances the blocking is partially attributable to inflammatory exudates deposited in the gut wall. In syphilitic as in other connectire-tissue strictures involing the intestinal tunics, the peritoneum is thickened and the bowel is usually bound to the adjacent structures by adhesions or exudates.

Kleinschmidt observed this connective-tissue involvement of the scrosa and interspersed nodular villus formations, and upon cross-section found the lymph-ressels packed with round cells, the muscular coats disturbed, and the muscle bundles torn apart by invading connective tissue, but he did not find the diffuse infiltration of the mucosa described by others. According to his interpretation, the plate-shaped thickenings noticeable in gummata are due to a hypertrophy of the submucosa following an infiltration which originates in the arterial adrentitia.

Syphilitic strictures involving the small bowel or colon may result from congenital or acquired lues. and are encountered very much less frequently than stenoses of the rectum (Fig. +t). In this class of cases the patient suffers from an obstructive diarrhea. and the number and consistence of the stools depends upon the number of constrictions, the degree of obstruction aused by them, and upon the extent of the complicating ulceration. Obstructice diarrhea of syphilitic origin may be caused by a gumma which projects into the bowel, resulting in a narroi circular constriction (a cicatricial ring), or by a tube-like narrowing of the gut induced by a probnged subacute inflammation which results in fibrosis and thickening of all the intestinal coats, slowing a tendency to contract and form the so-called gas-pipe intestine (tubular stricture). Strictures in all parts of the intestine are invariably complicated by ulceration at and above the constriction, and when the ulcers are superficial the evacuations contain a small amount of admixed pus, blood, and mucus, but when they are extensive and deep the discharge is copions and the movements are fluid and freptent. Sometimes, when the lesions are penetrating or crater-like, the discharge of feeal or other inlectious matter becomes pocketed in them, infection takes place, and terminates in abscess or fistula, or perforation occurs and the pationt 
suffers from a localized or general peritonitis, possibly pyostercoral abscess and adhesions, and one or all conditions may interfere with the functionating power of the intestine.

Pathology OF Axorectal Siphilas.- All of the luetic lesions (proctitis, syphilitica, secondary and tertiary ulceration, gummata, and strictures) found in the small and large intestines are encountered in the rectum, but more frequently and in an aggravated form. In addition, one sometimes has to deal with chancres, luetic condylomata (Fig. 45) of the perianal skin, and the fissures of congenital syphilis. Owing to trauma of the inflamed and ulcerated mucosa, lodgment of feces under the edges of undermined ulcers during defecation, mixed

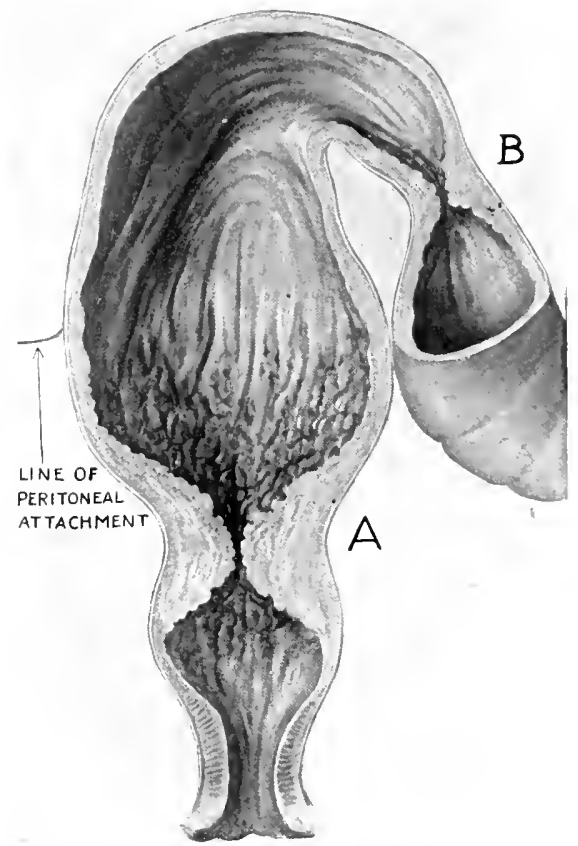

Fin. 44.-Multiple syphilitic stricture: $A, \ln$ rectal ampulla; $B$, in sigmoid flexure (alfer atuthor's case).

infection, and retention in the lower bowel of foul discharges, intestinal syphilis is met in its worst form in the rectum, and complications that do not occur above are often encountered here-viz., numerous polypoid-like excrescences, abscess, fistula, hypertrophied papilla, skin tags, fissures, erosions of the skin, and wart-like growths, which involve the mucosa, integument, or both, and the anal canal and skin of the buttocks are constantly bathed hy an acrid discharge having a clisgusting odor.

Condylomata occur in patches, have a stem-like attachment, and club-like listal extremities. These growths are highly infectious, and 
new collections of warts appear upon part of the opposite healthe buttoek- when condylomala are not indilted. The atther treated at man

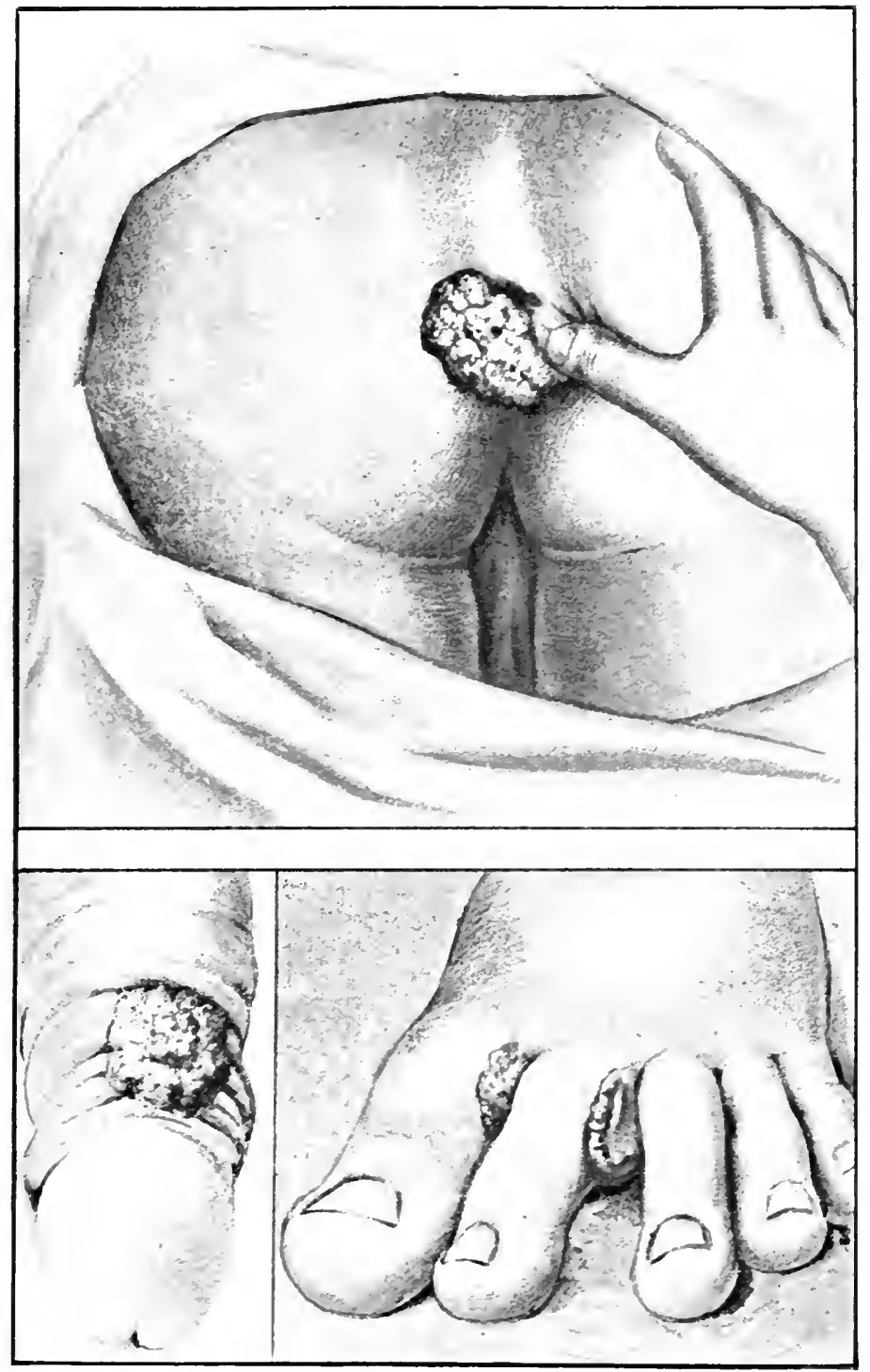

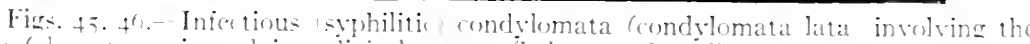
anus (abote): penis and interdizital spaces (betow). (Complication- if a luetic coliti(after author's ate).

who infected his toes and penis by scratching after having treated hianal condylomata (Figs. $\left.45,4^{6}\right)$. These excrescences are calued $1, \mathrm{~s}$ the syphilitic discharge from the rectum. 


\section{CHAPTER XXII}

\section{SYPHILITIC ENTERITIS, COLITIS, AND ENTEROCOLITIS (INTESTINAL SYPHILIS), DIARRHEA IN (Continued)}

\section{SYMIPIOMS, DIAGNOSIS, PROGNOSIS}

Symptoms.-The gastro-intestinal symptoms of syphilitic do not differ materially from those of catarrhal. entamebic, bacillary. and other forms of colitis accompanied by inflammatory and ulcerative changes in the mucona or strictures in the small or large intestine. Syphilitic colitis can usually be distinguished from other infectious diseases of the bowel by the constitutional manifestations of the affection-mucous patches in the mouth, throat, and about the anus, falling out of the hair, skin eruption, and getting a history of the patient's haring had a chancre.

Sufferers from congenital syphilis usually exhibit stigmata, riz., Hutchinson's teeth, enlarged fontanels, etc., which, in connection with the above symptoms. indicate that the gastro-intestinal disturbances-diarrhea, cramps, pus. blood, and mucus in the stools-are induced by syphilitic involvement of the intestine. Persistent diarrhea incident to lues is occasionally secondary to enteritis syphilitica, but in the rast majority of cases is caused by inflammatory or ulcerative le-ions of the colon. siamoid flexure, and rectum. and the frequency of the stools and amount of the contained discharge is very much greater when the lesions are situated low down than when high up in the bowel.

Intestinal syphilis when congenital may excite a diarrhea which manifests itself at birth or later, and the frequent and fluid movements may continue indefinitely until the child dies from exhaustion or recovers as the result of intelligent antisy philitic treatment.

In so far as the author has been able to observe, the character of the stools in syphilitic subjects suffering from diarrhea vary, dependent upon whether or not the lues involves the small intestine alone or in conjunction with the stomach, or is complicated by digestive disturbances or other infectious diseases of the intestine, such as tuberculosis and dyentery. L'sually children who suffer from luetic diarrhea show the more common manifestations and stigmata of congenital syphilis, such as Hutchimson's teeth, ocular disturbances, cachexia, snuffler, melena, erosions of the mouth. noee, or throat, pemphigus syphilitica. fissures or extensive ulcerated areas in the anal canal, at the anus or vaginal outlet, together with enlargement of liver, spleen, and lymphatic glands. The stools are fluid, contain a variable amount of mueus slightly stained with blood, and induce tenesmus when evacuated. The movements are offensive and irri- 
tating, and cause considerable burning pain during and following defecation through their irritative effect upon the raw surfaces of the anorectal region. When there are gastric disturbances the eracuations become exceedingly- frequent and contain consielerable quantities of pus, blood, or mucus and remnants of undigested milk and other food. Infants and childen afflicted with congenital syphilis, exeept when carefully handled, suffer greatly from excoriations of the mouth, raw genitals, and erosions of skin about the anus, owing to the irritation caused by the acriel discharge which of frecpuently bathes them. Pain from this source is much less in children who undereso specific treatment. and have the excoriated parts frepuently lathed and dusted with talcum and calomel (ecpual parts), or smeared over with an ointment containing calomel or mercury in some form, in combination with orthoform, analgin, or encain.

Colic is a common manifestation of intestinal syphilis in adults and children, but the latter suffer from it more frequently and severely than the former. When the stomach is involved in the syphilitic process the patient has more or less nausea and romiting, but comparatively little gastric pain. Nearly all infants and children who suffer from intestinal or gastro-intestinal lues are weaklings. Some rapidly improve when they are properly feel and put on specific treatment. Others continue to decline in spite of all therapeutic measures.

Syphilis of the snall intestine and colon is encountered more rarely. in adults than in children, and but few cases of chronic diarrhea from this source have been reported. Cinsergently but little is known of the symptom-complex of intestinal lues causing loose movements.

Except for the ordinary indications of syphilis. there is very little difference between the manifestations of intestinal lues and those induced by entamebe, tubercular and other bowel affections, accompanied by inflammation. ulceration, or stricture of the colon, sigmoid flexure, and rectum.

When diarrhea is due to uncomplicated enteritis syphilitica the patient may have from two to four stools daily, which contain considerable mucus, possibly a tinge of blook, but no pus. Where there is both inflammation and ulceration of the mucosa, the daily stools increase proportionately with the number and extent of the ulcerated areas. When the elestruction of tissue is moderate the patient may not have more than five or six movements daily, but in very bad calsen he may have anywhere from eight to twenty fluid moxements which contain a considerable amount of mucus, pis, and blood.

In cases where the discase has been allowed to go untreated, and one or more strictures have formed in the small intestine or colom, the patient suffers from obstruction in ardelition to the diarrheal manifestations mentioned abose. In the beginning of strieture constipation is present to a greater or less degree, then there is constipation alternating with diarrhea, and still later, when the lowel becomes almos occluded, diarrhea predominates because nothing but tluid stools can get by the constriction. 
The author has known patients who suffered from stenosis of the small howel to pass perfectly formed solid movements, but the feces e-caped through the stricture and passed into the large bowel, where they remained until they became solidified. Such instances are of rare occurrence because in this class of cases the nervous mechanism controlling the gastro-intestinal tract is disturbed, and this leads to frequent and prolonged peristaltic contractions, which, in turn, hurry the feces through the lower bowel as soon as they pass the obstruction.

Flatus, to a greater or less extent, complicates syphilitic diarrhea induced by luetic enteritis and ulceration of the small intestine or colon, but when the patient suffers frequenty and greatly from gas distention and meteorism, the gut is usually strictured. A constricted lowel is very erratic, and at times, when in a quiescent state, flatus and feces may pass the block freely and cause but little discomfort, but when it is irritated through the presence of gas, a fecolith, or acrid discharge, enterospasm sometimes intervenes. and there is a simultaneous contraction of the circular and longitudinal muscle-fibers of the intestine in the neighborhood of the stricture, which causes cramps in addition to the discomfort consectuent upon the gas and feces retained above the contracted segment of intestine. When enterospasm lasts for several hours or longer the patients also suffer from nausea, romiting. and the usual manifestations of intestinal obstruction and autointoxication.

In marked cases of stricture sometimes it is easy to feel and see the strong peristaltic contractions of the intestine through the parietes when peristalsis is stimulated by the application of electricity or by a sudden and hard tap of the finger made upon the abdomen near the stricture.

Lues frequently involves the liver, spleen, lymphatic glands, muscles (sternocleidomastoid), bone. testes, and the nerves or their centers. causing tabes, paresis, trophic disturbances, etc. Knowing this, in studying chronic diarrlea supposedly of luetic origin it is well to examine the organs and regions named, and to be on the lookout for manifestations elsewhere that might be caused by syphilitic invasion in parts other than the intestine, which, when found, are of great assistance in clearing up the diagnosis.

The author believes rhat in the adult, when the syphilitic proc$\therefore$ attacks the intestine sufficiently to produce chronic diarrhea, evidences of luetic involvement in other parts of the body can be found when diligently sought for. In a case he saw with another physician, that of a negro woman thirty-two years of age, there was a deep. clear-cut or punched-out gummatous ulcer in the center of the forehead and also numerous and extensive luetic ulcers, phagedenic in character, about the anus and vulva.

The early manifestations of syphilis are seldom demonstrable in persons suffering from intestinal syphilis, but mucous patches of the mouth. throat, and anus, ocular disturbances, ulcerating bone lesions. 
and syphilids have been frepuently observed in connection with chronic luevic diarrbea.

As a rule, individuals who suffer from syphilitic diarhea are not debilitared, suffer less severely from frepuent fluid movements, cramps, gas distention, and intestinal intoxication than persons aflicted with the other types of enterocolitis.

Luetic lesions-catarrh, ulceration, or stricture located in the lower sigmoid flexure and rectum-invariably cause more distress than when located in the colon or small intestine lreatuse they incite an almost incessant desire to stool, bearing-dor'n sensations, weight and fulness in the boaiel, burning pain in the rectum, frecuently complicated by abscess, fistula, and cxcoriation of the perianal skin, and incite a more ageravated trpe of diarrhea.

Diagnosis. - In sonfe instances it is comparatirely easy to diagnose chronic diarrlea induced by luetic lesions of the bowel, but in others it is extremely difficult or impossible to differentiate syphilitic from other types of colitis causing chronic diarrhea. With a negative history as regards chancre, absence of congenital stigmata, and the characteristic manifestations of aecquired syphilis, and there remains a doubt as to whether or not the frecpent stools are incited by syphilitic or other lesions, the patient should be temporarily placed upon an antispecific treatment. If under such circumstances he rapidly improves after having failed to do so under other lines of treatment, one is justified in attributing the loose movements to intestinal syphilis. Mercury prescribed for the relief of intestinal ulecrative lesions other than hetic invariably aggravates the diarrheal condition, but when the loose movements are caused ly syphilitic ulcers, the number of daily movements begin to diminish as soon as the physiologic action of the drug is manifest. In this class of cases the specific treatment works like magic, and often quickly overcomes a persistent diarrbea of rears' standing which had previously been considered hopeless.

The diagnosis of syphilitic diarrhea is comparatively easy to make in infants and children who have inherited lues and show avidence of it in deformed teeth, delayed closure of the fontanels, enlarged liver, spleen, and glands, "smuffles," mucous patches about the mouth, throat, vagina, rectum and anus, and other stigmata of congenital syphilis.

Again, when diarrhea does not respond to dietary and therapeutic measures ceffective in the treatment of loose movements from other gastro-intestinal disorders in children or adults who exhibit typic manifestations of congenital or acquired lues-viz., chancre, constiuttional symptoms, eruptions of the skin or mucous membranes, lymphoid enlargements, falling hair, iritis, or tertiary symptoms-the pattient, in all probability, is suffering from a luetic enteritis or colitis. The author has treated patients who suffered from syphilitic diarrhea which was complicated by entamebic or bacillary infection, which made the diagnosis difficult.

When attempting to reach a diagnosic in cases of diarrhea supposedly due to syphilis, the patient should be stripped and carcfully 
eximined to find out if he has external signs of the disease. enlargement if the spleen. liver. lymph-nodes. or a flat saucer-like swelling (gummatou- tumor anywhere along the large or small bowel. One must aloo starch for nodulated stellings. which sometimes indicate the lication of spphilitic strictures. because in such cases the bowel is sausage shaped and feels bogg! when palpated.

Spirochata pallida the etiologic factor in lues) when found offers convincing proof that the patient has syphilis. but since it is discoverable mainly in the incipient stage of the disease, and is seldom found in the fecal discharge or scrapings from svphilitic intestinal lesions. it is of very little or no importance as a diagnostic aid. and cannot be depended upon to help differentiate lutetic from other inflammatory diseases of the intestine.

In the majority of instances it is the later lesions of syphilis which induce the diarrhea, and spirochetes are seldom found, if at all, in gummata and other late lesions of lues. or in the earlier stages of the disease where the patient has been subjected to a thorough course of antisyphilitic treatment.

ITassemann's reaction is extremely helpful for clearing up the diagnosis in cases of colitis of suspected sphilitic origin, and this test is more important than Spirocheta pallida as a diagnostic aid.

The Massermann blood reaction is not infallible. for negative results hare been obtained where patients had syphilis with and without having undergone antisyphilitic treatment. Positive reactions have also followed Wassermann's test in other diseases. In the vast majority of cases. however, when one or more positive Massermann's have leen obtained, one is justified in holding that the patient has syphilis: and that he has not when tests are repeatedly negative unles: the patient has been under treatment. Nassermann's reaction $\mathrm{i}$ s valuable during the treatment. for by it one can determine when to begin or stop medication.

The Nasermann serodiagnostic reaction is of little use to the busy practitioner because of the sreat amount of care and time required to properly prepare for and carry out the test. which should be done in the laboratory.

There is no longer any question that lues pares the way both for uberulosis and cancer. Certainly these distressing ailments occur in sphilitic subjects with a frequency which cannot be otherwise explained. At time- it is exceedingly difficult to differentiate a tubercular from a syphilicic diarthea. since hoth produce almost identical manifestations. but a rational diagnosis can he made when sufficient time is taken and the patient i- carefully examined from exery standprint. A luetic subject gives a history of having suffered from one or more of the manifertations of the disease, his color and general appearance are comparatively normal, he can digest his food fairly well, and has sufficient strength to attend to his ordinary duties. while a tubercular subject having chronic diarrhea is very ill, has a sallow complexion, is emaciated and feethle, has poor digestion, runs an irreg- 
ular cemperature, and of ten has a comgh, laryngitis, hemoptysis, rectal fistula, or other indications of a localized tulereular process in the respiratory or gastro-intestinal trate manifestations not observed in persons suffering from intestinal syphilis.

The number of evacuations induced by tubereulosis and syphitis are diminished by colonic lavage, but patients having syphitis respond more quickly to antiluetic treatment than tuluercular sulpjects do to other therapeutic measures. In syphititic diartea mereury hats a specific action, but does litte or no goorl when the frectuent movements are callsed by tubercular lesions in the intestine; and, on the contrary, tubercular subjects do well when placed upon Russell's cmulsion, corlliver oil, a milk-and-egg dien, and when competted to sleep with the windows open and spend their time in the open air. precerlures which do little or no good toward improving the condition of patients sulfering from syphilitic diarrhea.

In both tuberculosis and syphilis of the small lowel and colon diminutive notules hat been observed in the peritoneal and other tunics of the bowel which have been mistaken the one for the other, but such swellings are encountered very much more frefuently and in larger numbers in uluercular than in syphilitic subjects. Again, inlestinal fues is sometimes present as satucer-shaped infiltrates of large size, originating in the mucosat or submucosa. I inder such circumstances, according to Brunner, a differentiation can le macle between the grayish-white tuberctes of tuberculosis and the opactue yellowish nodules of syplitis by inspecting the cliseased yut, and, in the living. hy ascertaining if there is a general lymphocytosis, enlargement of the spleen, inflammation of the hepatic veins, or other frepuent manifestations of constitutional lues. The Wassermann reatetion is of value in this class of eatses in determining whether or not the patient has stphilis, but the spirochetes have no diagnostic value here because they cannot be found except when the diarthea occurs during the early stages of lues.

()wing to the fact that a tuberculin reaction may le oblatinect losth in luetic and tubercular individuals this diagnostic aid is mot to bo reliect upon in determining the cause of diarrhea in doubtful canes.

Melenu is a common manifestation of congental intestinal syphitis. Ender such circumstances the covetations may be black form reataned blowe or, in exceptional instances, the stools contain more or less bright red hlood.

Macroseopic and microsoppic examination of the stools is of great

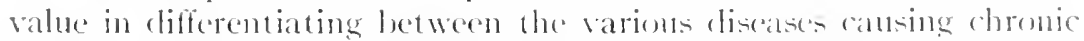
diarrhea. In so far as lues is concerneel, examination of the feces and discharges is newetiofe. Conseguently, when the subject has syphilis, and neither entamela nor dysenteric bateilli can be fonnd in the discharge to account for the condition, a tentative diagnosis of syphilitic colitis is justifiable, and the patient should be given salvalsan or a course of mercurial treatment, which rapiolly improves his condition when lues is responsible for the trouble. 
When the loose movements are the result of entamebic, tubercular, or gonorrheal colitis the microscope can be relied upon to clear up the diagnosis in many instances, because an examination of the scrapings and discharge from the ulcers will reveal the entameba, the bacilli of Shiga, Flexner's tubercle bacilli, or Neisser's gonococci, etc. Microscopic examination of the feces is also of assistance in clearing up the diagnosis between infections diseases of the colon, membranous colitis, and other inflammatory conditions of the intestinal mucosa. Finally, with the aid of test-meals and microscopic examination of the stools one can determine the presence or absence of undigested food remnants and determine whether or not the diarrhea is due to gastrogenic or enterogenic disturbances. In suspected cases of gastrogenic diarrhea the stomach contents should be analyzed to determine if there is an absence of hydrochloric acid or other abnormality of the gastric juice which might be responsible for the loose movements. Now and then a fecal examination will also indicate that the disturbance is in the liver or the pancreas.

Macro- and Microscopic Findings.-It is not difficult to distinguish between lues and tuberculosis of the bowel when a segment of gut can be had for examination, because when syphilis is the cause of the trouble one or more extensive flat gummatous swellings are usually present, which consist largely of small round-celled infiltrations, and involve one or all of the tunics of the gut and frequently include the mesentery. The mucosa may show a few or many superficial or deep crater-like ulcerated areas, and the peritoneum is greatly thickened and attached to adjacent loops of the bowel or other structures by exudates or band-like adhesions. Diminutive nodules have been observed in intestinal syphilis, but usually when numerous small tubercles are present they indicate the tuberculous nature of the disease. and the tissue should be examined microseopically for tubercle bacilli and giant cells, which, when found, indicate a tubercular process. Too much reliance, however, cannot be placed upon the presence of the giant cells, since a few writers upon syphilology maintain that they are to be found in the syphilitic tissue, and both tuberculosis and syphilis have heen known to attack the bowel in the same vicinity.

Proctoscopic and sigmoidoscopic examination is of little use in determining the presence or absence of syphilis in the small intestine or colon, hut is of great help in clearing up the diagnosis when the disease is situated in the lower sigmoid flexure or rectum, because when these segments of gut are involved in the luetic process the inflammation, ulceration, or stricture responsible for the chronic diarrhea can be seen and studied at leisure.

Blood examinations are unreliable when differentiating between tuberculosis, syphilis, dysentery, and other ulcerative lesions of the colon inducing chronic diarrhea, because patients suffering from these affections are usually anemic and show about the same blood changes as regards the hemoglobin, red blood-cells, and leukocytes. During the earlier febrile stage of lines a lymphocytosis is generally observable, 
but this is of little if any assistance in clearing up the diagnosis in cases of chronic diarrhea, because when the loose nurvements are the result of lues the diarrhea occurs in the later stages of the diseatse after the lymphocytosis has disappeared.

One cannot rely upon the change in number of exsinophils in the diarrheas mentioned. but they are of diagnostic value in cases when the parasitic origin of the disease is suspected. beculdse in certain parasitic affections of the intestine the eosinophils sbow at tenclency to increase greatly in number.

Crinary analysis is seldom of diagnostic value in this class of cases. There is evidence to support the belief that lues may incluce a pancreatitis accompanied by sugar in the urine, which rapidly disappears when the patient is put upon antistphilitic treatment.

Acquired anorectal syphilis may manifest itself in the form of a chancre, catarrhal proctitis, stricture, superficial or deep ulcers.

Chancres and the fissures of congenital lues are readily detected by separating the buttocks and inspecting the anus.

[leers can be detected with the finger, but their number, size, and condition can be more accurately determined by inspecting them through the proctoscope.

The location and characteristics of strictures located within 3 inchen $(7.5 \mathrm{~cm}$.$) of the anus can be ascertained by digital examination, but$ when situated higher up the diagnosis is made through the sigmoidoscope or with the airl of Wales' bougies, neither of which should be forced through the constriction until it has been inspected and the size of its caliber determined, because many deaths from rupture and peritonit is have followed the introduction of instruments through stenoses located about the peritoneal attachment to the rectum.

Digital examination of the rectum should never be neglected in this class of cases. because ulcers. syphilitic excrescences, and strictures located within 3 inches $(7.5 \mathrm{~cm}$.) of the anus can be readily detected with the finger and afterward riewed through the proctoscope.

The anus and perianal skin of persons suspected of having syphilitic colitis (diarrhea) should be carefully inspected, for if the patient has colonic or rectal lues he will complain of anal fissures and excoriations of the adjacent skin caused hy the acrid discharge pasing oser them.

Finally, when a patient gives a history of having had a chancre. falling out of the hair, a secondary eruption, sore mouth and throat. or has the stigmata of inherited lues, and suffers from chronic diarrhea. when the stools contain pus, blood, and mucus, and ulcers are visible in the rectum and sigmoid thexure through the proctoscope. one is perfecty justified in making a diagnosis of syphilitic colitis, particularty when the stools do not contain entamebas, dysenteric lacilli, helminths, etc.

The prognosis in cases of syphilitic diarrlea is favoral,le in some and grave in other instances, depending upon the type of the luetic lexioncausing the trouble and the extent to which they have progresied.

Enteritis syphilitica is not a serious affection, except in the cate 
of infants and old and debilitated individuals. Persons suffering from this complaint may recover spontaneously, but diarrhea, when persistent, is always more quickly controlled by combining the antisyphilitic with dietary and other therapeutic measures useful in overcoming intestinal catarrh and ulceration.

The prognosis in this class of cases is farorable, since the disturbance in the mucosa is, probably, similar to that of the skin in the secondary stage, which may disappear without treatment. In the later periods of intestinal syphilis, if the enteritis has become chronic, specific remedies and colonic irrigations can le relied upon to heal the lesions and diminish or arrest the frepuent movements.

The prognosis is always, more serious when the bowel is involved to a considerable extent by ulcerated areas or gummata, yet under such unfavorable circumstances antisyphilitic treatment nearly always improves the patient's condition and at times effects a permanent cure. Elsewhere the author has reported at length a case of chronic diarrhea induced by a large gumma that caused almost complete obstruction, yet the patient made a complete recovery following a course of specific treatment, and he has also succeeded in correcting diarrhea incited by syphilitic ulceration of the intestine in the same way. When the luetic process has produced one or more annular cicatricial stenoses of the bowel as the ulcers heal, or through the formation of a long tubular stricture when the sclerotic process is diffuse and involves the entire thickness of the bowel, antisyphilitic remedies are useless because they will not cause absorption of the scar and other dense tissues in and about a constricted segment of the gut, but they minimize or arrest the luetic process in other parts and prevent the formation of new strictures. When the bowel is partially or completely occluded by a syphilitic stricture in the small intestine or colon nothing short of surgical intervention will relieve the diarrhea and other symptoms of obstruction. In order to afford relief. and permit the patient to permanently recover or obtain relief in this class of cases, it is necessary either to $(a)$ remore the stenotic gut, $(b)$ establish an artificial anus above the stricture, or $(c)$ exclude the involved segment of intestine from the fecal current, all of which measures the author has practised with satisfaction at different times.

When the leutic process extensively involves both the small and large intestines, anel deep penetrating ulcers or multiple strictures are present, and when the disease has extended to neighboring organs or has resulted in the formation of simple or fecal fistula accompanied by copious discharges, temporary relief may be afforded the patient, but the prognosis is very grave and it is only a guestion of time when he, largely through exhaustion, must succumb to the disease.

Extirpation of the diseased bowel affords immediate relief, but when the patient still suffers from enteritis syphilitica or luetic ulceration of the large intestine, colonic irrigations and antisyphilitic treatment must be continued following operation unt il these conditions have been corrected, or the relief obtained will be of short duration. 


\section{CHAPTER NXVII}

\section{SYPHILITIC ENTERITIS, COLITIS, AND ENTEROCOLITIS (INTESTINAL SYPHILIS), DIARRHEA IN (Concluded)}

\section{TREATMENT}

\section{MEDICINAL (INCLUDING SALVARSAN), SURGICAL}

Medicinal Treatment. - The medical treatment of intestinal luetic lesions (Fig. +7 ) causing loose morements should be the same as that employed in the treatment of syphilitic lesions involving other parts of the body, except that it should be reinforced by eolonic irrigations. Here we hate nothing to do with the prophylactic and preventive treatment of lues, because the opportunities for this have long since passed in this class of cases.

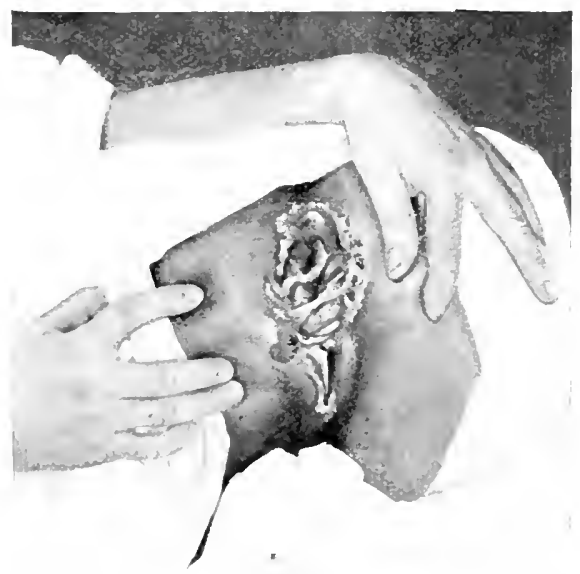

Fig. 47--Syphilitic coloproctitis complicated by extensive anotulvar uheration.

Nore ean be accomplished with drugs in the treatment of srphlititic than in tubercular, helminthic, dyenterie, or any other type of ulcerative colitis causing diarrhea. The usofulness of the antisyphilitic treatment has been demonstrated many times ly the author in congenital and acquired syphilis in cases where dianrhea had been incited by syphilitic inflammation or ulcers of the intestine and gummata causing the obstruction.

From time to time various adjurants and sulstitutes for metcury and the iodid of potassium have been suggested by syphilographers. 
some of which are probably more easy to employ, but their curative action is not superior to that of the older drugs. Of the newer medicines recommencled in the treatment of intestinal and other syphilitic lesions are arsacetin, atoxyl, soamin, kharsin, and asodyl, which belong to the arsenic gromp. These agents are of assistance in minimizing the effect of the virus and in toning up the system, but it is questionable if they have the specific action attributed to the remedies usually employed in the treatment of lues. Because of their non-specific action in this class of cases and their tendency to accumulate in the body in toxic amounts and produce distressing and dangerous manifestations-viz., migraine, deafness, dizziness, blindness, and retention of urine-they should not take the place of mercury and the iodids, but if employed to reinforce these remedies they should be given in smaller quantities. Of the arsenic compounds, atoxyl and arsacetin are the most reliable, and the best results are obtained when they are given in 5- to 8-gr. (0.30-0.50) doses every other day for two weeks, then discontinued for the same length of time, when again administered for a like period. The treatment should be continued in this way until the desired results are obtained.

Debilitated and anemic syphilitic subjects do better when the specific treatment is reinforced by dietetic measures-tonics, a change of surroundings, hydrotherapy, mild forms of electricity, or exercise in the open air. The usual tonics-arsenic, iron, coll-liver oil, Russell's emulsion, etc.-are useful for upbuilding the patient's general health, but for arresting hemorrhages of the mucosa and skin we have no more reliable remedy than calcium chlorid, grs. x to xx (0.60-1.30), administered three times daily.

Salvarsan (Ehrtich) is extensively employed in the treatment of syphilis, because with it one can in a few tays destroy spirochetes and alleviate symptoms which would by the older or mercurial potassium treatment require months to accomplish.

When employed shorty following infection it causes chancres to disappear and forestalls later manifestations. It is reliable in the first and second, helpful in tertiary, but is of lit tle use in the destructive or terminal stage of syphilis, except when employed in conjunction with potassium iodid or iodipin. A cure may be effected by a single large dose, but better results obtain when salvarsan is repeated in smaller amounts covering a period of from three to six weeks, and when the initial dose is followed by mercurial treatment. In the later stages of the disease, where lues is destructive, causing infiltrates or impairing the walls of blood-ressels which would lead to necrosis of the tissues, salvarsan is best reinforced by the iodids, which help to arrest the process and favor absorption of the deposits.

Absorption follows when salvarsan is injected beneath the skin into the muscles or aeins, but the intravenous method is preferable because it induces less pain, is seldom followed by sequele, and gives quicker and better results. This procedure must be practised with the care observed in other operations, and the patient should remain 
in the house for at least a day, otherwi-e infection or seriou-complications may follow. When properly armini-ered in -uitable fore-the reaction and subsefuent symptrms are unimpurtant, lut when the remedy is carelesily prepared or injected. wr is given in very laree doses, the patient sometimes complain- of vertigo, gastric rli-turbanceand rliarrhea, hish remperature. comvulsons, and death have -ometimes oecurred.

Elimination begin- in from thirty--ix to forty-eight hour-, and the late effects of salvarsan are irritation of the kidney:- transitory alluminuria, acute nephritis, gastro-intestinal irritability, and secasionally the organs of special sense have been affected and blindnes or dealnes has followed the treatment. complications thourht to have been cauned more by the disease than the remedy.

The treatment should be continued for weeks or month- until the spirochetes have disappeared and Hasermann" $^{\circ}$ blood reactions are repeatedly negative. Injections should subsequently be made when spirilla are discovered or the patient exhibits a positive Mas-ermann. A single injection may be employed in the symptomatic, luut repeated doses are necessary in the curative treatment of intestinal lues or elsewhere.

The average dose is ar. s $(0.60)$ for men and or. vii- 0.50 . fur women, and should be repeated as weth injections. extending oser a period of from three to five week- For chitdren eight year- old and upward gr. $v(0.30)$ is the usual amount, while the dose for infants having inherited syphilis is from gr. $\frac{1}{5} / 0.01$ to gr. $\frac{1}{3}(0.02$ ).

Salvarsan is unreliable in the treatment of syphilis complicated by tabes or paresis.

Patients afflicted with syphilitic colitis nearly alway improte following salvarsan injections. but this remerly is ineffective in thiclas: of cases except when reinforced by medicated colonic irrigationwhich cleanse the inflamed and ulcerated muco-a of teces. toxins. and irritating discharges, thus minimizing the part played by mixed infection.

Xeosalvarsan (a condensation of salvarsan and sorlium formalrtehyor sulphosylate from Ehrlich's laboratory) is better tolerated. cau-e less reaction, and appears to be as effective as salvarsan. The indications for and technic of administering nersalvarsan are the ame an for salsarsan, and the dee for men is gr. xiij to xt 0.750 .90 . and for women. gr. $x$ to xiij $10.60-0.75$.

Mercury and potassium iodid. alone or in combination. barring salvarsan, are the remedies par excellenes in the treatment of inte-tinal and other luetic lesions encountered in the various stages of syphilis. Mercury and the indids have a specifie action in most in-tance- leut now and then patients suffering from coneenital or acquired syphiliwith chronic diarrhea go from bad to wore under antisyphilitic treatment, both when it is instituted in the earlier and later stage- of the disease.

Syphilitic diarrhea nearly alway- occurs in the later-tages of lues. 
and because of this it is advisable to place the patient on the combined mercury and potassium iodid treatient at once to arrest the disease and favor healing of the inflamed and ulcerated mucosa or the absorption of gummatous infiltrations when present. The dosage of these drugs must necessarily be varied, for some syphilitic subjects can stand them in larger quantities and for a much longer time than others. Few persons can take mercury or the iodids alone or combined for months or years without suffering seriously from constitutional manifestations caused by them. Consequently, it is advisable to administer these remedies for a time (wo to three months) and then to discontinue them, according to indications, for from two to six weeks, after which they may be renewed and continued, according to this alternating plan, as long as may be necessary. In the later stages, as their influence upon the disease becomes more and more manifest, the periods of active treatment should be shortened and those of rest lengthened.

Considerable care is necessary when administering these drugs to persons having syphilitic diarrhea, because both mercury and the iodids are inclined to irritate the mucosa under farorable circumstances, and when strongly pushed in this class of cases they usually aggravate the intestinal trouble and augment diarrhea.

Potassium iodid is usually given internally, because when injected beneath the skin it is irritating and frequently causes abscess.

When potassium iodid is given by nouth the patient should be instructed to begin with m 10 ( 0.60 c.c.) of a saturated solution of the salt three times daily, and increase the close one or more drops daily until the physiologic effects of the drug are produced, when, after a short period, the dose should be gradually diminished or the drug discontinued for a time. The author has known potassium ioclid to work wonders in cases of syphilitic ulceration complicated by persistent diarrhea, and has treated one patient for an extensive gummatous infiltration causing obstruction of the bowel who was completely cured by this agent, and in two other instances he has greatly reduced the size of summatous swellings involving the intestine. In these cases no mercury was given, and the good results were attributed solely to the potassium iodid, though the sufferers had taken antisyphilitic treatment hefore they came to him. Mercury exerts a beneficent influence in arresting and preventing extension of the luetic process, but it is not so reliable as potassium iodid for hastening the absorption of inflammatory exulates and gummatous tumors of the gut.

Iodipin (combination of iodin chlorid on sesame oil), made in two strengths, io and 25 per cent., is practically as effective, and is a good substitute for iodids in the treatment of syphilis, because it can be administered for a longer time without producing iodism.

The author has obtained good results in the treatment of syphilitic colitis from the 10 per cent. preparation, administering it in $5 \mathrm{j}$ to $5 \mathrm{iij}$ (4-I2 c.c.) doses three to four times daily, and the 25 per cent. solution when 30 to 90 me $(2-6$ c.c.) were injected. 
There is also a solid preparation of iodipin (containing to per cent. of iodin) which is usually prescribed wo to three times daily in 30-gr. (2.0) doses. The drug should be continued until its full pleysiologic action is obtatined.

Mercurial preparations can be relied upon in the treatment of syphilitic lesions of the intestine causing diarrhea excepting gummata. Mercury may be administered by mouth, inunction, hyporlemically, fumigation, intravenously, or in the form of a suppository, lut of these methods the first three named are the most retialole.

Formerly mercury was prescribed in the form of a tablet or pill, but, owing to the tendency of the drug to cause gastro-intestinal disturbances when administered by mouth, many physicians now give it in the form of an inunction or inject it beneath the skin or, preferalsly, into the muscles. Mercury, in the following forms and dosage, has been successfully employed at various times in the treatment of syphilis involving the intestine or other organs, viz. bichlorid of mercury, gr. $\frac{1}{32} 10, \frac{1}{3}(0.002-0.008)$; liniodid of mercury, gr. $\frac{1}{50} 10 \frac{1}{10}^{1}(0.0012-$ $0.006)$; protiodid of mercury, gr. $\frac{1}{6}$ to $\frac{1}{3}(0.01-0.02)$; and tannate of mercury, gr. j to $\mathrm{ij}(0.065$ O.I.3). When the preparations named do not meet the indications or disagree with the patient, a blue pill, calomel, or the peptonate, sozoiodolate, carbonate, or salicylate of mercury may at times be substituted for them. Many syphilographers prefer mercury as an inunction, and the favorite preparations for this purpose are the oleate and blue ointment. The former is liked best by fastidious patients, since it does not discolor the linen, but larger quantities of the oleate are required because it is weaker. These ointments may be applied to any part of the body, but regions devoid of hair should be selected, because rubbing in of the ointment causes less discomfort and there is less danger of infection and furunculosis where hair-follicles are few.

The inunction is best made at the inner or outer sides of the thighs, arms, the ablomen, and back. Preferably the inunction should be driven in immediately following a hot bath while the pores of the skin are open, which explains why syphilitic subjects who take tratment at the various hot springs improve rapidly. After the rubling at night the patient should retire, and next morning remove all evidences of the ointment by bathing the parts with dilute alcohol or hot water. The treatments usually consume from twenty minutes to half an hour and should be given three times weekly or more frefuently, and continued for one to three months at a time.

Preferably, mercury should be administered by the injection method, because the treatment is cleanly, inconveniences the patient but litte, causes but slight pain, is rarely followed loy gastro-intestinal disturbances, one can accurately measure the amount administered. and the action of the druy is more lasting. An ordinary glass syringe. fitted with a gold or platinum needle, can le used, but the needle should have a large bore when gray oil or Iampkin's cream is used. The salicylate and bichlorid are more often employed than other prepara- 
tions of mercury, and the injections cause less trouble and give better results when made into the gluteal muscle. The treatments should be made once a week or at longer intervals when they disturb the patient, and for as long a time as may be repuired. Pain from them may be minimized by combining the drug with encain or cocain. The average dose of the salicylate of mercury is gr. $\frac{3}{4}(0.045)$, but the amount should be increased when there are destructive lesions in the bowel and administered at least three times weekly. When salivation and irritation of the gastro-inteslinal tract and kidneys become alarming, the quantity of the drug should be reduced or it should be discontinned.

In cases where tuberculosis attacks the intestine of a syphilitic subject the succinate of mercury should take preference over the mercurial preparations alove mentioned.

For the convenience of the busy practitioner the author has incorporated below a few formulie of known value in the treatment of luctic diarrhea and other manifestations of syphilis. Most of them include the mixed treatment in some combination, for mercury alone is rarely indicated, since persons suffering from luetic diarrhea have usually passed the early stages of the disease and require both mercury and the potassium iodid. The dosage of the formula given may be increased or decreased according to the necessities of the individual case,

R. Hydrargyri iodidi llavi

Ferri et quinina citratis.

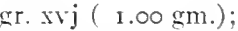

Extracti hyoscyami

Divide into pilulas No. lx.

Sig.-One pill three times daily.

R. Ilydrargyri iodidti rubri.

Potassii iodidi.

Tincture cinchone compositi gr. xij ( $0.75 \mathrm{gm}.) .-11$.

Aque...................

Sig.- Teaspooniul three times a day.

R. Hydrargyri chloridi corrosivi...

Potassii iodidi

Ferri et ammonii citratis................

Tincturæ gentianæ composite........ q. s. act. $\tilde{J}^{3}$ iv (1 20 c.c.). - II.

Sig.-Teasponful three times a day.

R. Hydrargyri chloridi corrosovi

I'otassii iodidi.

Fluid extracti sarsaparitle

gr. j-iij $(0.065-0.195 \mathrm{gm}$.$) ;$

$\overline{3}$ ss-iss $(15-45$ c.e. $)$;

Jiij (9o c.c.);

qs. ad. $\overline{3}$ ir (1 20 c.c.). - M.

Or,

Syrupi sarsaparilla compositie

Iqua ....

Sig.-Teaspoonitul three times a day.

gr. j-ij $(0.065-0.13 \mathrm{gm}$.

ijes (Iо c.c.);

予 $\mathrm{ij}(60$ c.c.).

$3 \mathrm{iv}(120 \mathrm{cos})$

q. S. ad. -11

Surgical Treatment.-When antisyphilitic treatment and other therapentic measures heretofore recommended, reinforced by medicated colonic irrigations, fail to heal luetic lesions in the bowel and re- 
lieve chronic diarrhea, surgical intervention is imperative if the patient is to be permanently cured. Operations may be, but are not always. effective, since frecuently a postoperative course of treatument is necessary to complete the cure. When the lwow is highly ulecrated, diarrhea is persistent, the stools contain considerable pus, blocel, and mucus, and the patient suffers severely Irom intestinal anto-intoxication and infection, appendicostomy (see Fig. 159), cecostomy (see Fig. I6 t), on the author's enterocecostomy (see Fig. I64) are indicaterl, because in such cases the colon is highly inflamed and ulcerated and nothing short of through-and-through bowel irrigations will heal the lesions and relieve the patient. Subsequent to the establishnent of an inket above. by inserting a proctosepe the fluid can be made to enter the appendix or cecum, and readily find its way through the colon and ont at the anus, and in doing so dislodges and washes out toxins, foot remnants. irritating feces, and acrid discharges. Better results are obtained when the patient's position is changed from time to time during the treatment to insure the fhuid reaching all sides of the diseased gut. Strong irrigants are required when lesions are numerous and extensive, but their strength is best reduced as healing takes place. which is indicated by the diminished number of evacuations and amount of pus, blood, and mucus in the stools. The colon should be washed out once or twice daily in aggravated cases, hut as the condition improves colonic flushings need not be made more than three times weekly:

When the movements are extremely foul, an ichthyol ( 5 per cent.) or a hydrogen peroxid (Io per cent.) solution can be relied upon to disinfect the intestine and relieve symptoms. In the arerage case horic acid (3 per cent.), ichthyol (2 per cent.), potassium permanganate (I per cent.), and protargol (5 per cent.) irrigating solutions are effective, but when diarrhea is persistent and the discharge is abundant, their strength should be increased. There are many other effective irrigants which one can employ following appendicostomy and cecostomy in the treatment of syphilitic and other forms of ulcerative colitis, but these and the technic of their administration have been given in Chapter XLI, devoted to Colonic Irrigations and Enemata.

Before the advent of appendicostony and cecostomy, enterostomy and colostomy were occasionally resorted to, but they have been abandoned because patients object to having their feces discharged through the side, and because a second and dangerous operation is necessary. to close the artificial anus after the lesions have healect.

Intestinal (colonic) exclusion (see Figs. 177, I78, I8I) is sometimes practised as a substitute for appendicostomy and cecostomy, or is resorted to following these procedures where the lesions have not healeed to exclude the inflamed and ufcerated segment of intestine, so that it will have an opportunity to rest and not be constantly traumatized by the feces. The author has, in a number of instances, succeedect in curing patients aflicted with syphilitic, atarrhal, and other forms of infectious colitis by this procedure. In some cases only a short piece of the gut was shist off, but usually the ileum was revered near the 
cecum, and after both extremities had been closed the proximal end was anastomosed with the sigmoid flexure or rectum (ileosigmoidostomy, ileorectostomy).

Unilateral (see Fig. I8I) and bilateral (see Fig. 177) exclusion have been resorted to, but the former is indicated more frequently than the latter when the small intestine. cecum, colon, sigmoid, or rectum are extensively inflamed, ulcerated, or blocked by strictures.

When less radical measures fail to relieve or cure the patient the diseased gut should be resected or extirpated, the procedures for which, named according to the segment of gut to be removed, are designated enterectomy, cecectomy, colectomy, sigmoidectomy, or proctectomy. Where stenoses are located in the lower 3 inches $(7.5 \mathrm{~cm}$.) of the rectum or below the peritoneal reflection, and the patient prefers a palliative to a radical or curative operation, the lumen of the strictured bowel can be quickly increased by forcible diulsion or proctotomy (internal or external) procedures, which are dangerous when the stenosis is in the upper rectum. For a description of the indications for the abovementioned surgical procedures the reader is referred to the chapters devoted to the surgical treatment of Diarrheal, Catarrhal, and Parasitic Diseases.

In concluding this chapter the author wishes to express his indebtedness to the works and publications of J. Brunner, Power and Murphy, Birch-Hirschfeld, Czernঘ̣, Fournier, Ehrlich, Fränkel, Jullien, Kleinschmidt. Oser, Hayem and Tissier, and Levaditi and Roche, the leading authorities upon intestinal syphilis. 


\section{CHAPTER XXVIII}

\section{ENTAMEBIC COLITIS (ENTAMEBIASIS, ENTAMEBIC DYS- ENTERY), DIARRHEA IN}

\section{DEFINITION, GENERAL REMARKS, HISTORY, ETIOLOGY, GEOGRAPHIC DISTRIBUTION, MORPHOLOGY, CULTURE AND CLASSIFICATION OF ENTAMEBA}

Definition.-Entamebic colitis (dysentery) is a sporadic or enclemic infections disease of the colon (and sometimes terminal ileum) characterized anatomically by marked inflammatory and ulcerative lesions in the mucosa, clinically by frecpuent fluid stools containing an abundance of mucus, blood, and at times pus, and subjectively by abdominal pain, tenesmus, and constitutional manifestations, as prostration, anorexia, and fever.

The author is aware that this definition is unsatislactory, as would be all others which attempted in a single sentence to explain what is understood by various investigators as dysentery.

One might as woll attempt to get up a single definition which would define scarlet fever, whooping-cough, chicken-pox, etc., as to seek to accomplish the same for dysentery, because of its variegated etiology, changeable intestinal pathology, and the dissimilar manifestations in different forms of the disease.

General Remarks. - Two decades ago this affection was believerl to be constitutional, but we now know that it originates within the bowel (from Entamixa histolytica), and when general disturbances arise they are secondary to local infection in the colon and exceptionally the lower ileum.

I'ntil a few years ago the term "dysentery" was universally applied to all bowel disturbances represented by the symptom-complex-viz.. frequent bloody eaduations containing mucus, with abdominal pain and rectal tenesmus-and, unfortunatcly, the custom still olstains in some quarters.

The practice of regarding lowel affections accompanied by thene symptoms as dysentery has leel to great confusion and improper treat ment. hecause the term means nothing from an etiologic or pathologic standpoint.

Small intestinal affections are rarely accompanied by these manifestations, but there are many diseases of the colon, sigmoid thexure, and recum, as nom-specifie (catarhal), tuhercular, syphilitic, and gonorrheal colitis, in adelition whe so-called dysenteric group) (entalmebic, bacillary, flagellate, ciliate and coccidic, and helminthic colitis), any and all of which may augment the number of evactutions, callet 
straining, the discharge of blood and mucus, and incite localized intestinal or general abdominal soreness, pain, and tenderness upon pressure.

Formerly patients might have suffered long and severely from any of these affections, and a diagnosis of dysentery would not have been thought of until the dysenteric symptom-complex became manifest, when instantly it would be diagnosed as such.

With our present knowledge it seems to the author that it is time to stop calling intestinal affections accompanied by the above symptom-complex dysentery, and classify them according to their etiology and permit the word dysentery to become obsolete.

Since colitis-slight, moderate, or severe-represents the chief organic change in most, if not all, bowel disturbances accompanied by diarrhea. mucus, pus, and blood in the stools, tenesmus, meteorism, and abdominal discomfort or pain, the author would suggest that, as a means of designating the various affections of this class, the term colitis be employed with the etiologic factor in the disease used as a prefix.

Such a grouping seems practicable because the name would then mean something, since it would convey an idea as to the etiology, pathology, and symptomatology, and indicate the treatment of the intestinal condition (colitis) referrect to.

Based upon this understanding, inflammatory and ulcerative lesions of the colon could then be classified, for example, as (a) a nonspecific (catarrhal) colitis; (b) tubercular colitis; (c) syphilitic colitis; (d) gonorrheal colitis; (e) bacillary colitis; ( $f$ ) entamebic colitis; $(g)$ helminthic and balantidic colitis, coccidial colitis, and flagellatic colitis.

Organisms Associated with the Specific Agents of Dysenteric Colitis.- It has been stated elsewhere that bacilli of the colon group, streptococci, and other forms of intestinal bacteria have been frequently encountered as causal or aggravating factors with the specific agents (entamebe and bacilli) of colitis (dysenteric), and here the author wishes to call attention to other parasites and organisms which have been found associated more or less frequently with different types of the disease.

Anderson ${ }^{1}$ investigated the convicts at Port Blair to determine how many of them harbored intestinal protosoa. He studied 920 of the 2539 cases of dyentery admitted to the hospital during 1905 , and found that amebe were associated with flagellates (trichomonas and lamblia) in 455 instances, and that the latter were present in 29 cases where amebe were absent. Balantidium coli were observed in combination with flagellates and amehe four times; alone, once. Of 2 Io individuals afflicted with other ailments, in 7 there were amebe, in $5 t$ amebx and flagellates. and in 80 flagellates only, which gives one an idea as to the frequency with which protozoa are harbored within the bowel in districts where entamebiasis is endemic-and no doubt protozoa frequently complicate bacillary colitis. The author has treated several

'Brit. Med. Jour., 1008, vol. ii, p. 1224. 
patients who harbored dysenteric bacilli or entameba and helminths (tape-, round,- or pin-worms).

Historic Note.- Dysentery has attracted the antention of medical men ahnost continuously since it was first described by flerolatus, shortly following an epidemic which ocoured in 430 B. C., and its importance has been particulaty emphasized by (aden (I6) A. D.). who pointed out the clinical manifestations of the ailnent. Since then, Aretens, Celsus, Sennertens, Benivient, cruveilhier, Rokitansky, Virchow, Wookward; and later, Lambl, Loesch, Kartulis, Quincke, Roos, Muster, Osler, Stengel, Stocklon, Harris, Conncilman, Lafleur, Ogata, Shiga, Russell, Flexner, Hiss, Barker, l)wal, Bassett. Vedeler, Musgrave, Strong, Hartmann, Whitmore, Craig, Siler. Kruse, Gant, and others have contributed valuable information to the subject.

Benivieni ( 506 ), held the first autopsy on a patient who died from dysentery, but prior to this Galen showed that the disease was confined to the colon. Sennerteus $(1584)$ pointed out that dysentery might be epidemic or sporadic, and characterized by frecuent bloody stoots and abolominal pain conserpent npon intestinal ulceration.

Early in the present century Cruveilhier and Rokitansky for the first time elescribed dysenteric changes in the bowel, and their findings were exemplified later by Virchow, and more recently by a unmber of investigators mentioned in the text.

At present we are fairly familiar with the pathologic changes common to the disease and know that it is caused by Entamaba histolytica.

Historic Data of Amebe and Entamebe.-Amebre were discoverer in the dejecta of a patient suffering from diarrhea more than fifty years ago, and they have since been investigated by a number of the world's best authorities, and yet we do not know positively how many varieties there are and which are pathogenic and which are not.

lambl ( 1860 ) found ameba in the feces of a child who suffered from diarrhea, accurately described the organisms, and intimated that possibly they were an etiologic factor in the bowel disturbance. Ten years later Lewis ( 870 ), while investigating cholera, observed ameba which morphologically corresponded to Lambl's, but attributed no etiologic importance to then. Loesch (1875) encountered in the dejecta of a patient afflicted with chronic recurring dysentery active organisms of a like character and named them Ameba coli. The and at were observed at various times while the lysenteric procest was active, and boesch considered them responsible for the disease, and to demomstrate their pathogenicity he injected infected feees into the rectum of a dog and succeeded in producing utcerative and inflammatory hesions of the mucosa, the discharges from which contaned organisms similar in every way to those found in the patient's stool. This investigator showed conchusely that the Amoba coli, which are now helieved to be the same as Schaudinn's Entamoxba histolytica, were al castative factor in amebic dysentery.

1) uring the next ten years numerous investigators, inchuling Leuckart, Perroncito, Cumingham, Koch, Grassi, Kartulis, Normand, 
Blanchard, Hlava, and others, found amebe either in the dejecta from the normal bowel or feces of patients suffering from dysentery. Grassi encountered them alike in both healthy and abnormal feces. About this time the pathogenicity of these organisms became the subject of hot debate because some investigators maintained that amebx were the inciting factors in dysentery, and others that they were of little if any importance as an etiologic factor in the disease.

Those who argued against the anebre being causative factors in the disturbance offered the following reasons for the belief: (a) The organisms were often found in the dejecta from healthy intestines (Cunningham, Perroncito, Grassi, Massciutin, etc.); (b) encysted amebe could be swallowed without causing the disease (Calandruccio); (c) the injection of garden mold into the rectum of cats excited an active colitis, the stools from which contained amebre (Gasser); and (d) dysenteric matter, free from the organisms, when injected into the intestines of man and animals, produced dysentery, and the consequent discharges contained amebie (Sorga).

Kartulis ( $1885^{-86}$ ) succeeded in demonstrating the presence of amebe in the dysenteric stools of the living and in sections made from intestines of patients who died from the disease, but met with little or no success in cultivating them. The organisms were absent in fecal discharges and microscopically examined gut in a large number of individuals who died of tuberculosis, typhoid, typhus fever, and bilharzia, but in 50 consecutive autopsies, performed on subjects who had suffered from the characteristic manifestations of Egyptian dysentery, he found amebx in every instance.

These and other findings convinced Kartulis that these protozoan agents were the undoubted cause of the disease, and, further, that amebe were proportionately much more numerous in malignant than in the benign type of dysentery, and that frequently there is a direct etiologic connection between amebic dysentery and abscess of the liver.

Hava (I887) in 60 cases confirmed the views of Kartulis as to the pathogenicity of amebic dysentery, and by injecting infected feces into the rectums of animals obtained positive results in 2 out of 17 dogs and 4 out of 6 cats experimented upon.

Osler (1890) was the pioneer in this work in the L'nited States. He discovered amebe in the dejecta of a patient afflicted with chronic dysentery, the morphology of which closely resembled the organism clescribed by Kartulis. In the same year, and in quick succession, Musser, Stengel, and Dock made similar discoveries.

To Councilman and Latleur belongs the credit of $(a)$ first vividly describing amebe; $(b)$ pointing out the character of pathologic changes incited by them; (c) assembling the characteristic manifestations of infections dysentery; $(d)$ calling attention to the points of differentiation between catarh and amebic dysentery; (e) showing that pathogenic Ameba dysenterice (Councilman and Lafleur), now regarded as Amoba histolytica (Schaudinu); and harmless organisms, probably Amaba coli, designated hy Schaudinn as Entameba coli, may be en- 
countered in the same case or in different individuas; and (f) demonstrating that amelie dysentery could be produced in cats be injecting into their rectums pus containing amebe taken from an abseces in the liver.

Numerous investigators, including Duincke and Roos (1803), and Kruse and Pascpuale (esot), confirmed the findings of (ouncilman and Lafleur in the main, and have demonstrated conclusively that there are two types of amelne, one pathogenic and the other non-pathosenis. and that anebic dyentery can be producerl by injecting fecen containing the pathogenic variety into the rectums of cats or doges.

Casagrandi and Barbagallo ( 1897 ) claim to have ionlated several varieties of anebar, but do not consider that they are etiologic factors in dyentery. These investigators maintain that there is a marked difference beween the amebe of man and the organisms found in fresh water, and suggest the name Entamabe hominis for amelye of the A maba coli variety, an arrangement not altogether satisfactory because different species exist in the same group.

Strong and Musgrave (1900), Ifarris (I90I), Jürgens (I902), Musgrave and Clegg (I904), and others have succeeded in demonstrating the pathogenicity of amelye in dysentery and abscess of the liver. and Jürgens confirmed the findings of Councilman and Latleur as regards the presence of both pathogenic and non-pathogenic antebe in the stools of individuals suffering from dysentery.

Schaudinn ( 1903 ) encountered both harmless and disease-producing amebes. The former, which was met with in the dejecta of both healthy and individuals suffering from dysentery, he named Entamoba coli (see Figs. 50, 51, 5.3), and the latter, the chice etiologic factor in true dysentery, he designated Entamaba hystolytica (see Figs. to, to, 52). His studies indicate that the morphology, method of reproduction. and life-cycle of non-pathogenic differs widely from that of pathogenic entamebe.

To demonstrate that Entamaba coli were harmless and Entamaba histolytica were the cause of dyentery he, on different occasions. swallowed both varieties, with the result that the former causerl no inconvenience, while the latter incited inflammatory and ulcerative intestinal lesions. Four vears after these experiments schatulinn was still suffering from dysentery, and died from an absecsin the sigmoid flexure which was thought to have originated from the infection.

Craig, (I903), Hartmann, Jürgens, Kartulis, and most investigators of amelic and entamebic dysentery and zoologists have confirmed the position taken by Schaudinn and adopted his classification. Muserave and clege, while agreeing in the main with Schatudinn, differ with him in that they hold that all amebe or entamebe are potentially pathogenic, and that so long as they are present in the bowel the patient hats amebiasis.

Viereck (1006) described a new pathogenic species of anelna assuciated with dyentery which he called Entumela tetragena, and Craig. Hartmann, and Prowazck have confirmed his findings. 
Craig (1906) discovered a pathogenic organism possessing both a flagellate and ameboid cycle of development, to which the name Paramere hominis was given.

Prowazek (1904) isolated the Entamaba buccalis; Castellani (1905). the Entrmaba undulans; lesage (Igos), the Entamaba tropicalis; Ganducheau (1908), the Entamaba phagocytoides; Elmassian (1909), Entamaba minuta, and Koidzumi (1000). the Entamaba nipponica, which differ in their morphology and life cycle. No doubt other varieties of entamebre have or will be discovered which, like the above, are pathogenic in man and aninals, on the one hand, or, on the other, harmless when encountered independently or together.

Etiology.-While true dysentery is a specific disease, the symptomcomplex of the affection. frequent bloody movements, abdominal pain, and tenesmus resulting from other causes, are frequently mistaken. diagnosed, and treated as dysentery.

Indoubtedly the chief factor in dysentery is contaminated iiater used for drinking and cooking purposes. In some instances the water is renclered impure through the emptying of sewage into the stream, and in others wells and springs are polluted through the drainage from stables, privies, and unhealthy ponds, which finds its way into them by gravitation.

Strong (I902) collected numerous amebe from water, but did not prove their discase-producing power: but Musgrave (1904) induced dysentery in a monkey with water amebe, but failed to infect cats by the injection into their rectums of organisms taken from the monkey's feces.

Recent investigators have shown that water containing ameba is not harmful, and was demonstrated by Brown, who examined the water of ships arriving at eastern ports following long tropical rosages that contained amebe in large numbers. but which did not cause diarrhea or dysentery among the crew who constantly used it. In tropical countries rain-water collected in tanks usually contains amebe in abundance, but thes are not harmful. Turbid is no more dangerous than clear water, because either may harbor encysted or harmless entamebas.

The opinion prevails among the public that filtering of water with household filters protects them against dysentery and other infectious diseases, but Brown insists that if once a filter becomes infected it is always a perpetual souree of danger until sterilized. which is often difficult or imposible, consequenty, when a filter is employed it should le used to clear the water before and not after it has been boiled.

Fresh aegetables from gardens which receive drainage from neighboring toilets and privies and those which have been fertilized with diluted human fecal matter, as is sometimes practised by Chinese and other gardeners, have been known to cause dysenteric colitis in a number of instances. Nusgrave and clegg have demonstrated that five or six washings of lettuce, tomatoes. cucumbers, radishes, etc., were insufficient to remose the amebe from salack, which indicates that 
these organisms frequently gain entrance to the alimentary tract alive or in the encysted state through the medium of uncosked forst.

Formerly it was thought that onee the soil harl been polluted with the fecesof elyenterie patients it remained so for years, and it was shown that during wars the discane hats repeatedly broken out at different times when soldiers have recamped upon infeeted ground, though gears had elapsed since the preceding epidemic had oceurred. Authorites now agree that entamebic dyentery is spread by tlies and individuals who harbor entamelsa.

Exposure to a high temperature, sudelen change from a hot to at colder climate, unhygienic surroundings, poor and infected food, disipation, living in bow-lying, swampy countries, exhatstion, debility and malaria, anemia, ptomain-poisoning. catarrhal and ulecative conditions of the intestine-one and all pare the way for or aggravate dysentery.

Classes.-Dysenterý attacks all classes alike, but is most common and fatal among the poor, who are badly nourished, live in an unhealthy atmosphere, follow arduous occupations, or constantly expose themselves.

Age.-Dysentery is most frequenty encomtered in persons between twenty and hirty-five years, although it may occur in the aged or very young children, as was shown by Amberg, whose patients were two and one-half, three, four, and five years of age, and there is on record a case of amelic dyentery which occurred in a breast-led Chinese baby. Children are less apt to become infected than adults because they are not so freptently exposed and their acid (milk) stoxis are unfarorable to amebe, which thrive in an alkaline media.

Sex.-The disease is much more common among men than women. since they are more frecpuently exposed, are often segregated in harge numbers while working on railroads or in mines, ete., and because their duties more frefuently call them to distant comtries where bacillary or entamebic colitis is endemic. Of the 200 cases of amebic dyentery reported by Strong, there were 177 men and 2.3 women, and this authority avers that in the eastern tropics dysentery occurs in the ratio of 5 males to I female.

Racial Predisposition.-In this country it appears that negroes are less apt to become infected than whites. for Simon's 50 cases. treated in Vew Orleans, where the blacks are numerous, included t2 white and s neero patients. While Europeans are prone to ameliasis and other forms of elyenteric colitis, the natives (particularly soldiers) of tropical and semitropical countries suffer from the diseatse sery much more frecpuently, because they live in the midst of poor hyegenic =urroundings, are carelest as to the water they drink, and because they often eat impure and indifferently prepared loxels.

Occupation. - Vocations which leat to exposture, exhatustion, and the spending of time in districts where malariat and dyentery are onmmon render the subject more liable to the diseatse. hut otherwise accu-

IJohns Hopkins Merl. Bull., roor. 1) 3.5.5. 
pation is unimportant as a predisposme cause of dysentery. As a matter of interest it may be stated that in Simon's series of cases there were 25 laborers, 3 farmers, + merchants, 2 beggars, and a rabbi.

It has been said that dysentery is a disease of war, famine, and exposure, and this is largely true because the disease has been encountered in its most epidemic and malignant forms during war times in different countries, but it has proved most destructive where the fighting has taken place in tropical or semitropical countries, as will be seen by a glance at the statistics given under the section deroted to the distribution of the disease.

From our present knowledge of the causation of dysenteric colitis it would appear that exhaustion, overheating from long marches, insufficient and improperly cooked food, irregular time for eating, sleeping upon the damp ground, malarial surroundings, and climatic and atmospheric conditions, etc., play no part in the actual causation of the disease, as we formerly believed, but weaken the subject, diminish his resistance, and render him unable to combat Shiga's and other bacilli or entameba, the specific agents of dysentery (colitis), when they find their way into his intestine.

While bacillary and entamebic colitis (dysentery) is prone to break out during war and often causes more deaths than the fighting, it has been frequently encountered in endemic and epidemic form in asylums, prisons, barracks, and other places where people are assembled in large numbers and remain anywhere in the same place or locality for days, weeks, months, or years.

The disease is likely to break out among such assemblages for several reasons-riz.: (a) it is difficult to obtain a sufficient amount of pure water for drinking and cooking purposes; $(b)$ toilet facilities are inadequate; $(c)$ the diet differs from the normal; $(d)$ food must be collected in enormous quantities, and it is difficult or impossible to prerent it from spoiling; $(e)$ cooking is insufficient or bad; $(f)$ their manner of living subjects to exposure; $(g)$ they have irregular hours for eating and sleeping: and $(h)$ the chances are that one or more of the assemblage are diseased or act as carriers for the specific agents of dysentery.

During camp life the probabilities are, in the majority of instances, that dysentery starts through infection brought about by flies or contact (usually the fingers), and not through contaminated water, regetables, or food.

Jehle and Charlton hold that bacillary drsentery is generally due to the eating of spoiled foods, hut this Kruse questions. It has been demonstrated that certain individuals are carriers of dysenteric bacilli and entamebre, the same as others are of the typhoid bacillus, and that healthy individuals may contract the disease from them under favorable conditions.

Craig estimates that 50 per cent. of human beings harbor amebx, but this signifies nothing, since they are harmless.

Pringle holds that the contagion may directly pass from one per- 
son to another in the same tent or room, and from them to others through the medium of rotten straw, clothing, etc.

Persons afflicted with dysenteric colitis have freptent fluid evacuations, and often the feees are discharged with foree and before the patient is in a proper position, with the result that the toilet seat hete comes soiled and infected, and it is not unreasonable to suppose that healthy individuals may contract the disease through the anus or buttocks coming in direct contact with the batilli or and be of such deposits, because it has been shown that these oryanisms retain their pathogenieity for hours or longer when the stools remain warm, as would be the case in temperate or tropical climates. It has also been shown that an ice-cold temperature renders amebe temporarily inactive, but that they again assume their pathogenicity under favorable conditions. The crossing of a river in Africa by French soldiers was immediately followed by an epidemic of dysentery, and it was formerly thought that the infection occurred from direct contact with the water or food which was contaminated by it, but this view has been discredited.

In camps flies are an important factor in spreading the disease by collecting the feces upon their feet and legs and carrying it 10 and infecting the water, milk, or food that is being consumed by healthy individuals, and by depositing infective material upon their hands, mouths, or clothing. Ancha and probably dysenteric bacilli like tubercle bacilli are rendered inactive, but are not destroved by the gastric juice, and when introduced into the body with water or food they are practically harmless until they have found a lodging-place at the ileocecal valve or in the appendix, colon, sigmoid, or rectum, which they infect under favorable conditions as soon as their vitality has been restored.

The author agrees with Jelks, in that the infection frequently originates in the lower bowel (often upper surface of the reetal valves), from whence the disease extends upward to attack the colon and possibly the ileum.

Climatic and Seasonal Prealence.-Bacillary and entamelsic dysentery (colitis) have been encountered in tropical, semitropical, and in colder climates, but the former is more prevalent in non-tropical countries, because dysenteric bacilli are not so easily affected ly variations in the temperature and atmosphere, while the warmth and moisture of hot climates favor the development of and pathogenicity of entamelya.

Brown has pointed out that entanebic dysentery is more manifest during the cool season, but says that this is due more to an accentuation of the symptoms and tendency to relapse than it is to augmented infection which is prone to oceur during the hot and wet montls. Heary and prolonged rainfalls favor the spreat of dysentery; this was show following the great Manila flood (root), when the fisease lecame epidemic. In this way organisms which have collected in various places are washed out and pass into wells and streams to penllute the water used for drinking and cooking purposes. 
Entamebic dysentery (colitis) may quickly follow the entrance of active pathogenic entameba (Entamaba histolytica) into the intestine, or the disease may develop considerably later, when they are encysted, in patients who are debilitated, suffer from some other intestinal affection, or conditions arise which would incite them to become active and cause the dysenteric symptom-complex, diarrhea, abdominal pain, tenesmus, blood, mucus, and pus in the stools.

The virulent Shiga and less virulent bacilli (Flexner's, Strong's, Dural's, Hiss', and Russell's) appear to be able to withstand the elements for months or longer, and are then capable of setting up a bacillary colitis after they reach the bowel. At present some of the leading authorities assume the existence of hosts or intermediary carriers, as in typhoid fever, in the bacillary colitis, but a study of the statistics indicate that in most instances this type of dysentery is carried to the intestine by water, milk, or infected food.

Geographic Distribution of Dysentery (Entamebic and Bacillary Colitis).-Dysentery, formerly considered a disease of hot or semitropical countries, has in recent years been encountered in nearly all parts of the civilized world in epidemic, endemic, and sporadic forms. Dysenteric colitis undoubtedly occurs with greater frequency in warm and tropical countries, particularly in districts which are damp, poorly drained, and where the surroundings are unhygienic and the inhabitants are more poorly nourished than elsewhere. Consequently, it is prevalent in the Southern States, Cuba, the Philippines, Japan, China, Mexico, South Africa, India, and South American countries, but it has been met with in northern Germany, Russia, Greenland, and in the States above Mason and Dixon's line. Helminthic, balantidic, coccidic, and other types of infectious colitis are also common in tropical countries.

Both bacillary and entamebic dysentery (colitis) have been frequently encountered in European countries, the United States, and, in fact, nearly the world over, but the former is more prevalent in Great Britain, Germany, Russia, the United States, and Japan than the latter, which is extremely common in the Philippines and Panama.

Bacillary colitis (dysentery) is more destructive in Japan than in any other country; occurs in epidemic form, and as many as 60.000 cases have been reported within a rear traceable to bacilli carriers, or, as Shiga puts it, explosive epidemics brought about through the pollution of water by dysenteric dejecta.

During war soldiers are frequent sufferers, and many of them die of the disease (entamebic or bacillary dysentery), an example of which is to be found in the statistics of Woodward, who collected 259,07 I cases of acute and 28,45 I of chronic dysentery in the Federal service during our Civil Mar. During the Boer War, from October, I 899, to September, I900, I I, I 43 Englishmen were admitted to the hospital suffering from dysentery, of which number $5+6$ died, and the proportion of soldiers afflicted with the disease and the deaths following were very much greater during the siege of Lady'smith and Mafeking. 
Strumpel states that 30 per cent. of all deaths oceurring in the Anglo-Indian Army are due to dyentery. Shiga cetimates the number of cases reported in Japan in ten years ( $1800-1000)$ as 875,000. of which no less than 231,000 were fatal, at mortality of 26 per cent.

Dysenteric colitis is exceedingly commons in Hong Kong. (extrinChina, East Indies, and Sumatra, due largely to amelic and hacillary infection.

In the northern Lnited States epidenic dyoutery rarely occurs. but is encountered in the endemic and sporatic forms quite frepuently in the South. If one is to judge by published calses, the rliceane forsmerly occurred very rarely in this country, or it was incorrectly diatsnosed, but torlay it is met with quite often.

Dysentery (entamebic and bacillary) is often brouglet here and widely distributed by soldiers, merchints, and others who come from the Philippines, Panama, etc., or other infected districts. The discate is endemic in certain sections of the Southern States and in some arylums, penitentiaries, and other public institutions, and sporadic cases of both bacillary and entamebic dysentery or colitis have been encomntered throughout the States.

The author has in recent vears treated 20 patients afflicted with dysentery ( 2 amebic and 8 bacillary) who never had been out of the States, or, in so far as they knew, come in contact with individuals suffering from the disease. Of this number, i I had always lived in the Eastern States (New York, New Jersey, New Hampshire. Connecticut, Ohio); the home of 3 were west of the Mississippi River (Missouri, Kansas, New Mexico); I was from Iowa, while the remainder came fron Tennessee (I). Louisiana (2), Kentucky (I), and Mississippi (I). At the ratio with which bacillary and amebic dyentery is increasing. unless radical means are taken 10 prevent its spreading, the disedse will soon be classed with the more common affections of the gastrointestinal tract.

Culture of Entamebæ.-Investigators have met with more success in cultivatiog non-pathogenic than the pathogenic parasitic ameloce encountered in the human alimentary canal; in fact, some of the most reliable authorities in this work maintain that the latter have mot been successfully cultivated, while others insist that they have.

Numerous attempts have been made hy Craig to cultivate the Entamaba histolytica, E. tetragena, anel E. coli taken from the stoulof patients which contained them, and bis effort failed in every instance.

Free nor-pathogenic amebe wsually contaminate the water and many green vegetables in all parts of the wortd. It is probable the e organisms have been cultivated in the tropics, though attempts in this direction have failed in this country.

Musgrave and Cleger atmit parasitic entameblat are not cultivalule alone. but are in symbiosis with certain bacteriat. Cats inombated with such cultures rematin well, hut monkeys showed dyenteroid symptoms and anche in the stools. 
Lesage carried a growth of Entamaba histolytica through a series of 66 subeultures along with bacterium, but if he succeeded in producing dyentery in animals with the cultures he does not mention the fact.

Noe suceceded in cultivating entamebre from liver abscesses, water, and lysenteric stools which differed in some but resembled Entamaba histolytica in most respects, and Ganducheau rendered rabbits immune to amebic infection by cultures of entamebe resembling the latter through peritoneal injections.

Parasitic amebe, common to man, grow well in connection with Bacillus coli, B. typhosus, B. rubra, B. fluorescens, Staphylococcus pyogenes, and ibrios of cholera.

In cultivating amele derived from feces Musgrave and Clegg proceed as follows: "The surface of the special culture-medium, contained in Petri dishes, is smeared with a culture of Bacillus coli or other bacterium with which amebe grow well, and the feces is then smeared in concentric circles upon the surface of the medium or in streaks across it. The plates are then kept at room temperature and examined upon successive days for at least a week. The amebe can be easily seen upon the plates, as they wander from the material in the spread out in to the surrounding medium, and the course that they take can often be followed by the development of colonies of the bacteria, which they carry with them."

Craig ${ }^{1}$ holds that the entire subject of the cultivation of the parasitic ancber is in a chaotic condition, and much more work will have to be done before it can be accepted that any of the parasitic species of man have been cultivated, and believes that organisms cultivable on artificial media are free-living and not the parasitic amebe found in man.

Nalker, who has given considerable attention to the cultivation of intestinal amelox, maintains that (a) parasitic amebe thrive only on a solid medium and one which is neutral or alkaline; $(b)$ that bacteria are essential factors in their cultivation, and $(c)$ that they multiply most rapidly in the presence of moisture, free oxygen, and a temperature of from $20^{\circ}$ to $25^{\circ} \mathrm{C}$.

Classification of Amebæ and Entamebæ.-Since the publication of Craig's work important changes have been made in the classification of amebe and entamebe, largely through the investigations of Hartmann, ${ }^{3}$ Whitmore, ${ }^{4}$ and ('alkins."

Ilartmann's Classitication:

Order: Amabinal Ehrenberg.

Cienus: Amabal Bory, 1822.

Cienus: Vahlkamptia Chatton, i012.

Genus: Entamola Leidy, 1879, (em. Schaudinn, I903.

1 The Parasitic Anchae of Man, 1011. p. 00.

2 Ibid.. I01 1 .

${ }^{3}$ Kolle u. IVassermann $:$ Handbuch d. pathog. Mikroorganismen, vol, vii Hartmann and Whimore, Archiv. f. Protistenkunde, 24, P. is2.

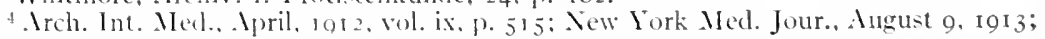
The Post-Graduate. November, 1013.

${ }^{5}$ Trans. XI International Comer. on Hyeg. and Demog., to 3 , vol. ii. p. 287. 
Definition of the Order. - Organisms which during their enture regetative hife show greatly changing form and move by su-called psearlopodia.

These peculiarities are brought about by the absence of inner stedetal elements and the naked surface. Differentiation inte ectoplasm and entoplasm, as well as contractile vacuoles, are not present in all forms; single or multiple nuclei (generally true caryosome nuclei) are present; reproduction is ly simple into two or multiple divisions, and hologamy, merogamy, and autogamy have been mentioned as fertilization processes.

Calkins' Classification.-Calkin's splits up the old genus amebe into allied genera as follows:

$\begin{array}{lc}\text { Ameba, } & \text { Craigia, } \\ \text { Vahlkampfia, } & \text { Tremastigameba, } \\ \text { Nägleria, } & \text { Entameba, } \\ & \text { Parameba. }\end{array}$

Points of Differentiation Between IIartmann's and Calkins' Classification.-Organisms which ordinarily grow as amebe, but which under certain outside intluences become flagellated, are considered as flagellata by Hartmann and as amelne by Calkins. The flagellate organization is the higher in Hartmann's opinion, and this flagellate stage is entirely characteristic for the particular species, the Hagellates in this stage being much more different than are the differences in the amelot state. Therefore, this flagellate stage cannot be considered as an adaptation to fluid media or any such change; it is a hereditary character, and is gone through with by the same organism under the same conditions, always in the same way. Many of the undoubted Hagellates, as Trichomonas intestinalis, frequently appear as ameboid organisms in the stool, but their position as flagellates is unquestioned: consequently, these ameboid organisms are taken ont of the group of pure amebe and grouped with flagellates by Hartmann-viz.. Paramoba eithardi Schaudinn, Paramoba hominis Craig (Cragia hominis (alkins), and Trimastig-amoba philippinensis Whitmore, organisms, all of which may be found in the stool.

Definition of the Genus Ameba.-Large forms, $100 \mu 10$ I mm., haring one or more contractile vacuoles and one or more nuclei, the mucleus containing a large caryosome or scattered gramules of chromitin.

Definition of the Genus Vahlkampfia.-Small amelue, 5 10 $20 \mu$. which move by a single pseudopod pushed out at the side, have one or more contractile vacuoles, typic nucleus of the genus and a large caryosome with variable development of the onter nuclens with or without a nuclear membrane. The nucleus divides ly pomitomis and

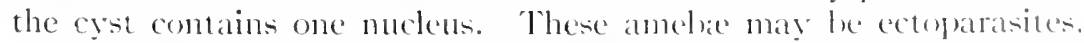
semiparasites, or establish themselves as parasites for a time, are commonly grown from feces, water, ete., may he confused with entatmeba on that account, and this genus has catsed the existing confusion relative to the cultivation of cntamelat. 


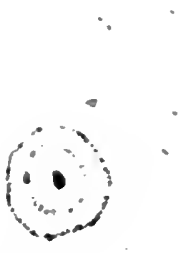

I

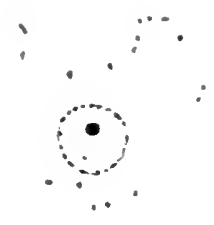

2

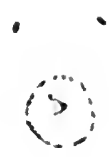

3
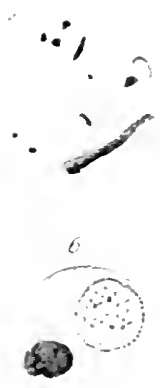

9
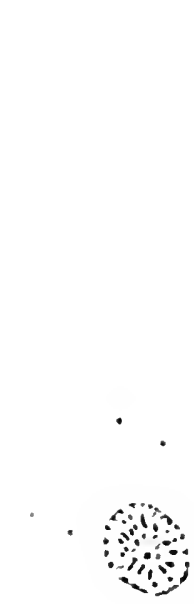

4

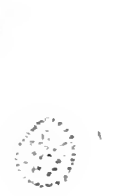

7

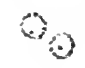

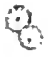

10

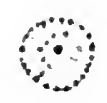

5

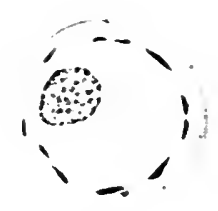

8

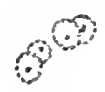

17

Fir. 48.-Entameba histolytica: r. Vegetative form. showing the cyelic changes in the nucleus. Above. to the left. is a hyaline peudopod. The original outline of the aneba show at a slightly higher level. 2. Vegetative form. showing coli type of nucleus. 3. Vegetative form. showing spindle-shaped caryosome. + Becrinning encystment. 5. Indivilual with lare vacuoles in the crtoplasm, and indistinctly staining nucleus. Probably degeneratine form. 6. Degenerating form. The nucleus has lost its structure; the chromatin is collected in masse in ide the nuclear membrane. Chromidia in the crtoplasm. - Beginnine encystment. There is a large racuole in the cytoplasm. The nucleus $\mathrm{i}=$ poor in chromatin; there $\mathrm{i}$ - no caryosome. and there is a thin laier of fine granules of chromatin around the periphery of the nucleus. There is no evidence of nuclear membrine. 8. Berinning encrstment. Large vacuole and numerous chromidia in the ytoplatm. 9. Like - but there is no vacuole and there is a large chromidium in the ytoplism. ro. ir. Complete cyst- with four nuclei. (Whitmore.)

lll figure: are from preparations fixed with sublimate-alcohol and stained with Heidenhain's or Rosenbusch's iron-hematoxylin.

The drawing were made with Abbe's camera lucilla, with the drawing-table at the level of the stage of the microsope. Zeis apochromatic obj. 2 mm. Compens. oc. I 2. Matenification about 1050 . 
Definition of the Genus Entameba.-Amebre, 20 to $100 \mu$. The nucleus is characteristic, there is a small caryosome, well-eleveloped outer nuclets, and a double-contoured nucleatr membrane. The earyosone shows cyclic changes, the cysts are polynuclear, and there is generally no contractile vacuole, for the parasitic ance be and marine forms do not, as a rule, have a contractile vacuole, while these living in fresh water do. Fresh-water amele having a contratetile vacuble lose it when placed in sea-water and regain it when returneel to fresh water.

Entamelor (histolytica) are the parasitic ameba and usually spread from host to host by cysts (Fig. +8) which they form.

Craig's classification ${ }^{1}$ included several entanel)e, viz., Entamaba coli, E. histolytica, E. buccalis, E. tetragena, E. phagocytoides, E. tropicalis, E. minuta, E. nipponica, E. kartulisi, E. undulans, E. pulmonalis, E. urogenitalis, E. minrai, E. gingizalis, E. dentalis, and his Paramaba hominis. but recent investigations have shown that E. histolytica causes entamebic colitis (dysentery).

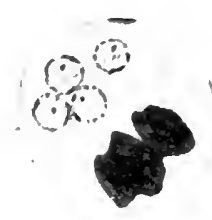

A

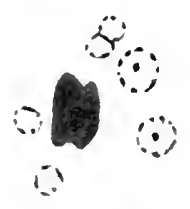

B

Fig. 49.-Entamceba histolytica cysts: 1. Complete four-nucleated cyst with chromidia. B. Six-nucleated cyst. Two of the nuclei are large (the two that have not yet divided). This is an abnormality similar to the Entamobal coli cysts. with more than eight nuclei. (Whitmore.)

The recent investigations of Hartmann and Whitmore indicate that there are but wo entameba-Entamaba coli and E. histolyticaparasitic in man, of which the former is harmless and the latter pathogenic (Figs. 49, 50). These authorities hold that entamebiasis is invariably cansed by Entamoba histolytica, and that having been seen in a different light by other investigators variots names had been given it-viz., Entamaba tetrasena. E. Minuta, E. nipponica, E. tropicalis, E. phagocytoides. E. undulans, and E. kartulisi, types which do not exist and must fall from the classification.

The cyst formation, as described by Schaudinn and confirmed hy. Cragi, falls, while that given for Entamobe tetragena (four-nucleated cyst) holds, and since E. tetragena and E. histolytica are the same, the latter name is retained hecatise of its priority.

Entamaba gingivalis, E. urogenitalis, and E. dentalis are doubuful, and E. miurai and E. pulmonalis are not considered valid species. while E. buccalis (harmless) stands as given by Craig.

Paramaba hominis (Craig) is taken out of the Order Amebina and is put in the llagellate Order Chrysomonadina.

${ }^{1}$ The Parasitic imebe of Man, roI 


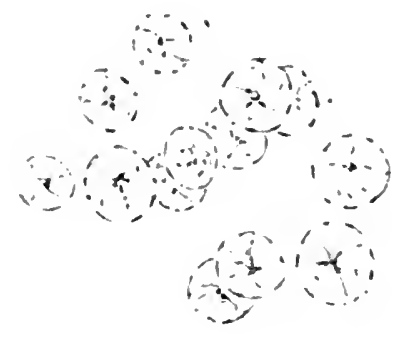

\section{1}

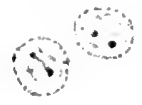

2

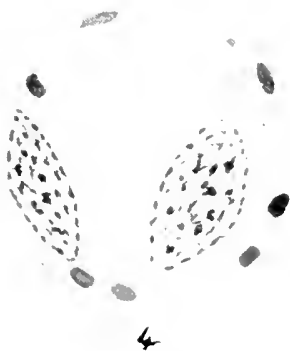

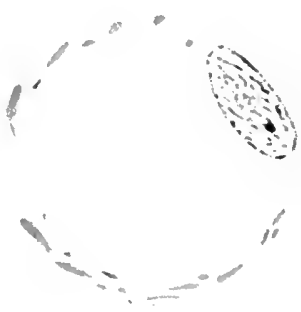

3

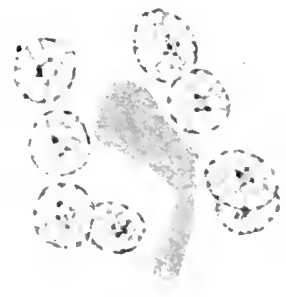

5

Fir. 50.-Entameba coli: I, Cyst with thirteen nuclei. This is an abnormality which is not uncommon in Entamuba coli. (Cysts with up to sixteen nudei.) 2. Vegetative form with two nuclei, beth nuclei in division. 3, Beginning of multiple divisien. Cyst with large vacuoles; nucleus early in division. 4. Cyst with large racuoles. The nucleus has completer the first division. 5. Complete cyit with eight nuclei. The large chromidium is rather unusual in the complete coli cyst. (Whitmore.)

Morphology of Amebæ and Entamebæ.--The morphologic characteristics of parasitic amela and entamebe encountered in the human intestine are understood in a general way, but otur knowledge relative 
to the peculiarities of the different varieties is not yet sufficient to enable one to closely distinguish between them. The lask of differentiating one entameba from another has been make more confusing by the varying description given by investigators of the individual types. and, further, because of the many kinds of non-pathogenic free-living amebe which gain entrance to the howel, appear in the feces, and are often mistaken for the parasitic organisms.

Entancbe are of variable size during the developmental stages, are continually changing their shape when in motion, and assume an ovoid or spheric form when at rest.

Some authorities (Schaudim, (raig. etc.) matintain that it is not difficult 10 differentiate between Entamesha coli (Fig. 50) and E. histolytica (Fig. fis), while others (Musgrave. (Clegg, Strong, etc.) hold that the reverse is true.

In the following smmmary relative to the significant characteristics of Entamobat coli and E. histolytica Park $^{1}$ has concisely given the dis-

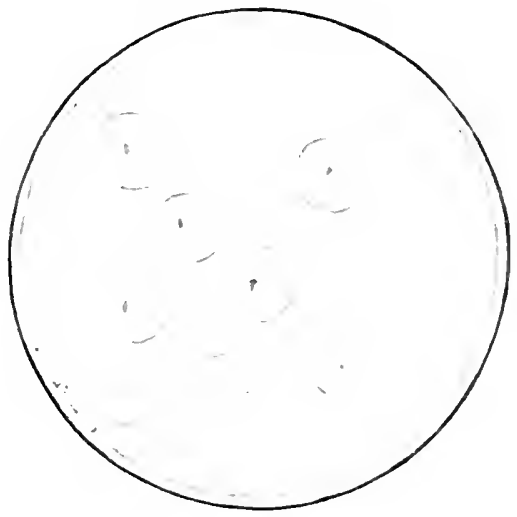

Fig. 51.--Fncysted Entumechit coll.

tinctive features of parasitic entamebre in man- iz.: (1) F. coli is, on the whole, smaller than E. histolytica; (2) its ectoplasm is so small in amount and so slighty differentiated that it is only seen when the organism puts forth pseudopods, while the cortical zone of E. histolytica is wider and is distinctly differentiated from the entoplasm: (3) the pseudopods of the former are small, rounded, delicate. and not highly refractive, those of the latter are larger, finger shaped, firmer, and more highly refractive, thus indicating the power of the oreanisu to penctrate its host s tissues; (4) the nucleus of li. coli is rery distinet in life as well as in stained spreads, due to a definite membrane, a more distince karyosome, and much chromatin, which is distrihuterl throushout the nucleus with more of a collection about the periphery; the nucleus of E. histolytica, on the other hand, is seen with difficulty during life, and stains faintly, owing to its delicate membrane, its small amount of chromatin, and small karyosome, the chromatin is 
collected about the karyosone and the periphery of the nucleus; the nucleus, moreover, is much more variable in shape in the active organism than is that of E. coli; (5) the entoplasm of E. coli is less granular and vacuolated and contains fewer red blood-cells than that of E. histolytica, which sometimes shows immense numbers of these blood-cells.

Motility.-As a rule, entamebe are constantly on the move, frequently change their form and arrangement of their neoplasm, but pathogenic entameba, such as the Entamoba histolytica, are actively motile (Fig. 52), while non-pathogenic E. coli are very sluggish in their movements-distinctions valuable from a diagnostic standpoint.

Locomotion in both instances is effected through the projection of ectoplasmic pseudopodial extrusions which repeatedly present at different points on the circumference of the organism. The pseudopodia of Entamoba coli are grayish, and gradually extend as the entoplasm flows into them and produces a slow but progressive motion; or, on the other hand, simultaneously project at various points, which induces motility but no advance of the organism.

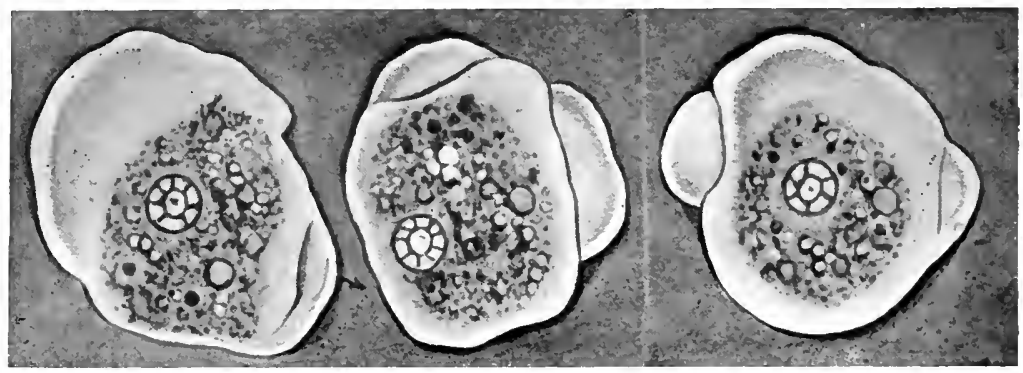

Fig. 52.-A living Entamøeba histolytica shown in three successive ameboid stages (Hartmann).

The pseudopodia of Entamoba histolytica, at first devoid of entoplasm, are more clear, refractile, thick, and resistant than the short and rather blunt projection of the $\mathrm{E}$. coli.

According to Craig, Entamoba histolytica possess three forms of motility: (1) active progressive motion; (2) the extrusion of pseudopodia without progression; and (3) movements of the cytoplasm.

In both species of entamebe the appearance and shape of the pseudopodia vary in the different stages, but at no time are they like those of the free-living or non-parasitic variety.

The motility of pathogenic amebe is most marked at the bodily temperature, and their activity decreases in proportion as it rises or lowers, a peculiarity which enables them to be differentiated from the saprophytic varicty, which more strongly resist heat and cold.

All entamebe travel faster in a thin than a dense media, but $E$. histolytica have a greater driving force and power to displace objects blocking their course than $E$. coli, and generally progress minterruptedly in a definite direction; less motile, they may move for a short 
distance one way and then turn and proceed sowly in another, which enables the eye to follow them for a consicteralsle time.

Duration of Life and Resistance. - Both E. coli and E. histolytica may exist in the alimentary tract for weeks. but gradually beerme less active outside the body, even in the presence of a firvorable temperature, and eventually die, though the latter in more pugnacions than the former, and bave been known to live several days and when death ensues both shrink up and become ovoid in shate. (ertain of the free-living or non-pathogenic amebe can maintain their vitality at higher and lower temperatures than those parasitic in man.

Several investigators have pointed out that entamelse can he destroyed hy strong electric currents, $x$-rays, and chemicals. ant that they respond in one way or another to mechanic, electric, and chemic stimulation.

Park has pointed out that while pathogenic entameba are demotilized they may still be infective after freezing, and Musgrave, at a suitable temperature, succeeded in keeping an encysted culure from dyenteric dejecta for a month and a half, when it still remained viable.

Reproduction.-But little has been addeel to our knowledge concerning the reproduction of entamebe (Figs. 50 and 53 since the publication in 1903 of Schaudinn's investigations; the exact manner in which it always occurs and the different varieties is not positively known.

There are four methodis of reproduction-simple dizision, schisogony, gemmation, and reproduction within a cyst, and these Craig has described as follows:

"Simple Diatision.- This method of reproduction is a-exual in nature, and consists of a primary division of the nuclens. which may he either mitotic or amitotic in character. followed by the division of the cytoplasm, two amebe being thus produced. This method of reproduction occurs in all species of amebet so far as is known.

"Schicogony.-This method of reproduction is also asexual in nature. and is accomplished by the division of the karyosome by printive mitosis, after which the muclear chromatin becomes collected around the nuclear membrane, while the cytoplasm rids itself of all foreign material. When this is completed, the chromatin divides into from four to eight litte masses, the nuclear membrane ruptures. and the chromatin masses become free in the endoplasm. Finally, the cytoplasm divides into as many parts as there are chromatin manese and a number of daughter amelox are thus formed (Fig. 53. 5 \%

"Reproduction Ifithin a Cyst.- When conditions are unfarorable for the vegetative existence of andeb they underge ency-tment and reproduction occurs within the cysts. The method of reproduction within a cyst varies in different species. and oceurs in two clifferent ways: either ly the primitive mitosis of the muclens, followerl ly antugamous fertilization. or hy the nuctear chromatin hecoming dispersecl throughout the cytoplasins. followed by the formation of minute burlo

1. Irbeiten aus dem Kaiserlichen (jecundheilamte, roo3. 
contaning chromidia, which are separated trom the parent body and form spores by the secretion of a resisting membrane. In this condirion they are incapable of undergoing further development until surrouncled by conditions faroralle to regetative life. These methods of reproduction will be considered more fully in the discussion of individual amebe."

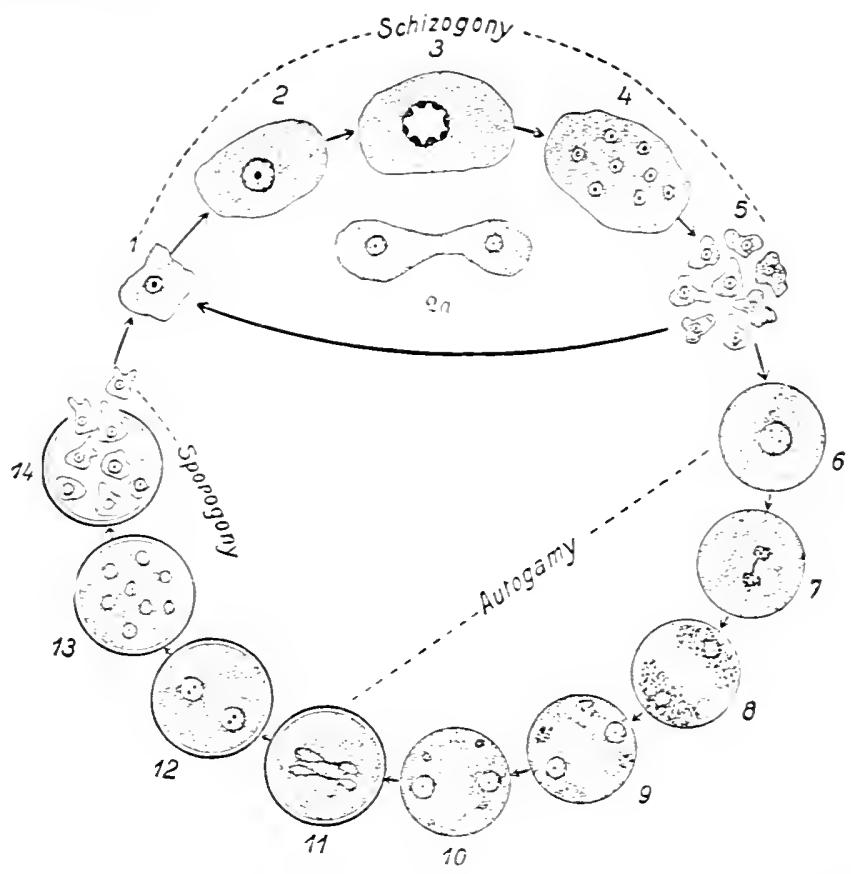

Fic. 5.-Diacram illustrating the life-crcle of Entamœba coli: 1. Young ameba; $\therefore$ arlult ameba: 26 . reproduction by simple division: 3. the division of the chromatin of the nucleus into eight aroups collected upon the suriace of the nuclear membrane: 4 . complete nuclear division: 5. shizocony or division of the ameba in to eight daughter amebæ; 6. commencement of $y \rightarrow t$ formation: 7 . primary division of the nucleus within the crst; s. incumplete cell-division and formation of chromirles: o. formation by the chromides of two pairine nuclei: 10. iormation of two reduced nuclei by each pairing nucleus: I I. division of each of the reduced nuclei into a free nucleus (male) and a stationary nucleus female: I2. two nuclear stage formed by the mercing of one free and one stationary nul leus: Is. adult crst containine eight daughter nuclei iormed by the repeated division wit the new nucleus: if the adult cyst aiter reaching the intestine of the host. liberating the eisht amebe formed durine sporogony (Hartmann).

The differentiating points between the different species of entanebe as regard - their reproduction are too varied and complex for discussion in this work. and because of this the reader is refered to the published researches of schaudinn and the works of Craig. Park, and Brown, where they have been fully described. 


\section{CHAPTER XXIX}

\section{ENTAMEBIC COLITIS (ENTAMEBIASIS, ENTAMEBIC DYS- ENTERY), DIARRHEA IN (Continued)}

\section{PATHOLOGY (INCLUDING TROPICAL LIVER ABSCESS)}

THE changes which take place in the bowel in entamebic coliti(dysentery) vary greatly in different suljects, becalus in some instances the disease is acute and in others chronic, and, further, for the reason that this type of infection is excessively virulent in one inclivitual and less so in another, and, finally, owing to the fact that entamebic eolitis, acute and chronie, does not atways pursue an even course. for at times a diphtheric-like membrane may form on the mucosa, or the superficial tunics of the howel may hecome extensively involvert through a mixed infection or by a gangrenous proceson and slough.

I'sually, in the chronic rariety, small ulcers form and the destructive process extends by coalescence until large, irregular raw areas result, and the mucosa beeomes undermined and fistulous intercommunications form which connect the ulcers.

Some idea may be formed as to the character of the changes which occur in the mucosa and the bowel turing life by inspecting the lesions through the sigmoidoscope and examiuing the gut at autopsy. but one is seldom fortunate enough to secure a section of the diseased intestine for inspection and microscopic examination

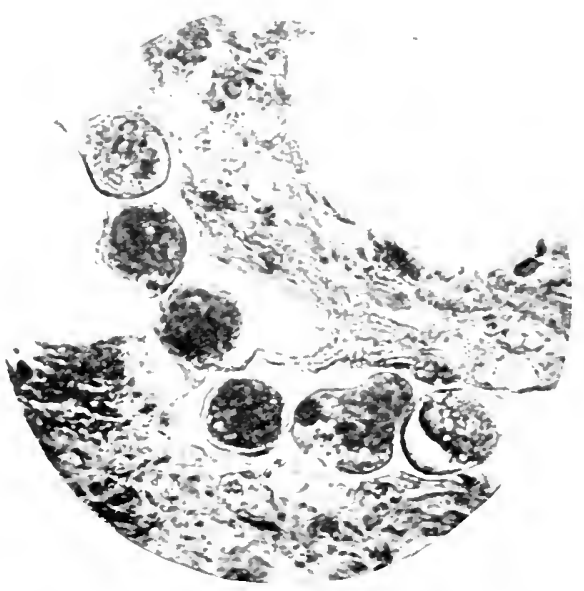

Fig. 54.-A section of the intesline, showing lintameba histolytica within the mucous membrane: $\times 750$ (Crair).

while the dysenteric proeess is active (Ficr. 5t): conseduently, onr knowledge concerning the pathology of entamelic colitis is not complete, though we know very much more about it than we lid a decate ago.

Entamebic has been oecasionally mistaken for bacillary colitis. lut the pathology of these forms of dysentery is of distinctively different that physicians familiar with them will not make this error.

Location and Distribution of the Lesions.-Entimulic colitis is primarily a disease of the colon, though in rare instances the specific 
organisms find their way through the ileocecal valve and infect the ileum. It is possible that entamebic ileitis may also be induced by Entamoeba histolytica, E. tetragena, etc., which gain entrance to the intestine through the drinking of infected water or eating of contaminated foods. When the ileum is specifically involved, usually not more than a few inches or at most 2 feet are affected. The lesions here and the manifestations arising from them resemble so closely those of the colon that a separate description of the pathologic changes and symptoms of entamelic ileitis is considered unnecessary.

Clinicians familar with the disease agree that the cecum, signoid, and particularly the rectum are the segments of the colon most frequently and severely involved by the infective process. Usually the trouble originates in the cecum and extends downward, but the author

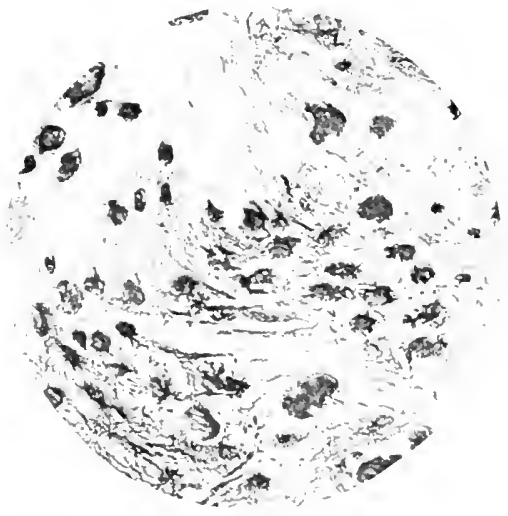

Fig. 55.-Entamebic ulcers of the sigmoin tlexure and rectum. has observed cases wherein the focus of infection was primarily in the rectum and gradually extended from thence upward and involved the different segments of the colon and cecum.

lesions located in the rectum are usually more extensive and persistent than those in the colon, but this is due to the trauma of the parts incident to defecation, prolonged retention of toxic feces in contact with the sores, and more active participation of streptococci, colon bacilli, and other obligate and accidental bacteria, and no good reasons have been adranced to show that the specific organisms (entameba) of dysentery are independently more virulent in the lower than the upper part of the large bowel.

In catarrhal, huetic, tubercular, traumatic, and, in fact, all forms of colitis, ulceration is most marked in the rectum and lower sigmoid ( 1 ig. 5.5$)$, and here, as in entamebic and bacillary colitis, the lesions are not more extensive because of their location or difference in the composition of the rectal mucosa, but to the constant trauma to which they are subjected plus a mixed infection participated in by the pathogenic and pyogenic bacteria found in this region.

The upper concave surfaces of the rectal adies afford an excellent temporary abiding place for both feces and entamebe, and, naturally, characteristic uleers are to be found in these localities. The writer has very frequenty observed lesions in their varying stages here, and Jelk, a close student, has found them in nearly every instance, but the lower or convex surfaces of the valves less often become ulcerated than the rectal wall.

In acute entamebic proctitis the mucosa of the lower rectum is 
swollen, edematous, red, sensitive, and occasionally projects slightly beyond the anal matrgin during defecation and straining. In the chronic form the membrane of the anal camal is less angry, but is irritated owing to the acrid discharge which is comstantly poured upon it from above.
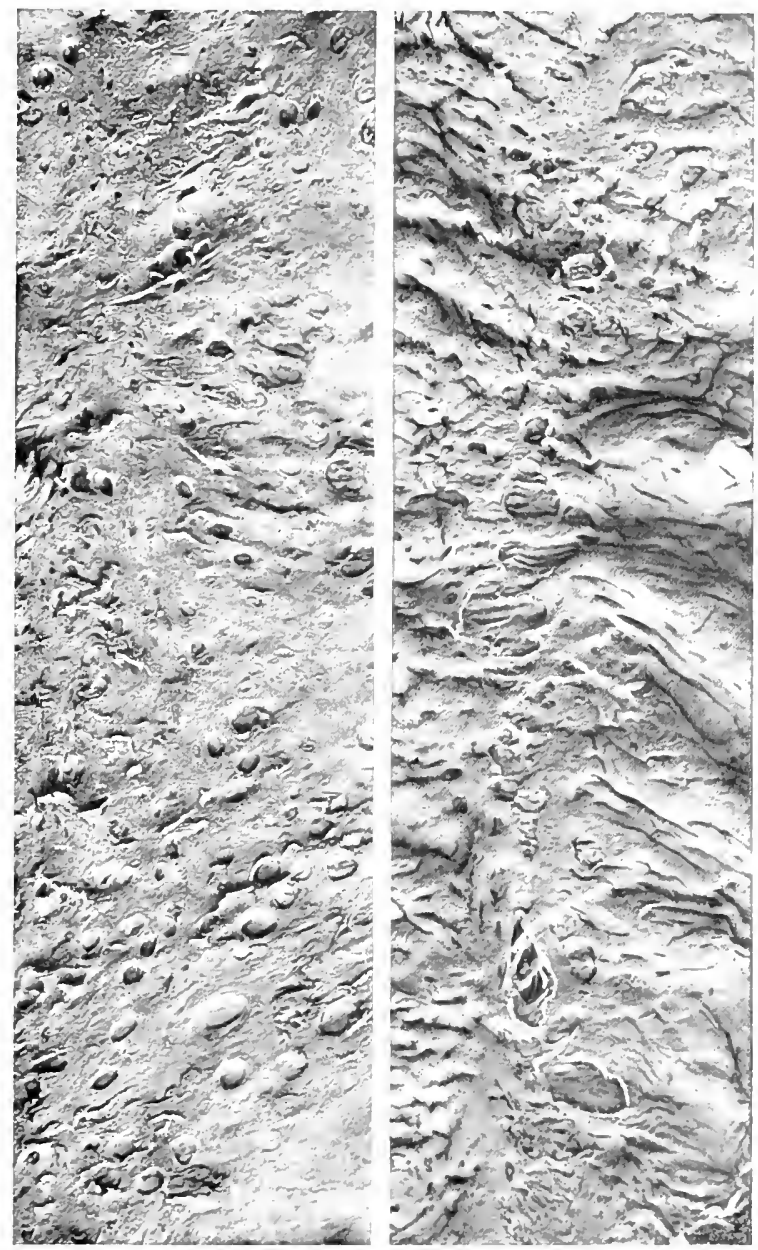

Figs. 56, 57.- Entamebic colitis (dyentery).1

Firg. 56.- Follicular ukeration and patches

Fir. 57.- Follis ular uhe ration. of preudomembrane.

Typic dysenteric ulcers have leen observed by the writer inmediately alrove the ants in a fow instances, lut, as a rule, characteristic lesions were not found to a greatl extent within the anal canal, but from the beginning of this narrow, tube-like ontlet lipwarel. The ulcers were more numeroms and extensive in the lower part of 
the true rectum, and gradually became fewer in number and less malignant the further up the examination was carried. In many instances the infection seemed to be confined to the lower bowel or sigmoid, but not infrequently the lesions evidently involved all or a liarge part of the colon (Figs. 56, 57). Clinical observation and autopsies have demonstrated that widely separated segments may be severally involved by the infective process, while intervening portions remain unharmed, and that unobstructive or straight pieces of gut, like the ascending, trans-

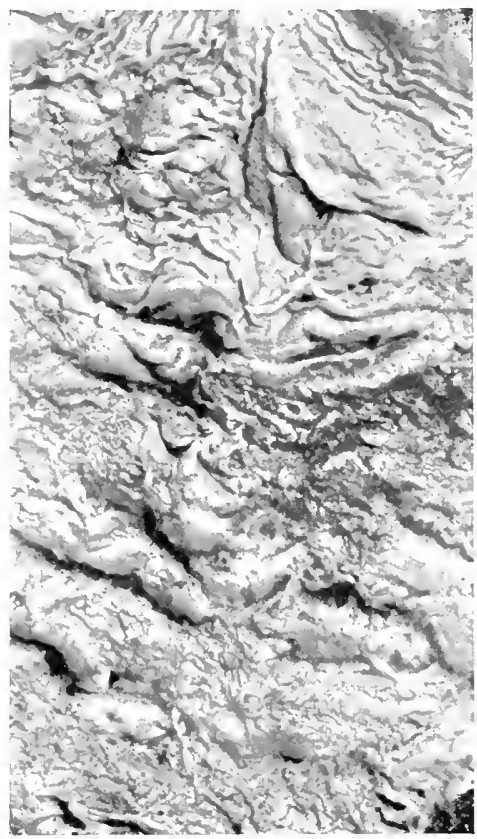

Fir. 3 S.-. Acute coloni entamebiasis. ${ }^{1}$ rerse, and descending colons, are affected less often and severely than are the cecum, flexures, and rectum, which longer retain or make passage of the feces difficult.

Brown holds that the entire colon is affected in 60 per cent. of all fatal cases of entamebic dysentery, and that under such circumstances the disease is most malignant in the rectum. Of Craig's 78 cases, 57 showed lesions in the cecum and rectum, while the intervening gut did not, and the disease extended above the ileocecal valve in luut two instances.

Except in the terminal gut, where the lesions are more pronounced owing to the fecal retention, irritation incident to defecation, and more active mixed infection, the changes consequent upon entamebic colitis are about the same in all parts of the colon and appendix.

The PATHOLOGY of entamebic colitis or dysentery can be more clearly and consistently studied by subdividing the disease into the (I) acute and (2) chronic varieties.

Acute Entamebic (Amebic) Colitis.-Acute entamebic dysentery (Fig. 58) may be mild or severe, be characterized by slight or grave changes in the bowel, and in one instance the patient may succumb to treatment or in another the condition may become subacute and eventually terminate in the chronic form of the disease. In farorable cases infection is light and incites a catarrhal colitis, which is first accompanied by several copious fluid evacuations daily containing an abundance of mucus and a slight trace of blood, abdominal discomfort, and rectal tenesmus, and later ly a persistent diarrhea, wherein the stools are smaller, with marked straining, and are composed exclusively of mucus and blood (pure or clotted). In the presence of catarrhal inflammation the mucosa is congested, has an intensely red color, is edematous, streaked 
with blood, smeared over with a glaty mucus, sensitive to the touch and presents hyperemic ridge-like folds, and the sulmucosil is somenthat inflamed and thickened. In this form of entamelic colitis the lowel lining may be deted here and there with petednal hemorrhages or superficial oroid ulcers, which respond more kindly to troatument than classic dyenteric lesions. In some instances of alente entamethic colitis the infection is virulent and the inflammation very aretive and extensive, in which case the ahsorption of toxin is so rapid that death may result from shock before the stage of ulceration, and, as brown says, on opening the colon the mucosa is found to be intensely engorged and swollen, is dark blue or purple in color, with rextensive aboriza-
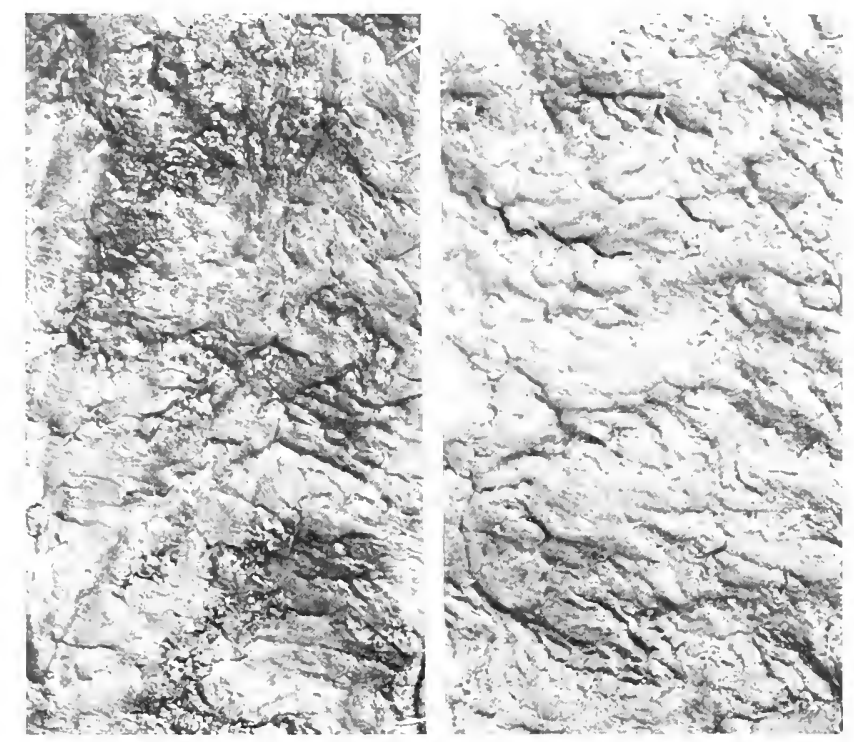

Figs. 50, 60.-Entamebic colitis (dysentery).?

Fig. 50.-Ulcerative stage.

Fig. 6o.- Ippearance of pseudomembranous (diphtheric) colitis before it begins to slough.

tions of congested vessels, and numerous extravasations are scattered over the surface. There is selem any ulceration or evidence of deep suppuration, but points of superficial necrosis, many of them alrealy beginning to break down, are abundant, and there is always an exers of mucous secretion, and here and there patches of exulate berome closely incorporated with the disintegrating mucosa and mite with it to form diphtheric membranes (Fis. 60). In the more acule and fatal (malignant) forms of loth entamebic and barillary colitis the highly inflamed intestine, when insperted through the sigmoidesente. presents about the same appearane, but, as the inflammation sulsides and the disease lecomes subacute or chronic, the differeness in the character of the lesions of the two types of infection are apparent.

1 Army Ned. Nuseum. 
So-called acute or fulminating dysentery, involving the mucosa (throughout the colon) in a necrotic process, and frequently causing death in from two to fire days, is, according to the author's belief, of bacillary origin in most instances, and will be discussed in connection with the pathology of that form of colitis.

In discussing the changes which occur in acute entanebic colitis nothing has been said concerning the manner in which entamebe gain entrance into the mucosa and submucosa and cause ulceration because the subject is to receive full consideration below in the section devoted to the pathology of chronic entamebic colitis.

The appendix very often becomes infected in entamebic and bacillary colitis, and treatment, barring appendicostomy, frequently fails because the nests of specific organisms can neither be destroyed nor dislodged, and remain to be occasionally discharged in to and reinfect the colon, sigmoid flexure, or rectum which have been healed by irri-
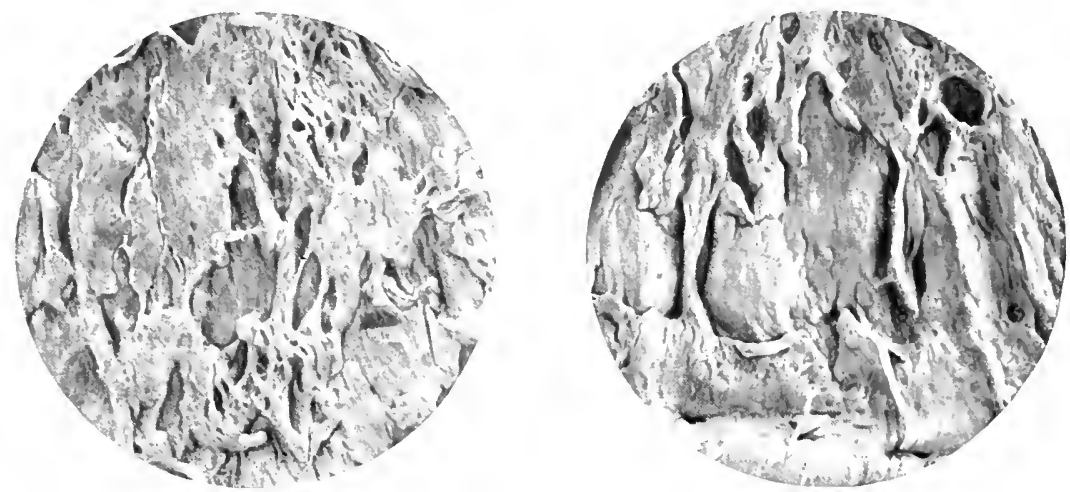

Fig. 6r.-Chronic entamebic colitis. Irregular entamebic (dysenteric) ulcers of the colon. Note how the mucosal is undermined. ${ }^{1}$

gation or medical treatment. Woolley and Musgrave estimate that the appendix is involved in 15 out of every 200 cases of entamebic dysentery.

Chronic Entamebic (Amebic) Colitis.-Entamelic colitis, whether it starts as an acute dysentery or insidiously and without producing the usual dysenteric symptom-complex, always becomes persistently chronic when it is permitted to go untreated, and occasionally so in spite of all remedies, and eventually severely injures or destroys the colon and causes chronic invalidisn or death. In some instances the discase assumes a relapsing, and in others a recurring, form, but in both the intestinal changes are very similar, though in one case the infection may be more virulent or latent than in another. It has already been intimated that the structural changes in bacillary are superficial and concern mainly the epithelial layer of the intestine, while entamebic colitis is a deep-seated affection, and imvolves the submucosa (first)

${ }^{1}$ Army Med. Museum.

${ }^{2}$ Reports to the Bureau of Government Laboratories, Manila. June, I905. 
and mucosa, and occasionally the muscular layers (lig. 61) anet peritoneal tunic. The former ype of infection progresses inaterd and the latter outward or toward the intestinal lumen.

It is a thoroughly well-stablished fact that the Entameter coli, which is considered hamestes. and the E. histolytica, of the pathogenic: group can and do frepuently remain free in the intestine for a short or longer time without inciting colitis or causing trouble, but when Entamolat bistolytica fund their way into the submucosa, multiply, and generate their toxins, they soon proluce the lesions and manifestations commonly associated with colitis or dysentery.

There has been considerable controversy as to the manner in which these organisms find their way through the mucous membrane inte the submucosa and exceptionally into the intestinal musculature. No doubt previous catarrh or other intestinal disturbances which lead to the formation of diminutive breaks, erosions, or uleers in the epithelial lining of the bowel favor this process, but these organisms, under favorable conditions, also possess the faculty of reaching the submucosa when apparently the continuity of epithelial covering has not been disturbed. Inder such circumstances they collect in Lieberkühn's follicles, and through the aid of their pseudopodia work their way through the walls of the crypts, the leader being followed ly other parasites in the manner practised by hat leships on parade, or, according to several investigators (Jurgens, Schaudinn, Dohter, etc.), entamebe penetrate the entire mucosa, finding their way through the interglandular tissue. Craig suggests that the organisms secrete a toxin which causes the intial destruction of the epithelial cells and paves the way for their entrance to the submucosa, and believes that this poison also participates in the formation of the grose lesions which follow. The examination of microscopic sections removed from the intestine during life and after death, both in the pre-ulcerative stage and where typic ulcers have formed, show conclusively that in this type of infection entamele alound in the submucrsa, and here inaugurate the inflammatory toxic and necrotic processes which (assisted by mixed infection) ultimately lead to the formation of the characteristic lesions observed in true entamebic colitis.

The writer has made a practice of grouping the lesions and manifestations of chronic entamebic colitis into three stages-viz., the pre-ulcerative, ulcerative, and destructive - which plan will be followed in discussing the pathology of the affection.

Pre-ulcerative Stage.- When conelitions faror extension of the infection, the colony of entamebse in the submucosa is constantly leing augmented through their multiplication hy schizogony and the frequent migration to the region of additional specifo organisms from the intestine. As a result of this activity, distention, generation of toxins, and disturbance to the circulation evidences of infection are soon seen in the overlying mucous membrane, though in rate instances and in some inexplainable way the destructive process subseles before any damage has been clone. The changes which take place 
in the mucosa and submucosa following entame! ic infection are subject to variation accordingly as they are modified by the virulence of the organisms, general health of the patient, local disturbance in the bowel, and extent to which ordinary and specific bacteria participate in the infective process. The disturbance may be confined to a small or large area in a particular segment of the gut, or appear almost simultaneously throughout the colon and, possibly, lower ileum, but usually the most active foci are to be found in the rectum or sigmoid flexure.

The disease early manifests itself in the form of hyperemic or edematous spots in the mucosa, which become elevated as the entamebic cellular infiltration and secretions in the submucosa increase and forge outward. These diminutive, ovoid swellings are usually located at the summit of the folds in the mucosa, and some of them present a hemorrhagic, others a vellowish, and still others a bright-red appearance, and in the affected region the mucous membrane undergoes a catarrhal inflammation, is covered with a glairy mucus, and when the papules dottect over its surface are incised they are found to contain a vellowish, viscid, sticky fluid composed of dead cells, mucus, and entamebe. In time the epithelium covering the nodulated areas becomes eroded as the result of local irritation, generated toxins, pressure or interference with the circulation, and the intestinal feces and bacteria find their way into the mucosa and submucosa, where they in conjunction with entamebe favor the rapid production of the ulcerative stage. In turn, the mucosa at the summit of the elevations undergoes necrosis, and yellowish, round, irregular-shaped lesions form, the floors of which are composed of the submucosa, and they are smeared over with a gelatinous secretion. Brown holds that in characteristic cases the submucosa becomes inflamed and infiltrated and the connective-tissue fibers proliferate. changes which lead to the formation of minute buttons of adenoid tissue which pusly up from below and appear as chusters or wart-like buds on the mucosa, and later that the central portions of these protuberances separate and pus exudes through minute openings in the apices of these mammillated growths, which are usually more pronounced in the sigmoid flexure and rectum. In either case the intial ulcers are raised above the level of the healthy bowel and the mucosa surrounding them is red and swollen.

Clecrative Stage.-Following the formation of ulcers the destructive process extends very much more rapidly, and the patient begins to complain of the true dyenteric symptom-complex-viz., diarrhea, mucus and blood in the stools, discomfort in the lower abdomen, and rectal tenesmus.

Inclependent of the fact that a vent has been formed through the mucosa, entamebe continue active in the submucosa, which becomes edematous and assumes a gelatinous greenish-yellow or whitish appearance, over which rests the intlamed mucosa, with its nodulated swellings, and punctate or larger ulcers encompassed by necrotic tissue. 
The specific organisms, aieled by other pathogende and pyogenic bacteria, their toxins, spheroidal infiltation, and strangulation of the blood-resiels, faror extension of the necrotic process downwarl inte the muscular tunic, then in the submucosa, anel, fonally, upward through the muscularis, mucosa, and mucous membrane, which is evidenced by papular enlargement, ecchymoses, or small ulcerated patches, with the healthy mucous membrane and arborization of the vesels showing between.

In long-standing cases the lesions are abundant, extensive, and characteristic, but in the beginning of the ulcerative stage of entalmelic colitis and later the uleers vary in shape, depth, and generibl appearance, and vary from a few confined to a small area to larste numbers seattered over considerable segments or the entire colon.

In the begimning they are usually superficial, from mustard-seed to split-pea size, round or ovoid in shape, have congested, thickened
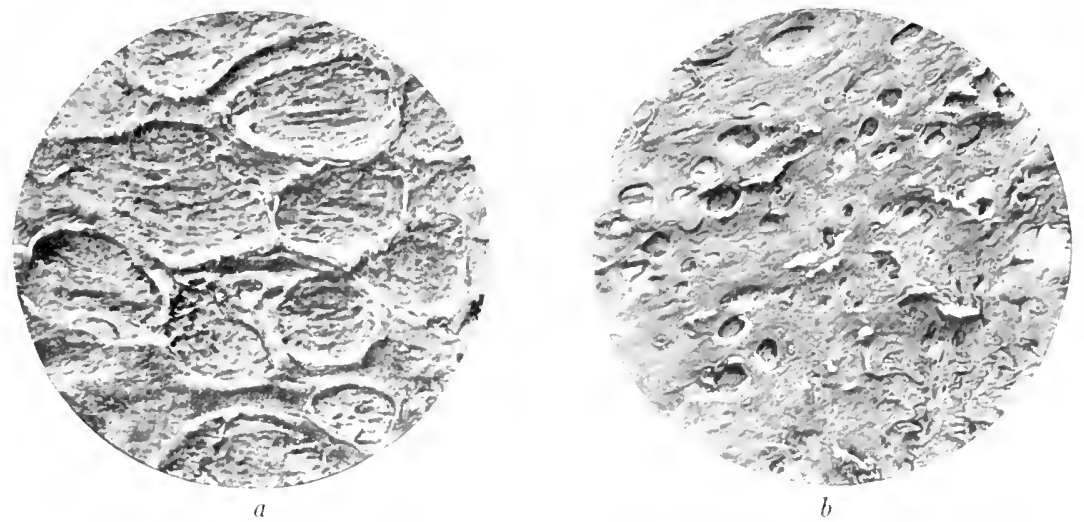

Fig. 62.-Entamebic ulcers of the colon: $a$, Round and ovoid (Harris" ulcers); $b$, follicular.

edges, a punched-out appearance, are of a grayish or vellowish tint. and are most commonly situated upon the summit of the folds in the mucosa.

Lesions of this ype are frequenty designated as Harris ulcers (Fig. 62, a and b), since he was the first to describe them.

Sometimes the sores are linear, follow no systematic course, and in conserpuence intersect each other and form the well-known stellate uleers. Either or both types of lesions may coalesce to form congaterl or larger and more irregular-shaped uleers with necrotic exlges, the bates of which ate congested, cosered ly gelatinous material. and formed by the submucosa or muscular tunie. These and the more serious lesions which form later show a tencleney to follow the course of the blood-ressels (or form at a right angle we the fong axis of the bowd), but not to the elegree observed in tulserculosis of the intestine.

Another type of lesion observed fairly often by the author in entat-

1. Army Med. Museum. 
mebic colitis is the so-called collar-button ulcer, which is characterized by a good-sized superficial round sore, joined to a much larger undermined ulcer (Fig. 63) in the submucosa by a narrow neck-like sinus, from which exudes a gummy secretion or pus which keeps the mucosa constantly irritated.

In some cases small and large lesions representing the different types of ulcers are present at the same time, as well as nodulated elevations between them, which later become necrotic and form sores. Inder such circumstances it is easy to understand how the ulcers are extended by continuity through the action of pathogenic and pyogenic bacteria and the constant formation of additional infected foci consequent upon the multiplication and nigration of entamebe and bac-

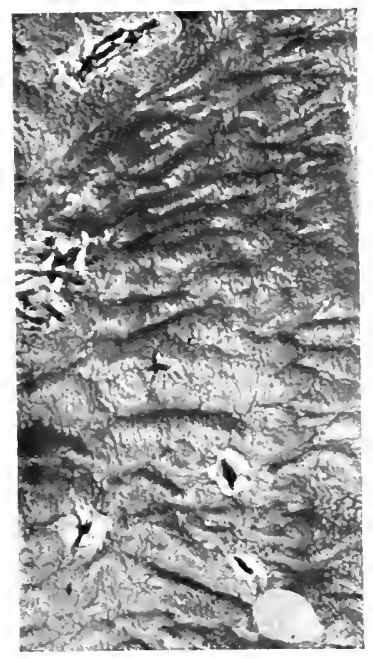

Fig. 6.3. - Entamebic colitis with perforating ulcers. ${ }^{1}$ teria, so that large ulcers or enormous raw areas are formed in the colon, sigmoid flexure, or rectum.

Irrespective of the characteristics of the lesions in the beginning, when entamebic colitis is fully developed classic undermined ulcers are present and usually dominate the destructive process. These ulcers are at first small and connect with much larger lesions in the submucosa, but later attain considerable proportions superficially and by undermining the mucosa.

Through the sigmoidoscope typic ulcers present a yellowish tint, reddened, indurated, elevated, uneven and undermined edges, and a necrotic or irregular boggy floor smeared with pus and blood, while the rims and floors of older and partially healed utcers are more rounded and smooth, though the adjacent mucosa is undermined. Lsually the mucous membrane and muscular coat in a large measure resist the infective process, which often destroys the submucosa to an extent that a probe, introduced through an ulcer, may be made to sweep around and beneath the mucous membrane from one-half to several inches. Exceptionally, the mucosa and musculature suffer more extensively, and at times the peritoneum is destroyed, perforation occurs, and the patient dies from peritonitis.

Then entamebic colitis is severe the serosa is usually discolored and thickened, though it is not directly involved by the ulceration.

Typic single entamebic ulcers may vary from I to + inches $\left(2.5^{-}\right.$ Io cm.) in diameter, but when they coalesce they may be very much larger and encircle half of the mucous membrane of the entire circumference of the bowel. Inder such circumstances the mucosa between the raw areas may remain normal, but usually it is involved in a catar-

1 Army Med. Museum. 
rhal inflammation or dotted over by erosions incident to the irritating discharge which constantly hathes it.

On account of the chronic character of the disease exudates deposited in the submucosa and musculature, induration which follows. and density of the peritonem, the bowel during life and after death presents a thickened and hardened feel when rollerl between the fingers, and when inspected, recent uleers can lx boated by hyperemic spots, and older or partially healed lesions ly contracted. whitioh areas in the peritoneum.

Destructize Stage.-In particularly virulent, neglected cases, and in those where the patient's general health or complicating local disturl,ances aggravate the condition, the persistent infectious procen inaugurated by Entamotba histolytica and augmented by the myriads of accidental and obligate bacteria in the bowel frefuently progresses to a degree which seriously incapacitates or destross the colon and induces an exhausting diarrhea, the loss of considerable blood, the absorption of pus and toxins, marked loss in weight. and formation of liver abscess or disturbances within the lung, brain. spleen, blood-ressels, or peritoneal carity when entameba reach them.

When the patient becomes thus sorely afflicted the author considers he has reached the destructive stage, and will rapidly grow worse and die if proper measures are not prompty taken to arrest the disease.

Naturally, owing to the lowered resistance of the patient and his inability to take and digest nourishing foods, the destructive procenow progresses rapidly, large areas of the mucous membrane are destroyed, the submucosa gives way, and extensive excarationform beneath the mucous membrane, which may retain their individuality or communicate with a number of other carities $1, y$ connecting sinuses (see Fig. 6I). Pockets that serve as collecting places for infectious material may lead to the formation of abscesses and fistula from which thick, yellow pus can be easily expressed.

In rare instances, owing to the virulent character of the infection. peculiar action of the toxins generated by the specific or mixed organisms, obstruction to the circulation, or due to other catise. small or extensive areats of the mucosa becone gangrenous and skugh. exponing the intestinal mu-culature (Fig. 6f), or at diphtheric membrane may form and cover the mucosa of the rectum, sigmoid flexure, or colon (Fig. 65). More often, however, in severe cases there are large ulcers with raised edges and long. trough-like sores in the intervening mucosa. lesions which give a buffalo-skin-like appearance to the mucou- membrane, which Craig regards as fairly common and characteriotic of entamebic colitis.

The author has treated wo patients for entamelic colitis who, in addition to the usual lesions, suffered from numerous polyps. which were fragile, varied from peal to clive size, and were acattered ofer the 
mucosa of the rectum and sigmoid flexure. Some showed as elevations, while whers were attached by pedicles $\frac{1}{2}$ inch $(1.25 \mathrm{~cm}$.) or more in

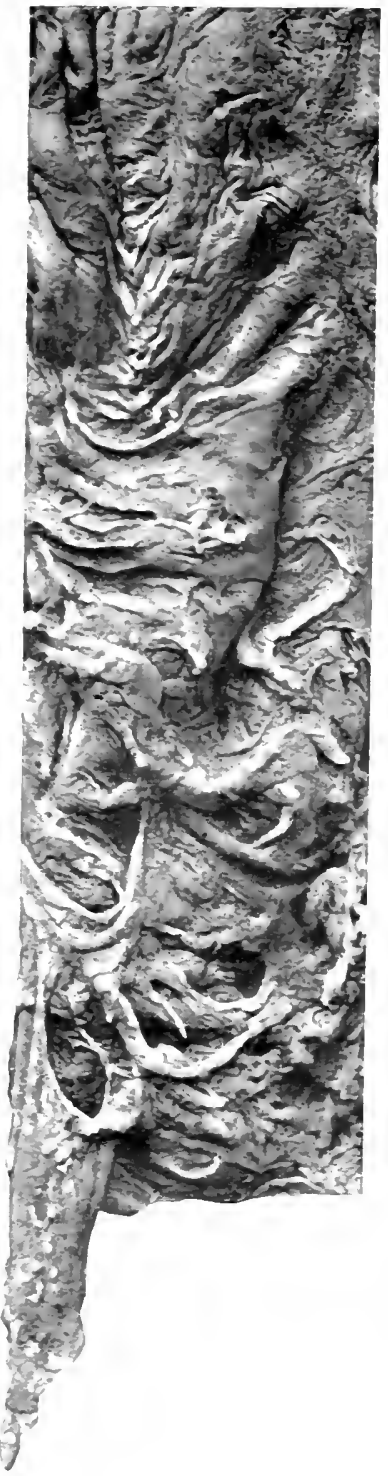

Fig. 64.-Fntamebic colitis (dysenteric of the decending colon complicated by gangrenous slourhing of the pseudomembrane diphtheric and mucusa. ${ }^{1}$ length. The discharges from the bowel in these cases were particularly offensive.

These growths (Fig. 67) or excrescences were not attributed to the entamebic infection, but to

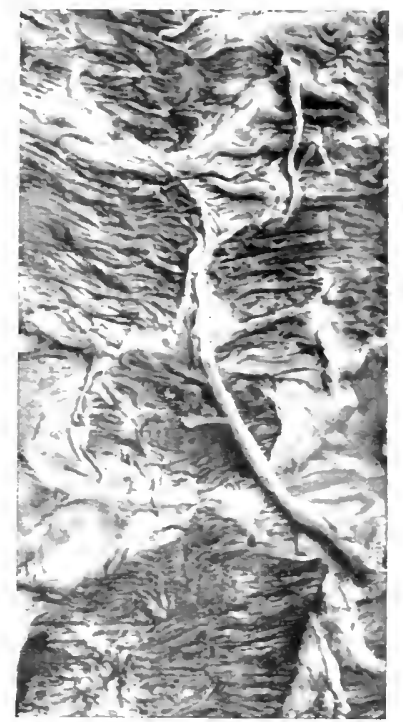

Fig. 0.-- - Clcers and slough in a case of acule entamebic dysenteric colitis. ${ }^{1}$

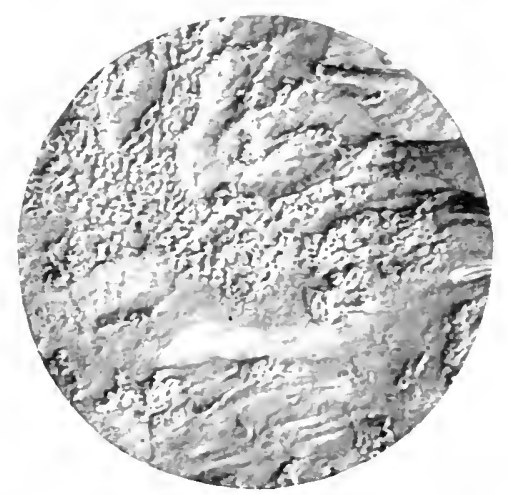

Fig. 6ti. - Colonic pseudomembranous patches in a case of entamebic colitis. ${ }^{1}$

hypertrophic changes consequent upon prolonged irritation of the mucosa incident to the acrid discharge which hathed it, because similar 
cases had previously been treated by him which were undoubledly due to other chronic, inflammatory, ancl supporative discases of the colon.

Musgrave maintains that sometimes in neglected cases of entameloc colitis a calarrhal condition prevals, the intestine atrophies (enteritis chronica atrophicans), the mucous folds disappear, and the gut becomes thinner and longer.

Microscopic Appearance of Entamebic Ulcers.-Thebowel, when sectioned through an uleer, reveals the elevated character of the lesion, the surrounding muessal congested, swollen, edematous, or necrotic, the blood-ressels strangulated through pressure of the exudates, spheric cellular infiltration evervwhere, lymph-channels dilated, thickened and misshaped, and entamelae in large numbers in the necrotic edges of the sores, granulating tissue, and submucosa, but the parasites are encountered in greater numbers in fresh and round than in the old, larger, and more irregular lesions, and particularly at points undergoing degenerative changes.

Entamebe may lee encountered in chains, few in a group or en masse, where they are accompanied by leukocytes and other evidences of inflammatory activity, and confined to the regions named or encountered in the blood-and lymph-ressels.

In characteristic instances the vascular and lymphatic systems in the congested, infiltrated, or degenerating submucosa are enlarged, thickened, and abnormally arranged, and usually, as has been pointed out by Brown, the endothelium is shed, and dense thrombi, formed of corpuscles, coagulated lymph, and entamedre, block the lumen. At this stage of the morlist process, howerer, entamelae are very difficult to identify, and unless the preparation is properly stained they may be easily confused with endothelial cells or other mor-

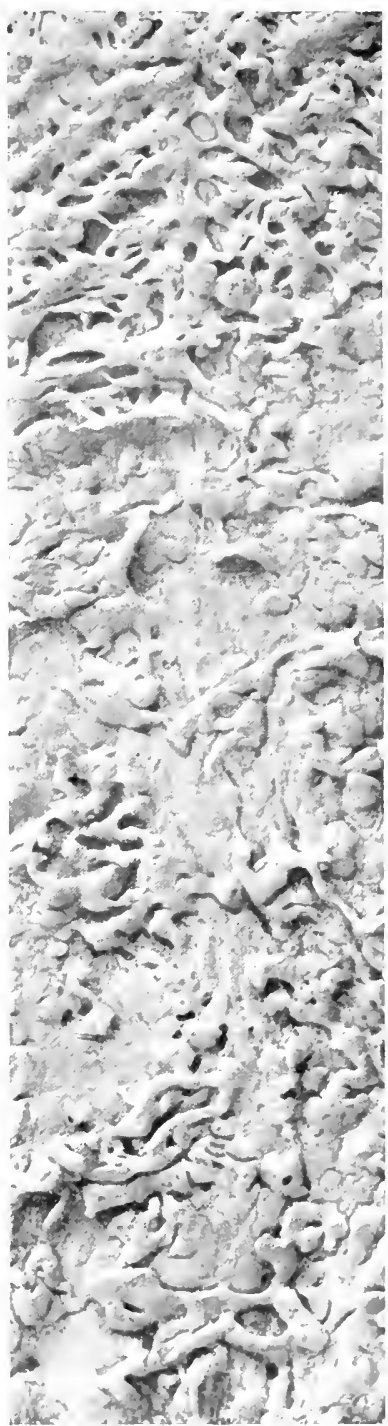

Fix. 67. - Pseudopolypcomplicating entimebic coliti: (dyentery).

bid products. The closed follicles are generally inllamed and infiltrated, but show no distinctive alteration, and only in exceptional instances do they contain entamebit. 
The presence of different varieties of bacteria encountered in the tissues and discharges in the vicinity of entamebic ulcers demonstrates that the suppurative and necrotic changes are the result of mixed infection.

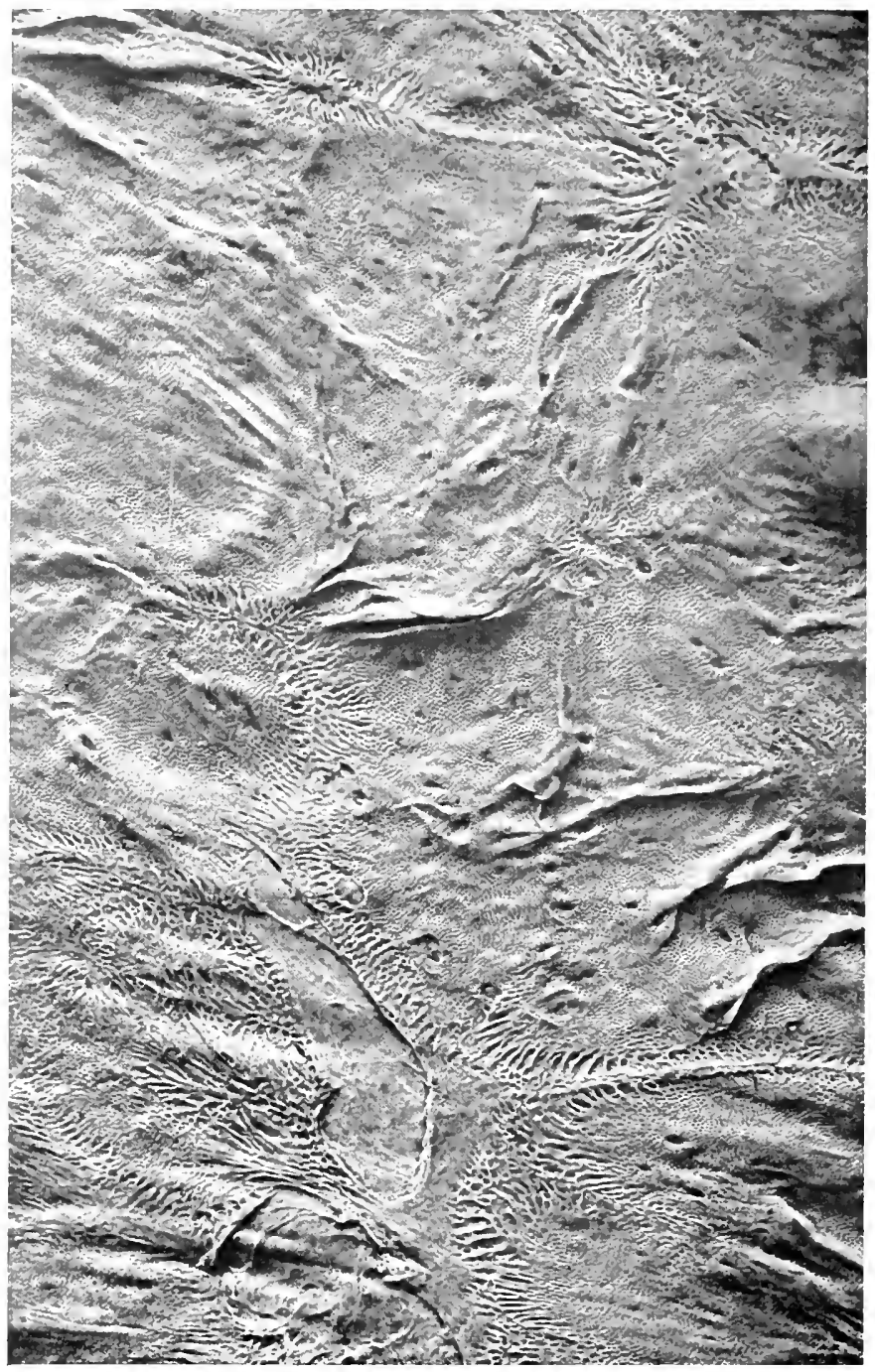

Fig. 68.-Peculiar extensive distortion of the mucosa incident to cicatrices in al case of chronic entamebic colitis. Note healed and unhealed ulcers. ${ }^{1}$

Healing Tendencies of Entamebic Ulcers.-Except in farorable or properly treated cases the lesions of entamebic colitis exhibit but a slight tendency to heal because they obtain but little rest, owing to the accompanying exaggerated peristalsis, irritation caused by the ${ }^{1}$ Army Med. Museum. 
feces, and the furtherance of the infection by entamelye and bacteriat constantly present in the gut.

When the bowel is drained and frepuenty irrigated with antiseptic and stimulating solutions, or in some catses under internal medication, the superficial ulcers clean up, becone covered with bealthy granulations, and rapielly heal, while, at the same time, the excavations beneath the mucose simulaneonsty close and the bowel is lefe practically in a mormal condition, exeept that the destroyed glands are not reprodicerl.

Frepuenty, owing to reinfection and other causes, the ahmos on entirely healed sores often completely or partially break down, when the patient again suffers from the ustal manifestations of the disease.

Occasionally bealing is characterized ly the formation of a thin or thick layer of scar-tissue, which contracts and puckers the atjacent mucosa (lig. 6s), or, when the destruction of tissue hat heen consilerable and involved nearly the entire circumference of the gut, parttial or complete strictures form. 


\section{CHAPTER XXY}

\section{ENTAMEBIC COLITIS (ENTAMEBIASIS, ENTAMEBIC DYS- ENTERY), DIARRHEA IN (Continued)}

\section{SYMPTOMS, COMPLICATIONS}

Symptoms. - Lisually the manifestations of entamelic colitis are fairly characteristic, but in some instances they are confusing because of their close resemblance to disturbances consequent upon dietary indiscretion, ptomain-poisoning, enterocolitis, and inflammatory and ulcerative lesions of the colon of tubercular, bacillary, luetic, traumatic, or gonorrheal origin.

The symptoms are variable in different people and stages of the disease, and one person may remain practically well, another suffers moderately from abdominal discomfort, diarrhea, pus, and blood in the stools, while another soon becomes dangerously ill from toxemia and aggravated dysenteric manifestations. Statistics indicate that the disease begins acutely in from 50 to 60 per cent. of the cases.

When analyzing the symptoms in suspected cases of entamebiasis it is well to remember that in some instances patients are infected by both entameba, Shiga's, Flexner's, or other bacilli, and the myriads of pathogenic and pyogenic micro-organisms in the intestine may also actively participate in the disturbance as soon as the mucosa is broken, and the sufferer may have some intercurrent disease which may modify the symptons.

Entamebic colitis is characterized by a catarrhal inflammation, ulcerative processes, a diphtheric membrane formation, or the superficial tunics may become extensively involved in a gangrenous process and slough, leaving the bowel denuded over long or large round areas.

Bacillary less often becomes chronic than entamebic colitis, and the earlier symptoms of the former are usually more distressing and dangerous than the latter; in other words, in the average case bacillary is most dangerous in the early stages and entamebic infection in the later periods of the disease. Irrespective of whether or not the onset of the disease is mild, moderately seiere, or dangerously acute, entamebic colitis generally becomes chronic and greatly debilitates or kills the patient after months or years of suffering, while in bacillary colitis he usually recovers or succumbs to the infertion within a few days or weeks, and relapses complicate the former more often than the latter.

Latent Types of Entamebic Colitis (Dysentery).-Ordinarily this form of colitis is very active in the initial stage and characterized by the dysenteric symptom-complex-viz., diarrhea with blood and mucus in the stools, abdominal discomfort, rectal tenesmus, etc.-but less 
often (30 per cent., Brown) the disease makes an insidious onset and the patient suffers from anorexia, minor digestive disorders, lack of desire for work or amusement, and possilyly constipation, manifestations which would not leat one to suspect entamelse infection unless he had had a previous experience with this ype of discase, or knew that the patient came from a country where lysentery was enelemis. The author has observed cases where entamedse abounded in the dejectal, and yet there were few lesions in the rectum, sigmoid, or colon, and the patients dial not complain of the usual dysenterie semptoms; and has treated patients where entamele were present in the stocks, the bowel extensively ulcerated, smeared with mucus, and who did not suffer from diarrhea and bloody stools or the ordinary signs of dysentery, but, as a rule, he has observed that dysenteric manifestations appear simultaneously with the lesions and becone worse proportionatcly as the ulcers increase in size and number. Other patients have been studied where the disease became acute after having remained latent for weeks or months, and the author in a number of instances has known individuals to suddenly develop acute symptoms of entamelic colitis who had been previously cured, the result of reinfection from contaminated water, food, or entancbe which had found lodgment in the appendix, glands, or submucosa. From what has freen said, the necessity of bearing in mind that the infection may have been latent in cases simulating entamelic colit is becomes obvious, and the stools should be examined for entametae whether or not the patient suffers from diarrhea.

Vecropsy statistics of the disease show conclusively that many persons have suffered from liver abscesses and died from dysentery who were not suspected of having it, owing 10 the constipation and other symptoms complained of.

Classification.-For convenience of study entamelic dysenteric coitis may be subdivided into three types-viz.: (1) mild: (2) moderately severe: and (3) aggravated, any and all of which varieties may insensibly pass into the chronic or characteristic form of the discase.

Mild Type.-In this variety of entamebic colitis the affection comes on insidiously and the health of the patient remains fair, though he appreciates that there is some intestinat disturbance, a loss of appetite, and suffers slighty from diarrhea unaccompanied by tenesmus, the stools showing semisolid or fluid, some mucus, and but little bleorl. In some instances he sulfers from alternating diarrbea and constipation, and does not exhibit the typer stmptom-complex of dyentery for weeksor months (if at all), but, as a rule, the manifestations beeone fairly characteristio in a short time.

Hoderately Serere Type.-This type of entameloce colitis is most common, and is ushered in ly diarthea, aldominal pain, tenesmus. mucus and blood in the stools in varying amomots when the attark appears abruptly, and the patient frecpently complains of chilly sensations, natsea, a ferer wherein the temperature varies Irom $10 \mathrm{I}^{\circ}$ to Iof F., and a slightly increased pulse. The evatedations in the legein- 
ning contain a slight amount of fecal matter, but later become frequent and fluid, and are composed chiefly of mucus and more or less blood, and their passage incites an aggravating tenesmus, and there is an almost incessant desire to empty the bowel, which, when accomplished. affords but slight if any relief. The acute manifestations, when toxemia is marked, persist for two or three days or longer, and may endanger life by bringing about a subnormal temperature and collapse of the patient. or they may abate and he will suffer from the usual dysenteric symptom-complex only. When the disease lasts for a considerable time there may be intermissions and exacerbations of the chief symptoms. but, except in the latent cases referred to, diarrhea prevails, though the stools vary greatly in number and character. In moderately severe cases with an acute beginning the evacuations are thin, have a disagreeable odor, are fairly copious, and vary from five to ten daily for the first few days, after which they become smaller and may either diminish or increase in frequency. Often the patient will pass from ten to thirty stools in twenty-four hours, made up of mucus, blood and necrotic tissue, and exceptionally a fibrous membrane. In the beginning and later, pressure over the colon elicits soreness and the sufferer complains of pain in the sigmoid or other regions and of vesical and rectal tenesmus. Ordinarily there is very little gas distention, but when bleeding is profuse and blood is retained, and when ulcers are present which incite enterospasm, gas backs up and causes considerable discomfort or severe colicky pains. Untreated and improperly treated cases of entamebic colitis gradually or rapidly go from bad to worse, and the patient exhibits evidences of the disease in many ways - riz.. loss of weight (20 to, 30 pounds), furred tongue, stomatitis, inefficient digestion, muddy or sillow complexion, anorexia, anemia, profuse perspiration. headaches, melancholia, muscular weakness, restlessness and disturbed sleep, malnutrition, weak, slightly accelerated pulse, exhausted state and a high $\left(102^{\circ}\right.$ to $104^{\circ} \mathrm{F}$.) or subnormal temperature, depending upon the degree of sepsis incident to the ulceration and mixed (streptococcic) infection, the formation of a liver abscess, or perforation and peritonitis.

In chronic cases diarrhea is frequently intermittent to a degree, but, as a rule, the stools are more uniform than at the onset of the disease, and undigested remnants are to be seen in the dejecta along with the mucus, blood, tissue débris, and pus when ulceration is marked or submucous abscesses have formed. In the arerage case the movements are multiform, and the accompanying tentemus, abdominal discomfort, and indigestion are so distressing that the patient is in a deplorable state and unable to obtain rest night or day.

Aggrazated Type.-The author regards as aggravated cases of entamebic colitis wherein the patient is dangerously ill almost from the beginning incident to the generated toxins, extensive involvement in a. diphtheric or gangrenous process, and where the mucosa and sometimes deeper tunics are destroyed over large areas or throughout entire 
segments of the colon. Malignant dyentery may be severe from the onset, develop upon the basis of a mileler type of infection, or in subjects afflicted with local bowel disturbances destroy the epithelium and make easy the entrance of entanelere, other micro-oreanisms, and their toxins into the deeper structures and blood-and lymphressels, especially in individuals in a run-down condition. Where the infection is virulent, toxins abound, a diphtheric membrane forms. or the inner layer of the bowel becomes gangrenous, it is generally conceded that, in acledition to entameler, other acciclental and obligate micro-organisms or helminths participate actively in the infection, and are partially responsible for the severe manifestations and extensive changes which take place in the gut.

In aggravated cases of entamebic colitis the patient sometimes complains of malaise, gastric disturbances, indigestion, and loss of appetite for two or three days before he becomes seriously ill, but, as a general thing, when the infection is virulent the sufferer begins to complain almost immediately of rigors, followed by fever, thirst, occasionally nausea and romiting, severe colic, marked colonic pain or tenderness on pressure, and diarrhea, wherein he has from ten to fifty passages in twenty-four hours, the first of which are slightly feculent, contain considerable mucus, and may or may not show traces of blood.

As a result of the absorbed toxins or sepsis which ensues the temperature nay be subnormal or rise to $102^{\circ}$ to $104^{\circ} \mathrm{F}$., the patient has a rapid, feeble pulse, dry, shiny skin, is bathed in a clammy perspiration, becomes restless, is sometimes delirious, and may pass into a comatose state or die in two or three days from exhaustion and collapse, or within a week from peritonitis when the bowel is extensively injured or perforated by the diphtheric or gangrenous process, but usually the urgent symptoms partially subside when the subject passes the danger-mark.

In diphtheric and gangrenous entamebic colitis the movements, which run from thirty to forty daily, subsequently diminish to half this number in fatal and cases which becone chronic, and the amount of the discharge, which at first is copious, gradually climinishes, until not more than one to four tablespoonfuls is encountered at a sitting. The stools are always offensive and contain little blood in the beginning, but later the quantity is markedly increased. and it may be a bright red if fresh, or a lark brown color when it has been retained; in other respeets, according to the nature of the case, the dejecta is made up chietly of mucus containing some pus and mucofibrinous casts of the bowel in the presence of a diphtheric inflammation, or necrotic tisste when the intestine is gansrenous.

Abdominal tenderness remains as the aculeness of the attack suldsides, while the terrific griping pains disappear, but the burning in the lower bowel and rectal tenesmus continte to make the patient uncomfortable during and following lefecation.

When sloughing has been extensive, exhatsting diarrhea and tine absorption of toxins sometimes may continute, with the result that the 
patient rapidly loses flesh, strength and power of digestion, and becomes exhausted, or, where the destruction of tissue is less extensive, acute malignant entamebiasis may insensibly pass into the chronic variety.

Chronic Entamebic Colitis (Dysentery).--From what has been said, it may be inferred that the mild, moderately severe, and aggravated types of acute entamebic dysenteric colitis may one and all subside into the chronic variety, which constitutes the form of the disease most frequently encountered in this and tropical countries. Persons afflicted with chronic dysentery complain of about the same manifestations as those having acute entamebic colitis, but they are less severe.

Frequently, in long-standing cases, toxemia and tenesmus are absent, and blood and shreds of tissue disappear from the stools, which are of a dull yellow color, and more homogeneous than the evacwations of acute entamebic dysentery.

Patients having chronic dysentery may go along for weeks or months and have about the same number of daily evacuations (three to ten), or, as the result of dietary indiscretions, violent exercise, taking cold, drinking ice-cold or alcoholic beverages, or without apparent cause, acute exacerbations may arise, and while they last the evacuations increase in number, the dejecta contain a greater amount of mucus, blood, and pus than formerly, and the patient complains bitterly of abdominal pain, soreness over the colon, tenesmus, and a constant desire to stool. Occasionally, in latent cases between acute attacks, the patient may suffer from constipation or costiveness alternating with diarrhea, and the evacuations often contain small scybala and are influenced by the character of food consumed, being more fluid and frequent (containing undigested food remnants under a mixed diet) and frothy when considerable starch enters into the meal. Some patients hold their own between acute exacerbations, but the majority have little if any appetite, digest their food improperly, are septic to a greater or less degree incident to the chronic diarrhea, and become exhausted and greatly emaciated, are afflicted with progressive anemia and have a glistening parchment-like skin, sallow, mucldy complexion, pigmented face, and a listless, careworn appearance. When toxemia is extreme the patient is nervous, has melancholia, feeble pulse, suffers from malaise, is indifferent to social and business affairs, presents a furred tongue, cracked lips, sordes, weak heart, tympanites, and in fatal cases, Brown says, "small quantities of loose and fetid dejecta are passed involuntarily, and of ten almost continuously; prostration greatly increases, and after a short period of unconsciousness death results from paralysis of the cardiac muscle."

Generally in acute and chronic entamebic colitis the movements have an alkaline reaction which favors the multiplication of entameba.

Other Symptoms.-In addition to the manifestations already mentioned patients afflicted with acute and chronic entamebic dysentery may suffer from cyanosis, heart failure, cerebral emboli, neuritis, 
chorea, muscular inco-ordination, paraplegia, inflammation of joints and tendon sheaths, enlarged lymph-nodes, boils, enterospasms, skin affections, thrombosis of the portal win or colonic mesenters. intestinal perforation, peritonitis. adhesions, angulations and thickening or stricture of the ant, jaundice, cholecystitis, bronchitis, hepatitis, pleurisy. edema of feet and ankles, albuminuria. cancrum oris, stomatitis, sastralyia. gastric catarrh. dyspepsia, ptosis, intussusception. perityphlitis, appendicitis, pericolitis, sigmoiditis, proctitis, hemorrhoids, fistulu. fissure. and small or large abscesses in the rectecolonic, sulmumeora. inchiorectal fosse, brain, liver, lung. spleen, hidneys, alveolar and buecal tissues. psoas muscles, and other organs or over the entire body in severe cases, when there is general sepsis and changes in the blood and urine.

\section{COMPLICATIONS}

Entamebic appendicitis frequently complicates entamebic colitis, and the same can be said of perityphlitis. and in such cases Entamahe histolytica are frepuently demonstrable in the appendicular wall or di-charge. Musgrave, in i 50 necropsies (amebic dysentery), observed appendicular lesions in $1+$ cases. in 6 of which the infection occurred from the cecum. In 2 instances the author failed to cure entamebic colitis owing to relapses thought to be due to reinfection from the appendix. for one of these patients was later cured by removing the appendix, and the other ly using it as an irrigating tube (appendicostomy). In the remored appendis entamebe were discovered in the contained gelatinous mucus and in the submucous tis-se. Jelk- has called attention to the frequency of appendicitis in this form of dysentery, and others have reported cases wherein the appendix was involved in a catarrhal inflammation. ulcerated or perforated. Perforation is comparatively rare in spite of the fact that entametic ulcers often involve extensive areas of the gut and penetrate deeply into its structure. In II cases recorded by Musgrave there were onty three perforations, one of which rocurred early.

Abscesses and Fistulæ. - In aggravated chronic cases of entamebic dyentery the mucus membrane is perforated at many points with ulcers which connect with diminutive abserese benteath the mucosa, which in turn communicate with other s through numeroum fistulee. When the pus fails to escape and collect in comsiderable amounts, jt may rupture and be voided by way of the lewel or form diverticula or pericolonic absecses. In the rectum perforation accalsionally occurs and the pus follows the bowel downward. and perianal or ischiorectal alsceries are formed.

Metastatic abscesses are of frecuent eccurrence in the liver. lex. often in the lorain, lungs. spleern, kidneys, and other structures far remote from the original source of infection, and occa-ionally, in the presence of marked sepsis. alsweses become generalized or the patient suffers from furunculosis. While entameleare at times found in thene septic foci. the probabilities are the infection is mixed. and that -uch absceses are due solely or in part $(0)$-treptocored. -taphylococci. 
colon bacilli, or others of the pathogenic and pyogenic intestinal microorganisms. Among the I I 9 cases observed by Musgrave, 22.6 per cent. had liver abscesses, 9 of which ruptured in to the lungs.

Hemorrhage.-Slight, moderate, or profuse bleeding occurs at one time or another in practically all cases of entamebic colitis. Dangerous hemorrhages are rare, and may be the result of the encroachment of a small ulcer upon a large vessel or extensive sloughing and injury to a number of arteries and veins, and it has been shown by Strong that bleeding often occurs coincident with hepatic abscess and that the coagulability of the blood is markedly below par. The bleeding may be caused by a dysenteric or mixed infection, ulcer, or thrombosis of the vessel. A hemorrhage from the colon is indicated by dark-brown or coffee-ground evacuations, and that of the rectum by a small or large amount of bright red blood in the movements.

Skin Affections.-During the acute stage of the disease the skin is dry and glistens, but within forty-eight hours it softens and the patient perspires freely. In the chronic form of the disease, particularly in the presence of extensive intestinal ulceration and marked toxemia, the integument is loose, has a disagreeable odor, assumes a sallow, jaundiced, or muddy, scrawny appearance, and Jelks has observed the skin to become edematous or involved by an erythematous papular or pustular eruption, and investigators, including the author, have known localized or general furunculosis and urticaria to complicate chronic entamebic colitis.

Perforation.- Since in entamebic colitis the destruction of tissue is confined chiefly to the submucosa, perforation is rare, and occurs when ulcers assume a penetrating claracter and when the bowel is distencled. Where leakage takes place, general peritonitis usually ensues and the patient dies quickly, but in farorable cases an abscess may form or the infected material may become blocked in by localized adhesions. Perforation occurred in but 85 of the 580 cases recorded by Beranger and Feraud.

Stenosis, Adhesions, and Angulations.-The sequelce of acute entamebiasis, in so far as they relate to the intestine, are not numerous or annoying, but they are common and seriously impair the functionating power of the bowel where the disease is chronic, inflammation is marked, ulcers are numerous and large, and when extensive sloughing of the gut takes place. Inder such circumstances the colon becomes thick and hard and is often made tortuous through contracting adhesions, which form and clraw it to adjacent structures, causing angulation. Strictures (single or multiple) of the colon and, more particularly, the rectum are quite common, and are induced by the contracting, cicatricial tissue of healed ulcers and sloughs. Ocelusions thus formed may be annular or tubular and cause partial or complete obstruction, with its usual train of symptoms. Occasionally intestinal atrophy is a complication of entamebic colitis, and sometimes an extensively ulcerated bowel heals and exhibits little or no evidence of the disease, but in most instances the bowel is left deformed or its function is 
Submucous, perirectal, and ischiorectal abscesses and fistulce are common, and follow an infection where septic material becomes lodged beneath the mucosa, edge of an ulcer, or is carried to the part through the circulation. Adenomata, papillomata, and sivellings of the mucosa are fairly common and are due more to trauma and bathing of the bowel with the irritating discharge than to the infection.

Hypertrophy of or contraction of the rectal ialies results from the prolonged inflammation or contraction of cicatrized ulcers. Now and then cryptitis and hypertrophy' of the anal papilla are induced by the prolonged irritation.

Rectal ulcers and strictures the result of entamebic colitis have already been discussed.

The sphincter muscle in dysenteric cases may be excited to spasmodic contraction by anal ulcers, or it may become fatigued and remain in a relased state and permit the feces to insoluntarily escape, when the patient suffers from continuous diarrhea and frequent evacuations.

\section{HEPATIC ENTAMEBIC (TROPICAL) ABSCESS}

Liver or so-called tropical abscess constitutes the most common and distressing complication of entamebic colitis, and results from a suppurative inflammation in the liver incited by the entamebre, which gain entrance to the liver through the portal vein or in ways described below. It has been conclusively shown that hard drinkers afflicted with dyentery suffer much more frequently from hepatic abscess than abstainers. The following statistics indicate that the percentage of liver abscesses in patients afflicted with entamebic colitis varies greatly, probably owing to the occupation and habits of the inclividual, exposure, virulence of the infection, climatic conditions, and whether or not the patient is a native of the country where the disease was contracted.

The routes of infection followed by entamebe in their migration to the liver are not positively known, but it is quite probable that they reach the substance of the organ in several ways-riz.: (I) Directly from the intestine by way of the duct; (2) through the portal circulation after having gained entrance to its radicals in the submucosa where the infective process is most active; (3) transperitoneally from the localized lesions in the gut; (4) the general circulation, evidence of which has been the finding of entanrebe in the vena cava and right side of the heart: and (5) lymph-channels.

Since hepatitis and liver abscess are rarely present in the early stages of dysentery, and do not occur until ulcers have formed, it would seem that the blood and lymph circulations are important carriers of entamebe from the bowel to the liver.

While the manifentations of liver abscess have been encountered in the various stages of entamelic lycentery, they rarely appear before the sixth week, and usually the indications of suppuration within the 
organ are not apparent in latent or chronic cares for several monthe or years following infection.

Kartulis observed liver abscess in 55 per cent. (4o0 antop-ies: Zancarol, in 59 per cent. (4+t cases); Hoodward, in 2 I per cent. (36), collected cases); Smith, in st+4 per cent. ( 45 cases); (comojhman and Latleur, in 21 per cent. (1 +29 collected (asts); MIusgrave, in 22.6 per cent. (110) cases): and (raig, in 5 per cent. ( $7+5$ cartes : and also 33 per cent. in a series of is fatal cases of amelic dyentery.

Strangely, and for no explainable reason, women and children suffering from dysentery have liver absess proportionately very much less frectuently than men.

The published records indicate that in from 75 to to per cent. of all cases of tropical (liver) alsscess entemebe Entamel a histolvica are demonstrable in the dejecta, or a history of the patient- having previously had dysentery can be obtained.

Number, Size. and Location of Lizer Abscesses. - Entamebic hepatic abscesses may be single or multiple, and when there is more than one the number may vary any were from two to thirty. Zancarsl found liver abscesses single in 60.2 per cent. (562 Egyptian cases), and Rodgers observed multiple abscesses in 32 per cent. of the patient= treated by him in India.

TABLE SHOWING THE NEMBER AND LOCATION OF ABSCESES IX TWENTY-FOLR FATAL INFECTIONS WITI ENT.AIIEB.A HISTOLYTIC.A OBSERTED BY CR.IIG.

\begin{tabular}{|c|c|c|c|}
\hline $\begin{array}{l}\text { Number of } \\
\text { cases. }\end{array}$ & single. & Multiple. & Number. \\
\hline 1 & & yes & 6 \\
\hline 2 & & yes & 8 \\
\hline 3 & & yes & + \\
\hline 4 & & yes & $\mathrm{I}_{3}$ \\
\hline 5 & & yes & 8 \\
\hline 6 & yes & . & I \\
\hline 7 & yes & & I \\
\hline 8 & & yes: & 2 \\
\hline 0 & yes & & I \\
\hline IO & . & $y e-$ & 17 \\
\hline I I & & yes & $\begin{array}{l}\text { Too numer- } \\
\text { ous to } \\
\text { count. }\end{array}$ \\
\hline I 2 & & yes & 10 \\
\hline 13 & yes & & 1 \\
\hline 14 & & yes & 3 \\
\hline I 5 & & ye- & 5 \\
\hline I 6 & yes & & I \\
\hline 17 & & yes & 3 \\
\hline IS & $y e=$ & $\ldots \ldots$ & I \\
\hline I0 & yes & $\ldots \ldots$ & I \\
\hline 20 & & yes & $\begin{array}{c}\text { Very nu- } \\
\text { merous. }\end{array}$ \\
\hline $2 \mathrm{I}$ & yes & & 1 \\
\hline 22 & $\ldots$ & yes & 30 \\
\hline 23 & yes & $\ldots$ & + \\
\hline 24 & yes & $\ldots \ldots$ & I \\
\hline
\end{tabular}

Right lobe. 5: leil kbe. I Kight lobe. s: leil lobe. o Right lobe, a left kute. I Right lobe. s: leit lobe. 5 Risht lobe. s: leit libe. 0 Richt lobe. o: leit lobe. I Rirht lobe. o: leit hbe. I Rirht lobe. 2 : leit lobe. Lobu- -pigelii kinht lobe. In: letit lobe. I

In both lobes

Risht lobe. 2: leit lobe. 3 Right lobe. I: leit lobe. o Right lobe. 3: leit lobe. o Riaht lobe. : letu lobe. o Riehs lobe. o: lefi hobe. 1 Risht lobe. 3 : leit lobe. o Right lobe. I: leit lobe. o Rirhi lobe. a: leit lobe. o

Risht lobe, all but 2

Risht lole. s: leit lobe. o Risht whe. za: leir lube. Right lobe, a; left lube. o Right lube. I; left lobe. o 
Hepatic abscesses may be microscopic, moderate, or enormous in size, and the pus accumulations may be small in one or considerable in another section of the liver, and the abscesses in different cases may be located in the various lobes of the organ, though the upper right lobe is most frequently involved.

In a series of 639 cases of hepatic abscess collected by Rouis the lesion was located in the right lobe in 17.8 per cent.; in the left lobe, 13.3 per cent.; and in the lobus spigelii in 0.3 per cent. of the cases.

Tropical liver abscesses may remain for a long time or assume enormous proportions without leaving their confines in some instances, while in others they rupture early as the result of coughing or vomiting when small or large, and the pus finds its way into adjacent structures and organs. Most of ten the abscess breaks into the pleura, pericardium, or lung, and less frequently pus is discharged into the peritoneal cavity, inferior vena cava, colon, stomach, kiclney, or lumbar region. Brown says that approximately to per cent. of untreated abscesses burst into the colon and I per cent. into the small bowel.

The following table, arranged by Craig, enables one to form some irlea as to the organs into which liver abscesses most fruquently rupture, and the proportion of cases in which this complication arises:

TABLE ILLUSTRATING THE SITE OF RUPTURL IN AMEBIC ABSCESSES OF THE LIVER.

\begin{tabular}{|c|c|c|c|c|c|c|c|c|c|c|c|}
\hline Observers. & $\begin{array}{l}\text { Cases } \\
\text { of } \\
\text { liver } \\
\text { abscess. }\end{array}$ & $\begin{array}{c}\text { Cases } \\
\text { of } \\
\text { rupture. }\end{array}$ & $\begin{array}{l}\text { Peri- } \\
\text { car- } \\
\text { diun. }\end{array}$ & Pleura. & Lung. & Colon. & $\begin{array}{l}\text { Stom- } \\
\text { ach. }\end{array}$ & $\begin{array}{l}\text { Bile- } \\
\text { ducts. }\end{array}$ & $\begin{array}{l}\text { Vena } \\
\text { cava. }\end{array}$ & Kidney. & $\begin{array}{l}\text { Lum- } \\
\text { bar } \\
\text { region. }\end{array}$ \\
\hline Waring. & 300 & 68 & 14 & 28 & 15 & 2 & I & I & 3 & 2 & 2 \\
\hline Dutroulau. & 06 & 25 & 2 & IO & 7 & I & I & $\ldots$ & $\ldots \ldots$ & $\ldots \ldots$ & 4 \\
\hline Rouis.... & 102 & 54 & I I & 17 & I & 3 & 0 & 2 & & & \\
\hline Haspel ... & 25 & 6 & + & 2 & & & & & & & \\
\hline Camboy. & Io & 3 & & 2 & & & & & & & \\
\hline Howard. & 0 & 5 & 5 & & & & & & & & \\
\hline Craig... & $2 t$ & 7 & 2 & 5 & & & & & & & \\
\hline
\end{tabular}

Histopathologiy of Extanebic Liver Abscess.-The liver is considerably swollen when there is hepatitis, and characterized by diminutive and small or nodulated enlargements when abscesses are forming or pus collections are already present. In the beginning such raised areas are light colored, bloodless, and firm, and where sectioned present a lardaceous appearance and contain a grayish, semisolid fluid matter. The small tumors become encysted or coalesced to form larger swellings, the centers of which degenerate and form abscesses, which, when incised, are found microscopically and macroscopically to contain a semifluid yellowish or chocolate red matter, broken-down liver cells, shreds of necrotic tissue, red blood-corpuscles, scattering pus-cells, and entamebe when the disturlance is due solely to entamebic infection. Where the troulble is due to mixed infection the alsscess cavity is filled with ordinary or greenish-looking pus, in which are to be found entamebæ, streptococci, staphylococci, colon bacilli, and other organ- 
isms concerned in the septic process. In some instances the lischarge is reddish and, according to Brown, has a getatinous consistency and a "liverish" smell; in others and when libe stained it possesses at greenish hue, but when colon bacilli are active it has a decicledly fecal odor.

Hepatic abscesses may remain isolaterl or rupture inte each other through coughing, pressure, or sloughing until one or more anermous cavities are formed within the liver lobe. The retaining wall orntain entamelye and mat be rough as a result of undetached shred or latrer pieces of necrotic lissue, or smooth when the abscess hats existed for a long time. Occasionally the vessels and connective tissue of the liver, which resist the suppurative procese more strongly than the liver substance, are to be found stretcherl partially or completely across small or largé alscess carities. I'sually the walls of moderately small pus cavities are smooth, and those of diminutive abscesses cannot be defined.

The tissue concerned in liver abscess when sectioned and microscopically examined presents a varied appearance in the different stages of the disease. At first there is congestion of the part and an accumulation of white blood-corpuscles, connective-tissue cells, and occasionally entameles, and later, as degeneration takes place in the center of the swedling, necrotic tissue, fluid or semisolid matter, epithelium, white blood-corpuscles, micro-organisms, and lymphoid cells are demonstrable. Newly formed bile channels are often observed, and examination of the periphery of the small abscesses shows that the process commences in the interlobular areas. The wall of these alyscesses is formed by connective tissue considerably infiltrated ly leukocytes and young connective-tissue cells, and entamedx may sometimes be demonstrated within it. The liver cells in the immediate vicinity undergo necrosis, the capillaries are congested, and the bile-duets are often obliterated or encroached upon by rapidly growing connective tissue. In large abscesses which have a rather thick wall, sections show more or less necrotic material at the inner border, while externally fibrous tissue is very marked and resembles granulation tissue, the cells of which are mosty uninuclear. More externally there is a layer of less dense connective tissue, infiltrated with spindle and small round connective-tissue cells. This infiltration varies with the age of the abscess, and when the fibrous wall is very thick the infiluation is not as great as where the fibrous tissue is of more recent formation.

The entamebe found in the alscess wall in the zone of necrosis are generally near the border of the connective-tissue portion of the wall. which is infilerated by small round cells. From this it will be seen that entamebe are seldom found in old abscesses showing litte necrosis and a very thick, dense, filorous wall. They are found most often in mediumsized abscesses, presenting evidences of marked necrosis of the liver tissue (Craig).

Since entamebe are usually demonstrable in the pus or walls of small and large abscess cavities in almost every instance, and since 
mixed infection occurs in only about 50 per cent. of the cases of liver abscess, there can be but little doubt that entameba are the specific cause of this disease, and that later streptococci, staphylococci, colon bacilli, and others of the intestinal pathogenic and pyogenic organisms participate in the infection to the further detriment of the patient's health. In this connection it is interesting to note that a number of cases have been recorded where entamebe have been carried by the blood to other regions than the liver, which became infected and abscesses formed.

Sruptors AxD Diagxosis. - The symptoms and complications are variable in different cases, depending upon the duration, number, and size of the liver abscesses and manifestations of the accompanying colitis. Tropical hepatic abscesses may develop in a few weeks, or take months or years to form and produce definite symptoms, or they may become encysted and remain indefinitely without causing the patient any trouble. In some instances a hepatitis, which eventually results in the formation of an abscess, may occur shortly following entamebic infection of the bowel, months later, or not until the dysentery has been apparently cured. In addition to the usual dysenteric symptoms, the patient gives a history of having suffered from chilly sensations, followed by a temperature of $103^{\circ}$ to $104^{\circ} \mathrm{F}$. The temperature may be normal at one time and high at another, and these patients exhibit the usual indications of sepsis-viz., malaise, headaches, nightsweats, sallow complexion, and lack of desire for work or pleasure. In acldition to tenderness over the colon these sufferers complain of more or less severe pain in or near the liver, particularly in the subcostal region or midaxillary line near the ninth rib. When the abscess is forming pain is frequently of a sharp, shooting, or stabbing character and is felt in the affected liver lobe, but when formed and considerable pus has accumulated it is dull, disseminated throughout the hypochondriac region, and is felt in the back or right shoulder. The tumor causes sensations of fulness, weight and pressure, and is the cause of much discomfort during respiration, when the liver is bound to adjacent structures by adhesions. Naturally, the local and reflex pains induced by multiple are more frequent and severe than those caused by equal sized single abscesses. Discomfort in the epigastric region and disturbed digestion are of ten concomitant symptoms.

Tropical liver abscess patients, owing partially to the local lesions in the bowel, septic condition in the liver, and exhausting diarrhea, appear very ill, are greatly emaciated, have a muddy, unhealthy complexion, flabby skin, muscular weakness, discolored conjunctiva, and suffer from emaciation, nervousness, insomnia and indigestion, exhaustion, interference with the respiration, and occasionally coughing, pleuritis, or pneumonia.

The urinary chlorids and urobilin are increased and urea is diminished in the presence of liver abscess, and albumin may be present in the urine when sepsis is marked and the patient continuously exhibits a high temperature. 
While polymorphonuclear leukocytosis is oceatsionally noticealste in the later stages of the disease, it is not to be relied upon as a diagnostir sign. Brown says the total number of leukocytes is usually high and varies from fifteen to twenty thossand.

When, as the result of chistention or coughing, a liver alsecess ruptures into the lung the patient expectorates greenish or chesolatecolored matter, which contains necrotic tisste, pus-cells, liver substance, entameba, and pyogenic bacteria. Abscesses which hurst into the aldomen induce peritonitis and often cause death; those which open into the periearlium greatly interfere with the heart's action, but when they discharge into the bowel, troublesome manilestations seldom arise. For the treatment of entamebic or tropical liver absecess see Chapter XXXII. 


\section{('IAP'TER XNXI}

\section{ENTAMEBIC COLITIS (ENTAMEBIASIS, ENTAMEBIC DYS- ENTERY), DIARRHEA IN (Continued)}

\section{DIAGNOSIS, PROGNOSIS}

Diagnosis. - In this dass of rases the main thing is to differentiate chtamelie from other forms of colitis, hut, as a rule, lhis can be accomplisherl withoust serions troulde by obtatining a complete history, finding ent wheller or not the pat ient is or hats been living in the tropies, lands or sertions of the worth where entameliatsis is endemic, analyzing the lysenterie symploms, inspecting the bowel through the proctoseope, and eximinims the dejectate

When it is berme in mind that pattents alllicted with bacillary, lochminthie, halanticlic, coccidic, schistosomic, ciliate, or thagellate colitis complatin of manifestations similar to those of entamebic dysenfery, the importance of thoroughly examining the stods for entamebe and ofler sperific argatisms and worms is obvious. The finding of

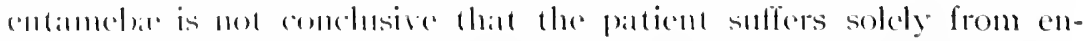
tamelic colitis, hecanse hambess lintamedna coli are often present, and oceasiontully pattents sulfer from a mixed infertion participated in by entamelor, Shiga, Flexner, or ofher bacilli, Balantidium coli, helmimlss, ole., which can be isolated by carefully examining through the

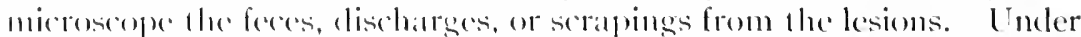

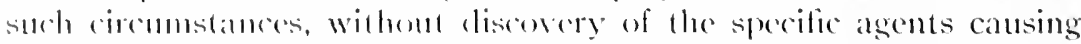
the trouble, a diagmosis is comfusing beanse the symptoms of the dillerent gypes of inferetion are marked or berome blended and difieult to distinguish. Alento is ansily recosnized, hut chronic entamebie dys-

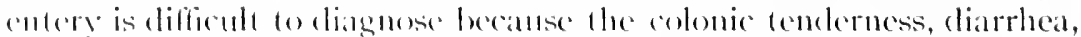

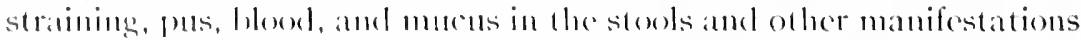
consefuneme 11 pen it maty dosely resemble those induced by chronic

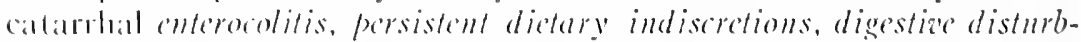
anres, gonortheal, balamtidic, syphilitic, bacillary, or mbercular colitis, stricture, camer, polypi, and intestinal obstrmition, bilharriasis of the rectum, hemorthoids, hemorthages, hedminths, efe., and because the

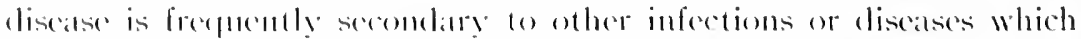

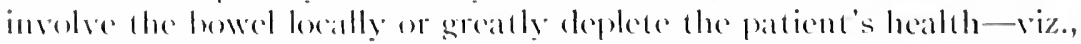
such as typhodel, lyphus, and scarlet ferers, cholera, sepsis, small-pox,

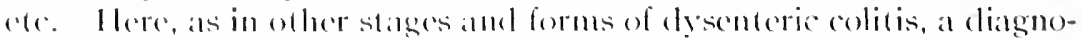

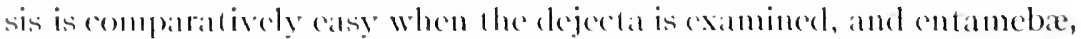
desenterie bacilli, or other specilic agents catusing the infection are isolaterl. 
A microscopic examination of the feces in patients suffering from bowel disturbances who do not suffer from diarrhea or blood and mucus in the stools occasionally demonstrates the presenee of entandede in the intestine and that the subject has a latent form of dysentery.

The lesions of entamebic infection are manifest as numerous large and small ulcers of the mucous membrane which connect with extensive intercommunicating carities beneath the mucosa, and are less seldom seen in the form of membranes or stonghs, while those of the bacillary type, when inspected through the proctesenee, usually show as a catarthat inflammation, superficial erosions or ulcers, diphtheric membranes, or gangrenous sloughs of the mucosa. Finally, contamelic colitis may be clifferentiated from the bacillary form by its nore insidious onser, oceasional latency, absence of early and profonnd toxic manifestations (high fever, rapid cmaciation, elelirium, coma, etc.), greater tendency toward chronicity, and becaluse it occurs less often in epidemic form, is not so frepuenty encountered among the inmates of asylums, barracks, prisons, and poor-houses; constitutional symptoms do not ustally appear in the beginning of the disease and the mortality is highest in the later stages, while in bacillary colitis cleath is most common within three or four days or a week.

Finally, liver alscess, a common complication of entameliasis, never occurs in bacillary dyentery except in the presence of a mixed infection, strictures and sepuelat are very much more freguent in the former than the latter, and antanelic infection is ustally confuned to the colon, while that of the bacillary type more of ten extends well up in the lower ileum.

Examination of the stools is very important and should be practised as a routine measure in all suspected cases of entamebiasis.

By a macroscopic examination one can determine the color, amount, and consistence of the evacuations and whether or not they contain pus, blood, mucus, membranous, and gangrenous tissue, undigested food remnants, foreign bodies, or large worms of the round. tape, and other varieties. but he cannot detect in this way the more minute organisms which cause dysenteric colitis.

A microscopic examination is very much more reliable, and is an imperative diagnostic aid in this class of cases, because when a specimen of feces has been olstained in the right way, properly prepared and mounted, and is carefully inspected through the microscope, a positive diagnosis is possible, since harmless (Entamolya coli) and pathosenic entamebe (Entamoda histolytica), dysenteric hacilli (Shiga, Kruse, Flexner, Park, Duval, Hiss, etc.), diminutive and larger helminths (with their ova), schistosoma, Balantidium coli, ciliates, flagellates, and coccidia, the active alologic factors in the different types of dysenteric colitis, can be detected.

Entamelse are prone to be overlooked when dead, lut when motile they are easy to distinguish, and are interesting to stucly because of the variations in extension of their psendopodia and their method of locomotion when traveling from one point to another. 
The best results are accomplished by examining the feces while fresh and warm in a room comfortably heated and with the slide upon a a arm stage when the life-cycle of the organism is to be studied, and upon an ordinary stage when the finding of entamebe is all that is desired. Preferably, salts should be administered several times previous to the examination to wash the specific agents free of the mucosa, a fluid should be selected over a semisolid specimen, and the mucus should he diluted as occasion demands to keep it from drying and the entameber active as long as may be required. Usually, it is advisable to examine the dejecta of from six to ten stools by the ordinary or drop method, to determine if specific agents are always present, their number, and whether or not they are associated with bacilli or other dysentery-producing micro-organisms. For routine purposes staining is not required because entancbu can be differentiated from red blood-cells by their much larger size (t to $8 \mathrm{~cm}$.), epithelial cells, pseudopodia, and active protoplasmic motion.

Feces admixed with urine and that which has been disinfected or obtained from patients who have been irrigating the bowel with stimulating or astringent agents, or have taken antiseptic medicines, is unfit for microscopic examination, because if entameba are present they will be inactive or dead and difficult to locate. Where the stool is to be brought to the office or hospital it can be kept at a proper temperature by placing it in a Thermos or a small bottle which is dropped into a larger one filled with warm water, but when an immediate examination is to le made the feces may be evacuated into a warm bed-pan.

While it is customary to examine the dejecta for the organisms, entameba are frequently found in the voided thin or gelatinous mucus, scrapings taken from the edges and base of ulcers, and sections removed from the bowel at the site of the lesions, but the author has obtained the best results from specimens obtained by the curet used through the proctoscope.

With care and the aid of the microscope a positive diagnosis can be made in nearly every case, a fact demonstrated by the author's fundings, which are in harmony with those of Hanes, who detected motile entamelae in each of $2+$ cases.

With the exception of the harmless Entamaba coli, other organisms of this class (E. histolytica) are pathogenic and under favorable conditions produce entamebic colitis. In so far as the diagnosis of dysentery is concerned, the differentiation between the various entamebre is uncalled for, but scientifically the one can be distinguished from the other by studying their morplology and methods of reproduction and by observing their motility, since it is claimed that pathogenic are very much more active than the non-pathogenic $E$. coli.

In summing up his conclusions regarding the points of differentiation between the various entamebe, Craig" says, "If in a freshly voided

${ }^{1}$ For further particulars relating to examinations of the stools see Walker, Phil. Med. Sci., August 1,3, 101,3.

${ }_{2}$ The Paratsilic . Imebe of Man, P. I97, I9II. 
specimen of feces we observe ameba showing slugerish motility, no distinction between the ecto- and endoplasm, or a very sieht distinction, and the presence of a nucleus having a well-rletined nuclear membrane and containing much chromatin, wo may diagnose the organism as Entamaba coli; under the same conditions. if we olserve

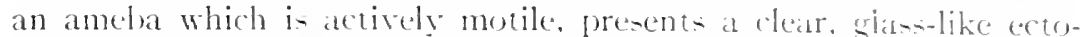
plasm sharply distinguished from the entoplanm, and a nucleus having a well-defined nuclear menlorane and a clear area surrounding the centriole, or a nucleus is absent, or, if present, show no, nuclear membrane and but little chromatin, the diagnosis will be E. histolytica."

When inspected at autopsy or through the simmoidoscope the le-ions of acute and subacute entamebic colitis are fairly characteristic and can be rea lily differentiated from bacillary infection. but in chronic cases acompanied by mixed infection the task in les- eats, because lesions conseguent upon the specific and pathogenic micro-organismof the intestine are somewhat similar in both varietic of dysentery. Entamebic nleers in proces of formation show as elevated reddish dots on the summit of the mucous folds, which later as-ume a grayish or darker hue when on the point of breaking down, or as superficial linear erosions which cross, coalesce, and undergo necrusis to form the classic stellate dyenteric ulcers. In neglected cases the raw areas (ulcers) are variable in shape, size, and depth, have a grayish necrotic base, reddish, raised, undermined edges, and lesions upon the mucosa connect with extensive carities bencath the mucosa. forming collar-button-shaped ulcers. Frequently the cxcarated areas bencath the mucous membrane join to make larger ones, abscesses form, and intercommunicating fistulix connect the one with the other.

Diphtheric membranes and gangrene of the mucesa are occasionally seen, but these destructive proceses complicate bacillary. very much more frequenty than entamebic colitis. When the mucous membrane is involved ly a catarrhal entamebic infection it is extremely. red, swollen, sensitive, at times erlematous, and is besmeared with is thin, clear, or thick gelatinous mucus. When the inflammation is les active and ulcers are numerous. small, or of fair size, the bowel lining is less red and thick and is abundantly covered with mucu- admised with feces, consicierable lolort, tissue lebris. and a slight am unt of pus, but in chronic dyenter $\mathrm{in}$ the presence of exten-ive le-ions. collarbutton uleers. submucous abscesses, and fistulie. the mucou-membrane is constantly bathed in yellow pus or a reddish-tinted discharge, composed largely of pus, blood, and mucus, and is sometime- eorered with a diphtheric-like or fibrinous membrane or unclerexes enengenous changes, and looks black or is to be seen hanging from the buwel in the form of necrotic strips or pieces.

Diagnosis of Liver Abscess. - Knowing the patient has or suffers from entamebic dysentery, in the presence of the alowe symptoms, and after a careful examination, it is comparatively eats un diagnose this condition, but in cases of latent dysentery, and where the als-cess forms 
insidiously and the symptoms of both infections are massed, the presence of suppurative foci in the liver are often extremely difficult or impossible to detect. Usually in these cases the entire liver or some of its lobes are tender, enlarged, and can be felt or seen bulging beyond their normal confines, and when pressed upon cause the patient considerable pain.

Formerly the aspirator was frequently employed, but this method of diagnosis is unscientific and dangerous and should be discarded. Naturally, in patients who suffer from colitis and disturbances within the liver the finding of entamebe in the dejecta points strongly to liver abscess, and more particularly when the patient exhibits the usual manifestations of sepsis.

Empyema, malaria, suppuration within the gall-bladder, kidney lesions, hydatids, subphrenic abscesses, and other diseases of the neighboring organs have at one time or another been mistaken for liver abscess, but with our present knowledge the diagnosis of this condition is in most instances made without much difficulty.

Prognosis.- The prognosis is good in some and unfavorable in other cases, depending upon the virulence of the infection, condition of the patient when attacked, duration of the disease (for it may run an acute, subacute, or chroulic course), and whether or not patients are neglected or ignorantly treated. When properly handled under favorable conditions entamebic colitis rarely ends fatally, but the prognosis is bad when the patient has not had proper care until the disease has almost completely destroyed the colon, invaded the liver, or led to mixed infection, and in persons afflicted with tuberculosis, liver, or other affections which destroy their digestion and vitality.

The author has lost no uncomplicated cases of the disease; hence, with the above-named exceptions, he considers the ultimate prognosis of entamebic dysentery good when the patient is treated according to the plans outlined elsewhere.

It is extremely difficult, however, to give a prognosis as regards the time necessary to effect a cure, because from what has been intimated it may be inferred that in a series of cases it may take days, weeks, months, or longer to completely eradicate the disease and prevent relapses.

Some patients completely cured of dysentery continue to regularly or intermittently suffer from indigestion or diarrhea owing to their nervous state, or the irritahle condition in which the gastro-intestinal tract has been left.

Prognosis of Liver Abscess.--Where the dysentery has been cured or it remains quiescent, and there is but one or two small or a single large liver abscess, patients make a very good or complete recovery, providing the abscesse's are prompty incised and drained. Unfortunately, those afflicted with liver abscess conseguent upon entamebic colitis are usually exhausted as a result of a long-continued diarrhea, loss of hlood, and their septic condition, or suffer from one or more enormous abscesses which have destroyed considerable areas of the 
liver substance and are operated upon with great difficulty. Brown says that from , 30 to 40 per cent. of all cases of postrlysenteric alderess of the liver prove fatal in spite of the modern surgiral procedures, but this mortality is rather high. Numerous instances have been recorded of patients who bave recovered following operations for absersoses which had destroyed the lobes of the liver to a large extent. Mach better results are oblatined when the infection is regarded as dual and the bowed and liver are simulaneonsly areated. The results recently oluaned in the treatment of liver alsecess leats one to hope that the mortality of this infection will be greatly reduced in the future.

While much hangs on the surgical technic employed, still more depends upon the early recognition and operation in this class of cases. 


\section{CHAPTER XXXII}

\section{ENTAMEBIC COLITIS (ENTAMEBIASIS, ENTAMEBIC DYS- ENTERY), DIARRHEA IN (Concluded)}

\section{TREATMENT}

PROPHYLACTIC, MEDICINAL, IRRIGATING, SURGICAL

Treatment.- While considerable progress has been made relative to the etiology, pathology, and method of handling entamebic colitis, thus far no routine treatment effective in all cases has been discovered, and there is no unaninity of opinion as to which are the best therapeutic agents to employ in this chass of cases.

The treatment must be prophylactic, supportive, medical, local, and surgical, according to the indications in at given case, dependent upon the virulence and duration of the infection, degree of bowel involvement, and condition of the patient's general health.

Prophylactic Treatment.-Prophylaxis against entamebic dysentery is important in this country, and more so in countries where the disease is endenic, because it usually results from the ingestion of pathogenic entameba which is avoidable when due precautions are taken. To obviate this type of infection or prevent its spreading when endemic and epidenic, it is necessary-viz., (I) to drink pure bottled or water after it has been properly filtered, boiled, and placed in covered containers free from parasites (when contaninated); (2) consume only vegetables ancl fruits from soils which have not been contaminated through fertilization with or the drainage of human excreta; (3) when bathing in impure water to prevent its entrance into the mouth or the rectum; (4) avoid ices and ice-cream which havor encystment of contained entanebe, which may later infect the bowel, (5) disinfect the dejecta of dysenteric patients; (6) avoid raw and improperly cooked foods, regetables and salads, cold meat, fish, chicken, etc., which have been arelessly handled, placed in unclean dishes, or left uncovered: (7) use care in the handling of these sufferers by isolating them, washing their linen, having them use the same toilet, screening their excreta from flies which would carry the infection elsewhere, and keeping them under observation and treatment for a reasonable time after an apparent cure, because they may be carriers of entamebe and be the means of infecting others.

It has been demonstrated that to a slight degree alcohol and acids minimize the danger of and effects of entamebic infection, hence it is advantagcous for strangers visiting tropical countries to consume in reasonable amounts dilute mineral acids, or Iloch, Moselle, Sauterne, Graves, and like wines with their meals, or, in their absence, whisky or brandy in solla and aërated waters. 
The chief prophylactic measures consist in cleanliness, avoidance of contaminated water and food, moderate exercise in the open air, and the correction of local or general manifestations which lessen resistance and render the subject more liable to infection, and change to a climate where the discase is less prevalent.

Supportive Treatment.-Supportive measures are not so frepuently indicated in acute as they are in chronic entamelic colitis (dysentery) where the patient has heen ill for a long time, the organs inperfectly perform their functions, and he is weak and emaciated. Mild forms of exercise are healthful, of which none is better than wabking, lut violent exercise is contra-indicated at all times, and one should be less active during relapses and exacerlotions. Rest in the house and, il necessary, in bed cluring acute attacks and in the subacute and chronic varieties is necessary when experience has demonstrated that getting up or attempts at work or going about exaggerates the dysenteric symptom-complex, for some patients remain apparently wefl while in bed and have relapses when about. It is also advisable to keep very weak and emaciated patients as quict as possible, particularly when they have heart complications. The author has found that the average individual afflicted with chronic and subacute dysentery improves faster and retains his strength better when he is permitted to exercise moderately in the open air or attend to business (when it is not too arduous) except during exacerbations. Since diarrhea prevails both when the patient is culet or up and about, it is best to keep him out of bed to avoid weakness and improve his mental state.

Dysenteric subjects should be properly clothed and kept warm, and should be warned against exposure, cold bathing, and ice-cold drinks.

Diet constitutes an important part of the treatment in some, but not in all cases. It is imperative that the dict be controlled in acute and during sharp attacks of chronic entamebic colitis, but patients who have suffered long from dysentery are of ten harmed by having them stick to a rigid fluid diet, and the author makes it a practice to allow them as much mixed food as they can properly digest to aroid the malnutrition which accompanies a purely fluid diet. It is advisable to have these patients eat more frequenty, consume less food at each meal than normally, and select nutritions articles of diet which will make up the loss to the body due to their protracted illuess. In very acute dysentery the intestine is violently inflamed, and it is advisalsle to temporarily stop all food and give complete rest to the mucosal unless some nourishment is necessary to maintain the patient s strength. Under such circumstances, and during marked exacerbations of subacute and chronic dysentery, the anthor prescribes fluid mutritive foods and prohibits solid and irritating articles of died or those which leave a coarse fecal residue. While the patient is extremely ill, broths, chicken soup, whey, egg-allnumen, and barley- or rice-water are desirable, but, as acuteness of the attack subsides, milk (p)aint, sour, peptonized, or difuted with barley-water, may be administored in liberal anounts along with a puree of rice, potatoes or beans. extracts 
of beef, or the juice of pressed meat, and occasionally macaroni or gravy. Later, when convalescence is evident, soft-boiled eggs. weak tea or coffee, milk-toast, fresh fish (not shell), rare roast beef and mutton, butter, and easily digested and non-irritating regetables may be gradually adcled to the diet. but berries and fruit should be prohibited until the acute symptoms have completely disappeared.

In chronic dyentery a liberal mixed tiet is indicated, but articles of diet difficult to digest and those which ferment or leave an abundant or coarse residue are contra-indicated, and foods of whatever kind should not be eaten very hot or very cold, because extreme heat and cold stimulate peristalsis. which tends to increased evacuations or induce enterospasm.

In long-standing cases meat should dominate farinaceous food because it is more nutritions. stimulates an active flow of the gastric and intestinal secretions, and thereby minimizes fermentation; but liver, kidneys, sweetbreads, and preserved or salted fish or meat are undesirable. Patients soon become tired of meat unless it is varied or occasionally consumed in connection with carbohydrates. Rare beef and mutton are more easily taken care of than when they are well done. Brown says, "As an auxiliary to a modified meat diet. somatose, a granular tasteless powder which is soluble in water, easily digested, and, for its bulk, highly nutritious, is one of the most useful of the solid peptones: while of the casein preparations the best known are protene, which appears to be practically pure casein, plasmon, in which there is over so per cent. of protein; and sanatogen. which is composed of casein and about 5 per cent. of added glycerophosphate of soda. Apart from their alimentary value-casein. bulk for bulk, is more nutritious than meat protein-these preparations are often of remarkable service in the treatment of the later stages of amebic dysentery, and are specially effective in counteracting fermentation and checking the absorption of intestinal toxins."

The following is a specimen of a modified meat diet suited to the treatment of an average case of chronic amebic dysentery:

7.30 A. M.: Tea, with milk and a little sugar; no bread. An orange, a few grapes, or a pear.

9 A. M.: Two very soft-boiled eggs. Tea or weak coffee and milk: a limited quantity of toast and butter. If eggs cannot be taken. fresh fish may be substituted; and protene or sanatogen may be given instead of tea or coffee. No bacon. ham. or preserved fish is allowed. tion.

II.30 1. M.: Ten ounces of clear soup, with plasmon or some other protein prepara-

I.30 P. M.: Six ounces of underdone roast beef or mutton, minced beef, chicken, rabbit, or calf's head, or game with macaroni. green regetables, or tomatoes. Any stewed or fresh non-carbohydrate fruit in season. Strawberries and rhubarb are specially suitable. Appollinaris or Perrier water.

5 P. 11.: Tea, with a limited quantity of brown bread and butter.

7.30 P. .1.: Clear or Julienne soup; a little dry toast. Fresh fish cooked in any way. but without flour. For convalescents (in addition), a little cold meat, chicken, or game. A pear or other fruit in season. Appollinaris or other table water.

ro.00 P. M.: Ten ounces of hot water.

1. Amebic or 'Tropical Dysentery, p. 225, I9Ir. 
Buttermilk and other sour milks, prepared through the agency of lactic acid bacilli, are palatable and frecuently serviceable alone or in conjunction with other foods in the treatment of dysentery. Alcohol and wines shoukl be prohilited except when stimulation is necessary or the appetite is to be improved.

Medical Treatment.-No specific drug for entamebic dysentery has yet been discovered, though several remedies have heen employed in the treatment of this condition which modify the symptoms, shorten the attacks, and diminish the freenency of relapses, of which ipecactanha and emetin are believed by many to posiess curative properties in this form of colitis. The author's views are in harmony with those of Strong, who holds that entamebiasis (amebiasis) is an affection of the colon, and it is unreasonable to suppose that drugs retain sufficient potentiality after passing the stomach and traversing 20 feet of intentine to permanently destroy all entameba.

The medical treatment should be begun with a liberal dose of castor oil. 3 j to ij (30.0-60.0); calomel, gr. iij to r (0.I 8-0.30); magnesium or sodium sulphate, $5 \mathrm{j}$ to viij $(4.0-30.0)$ : Rochelle salts, $5 \mathrm{j}$ to iv (4.0-I5.0), or a saline mineral water for the purpose of clearing the intlamed or ulcerated intestines of scybala, tissue débris, and irritating discharges. Thereafter curative symptomatic or antiseptic or tonic remedies are prescribed according to the indications in different cases.

In the beginning of very acute dysentery catharsis should be accompanied or followed by the administration of opium in some form to minimize the patient's suffering and diminish the frecunency and fluidity of the movements. Opium is also a valuable arljunct to the treatment in chronic entamebic colitis, for the purpose of preventing restlessness, securing sleep, allaying pain, peristalsis, and tenesmus. particularly in weak, emaciated subjects, and may be administered by mouth, hypodermically, or in the form of a suppository, alone or in conjunction with bismuth or belladonna, hot fomentations, turpentine stupes, and hot irrigations. Norphin, gr. $\frac{1}{4}$ (0.015), hypodermically, gives the quickest relief from all types of acute pain, but opium pills. gr. ${ }_{4}^{1}$ to $\frac{1}{2}(0.015-0.03)$, are more effective for controlling abdominal soreness, peristalsis, pain, and diarrhea, because they soothe and the contained resin acts beneficently upon the irritated gut, but when colic or enterospasm are severe or frequent complications, bellaclonna, gr. $\frac{1}{4}$ to $\frac{1}{2}\left(0.015^{-0.03}\right)$, should be prescribed independently or with opium.

When tenesmus is very severe it can be relieved by hot-water, saline. oil, or medicated enemata or irrigations, or by a 3 - or f-ounce (90120) injection of starch-water, containing laudanum, mxy (1.0), and belladonna, mx (o.6o), or by the insertion of a cocoa-butter suppository composed of morphin or cocain, gr. ${ }_{1}^{1}(0.015)$, and hetlartonna, gr. $\frac{1}{8}$ to $\frac{1}{4}(0.008-0.15)$, ats often as may be required.

Olive oil, liquid paraffin, or petroleum, mineral or hydrocarbon oils (albolin, benzoinal, and neutralol, etc.), administered in . $\mathrm{j}$ to ij (30.060.0) or larger doses once or twice daily, are soothing and sometimes do much toward relieving the dysenteric symptoms. 
Antiseptic remedies, such as bismuth subnitrate, subcarbonate, salicrlate, or subgallate, beta-naphthol, salol, benzosol (beta-guaiacol), saltpeter or thymol, in 10- to I 5-gr. (0.60-1.0) or larger doses, administered two or three times daily, are the most popular remedies of the class employed in the treatment of dysenteric colitis. Acetozone, I : 3000, consumed in liberal amounts, 1 to 2 quarts (I000-2000), as recommended by Musgrave, is useful, because the drink is slightly antiseptic and tends to minimize gastric and intestinal fermentation. While antiseptics possess germiciclal power and inhibit or prevent putrefaction and fermentation to a certain degree, and in some instances add to the patient's comfort, they cannot always be relied upon to lessen the patient's suffering or to cure his dysentery, because they are toxic when administered in doses of sufficient strength to kill the entamebe and bacteria participating in the infection. Bismuth and thymol preparations are most frequently prescribed in this class of cases, but the former sometimes does considerable harm when given in large doses for a long time, because it becomes oxidized and accumulates beneath the edges of ulcers where it cannot be washed out, or forms large bismuth enteroliths or putty-like masses which irritate or block the intestine.

In the tropics, where it could be borne, oil of turpentine, mo 30 to 50 $(2.0-3.3)$, has in some instances given very good results, but is objectionable because of its disagreeable odor and taste, but it possesses germiciclal powers and can be used to advantage with castor oil in cases of chronic dysentery complicated by scybala, constipation, or large collections of jelly-like or stringy mucus.

Astringent remedies, administered alone or in conjunction with opium, assist in controlling hemorrhage, diminishing the number of stools, and making the patient more comfortable, but accomplish nothing toward a permanent cure of dysentery. Of such remedies the author prefers the salicylate of guaiacol, tamalbin, tamigen or tannoguaiaform, tannopin or tamnocol, in doses varying from 5 to io $\mathrm{gr}$. (0.3-0.6), administered three or four times daily, according to indications, or the following combination:

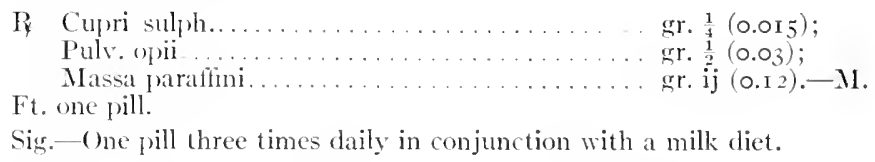

Acids minimize alkalinity of the alimentary tract and thereby hincler the multiplication of entamebs, and with this object in view the author has, with very good results, occasionally prescribed the mineral acids, animal and human gastric juice, champagne, sherry, alcobol, and sour wines, alone or in combination with digestive ferments.

Tonirs, such as strychnin, when the patient is weak and the heart is disturbed, and reliable preparations of iron, when he has lost considerable blood, are sometimes serviceable, but should be discontinued when disliket or they interfere with digestion. 
Ipeciccarh. - For many years ipecac has outranked other drugs in the treatment of entamebic colitis (drsentery), and some prominent authorities mantain that it is almost a specific in this affection, while others, including the author, hold that it has no curative power, but is markedly helpful when employed to guickly diminish the number of stools, bleeding, and tenesmus. The author has frequenty used this remedy according to the plans of those who know most about it, but in his hands it has proved valuable as a palliatice and disappointing as a curatice measure. In his cases it has afforcled great relief in acute, subacute, and chronic entamelic colitis. and in some instances the dysenteric symptom-complex has been partially or completely controlled in two or three days, and occasionally diarrhea, abdominal pain, tenesmus, and blood and mucus in the stools have disappeared for periods varying from a few days to several weeks; but relapses have sooner or later occurred in every case, except when the ipecac treatment was reinforced by intestinal irrigation or tropical applications. In some of the author's cases the number of motile entamebe in the dejecta were apparenty diminished by the administration of ipecac, but in every case they were found in the stools latter. following repeated examinations of the dejecta.

Authorities differ widely as to the modus operandi of ipecacuanha, but no one really knows how its beneficent action is brought about, for this may be accomplished by $(a)$ the direct action of the drug which attenuates or kills the entamebe; $(b)$ changes caused in the in testinal content which discourages multiplication of the parasites; (c) its soothing effect upon the inflamed or ulcerated bowel or in some other way. It is known that this remedy. when administered in small and repeated doses, acts as a tonic to the mucosa, but this would not account for the manner in which the drug diminishes the stools, minimizes bleeding, and lessens tenesmus. When it is known to be effective in a given case, the dysenteric symptom-complex is urgent, and until results are not obtainable from dieting, intestinal irrigation, and other therapeutic measures one is justified in employing ipecac, but under other circumstances it is to be disconntenanced because the drug is difficult to arminister, causes distressing nausea, vomiting or salivation, disturbs digestion, and in other ways interferes with the body. functions.

Usually ipecacuanha is contra-indicated in very acute dyentery in anemic, weak, and emaciated patients, pregnant women, those who have severe heart trouble, romit easily, and in children and persons severely ill from other affections. Authorities differ greatly as to the manner of administering and the dowage of ipecacuanha, but this is not strange, since it cannot be given advantageously in a routine way because of the variations encountered in flifferent cases.

To obtain the best results it is advisable to put the patient in bert. keep him on a milk diet or fast a few hours, ancl clear the bowel with Epsom or Clauber's salts, castor oil, or calomel prior to administration of the drug. In the beginning only moderate or large doses are effect- 
ive, but later, as the diarrhea, abdominal pain, tenesmus, and mucus and blood in the stools lessen, the dose should be gradually diminished. Naturally, the amount prescribed is not so great in moderate as in severe cases, when the author administers $45 \mathrm{gr}$. (3 $\mathrm{gm}$.) the first night and thereafter diminishes the amount $3 \mathrm{gr}$. (O.I $8 \mathrm{gm}$.) daily untit Io $\mathrm{gr} .(0.60 \mathrm{gm}$.) is reached, a dose which is continued daily one or several weeks until the lysenteric manifestations are modified, cease, or the patient cannot longer take the drug on account of nausea and romiting. Better results are obtained when the patient abstains from eating for a time after taking the drug, and limits the amount of solid food consumed during this part of the treatment.

In urgent cases two doses may be administered daily, and in mild ones one every other day will suffice. Ipecac in its natural state is difficult to administer, causes nausea and vomiting, and is often expelled from the stomach before it has accomplished any good, and because of this, and to aroid the necessity of giving an opiate to quiet the stomach, the author has been accustomed to administer it in kreatinized chocolate, salol-covered pills or in gelatin capsules. Nausea and romiting can often be prevented or minimized by keeping the patient quiet in a darkened room, with head lowered, restricting the diet, and applying an ice-bag or mustard plaster over the stomach.

Regarding the effects of ipecac the author's observations agree with those of Brown, who says, "In most instances a copious loose motion of characteristic yellow color and acid reaction is passed within three or four hours, and the patient experiences a marked sense of relicf. Similar discharges, almost entirely free from mucus and blood, are voided during the next two or three days, the intestinal functions gradually become normal, and the patient is soon convalescent."

EuETIx, the chief alkaloidal product of ipecac, first shown by Vedder to be very effective in entamebiasis, seldom if ever causes nausea or vomiting. Entameba which come in contact with it in the bowel and those placed in high dilutions of emetin appear to die at once, and recent experiments with the drug indicate that it is almost if not a specific in entamebic dysentery.

Emetin relieves the dysenteric symptom-complex (diarrhea, abdominal pain, blood, mucus and pus in the stools, and tenesmus) almost immediately without causing other manifestations irrespective of the duration of the disease or virulence of its symptoms. Emetin acts quickly, is more effective, and is more acceptable to patients than ipecac. Following its administration diarrhea frequently ceases within a week, pus, blood, mucus, and entameba temporarily or permanently disappear from the evacuations, and the patient rapidly gains in weight.

Rogers has shown that encetin does not improve bacillary colitis, hence in suspected cases of dysentery, where the patient's condition rapidly improves following its administration, one is justified in inferring that the infection is due to entanebr. The emetin saltshydrochlorid and hydrobromid-are employed in a solution which is 
injected beneath the skin two or more times claily, according to the exigencies of the case. Beginning with $\frac{1}{6} \mathrm{gr}$. (6.01), the dose is increased to $\frac{1}{3}$ or $\frac{1}{2} \mathrm{gr}$. (0.02-0.03), and is continued every few hours until from I to $1 \frac{1}{2} \mathrm{gr}$. (0.06-0.09), which equals from go to i.30 gr. (6.0-8.60) of ipecac, have been taken. Reported cases indicate that this amount of emetin is usually effective in obstinate cases of dysentery. The author has had a limited and fairly satisfactory experience with the remedy, but would advise that the drug be comlinued in small closes for a lew weeks after an apparent cure to forestall a possible relapse.

Local Treatment.-Rest, regulating the diet, and internal achministration of medicine are helpful, but alone or combined they are not nearly so valuable in the treatment of entamebic colitis (dysentery) as IRRIGATIONS and TOPICAL APPLICATIONs which come unchanged into direct contact with the inflamed and ulcerated mucosa. Many times the author has succeeded in curing patients by thorough irrigation who previously had been treated for a long time with other therapeutic measures withont benefit. Medicated irrigation is effective because it insures drainage of the polluted bowel and dislodges, washes ont, attenuates, or destroys entameba, colon bacilli, streptococci, and other pathogenic micro-organisms which participate in specific and later in mixed inlection, which follows denudation of the mucous membrane. This treatment, to be effective, must be employed sufficiently of ten to keep the intestine free from irritating discharges, débris, and feces, and to accomplish this copions irrigations are required and the position of the patient must be changed from time to time during the washing out, so that the fluid is made to come in contact with eiery portion of the infected mucosa throughout the colon and rectum. This irrigating treatment frequently fails because the amount of solution used is too small or does not reach sufficiently high, or the lavage is always carried out with the patient in the same position, which permits the medicated fluid to traverse one side of the bowel only and keeps it from coming in contact with the lesions on the other side.

The beneficent action of bowel washing in these cases is the more to the mechanic action of the fluid in cleansing the ulcers and intlamed gut of irritating material than to its temperature (extreme heat or cold) or the contained drugs.

Patients who have not been benefited by other thropentic measures immediately gain courage upon institution of the irrigating treatment because it brings about immediate partial or complete relief, and they feel as if the seat of their trouble has finally been located and is being treated. Enemata are preferable to no treatment, but continuous irrigation, according to the plans described below, are far superior to them. Frequenty in acute and practically always in chronic entameloic colitis the bowel is highly inflamed and extensively ulecrated, and in consequence the injection and retention of a sufficient amonnt of water or a medicated solution to reach all parts of the infected colon may induce considerable pain, coloptosis, or result in perforation of the intestine and peritonitis; hence the importance of being careful when 
administering large enemata of from 2 to + quarts (liters). When it is desirable to have the solution retained for a time this can be accomplished by inserting a self-retaining anal dilator, through the center of which runs a tube which can be clamped when the desired amount of fluid has been introduced. The injection may be permitted to flow out gently through the pipe when necessary, or be encouraged to come away at once by placing the patient upon a commode and remoring the dilator.

About the only time the author resorts to large and retained enemata is when he has reasons to believe that the ileum is also infected. Enique past experiences in the treatment of specific and other forms of inflammatory and ulcerative lesions of the small intestine, together with photographs (see Fig. I3I) of the small and large bowel, taken by means of the bismuth injection $x$-ray process, has convinced the author that occasionally if not frequently solutions under reasonable pressure can be made to pass along the colon through the ileocecal valve into the ileum. Cole, the New York röntgenologist. has taken a number of pictures for the author which plainly show that the bismuth injection often reaches the small gut when given as an ordinary injection without extra pressure, and this does not imply an incontinence of the ileocecal valve.

Irrigations and enemata should preferably be given at the body temperature or slightly warmer, for then they are soothing and induce the least discomfort, but in the presence of cramps the solution may be employed as hot as $110^{\circ} \mathrm{F}$., because at this temperature it relieves pain and causes relaxation of the intestinal musculature. Ice-cold. irrigations attenuate entamebre in the bowel and beneath the mucosa, but they frequently cause considerable pain, enterospasm, and a desire for their expulsion before they have accomplished their purpose, and, so far as the author has been able to observe, they do not permanently destroy entamebre. Formerly it was believed that long tubes were necessary for complete colonic flushing, but recently the author has confirmed Hanes' findings (see Fig. I27), that in nearly all cases it is impossible to introduce the tube beyond the middle sigmoid flexure. Enemata and irrigations can be made to reach all parts of the large bowel by pouring them through a proctoscope into the sigmoid flexure while the subject is in the inverted posture, by using the author's funnel-shaped proctoscope and pitcher (see Fig. I 24), or permitting the solution to flow into the rectum through a short, soft, hard-rubber, or metal tube while the patient remains in the Sims or lithotomy position with the hips well elevated. When the fluid does not pass rapidly or high enough it can be made to do so by elevating the irrigating container, straightening out kinks in the tube, changing the patient's position, or by masisaging the colon in an upward direction.

Frequently patients complain of severe abdominal pain following injections and irrigations, and this can be avoided, when it is not due to ulcers or distention, by holding the irrigating nozzle upward and letting the fluid run through it until all air is out of the tube before it is 
introduced. When this precaution has not been taken, usuaily the intense suffering of the patient can be promptly relieved by introducing a proctoscope or rubber tube and permitting the air and gas to eseape.

When the bowel is particularty sensitive, it is occasionalty and isable to precede the washing out by the administration of an opiate internally or in the form of a suppository, and when enterespasin is a complication, belladonna should be used in conjunction with it.

In chronic dyentery the bowel should be irrigated once or twice daily, according to indications, for several weeks or montlus or until entamelare disappear from the stools and the patient has apparently been cured, because these specific organisms are 10 be found fres in the bowel and embedded beneath the mucosa, and frequent and prolonged flushing is imperative if the intestine is to be permanently freed of them.

Critically ill and extremely weak patients shouid be recpured to rest quietly in bed cluring and following irrigation, but fairly strong individuals may be permitted to excrcise in the open air or attend to their work following the treatment.

When there is reason for believing that the rectum contains scyluala or softened feces, a small soapsuds or saline enema should be administered to cleanse the intestine before it is irrigated, and much better results are obtained if, when the bowel contains considerable gas, it is permitted to escape through a tube or proctoscope hefore the treatment is begun.

The flow of the solution is less apt to be interrupted by intestinal contractions when it is allowed to run in rapidly than when it is permitted to trickle in: and the inflow must be regulated to meet the indications in different cases. Inder all circumstances it is well to bear in mind that the tolerance of individuals to enemata and irrigations varies greatly, and that one can stand the introduction of a greater amount of solution than another.

The best results are obtainalle with a minimum disturlsance to the patient and the least inconvenience to doctor or nurse when the intestine is irrigated by means of a Cant (see Fig. 158 ) or Kemp) irrigator or a return flow colonic tube of the Jelks or Murray (see Fig. 120) types, and more particularly when from 3 to ro quarts (liters) of the solution are used in the process.

Occasionally, where the bowel is extensively ulcerated and it is desirable to obtain a prolonged application of the medication to the ulcers, it is advisable to block the anus and temporatily retain part of the solution, but in most instances the treatment is most effective when the fluid is permitted to flow directly through and out of the bowel, if during which time precautions are taken to see that it reaches all parts of the infected gut.

When administering enemata or irrigations no attempt should be made to force the proctoscope, sigmoidoscope, irrigator, or colon tube upward when its progress has been arrested, because the bowel may be perforated, the patient is made to suffer unnecessary pain, and the 
treatment will fail, since kinking of the tube prevents the upward flow of the solution. Sometimes this trouble can be overcome by withdrawing and reintroducing the tube and letting the fluid run rapidly, particularly when the opening is in the end and not at the side of the pipe. For further information relative to the technic of irrigating the colon and administering enemata the reader is referred to Chapter XLI.

Irrigants.-Water, saline, disinfecting, astringent, antiseptic, and specific (so-called) solutions of various kinds have been employed in entamebic and other types of dysenteric colitis, with the idea of dislodging, washing out, attenuating, or killing entamebe and other pathogenic organisms within the bowel, reducing inflammation, healing ulcers, lessening toxemia, removing débris and discharges, relieving pain, colic and enterospasm, and stimulating the local and general circulation, but many of those recommended are unsuitable because they are not effective, irritate the bowel, or cause shock.

Of the solutions which have been successfully employed in the treatment of entamebic colitis, the following, named in the order of their usefulness, have given the best results-viz., silver nitrate, ichthyol, thymol, potassium permanganate, protargol, argyrol and acetozone, I : 1000 to 2000 , and Jelks' formalin combination given below. These agents are germicidal to a certain extent, effectively cleanse the bowel, and encourage healing. When diarrhea is marked, bleeding is profuse, and the discharges are abundant the strength of the solution should be increased and afterward diminished as the condition of the patient improves. Ichthyol ( 2 to 5 per cent.) is the most desirable drug to employ when the stools are particularly offensive, and a solution of hydrogen peroxid (Io per cent.) is also useful here because of its soothing, antiseptic, and cleodorizing action, and an irrigation of iodin ( $\frac{1}{2}$ to 1 per cent.) may be employed for the same purpose, but is more irritating to the mucosa.

The bisulphate and other salts of quinin are thought by some to possess a specific action against entamebc (ameba), but the author's experience with the drug does not indicate that this is so, and he believes that the chief benefit derived from quinin solutions are due to their cleansing and healing powers.

The silver preparations should be employed very strong when beginning the treatment, and in cases where the gut is extensively ulcerated the author frequently injects I quart (liter) of water containing $30 \mathrm{gr}$. (2.0) of silver nitrate, and then as improvement follows reduces the amount $5 \mathrm{gr}$. (0.30) each day until Io gr. (0.60) is reached, and continued until a more soothing irrigant will suffice. Silver in the above strength causes a drawing or puckering sensation in the bowel, but seldom induces sharp pain, but when it does, this can be relieved by flushing the bowel with a warm normal saline solution. Argyrol and protargol are useful, but are rarely employed because of their cost. Acetozone and alphozone, when made up in an acid solution, possess decided germicidal powers, but are objectionable, owing to their irritating properties. The author has successfully employed 
Jelks' formalin boric solution, with which he has obtained satisfactory results. This irrigant is composed of 1 : 300 or $t 00$ of the formalin solution (which makes approximately a I : iooo solution of formaldehyd gas), to which he adds a tablespoonful of boric acid for each quart (liter). Jelks has also employed copper phenol-sulphate in conjunetion with the formalin solution because of its partsitic and astringent qualities, and claims that the formalin-boric acid solution is effective because it kills or inhibits entamebe, paramecium, and colon bacilli, the formaldehyd gas is absorbed, permeates the infeeteel foci, and restroys symbiotic bodies, which discourages the production of entameba.

Quite often it is advisable to alternate soothing with the antiseptic, astringent, or stimulating remedies just discussed, because the latter irritates the intestine and favors extension of more than minimization of the infection, and occasionally the author has been compelled to discontinue the usual irrigations and substitute a methylene-blue solution. oils, emulsions, etc., for them, which have a sedative action upon the mucosa and lead to an improvement in the patient's condition because of their tendency to quiet intestinal activity.

The favored soothing remelies employed by the author. named in the order of their usefulness, are crude petroleum, coal oil (refined petroleum), liquiel paraffin, so-called mineral (petroleum), olive and sweet almond oils, alone or in combination with bismuth, thymol, orthoform, iodoform or other sedative, astringent or antiseptic drug. starch, oatmeal or flaxseed tea, slippery elm and boric acid solutions. all of which are more effective when used hot. The following emulsion, with slight variations, has been used by the author with universal success for years to reduce inflammation, heal ulcers, minimize tenesmus, and quiet pain in the large intestine, and may be injected directly into the rectum or through a colostomy, appendicostomy, or cecostomy opening.
R Olive oil.
()j $(500.0)$ :
Orthoform
$5 j-i j(4.8)$
Bismuth sub. nit.
J) $\mathrm{j}(30.0)$. - M.

Sig.-Shake, warm, and inject 3 ounces or more into the bowel and permit it to remain over night.

One inexperienced with the formula woulel expeet it to be expelled in short order, but this never occurs when the emulsion is depesited in the sigmoid or higher, becanse it is frestuently retained for w wentyfour hours or longer and reeluees irritation, arrests peristalsis, lesente diarrhea, and diminishes tenesmus and pain.

The author cannot refrain from commending the cool-oil treatment of Hanes, because it is one of the best remedies we porsess for relieving and curing entamelic dysentery and for healing of her inflammatory and ulcerative lesions of the colon. It acomplishes the elesired results owing to its remarkable parasiticidal, sedative, and healing properties, and because it is absorbable, non-irritating. anel is retained for a considerable time. The best results are derived from coal oil when it is 
warmed and injected in liberal amounts- I quart (liter) or morethrough a proctoscope introduced after the bowel has been cleansed and the patient placed in the inverted posture (see Fig. I 22), which farors the displacement of adjacent organs and intestinal loops, escape of the intestinal gases, and the traversing of the entire colon by the oil. The treatment is still more effective when administered just before going to bed, or by having the patient lie down for a few minutes following its introduction when he is to be active later. The author has many times demonstrated that one or several quarts of coal oil can be projected into the bowel without causing pain or toxic manifestations. In fact, patients almost invariably feel better following the treatment.

Laudanum, mxx (1.3). or an opiate in some form may be added with advantage to starch-or rice-water or other injections when the patient has an extremely sensitive bowel and suffer intensely from pain and tenesmus.

The symptoms of entamehic colitis can often be modified or a cure effected more quickly when dieting. medication, and irrigation are reinforced by topical applications made to ulcers and the inflamed mucosa. Through the proctoscope and sigmoidoscope one can readily locate and treat lesions requiring special attention, and apply remedies to the bowel upward to the middle sigmoid, which assist in healing the ulcers and reducing intestinal irritability, pain, tenesmus, and diarrhea. Lsually one treatment daily is sufficient, and not more than two should ever be made. because frequent instrumentation may do more harm than good, particularly when it is done ignorantly or carelessly. Spraying the bowel with oil and alkaline soothing, antiseptic, and astringent preparations similar to the composition of those employed in the treatment of throat troubles affords considerable relief in the presence of acute and chronic sigmoidoproctitis and when there are erosions in the mucous membrane. Insufflation of the rectum with boric acid, antiseptic, astringent, or Rosenberg's powder, or the author's formula are useful in the same class of cases, viz.:

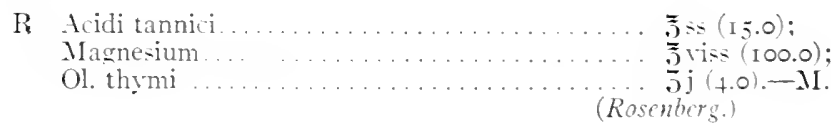

R Talcum pulv.

Hydrarg. chlor. mit.

Zinci stearati Acidi lannici;

jij $(8.0)$;

$5 j(4.0):$

āā $\overline{\text { jij }}(8.0) \cdot \mathrm{M}$.

Sig.-Insufflate through the sigmoidosope.

$\cdot($ Gunt. $)$

Prior to the making of topical applications, ulcers should be isolated after the bowel has first been cleansed of discharges and feces by wiping or flushing it.

Extensive and long-standing ulcers which do not respond to milder measures should be cauterized with the electric or Paquelin cautery or chemical agents. the most reliable and safest of which are 
silver nitrate or copper sulphate (5o per cent.) and pure ich thyol or the balsam of Peru. When ulcers are mumerous and of recent origin, they can be made to heal quickly by painting them three times weekly with a solution of silver nitrate, 10 to $1.5 \mathrm{per}$ cent.; ichthyol, 2.510 .50 per cent.; halsam of l'eru, 50 per cent.; protargol or argyrol, 20 per cent., and then irrigate the bowel on alternate days.

Frepuently dysenteric and other lesions of the rectum are extremely irritable, and the application of the above stimulating drugs aggravates them, under which circumstances they should be discontinued for soothing remedies, the most mniversally successful of which are methylene-blue, io per cent., and emulsions composed of oil, ( )j (500.0), which is soothing, bismuth subnitrate, zss ( 15.0 ), which covers and protects the lesions, and analgen or orthoform, $5 \mathrm{j}$ (4.0), which minimize intestinal irritability and pain. In making topical applications it is advisable to treat the lighest ulcer first and then gradually work downward toward the anus.

Dysenteric ulcers are always most numerous and large in the lower sigmoid and rectum, and since they usually respond readily to local treatment the importance of topical applications in this class of cases is apparent.

Treatments made to lesions at or near the anus cause considerable pain, and sometimes the upper rectum is very sensitive; consequently. when necessary, it is advisable to spray the ulcers with or apply a io per cent. cocain or eucain solution to them hefore the treatments are made. Care should be used to limit the caustic applications to the lesions, because when they cover large areas of the mucosa dangerous or fatal poisoning or sloughing of the mucosa may ensue.

Surgical Treatment.-Entamebic colitis becomes a surgical disease immediatcly in acute cases where the patient is profonndly poisoned, loses a dangerous amount of blool, or suffers incessantly from diarrhea and it is obvous that other measures will not bring prompt relief, and in subacute and chronic cases, where he does not respond to rest, dieting, internal medication, and irrigation from below. The author has had a large experience in the treatment of all forms of dysenteric colitis and with the various surgical procedures employed for the purpose, and his experience warrants the opinion that surgery is the most reliable therapentic measure suggested for the cure of this affection in the vast majority if not in all cases. Since clrainage is the essential feature in the treatment of entamebic colitis, is it not reasonable to maintain that operations which provicle a means of throughand-through irrigation will shorten the convalescence in all instances and frepuently effect a permanent cure when other measures have been faithfully tried or failed?

Since the appendix and cecum frefuently, if not always, contain nests of entamebae, it is obvious that these regions must be reached by the irrigant, and the specific organisms free in the bowel and embededed in the submucosa destroyed and re-infection of the gut below prevented. 
The author first attempts to accomplish this by irrigation. carried out by way of the amus, and frequent changes in the patient's posture; but. When for any reason. all of the infected areas are not reached by the solution. the patient fails to improve, and suffers frequently from relapese. he prompty operates and makes an inlet (appendicostomy ur cecostomy at the head of the colon. so that it can be frequently and thoroughly cleansed from one end to the other. and in addition, appendectomy is performed when indicated. The author has never failed to benefit dyenteric colitis by operation, but in four or five in-tances patients have had relapse- extending over a period of from three months to two rears before they were cured, and in one in-tance the operation appendicostomy utterly failed. In these cases. where the sursical treatment proved unsatisfactory. the entamebic infection axtended well into the ileum supposedly from the srmptons- and cure- which subequenty followed the author's enterocecostomy. or was complicated by balantidic or helminthic infection or some other debilitating disease.

Vaturally patitnt-who are operated upon when they are extremely weak and emaciated. and whose digestive organs are greatly impaired. convale-ce very much slower than persons who have been ill only a short time and are comparatively robus. The establishment of an artificial inlet in the upper colon is of the greatest importance to the patient. both because the treatment rapidly improses his condition and he is independent of doctor and nurse, since he can effectively irrigate the bowel thereafter without their assistance.

Operations of this clas immediately influence the bowel in some way probably through the mind, for very often the diarrhea is greatly improved before flushing is besun. Following appendicostomy', the author"s enterocecostomy and cecostomy the number of eracuations become rapidy fewer, and the manifestations of toxemia begin to improve as soon as through-and-through irrigation is instituted. In aggrarated cases the number of evacuations (twenty to thirty daily) are lesened by one-half. and the amount of pus and blood passed are very greatly decreased by the first weth and not infrequently at the end of the second abdominal pain and tene-mus cease. there is a slight amount of blood and pus in the dejecta, and the patient does not have more than two or three morements daily. Lsually the diarthea is cured and entameba completely disappear from the stools within a month or sis weeks: but since relapses have occurred when the artificial opening- were closed ton soon, the author now leaves them open for at least six month-. and all the time when the patient lives in tropical countries where entamebic colitis is endemic.

$A=$ a rule. when marked improrement does not follow appendicostomy or cecostomy and through-and-through irrigation. it is because all side of the howel are not reached by the fluid, the disease extends to the small gut, digertion is impaired, tuberculosis of the lung or bowel is a complication. or the entamebic is aggravated by balantidic, helminthic, or other specific infection or distase which delays healing of 
the bowel or depletes the patient's health. Uncler such circumstances the position of the patient should be frequently changed during the flushing, or the local treatment should be reinforced hy therapeutic measures which will correct the associated ailments.

To a certain extent these operations improve the diurlecic condition by the arrest of peristalsis through the cecal suspension. Appendicostomy, cecostomy, and bowel-washing improve but do not cure patients where the intestine has been extensively involved by sloughing or ulceration which has resulted in partial or complete stricture at one or more points, for in the presence of stenoses, diarrhea, pus and blood in the stools, and straining continue.

When establishing an artificial opening to provide for drainage, except when the appendix is used, appendectomy is advisable, for when the organ contains entamebe and is not removed reinfection from this source is sure to follow.

Cecostomy and appendicostomy are practically devoid of danger except in cases which are already moribund, and because of this one should not hesitate to operate early, particularly when other measures have proved ineffective.

The irrigants and sedative remedies employed following these operations are the same as those recommended for entamelic colitis, where the solution is introduced through the anus. With an artificial inlet at the head of the large gut and a perforated self-retaining anal dilator in place one can practice through-and-through colonic irrigation until the intestine is completely freed of irritating gas, toxins, discharges. and feces, and the lesions are left perfectly clean by using, if necessary, several quarts of the fluid. In this way stronger solutions can be used, and less discomfort accompanies or follows the irrigation than when the flushing is clone from below, because the irrigant passes directly through the gut and is not retained to cause distention.

The following surgical procedures, named in the order of their usefulness, have been employed with more or less frequency in the treatment of entamebic and other forms of colitis:

(I) Cant's enterocecostomy, which provides a means of simultaneously or separately irrigating the colon and small intestine.

(2) Cecostomy.

(3) Appendicostomy.

(4) Appendicocecostomy.

(5) Exclusion (colonic).

(6) Enterostomy.

(7) Colostony:

(8) Resection and amputation.

The technic and criticism of and indications for these operations in the treatment of dysentery and other inflammatory and ulcerative lesions of the intestine which cause diarrhea will be fully (liscussed in Chapters XLVIII-LI, devoted to the Surgical Treatment of biarrheal, Inflammatory, and Parasitic Diseases of the Gastro-intestinal Tract, and require no further elucidation here. 
Treatment of Tropical or Entamebic Hepatic (Liver) Abscesses. - Liver abscesses arising from entamebic (dysenteric) colitis, discussed elsewhere, may be multiple, small or single, and large, but the former are more often complicated by general sepsis, and are not amenable in most instances to surgical treatment.

Tropical abscesses (caused by Entamoba histolytica) are a common complication of entamebic colitis (dysentery) in all stages of the disease, and have in a few instances been observed in connection with bacillary colitis. Tropical abscesses are usually single and large, but may be multiple and small. In more than 50 per cent. of cases they are located in the right liver lobe. They vary from a small lemon to a large apple size, are oroid in form, fluctuating, and contain a reddish or green tinted fluid, blood-clots, degenerated liver tissue, pus or gangrenous tissue, according to the acuteness and virulence of the infection.

Having discussed the etiology, pathology, diagnosis, and prognosis of tropical liver abscesses in connection with entamebic dysentery (see pp. $364-369)$, the author will now point out the best methods of handling them.

Surgical Treatment.-Some surgeons aspirate liver abscesses, but this is a pernicious practice, and, except for diagnostic purposes, should be abandoned in favor of radical operations, which enable the surgeon to explore, clean, and drain the abscess and determine if there are other infected foci.

In exceptional cases where the abscess bulges the skin and deeper structures 11pward, it should be incised, evacuated, and drained, using a large tube.

High abscesses are most easily reached by removing portions of the ninth and tenth ribs posterior to the midaxillary line, those farther back by a cut along the posterior line of the scapula, and those situated lower lown by a vertical incision through the right rectus.

When the organ has been exposed the liver tissue is bored through with suitable forceps until the abscess cavity has been entered, following which a fairly tight-fitting drainage-tube is inserted and packed about with gauze. Finally, the wound is closed at each end. 


\section{CHAPTER XXYIII}

\section{BACILLARY COLITIS (BACILLARY DYSENTERY, ASYLUM DYSENTERY), DIARRHEA IN}

\section{HISTORY, ETIOLOGY}

\section{HISTORY}

BACILLARY colitis (dysentery) was first described superficially by Chantniss and IVidal, but it remained for Shiga $\left.(\mathrm{I} s)_{7}\right)$ to discover and identify the organism (Bacillus dysenteria. Figs. 69, 70) responsible for the loose and bloody -tools-viz., a bacillus of marked pathogenic properties which morphologically resembles the colon bacillus, but differs from it in that it fails of motility, lack-gas-producing qualities from fermenting sugars, is more pathogenic, posiesses a higher degree of irritability for the mucusi, and is not the same in its agglutinating

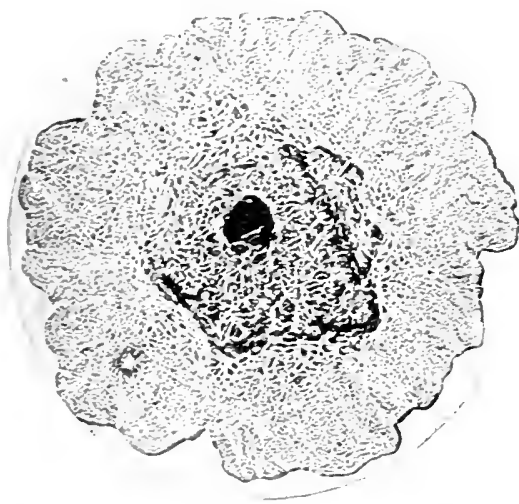

Fig. 69.-Bacillus dysenterix. Colony on gelatin, four days; $\times 20$. (Inerr.)

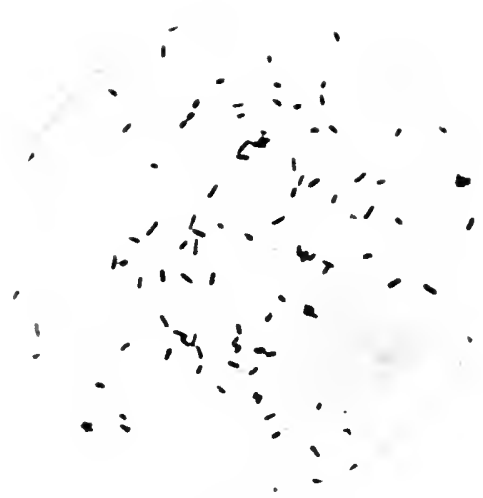

Fir. 7o.-Bacillus dysenterix fromagar culture. Fuchsin stain. Lettnow prep. (Kolle and Wassermann.)

characteristics. This pathogenic agent bears the name shiga and was invariably found by its discoverer in the stools or superficial layers of the mucous membrane of persons afflicted with epidemic dyontery when the examination was made during the crises: hut shiga, in a number of instances. was unable to demomatrate the presence of his bacillus in the dejecta of healthy individuals and thwe afflicted with other gastro-intestinal affections.

Flexner and Strong, two vears later (1)00). While investigating dysentery in the Philippines, discovered bacilli some of which corre- 
sponded to the Shiga bacillus and others which differed materially from it. Shorty thereafter Kruse had a similar experience while studying dyentery among asylum patients: that is, he encountered the true Shiga organism and bacilli which resembled it from a culture point of view, but differed in their agglutinating and other distinguishing features.

IVithin the next three years Dural and Bassett. Park and Dunham, and Hiss and Rus-ell discovered bacilli which they believed to be an etiologic factor in dyentery which differed in one way or another from the organism originally described by Shiga. These and other investigator- conceded the individuality and pathogenicity of Shiga's bacillus in some epidemics of dyentery. but found that in others a different organism independently caused the disease.

While investigating a dysenteric epidemic at Seal Harbor, Maine, and other widely separated places. Park and Dunham discovered a bacillus somewhat like Shiga's, but which differed in its agglutinating characteristics and produced indol in a peptone solution. Dural and Bassett, while examining the dejecta of patients afflicted with summer diarrhea, found an organism which ther thought was that of Shiga, but which they later found to belong to the Flexner type.

After thoroughly investigating epidemic dyentery in Europe, Martini and Lentz made a report (I902) which supported the claim of Shiga in that his bacillus was the chief etiologic factor in true dysentery and does not ferment mannite.

They also pointed out that the Flexner. Strong. Kruse. Park. Duval, Hiss and Russell, and other bacilli differed from the Shiga organism in their glutins and power to ferment mannite, which the Shiga bacillus lacks. Vaillard and Dopter maintain that in France domestic dysentery (Dysenterice nostras) is always of bacillary origin. and that the serum of such patients agglutinates the cultures of the Shiga and Flexner organisms, but that this does not obtain in the amebic form of the disease, and sugrest that this, like Widal's method in typhoid, be employed to differentiate between the bacillary and amebic forms of dyenteric colitis.

The Germans claim that true dysentery is caused by the Shiga bacillus, while Americans maintain that it may be induced by either this, the Flexner-Philippine, or other types of bacilli. In order to establish the causative relations of the different forms of bacilli to dysentery Park thoroughly investigated several acute epidemics of the disease that occurred in lew lork City, Sheepshead Bay. Seal Harbor, Me.. Orange. I. I.. and at Riker's Island penitentiary. In all cases the patients suffered from typic dysenteric stools, but in some instances the disease was more serere than in others. These investigations demonstrated that in some places the disease was calsed by the Shiga type and in others by bacilli of the Flexner-Philippine variety.

The Shiga organism was usually encountered when the disease was serere and the mortality high. but was absent in cases of diarrhea except in the presence of typic dysenteric symptoms, while bacilli 
of the Flexner variety were discovered only in the dejecta of patients who suffered from the milder forms of dysentery (colitis).

In discussing the relationship of the mannite-fermenting varieties to dysentery (lexner, Strong, Kruse, Park, etc.) Park says: "The cultures isolated by us from over to cases were found to fall bargely into two distinct types, one of which differs from the Shiga hacilhus more radically than the other. The variety nearest to the Shiga bacillus has the characteristics of the culture which was isolated by us at beal Harbor, Me., in August, 1902, and the other variety is represented by the Flexner-Philippine type.

"The first type differs from the Shiga bacillus in its agghtinating characteristies, and in that it produces considerable indol in a peptone solution and ferments mannite with the production of acids. The second type differs in these points, and, in addition, in its agglutinating characteristics and in fermenting chemically maltose in peptone solution."

Abe isolated from the stools of dysenteric patients in Japan an organism which was neither a known bacillus or entameba which he consiclered the cause of the trouble. These bacilli were more or less agglutinated by the blood-serum of patients affected with dysentery down to a solution of I : 10,000, which did not obtain with the colon bacillus. Morphologically, these bacilli resembled the colon group and had a lively motion, formed gas in grape-sugar media, and coagulated milk. Animal experimentation showed the organisms to be weakly pathogenic for rabbits, guinea-pigs, mice, and sparrows when applied subcutaneously and within the peritonemm, and non-pathogenic when administered by mouth.

Bacillary colitis (bacillary dysentery) in some instances is probably due to a mixed infection, since the Shiga and other varieties of dysenteric bacilli have been found associated in the same case a number of times, and these organisms have been discovered along with entamebe and other parasites in the dejecta of patients afflicted with the disease.

From the foregoing it would seem that we are justified in assuming that dysentery may be caused by bacill of the Shiga, Flexner, Kruse. Strong, Hiss, Duval, or Park varieties and possibly their strains. Recent investigations indicate that there are still other organisms concermed in the production of summer diarrhea and other inflammatory and ulcerative lesions of the bowel which will in all probability be isolated in the near future. Nodoubt in many instances the bowel clisturbance results from or is aggravated by the above-mentioned dyenteric bacilli, colon bacilli, streptococci, staphylococci, and other organisms.

According to Park, there are three varieties of bacilli directly concerned in the production of dysentery: (a) the non-mannite group; (b) the mannite-forming gromp (but not maltose); and $(c)$ the variet which ferments both mannite and maltose.

Kruse (I900) suggested that bacillary dyentery should be grouped under two headings: (I) True dysentery, caused hy the organisms of the Shiga-Kruse variety, and (2) Pseudodysentery, incluced by bacilli 
of the Flexner, Strong. Park. Dural. Hiss, etc. types; but this arrangement has not been generally accepted. probably because it has been demonstrated that the latter or mannite-termenting varieties of locilli are unque-tionably the specific agents of epidemic and endemic dyentery in a fair proportion of cases.

Kruse =elected the term psendodysentery because the disease associated with the Flexner. Strong, Park. Hiss, and Dural bacilli is encountered less often in great epidemics and is rery much less malignant than dysentery consequent upon the shiga-Kruse organism. He admits, however, that his pseudodyentery may occasionally assume an equally grave character. both in epidemic and individual cases, under which circumstances all the clinical signs of the true disease are present.

Park prefers to restrict the name dysentery to the disease caused by bacilli of the shiga class, and to apply the term paradysentery to dysenteries induced by the other varieties which more closely resemble the colon group of bacilli. in that they produce indol and have a greater range of activity in fermenting carbohydrates. but his arrangement is not altusether satisfactory.

Since the different bacilli concerned in the production of the disease posess individual characteristics. would it not be better to group them all under one heading. dysenteric or dysentery) bacilli, and then identify them in different cases where they are the inciting cause?

With this under-tanding there would be less confusion, and dysentery would fall under two chief classes-riz.. entumebic and bacillaryform - f colitis ea-ily distinguished from those induced by Balantidium coli, coccidia. flasellata, ciliata, etc.

Classification of Bacilli Concerned in Bacillary Colitis (Dysentery). - Shiga ha-formulated five types of dysenteric bacilli-( I) splits only dextrose Shiga : 2 splits dextrose and mannite: (3) splits dextrose, mannite, and saccharose: $\mid 4$ splits dextrose, mannite, saccharose. and malto-e: 5 behave- like + ' except during its growth in mannitelitmus-peptone and water the original acid reaction later becomes alkaline. He does not agree with Lentz that these organisms can be differentiated exclusively upon their mannite-forming characteristics, nor doe- he believe that any of them should be designated as psendodysentery bacilli, as compared with trut dysenteric bacilli, according to the plan of Kruse.

Marceau claims to have discovered two new varieties of bacilli. one motile, the other immotile. which belong to the aërobic type, and are characterized by the ahsence of acid and gas formation, which he belieres to hare a cau-al relation to dysentery.

Lpon the basis of our present hnowledge relative to the organisms concerned in the caustion of bacillary dyenteric colitis, it is impossible to clasify them in a suti-factory manner because they differ in their mannite-fermenting, chenical, agglutinating, and other characteristics, and because new organisms believed to have a causal relation to the distate are almos constantly being added to the list. 
In a general way so-called dyenteric bacilli can be gromped under two headings, viz.:

(1) Non-mannite fermenting, as represented by the Shiga bacillus; (2) mannite fermenting, typified by the Flexner-l'hiliprine bacillus and related strains as represented by the organisms of Strong, l'ark, Hiss (Y), Duval, Abe, Dopter, Cierswalel, Morcul, Deycken, etc. Calmetti and Maggiora have also encountered the Betcillus pyocyaneus both in dysentery and infantile diarrhea. Dunham has described a minute microegcens which he isolated from the blond, liver, spleen, kidney, and bile in several cases of asylum dysentery, and Manson says there are grounds for supposing that this organism mat prove to be responsible for at least one variety of the discase occurring in these institutions.

Shiga bacilli are more often concerned in great epidemics in asylums and elsewhere than the lexner variety, which is more of ten enconntered in endemic dysenteric colitis; but both possess fullities which permit them for a long time to withstand variable temperatures and other tests, or to be transported from one place to another by carriers and to canse colitis under favorable conditions.

As a rule, a single organism is the cause of the trouble in the same epidemic, though the type is prone to differ in other outbreaks of the disease; but cases have been recorded where two varielies of bacilli, or one and contamelic, have heen encountered in the same individual, which would indicate that a mixed specific infection had taken place. No doubt the lesions inaugurated by dysenteric bacilli are materially furthered by bacteria, coli commune, streptococei, staphylococci, paratyphoid, and other accidental and obligate micro-organisms within the bowel; microbes which are also capable of preparing the field for specific agents of dysenteric colitis.

Most investigators concede that the bowel lesions and manifestations consequent upon infection by Shiga bacillus are more serere than those caused by the Flexner and related organisms.

When it is desirable from a clinical standpoint to indicate the difference in malignancy between the two types of infection, this could be done by designating the former (Shiga type) as bacillary dysenteric colitis, and the latter (Flexner and related types) bacilleny dysenteroid colitis (author).

The lifferent characteristics of dysenteric bacilli and allied groups of micro-organisms have been concisely given on p) 3os. (After Manson $\left.{ }^{1}\right)$.

In addition to the culture-media and other tests shown in the table, the effects of Cram's stain, bile salt loroth, mannite nutrose broth, raffnose nutrose nedium, salicin nutrose medium, calfein medinm, and agglutination with enteric fever serum were atso tried. The effects were as follows: Gram's stain. "decolorizerl" with all nine bacilli. Bile salt broth, "acid, no gas," for the first eight bacilli; "acid and gas" for the Bacillus coli communis. Mennite mutrose broth,

\footnotetext{
1 Tropical Diseases, $1907, \mathrm{Pp})+3 \mathrm{~S}, 430$.
} 


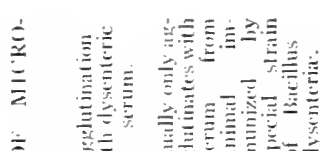

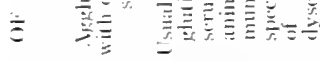

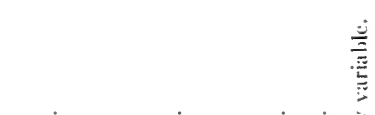

产

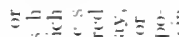

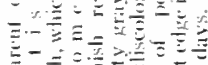

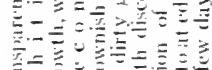

产

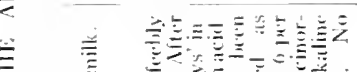

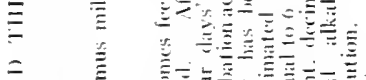

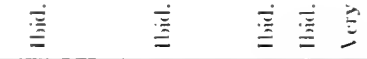
言 言 容

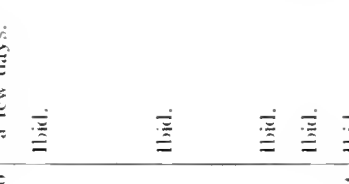

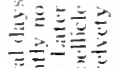 \\ 要要}

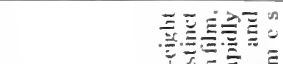

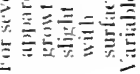

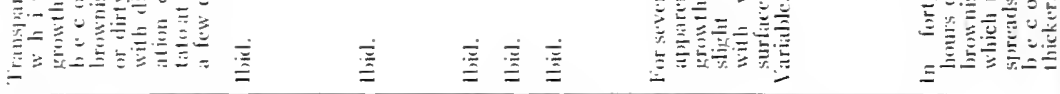

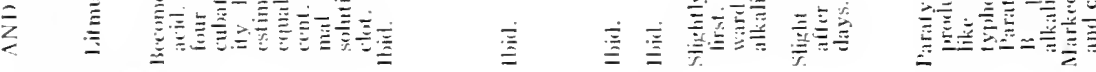

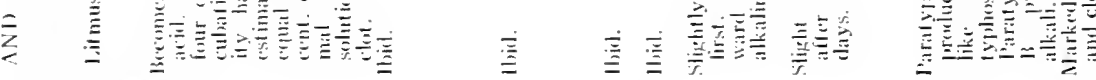

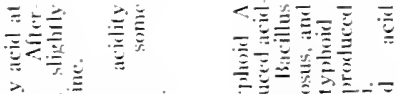

$\stackrel{=}{=}$

$\overline{\underline{E}}$

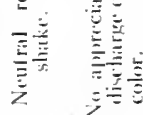

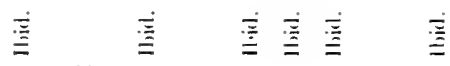

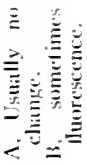

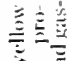

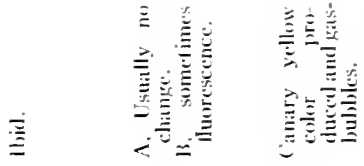

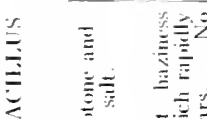

$\cong$

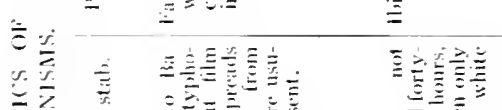

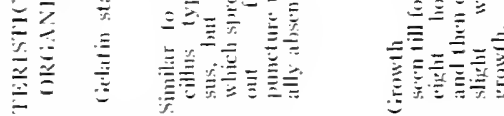

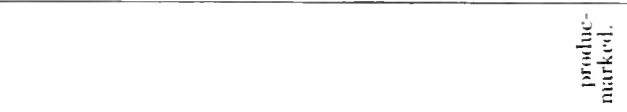

竞

言

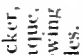
童至

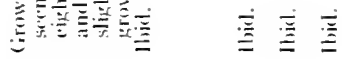

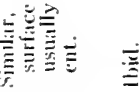

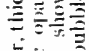

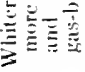

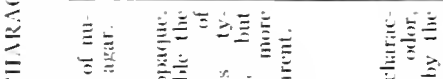

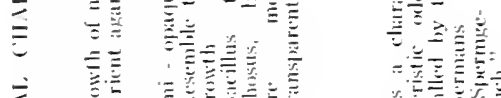

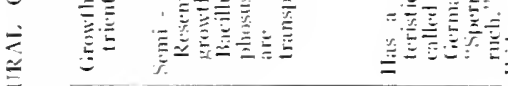

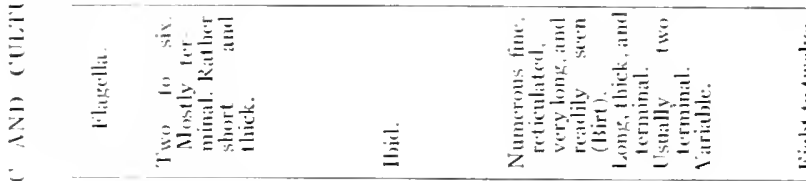

3
0
0
0

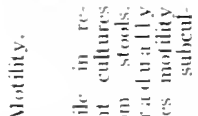

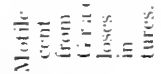

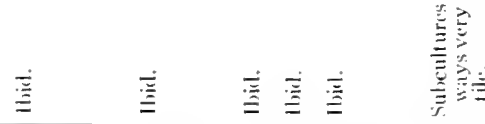

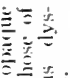

尊尊

寅

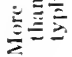

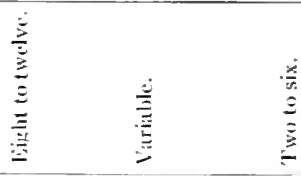

章

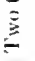

$\div 3$

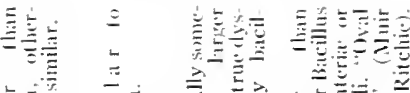

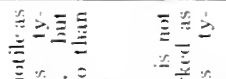

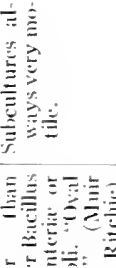

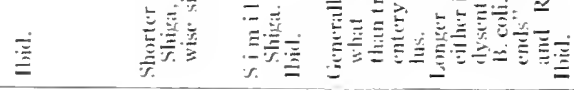

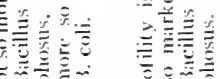

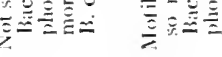

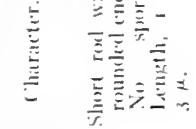

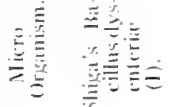

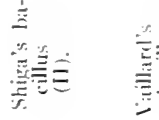

焉

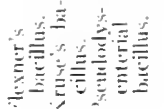

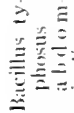

$2=$
$z=$

鴊

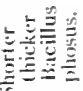


"unchanged" for the first five bacilli; "groweth and acid" for the pseudodyenterial bacillus; "acid" for the last three hateilli. Raffrnose nutrose medium and also salicin nutrose medinm. "aciel and grom th" for the pseudodysenterial bacillus: "nuchanged" for the other eight bacilli. Caffein medium. "growth" with the Bacillus typhosus ahedomimalis and the paratyphoid hacilli; "no growth" with the other seren bacilli. On testing with enteric ferer serm the agghtination result was "marked" with the Bacillus typhesus abdominalis and "nil" with the other eight bacilli. 


\section{CHAPTER IXXIV}

\section{BACILLARY COLITIS BACILLARY DYSENTERY, ASYLUM DYSENTERY) DIARRHEA IN Continued)}

\section{PATHOLOGY}

BACILLARY colitis (dysentery) occurs frequently in epidemic form in the tropics. Japan. China. India, Europe, and this country in asylums, among soldiers during war times), where large numbers camp together, and in children during the summer month.

The bacilli chiefly concerned in its production are the Shiga, Flexner-Harris, Kruse, Strong. Hiss and Russell. Park. Dopter, Duval, etc.. but the manife-tations and pathologic changes induced by the Shiga organi-m- are more serious and characteristic than those consequent upon infection incited by the other above-named bacilli.

Drsenteric colitis was thought to be due in a vast majority of instances, to entamebe amebe), but recently it has been shown that bacillary colitis is a world-wide disease, and that bacilli cause intestinal infections here. abroad, and in the tropics rerv much more frequently than wa- formally supposed. and that this form of colitis is often mistaken for amebic dy-entery.

Frequently particularly in the tropical and semitropical countries) colitis results from a dual infection, when both pathogenic entamebre and bacilli are present in the tisules and dejecta. Inder such circum-tances the le-ion- are not characteristic of either bacillary or entamebic inflammation of the bowt. and finding of the specific organisms in the stool is necenary to complete the diagnosis.

Bacillary dyentery i- esentially an acute disease, and the patient usually dies or recovers quickly, and though the inflammatory process and accompanying toxemia are intente while the infection rages, the destruction to the bowel tunics is not nearly so marked as occurs in entamebic colitis. which is usually a chronic affection complicated by recurring infections and progresive encroachment upon the mucous membrane and submucosa.

While con-iderable has been added to our knowledge concerning the pathology of bacillary colitis, the subject still remains somewhat obecure an regards the structural changes which accompany the disease in both adult- and children. In fact, the descriptions given by the authoritien of the patholosy and manifestations incident to this type of infection vary to such an extent that it is often difficult to realize that they are discus-ing the same affection

The manifcitations and pathologic changes which accompany bacillary colitis vary wiklely, and may be slight, moderate, or secere, according to the virulence of the infection. 
Peculiarly, in fatal cases the bowel often exhibits but minor, if any, structural changes, especially in infants and children. Here, as in entamebic colitis, the initial abrasions or ulcers are directly due to specific agents (dysenteric bacilli), but the subsecpuent or more extensive lesions are chiefly caused by the artivity exhibited by other pathogenic and progenic intestinal micro-organisms and their usins, which cause a mised infection.

Epirlemic or bacillary colitis is an acute affection which extends over a period varying from a few dars to weeks. and which distinguishes it from entamelsc colitis, which ordinarily endures for months or years. The onset of the former is sudden. and the early manifestations and tisoue changes are intense from the first, while the latter comes on insidionsly and gradually invalidizes, and patients afflected with bacillary are less often subject to relapses (reinfection) than those who suffer from entamebic colitis, though occasionally the disease runs a protracted course.

Owing to intensity of the inflammatory changes in the bowel and marked toxemia incident thereto frepuently the incipient stage of bacillary infection is characterized by high temperature, anorexia. exhausting diarrhea, and general prostration, but after a few days the symptoms become subacute. the intestinal manifestations less distressing. and by the end of from two to four weeks the infective processubsides, the patient gradually recovers his healthy state, and the bowel asiumes a normal appearance.

The absorption of toxins in bacillary colitis is evidently a dangerou. element because frequently adults and children die without discorerable psendomembranes or microscopic lesions in the intestines.

Some of the prisons formed by the Shiga. Flexner, and Lruse bacilli. etc., ace directly upon nerve-centers to paralyze the extremities and bladker or produce other nervous phenomena, and others upon the gastro-intestinal tract. and cause the typic symptoms of the discase (diarrhea, blowly stonls, cramps. etc.).

The infective process is usually confined to the colon, and is most destructive in the sigmoid flexure and rectum. though it involves the lower ileum more frequently and to a greater length particularly in children) than entamebic colitis.

According to Shiga, there are "ascending and descending" typen. the former is the most common, and the infection extend-upwart from the rectum; while the iatter. or high type, starts in the ileum or upper oulon and works downward.

The mortality from bacillary is very much higher during the fir-t two or three weeks than it is from entamelic colitis. hut ultimately as many or more deaths oecur from the latter ats the former. leacatede entamelic drsentery is progressive and destructive to the interine and general health of the patient.

Dysenteric bacilli primarily attack the epithelium and then gratually penctrate decply into the mucosa in contradistinction to entatmeba, which first pass through the mucous membrane to the sulp- 
mucosit, the center of their activity. from which point the destructive proces works upward and through the orerlying membrane to form ulcers. Knowing this, it is easy to understand why the lesions of bacillary are more superficial and less devastating than those of entamelic eolitis.

Deep and extensive uleers rarely complicate the disease except when it is chronic and there is a mixed infection or an intense infiltration of the intestinal tunics, the blood-resets strangulated, and where coagulation and necrosis have taken place or extensire sloughing (gangrene) has occurred.

ln serere types of the infection the submucosa beeomes congested, edematous, and thickened, but rarely undergoes necrosis, and the lesions seldom involve the intestinal musculature and peritoneum enough to cause perforation. as sometimes occurs in entamebic colitis.

It is impusible to give a description of the pathology of bacillary colitis which would fit all cases, because the changes in the tissues frequently vary in different individuals, or in the same case, where the patient is run down, the infection is slight, or extremely virulent in different stages of the disease.

Bacillary colitis may manifest itself in the catarhal, ulceratide. and psendomembranous forms. hut small or extensive diphtheric-like patches are present upon the mucosa in most cases.

Catarhal bacillary colitis, the mildest form of the infection. is more common in children than adults, and is characterized chiefly by hyperemia, swellins. and edema of the mucous nembrane. which. in addition, show: petechial hemorrhages and hyperplasia of the lymph-follicles.

Oecasionally necrosis takes place in the superficial mucosa to a slight degree. Which results in the formation of erosions or shallow ulcers. under which circumstances the mucous membrane is seen covered with blood-streaked glairy mucus, alone or admixed with pus. when the inflammation is purulent.

Microsopic examination of the stools demonstrates the presence of mucus, red blond-corpuscles an unusual number of leukocytes, various shaped epithelioid cells. and occasionally necrotic tis-ue and pathogenic hacilli.

Clectatie bacillary colitis is encountered more frequently than the form just described. and the ulcers may appear quickly when the infection is of a virulent type, or secondarily in the presence of catarrhal inflammation or formation of false membranes. but the lesion- are not destructive except when other micro-organisms than the sperific hacilli (Shiga-Flexner. etc.) actively participate in the infective proces: consequently. when the bacillary inflammation subsides the bowel shows little or no evidence of the infection.

Both caturrhal and ulcerative lesions are common to the lower ileum and colon, and are hut rarely encountered in the upper small intestine. When ulcerated the mueosa may be of a dark bright red, purple, or dark brownish color in different cases, and the lesions may be single or in groups, small or large, superticial or deep, ovoid, encircling or 
irregular in shape, have clear cut, elevated, or modermined edges and clean or necrotic bases smeared with mucts, according to the viru lence and stage of the disease and patt played lo mixed infection (Fig. 7 I).

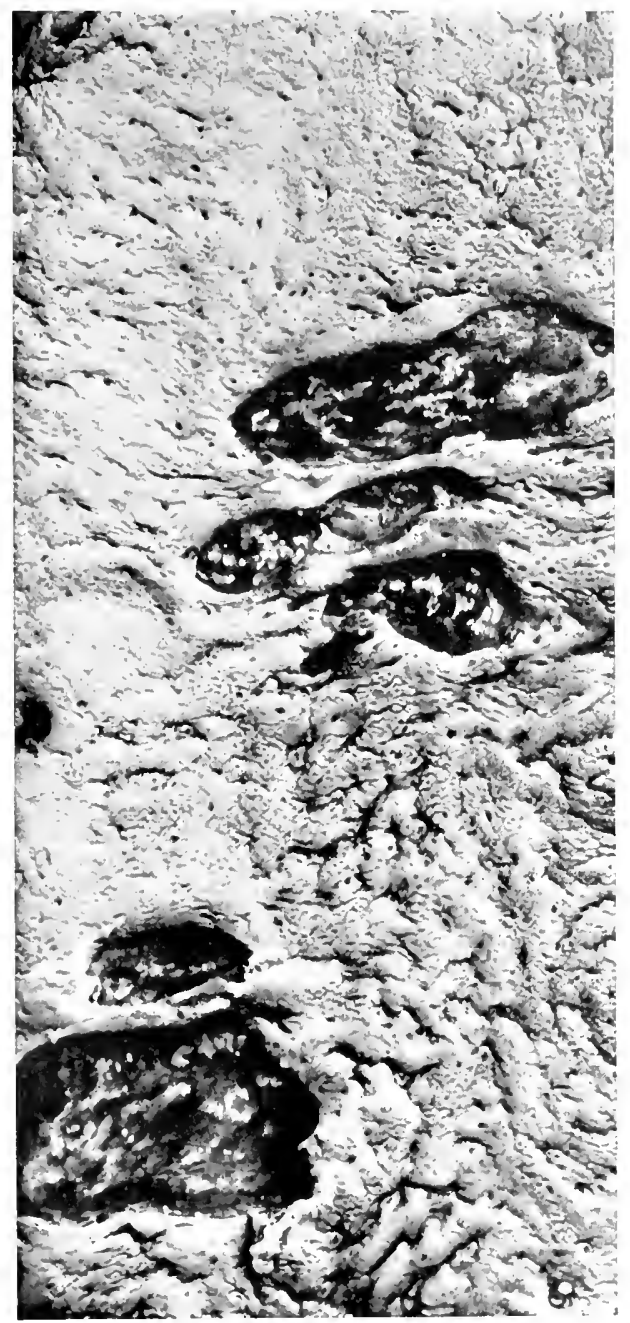

Fig. 71.-Chronic colonic bacillary (o)litis (bacillary dysentery) with diphtheric ulcers.?

Sometimes diminutive and large uters are present, or coalescence can be seen taking place between adjacent lesions, and oceasionally the colon is dentred of its mucosa from one-thirel to onc-lalf its extent, in which case the ulcerative stage has usually been precerled by a deep-seated, diphtheric inflammation which resulted in gangrene and sloughing of the tissues.

I. Irmy Med, Museum. 
The solitary glands and agminated follicles are usually swollen. edematous, and may undergo necrosis, and the mucous folds are congested, thickened, and sometimes present as corrugated-like transerse ridges, while the mucous membrame between them may show dark, petechial hemorrhagic areas or diminutive or larger grayish

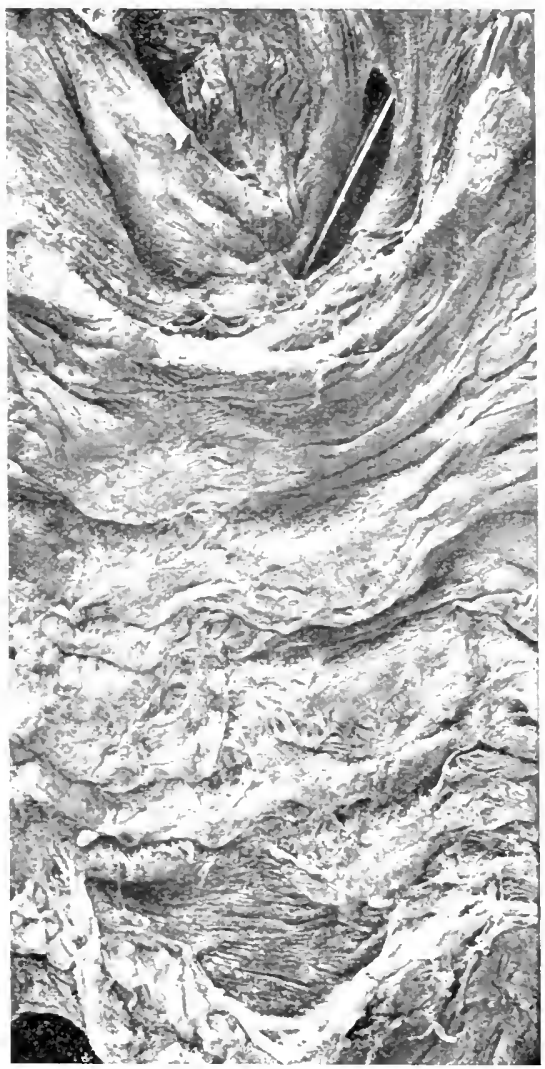

Fig. 72.-Bacillary colitis (bacillary dysentery) with peculomembranums (diphtheric) sloughing of the cecum. ${ }^{1}$

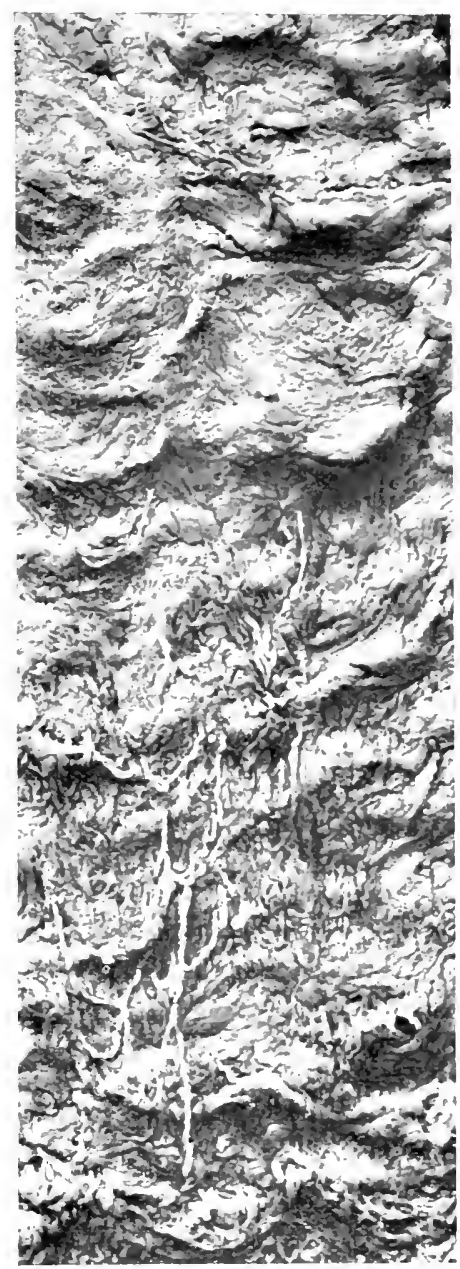

Figr. F.- Sloughing preudomembranous (diphtheric) dysentery of the rectum.

membranous patches, which when wiped off leave a visible highly rectened mucosit.

The ulcerative and inllammatery besons seldom involve the submucosa or deeper structures; conseguenty, the intestinal wall is not

1. Army Med. Nuscum.

"Med. and Surs. Hist. Mar of Rebellion. 
so thick as in entamelic colitis, and peritonitis is a rare complication, though the peritoneum freepuenty appears congented, thickened, and moist.

In exeeptional cases of bacillary ulceration, where the rectum and sigmoid are ridelled with lesions, he lowel is comstantly lathed in at bloosly mucopurulent discharge, the mueoun membrane and skin of the perianal region is excoriated, and when seeds, feces, or infortive

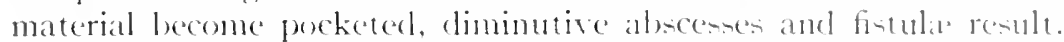

In the rapidly progresive or fulmimating type of lacillary onlitis (dyesentery), which frepuently ends fatally within the firet week, ulceration may or may not oceur, but toxemia invarialsy features the disease and often catteses death.

In these cases the mucosit appears intensely intlamerl. swollen, and superficially necrotic oxer extensive areas, or throughout the colon ant occasionally the lower ileum, and exhitits highly colored, small hemorrhagic areas amel clevated, congested, enlarged solitary follicles or a characteristic diphtheric memhramous covering.

The necrotic membrane which forms here is composed chiefly of coagulated mucus, epithelium, leukocytes, red blood-corpuseles, live and deat micro-organisms, and tisole débris, and is detachable in some instances without injury, lut in others. where the inflammation and destructive process is deep seated, removal of this petuclomembrane leaves a granulating, superficially or extensively ulecrated mucosa (Fig. 72).

It has not been condusively shown whether the infertive procest starts in the lymph-follicles or is incident 10 the irritative action of bacilli and their toxins upon the mucosa, which results in the destruction of the epithelium and formation of an exuelative membrane. which later sloughs.

Statisties indicate that in epidemic hacillary colitis uleers occur in 50 per cent. in adults, and 25 per cent. of the cases in infante and children appear at the end of the first week, are active for two weeks. and show a tendeney toward healing in from three to five weets.

During a serious epidemic of hacillary dyentery at the l)anvers (Mass.) State Hospital (rgos), of 16 deathe recorderl, the aecompanying statisties give the time patients lived after the infection started:

Cases dying in from six to eight days after onset . . . . . . .
Cases dying in from eleven to sixteen days after onset . . . . . 6
Cases dying in from twenty-one to thirty-tive days after onsel . 3

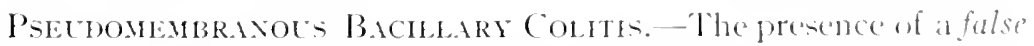
or diphtheric membrane is the pathognomonic sign of hacillary inflammation (Shiga, Flexner, Kruse, etc., types) in the vast majority of instances, and forms when the mucosid is involverl in at caltarthal hemorrhagic or true diphtheric process in anywhere from ene 10 two weeks (Figs. 72,73). This pseudomembrane may embrace the -ubstance of the mucosa or form upon it, in which case it can les removed with a curet or wiped off through the sigmojectosene with a swat, lut in 
either case necrosis ensues to a slight or serious degree, and the diphtheric covering gives way when the highly reddened, smooth, or uneven ulcerated underlying mucosa becomes visible. This condition prevailed in is per cent. of the 28 cases of the disease in infants investigated hy Howland, and in 26 per cent. of the 9 adult cases examined at the Damvers Hospital.

The pseudomembrane, which may cover a small area, a single segment, or all the lower bowel, is alive with a specific bacilli (dysenteric), cocci, and other micro-organisms. In the underlying congested mucosa are to be found swollen and degenerating solitary follicles, necrosis of the interglandular structures, dilatation, and partial or complete strangulation of the blood-ressels, and in aggravated cases thickening, edema, or breaking down of the submucosa.

In rare instances the necrotic membrane embraces the superficial intestinal tunics to a greater or less extent, and comes away in strips or casts shaped like the bowel, following which the intestine may remain thick and hardened, undergo atrophic changes, or strictures may form at points in the bowel where the destruction of tissue has been extensive.

Intestinal stenosis is a complication of entamebic very much more frequently than bacillary colitis, and in either case the stricture is located more often in the rectum than in the colon or sigmoid flexure.

Liver abscess is rarely induced by bacillary infection, but myocarditis, cardiac hypertrophy, valvular and circulatory disturbances, nephritis, phlebitis, paraplegia, hepatic congestion, meningitis, cholelithiasis, and bronchopnemmonia have been more or less frequent complications of the disease, and it has been noted that bacillary colitis lowers the resistance of the patient so that he readily contracts other infectious diseases, such as typhoid ferer, pneumonia, tuberculosis, cholera, and diphtheria. 


\section{CHAPTER XXXV}

\section{BACILLARY COLITIS (BACILLARY DYSENTERY, ASYLUM DYSENTERY), DIARRHEA IN (Continued)}

\section{SYMPTOMS, DIAGNOSIS}

Symptoms.-In this condition the manifestations are variable, the disease may be very acute or chronic, or more virulent in one case than another, the symptoms are worse and the mortality greater when the infection is induced by Shiga's than when it is incident to the bacilli of Flexner, Park, Strong, Hiss, Duval, or related organisms.

In some instances patients are desperately ill throughout the attack, the accompanying symptoms are out of all proportion to the intestinal lesions, and they may be difficult or impossible to discover. Children are infected with Shiga's bacillus less often than with other types of dysenteric bacilli.

The catarrhal form of bacillary colitis may start as a mild diarrhea accompanied ly slight colicky pains and intestinal soreness, the patient will have from four to six movements daily, which in the beginning are copious and semisolid or mushy, and may contain considerable bile, some mucus, and little if any blood, and defecation causes very little straining and is almost painless. Later there is a loss of appetite, a feeling of malaise, and the stools become fluid, smaller, very much more frequent (ten to fifty daily), and are composed chicfly of mucus tinged with blood, and their expulsion, which is accompanied by griping pains. induces considerable straining and leaves a burning sensation in the rectum. In favorable cases the manifestations may subsicle in a few days and the patient make a complete recovery, or the infection may become active locally or extend to other segments of the bowel, under which circumstances the dysenteric symptom-complex becomes exagserated.

Ordinarily, bacillary colitis is of a more sudden onset and of a severer type, in which case the patient is extremely ill from the beginning, and complains of symptoms which indicate that both the bowel and general system are profoundly affected by the infection and alssorption of toxins, both when the disease is restricted to the colon or involves the large and small intestine, with the result that the sufferer becomes dangerously sick within forty-cight hours and not infrequently dies in from three to seven days.

Deplorable cases in the beginning or later exhildit chilly sensations, intermittent temperature, which varies from $\mathrm{IOI}^{\circ}$ to $\mathrm{IO}^{\circ} \mathrm{F}$., erratic, weak, fast pulse (Ioo to I50), anorexia, thirst, hoarse voire, glazed red tongue, cold dry skin, aching body, prostration, restlessness, head- 
ache, severe cramps or disseminated abdominal pain, colonic tenderness on pressure, sometimes nausea, vomiting, delirium, congestion of the liver, and an exhatisting diarrhea characterized by diminutive, $\overline{3}$ ij to $\bar{\jmath} \mathrm{j}(8.0-30.0)$, frequent (ten to fifty daily) passages, which in the beginning are feculent, then seromucous, and finally composed almost entirely of mucus, tissue débris, and blood, which shows bright red if fresh or as "coffee-ground"-like stools when long retained. Within two or three days in aggravated cases the tongue becomes furred, the skin moist and relaxed, and the serious condition of the patient is evidenced by exhaustion, collapse, delirium or coma, resulting from the absorption of toxins, sepsis, or degree of fatigue which follows the loss of sleep, inability to digest food, cramps, diarrhea, rectal tenesmus, and small or considerable loss of blood.

Death ensues in from 5 to 20 per cent. of the cases within three to seven days.

In favorable cases the manifestations consequent upon the infection usually quickly or gradually subside and the patient gets well in from two to five weeks, but occasionally the acute insidiously passes into chronic dysentery, under which circumstance the evacuations continue abnormally frequent or loose and contain more or less mucus, blood, and pus. The frequency and fluidity of the stools and the amount of contained mucus and blood are invariably augmented when the patient takes cold, and indulges in dietary indiscretions, active exercise, remains in the broiling sum, or consumes alcohol or drinks lemonade, highly charged water, or ice-cold beverages.

When the bowel is widely ulcerated or extensively involved in diphtheric or gangrenous processes, mixed infection ensues, and the septic condition of the patient is observable in his blood changes (leukocytosis, etc.), sallow or muddy complexion, rigors, irregular high temperature, and the formation of diminutive or large abscesses which open in to the peritoneal cavity or through the abdominat wall. While sepsis independently is conducive to diarrhea, the frequency of the evacuations are proportionate to the extent of raw areas in the mucosa. When ulcers are numerous or entire sections of the mucous lining of the bowel slough the evacuations are incessant, foul smelling, and composed of mucus mixed with fresh or clotted blood and fibrinous membrane, or offensive necrotic sloughs variable in size and shape.

In deplorable cases of bacillary colitis (dysentery) the patient becomes chronically ill, convalesces slowly, has occasional relapses, and may suffer from indigestion and persistent diarrhea after the specific bacilli which caused the trouble have completely disappeared, and there may be an irritable, inflamed, or ulcerated state of the mucosa, or presence of strictures, adhesions, angulations, and other sequelie.

The COMPLICATIONS and SEQUELE of bacillary are not so frequent or distressing as those induced by entamebic colitis, and liver abscess never complicates bacillary colitis except in the presence of a mixed infection, but hepatic congestion is a frequent manifestation of the 
affection. Perforation and peritonitis are rate, since the destructive process is usually confincel chiefly to the superficial mucosi, luut strietures oecur occasionally when the mucous membrane has been denueded at several points or over large areas by ulcers or extensive sloughing.

Inflammation of joints and tendons, bronchopnemmonia, pleurisy. albuminuria, nephritis, phlebitis, perirarditis, endocurditis, ialiular disturbances, meningitis, paraplegia, neuritis, splenic enlargement or abscess, choletithiasis, impairment of the arterial tunics, enlarsement of the mesenteric lymph-nodes, intestinal ptosis, adhesions and angulations, appendicitis, pericolitis, sigmoiditis, proctitis, hemorrhoids. adenomata, enlarged anal papilla, fissures, ischiorectal abscesses, fistulce. and erosions of the mucost and gluteal skin have been enconntered at one time or another as complications of bacillary colitis.

The blood changes in bacillary dyentery are not very important. since in the beginning there is but slight increase in the white bloodcorpusetes and fymphocytes, which thereafter gradually diminish and become normal in a week or ten days. Except in so far as influenced by the patient's high temperature, toxemia, and a septic state, the urinary complications are unimportant and do not deserve special consideration; deviations from the normal, calsed by the conditions mentioned, influence one in diagnosing this affection.

Diagnosis. White the alove-enumerated symptoms help one to arrive at a conchusion, they are not sufficiently accurate to warrant one in basing a diagnosis upon them. This condition has occasionally been mistaken for diarrhea incident to dietary indiscretions. worms, and balantidic infection, but most often it has been confused with entamebiasis, which must be excluded before a diagnosis is made.

Bacillary differs from entamebic colitis in that (a) it occurs in epidemic form more frequently (particularly among the inmates of asylums, prisons, barracks, railroad buildings, camps, etc.): (b) has a more sudden onset; $(c)$ is accompanied by a serere type of toxemia; (d) more frequently attacks children; $(e)$ is of shorter duration; $(f)$ is most dangerous during the first week or ten days: (g) very often complicated by diphtheric or gangrenous intestinal changes: (h) is ruite often complicated by inflammation of the joints and tendon sheaths: (i) usually invotves the superficial mucosa only; ( $j$ ) is less often complicated by relapses or passes into the chronic form; $(k)$ more quickly subsides or kills the subject; and $(l)$ the patient s blood-serum agghtinates with Shiga's, Flexner's. Dural's, and other dyenteric bacilli, white the serum of porsons afflicted with entamebiasis does not agghlutinate with entameber.

In all cases a tentative diagnosis must be confirmed by funding of the specific (Shiga, Flexner, l'ark, l)urat, or Hiss, etc.) becilli in the dejecta and developing it ly cultivation so that it can le distinguished from other dysenteric bacilli, which is not alwas eas when the dejecta is fresh.

Macroscopically, one can determine the color and consistence of the feces and whether or not they contain mucus, pus, blood, shreds of 
tissue, gangrenous sloughs, undigested food remnants, or larger worms, but it is imperative that the stools be microscopically searched to demonstrate whether they contain diminutive helminths (or their ova), Balantidlum coli, flagellates, ciliates, coccidia, or entamebe, which may induce manifestations closely resembling those of bacillary colitis.

The agglutinating test which can be relied upon to differentiate bacillary from entamebic colitis cannot be successfully employed in every case to distinguish between Shiga or true dysenteric bacilli and so-called pseudo- or parabacilli, as represented by the Flexner. Hiss. Park, Dural, etc., types, because the latter may agglutinate to a certain extent with the blood-serum of healthy individuals and not always with that of dysenteric patients, and becatuse it is sometimes difficult to differentiate between the specific organisms of the last-named group.

Martini and Lentz (I902) did much to clear up the subject when they pointed out that bacilli of the Flexner. Hiss, Park, and Dural strains possess glutins and a power to ferment mannite which were lacking in the Shiga organisms. Shiga bacilli are detected proportionately more frequently in seiere, and the Flexner organisms in the milder cases (pseudodysentery) of bacillary colitis, and the former most often causes the infection in adults and the latter in children.

Park groups dysenteric bacilli into $(a)$ the non-mannite; (b) mannite fermenting (but not maltose); and (c) varieties which ferment both mannite and maltose, and Flexner also recognizes these types differentiated from each other as follows:

"( I) Shiga type attacks glucose without action on other sugars, including mannite and lactose. (2) Flexner-Harris type attacks glucose and mannite. (3) Hiss and Russell bacilli attack glucose and mannite. No action on dextrin and lactose."

Park has described a lacillus which produces indol in a peptone solution and differs from the Shiga in its agglutinating characteristics, and is distinguishable from the Flexner organism in other ways.

The progress now being made in intestinal bacteriology indicates that ere long other bacilli concerned in the causation of true dysenteries and the summer diarrheas of infants and children will soon be discovered, as well as the means by which the various organisms can be clearly distinguished from each other.

The infection of animals with supposedly dysenteric bacilli as a confirmative diagnostic measure has thus far proved disappointing and unreliable.

Manifestations closely resembling those of bacillary colitis are also encountered in hemorrhagic, diphtheric, and catarrhal affections of the colon, and in ptomain, mercurial, and bacterial poisoning acting through the blood. Toxemias may also be induced by pathogenic accidental and obligate intestinal micro-organisms, such as the colon, pyocyaneus, proteus, and airogenes bacilli, streptococci, and staphylococci when they are unduly virulent there are intestinal lesions, and the patient's resistance has been lowered. or he has stomatitis, angina, pneumonia, or sepsis. 
The blood changes of bacillary colitis resemble those of typhoil. in that there is an absence of polynuclear teukocyouis and a slight lymphocytosis in the beginning. which diminishes to the normal in from a week to tem days.

Kruse, who regards his hacillus as being in the some class with Shiga's, maintains that it can be differentiated from pandelsacilli (Flexner, Hiss, Park, ete.) when cultures are made with the litmusmannite culture-media of Lentz, and beeanse his and the Shiga bacilli form a soluble toxin in analogy with the diphtheria bacillus in contritdistinetion to the others.

The agglutinating is the most reliable test for detecting bacillary colitis, though the finding of the bacilli in the stools or other organt after death points strongly to them as the canse of infeetion. In the Danvers epidemic this test proved positive in the first week in 50 per cent.; in the second, in 92 per cent.. and in the third and fourth weeks in soo per cent. of the cases. The conclusions regarding the value and variations of the agglutinating test for dysenteric bacillary colitis arrived at by Richards. Peabody, and Canavan, ${ }^{1}$ who had frequent occasion to apply it during the Danvers Hospital epidemics. are clearly emphasized in their report, riz.:

"(1) Sera of I 5 dysenteric patients were tested for agghutination with stock cultures of the Shiga and Flexner-Harris types of Bateillu. dyenterixe. Eighty-one per cent of these sera gave positive agglutination reactions, in the sense that they reacted with Shiga (32 cases). with Flexner-Harris (37 cases), or with both $2+$ cases) were negative.

"(2) Ten control sera, from persons without olstainable history of dysentery, proved negative except in one instance (positive with the Flexner-Harris type, $\frac{1}{x_{0}}$ ).

"(3) A positive reaction was obtained in I case of + tested on the first day of symptoms, in $\mathbf{5 2 . 6}$ per cent. of all cases (19) tested during the first week, and in 92.3 per cent. of cases (1 3) tested during the second week. The cases (19) tested during the third and fourth weeks after onset were all positive to Shiga, Flexner-Harris, or both. The pereentages of positive reactions during subsequent weeks declined somewhat gradually.

*(4) The serum of a case showing the Shiga type on cuhure may agglutinate in higher dihution a stock Flexner-Harris type than a stock Shiga trpe. One serum from a case whose stools yelded the Park-Hion type of Bacillus dysenteria in this epidemic failed to agglutinate the stoek cultures of either Shiga or Flexner-Harris.

"(5) Analysis of the table of agghtinations shows that there wat a sharp increase in the number of catses with seral agglutinating the Shigat type (It), as compared with the sera agglutinating the Fexner-Harritype $(7)$, during the first two weeks in August, corresponding with the highest elevation in point of morbidity. The cases arising during other weeks are either too few for deductions or else show a matried preponderance of Flexner-Harris agglutinators. It is posiblele. lut cammot

${ }^{1}$ Buston Merl. and Surr. Jour.. p. O8r. November Ir, 1000. 
be proved, that the first prominence in the curve (first phase of epidemic) corresponds to an invasion of organism of the Shiga type. whereas organism of the mannite-fermenting type may be found throughout the epidemic. This point shoult be considered in future work.

"(6) Serial agglutination test- were carried on in 38 cases: (1) During disease or convalescence. I 90, : (2) in February, 1909; and (3) in March, I909. The cases were chowen at random as clinically typical cases of dysentery. Only I of the 38 cases was negative in all three serial tests. This high percentage (97 per cent.) of positives with employment of serial tests may be contrasted with the percentage (or per cent.) obtained from cases tested during the disease alone. The results are interesting and capable of several explanations-such as derelopment of agglutinins(?), persistence of organism producing agglutinins in the absence of symptoms(?), reinfection(?), crossed agglutinin formation (Bacillus coli)(?).

$\because y)$ Several instances occurred in the several tests of the development of agglutinins for Flexner-Harris that required many months to recover from dysentery. In some instances these agglutinins were transient $(e . s$. present in February, absent in March). No instance of de no:o development of Shiga agglutinins (either persistent or transient, was observed during February and March, I909, examinations.

"(8) Conclusions 5. 6. and 7 point with some probability to the hypothesis, already adranced, that the Shiga type of Bacillus dysenterice is a true parasite. whereas the other (mannite-fermenting) types are frequently normal inhabitants of the intestinal tract."

Finally, in acute cases of bacillary colitis the intestine, when inspected through the sigmoidoscope, appears highly colored, edematous, and the mucosa is broken at one or many points by superficial erosions, is covered by a whitish fibrinous membrane with hemorrhagic markings or involved in a gangrenuus process, but in chronic dysentery numerous ulcers variable in size are frequently seen. Lsually the rectum is sensitive to digital examination and the bowel feels warm or hot to the finger, particularly during the acute stages of the disease. 


\section{CHAPTER XXNT}

\section{BACILLARY COLITIS (BACILLARY DYSENTERY, ASYLUM DYSENTERY), DIARRHEA IN (Concluded)}

\section{TREATMENT}

\section{PROPHYLACTIC, SUPPORTIVE, MEDICAL. SERUM, IRRIGATING AND LOCAL. SURGICAL}

Tue symptomatic is about the same, but the curation treatment of bacillary differs materially from that of entamebic colitis (dysentery). This is due largely to the faets that the former is decidedly more acute, is accompanied by a high degree of toxemia which clemants immediate consideration, and the disease responds quickly to serotherapy.

The rational treatment of bacillary colitis embraces $(a)$ prophylatic precautions; (b) supportiae therapentics; (c) internal mediation; (d) serotherapy; (e) colonic irrigation; and (f) surgical measures, but all these methods of treatment are not reduired in every case.

Prophylactic Treatment.--In many respects the prophylaxis of bacillary is similar to that of entamelic colitis discussol elsewhere. In England the percentage of asylum inmates whosuffer from this type of dysentery is great, and the mortality of these afflicted is alont 2.5 per oent. The tisease is not so common or fatal in our prisons, barracks, and insane asylums, but prevails to a mueh greater dexree than is believerl, and when once it appears in these institutions in epietemic form it is likely to reappear many times after heing apparenty stamped out unless rigid precantions are taken to destroy everything which might be infected, and isolate patients who are carriers of Bacillus dysenterice, becande it is not known under what conditions and how lons thene organisms may live in or outsicle of man.

To prevent the infection from spreading, dysenterie patients should not be permitted to go from one ward or house to another, 1 sie the same toilet, or handle the forel, water, drinking-cups or dishes of healthy individuals, or sleep with them. Dne precaution should be taken to see that drinking-water and the fores is property protected against contamination, and that the stoolsof dyenterie patients are thoroughly disinfected and tlies are prevented from spreating the disease. P'asteurizing or otherwise protecting milk against infertion is most important during the summer months. and more particularly when it is to be consumed by infants and children. Finally, cleanliness from cellar to garred must be enforeed in the presence of individual and cpidemic cases. 
Supportive Treatment.--Supportive measures are not so universally imperative in bacillary as they are in the treatment of entamebic colitis, because properly (serum) treated patients recover from this affection before they have become greatly tmaciated, anemic, and run down as the result of persistent diarrhea, loss of blood, and mixed infection. When, however, bacillary dyentery betomes chronic, one should build up the patient's health as much as pos-ible by giving him nourishing food, tonics, and having him exercise mildly in the sunshine, but without ageravating the simptoms.

In this form of colitis REsT in the house and. preferably, the bed is imperative during acute crises. becalte of the toxic condition and profound illness of the patient. but when the affection is chronic this is not necessary or adrisable. except during exacerbations of the symptoms and where it has been demonstrated that getting up or exercising augments the diarrhea. mucus, and blood in the stools.

In acute virulent bacillary dyentery regulation of the DIET is important, and all food. excepting milk or water, should be discontinued for from twenty-four to forty-eight hours, and for the next two or three days the diet should be confined to liquids. such as milk. wher, broths, soups, expressed beef-juice and meat extracts. strained gruels. Whites of eggs. etc., after which the amount of nourishment may be increased and a mixed diet gradually permitted in proportion as the acute symptoms modify. The author does not believe in starving or confining patients afflicted with chronic dỹsentery to a licquid or semifluid diet. but makes a practice of letting them have as abundant and mixed diet as they can take care of. for these sufferers are often porly nourished, anemic, and run down. Many time- he has taken individuals afflicted with chronic dyentery who had been living on liquids for a long time without benefit. and immediately started them on the road to recorery by prescribing a generous mixed diet and keeping the howel clear of irritating discharges and feces. This affection is confined chiefly to the colon, and does not interfere with digestion when gastro-intestinal peristalsis is arrested, which can be accomplished by intestinal irrigation or by means of oil emulsions which contain anti-eptic healing, astringent, or soothing drugs according to indications. In the absence of bowel-washing it is necesary to pay a great deal more attention to the diet.

In acute, and sometimes chronic, bacillary colitis, where, as a result of profound toxemia. loss of blood, or exhaustion incident to persistent diurrhea. pain, or tentemus. the patient becomes extremely weak, anemic, nervous, or the heart filters. the temporary administration of alcohol. whishy, brandy, wine, or strychnin is advisable to brace him up; tonics such as arsenic, strychnin, iron preparations, or Russell' = emulsion are nccasionally indicated until he has been strengthened and the dysenteric smptom-complex has ceased or been greatly modified.

Medical Treatment.-The medicinal treatment is symptomatic, since no drug has been suggested which acts as a specific for this affec- 
tion, though a few authorities maintain that it can be cured by ipecatcuanha when properly administered.

At the outsed in the acute, subacute, and chronic forms of hacillary dysentery the treatment, of whatever kind, should be inaugurated by thoroughly cleansing the bowel of lissue débris, discharges, scybala, and fluid feces by the administration of lileral and, if necessary, repeated doses of castor oil, $3 \mathrm{j}$ to $\mathrm{ij}(30.0-60.0)$, magnesium or solium sulphate, 5j to viij (4.0-30.0), or calomel, gr. ij to iij (0.12-0.18).

Unquestionably, ipecac is the most generally emplored and useful remedy that we have for this affection, becalse its administration nearly always diminishes the number of evacuations, minimizes pain and tenesmus, and lessens the amount of pus and blood in the stools. While it camnot be clenied that the drug brings about these beneficent results in many instances, the fact remains that ipecac neither destroys specific bacilli in the dejecta, prevents relapses, nor affects a permanent cure.

The best results are obtained from ipeeac when 45 gr. (3.0) are administered the first night, and the amount thereafter is decreased $5 \mathrm{gr}$. (0.30) daily until $10 \mathrm{gr}$. (0.60) are given, an amount which may be continued nightly until the dysenteric symptoms abate or cease. The value of the drug may be enhanced log having the patient rest quietly in a darkened room, take a liberal dose of castor oil, calomel, or salts, and fast for a few hours prior to its administration and remain inactive and take but little food for a time thereafter. When the medicine induces persistent nausea and vomiting this may be minimized by placing a mustard-plaster or ice-bag over the stomach, lessening the doses or increasing the length of time between them. and giving the drug in creatinized or chocolate-covered pills or in gelatin capsules. In subacute and chronic bacillary colitis the amount of ipecac administered should be diminished to one-half or less than that recommended above for the acute variety. 'This class of sulferers at times complain bitterly of abdominal pain, cramps, tencsmus or almost incessant evacuations, and often collapse unless relieved, which is most satisfactorily accomplished by morphin, gr. ${ }_{3}^{1}$ to $\frac{1}{4}(0.008-0.015)$, hypodermically; an opiate pill, gr. $\frac{1}{8}$ to $\frac{1}{4}(0.008-0.015)$; or laudanum, mxx (1.3), alone or in combination with belladonna, gr. ${ }_{4}^{1} 10{ }_{4}^{1}\left(0.008^{-}\right.$ $0.015)$, when enterospasm is a complication.

In the presence of persistent active fermentation and putrefaction. bismuth subnitrate, subcarbonate, and subgallate, hymol, heta-naphthol, salol, and like remedies in 10- to 15 -gr. (0.60-1.0) doses, three times daily, assist in preventing the formation of gases. minimizing bacterial activity and molifying the dysenteric symptoms, and similar benefits are derived from an acidified acetozone solut ion, $1: 3000$, when it is consumed daily in amounts not to exceed I or 2 cularts (liters). Antiseptic remedies of this class probably possess some germicielal power, but if they should be anministered in doses of sufficient sime to kill the dysenteric bacilli death would ensuc from poisoning. Bismuth preparations, administered in successive and large doses (30 
sr. or more), several times daily, undoubtedly lessen the number of evacuations for a time, but later increase them. because it becomes impacted in ulcers. acts as a constant source of irritation or accumulates in the form of hen s-egg-sized enteroliths or larger putty-like masses, and is retained to block or traumatize the already sensitive hemel. Consequenty, this drug should not be long employed in the smptomatic treatment of this and other forms of ulcerative colitis.

A-tringent remedies, sallic acid, tannalbin, tannigen, and the salicylite of guaiacol. given in IO-gr. (0.60) doses three times daily, may le prescribed in urgent castes with the idea of modifying or controlling the diarrhea, but are useless as curative agents. Patients suffering from bacillary colitis would have their symptoms modified sooner and be cured more quickly if physicians would substitute serotherapy and intestinal irrigation for the old-time remedies so often mistakenly prescribed for this condition.

Serum and Vaccine Treatment.-The unefulnes of dysenteric sera in the treatment of bacillary colitis has been thoroughty proved, and they are as neces-ary for the cure of this disease as antitoxin is for diph theria. Their emploment is imperative lecaute they effectively relieve or cure this type of dysentery without danger of causing local or constitutional disturbances or leaving annoying sequele. Properly prepared sera shorten the attack by half, immediately modify or arrest the dysenteric manifestations, and the subcutaneous injection of the serum i- usually followed by recovery in mild cases in from two to three, in serere case in from three to four, and in grave cases in from four to six days. but when the serum is not sufficiently powerful or the patient does not readily respond. more than one injection and from seven days to two week- may be required to bring about the cure, owing to the influence of the patient : age (thirty to fifty years being farorable) and resistance.

Thi- treatment is not so effective when the subject, in addition to dy-entery; suffers from lues. tulserculosi-. or other infectious or wasting disease, and convalesence can be hastened by having the patient remain quitety in bed, consume fluid and non-irritating foods, and wash out the bowel daily with hland mild astringent or antiseptic solutions. except where experience demonstrates that irrigation is harmful.

Bacillary dy-entery rarely parse into the chronic state and relapses are unusual following the serum treatment, and where subsequent atrack- occur they are mild. far apart. and are easily and quickly controlled by repeating the injection. Since the serum is harmless, and tends to relieve present and prevent future attacks, the author considers it advisable to continute administering it at intervals of two or three days or fonger, according to indications. over a period varying from two to eight weeks in patients apparenty cured, with the idea of fore-talling possible future trouble, and w repeat the injections at shorter intervals in tho who suffer severely from colic, hemorrhages, diarthea, or toxic manifestations. 
A positive diagnosis of bacillary eolitis should le made before the serum is employed, becanse it is useless in the treatment of entamebic, balantidic, helminthic, coccidic, ciliate, and flagellate dysenteries.

It is also neecesary to isolate the calusative organism in bacillary colitis, since a serum which is curative in the presence of an infeetion induced by the Shiga or Lruse bacilli dees not always arcomplish the desired result when the dysentery is incited by oreaniom of the Flexner. Park. Dual, and Hiss yyes. It is also impertant to determine whether or not bacillary is complicated ly entamelsic, helminthic, or other form of dysentery, and if there is mixed infection participated in by pathogenic intestinal micro-organisms, because thus far no serm has been discovered which would bring about complete relief under such circumstances.

Shiga first and others since have succeeded in producing relialsle dysenteric sera by isolating bacilli responsible for colitis and injecting them subcutancously or into the veins of horses, rabbits, goats, and other animals. Serum obtained from animals immunized in this way has a decided antibactericidal action. Todd and Rosenthat suceeeded in obtaining a soluble toxin from cultures of the Shiga and Kruse bateilli, with which he immunized animals and produced a serum posicsing decidedly antitoxic powers. Vaillard and Dopter, using this discovery. immunized horses by inoculating them on alternate weeks with progressive doses of living bacilli and bacterial toxins. and in this way obtained a serum having both antibactericidal and antitoxic qualities.

Attempts have been made to produce a serum which would prove effective in all types of bacillary dyentery and in the presence of a mixed infection by immunizing animals with cultures of the shiga. Kruse, Flexner. Park, Dural, Hiss, colon, and other hacilli, but the results have been disappointing. Serum prepired from either the Shiga or Kruse organisms is effective in the treatment of colitis induced by either, and is helplul in other forms of bacillary dysentery, but the serum from animals immunized ly cultures of the Ftexner, Duval, Park, Strong, or Hiss bacilli, etc., will not relieve or cure a bacillary colitis consefuent upon the first-named organisms. conseffuently it is advisable to use in the individual case a serum derived from the type of bacilli causing the dysentery.

Like other therapeutic agents, the amount of sermm preseribed must be varied according to its strength, the age of the patient, and his resistance.

The serum is usually administered subcutancously, and 20 c.c. is the dose ordinarily given to atults, but half this amount is recpuired for children, though oceasionally a smaller quantity will suffies. In mild cases one injection is effective, in severe ones the treatment should be repeated in six hours, and in grave cases the injections maly le math twice daily for two or three days or until the patient sondition is markedly improved. When he is dangeromsly ill from hypertexicity or other evidence of the infection, from to to bo c.c. or more mal be 
administered, and the injections repeated on the following day when inclicated.

The serum treatment is most useful when employed early, and should be instituted not later than the thiret day if a diagnosis has been made and the serum can be obtained.

When the serum is used in time the mortality of bacillary colitis is from 33 to 50 per cent. less than when other methods of treatment are practised, and Kruse with it reduced the mortality of summer diarrhea from is to 5 per cent. The remarkably good results of the serum treatment are shown in the accompanying summary of the 96 cases of bacillary dysentery treated by Vaillard and Dopter, viz.:

\begin{tabular}{|c|c|c|c|}
\hline & Stools in twenty-four hours. & Number of cases. & Deaths. \\
\hline I. Ordinary cases. & $\ldots$ I 5 to 20 & 50 & 0 \\
\hline 2. Severe cases... & 30 to So & IS & $\circ$ \\
\hline 3. Grave cases.... & .... So to I 50 & 24 & o \\
\hline 4. Very grave cases & $\ldots I_{50}$ to $2 \mathrm{SS}$ & 4 & I \\
\hline
\end{tabular}

Permanent Immmity-Dysenteric (bacillary) sera will relieve or cure bacillary colitis and tend to prevent relapses and reinfection when the patient has had several injections, because under such circumstances antibodies form more readily, which partially immunize the patient. 'Thus far no bacillary produced a'accine has been brought out which when injected permanently insures against infection from all the types of dysenteric bacilli. Shiga at one time practised mixed active and passive immunization (bacilli plus immune serum on 10,000 individuals), but this fid not decrease the number of infections, although a lower mortality was obtained.

Good results have been reported from combined vaccine and serum therapy, but from what has been said it may be inferred that these agents are more curative than preventive as regards bacillary colitis (dysentery).

Irrigating and Local Treatment.-Irrigation and topical applications are indicated often in bacillary colitis, hut they are not as necessary here as in the treatment of entamebic dysentery, because the former freduently responds to sera and less often passes into the chronic ulcerative state, and for the reason that in this affection the mucosa is so very irritable and sensitive that irrigating solutions are frequently expelled before they have accomplished their purpose, or when retained do more harm than good, except where the mucosa is extensively ulcerated.

The technic of using and the solutions employed in bacillary are the same as those already given for entamebic colitis, except that their strength should be weaker in all but the chronic form of the disease, where ulceration is extensive and mixed infection is evident. Consequently, silver nitrate, thymol, potassium permanganate, protargol, argyrol, and acetozone, in strengths varying from $1: 2000$ or $^{-} 3000$, are to be recommended in this class of cases during the acute and subacute stages, and stronger in long-standing cases. The duration 
of the irrigations and their frequeney must be vared to meet the indications or be discontinued when they fail to minimize the infection. modify the symptoms, make the patient extremely nervous, or ageratvate the dysenteric symptom-complex.

Soothing remedies usually accomplish more and in a sherter time than cauterization, astringents, or stimulation. The most usoful agents for diminishing intestinal irritability, quicting peristalsis, and minimizing the frepuency of the evacuations are warm or hot crude petroleum, coal oil, liquicl paraffin, or olive, sweet, almonel, or mineral oil

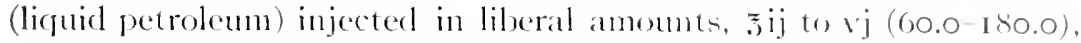
at night, and allowed to remain tmil morning or longer. Folkowing washing out of the bowel with a normal saline solution. Irrigants of strained gruels, flaxseed tea, slippery-ehm hark, or starch-water and mild boric acid solutions are also comforting and healing. In the presence of extrenc intestinal irritability the nightly injection of from 亏iij to is (90.0-120.0) of the following emulsion quickly lorings relief:

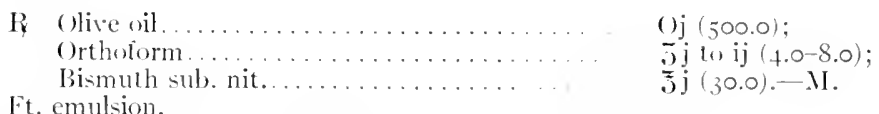

Sig.-Warm, shake, and inject $\overline{3}$ ij to $\vee j(60.0-180.0)$ at night.

Solutions and oils should be used warm or hot because of the soothing effect of the heat, and not cold, because enemata and irrigations having a low temperature often incite enterospasm and are promptly expelled. For reasons given elsewhere it is impossible, as a rule, to insert a colon-tube beyond the middle sigmoid (see Fig. I6), and, owing to this, and because fluids introduced into the rectum ly means of a short tube or irrigator can be made to reach all parts of the colon when the patient is in the Sins or lithotomy postures with the hips elevated, the employment of lengthy pipes is to le discountenanced for irrigating purposes. Long tubes are also objertionable because they frequently kink, induce consideraine pain, or maly perforate the intestine when it is ulcerated.

Oils and cmulsions can be injected by means of a l)avidion or fountain syringe, but a piston syinge is preferable, bectuse with it the necessary force can be used at the right time, since oil preparations are thick and difficult to propel and readily spoil the values or rot the tubing of other syringes.

When oil treatments are being mate he the attending physician the patient should be placed in the inverted posture (see Fig. 122), the proctoscope introduced, gas permitted to escape, and the warm southing oil or emulsion should be poured directly into the upper rectum or sigmoid flexure, from whence it immediately makes its wat around the colon to the cerum; but when it does nos, this can le accomplistued by massaging the colon or changing the pationt is posture.

Soothing are usceful, but stimulating topial applications do more harm than good in all but cases of chronic bacillary colitis with ulcera- 
tion. Consepuenty, the Paquelin electric and chemic cauterizations are contri-indicated except in the presence of mixed infection and old ulcers that are not benefited by less radical measures. Rectosigmoidal irritability can be materially reduced and dysentery markedly improved hy mopping the inflamed mucosa, erosions, or ulcers with warm oil, sedative oil emulsions, methylene-blue (ro per cent.), or extremely mild preparations of silver, 3 per cent.; ichthyol, 6 per cent.; halsam of Peru, Io per cent.; protargol or argyol, 5 per cent., two or three times weekly after the bowel has been washed out with a normal saline solution.

In making topical applications extreme caution is necessary, otherwise injury caused by introduction of the proctoscope or sigmoidoscope through which they are to be mate will counterbalance the good effect of the medication. specula should never be employed for this purpose because they trammatize the rectum and induce much unnecessary pain.

Surgical Treatment.-Surgical measures are rarely required in the treatment of bacillary colitis (dysentery) properly treated because it is curable by serotherapy alone or when supported by colonic irrigation. In neglected cases, and where the patient has been badly handled and the disease has become chronic, mixed infection prevails, numerous large deep ulcers have formed, extensive sloughing of the mucosa has occurred, or strictures have resulted from the healing of raw areas, and when hygienic measures, dieting, medication, serotherapy, and irrigation from belww fail to effect a cure, appendicostomy (see Fig. I64), cecostomy' (see Fig. I55), Gant's enterocecostomy (see Fig. I56) (which provides a means of simultancously or separately irrigating the small and large intestine), enterostomy, colostomy. resection, or amputation are indicated. Of these procedures, the first three are frequently and the remainder are seldom required in the treatment of this condition.

Their advantages and disadvantages need not he discussed here becatse they have received due consideration in the chapters devoted to the surgical treatment of entamebic. which is practically the same as that of bacillary colitis, and the technic of these operations has been fully described in the chapters on the Surgical Treatment of Diarrhea.

Overmuch cannot be said regarding the importance of throughand-1hrough irrigation, as one is cnabled to practice it following appendicostomy, ecostomy, and the author's enterocecostomy.

The irrigants, medicated solutions, oils, and emulsions indicated in becillery are the same as those employed in the irrigating treatment of entamebic colitis, to which the reader is referred. It is essential that the solution should be warm and abundant, and be permitted to rum freely through the colon and out of the rectum through a proctoscope until the bowel is thoroughly cleansed of offensive discharges, toxins, and feces, and that during the irrigation the position of the patient be changed from time to time to make sure that the solution comes in contact with all lesions on all sides and along the entire course of the infected intestines. 
Frequently the appendix afforels a lextging-phate for dyenterio bacilli and the patient repeatedly suffers from reinfertion, and in such catses appendectomy is inflicated in comnertion with recontonte and after appendicostomy following a cure.

Artificial openings, establisherl with the irlea of fatcilitating through-

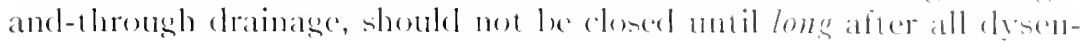

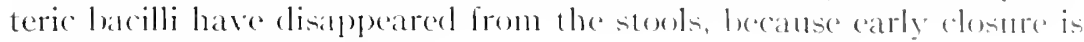
often Collowed lig relapses.

In suitable cases strgical measures, reinforced by affective irrigattion, frequently enalse one in a short time lo cure pationts who have been treated for months or years without lenefit by dieting, internal medication, and attempts at irrigation from leden, and the anther foels that he camot recommend appendicostomy and cecostomy too strongly in this class of cases. 


\section{CHAPTER XXXVII}

\section{HELMINTHIC COLITIS (PARASITIC COLITIS), HELMINTHI- ASIS, HELMINTHIC DYSENTERY, DIARRHEA IN}

\section{CESTODES, NEMATODES, TREMATODES (TENIASIS, UNCLNARIASIS, HOOKWORM DISEASE), ASCARIASIS, OXYURIASIS, TRICHURIA- SIS (TRICOCEPHALIASIS), SCHISTOSOMIASIS, TRICHINIASIS}

Helminthiasis induced by worms and fluke-worms is frequently complicated by chronic diarrhea, abdominal pain, and blood in the stools independently and when associated with entamebic or bacillary colonic infection. Hehminthic colitis is frequently encountered in tropical and semitropical countries, has occasionally been met with in the States, and is said to be the cause of Cochin-China diarrhea, which kills thousands of Chinese yearly.

When considering helminthic diarrhea (dysentery) it is well to bear in mind that increased movements incited by worms in the intestine do not always result from colitis (catarrhal or ulcerative), for they may be induced by exaggerated peristaltic activity or glandular hypersecretion where helminths cause local irritation without lesions, or make the patient nerrous, which leads directly or indirectly to digestive disturbances, intestinal irritability, and loose movements.

Where helminths are detected in the stools in connection with entameba (histolytica) or dysenteric bacilli (Shiga's, Flexner's, etc.) in patients suffering from diarrhea a diagnosis of entamebic or bacillary colitis is justified, because when either infection is manifest it is more likely responsible for the frequent fluid and bloody evacuations than worms.

Helminthiasis is as frequently characterized by constipation as diarhea, and in some instances the patient suffers from alternating loose morements and obstipation.

Active intestinal worms may incluce obstipation by forming balllike masses and hlocking the bowel (see Fig. 88), or by causing local irritation and inciting the simultaneous contraction of the circular and longitudinat muscular fibers, thus producing enterospasm and spastic constipation.

In so far as the symptoms are concerned, there is not a great deal of difference between entamelic, bacillic, and helminthic colitis (dysentery), and hecause of this a thorough macroscopic and microscopic examination of the dejecta is necessary in suspected cases to clear the diagnosis.

The author has no intention of going deeply into helminthology, 
since his object here is to show the relation of intestinal worms to diarrhea, call attention to the similarity in the symptoms of entatmelse, helminthic, anel bacillic colitis, briefly ombline the mothoeds of differentiating one from the other, and stmmatize the sillent features in the treatment of helminthiasis complicated he diartheat.

In so far as they relate to diarhea and colitis (dyosentery), intestinal helminths (worms) may be grouped under three headings:

( I) Cestodes (llat worms).

(2) Nematodes (round- and thread-worms)

(3) Trematodes (fluke-worms).

\section{CESTODES (TAPEWORMS)}

Tapeworms are long, flat, segumented, of a whitish color, and vary greatly in widh and length. Of the many varieties parasitio in man the Trania solium (pork tapeworm), T. saginata (becf tapeworm),

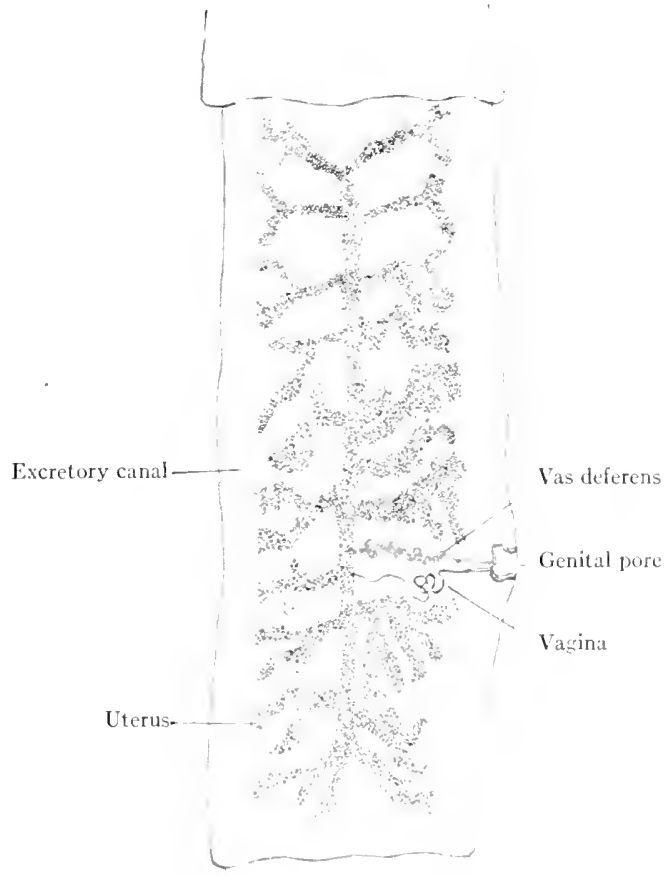

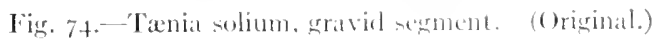

T. lata, Bothriocephalus latus (fish tapeworm), and T. nana (dmatre tapeworm) are the types most frepuently responsible for diarthea andel other intestinal disturlances.

Tania Solimm (Fig. 7t). - This parasite, usmally designated as the pork or armed tapeworm, varies from $6 \frac{1}{2}$ to 13 fere $(2+m e t e r s)$ in 
length, has 800 to 900 segments, and a small spheric-shaped head about the size of a pinhead. It is very common in Germany and countries where improperly inspected or cooked pork is freely consumed, and is rarely encountered in Jews and Mohammedans, who do not eat hog mat.

Cysticerci exist in measly pork, and when eaten uncooked the cyst wall hreaks fown alter reaching the intestine, and this permits the contained head of the parasite to become attached to the mucosa, from which the worm develops as segments form.

Tenia Saginata (Fig. 75).- This tapeworm (beef), the most common of all in America and throughout the world, is unarmed and considerably longer, thicker, and wider than the Tania solium; varies in length Irom 13 to to feet ( $4^{-1} 2$ meters), has from 1000 to $3000 \mathrm{seg}$ -

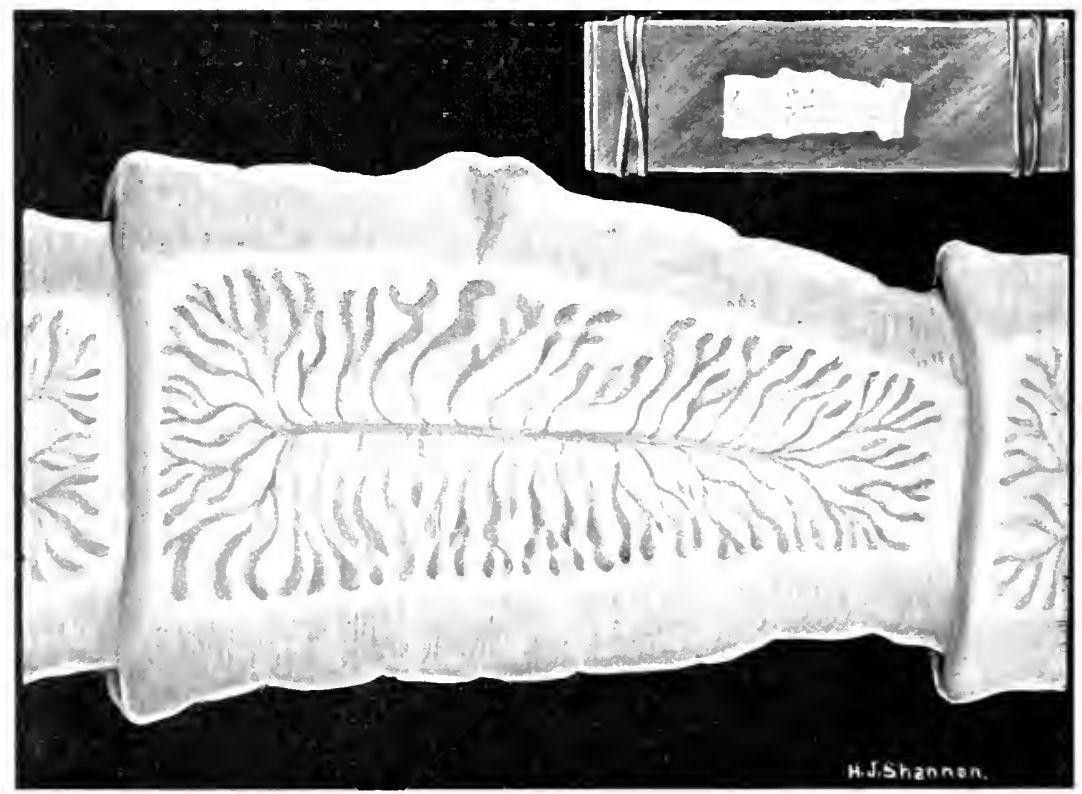

Fig. 75.-Tania saginata. Appearance of gravid segment without magnification. Method of making diagnosis by pressing gravid segment between two glass slides. (Original.)

ments, and possenses a darkly pigmented cuboid-shaped head deroid of hooklets. The cysticerci are derived from raw or improperly cooked beef and rapidly develop in the intestine, segments sometimes appearing within two months following infection.

Siler holds that three weeks' cold storage destroys cysticerci so that the beef cannot inlect the consmmer. Stiles estimates that Taenia saginata grow 13 segments daily and produce $150,000,000$ eggs in a vear.

Tania Lata, Bothriocephalus Latus (Fig. 76).-This, or the fish tapeworm, which possesses a head shaped somewhat like that of a 
pig, is the longest of the cestexless since it meatsures from $6 ! 30,32$ feet (2-10 meters) ordinarily, and exectumionally 65 feet (20 moters) in length, and is composecl of from 2000 to foo sexgments. It is thin

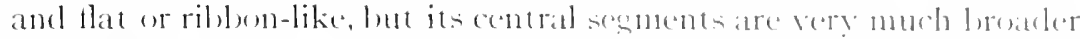

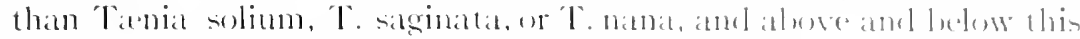
point they gratlually lecome longer and more natrom. The sogments grow rapilly in the lowed, and frome 2506 30 are prodeced datily, and Siler says they are dischareed in chains rather than singly.

The infection is derived from frike, perch, salnom, and other fresh-water fish, and it has leen estimated than in certatin sections of Japan and Turkestan 20 per cent. of the inhalitants are infected. This parasite has for a mumber of vears leen frequently encountered in Switzertanel, (iesmany, Russia, Polanel, Holland, swerlen, Madagasear, and South Africat, where the eating of raw fish is common.

Tania (IImenolepis) nana (Figs. T. 78).-This parasile, because of its small size, is called the dawef lapeworm: formerly it was believed to he of very rate occurrence, lut since 1894.50 or more cases

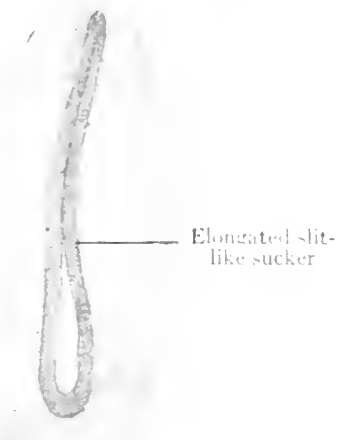
have been reported. It has also, leen encountereel in Egynt, siam, Japan, and Itaty, where Calanelruccio says 10 per cent. of the children are infected loy it.

Tanice nand are very fragile, and except when mumerous are often overlooked in the feces. They are compesed of from 100 to 200 segments, $\frac{1}{5}$ to $\mathrm{I}_{4}^{1}$ inches $\left(5^{-3.35} \mathrm{~mm}\right.$.) in lengelu, and the seetions. barring those at the tail, are broutler than lone. The exese after being swallowed find their way uo lower smatl intestinal villi.

Practically all tapeworms make their hebitat in the -mall intes-

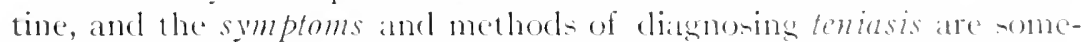
what similar, irrespective of the type of worms catuing the troulde.

Symptoms.- Titfeworms in the intestine maly (abtuc little or no) inconvenience, or slight or matred disturlaness. Patients whos suffer from chronic frefuent abetations and diarthea alternating with constipation, hunger, and complain of anorexia, natusea, romiting, itching of the nose or ants, colicky pains after atling sour fext, combulsions, palpitation, hives, hysteria, and lympanites should lx suspec-

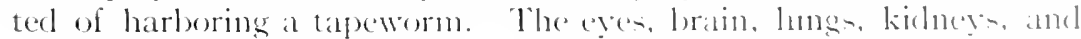
other structures remote from the intestine maty abse beeme invelued by cysticerci or larvale which reach thems.

Diagnosis. Tentasis is comparatively atsy to diagnote when several of the aboxe-mentioned typic manifentations of the liseded are present and the stools atre macroserpically and microsentically examined, which in such cases reveal the whole worm, mature segments, 
parasitic ova, or Charcot-Leyden crystals. When the dejecta do not contain samples of the worm or its exgs, they can be brought down for examination hy the alministration of an active cathartic, and when found the species to which they belong an be cletermined.

Treatment of Teniasis. - The prophylactic treatment consists in not eating improperly inspected, raw, or unclerdone pork, beef, and

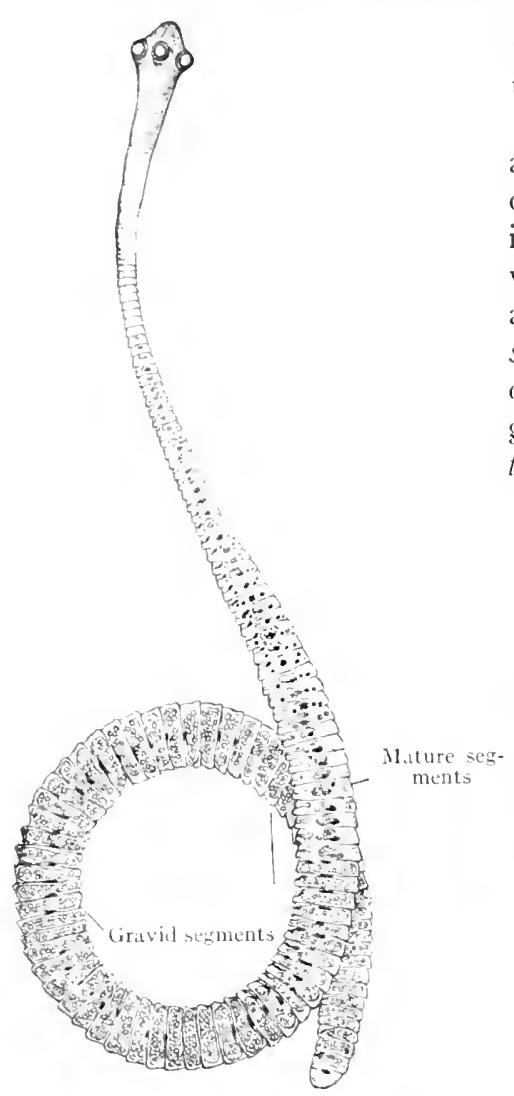

Fier. 77.-Tánia (Hymenolepis) nana. (Original.) fresh-water fish, 1101 drinking contaminated water, nor sitting on toilets used ly infected persons.

Sometimes tapeworms are easy and at others they are extremely difficult to expel. When a patient is ready to be relieved of the worm, first, he should be placed upon a fluid diet for twenty-four hours; second, the bowel should be cleared of feces and mucus by a hydragogue cathartic (salts or calomel); third, a teniafuge or antheminthic,

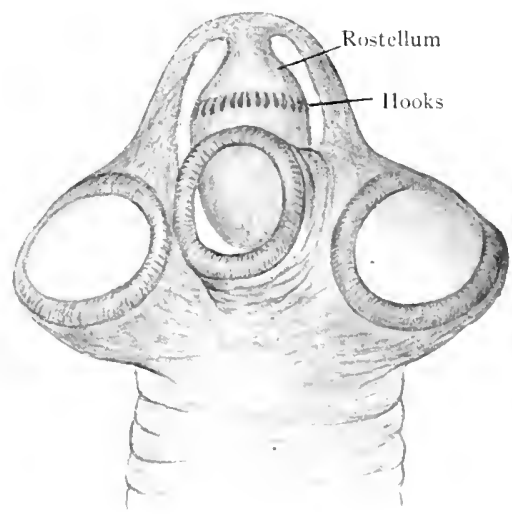

Fin. 7S.-Head of Tania (Hymenolepis) nana. (Original.)

such as male ferm, jos to ij (2.0-8.0); pomegranate root, fluidextract, 5 ss to ij (2.0-8.0); pumpkin seed or pelletierin, gr. v to vij $(0.30-0.50)$, should he administered to dislodge the parasite; and fourth, a purgative, castor oil, o j (30.0), or a liberal dose of calonel followed ly salts should be given shortly following the teniafuge to catse expulsion of the worm. The dejecta should be deposited in a berl-pan or chamber filled with warm water and the parasite perinited to come away undided. When it is thought that all or at part of the worm has been expellecl, a thorough examination should be made for the head, which when found indirates that the treatment has been successful. 
When cathartics fail, saline enemata will ocrat-ionally free the bowed of the parasite.

The treatment is very serere and should not be repeaterl until several weeks have elapsed, so that the patient can resain his -trength and the mucosa become normal. In very olstinate rater. where malle fern alone does not prose effective, Cohnheim recomments the following vermifuges:

P. Extracti filicis maris ather.

Chioroformi

() leti ricini

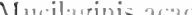

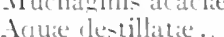

Ft. emulsio

Sig.-Introduced through at stomach-tube tarly in the morning.

R. Graniti corticis

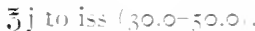

Mac. per hor. xil cum 200-300 aq. dest. deincle coque ad remanentiam. I50.0.

Sig.-Drink or preferably, introduce it through a stomach-tube early in the morning

\section{NEMATODES ROUND OR UNSEGMENTED WORMS}

Nematone worms are crlindric in form, dexoid of segmentation. and are variable in wize and thickness. The more common parasites of this group, while frequently encountered in America. Show a preference for tropical climates. where in some sections the dejecta of the inhabitants contain one or the other forms of nematode- or their ora.

\section{UNCINARIASIS ANKYlOSTOMIASIS. HOOKWORM DISEASE. DIRT. EATERS' ANEMia, DiarRHEA IN}

Encinariasis, or what is more commonly termed hookworm disease. has been encounzered more or lem freeguently in most tropical and temperate climates, but the affection show - a prexalence for tropical regions where the atmosphere is mojist and the soil sandy.

Intestinal medicater irrigation is aneful in these canes when the colonic macosa is inflemerl or ulcerated and the patient suffers from diarrhear.

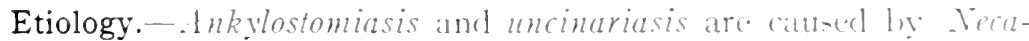
tor americanus (Stiles, I902) or Ankylostoma duodenale 1)ulini. Injh. small worms belonging of the family strongyloblet. The former is the parasite which causes the trouble in this country, and the hatter. in England (firis. 79 , ro).

The Ankylostoma duodenale (Fier. 7 ) is round, inch or lea in length, and taper- enward the powerior extremity, is harbored chictly in the small inte-tine. particularly in the jejunum lese often in the duodenum), and rarely reaches the ileum or colon. It attarcher it atl to the mucosil, from which it withdraw- the litoos apon which it feeds. and leaves oozing spots when its position is changerl: it thrives upur

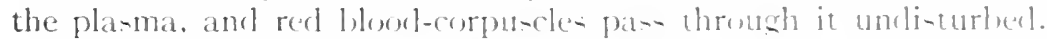

Female hookworms (Fig. nz) are smaller. but three times more 


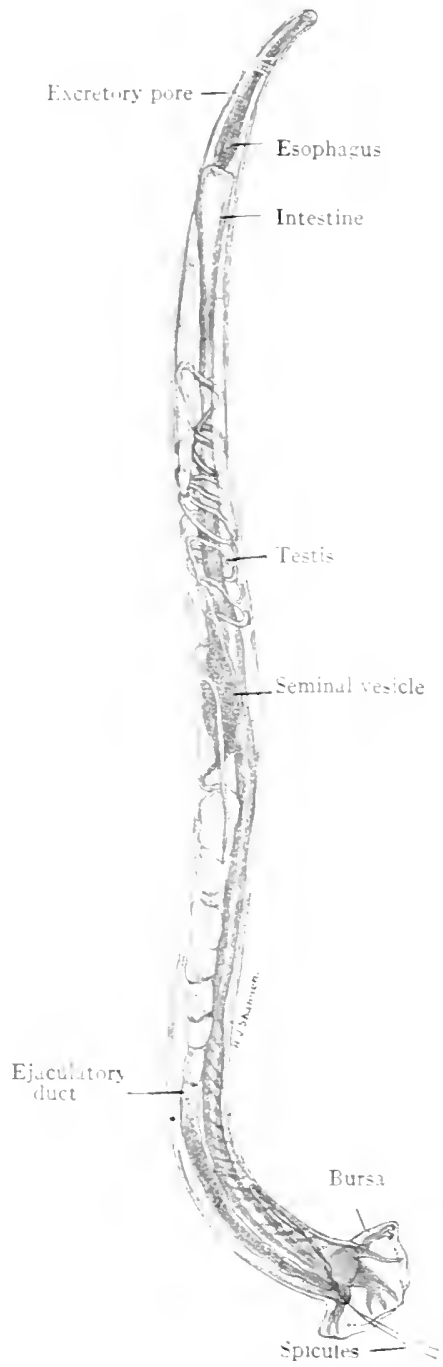

Fis. zo.- Male Inkylostoma duodenale. (Originat.)

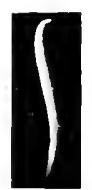

Fir. S1-Male Necator americanus, natural size. (Original.)

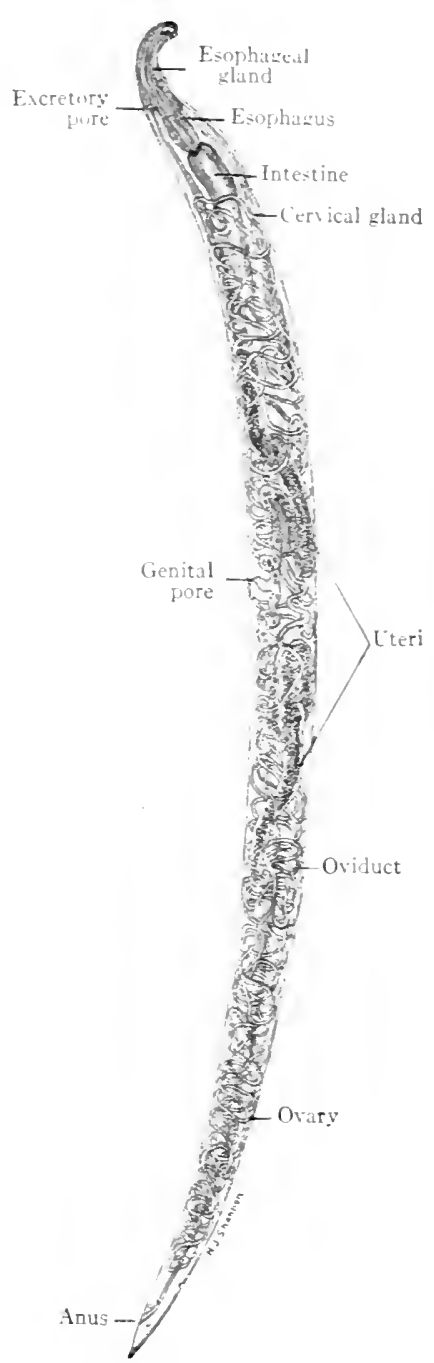

Fir. 8o.-Female Necator americanus, lateral riew. (Original.)

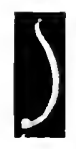

Fig. 82.-Female Necator americanus, natural size. (Original.) 
common than males (Fig. 8I), and both present a grayish appeatrance when empty or brownish when distended with hlockl.

The Necator americanus, the chice etiologic factor in uncinariasis in the Inited States, is shorter and more slender than the $1 \mathrm{mk}$ yostome duodenale, which it closely resembles, but in this species the female is slightly larger than the male.

Lncinariasis or ankylostomiasis resulte from infection $\mathrm{l}$, the larace of the inkylostoma duodenale or Veator americamms, which gain entrance to the circulation chiefly through the skin, from whence they are carried in a roundabout way to the small intestine, where the develop: may also be conveyed directly to the alimentary trate in contaminated water or food.

Pathology.-The pathologic changes induced in the bowed loy an$k y$ lostoma duodenale and Vecator americanus vary according to the magniture of the infection, but usually there is a congested mucosa, dotted over with hemorrhagic spots, which indicate the points of attachment of the hookworm and how the loss of blood responsilile for the anemia occurs.

Symptoms. - The manifestations of uncinariasis are mainly those of anemia from other causes, and in severe cases the patient often suffers from frequent evacuations or diarrheat, alternating with constipation, has a perverted appetite, and relishes chirt, charcoal, wood, cloth, etc.

Children afflicted with ankylostomiasis or uncinariasis are undeveloperl, adults are weakened, have very little cnergy, and the blood changes vary with the different stages of the disease and severity of the infection, but in a general way they

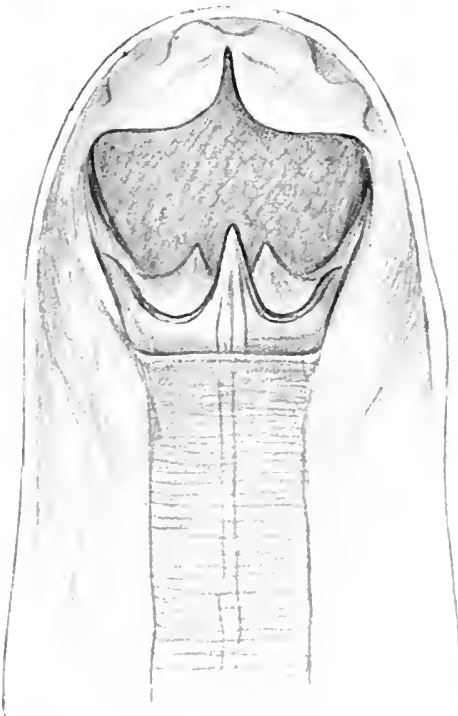

Figr. 83.-Head of Necator americanus. resemble the blood-picture presented in chronic ancmias. Cardiac disturbances, flificult breathing, ascites, and mental incapacity are characteristic in old and neglected cases.

Diagnosis. - In regions where hookworm disease is enclemic the nature of the trouble should be suspected in atl ancmic patients hasing a perverted appetite. A careful examination of the dejectat and

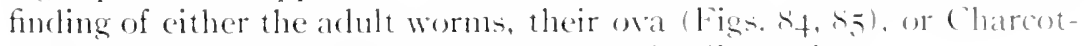
Leyden crystals is necesiary to complete the diagmosis.

Prognosis.-In Porto Rico it is said that one-thire of the death- are due to uncinariasis. When the parasites are numerous and the patient goes untreated the disease progresies rapidly, and he succumbs to dropsy and heart or lung complications, but when the infection is 
not -evere the -ufferer may live for a lone time or permanently recover when he i- properly treated and the worm and their watare destroted ur expelleet.

The treatment of hookworm diatalet has been very gratifying where thymol. Deta-naphthol. gr. Xi 1.0., or encalypus have been emploged. but of these therapeutic asent- thymol is the most reliable. To obtain the heot result with this drue the patien should he confined to a liquid diet for two days and have the bowel cleared

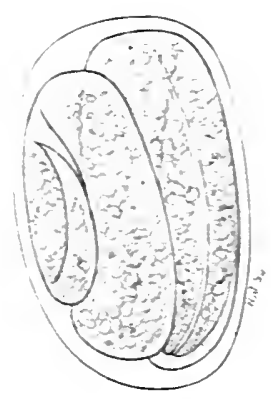

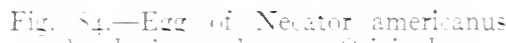
developing embryo. Orizinat.

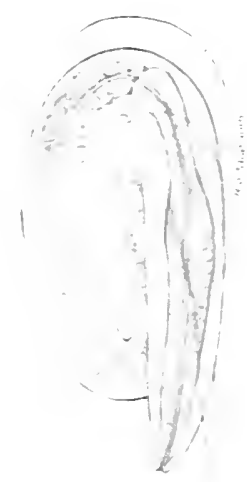

Fit. 5.-Egr of Necator americanus embryo escoping irum shell. Original.)

Ly a saline. On the next morning four i 5-gr. I.o doses of thymol should be administered one hour apart, following which a second saline is given to wash out the drus and bring the pard-ites away.

\section{TRICHINIASIS, DIARRHEA IN}

This affection i- canted lis Tribinella spiralis and comes from eatin infected purk. bise-tion fret the larve from the infected meat. which pas into the intertine. where they remain and within a week profuce numerou- embryos. Living embryo leave the bowel and misate to the musch liy different routes. and in ten days or two week - thereafter a-cume a larial form. irritate the tissues. and in a few weth- become encysted, when they may remain harmles for years or caute local irritation.

Symptoms. - When sastro-intestinal disturbances and diarrhea are cau-ed hy trichina the manifestations ari-e within three days followins eating of the infected meat. or during the time the embryos are journeye from the bowel to the muscles.

The treatment con-ist: in avoiding infected meat and freeing the intertine of the infecting agent- hy thorough catharsis shortly followins their comsumption. ( astor oil or calomel in large doses, followed ly an active saline in the morning are usually effective when reinforced by a santonin colonic irrigation. 
ASCARIASIS, DIARRHEA IN

\section{ASCARIS LUMBRICOIDES (ROUND OR LUMBRICOID WORMS}

The parasite Ascaris lumbricoides (Figs. 86, 87) is cylindric in fom, pointed at both extremities, variable in size and length, hats an outer corrugated exterior, closely resembles the ordinary earth-worm, and

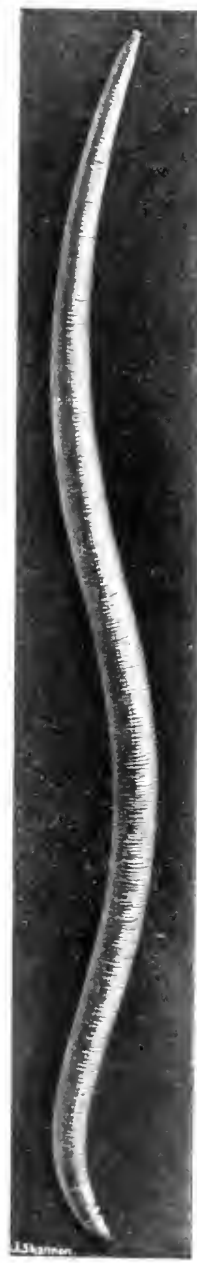

Fig. 86.-Female adult Ascaris lumbricoides. (Original.) is frecpuently parasitic within the bowel of man, particularly children between the ages of three and ten years. Ascaris lumbricoides usually finds it habitat in the small intestine, though it has been known to wander into the duets, stomach, esophagus, appendix, and, more frecpuently, the colon, where in the author's and Wheland's (Figs. 88, 89) cases the parasites accumulated in large numbers, became knotted, and caused intestinal olstruction.

Usually in this country not more than half a dozen are harbored by the patient, but in tropical countries hundreds may be present at the same time, and a large number may be voided daily.

The infection occurs from eggs which gain entrance to the body through water or food, and

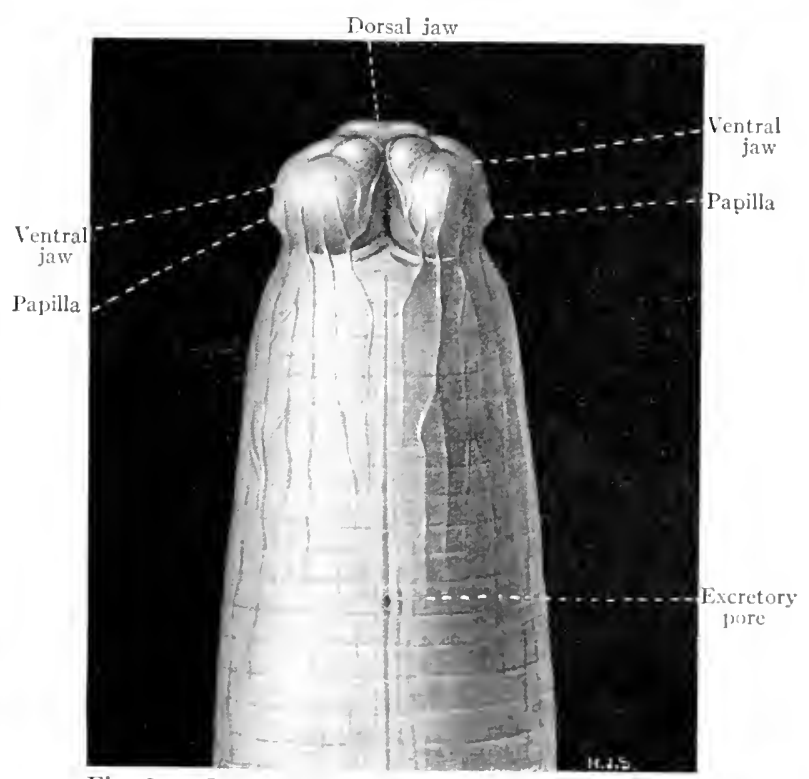

Fig. 8,-- - Ilead of Ascaris lumbricoides. (0)riginal.)

follows the dissolving of their shell-like covering ly the gastric juice. The worms attain maturity in about a month from the time eggs appear in the feces.

Symptoms.-Occasionally Ascarides lumbricoides give rise to no, symptoms, but more often they are accompanied by irritability, 
renlessness, irregular appetite, disturbed sleep, nausea, romiting, itching at the nose or anus, congestion of the intestinal mucosa, colicky pains, indigestion, eonstipation or diarrhea, and oceasionally convulsions in the very young. In exeeptional instances they migrate

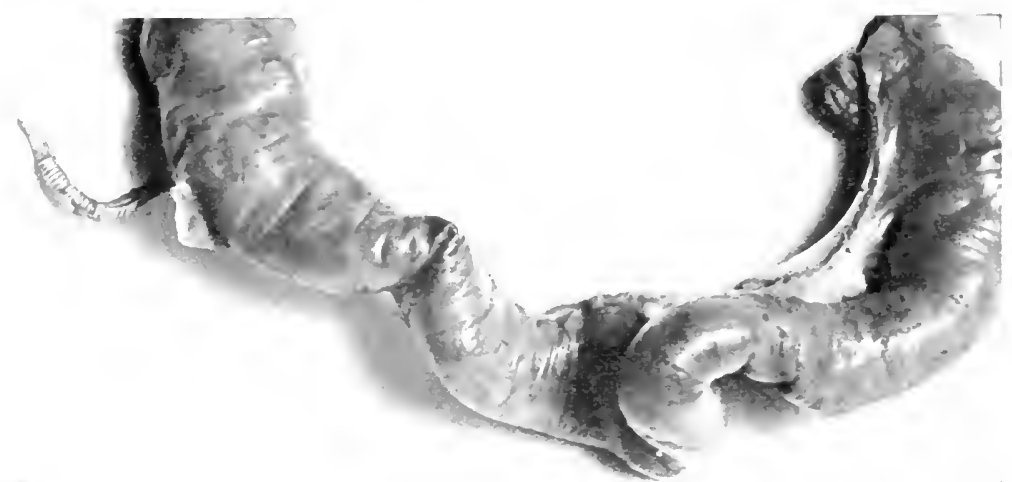

Fig. s. - Complete obstruction of the small inlesline and perforation caused by" Ascaris lumbricoides. Appearance of bowel before it was opened.

t1) the liver and ducts or perforate the intestinal wall (Fig. 88), and give rise to peritonitis or collect in masses and cause obstruction and other grave manifestations.

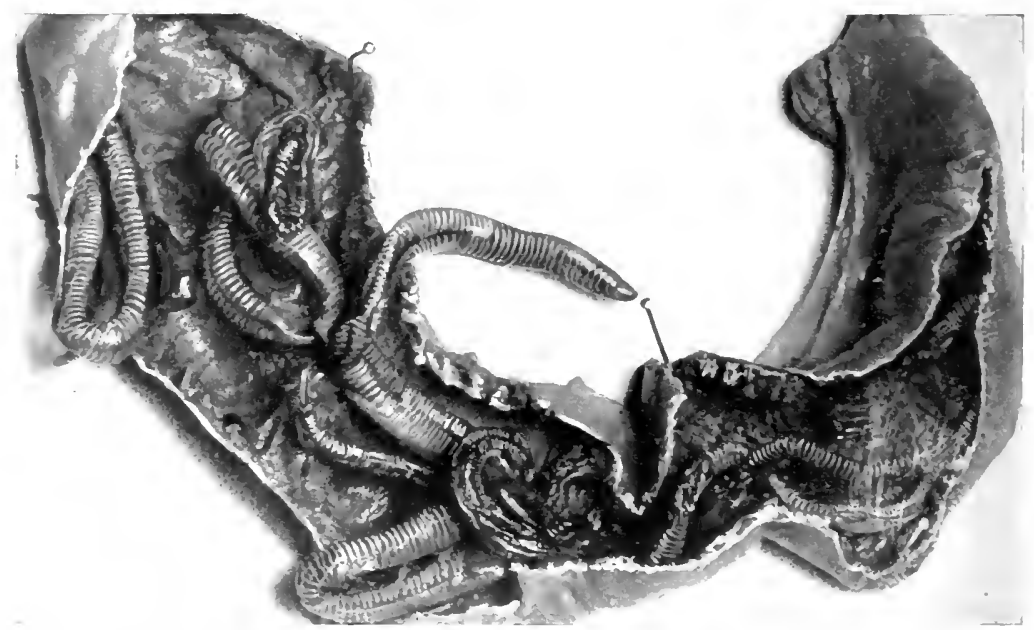

Fig. So-collection of Loraris lumbricoides which caused complete ob-truction and perforation of the small inteatine. Appearance of cut after being opened.

Diagnosis. In the presence of the above stmptoms ascariasis ought to be suspected, and the stools should be repeatedly examined macroscopically for athlt worns and microscopically for their ora. When located in the lower sigmoid or reetum Ascaris lumbricoides can be detected through the sigmoidoscope. 
Treatment of Ascariasis. - The elejectat of persons harboring these

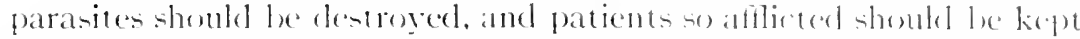
clean (particularly their hands) to prevent extemsion of the infection 10 others.

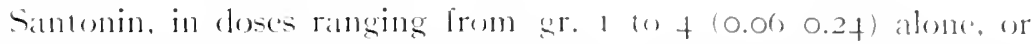
in conjunction with medium-sized doses of castor oil or calomed, all-

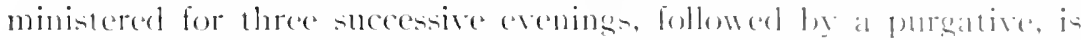
the hest-known agent for expelling the werms, particulaty when the patient's intestine has been emptiod betore the treatment is feerun. The amount of santonin atministered to children should le alsout one-sixth that given an arlult, and the treatment can be repeated alter a few weeks hase elapsed. Cohnheim recommends the bollowing formula:

1\% Santonini I Calomel , $\bar{a} \bar{a} \operatorname{cr}$. ss to is:s $(0.03-0.10)$;

Saccharianin

Fi. puls: Dos. vi.

Sir.- I powder night and morning.

P olei chenopodii ,

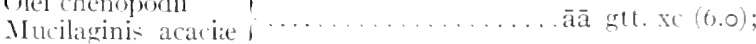

Aque distillata

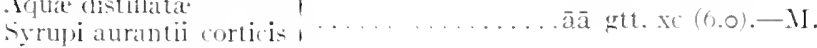

Ft. emulsio.

Sig.- One-half teasjoonful three times daily:

When the worms are almost lifeless or cling closely to the bowel, their expulsion can be hastened by frectuent enpious high siline enematil.

Asciris c.tris and A. Mrstax, so eommon in dogs and cats, have been encountered in the intestine of man, but so selelom that their further consideration is mnecessary.

\section{OXYURIASIS, DIARRHEA IN}

\section{OXYURIS VERMICULARIS PIN- OR THREAD-WORMS}

The parasite of oxyuriasis is so small, $\frac{1}{10}$ to $\frac{2}{3}$ inch $(3-5 \mathrm{~mm}$.), that it is freefuently overlooked, except when the examination is esunducted with a magnifying glass. Pinworm infection is farlycommon throughout the worlel, lut children suffer from it more frepuently than atults. Their habitat is the small intestine and cocum, where, after impregnating the opposite sex, male worms usually dice fut, as siler sigs. the latter sometimes reach the cecum and appendix in large numbers. Female worms (Fig. ()o migrate to the cecum, colom, and particularly the rectum. from whence they are ejecterl with the feresor worm the ir way through the anus, after which they pasis a the perianal thin. gluteal folds, vagina, or elsewhere, depositing lheir eges and call-ing itching and irritition.

Symptoms. - The chiof manifestations of the Oxyuris ermicularis is intense perianal itching (pruritus), nerveusuess, and, when they are 
particularly" numerous, dysenteric stools, restlessness, insomnia, itching of the nose, and in children chorea or convulsions.

Diagnosis.-Frequenty the author has discorered these worms both by macroscopic examination and with a hand magnifying glass.

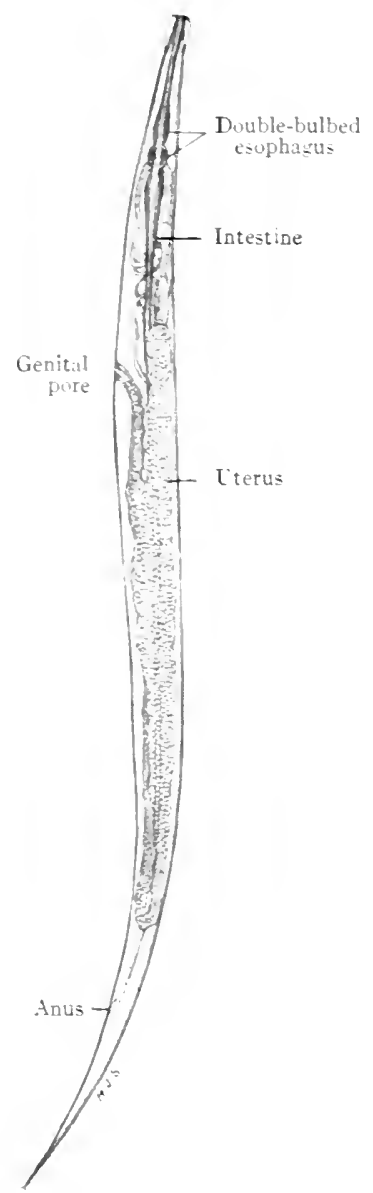

Fig. oo-osyuris rermiculari. female. Uriginal.) There are times when they are not present in the anal folds, and one must rely upon finding their ora (Fig. (9) in the stools with the aid of the microscope, and if nectssary scrapings from the mucosa and perianal skin should be examined.

Treatment.-Care must be taken to prevent reinfection, otherwise parasites or their ora will find their way into the body again (by way of the mouth or anus) in patients who bite their finger-nails or scratch the buttocks.

In some instances repeated purgation serves to clear the intestine of threadworms and their ova, but usually santonin, gr. j to iv $(0.06-0.24)$, or some other reliable anthelmintic, in conjunction with frequent copious saline, $5 \mathrm{j}$ to $\mathrm{Oj}$ (4.O500.0), acid-rinegar, $5 \mathrm{i}$ ss to $\mathrm{Oj}$ (6.0$500.0)$, or astringent-quassia, $\bar{j} \mathrm{j}$ to $\mathrm{Oj}$ (30.0-500.0). turpentine. $5 \mathrm{j}$ to $\mathrm{Oj}(4.0-$ $500.0)$, benzin, एu to $\mathrm{x}(0.30-0.60)$, or

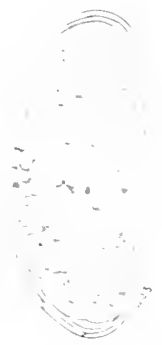

Fig. or.-Egg oi Oxyuri- rermicularis, (Original.)

ichthyol 5 per cent.) irrigations are necessary to effect a permanent cure and relieve proctitis. Satisfactory and quick results have been obtained in several of the author's cases by through-and-through medicated or saline irrigation following appendicostomy and cecostomy.

Direct bowel treatment following the establishment of a cecal or appendiceal vent is particularly valuable when, in addition to worms, the patient suffers from entamedic or bacillary colitis. 
Trichuriasis, Trichocephaliasis, DIARrheA IN

TRICHURIS TRICHIURA (WHIP-WORM, TRICHOCEPHALUS DISPAR, TRICHOCEPHALUS TRICHIURUS, TRICHOCEPHALUS HOMINIS , ASCARIS TRICHIURAE

This helminth, owing to its resemblance to a black snake or whip, is commonly designated whip-worm. Its distribution is worliwide,

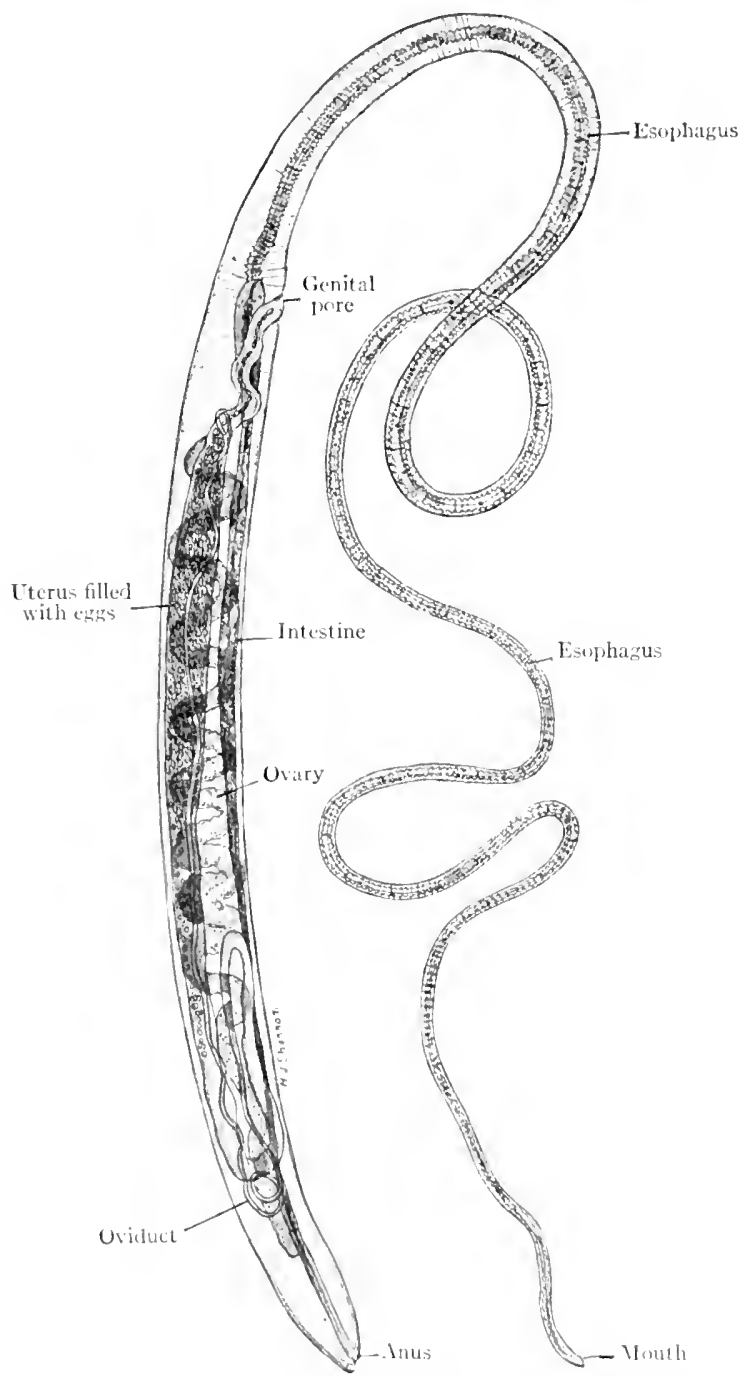

Fig. 02.-Trichuris trichiura, female. (()riginat.)

but infection from this species of worm is very common in the treppes, and in some combries more than half the population hathor them. 
The TRICHCRCS TRICHILRes ( $\mathrm{Fi}$ 92) is harbored chiefly in the cecum, but exceptionally it is encountered in other parts of the colon and rectum, and causes little trouble other than slight intestinal disturbances, except when numerous, and they cause anemia, penetrate the intestinal wall, or transfix mucous folds and provide a way for infection ly the intestinal organisms.

Symptoms. - Mild induce slight or no manifestations, while heary infections are characterized by digestive disturbances, the dysenteric symptom-complex, abdominal pain and frequent eracuations containing blood and mucus; restlessness, headache, and anemia.

The diagnosis is made by finding the ova or whip-worms in the dejecta.

Treatment.-Whip-worms are more difficult to eliminate than other helminths, and no specific agent has been discovered for them, though thymol, gr. Xv. (I.O), administered three times daily, gives the best results.

\section{STRONGYLOIDOSIS, DIARRHEA IN}

\section{STRONGYLOIDES INTESTINALIS STRONGYLOIDES STERCORALIS, ANGUIL- LULA STERCORALIS, RHABDITIS INTESTINALIS, SEPTODERA IN- TESTINALIS}

The nematode of strongyloidosis closely resembles a diminutive snake and differs from other microscopic worms in the manner of its sudden flash across the microscopic field; it abounds in Cochin-China and ltaly, has been encountered less often in different sections of America, Asia, and the Philippines, is more prevalent in tropical climates, and is frequently associated with anemia.

Norms of this class find a habitat in the upper small intestine and show a tendency to penetrate the mucosa and glands of Lieberkühn, where they derive nourishment and deposit eggs which develop in the gut wall.

Symptoms. - Strongyloides intestinalis may be present and cause very little discomfort, but usually are manifest in great numbers and cause irritative diarrhea (dyentery), evidence of which is found in the fact that they are abundantly present in Cochin-China diarrhea, and the number of embryos found in this type of infection is enormously augmented when the evacuations are very frequent.

The diagnosis of Strongyloides intestinalis is determined by detecting their ova or larve in the evacuations; the former are rarely discoverable except following active purgation, and the latter must be carefully protected in water, otherwise they will die before fully developecl.

Treatment.-Measures which will prevent infection taking place by way of the mouth or through the skin are indicated, and thymol, gr. Xr (I.O), should be arlministered as often as required to cause expulsion of the worms and their products.

Csophagostona BRumpti-This worm was discovered by Brumpt (1902) in cyst-like deposits located in the cecum and colon of an 
African negro. It may be found free in the loned or as encysted larvat beneath the mucosa; endirgements which vary from pinhead to hazelnut size and contain a coilect-up parasile. little is known of thoir symptomatology and pathology further than that they penetrote ihe gut, are blood-suckers, and cause anemial and irritation, nervousness. and diarrhea.

Trichostroxoluse- Three types of this parasite - Trichostronsylus instabilis, T. proboluris, and T. vitrinus - are comnunly found in the duodenum of animals, sometimes accidentally find their war into the intestine of man (Egyptian peasants), hut have no pathologic significance.

The Trionontophore's DImINetes has been encountered twice in the large intestine of man, and the srmptoms protecerl he it were-imilar to those of hookworm, and because of this Siler recommends thrmol in their treatment.

Physaloptera (caucasica and mordens) have been discorerel in the intestine of man, but in so far as known do not cause diarrhea.

Strongylus gibson $i$ is a rare species of intestinal paratsites which wats encountered in the dejecta of a Chinaman. Its symptomatology is unknown.

\section{TREMATODES (FLUKE-WORMS)}

\section{SCHISTOSOMIASIS, DiaRRHEA IN}

Etiology.-This affection is fairly common in tropical and semitropical countries. From Igof-og inclusive Brayton discovered lof cates among laborers working upon the Panama Canal, lut intestinal schistosomiasis is comparatively rare, and when it results from infection by the Schistosoma japonica. S. mansoni, or S. hamatobium the manifestations closely similate those of entamebic and bacillary colitis (dysentery), for which it is often mistaken.

There are many different kinds of fluke-aiorms parasitic in man, but only a few have been associated with diarrhea, and some of these. though not harbored in the bowel, reflexly or otherwise, induce diarrhea and other intestinal disturbances. Among these are:
(1) Schistosomide (bloor-llukes).
S.histosoma japonicit.
S. mansoni.
IS. hamatobium (Bilharzia hamatobia).
(2) Ctudorchis üatsoni (I'arumphistomus üatsoni).
(3) Fasciolata ilocana.
(4) Fasciolopsis buskii (Distoma bustii).
(5) Heterophyes (Distoma heterophyes).
(6) Gastrodiscus hominis ( 1 mphistoma hominis).
(7) Fasciolopsis fullchorni.

Schistosoma Japonica.-In Igot Katsurada discorered the Huke. Since then it has been repeatedly encountered in China. South Africa. the Philippine Islands, and Central Japan. This type of schistosomiasis is endemic, and the trematode causing it closely resembles Schistosoma hamatobium, but differs in that it is smaller. and the skin 
of the male parasite is smooth and devoid of spines and the ventral sucker is larger than the oral.

Pathology.-Schistosoma japonica infection is characterized by enlargement of the liver and spleen, anemia, cachexia, diarrhea, and exhaustion. Ova have been encountered in the stools, portal vein, liver, mesenteric glands, mesocolon, and intestinal wall.

It is thought that the habitat of the Schistosoma japonica is in the arteries and that of the $S$. hamatobium in the venous system. In Katto's case ova were widely distributed, being found throughout the submucosa from the cecum to the anus, liver, gall-bladder, pancreas, mesenteric glands, and tunics of the mesenteric vessels.

In addition to the above changes in this case there were eviflences of recurring peritonitis, enlarged appendices epiploica and mesenteric glands; obliteration of the rectoresical pouch and marked thickening of the colon throughout the mucous membrane, which was congested, inflamed, friable, marked by erosions, and necrotic; the bowel wall was cartilaginous-like and the rectum was enormously enlarged, hardened, and $\frac{3}{4}$ inch $(1.87 \mathrm{~cm}$.) thick at the point where it adhered to the bladder. The sigmoid was similarly affected and became thinner from below upward. The posterior bladder wall was thickened, but the organ was otherwise healthy. When sectioned the rectum resembled cartilaginous tissue.

In Katayama's case, in addition to the above, the brain and its covering were involved and contained ova. It has been pointed out that Schistosoma japonica does not involve the bladder, and in making a diagnosis this type of infection must be differentiated from Ankylostoma duodenale.

Schistosoma Mansoni.--In I903 Manson treated a West Indian, in whom he detected lateral spined ova, who never suffered from hematuria, and suggested the possibility of there being a new species of schistosoma; later Sambon discovered a fluke which differed from others in its distribution, pathology, appearance of its ora, and which had lateral spines, and named it Schistosoma mansoni.

The authorities have not as yet accepted this new species, which resembles Schistosoma hamatobium, and will not, according to Siler, until it has been demonstrated that the adult flukes are anatomically dissimilar.

Manson say's Schistosoma mansoni inhabits chiefly the mesenteric veins, and its ova are deposited in the submucous layer of the rectum and give rise to dysenteric symptoms-diarrhea, tenesmus, and mucus, blood, and ova in the stools. Sometimes in such cases small, large, branching soft growths are to be felt inside the sphincter ani. They resemble adenomata, have been mistaken for piles, and may extend up the bowel as high as the sigmoid flexure. On tearing up these growths ova can be seen in the débris.

The eggs of Schistosoma mansoni have also been found in the liver, giving rise to a peculiar form of hepatic cirrhosis.

Schistosoma Hamatobium (Bilharsia Hamatobia, Distoma Hama- 
tobium). -This fluke (Figs. 93, 94), so frequently responsible for hematuria in the natives of Africa, Algeria, Syria, Persia, and particularly Egypt, where half the population sulfer from the infertion, wats discovered by Bilhare in 1851 . Siler reports having seen 2 calses in New York (ity, and in both instances the discatse was contracted in touth Africal.

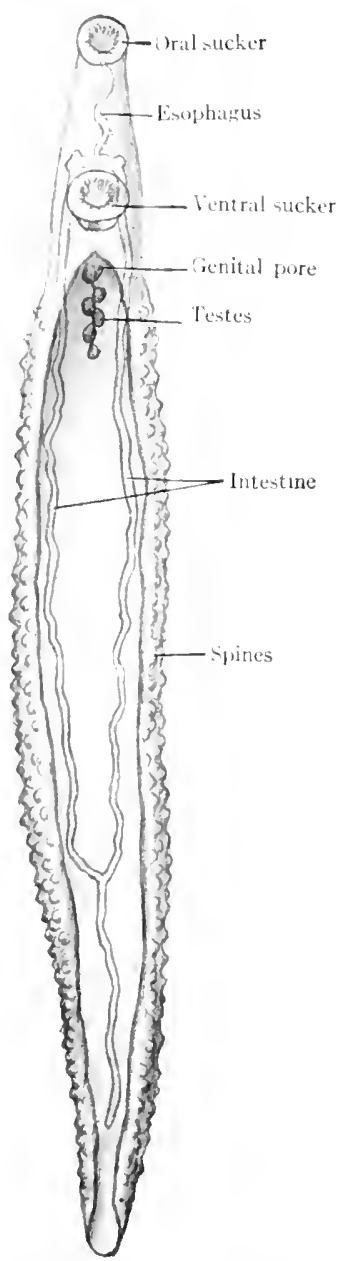

Fig. 03. - Schistosoma hamatobium, male. (Original.)

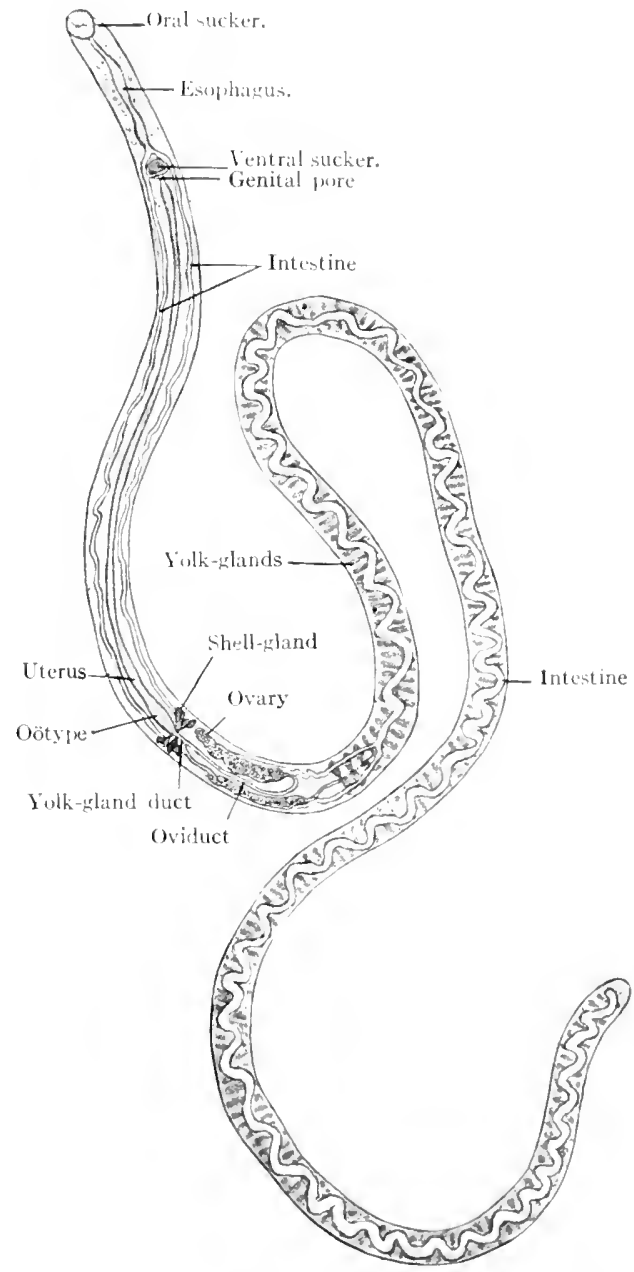

Fig. 0. - Schistosonat hamatobium, female. (Uriginal.)

These fluke-worms sometimes fund their way into the intestinal canal and thence pass onward to the portal rein, or they may gain entrance in other ways and migrate to the mesenteric, hemorrhoidal, vena cava, and other veins, where they are found in small or large numbers. 
Manoun relates that Loos has seren the submucous tissue of the bladder so fill of worms that a pair could be found in every area of $\frac{1}{5}$ inch $(0.5 \mathrm{~cm})=$. quare.

Symptoms. - The manifestation of schistosomiesis vary according to the degree of infection. and whether or not the Hukes are centered about the blatder (urinary schistosomiasis) or the bowel (intestinal. schistosomiasis.

In urinary schistosomiasis (endemic hematuria. bilharaiasis) hematuria is an tarly manifestation, and freftent micturition, straining, backache, perineal pain, and crstitis soon supervene; later, when ova enter the resical tunics, they become ulcerated or dotted orer with papillomata. Finally, the ureters and kidneys may become involved or abseeses form which result in urinary fistulit. When the rectoresical region is insaded and flukes enter the rectal reins the patient invariahly suffers from an aggravated type of diarhea, and where the ragina is involved the mucosa becomes thickened and cauliflower-like excrescences form, which when sectioned reveal both ora and adult parasites. These Hukes have also been frequenty en-

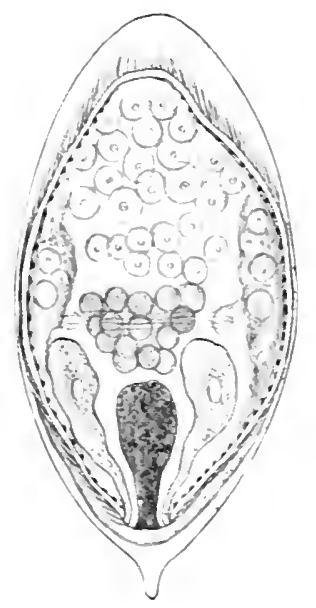

Fig. 05.- Developmental furm of schisusoma hremawbium ees =howing mirocidium. (Oricinal.) comtered in the lungs and kidneys. and their ova in varions parts of the body.

INTESTINAL SCHISTOSOMIAS is characterized by the so-called dysenteric group of symptoms-abdominal pain, watery evacuations containing mucus. pus. and blood, and tenesmus. When adult worms enter the veins of the walls in the large intestine and, more particularly, the rectum the gut becomes sreatly thickened, indurated, and gives a grity sensation when incised. Llceration of the rectal mucosa is very common. and when the infection result from Schistosoma. mansoni frequently papillomata can be seen projecting into the gut lumen.

These polypoid-like growths may extend high in the bowel or be large, located just above the sphincter ani. and be mistaken for hemorrhoids. Br incising and cureting such neoplasms adult fluke-worms and their ora can be exposed for examination.

Thickening of the liver, spleen, and peritonem is common, and in some instances the sacrococcrgeal region becomes infiltrated with ora and subcutaneous fistulie form.

The diagnosis of schistosomiasis is usually easy when a routine examination is made of the dejecta of patients who suffer from the dysenteric group of simptoms. particularly when the sufferer comes from a conntry where schistosomiasis is endemic.

Macroscopic and microscopic examinations of the stools for eggs Fir. 95) enable the examiner to exclude bacillary and entamebic dysen- 
tery, and demonstrate that the infertion is due to schistosomide, and determine which of the species is responsible for the trouble. When the colon and sigmoid thesure are involved, owing to its thickened and inchurated condition the gut can be plainly outlined lyg palpation, and when the diseare (as it ustrilly does in Schistosoma mansoni infection) attacks the rectum, the thickenerl ulcerated mucosit, hatelened bowel wall, and papillomatous growths can be noted by digital examination, and these and other lesions of the sigmoid and rectum can be aceurately inspected through the sigmoidoscope.

Urinary schistosomiasis (Schistosomal hamatobium infection) should be suspected in Egyptian and South African paticuts who suffer from hematuria and dysentery, and the urine and blood should be examined for special llukes and their ova, which can lue differentiated from the exgs of the other types owing to the arrangement of their spines.

Prognosis. - When schistosomiasis is discovered early and the patient is prevented from becoming reinfected the prognosis is good, but in neglected cases, where flukes and their ova have been permitted to accumulate in large numbers and enter the circulation and tissules in widely separated parts of the body, the subject's condition becomes deplorable or death ensues.

Under favorable conditions the symptoms induced by the ovid or adult fluke-worms may persist months or years after the patient has been removed to a non-infected clistrict.

Treatment.-Schistosomiasis is often caused by contaminated water, and because of this in suspecterl regions the water should be boiled or filtered, and precautions should be taken to prevent toilets and barns from draining into the water-supply.

No specific has been discovered which will destroy schistosomidie without poisoning the patient or injuring the rectal or vesical mucosal. By taking a slow course much can be done to add to the patient's comfort, heal lesions in the mucosa, minimize or get rid of the adult worms and their ova by irrigating the bladeler and intestine with mild antiseptic irrigations, keeping the patient fuiet, having him abstain from indiscretions, restricting his diet to bland nutritious foods, and giving him urotropin, gr. v (0.30), three times daily, when hematuria is a complication.

Methylene-lilue in 3-gr. (O.IS) doses or fludextract of male fern, mev (0.30), three times daily, have proverl useful in these cases.

- Stones may be crushed or washed out ly suprapubic or, preferably, perineal cystotomy and lrainage.

In intestinal schistosomiasis where infection is mild a great deal can be done to relieve the patient and minimize or get rid of the parasites by ichthỹol, balsam of Peru, permanganate of potassium, 2 per cent.. or weak silver irrigations introducel through the anus or an appendiceal or cecal opening. The inflamed and inlecrated rectal mucosal of the lower bowel can be benefited or healed by fairly strong topical applications or electric cauterization, and papillomatis within caln be 
snared or clamped off with a Gant valve-clamp. When the rectum and sigmoid are extensively involved and the mucosa is covered by numerous excrescences an artificial anus or extirpation is indicated, and in cases where the colon is similarly incapacitated it should be remored or excluded by dividing the ileum and uniting it below the seat of infection to the sigmoid flexure or rectum.

Where adult worms or their ova find their way into the subcutaneous structures in the coccygeal and sacral regions or elsewhere about the rectum, accumulate, and form abscesses and fistula, the latter should be incised, cureted, and drained.

Cladorchis Watsoni.-This tluke was discovered by Watson in a German Nest African negro, who succumbed to starvation and obstinate diarrhea, wherein the evacuations were frequent, fluid, bile stained, and contained many yellowish, translucent, and ovoid-shaped parasites. Autopsy revealed a jejunum filled with live and dead trematodes (flukes), some of which were attached to the congested mucous membrane, but the parasites had not affected other segments of the bowel or other organs.

Fasciolata Ilocana.-This parasite was discovered by Garrison in 1907 , who examined the dejecta of 5000 natives living in the provinces of the island of Luzon, and fomnd it in 5 instances. In these and other cases the eggs of this fluke were observed in the stools, and in one instance, with the aid of male fern, twenty-one parasites were expelled.

The manifestations incident to the parasites were unimportant.

Fasciolopsis Buskii.-This parasite has been encountered more frequently than the above, and shows a prevalence for India, Borneo, Southern China, and Sumatra; its habitat is the small bowel, and it induces diarrhea, probably through the local irritation which it induces.

Heterophyes.-This, the Egyptian intestinal fluke, was discovered by Bilharz (I85I), at Cairo, in the bowel of a child. More recently it has been encountered fairly often in Egypt and occasionally in Japan. This fluke is probably more common than is beliered, but is rarely detected because the intestinal disturbances induced by it are slight, the nature of the infection is not suspected, and because dejecta are not examined.

Gastrodiscus Hominis.- This fluke has been encountered less than half a dozen times, and then in India. except in the case of a Filipino, and little or nothing is known of the manifestations incited by it. Lewis and MacConnell $(1876)$ at autopsy discovered it near the ileocecal vale, and Simpson ( 1857 ) found the parasite in the cecum and ascending colon of an Indian who died from cholera. It has suckers, enabling it to cling to the mucosa, which at the points of lodgment is congested and swollen. When parasites are present in large numbers they induce considerable irritation and diarrheal manifestations.

Fasciolopsis Fulleborni.-This parasite has been encountered once by Rodenwalt (I909) in a patient who suffered from a typhoid-like 
fever, and the diagnosis of these trematodes consists in finding either their eggs or adult tlukes in the dejecta during life, or in the fects or bowel at autopsy.

When repeated 15-gr. (1.O) doses of thymol proves incefiective, the treatment recommended for tapeworms usually brings relief.

From what has been said concerning HeLuixtusiss the importance of a routine macroscopic and microscopic examination of the dejecta of persons afflicted with diarrhea is obvious, for in this way only can ore determire if there is a helminthic infection, and if so, whether or not it is complicated by an entamebic, bacillary, or protozoal colitis which would aggravate the patient's condition. 


\title{
CHAPTER XXXVIII
}

\section{PROTOZOAL (PARASITIC) COLITIS (PROTOZOAL DYSEN- TERY), DIARRHEA IN}

\author{
BALANTIDIC, FLAGELLATE, CILIATE, COCCIDIC
}

Axim.uL protosod in one form or another have been frequently found associated with ordinary diarrhea (colitis) and the dysenteric symptom-complex. frequent stools containing blood and mucus, rectal tenesmus, and abdominal pain, and in some instances two or more varieties of protozoa have been met with in the same case.

Of the numerous types of protozoa, those most frequently observed under the above conditions are: (I) amebre and entamebce (rhisapodes); (2) Alugellates (mastigophores); (3) ciliates (infusoria, Balantidium coli), and $(4)$ coccidia (sporozoa).

\section{AMEBAE AND ENTAMEBAE}

The characteristics and causal relation of these protozoan organisms to colitis (dysentery) have already been fully discussed, and a further consideration of them would be out of place here.

\section{FLAGELLATES}

Flagellates possess whip-like processes (flagella) which aid them in their locomotion and feeding. Parasitic flagellates thrive in tropical and semitropical countries, where they are widely distributed. The organisms of this class which have been most frequently discovered in persons suffering from diarrhea and dysentery, and which are believed to be the cause of or an aggravating factor in these conditions, are: (1) Cercomonas hominis; (2) Trichomonas hominis, and (3) Lamblia intestinalis (Figs. 96-98). The parasites gain entrance to the body through the drinking of polluted water or eating food contaminated by infected mice or rats. The pathogenicity of flagellates has been established, and the degree of howel disturbance consequent upon them is directly proportionate to the number of parasites present, which explains why a flagellate colit is in one instance may be very mild, and in another extremely severe and characterized by the dysenteric symptom-complex.

Apparently, flagellates find a habitat in both the small or large intestine, where, through attaching themselves to the mucous membrane, they incite peristalsis, augment the secretion of mucus, and induce diarrhea rather by irritation than ulceration, as manifested in bacillary and entamebic colitis (dysentery). 
Individuals harboring flagellates occasionally suffer severely from diarrhea, and their stools contain an ablundanee of blosel, mucus, and sometimes pus. Lneter such cireumstances lhere mat le at dual infection, when Entamepta histolytica, bacilli of the Shige-Flexner or other type, or Balantidim coli are found in enjunction with flagellates, which would help to accoune for the serionsnes of the infection.

simon has olserved 50 cases of amelofe dyesentery wherein in a large proportion of the stools he found Cercomonas hominis and Entamerba histolytica.
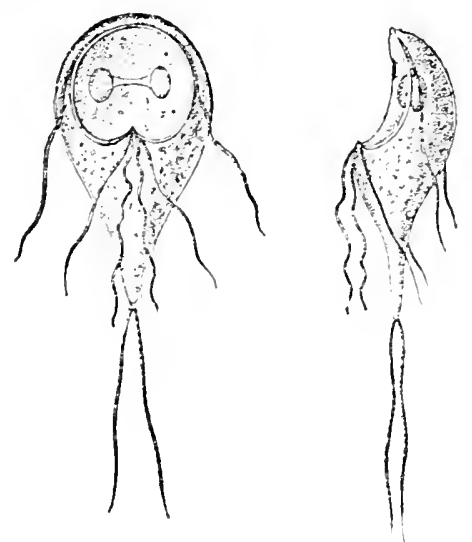

Fis. of.-Lamblia intestinalis, front and profile riew. (After Grassi and Skéviakov.)

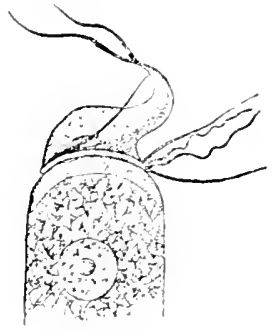

Fig. 97.-Lamblia intestinalis attached to an ejithelial cell. (After Cira-si and Skéviakor.)

It is reasonable to suppose that as a result of the trama or erosions to the mucosa induced by flagellates, mixed infection promptly ensues. participated in by colon and paratyphoid bacilli, streptococci, staphylococci, and other intestinal organisms which under favorable conditions manifest pathogenic propensities, to the end that numerous lesions variable in size are formed. Some investigators hold that flagellates are capable of affecting marked organic changes in the bowel, but
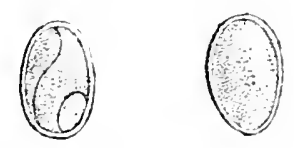

Figr. 98.-Cỵsts of Lamblia intestinalis. (After Grasci and Shériakor.)

Roos maintains that, while they undouldedly induce obstinate infestinal disturbances (dysentery, cte), they do not seem to posiesin independently the power of calsing deep intestinal lesions. Flagellates apparently do not penetrate the mucosa and lase been observed in the stools of healthy individuals, which would inclicate that. like entancber, they may be harbored within the intestine without harm until favorable conditions develop which would incise them to activity. 
Flagellates may, with the microscope, be seen in fresh dejecta actively motile or encysted.

Lamblia intestinalis are not always present in the stools, and for diagnostic purposes it is necessary to remove them from the intestinal epithelium to which they cling.

Spirochetes are varied and difficult to classify, because in some respects they resemble protozoa and in others bacteria. It is known that the Spirochcta pallida is concerned in syphilitic enterocolitis, since it has been discovered in the dejecta, discharges and scrapings taken from ulcers, and persons afflicted with intestinal syphilis. Other types of spirochetes (flagellates) have been so frequently observed by different investigators, in connection with inflammatory and ulcerative bowel affections, designated as spirillar dysentery, spirillar enteritis, diarrhee a spirochates, spirillosis intestinalis, etc., that they are now looked upon as a comparatively frequent cause of intestinal disturbances characterized by frequent bloody stools, abdominal discomfort, and rectal tenesmus.

Spirochetes have been frequently observed in the healthy dejecta and in the stools of patients suffering from colitis, and more particularly in the infantile form, as found in the tropics. "There can be little doubt that in various morbid conditions spirochetes find the surface of inflamed mucous membranes highly favorable to their growth, and that they multiply with extraordinary rapidity; but no fatal case of spirillar diarrhea has been described, nor do any definite postmortem reports appear to have been published. The pathology of the condition is, indeed, very doubtful, and it is questionable whether any etiologic function can be ascribed to the organisms" (Brown).

\section{CILIATES}

Ciliates, the most complex of protozoan parasites, have in recent years assumed considerable importance, because investigators who have been studying the relation of parasites to dysenteric and other intestinal affections have so frequently detected them in the dejecta alone and associated with Entamceba coli and histolytica, Shiga's, Flexner's, and Strong's bacilli, and other harmless and pathogenic micro-organisms.

Of the infusoria, the Balantidium minuteum (Fig. 99), Nyctotherus faba (Fig. IOO), N. giganteum, N. africanus (Fig. IOI), and Chilodon dentatus are the only types which have been observed in connection with dysenteric colitis in man. The Balantidium coli deserves special consideration here because of the frequency with which it has been encountered, while the other ciliates do not, and will not be discussed further because of the infrequency with which they are associated with intestinal disturbances complicated by diarrhea.

Balantidium Coli; Paramœcium Coli (Fig. 102).-The balantidia are the most important of the ciliates, and these infusoria, which are the largest of the protozoan parasites, were first described by Malmsten in $\mathbf{I} 857$, but the organisms and their relation to intestinal lesions 
and diarrhea were not carefully studied and determined until the present decade, and there remains yet much wo be leatred concernine their distribution and manner in which they gain entrance to and infect the colom.

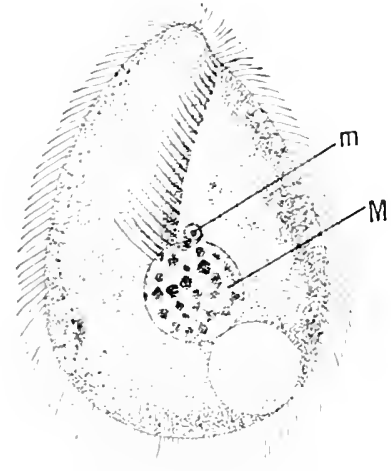

Fir. go.-Balantidium minuteum: M macronucleus; $m$. micronucleus. (Aiter Schaudinn.)

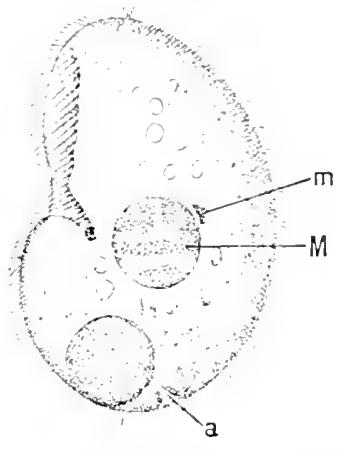

Fig. 100.-Nictotherus falsa: M. macronucleus; m. micronucleus; a, anus (After Schaudinn.)

The investigations of Strong, Musgrave, Opie, Brooks, Mitter. Doflein, Noe, Eberlein, and Bundle hare, in recent rears, alded greatly to our knowledee concerning the Balantidia coli. These infusoria have frequenty been discovered in the intestines of swine and

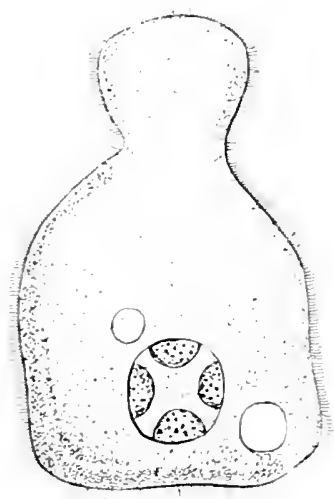

Fir. ror.-Nyetolherus africanus Castellani. (Castellani and Chalmers.)

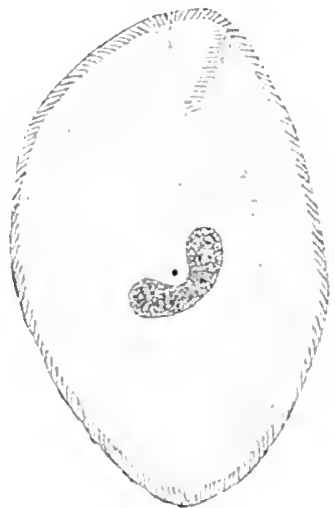

lïr. I02. Batantidium coli. from an ulcer of the large intestine of nan. Marsnitied, too: I. Braun and Jicike.)

less often in man, and in the former the halditat of thene oryanisms is in the cectm, hut in human beings they show a prevalence for the lower iletum, cectum, and rectum.

An epidemic of dysentery which oecurred among the orangentangs of the New York Zoölogical Park Was ascribed ln llarlow Brooki 
to Balantidium coli, and the symptoms and lesions were similar to those of balantidic colitis in man. The organism is less widely distributed than entanelye or the Shiga. Flexner, Strong. Kruse, ete., bacilli: nevertheless, it has been encounterel in one or more instances in Swerlen, Finland, Scandinavia, Rusia, Germany, Philippine Islands, China, Italy, the Lnited States and other sections of North America, and the author has observed a cate from New Jersey.

The recorded cases of balantidic colitis or dysentery show that thus far the disease has been met with more often in the first-named or colder countries than in warmer climates. 1nut some of the best investigator believe it will eventually he classified among the affections common to the temperate zones and tropics.

Balantidic infection supposedly results from the drinking of water, milk, or eating food contaminated hy the organisms, and it has been demonstrated that they can survive in water many hours.

Balantidia are most frequently found in individuals who have worked among hogs, made sausage, or eaten pork, and have never been encountered among Jems or Mohammedans in the tropics, and because of this some authorities hold that the discase is frequently, if not always. contracted from swine. The statistics of Musgrave's II collected cases support this riew, because 25 per cent. of the patients gave a history which pointed to infection from this source.

The manner in which the infection takes place has not been definitely shown. and it is thought that some active balantidia are destroyed by the gastric juice, and that others are protected by encystment or attenuated by the gastric contents and do not infect the gut until they reach their favorite places of habitat-viz., the lower ileum. cecum, or rectum.

Bowman has carefully investigated human balantidia relative to this point, but was unable to find evidences of encrstment: nevertheless he belieres that if man is infected through pork, it probably results from the resistant encysted forms.

Cultivation of the organisms has not proved satisfactory, though balantidia have been known to live from one to six days on suitable media. A number of attempts have been made to infect animats with balantidia, but nothing has come of the experiments. Bowman persistently tried and failed. though he injected infected feces into the rectum of a monker, introduced them into the large bowel through a colostomy opening. and sutured a section of an ulcer removed from a patient suffering from balantidic colitis to the animal's colon. but the result- were negative in ach instance.

Balantidia have been repeatedly found in the feces from the intestine of healthy individuals, persons who suffered slightly from diarrhea. and patients afflicted with severe ulecrative colitis, where they evelenty were inciting factors. From this it appears that under varying conditions these organisms may he harmless, induce mild intestina? disturbances, or cause malienant dysentery (colitis), but most often they have been associated with proctosigmoiditis. 
Altogether about 125 cases of so-called balantidic dysentery (colitis) have been recorded, and of these instances the Balantidium coli was evidently the chief etiologir fartor, lecante it was rarely encountered in the presence of other parasites, helminths, or the Shiga. Flexner, Kruse, or other dysenteric bateilli, and almost never in entjunction with the Entamoba histolytica. The author has treated a few patients whose feces contained entameber and balanticlia and Hanes has treated several.

In Musgrave's collected cases (117) there were 2 (hildren and I 5 adults, and except in 2 instances the patients suffered from diarrheat or the dysenteric symptom-complex.

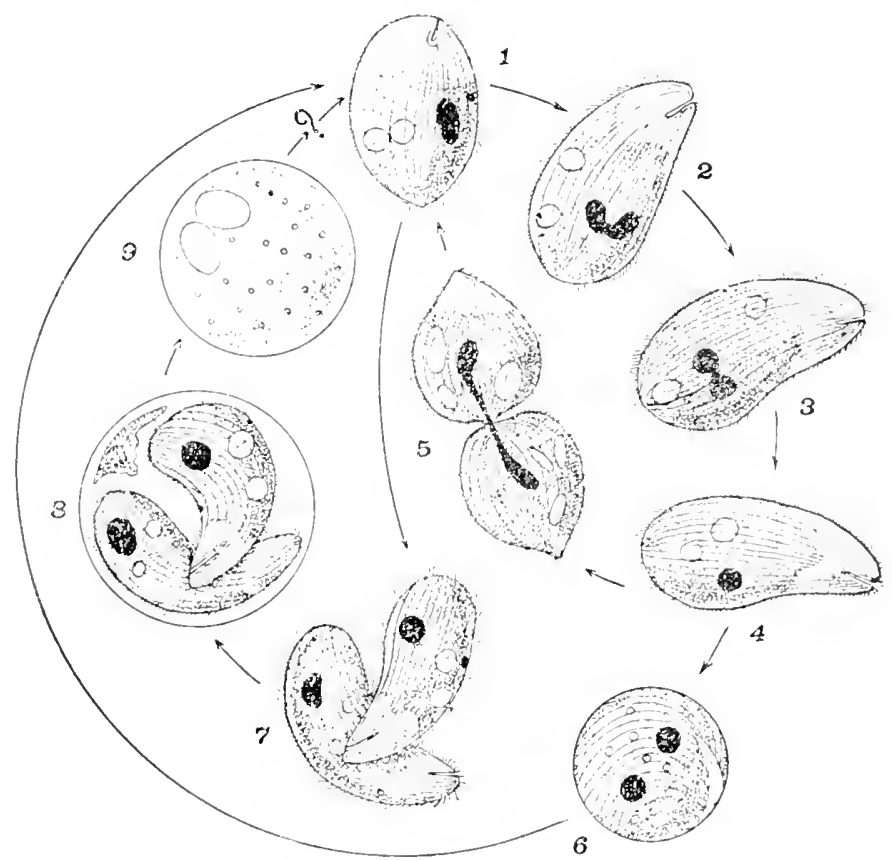

Fir. 103.-Reproduction of Balantidium coli: 1, 2, 3, 4, 5. Ascxual reproduction by sextuple generation; 6 , resistant cyst with a single individual; 7 . coprulation of two infusoria; 8 , reproduction-cyst; 9 . cyst contents.

The Balantidium coli has an egg-shaped body and measures from 0.07 to $0.1 \mathrm{~mm}$. in length by 0.05 to $0.07 \mathrm{~mm}$. in wiclth, dimensions which make it possible for the organism to be seen with the natkerl eve in mucus collected from the rectum. It is enveloped by transparent protoplasm studeled with cilia, which are factors in its forward and rotary motions, and which at the peristome asime greater length, where they aid in corralling food elements. The organism is rounded at the anterior end (the location of the funnel-shaped slit or peristome), upon the ventral surface of which is situated the culdesac-like 
mouth, which sone believe ends as a furrow, and in others leads to a gullet, and is round but broader at the posterior extremity, wherein is located the anal outlet.

Balantidia possess a large bean-shaped macronucleus and a small round micronucleus (near the anterior pole), and in the glandular protoplasm which fills its interior are regularly found two or more contracting vacuoles and occasionally hat and starch granules and red and white corpuscles.

Reproduction of the Bakantidium coli takes place by budding, simple transierse division, and sexual conjugation (Fig. I $0_{3}$ ). " In unfavorable conditions of life, such as it is likely to meet when expelled from the intestine, and possibly also as a normal stage of sexual reproduction, balantidium encysts and form minute sporocytes of exceptional vitality and tenacity. These are doubtless the medium by which in ordinary circumstances infection is conveyed from host to host" (Brown).

In fluid dejecta this protozoan parasite is markedly active, evidences of which are to be seen with the eve or microscope by its almost incessant forward and rotary movements, and the form of the organism changes to meet conditions when during its travels it meets with
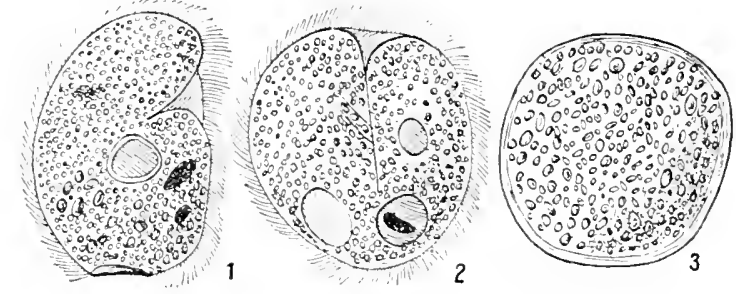

Fig. 10.4.-Balantidium giganteum. (After Krause.)

an obstruction, as, for instance, a projection of it may become narrow and like a pseudopod, which, if sectioned, wonld resemble the budding reproduction of the organism.

The Balantidia coli, like other ciliata and flagellata, are usually encountered in pairs (Fig. Iof) or large numbers, both in prepared feces and the stained section of intestinal ulcers taken from patients afflicted with balantidic colitis.

"The rapid circular motion of the peristome cilia seems to exert an attraction which brings the organisms in contact. I have observed many times in fresh feces two of them moving along rapidly in the fecal material; suddenly, on approaching each other, dart together and remain in this position for a considerable period of time, though no interchange of material could be seen taking place between them and no evidence of reproductive change" (Bowman).

In persons who harbor balantidia the organisms may be constantly or intermittently present in the feces, and usually manifestations of the discase are more marked in proportion to the number present, though cases are on record where they were abundant in the stools and the patient suffered from nothing more than a mild proctosigmoiditis. 
IIistopathology. - The changes which lake place in the bowel incident to balantidic colitis are variable, being sliegle in some and extensive in other cases (see lig. IOg), and it alphear that the are influenced by the patient s physical condition at the time of infertion, other enmplicating diseases, and virulence of the infertion, for, ats has already been shown, balantidia have been observed in the feres and intestine of healthy individuals, those who suffered from mild trpes of diarthea,

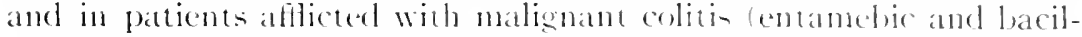
lary), as evidenced hy frepuent evacuations containing an alsundance of mucus, pus, blood, with temesums and characteristic aldeminal pains.

Autopsies have been made in about 35 cases of halanticlic colitis, in some of which there was a catarrhal coneretion of the mucosat, in

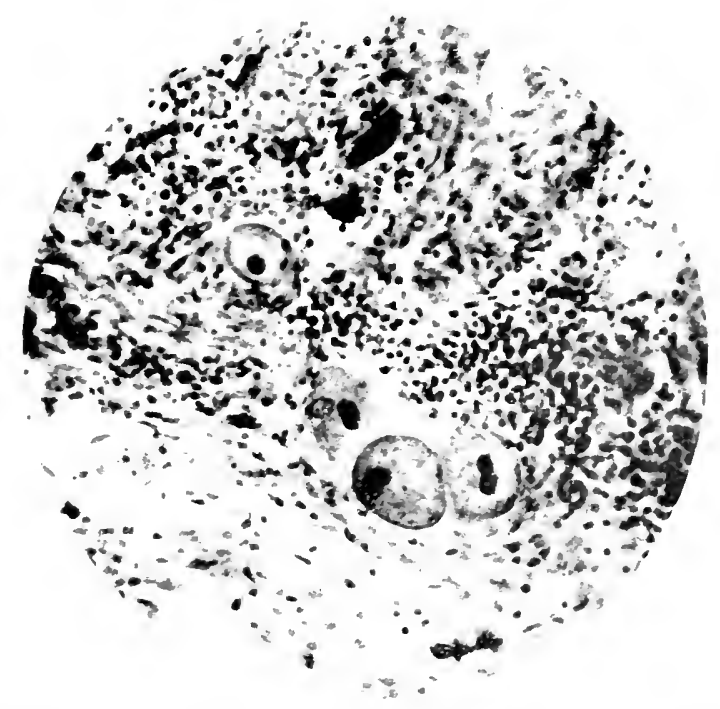

Fig. I05.-Balantidium coli pasing from mucosa throuen the muscularis mucosa. ( liter Strone.)

others diphtheric patches, and still others (which onstituled the majority) where an extensive ulceration marked the discats (see lig. Ior).

The disease is insidious and the tissue changes are slow but conttinuousty progressive long after the infection has lecome extensive, and the lesions in many respects resemble these of entamelic colitis, which form very much more rapielly. Balamidic uleers have leen known to involve the lower ileum (exceptionally) along with the laree intestine, but the disease is most destructive in the colon (Fin. 105), and more particularly the cecum and rectum, where the lexions are numerous and deep as a result of necrosis which occurs about the infected areas.

Balantidia are not solely responsible for the excavated uleer-present, because here. as in other bowel disturbances accompanied hy displacement of the epithelium, there is a mixed infection participaterl 
in both by the specific agent (Balantidium coli) and the colon or paratyphoid bacilli, streptococci, staphylococci, or other pathogenic organisms in the gut. The ulcers (Fig. I06) of balantidic colitis are, if anthing. more virutent than those of entamebic (histolytica) infection, tend to a more prolonged chronicity, and do not respond as readily to treatment.

In a case observed hy the author the bowel from the upper extremity of the anal canal to the highest risible point of the sigmoid (through the sigmoidoscope) was involved in the destructive process; the ulcers were ton numerous to count. and were situated in close proximity to each other or orerlapped in some instances. The mucosa. as seen at the site of recent lesions, was smeared with mucus,

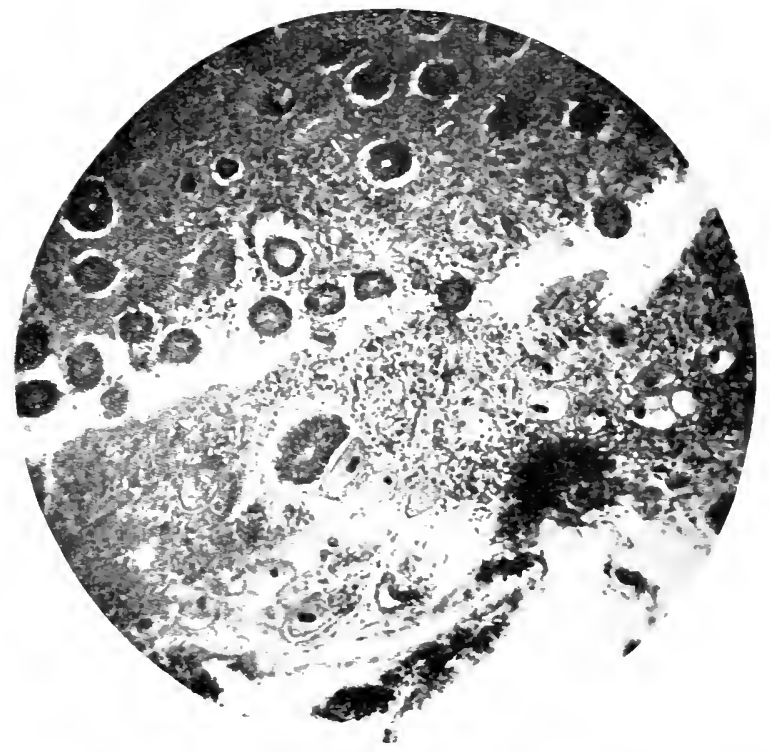

Fir. rob.-Balantidium colt in submucosa and surrounding tissue. (After Strong.)

tinged with blood, appeared darker than the healthy membrane. and it was thought that this was due to necrosis, since the tissue overhanging the edges of formed ulcers was necrotic. A number of infected areas were motermined, and in some instances yellow pus could be expresed through the ulcers when the adjacent mucosa was compressed with the finger, which showed that pyogenic bacteria had been active and diminutive abscesses had formed.

The dimensions of the lesions were not always the same, but typic ulcers varied from 0.4 to 3 or $5 \mathrm{~cm}$. in diameter, and differed from tubercular and syphilitic ulcers in that they exhibited no inclination to follow the direction of the hlood-ressels or collect in large numbers upon the mesenteric side of the bowel.

As a result of undermined and indurated rings about the sores 
the unbroken mucosa was thickened and presented an mneren, somewhat fobulated appearanee. The lesions of balantidie colitis oceat sionally penetrate the bowel tunies down to the seresia (see Fig. 100), and have, at least in one case, catused perforation of the cermun.

The anthor judged that in eases observed he him the entire colon and cecum were invelved like the sigmoid flextre and rectum, becatse the bowel could be plainly out lined by palpation owing we its indurated and thickened condition. Diarrheat wat persistent, luere Wals an abundance of mucus, pus, and blesel in the stoxls, and the patients complained of tenderness and pain when pressure was made ores the colon.

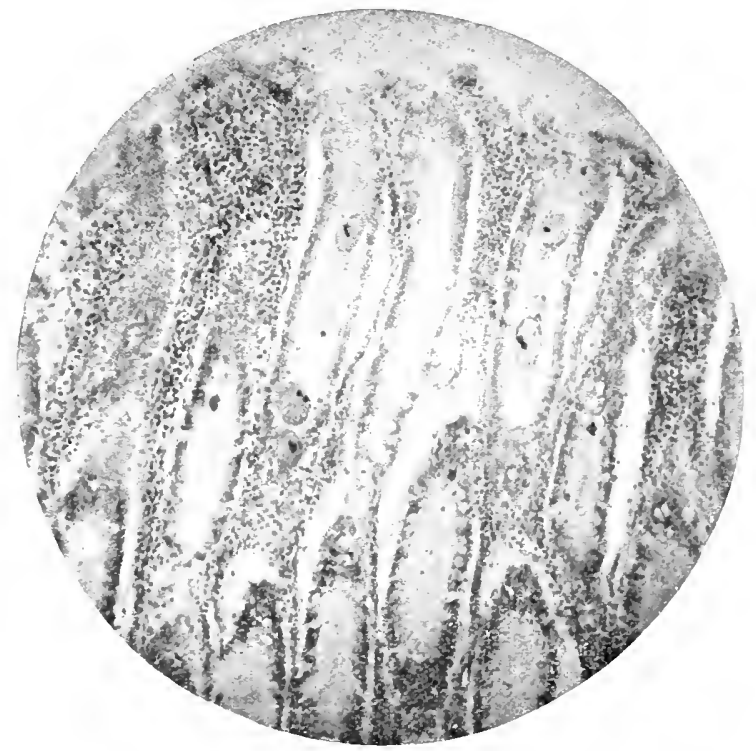

Figr 10\%,-Parasite- (balantidic) in mucosial. (After Strong.)

Bowman, who hats studied clinically 10 cases, 3 of which came to autopsy, says: "There is nothing to distinguish the late ulcerations

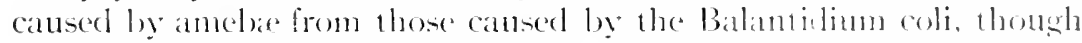
the early andic uleers appear to be somedines mone punctatle in character."

Balantidia, either alone or in conjunction with other organisms. have the power of penetrating the intestinal wall in some mamer, for they have been encommered free in the intestinal musesil, sulmustost,

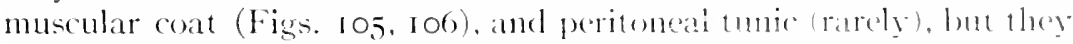
are met with much nore fresuently anel in latger numbers in the sulsmucosat than elsewhere, aud are surrounded with small cell infildation and eosinophits abound in laree mumbers.

Apparenty, balantidia can penctrate lhe normal mucous mem-

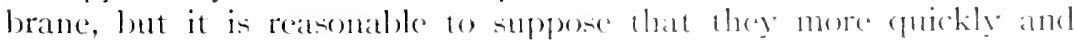
easily reach the deeper layere of the bowed when the epithelium hate 
already been disturbed or broken by catarrhal or other intestinal lesions prior to their coming in contact with them. Strong holds that the penetrating action of balanticlia is chiesly mechanical, and that their progress is closely followed by pathogenic bacteria which cause necrosis.

Balantidia hav abo been found in large numbers in the intestinal blood-resels, which are dilated and cause hemorrhagic areas, and reliable investigators incline to the belief that they may be transported to the liver and other organs through the lymph-channels, because parasites apparenty identical with them have been discovered in liver absceses, and it ha- been demonstrated that they frequently reach the gland of liaberkihn (Fig. 108).

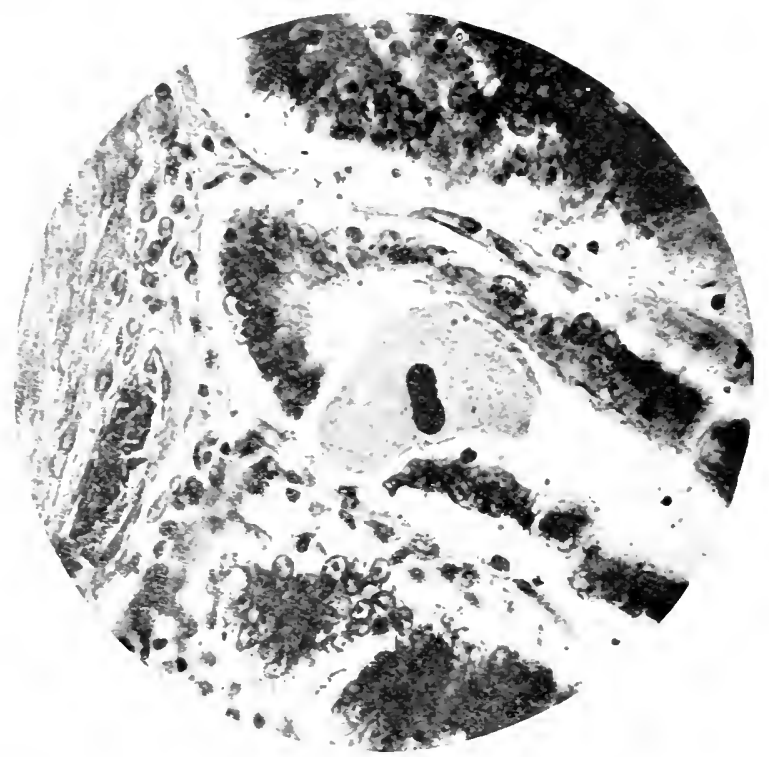

Fir. 108.-Parasite (Balantidium coli) passing through walls of a gland of Lieberkühn. (After Strong.)

"Where many of them are gathered together in the subglandular tissue, a surrounding zone, con-isting of plasma-cells and lymphocytes, is found, with an alnormal number of eosinophils, but with very few polymorphonuclear leukocyter preent. On the other hand, the cellular constituents of the necrotic material covering the ulcer consists largely of polymorphomuclear leukocytes. This fact would seem to indicate that the parasite, on first entering the tissue, causes a lowgrade infammation infependent of any bacteria which it may have carried with it. The intestinal hacteria are the terminal invaders and cause necrosis of the tissue already undermined and weakened by the balantidia. Lndoubtedly many of the primary ulcers are caused by orsanims entering the glandnlar laver, but. having once entered, ulceration may be the result of another process. On studying sections 
quite remote from any ulceration and though apparently uninjures mucosa, the organism may $b e$ fomel invaling the submucosal and muscular strata, while in the mucous later none are fombl. Where they are foumel singly there mat be no evielenes of inflammatory reatetion, but in other areas, where sereral of them are wathered to gether, there may be many round cells forming a surrounding zone, and in still other situations marked evidence of inflammatory reatetion. In a study of many sections all stages in this intlammatury process may be seen. From the single balantidium, which semingly has pushed itself medhanically through the tissue with litte cell reaction, to gromps of the parasites with surrounding zones of frmphocytes and plasma-cells which have infiltrated the mucosa itself, demonstrating a reaction which, with the invasion of intestinal hacteria, will terminate in ulceration.

"The organisms may enter the mesenteric lymph-glands and cause" necrosis. In one of many cases they were fomnd very numerous in one of the sectional glands, and there was some increase in the polymorphonuclear elements present, but no bacteria could be demonstrated and there was no evidence of tuberculosis. If the organism can invade the mesenteric lymph-glands, invasion of the lungs and fiver would seem much more probable" (Bowman).

Symptoms. - The manifestations of balantidic colitis (dyentery) come on slowly, and are similar to those of other forms of dysenteric colitis; conserfuenty, it is frecuently impossible to distinguish fretween them. The disease is nearly always chronic when recognized, hecaluse the disturbances at the inception of the infection are mild and the mature of the ailment is not suspected, in consequence of which the stools are rarely examined. At first balanticlia incite a catarhal coloproctitis, accompanied by an increased number of evacuations containing an abmormal amount of mucus and often undigested ford.

Shorty the patient begins to suffer from altermating attakes of diarthea and constipation, which, in turn, are followed by intermittent diarheal crises characterized by frepuent fluid evacuations and a comsiderable amount of pus and some blood in the dejecta. In neglected cases the attacks become more frecpuent and severe, and eventwally the patient presents the dyenteric symptom-complex-riz., Irequent bloody movements containing mucus, with tenesmus, aldelominal soreness, and pain on pressure (particutarly alomg the colon), anorexia, loss of weight, and digestive disturbances which contime during acule attacks, manifestations which almost completely subsicle in the intervats between the crises.

The attacks gratually lecome more frepulent and serere as the disease progresses, and after several momths the stonk beeome very mumerous, oflensive, and are composed chichly of blool (pure or in clots), mucus, pus, and debris consisting of necrotic tistue. By this time the patient is in a deplorable condition, and usmally suffers markedly from mental and loxtity exhauston, emariation, anemia, palpitation, indigestion, abdominal discomfort and pain, very fro- 
guent eracuations, colic, distresing tenesmus, occasionatly edema of the feet and ankles, and nansea or vomiting which are marked and complicated by abdominal distention in the presence of perforation and peritonitis.

Necropsies upon patients who hate dieel from balantidic colitis (dventery) have in different cases shown the bowel in various statesviz. congested from catarhal inflammation. partially covered with diphtheric patches, and extensizely involved hy uleration (Fig. Iog), varia-

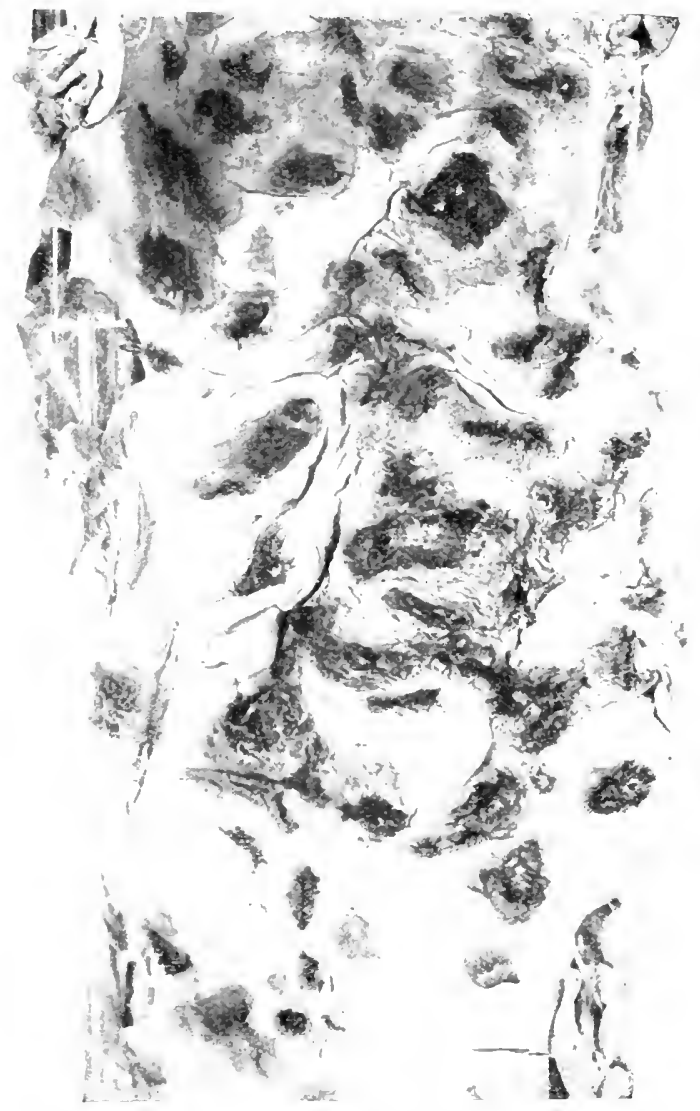

Fin. 100.--Photugraph of colon showing ukeration due to infertion with Batantirtium coli. (Aiter Bowman.)

tions which the author believes to represent stages of the disease under varying conditions rather than different types of infection.

Balantidia are usually discoverable in the dejecta while diarrheal prevails, and the number present seem to bear a direct relation to the severity of the attack, but the stonls contain few or no organisms during the quiescent periods of the disease.

Leukocytes may or may not be present. but eosinophilia characterizes the disease, which in some cases is partially accounted for by complicating helminths.

Liver abscesses, adhesive pleuritis, and enlarged mesenteric lymph-nodes have also complicated the affection.

Diagnosis.- Balanticlic ulcers can be plainly inspected through the sigmoidoscope. but it is often impossible to differentiate the lesions from those of other ypes of specific infections or coltis, consequenty the diagnosis depends mainly upon a careful macroscopic and microscopic examination of the dejecta, which will reveal the balantidia .mel shoukl eomplete the diagnosis in the absence of entamebe, helminths, and Shiga's, Flexner's, Strong's, ete, bacilli. 
The prognosis of balanticlie dyesentery (rolitis) is nest genel in any.

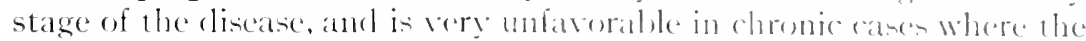

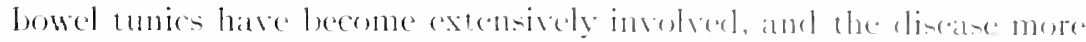
often terminates fatally than either hacillary, entamolis, or helminthic colitis (elyentery).

An analysis of the statistice concerning balantide colitio inclicates that the mortality in this allection is aluent 30 pere cent., lut this mortality is evielenty high, sinere the affection in many instances mato

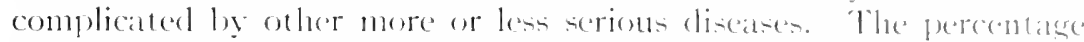
of permanent cures was approximately the same (30 per cent.) and slight or marked temporary improsement oremperl in the remaining cases. Naturally, a goosl many patients affleterl with halanticlic colitis are very weak and freptently attacked by one of the areute diseases, which may catuse their death.

TREATMEXT. - The therapeutic indications in balantidie are prastically the sance as those employerl in the treatment of entamelise colitis (dysentery) discussed in (haper XXXII, to which the reater is referred.

\section{COCCIDIA}

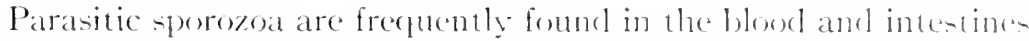
of cattle, rabluts, dogs, cats, pole-cats, rats. mice, and occasionally in the human alimentary tract. Three squecies of roceidia have lieen olserved in man, viz. Coccidium cumioli. C. hominis, and C. bisemimum. These oreanisms have often been discoserest in the feres on intestinal epithelium by investigators of entamelic, bacillars, and ot he forms of infectious colitis, and, while their pathosenticity in diarrleal affections has not been positively prosed, there is every reaten for believing that they bear a catial relation to such affections. and il non. they at least ageravate them.

Infection is accomplished by contaminated food, inhalation, and the bite of inserts.

The symptoms conseguent upon parasitic coecidia are anorexial. emaciation. Fever, diarrhea, with or without blow and mucus in the stools, and sonetimes a yetlowish mucoid natall and hucral dischatrece (in animals).

The diasnosis of eliarrhea from this source, in the athernee of ather causes, is based upon finding cocridia in the storks. intertinal epithe. lium, gall-rlucts, or liver.

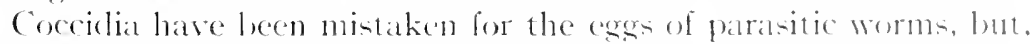

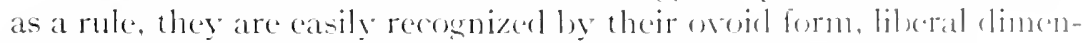
sions, and punctate depresion at one or the other extremity.

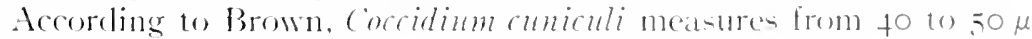
in its long, and from 22 to $28 \mu$ in its short, cliamoure, and c'. hominis, from $2510,301, y 151020 \mu$.

If the reader wishes further knowledgeconcerning the clat-ification. habits, and morphology of coccilia, he can find it in brown and Luhe's "Practical Parasitology," and Park's "Pathogenir Bacteria and Protozoa." 


\section{CH.APTER MAXIX}

\section{GONORRHEAL COLITIS AND PROCTITIS (INTESTINAL GONORRHEA), DIARRHEA IN}

Goxorrhe.AL inflammation involves the colon and rectum more often than is senerally supposed, is encountered in women and children more frequently than in men, occurs in the various walks of life and in all lands. but is observed more often in Europeans and Asiatics than in Americans because a greater percentage of the former are pederasts.

Conorrhea here as elsewhere is caused by the gonococcus of Neisser, and the anal or rectal mucosa first becomes infected. from whence the inflammatory process extends upward to the sigmoid flexure and colon.

In 75 per cent. or more of the cases the infection is limited to the anal canal. rectum, or sigmoid flexure, is acute, and accompanied by frequent eracuations and marked tenesmus. Chronic diarrhea may be a sequel of gonorrheal proctitis, but under such circumstances the stools rarely contain gonococci, and frecpent morements are incident to catarrhal inflammation or ulcers started by the original and continued by secondary or mixed infection.

On account of the cloce relation between the anus and ragina, and the cartes manner with which individuals having gonorrheal urethritis or vaginitis care for themselves (particularly the fingers), it is remarkable that the rectum is not more frequently infected when they scratch or wipe the anus. The author has treated many adults for gonorrheil proctitis contracted in the latter way. handled 3 men, admitted pederats. who caught the disease during rectal intercourse. and ha- treated o children ( 2 loy $=$ and + girls) for gonorrheal colitis (diarrhea) infected by nurses or governesses.

Pathology.- The pathologic changes of the mucosa in gonorrheal culoproctitis are less serious than those of tubercular, syphilitic, entamebic, or bacillary colitis because the ravages of mised infection are les. In the cases the mueous membrant within the infected zone is very highly inflamed, sensitive, greatly swollen, often edematous, smeared wer with foul-smelling pus, and occasionally marked by erosions. but is seldom extensively ulcerated.

The symptoms of gonorrheal differ from those of other types of coloproctitis in that the manifestations complained of are located chiefly in the lower howel. During the acutestage, tenesnus, burning pain, and sensations of weight and fulnes in the rectum are constant, and the patient has a never-ending desire to stool, which is not relieved hy an evacuation. The movement- are occasionally mushy or semi- 
soliel, but, as a rule, they are fluid, foul smelling, and romposed alumest entirely of thick yellow pus and muens. The acute manifestations ustually modify and quickly disappear withoun leaving secfurela under the treatment omblined betow.

Diagnosis.- comortheal coloproctitis can be diagnosed liv the acute symptoms characterizing the inflammation, inspecting the highly onngested and edematous rectal mucosia through the proctosecope and funding genococei in the discharge or feces.

Treatment.-Comortheal coloproctitis can, in the average case. le. quickly relieved and cured by keeping the patient in bed, restrieting him to a thuid or semisolid diet, applying ice to the spine, and frecfuently irrigating the colon and rectum with a hot $\left(100^{\circ}\right.$ to $110^{\circ} \mathrm{F}$.) ichthyol, permanganate of potassium, balsam of Peru (I per cent.), or argyrol (5 per cent.) solution.

When he complains bitterly of pain in the rectum, spasmedic contraction of the external sphincter and hevator ani mucles, or difficult micturition, the insertion of a suppository containing morphin or cocain, gr. $\frac{1}{4}$ (0.008), and belladomna, gr. 1 (0.015), brings immerliate relief. In adelition, much can be done for the patient's comfort and diminishing the intlammation he injecting a hot emulsion composed of olive oil, Jiij (90.0), and bismuth, jss (2.0), nightly. "The warm oil soothes the mucosid and quiets the irritable muscles, and the bismuth forms a coating over the mucosa and protects it from the feces, discharge, and toxins.

As the acute nanifestations become less troublesome the patient may be permitted to go about and resume a mixed, non-irritating, regular diet, but the irrigations should be continued at short or longer intervals until diarrhea ceases, gonocoeci disappear from the stools, and evilences of the inflammation are no longer visible through the proctoscope.

Appendicostomy and cecostomy, useful in the more malignant types of specific colitis, are not required here, except in rare instances where a catarrhal inflammation or ulcers of the mucosa remain after the gonorrhea has been cured. 
CHAPTER XL

\title{
MYXORRHEA COLI, MYXORRHEA MEMBRANACEA MYX- ORRHEA COLICA, DIARRHEA IN
}

\author{
MEMBRANOUS ENTERITIS AND COLICA MUCOSA
}

\section{HISTORY, GENERAL REMARKS, ETIOLOGY, PATHOLOGY, SYMPTOMS, DIAGNOSIS, TREATMENT}

IT is difficult to define membranous colitis and mucous colic because they are not discases, but a symptom-complex, characterized by copious periodic mucous discharges, abdominal discomfort, or cramps and constipation (spastic), characteristics which vary greatly in different cases and under varying circumstances, and, finally, because the same patient may at one time seek relief from mucous colic or at another from excessive mucomembranous eracuations.

Ixorhea coli occurs more frequently in women and in asthenic individuals than laborers, but is exceedingly rare in the very old and young.

The nomenclature of these conditions is confusing, and authorities of repute have coined more than twenty captions to indicate their interpretation of mucous colic and membranous colitis-riz.: membranous enteritis (1)aCosta), colica mucosa (Nothnagel), tubular diarrhea (Mason Good), pseudomembranous enteritis (Cruvelhier and Laboulbene), myxonemosis coli (Ewald), myorrbea (neurosa) coli (Albu), mucons disease (Whitehead), intestinal neurosis (Sireder), croupous enteritis, mucous croup, and colitis pseudomembranacea, etc.

Owing to the fact that mucus responsible for these conditions is secreted or collects within the colon, and to eliminate the confusion which exists concerning the nomenclature of so-called membranous enteritis and colica mucosa, the writer suggests that the caption mirorrhea coli be applied to all mucous stools, myorthea membranaced to evacuations characterized by strings, strips, or bowel-like membranous mucous casts, and myorrlea colica to mucoid collections including colic. Then, by adding the prefix "myorrhea" to the factor responsible for the hypersecretion or retention of mucus, myxorthea coli could be classified as follows, viz., Myorrhea catarmalis, $M$. constipatia (e.constipatione), M. spastica (enterospasm), M.traumatica, M. infectiosa, M. Ayspeptica, M. toxica, M. obstructiosa, M. neriosa, M. organost, and $M$. parasitica, ete.

Etiology.-Formerly, authors attributed myxorrhea colica and myxorthea membranacea to neuroses which led to the hypersectetion of mucus, and later Nothuagel claimed they might be caused by neuroses 
or intestinal catarrh, but these manifestations may also lee incluced by a variety of conditions and experimentally.

Clinicians agree that nerous phenomena-viz., hysteria, neurasthenia, melancholia, and trophoneurosis-are frequently observed in persons who sulfer from myxorthea colica and myxortuea membranacea, and the atuthor is willing lo concerle that oreanic or functional nervous affections or psychic disturbanees may incluce or aggravate them.

The chief claims in favor of the neurotic origin of myxorthea membranacea and myxorthea coliea are based upon the nervensness of such patients and the apparently normal appearance of the mucosit which frepuenty obtains during life and at autopsy in such eases.

The author offers the following argument to prose that mucous colic and membranous colitis are freguenty produced in other ways. viz.:

(I) Few patients afflicted with insanity, hysteria, melancholia, or other nervous affections sulfer from myxorthea coli.

(2) Mucous discharges may occur independently or complicate nervous discases.

(3) Myxorthea colica and myxorthea memlsranacea maý obtain for vears without the patient becoming nervous.

(4) Frequently neurogenic disturbances do not appear until months or years after the onset of myorrhea coli.

(5) Correction of associated neurogenic disturbanees often fail. while therapeutic measures directed against local or general disease causing intestinal irritation succeeds.

(6) The frefuency of mysorrhea membranacea and colica in women. and the fact that they are usually encountered between the twentieth and forty-fifth gears, indicate that abnormal menstruation sometines influences the hypersecretion of mucus.

(7) Atonic or spastic constipation usually precede or accompany mucous stools, and are probably a factor in their causation, since they frequently cease when regular evacuations are established.

(8) Copious discharges of memliranous or jelly-like mucus with and without colic follow frecuent or prolonged alum, sifver nitrate, copper sulphate, mercury, tannic acid, glycerin, and ioclin colonic irrigation.

(9) It has been demonstrated experimentally that the secretion of mucus can be augmenter hy iodin, silver nitrate, or alcohol applications to the intestinal mucosa, reflex action blotugh other organs or the solar plexis, and injecting rablots with solium oxalate, subjecting them to infection, having them eat indigestible foud, and stimulating the intestinal nerves with electric currents.

(ro) Patients suffering from myxorrbea coli usually have previous! suffered from gastro-intestinal or systemic disturbances.

(I I) Owing to the similarity of mucous discharges in intestinal catarrh and mucomembranous colitis it is often imposilnte to dissociate them. 
(12) Mucus may be constant in colitis, but when mucous stools appear periodically, colic ensues, and the mucoid discharges are membranous, it indicates that myxorrhea colica or myxorhea menbranacea are secondary to intestinal catarrh.

(I3) Mucomembranous evacuations and colic have followed fermentation and putrefaction, and very likely are caused by excessively active colon bacilli or other intestinal micro-organisms or their toxins.

(If) Myxorrhea membranacea and myxorhea colica are occasionally associated with intestinal helninths which irritate the mucosa.

(15) Intestinal stasis and auto-intoxication frequently complicate myxorrhea membranacea and myxorrhea colica and probably cause them in some cases.

(16) Myxorrhea colica and myxorrhea membranacea are occasionally manifestations of foreign bodies (intestinal sand, enteroliths, bismuth accumulations, and scybala) which traumatize the intestine.

( 17 ) Colonic coprostasis is accompanied by nerve phenomena and mucous evacuations, which disappear when it has been corrected.

(18) The expulsion of jelly-like or inspissated mucus is not characteristic of particular affection, but is a reactionary phenomenon, occurring under varying conditions, and Tremolière says, "Mucous stools do not represent a specific factor more than does mucous sputum a specific disease of the respiratory tract."

(I9) Mucous colic and membranous colitis may be secondary to defective metabolisn in phlegmatic or nervous individuals.

(20) It has been demonstrated by bowel inspection that myxorhea colica and myxorrhea membranacea may be induced by organic changes in the mucosa.

(2 I) Finally, it is sometimes difficult to determine whether myxorrhea coli is dependent upon affections of the nerves, systemic disturbance, inflamed mucosa, or obstructing lesions, lecause one or all may be factors in the same case and produce the symptom-complex of myxorrhea coli.

The author has treated patients for myxorrhea coli where multiple and widely varying factors played a part in its production, hence he is constantly on the lookout for more than one cause.

Myxorrica coli not infreguently complicates atrophy and atony of the large intestine, and has suddenly appeared during and following diphtheria, scarlet fever, measles, ptomain-poisoning, influenza, typhoid fever, chemical poisoning, and disturbances of the liver, gall-bladder, pancreas, and kidneys.

Gastrogenic disturbances (achylia gastrica, hyperchlorhydria, and cancer) which unbalance the secretion, lead to its stagnation, or interfere with gastric motility, occasionally lead to mucomembranous colitis, and myxorrhea coli has accompanied enterogenic disturbances which modify the succus entericus.

Frequently, myxurrhea colica and myxorhea membranacea are symptoms of catarrhal, syphilitic, tubercular, balantidic, entamebic, coccidic, Hagellate, bacillary, helminthic, and other types of colitis. 
Myxorrhea coli may also be induced by seybla, purgation, irritating drugs, enteroclysis, massage, vibratory treatments, or anblhing which irritates or inflames the mucosia or causes constipation, intertinal obstruction, enterospasm or fecal retention, and hat been known to follow rough handling of the intestine, abtominal viscera, and pelvit organs during operat ion and careless introluetion of the sigmodrearope.

Individuals afflicted with myxorrhea membranacea and myxurthea colica have a lowered vitality, are under weight, act sowly, and frequenty suffer from anomalies of the teeth. nails. or hair, movable kieneys, enteroptosis, uterine procidentia, hemorrhoids, varicone veins, hernia, insufficiency of the tisstes (connective, nervous, and musular), and nutritional trophie disturbances of the lymphoid and mucous glandular mechanism (actenoidism).

Rhinopharyngeal lesions (vegetations, ete.) and intestinal disorders compose the symptom-complex of adenoidism ofsersed in thyroid disease, which is an important factor in myorrhea membranacea and myxorrhea colica. Tremoliere holds that atrophy and hypertrophy of the thyroid gland are frequently associated with myorrhea coli, and when they are not, the liver, hidney, or some other organ is involved and causes faulty metabolism, malnutrition, or trophic changes, and myxorrhea menbranacea or myxorrhea colica.

Mysorrhea membranacea and myxorrhea colica may lue aggravated or caused by any of the following surgical diseases or conditions-riz.. consenital deformities of the bowel, foreisn bodies, relaxed abdominal walls, moable cecum, splanchnoptosis, malimant and non-malienant neoplasms, peritonitis, appendicitis, salpinsitis, dierticulitis, pericolitis, adhesions, tumefactions, iniagination (intussusception). ioliulus, extra-intestinal pressure, stricture. angulation. Lane's kink, abdominal aneurysm, rectocele. diseased or displacal neighboring orsans, mesenteric disturbances. hernia. cholelithiasis, enterospasm, obstruction by intestinal parasites, procidentia recti, hypertrophy of O'Beirne's sphincter, the rectal ialies, letator ani. and sphincter muscles. deriated coccyx and rectal affections (hemorrhoids, ulcers, or fissures).

The above diseases, singly or collectively, leat to the hypersecretion of mucus because ther narrow, block, or immohilize the intestine, interrupt peristalsis, induce obstipation and fecal impaction. augment pathogenic bacteria and their toxins, induce inflammatory and ulcerative lesions in the bowel, irritate the mucosa, wimulate the secretory and motor nerves to excessive activity. favor intestinal alutointoxication, or retain feces and cliselatrges until they leeome offensive and irritating.

Constipation may be congenital or appear early in life and nearly always is a complication of mucous colie and membranous colitis, hence the author believes these conditions are often ratsed he merhanic intestinal defects which lead to fecal retention. lineler such circumstances, owing to the small amount of food ingesterl by infants and young children, no trouble ensues in the heginning, but liter, when the diet is liberal, the feces are more bulky, they pass the olstruction with 
difficulty, stagnation takes place, and a hypersecretion of mucus tenstles.

The writer ha- ofserved myxorthea coli in a boy of three, a girl of five. and in six other children under twelve years of age, who suffered from postuperative sequele where attempts had been made to correct congenital deformities of the colon. reetum, or anus.

In studying the etiology of myxorhea coli one should keep in mind the relation of the colom to the diaphragm. kidney, liver, stomach. uterus, adnexa, bladder, and prostate. because the bowel is often displaced hy neighboring organs or becomes involved through the extension of disease from them.

Splanchnoptosis enteroptosis, coloptosis, nephroptosis, hepatoptosis. and gastroptusis is frequenty asociated with and evidently causes mxisthea colica and mxorthea membranacea, because stools and colic usually disappear when the organ have been replaced and anchored.

Appendicitis, peritonitis, pericolitis, diverticulitis, abdominal and pelic suppurations. typhoid ferer, and colitis frequently lead to mxorrhea coli because they faror the formation of exudates. bandular, broad or encircling adhesions or p-eudoperitoneal membranes. which agglutinate or bind the gut to neighboring organs or the parietes, twist. ansulate or press upon it. and lead to colonic irritability, fecal retention. and the hypersecretion of mucus.

Three times the author has cured myxorhea coli by removing bismuth accumulations from the colon or rectum, and has many times arrested mucoid eracuations by clearing the gut of scybala and fruit -tones. seeds, or skins.

The writer has a record of more than 200 patients operated upon for constipation induced by chronic invagination of the sigmoid flexure into the rectum, w whom 25 per cent. suffered from myorrhea colica or myxorhea membranaceal. and the majority of these sufferers were speedily relieved or cured ly sigmoidopexy. The frequency with which women suffer from mucomembranous colitis, and the good result- which follow the extirpation of uterine tumors, discased tubes and ovaries. and the correction of uterine displacements, demonstrates that female genital disturlonce- may cause myxorrhea coli.

Vesical and prostatic diseases may also augment the secretion of mucus in some instances by traumatizing the gut or exciting the levator ani muscle to frequent contraction.

In 75 per cent. of the case- of myxorbea coli treated by the writer the hyper-ecretion of mucu- was apparently caused hy adhesions. angulations. invagination, or mechanic bowel defects, inflammatory or ulcerative lesions of the colon or rectum. which increased the mucus or caused its retention until it became inspissated and membranous.

In concluding his remarki upon the etiology of myxorthea membranacea and mxorrhea colica the writer would reiterate that the hypersecretion of mucus is a common manifestation, complicates many chronic merlical and surgical diseases of the bowel, and that the amount. 
consistence, and form of the muroid eiacuations depend largely upen the irritable state of the intestine, enterospasm, shape of the :ult where the mucus accumulates, and length of time it is retained.

If internists and gatso-enterologists are aware of these facts, they fail to mention them, conseguenty their followers believe that myxorrhea coli (nysorthea membranacea and colica) are always induced by intestinal atarrh or neuroses, when they may be anterl by many other diseases and ronditions.

The pathology of myxorrhea coli is not understood berause it is not characterized by definite lesions, seldom kills, and has heen studied at antopsy only a few times where it complicated other fatal diseases. The author has observed myxorrbea membranacea and myxorrlea colica in patients where the mucosa was inflamed or ulcerated and where it appeared normal. Most of ten, however, it has leen encountered in individuals who had been sulfering from intestinal stasis, fecal impaction, and toxemia indueed by mechanic defects in the colon or rectum which caused fecal retention.

Symptoms. - Inder normal conditions the amount of mucus secreted is so slight that it is difficult to discover in the feces with the microseope, and when visible in the stools it is being abnormally secreted and the patient suffers from myxorhea. The manifestations of myxorrhea coli are variable in different cases and in the same individual at different times, but obstinate constipation and the periodic evacuation of large quantities of mucus or colic constitute the chief symptoms.

When hypersectetion is marked and mucus is long retained, the water is absorbed, and it becomes inspissated, gravish in color, accumulates in a large mass, or when compressed it assumes the form of bowel casts or strips, acts as an irritant, and excites mild peristalsis, which favors its expulsion.

Myxorrhea colica and myxorrhea membranacea may dominate the disease producing them, and clear jelly-like mucous evacuations may prevail for weeks or months, when suddenly a crisis sets in, the patient complains of cramps, and later the evacuations of mucomembranous strips or casts. Again, he may suffer indefinitely from mucous discharges induced by a variety of causes and never complain of myxorthea colica or myxorrhea membranacea.

Persons afflicted with myxorrhea membranacea and myxorrhea colica are sometimes bothered with impaired digestion, malaise, melancholia, nervousness and abdominal uneasiness, discomfort, soreness. pain or colic for a shore while before mucous stools appear, or remain well in the intervals between attacks of myxorthea coli.

Strips or calsts of mucus occasionally create crawling sensations and are mistaken for worms, hut when low down they are easily located through the sigmoidoscope, and are to be seen projecting through the rectosigmoidal aperture or hanging over the rectal vilves. When mucus becomes dry and collects in any form it causes irritation and leads to enterospasm (myxorthea colica), matked constipation, fecal 
impaction. gas distention and colic, which continue until it is evacuated through the sigmoidoscope or in some other way.

Diarrhea may be noticeable during attacks of myxorrhea colica or it may not occur until the obstruction incident to enterospasm has been relieved, and the backed-up irritating feces, scybala, discharges, and toxins find their way into the lower bowel and excite abnormal peristalsis and the hypersecretion of mucus.

Diagnosis.- - Esually in obstinate cases of myxorthea coli the history will show that the patient has been troubled for weeks, months, or years with gastro-intestinal disturbances. menstrual abnormalities, constipation, psychic or nervous phenomena, disturbances of the liver, pancreas or heart, or obstructing lesions of the gut prior to the attack, which indicate that the mucous discharges are secondary.

Myxorrhea coli is diagnosed with ease by the mucoid eracuations (made up of jelly-like or mucous strings, strips, or casts) which appear suddenly following attacks of nausea, indigestion, obstinate constipation, and abdominal uneasiness, discomfort, or soreness in patients who have otherwise been comparatively well.

Mrxorrhea colica must be elifferentiated from other types of intestinal obstruction, but in the presence of the enumerated symptoms, violent colic and the absence of pus or hlood in the stools, one is justified in diagnosing this condition before and after the mucous collections have been eracuated. When an acute attack of myorrhea coli subsides the abdonen and intestine should be examined and the feces analyzed, with the object of ascertaining what is causing the hypersecretion and retention of the mucus. It is also important to determine whether or not the sufferer is afflicted with a nervous or other affection which would influence the myorrhea colica or myorrhea membranacea.

Treatment.-A routine treatment of myxorhea coli is impracticable, owing to its varied etiology, and because the patient may seek relief from either myxorrhea membranacea (mucous casts) or myxorthea colica (colic).

During acute attacks curative measures should be held in abeyance. for the patient desires immediate relief from the discharge of mucous casts or masses or colic from which he suffers.

When the patient seeks a cure, the writer informs him that myorrhea coli is a manifestation of some other affection, and that, after the crisis is passed, prolonged treatment or an operation may be required to effect a cure.

Where tenacious mucus collects in the lower sigmoid or rectum it can be removed with a blunt scoop or swab and cotton, following the introduction of the sigmoidoscope. but when higher up, rest in bed, a liberal dose of eastor oil. hot-water drinking. abdominal fomentations, and hot high colonic enemata are indicated to soothe the mucosa and cause the intestinal musculature to relax, which in turn overcomes constipation, farors expulsion of the mueus, and relieves the patient mentally and phỵicallỵ. 
Strychnin, gr. is (0.001), lowler's solution, we (0.30), extract of phỹsostigma, gr. ${ }_{4}^{1}$ (0.015), hypophosphites, Ruscell's emulsion, ansl other nerve, muscular, or general conics are inclicited when the sufferer is nervous or rum down, and the treatment is more ffective when sup)ported by hyclrotherapy, abdominal matsage, viluallom, or electricity when the bowel is sluggish.

Where the bowel is semsitive, highly irritalule, and enterospasm is a complication, belladomm, mx (0.60), relieves the muscular spasm, lut wher colic is severe it should be reinforeed by eodein, er. ? $(0.0,3)$, or morphin, gr. $\frac{1}{4}(0.016)$, achinistered every three or four hours to arrest pain and induce slecp.

Between attacks of myxorthea membranacea and myxorthea colica liquid paraffin or albolin, administered in the morning and at night in liberal doses, of $\mathrm{j}(60.0)$, lubricate the intestine, minimize constipation, and faror the expulsion of mucus before it becomes inspissated and irritating.

It is artisable in some cases to control the diet, but a routine dietary is impracticable becatse of the varied etiology of mycorrhea coli. D furing attacks of myorrlyea colica better and fiucker results are obtained when the patient eats more frequenty and consumes chicfly hot Huids, milk, soup, purcés and meat juices, and abstains from fookts which leave a large residue.

Following an acute attack of myxorlea membranacea, forced feeding and a diet composed mainly of veretaloles (cellulose) in conjunction with rest in bed, afer the plan of Von Noerden, ustally corrects constipation, which is followed by cessation of the mucomembranous evacuations, and in uncomplicated cases the myxorrhea does mot recur while the patient has normal motenents.

A heavy, coarse diet temporarily causes sensations of abdominal fulness and discomfort, but they disappear spontaneously. Where constipation ensues from oeverdigestion, the administration of resulin, pieces of cork, or seets increase the luth of the feces, stimulate peristalsis. and usually bring about normal evacuations.

As soon as myxorrhea coli and constipation have heen relieverl, the patient should return to his regular diet and discominue the regulin. A cellulose or vegetable diet is out of place where the hypersecre-

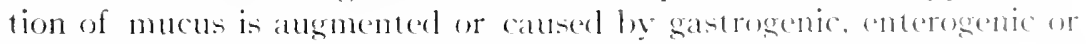
neurogenic disturbanees, or colitis, hecaute it makes lie fores bulky and irritating to the hypersensitioe, inflanede or ulcerated mucosi, augments the secretion of mucus, and hat a temeleney towated enterospatsm and colic.

Drastic cathartics are contra-indicated during crises of mucous colic because they cannot drive feces thrombh the blocked gut and increase the patient's suffering.

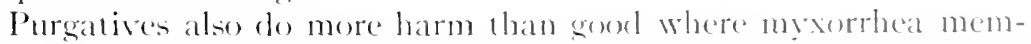
branacea or myxorrhea coliea are complicated by chromic intestinal obstruction from whaterer coutse, since they stimulate violent peristalsis and do not secure the coreted evatuation. Luder such circum- 
stances comfortable stools can be obtained by hot-water drinking and the administration of mineral oil, salts in small repeated doses. fluidextract of cascara sagrada, mpx (I.O), or a milder dinner pill to soften the excreta, or bellatomna, wex (0.60), to soothe and relax the irritable intestinal musculature.

Astringents (tannalbin, tannigen, ichthoform, and bismuth subgallate), gr. x (0.60), may be called for in the treatment of myxorrhea coli complicating colitis when diarrhea is troublesome, but should be discontinued when the stools become normal.

When myxorrhea membranacea is associated with inflammatory, ulcerative, or obstructive lesions of the colon, the patient can be quickly improved hy treating the mucosa with medicated solutions or vil introduced through the anus or through an appendicostomy or cecostomy opening, which insures their reaching all parts of the bowel.

Long colon tubes are not dependable for irrigating purposes because they double up in the sigmoid flexure or rectum, prevent the solution or oil from passing into the bowel, and cause pain. Medicaments can be macle to reach the upper colon by placing the patient in the exaggerated knee-chest posture, introducing the sigmoidoscope, and pouring the irrigant or oil directly into the bowel with the aid of a pitcher and funnel attached to rubber tubing (Fig. I I9), or by inverting the patient and emplosing the author's funnel-shaped proctoscope and pitcher, which enables one to pour a quart or more of solution or oil directly into the colon on account of the inflation and displacement of the viscera which enstes.

The author has experimented with many irrigants in myorrhea coli alone or complicated hy ulceration and diarrhea, but obtained the best results from boric acid (3 per cent.), ichthyol, balsam of Peru, potassium permanganate ( 102 per cent.), and argyrol (5 per cent.) irrigations, employed daily or three times weekly.

When the mucosi is sensitive and the musculature highly irritable, warm crude oil contilining bismuth should be substituted for or alternated with irrigation because of its soothing and healing action.

In addition, when the intestine is obstructed by adhesions, kinks, twists, pericolic membrane, extra bowel pressure, or stricture, etc., the lesion must be corrected before a permanent cure can be obtained.

In cases where the colon is permanently disabled, resection may be necessary, but when the patient is in a poor condition and cannot withstand a prolonged operation, the liseased bowel should be isolated by entero-amastomosis, unilateral or bilateral exclusion.

Colostomy has been successfully employed in the treatment of myxorthea coli, hut has heen abandoned in favor of appendicostomy, cecostomy, resection, or intestinal exclusion, because of its disgusting features and the serions secondary operation required to close the artificial ants. 
The prognosis of myxorrhea membrandeca and myxorthea colica is very good when cattsed by obstructing lesoms of the colon correctable by operation, but a longer lime is recpureel wo effect at cure when they are secondary to inflammatery or ulcerative lesions of the mucrest, and myxorrhea membranacea and myxorthea colicat are more difficule to permanenty eliminate when the hypersectetion of mucus is induced by gastrogenic, enterogenic or neurogenic disturbances, general disease, or atonic constipation. 


\section{CHAPTER XII}

\section{INTESTINAL IRRIGATION (ENTEROCLYSIS); ENEMATA IN THE TREATMENT OF DIARRHEAL, INFLAMMATORY, AND PARASITIC DISEASES OF THE GASTRO-INTES- TINAL TRACT}

Ix the treatment of diarrhea incident to inflammatory and utcerative parasitic lesions of the intestine of whatever kind, irrigation is perhaps the most reliable therapeutic measure at our command, while enemata, so useful in overoming constipation, play a minor part in these conditions. In this class of affections, enemata, large and small, are resorted to principally to prevent or dislodge fecal impaction where ulcers have healed and a stenosis has formed in the lower bowel, and

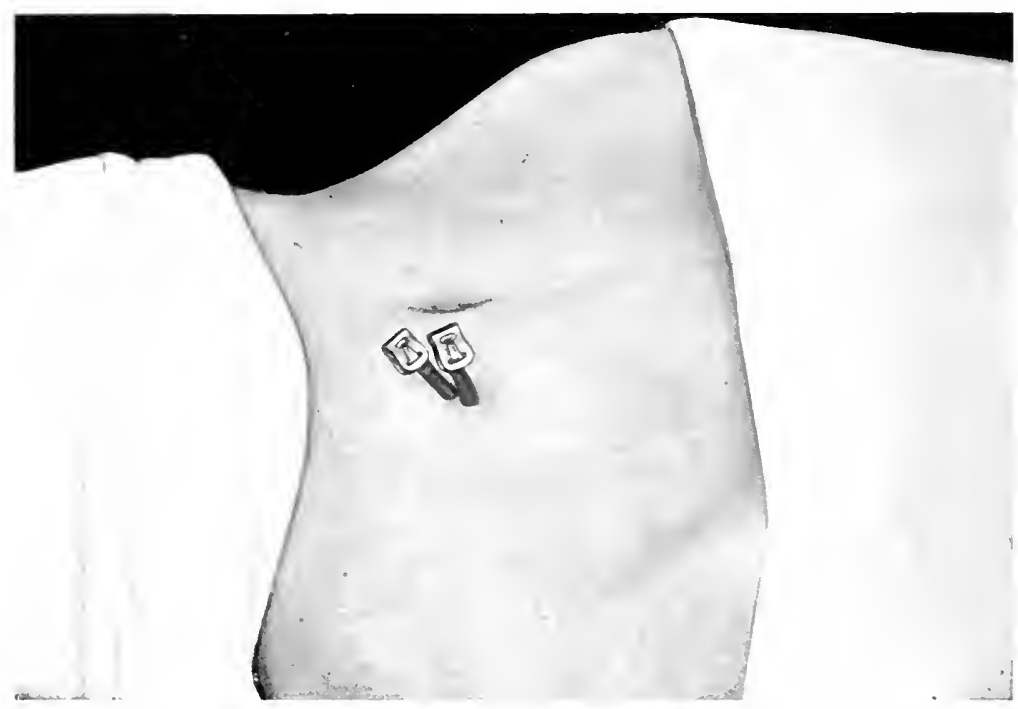

Figr. I ro.-Catheters closed with Cravat clamps. Gant's older cecostomy, which prorides for two-way irrigation.

for evacuating gas, putrefying food-remnants, discharges, and other irritants within the rectum.

Since one can accomplish these purposes by irrigation and otherwise benefit the patient, the author will not further discuss enemata, but will refer the reader to his former work, where their indication and techinic of administration have been given in detail.

${ }^{1}$ Cant, Constipation and Intestinal Obstruction (Obstipation), p. 23C, roog. IV. B. Saunders Co. 
Irrigations in the treatment of diarrheat and parasitic diseatses are employed chiefly to soothe the bowed, heal local lesions, prevent the formation of impacted fecal matsses, neutralize or wash out toxins, remove irritating pus, blood, mucus, tissue debris and feces, and relieve enterospasm, pain, and tenesmus. In this connection it might be well 10 remenber that enemata are uswally given with the ideat that they are to rematin until fecal accumulations are expefled, while irrigations are administered slowly, so that the fhud may be taken up lig the system, or it is permitted 10 run into and out of the bowel sinultane-

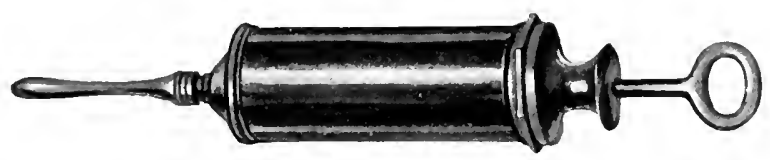

Fig. III.-Hard-rubber enema syringe.

ously, with the object of immediately cleansing the mucosa and stimulating lesions to heal.

Bowel flushing may be conducted from below by way of the anus, or above through an artificial opening, the operation for the making of which, according to its location, is designated cecostomy (Fig. I 5.5), appendicostomy (Jig. I6f), Gant's cecostomy with an arrangement for irrigating both the larse and small intestine

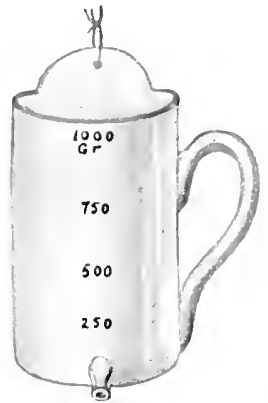

Fig. I 2.-Grarluated container for irrigat ing fluids. (Fig. I 56), or colostomy (Fig. 170).

In loa or rectal irrigation the washing is confined to the terminal extremity of the gut, and in high or colonic irrigation the entire large bowel receives treatment. When the solution is made to enter the arti-

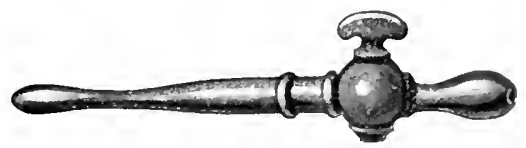

Fig. I 3.-Frrigating nozzle with stop-cork.

ficial inlet and pass quickly downward along the colon and ont at the anus the procedure is designated through-and-through irrigation or cnteroclysis.

The older plan of introducing the fluid from below is preferable where the solution reaches the entire diseased area, but in many instances, for one reason or another, this canmot be accomplished, and lesions in the upper portion of the colon are not reached and the treatment fails. Inder such circumstances through-and-thromgh irrigation should be inmediately provided for by one of the operative procedures named above and fully described elsewhere.

Appendicostomy or cecostomy is indicated immediately in the 
treatment of persons afflicted with diarrhea resulting from intestinal inflammation, ulevation, or hoth, who come from a distance and can remain hut a short time. those who are exhusted by frequent evacuation- and tenesmus or violently prisoned by retained toxins, and where the di-charges are enormous, irritating, and cause auto-intoxication, or hemorrhage is a dangerous complication. This method of completely Hushing the large intestine is more reliable and effective than rectocolonit when the fluid is introduced through the anus. but when there is an enterocolitis. Gant's eecostom y. with an arrangement for irrigating separately or simultanteus the small incestine and colon, is indicated, becaure lesions in all segments of the intestine can be treated, while followin appendicostomy and ordinary cecostomy the irrigation does not reach above the colon.

The frequency of the irrigation varies in different cases. and must he increased or decreased according to the condition of the patient. When the movement= are very frequent ten to twenty daily-). toxemia is alarming. bleeding profu-e. or the discharges are copious and foul, the sut -hould be Hushed two or three times daily for a short time, and then once or twice as the-e smptoms modify, and. finally, at the

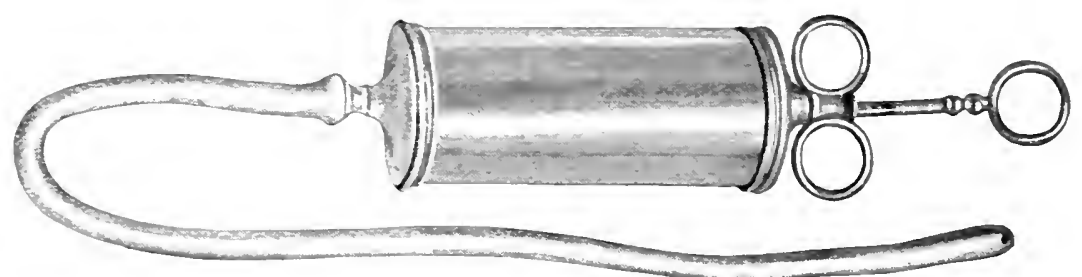

Fiz. If - Metal piston enema syringe and attached colon-tube.

end of a fer weeks, when the morements are fewer or normal and the patient has gained in weight and strength. his complexion has improved, and the discharges are slight. all of which manifestations indicate that the lesion are rapidly healing, irrigation is not necessary more than once daily or three times weekly. It has heen the author's practice to continue the treatment for several week- and sometimes muth-fullowing an apparent cure of the patient, because on several occarions where flushing was stopped too soon relape occurred.

When administering enemata the amount of fluid injected is usually limited to 2 or 3 quarts hecause of the distention pain which follows. and o quarts, the full capacity of the colon and rectum, is the larsest amount that can posibly be used. and this quantity is danger"us because it may rupture the intestine or cause ptosis or paresis. There is no limit, however. to the quantity of fluid which may be emploved when giving an irrigation where the solution is allowed to How into and out of the gut through a double or return-flow tube or irrigator. In this way it can escape as fast as it enters the howel, and the same olstains when the fluid is permitted to continuously flow into the bowel hy way of an artificial opening (appendiceal or cecal) abore 
and out at the anus through a proctoscope or pipe introtueet for the purpose.

This class of patients are miserable so long as poistmous foxins and discharges are retained, conseguently, the irrigation shomled be continued wntil they have been dislodged and wastred ont. When this is done regularly it is rapielly followed by a matked improsement of the patient's eondition. Onee these individuals have beens stecessfully irrigated, they regain confielence and fecel that they are on the road to recovery because something definite is being done to heal the bowel, and rejoice when they fincl out that it is no longer necessary for them to continue bismuth, opium, and other drugs.

Irrigation is not always successful because in some instances the local disease has almosit completely destroyed the mucosa, stemoses are present, systemic involvement has taken place, the patient is so deritalized that healing is impossible, or because the flushings have

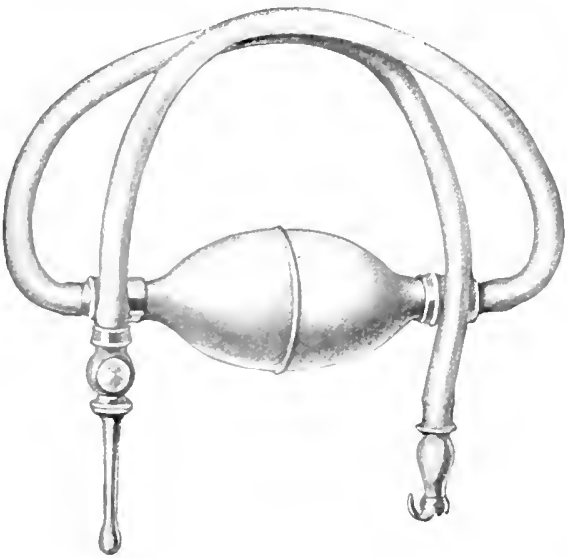

Fig. Ir. - Daridson's syringe.

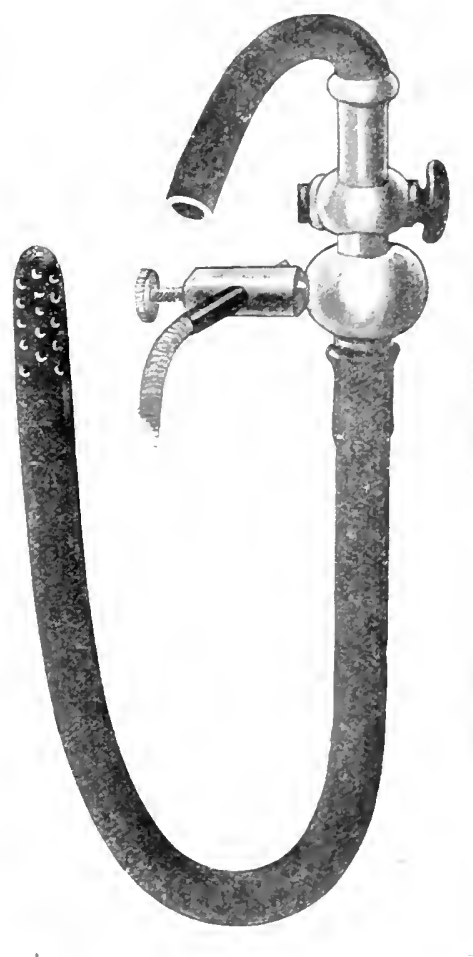

Fig. I 6.--Iydriatic edectrode.

been improperly conducted. When the flud reaches only one axtremity of the gut, or is permitterl to regularly rum orer the sime site of the bowel, a cure cannot be affected because some of the lesions are not reached. From this it may be inferred that the solution must les mate to pass from one end of the large bowel to the other and the position of the patient frecuenty changed, so that the solution comes in contart with every side of the entire length of the diseased incestine.

Irrigants.- Nater, normal saline, and medicaterl solutions and rils in one form or another have been sucessufnlly amplosed in the treatment of intestinal lesions inducing diatheal. Iatwater irrigat- 
tions have been recommended by Tuttle in entamebic colitis (dỵsentery-1, lut they have been discarded by the author because the sudden injection of water at a low temperature is always uncomfortable, frequently induces intense pain or enterospasm, and does not produce as good results as some of the other remedies to be recommended. If one were to remore entamebe and keep them in ice-water they would die, but the author doubts if they do in the colon (under this treatment', because here the ice-water quickly becomes warmer and is no longer fatal, though it may temporarily retard their activity. Equally good results have been obtained by flushing the bowel with hot water $\left(100^{\circ}\right.$ to $I 10^{\circ} \mathrm{F}$.). and the patient greatly prefers hot- to cold-water irrigation because the heat relieves pain, cramps, and soreness, and the water is longer retained in the intestine to be absorbed, improve the circulation, and cause the emunctories to become more active. The author does not attribute the beneficial effects noticeable following hot-or ice-water irrigations to the temperature. which has comparatively little bactericidal power once the fluid reaches the gut, nor does he believe that there has yet been suggested a specific remedy which can be relied upon to kill entamebe or other micro-organisms responsible for infectious colitis. He attributes the beneficent action of the irrigation to the mechanical action of the fluid. which, while being driven through the bowel. neutralizes and washes out retained toxins. cleanses the inflamed and ulcerated mucosa of foul discharges. débris, irritating feces, and relieves discomfort, distention, pain, and cramps.

Improvement invariably follows irrigation of the bowel with water, medicated solution-. or oil. but the good results do not depend so much upon their temperature or the nature or strength of the medicament as they lo upon the amount and frequency with which the fluid is used. No doubt antiseptic, stimulating, deodorant, and soothing medicaments are of value in the treatment of inflammatory, ulcerative. and obstructive lesions of the bowel when emplosed in conjunction with copious irrigations, but too much reliance should not be placed solely upon them.

The author will now enumerate and discuss the most useful medical agents that have been employed in the irrigating treatment of diarrheal, catarrhal, and parasitic diseases of the intestine.

Quinin (bisulphate). I : I000 or 1500. has been largely used in the treatment of entamebic and bacillary colitis (dy-sentery), but in the author $=$ practice it has not shown any specific action nor given better results than the remedies mentioned below. He will not particularize further because he has found that solutions which heal the inflamed or ulcerated mucosa in one form of colitis causing diarrhea will prove effective in another, irrespective of the cause or stage of the disease.

Naturally, the strength of the contained medicament must necessarily be changed to meet indications in the individual case because they are beit used very mild when the inflammation is slight, stronger 
when it is more marked and there are erosions and superficial ulcers, and quite strong when the lesions are large, numerous, and aceompanied by a profuse discharge of pus, heoxi, and mucus.

In the arerage case of ulcerative colitis cathing diarrhea the following solutions give very satisfatetory results:

In mild cases of recent origin daty irrigition with a hot normal saline solution, camomile, flaxseed, or oak-hark tea, pinus canalen-is or hydrastis ( I per cont.), borolyptol, potassium permanganate, syeothymolin or listerin (3 per cent.), salicylic acid, alum, zinc sulphate.

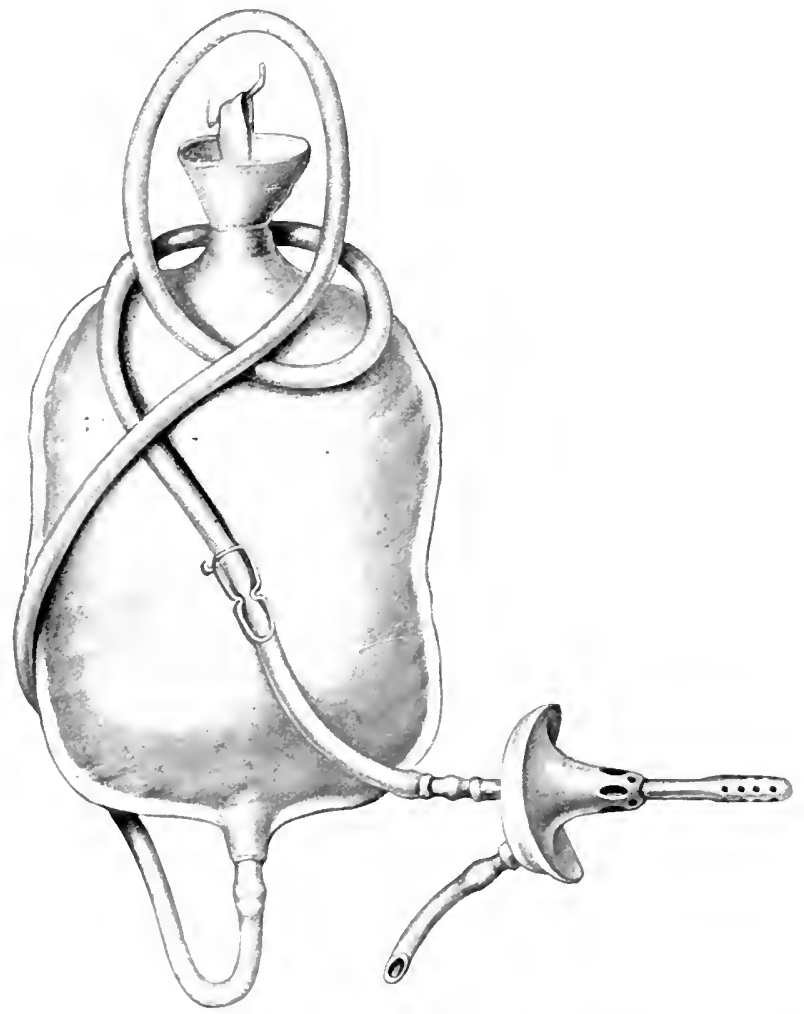

Fig. I 7.--Fountain siringe with return-flow irrigating attalchment.

copper sulphate or silver nitrate ( per cent.), looric acid or (arlabad salt (2 per cent.), or thymol (I : 2000), will refresh the patient and improve his condition.

When there has been an acute colitis which has sulsidect or there are evident erosions and fair-sised ulcers in the intestine which excite peristalsis and increase the evacuations it is well to empley lowe allowe remedies a little stronger or use the following irrigants, viz., ichulhyol. balsam of Peru, 2105 per cent.; salieylate of sorta, 3 to 5 per cent. or boric acid, 5 to Io per cent. : silver nitrate, $10 \mathrm{gr}$. to Oij $(0.60-1000.0)$; 
protarsol or areyrol. 2 to 5 per cent.: salicylic acid or tannic acid, I per cent.. or the following combination of krameria and soda, riz.:

R Fluiklext. krameriat Sodii biburalis 5iv 120.0 : il.

Sig.-Tablesponiul to the quart and irrigate daily or three times weekly. (Gunt.)

Originally krameria and water were uned. Hut the drug threw down a heary precipitate which proved very irritating, and it was found that with the addition of soda it formed a perfectly clear. non-irritating, blond-colored solution. The author use this formula more than any other in the treatment of the intestine when irritated. intlamed, or uletrated. because it is soothing. very healing. and never produces toxic manife-tations.

When the movement- are frequent as a result of severe chronic intestinal catarth. much can be done to reliere and cure the patient by the injection of the following combination. which is recommended by Cohnheim:

$$
\begin{aligned}
& \text { P starch } \\
& \text { Tannic acid } \\
& \text { Sir-Inject. }
\end{aligned}
$$

In aggraiated cases of diarrhea consequent upon ulcerative colitis of whaterer kind, when the patient has anyhere from ten to thirty exacuation-daily, there is an abundance of pus, mucus, more or less blecting. and he suffer incensely from exhaustion and auto-intoxication. more radical measures are required. Lnder such circumstance- the brwel is irrigated with a solution of silver nitrate. gr. XXI to OHj 2.0-I000.0\%. protargol. or argyrol 5 to 10 per cent.). after which it is flushed shortly with a normal saline solution to wash out any excen of silver. The former preparation is preferable because it is alway- effective and is much less expensive. The author employs the -ilver -olution in the above strength every other day for the first week. and as improvement follows reduces the drug weekly until only 5 gr. to the quart $0.30-1000.0)$ are used, during which time on alternate day the bowel is irrigated with the krameria or other solution. L'-ually by thi- time marked improvement is noticeable in the patient ${ }^{\circ}$ condition hy his improred appetite, gain in weight, clear complexion, the number of stools are greatly diminished, the discharge is lec.. all of which indications point to the rapid healing of the ulcers and non-aborption of toxins.

In very bad cases. next to silver irrigations. the author has obtained the beat realts from the emplorment of from 5 to 10 per cent. solutions of ichthyol or the balsam of Peru, or copper or zinc sulphate (1 to 2 per cent.), but prefers the former because the latter induce more pain and may cause toxic symptoms. Irrigation with a full strength luric acid solution is very servicealole in many cases, particularly when the patient is on the road to recovery, but it is necessary to watch its 
action, because in two of the atthor's cases typic poisoning oceurred. necesitiating its discontinnance.

Mlikl or soothing solutions should be substituted for strong ones

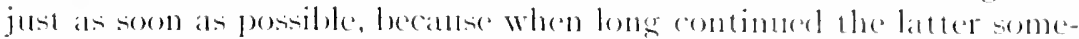
times irritate the mucose and ageratate insteat of improving the pattient's general combition.

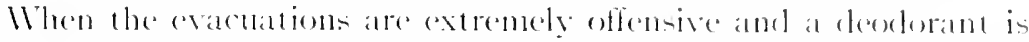

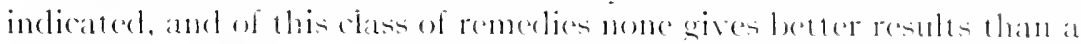
5 per eent. ichthyol or 1 per cent potatsium permanganate solution,

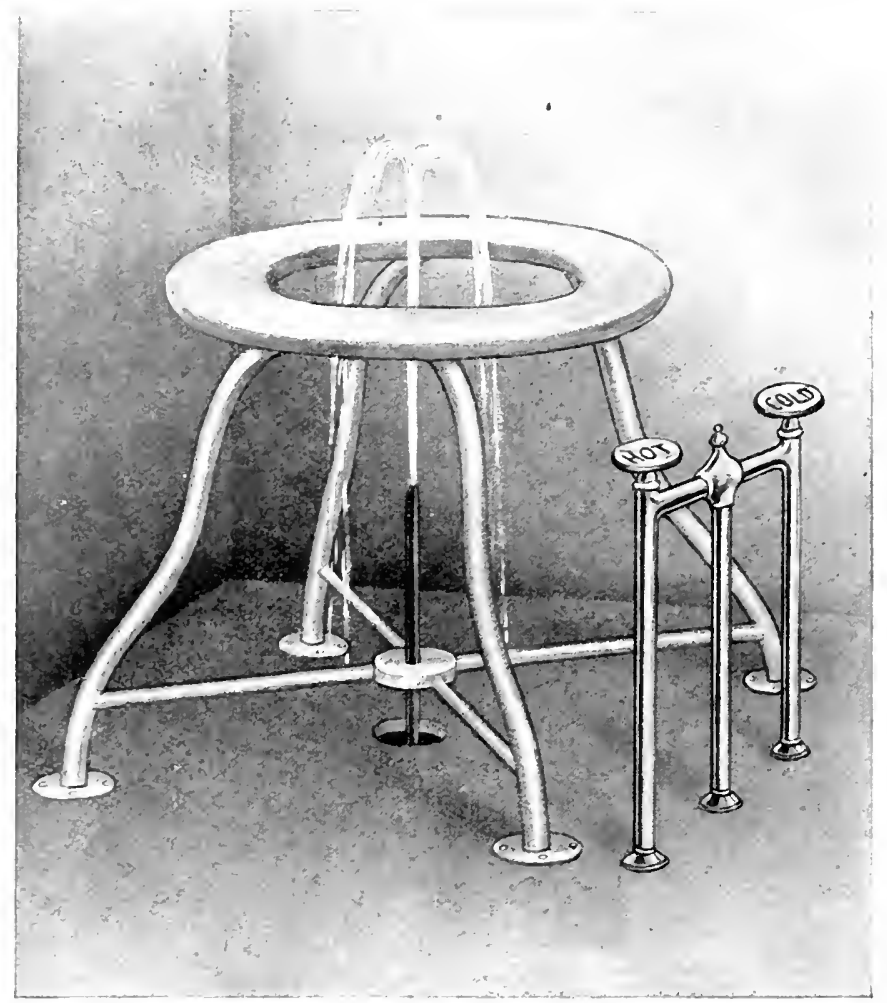

Fig. II8.-Author's anal (ascenting) douche

injected wo or three times daily, although the perosid of hyctrogen (2.5 to 50 per cent.), acetotartrate of alumintm, i 10 2 pere cent., hase prover good disinfectants in some instances.

Oils and emulsions alone, or when used in conjunction with one of the above solutions on alternate days are a great aid in the treatument of nearly all inflammatory and nlecrative lesions of the intest ine partticularly those of the colon and rectum). lecoutse the are somthing. healing, afford a certain amount of nutrinent, and favor alserpotion within the large bowel through their tendency of guiet peristalsis and prevent the frefuent evatuations. The efficate of the oil is ereatly 
enhanced by using it warm $\left(90^{\circ}\right.$ to $I 10^{\circ} \mathrm{F}$.) to arrest enterospasm and intestinal muscular rigidity, lessen pain, and by the addition of antiseptics, styptics, or sedatives, alone or combined with stimulating remedies, to favor healing of the diseatsed gut, diminish putrefaction, and minimize the patient's discomfort.

Oils, named in the order in which their usefulness has been proved by the author, are crude petroleum, olive, sweet, almond, and cottonseed, liquid vaselin and paraffin, neutralol, and others of the so-called mineral oil group, and kerosene. Ordinary oil is used in much larger quantities in the treatment of diarrhea than constipation, because

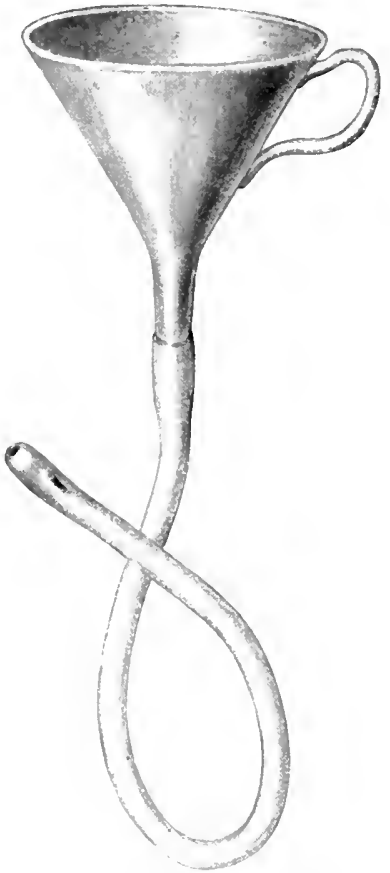

Fig. I rq.-Funnel and tube, useful when administering oil and thick enemata.

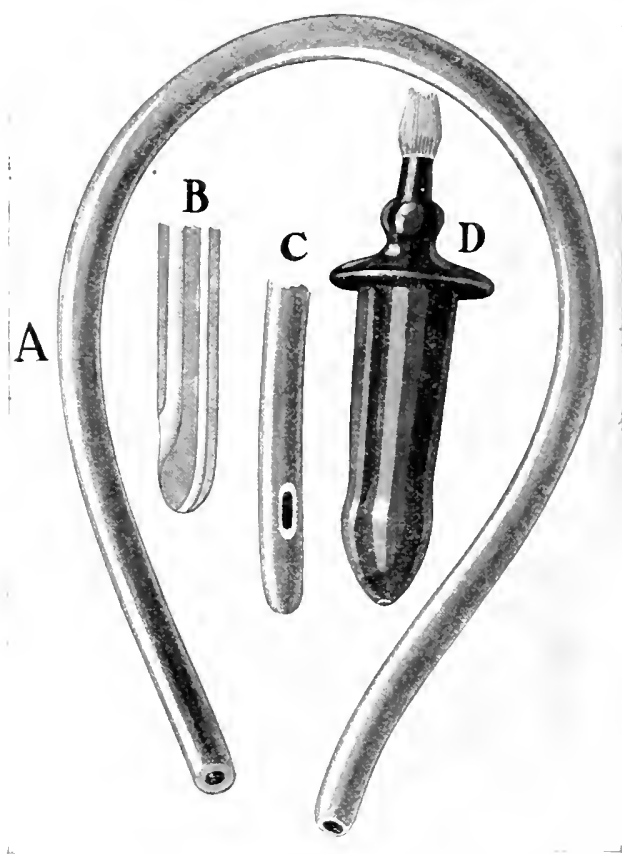

Fig. I 20.- Enema tubes: A, Ordinary colontube with opening in the end; B, Murray doubleflow tube; $C$, pipe with eye in the side; D, hardrubber dilating irrigator.

here it must be made to reach the entire surface of the inflamed and ulcerated gut, while in the latter it is usually injected but a short distance within the bowel to soften and lubricate the feces, that they may be easily expelled.

In the treatment of intestinal lesions, oil, like irrigating solutions, may be introduced either from below (by way of the anus) or through an artificially made opening at the head of the colon or appendix. A smaller amount of oil, however, is required, becaluse the irrigating fluid is permitted to flow in and out at the sane time or be expelled shortly after its introduction, while only a sufficient amount of the 
oil is introfuced to be retained and form an oily or protective conting to the sensitive bowel. Warm oil, becaune of its -osthine effect, is retained very much longer than cold, which is often quickly expetled because it caubes the bowed to contract. When it is injecterl into the colen or sigmoid Hexure warm it is u-ually retained wer night or for two or three days, but when, il it is intentionally or accielentally introdueed into the rectum only, expulsion takes plate quickly, the experienced physician know by this sign whether or not the ril has been deposited in the portion of bowel for which it was intenterl.

Oil. according w indications. may be introduced in amount-varying from a few ounces to 2 quarts or more. Hasnes having repeat tedly used as much as a gallon of herosene at one treatment. lut the using of so large a quantity is unclesirable. because all parts of the intestine can be reached by a smaller amount and with less discomfort to the patient.

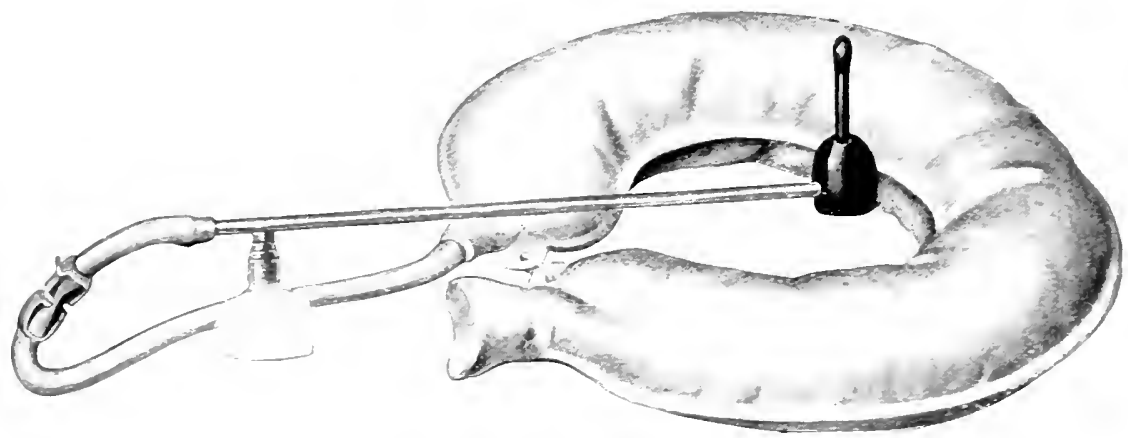

Fig. IzI.-Jamison's seat syrince.

The above oil preparations when continuotuly employed do not produce any unpleasunt manifertations, except that sometimes the parient can taste them and they are noticeable upon his breath or dribble through the anus.

In the majority of instances better results are obtained when each quart of the oil contain $\bar{j}$ ss to ij $15.0-60.0$ of bi-muth subnitrate or carlonate. because these chemicals have antiecptic properties, are sedative. and form a coating which protects the raw -urface of the gut, allays intestinal irritability, and le-en- peri-taltic activity.

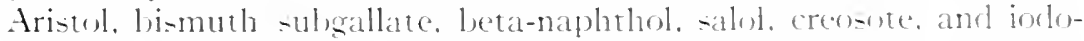
form in smaller amount - are al-o serviceable for the same purpesen.

The author knows of no remedy which i- more soothing and healing or gives better results in the treatment of diarrhea incielent to an inflamed and ulcerateel colon or rectum than the followine emul-ion. riz.:

P Puts: opii .

Iockorm .

Bismuth subnitrate

()live oil

Fle emulion.

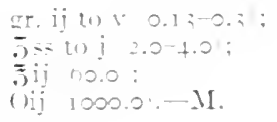

- i i

(i) $1000.0 \div-11$

Sir.- Wirm ant inject all or anỵ part. acurdina (1, indications. 
In this class of cases, when the stools are irritating and foul smelling, the adulition of ichthyol or halsam of Pert (5 per cent.) to the oil combination deodorizes the movements and arrests burning pain and tenesmus in the rectum.

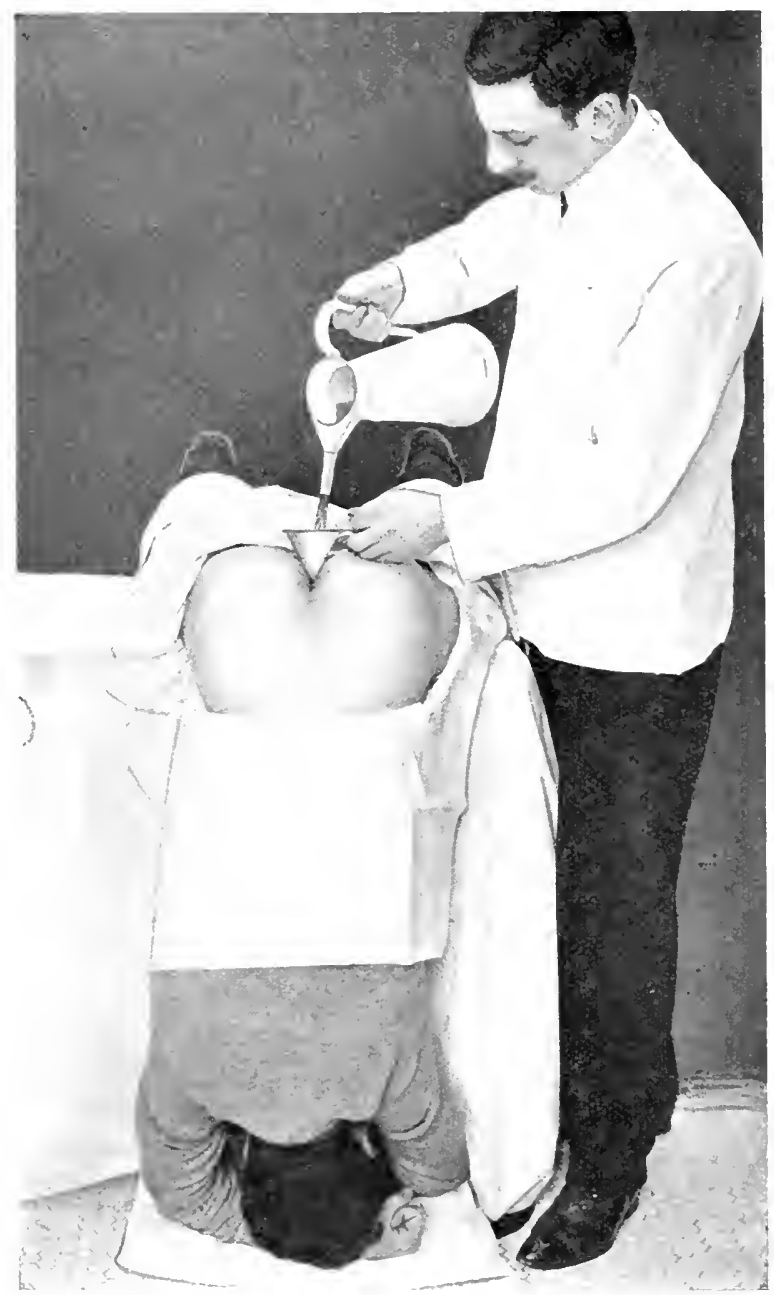

Fig. 1 22,--Nethorl of filling the colon with oil or solutions by mean of (rant's funnel pitcher and fumnel protoscope, while the patient is in the inverted posture.

Gelatin. When oil proves ofjectionable in any way, gelatin, which is non-irritating, should be substituted for it, because bismuth remains suspended in it for at consiclerable time.

Technic of Bowel Irrigation. - The technic of enteroclysis is not understood and is more difficult to carry out than is generally supposed by plysicins and nurses. "The essential features in the procedure are the placing of the patient in the proper posture and properly arranging 
the tube or apparatus by means of which the irrigation is to be conducted into the bowel.

The following, named in the order of their importance, are the positions (Figs. 122, 123) best suited for enteroclysis and colonic irrigation when they are to be administered in the offec-inierted, kneechest, dorsal, right and left Sims, and sitting.

When the bowel is irrigated by the atthor, his assistant, or nurse, the knee-chest or inverted postures are preferable because of the time saved, owing to the fact that the small intestine and pelvic organs fall away from the rectum and permit a rapid inflow of the fluid. In the former the inlet to the sigmoid flexure is quickly exposed by means of a proctosecope of suitable size, and the tube attached to the container is then introduced into the sigmoid flexure and the desired amount of thuid or oil is permited to flow into the bowel. Nhen the patient is placed in an inverted posture, an examining or the author's funnel proctoscope (Fig. I22) is introduced and the fluid or oit is poured directly into the colon, using the author's pitcher which has an attached funnel or proctoscope (Fig. 12t). Esually from I to 2 quarts can be introduced within five minutes, though sometimes gas or air prevents rapid entrance of the irrigant, a difficulty easily overcome by changing the position of the instrument

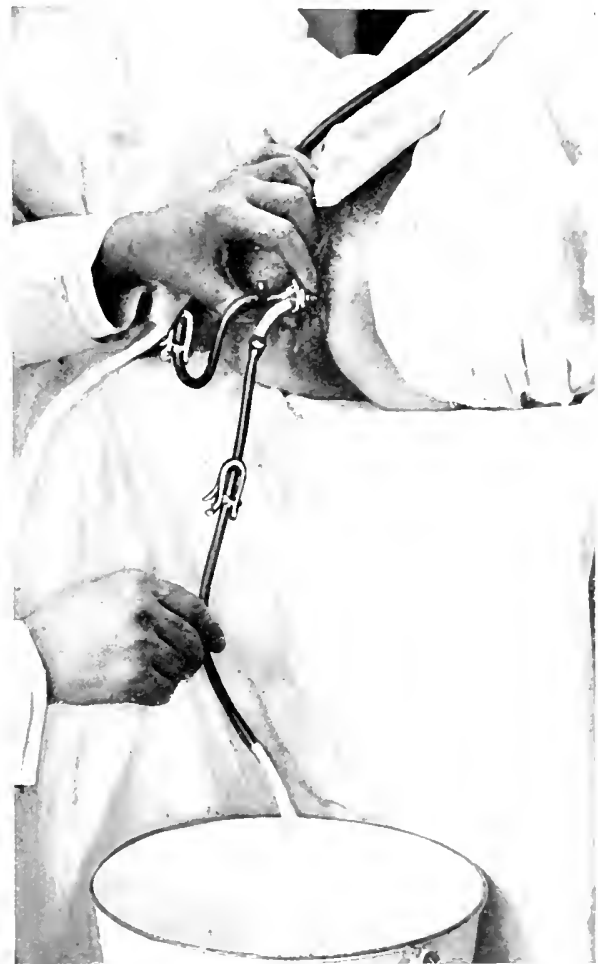

Fise. 1 22.- Method of irrigating the colon and rectum with a two-way irrierator. or withelrawing and reinserting it. When desirable, the proctoseopic end of the author's pitcher can be introduced into the rectum, and the thid or oil paseses into the upper bowel as soon as the obturator is removed (Fig. 122). This position is cumbersome and oljectionable to women, hut there can be no doubt of itseffectiveness. and once the patient has had the irrigation in this position it is preferred to others. In this and the kneechest posture, when the patient complains of the proctusenes a smatler one should be substituted, or, if necessury, it should he discarded and a rubber tube connected with a funnel or heary piston-syringe may be used instead (Fig. IIt). 
When patients irrigate themelves the dorsal, sims', or sitting postures are recommended. and they are told to use the one which gives the best results. but of these the fir-t two are the most reliable. Better re-ult- are obtained when the hip= are elevated and the patient's pustion is changed from time $t$, time if the water doe- not readily enter. Water or other fluds can be intruduced into the rectum and all parts of the colon quickly and with little discomfort to the patient by means of a suitable syringe if the person giving the injection will use a little patience. The solution can be made to move from one part wif the bowel to another by haring the patient turn from left to right. wr vice ver-à as ucca-ion demands. Because of the tendency of the abdominat or pelvic organs to drop downward and obstruct the bowel.

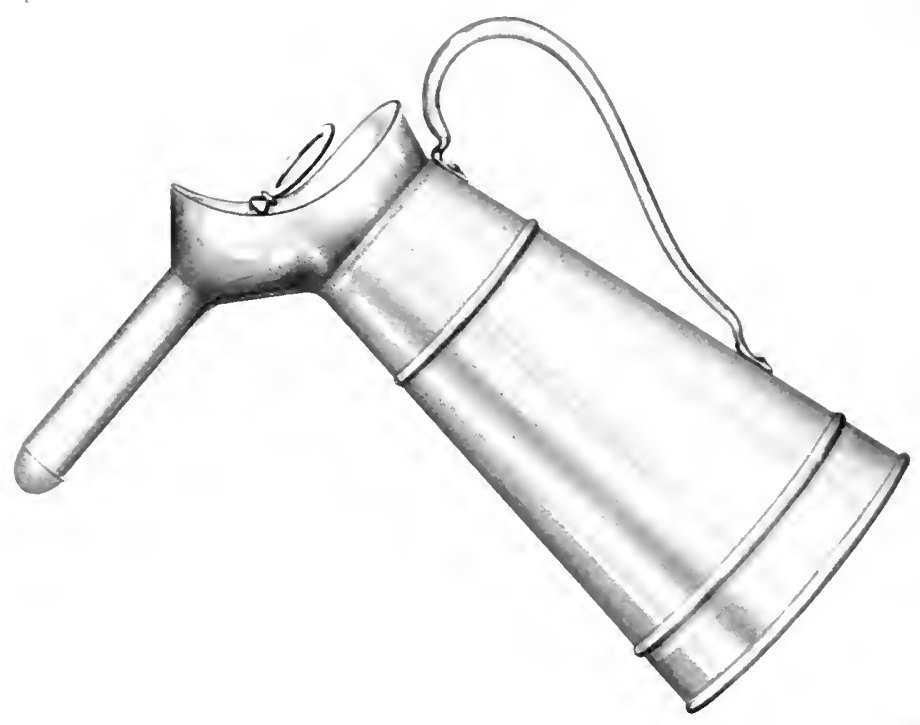

Fig. I - -Gant = combined iunnel protoscope and pitcher employed for pouring oil or sulutions diretly into the colon with the patient in an inverted posture.

the sitting posture is not adrisable for injections except when it is desirable to secure an immediate eracuation by throwing in a small quantity of fluid. If the patient is a child, enemata can be most effectively given with the child lying upon its abdomen and across the lap of the mother.

The quichest and nost satisfactory way of filling the colon with oil or water $i=$ to place the patient in the inverted posture and pour it in. using the author s funnel-shaped proctoscope and pitcher (Fig. I 24 . hut when the patient object - to the posture some other plan must be adopted.

The apparatus uned for the purpose of injecting irrigating solutions and wils into the bowel may be simple or eleborate. but the former, as a rule, are more durable, effective, and less expensive. 
The essential parts of any irrigating onthe are the container (Fig. I 12) and the tube (lig. 125, A) through which the fludel flows inte the bowel. The following paraphernalia are most genorally used for giving enteroclysis and high and low enemata-viz., llue fointain, Dalidson, and piston syringes (Jigs. I I $, 115,117$ ), and different kinds of special apparatus. Of these, the first two are the most satisfactory where the medium to be introluced is a thin Huid, while the two last are preferable when foree is reguired to inject oil or any thick solution into the colon.

The tubes through which the water is carried inte the bowel are variable in size, shape, and length, and may be made of metal and soft or harel rubber, but should invariably be smooth and of such size and shape as not to cause irritation or pain. Irrigating tubes shoukl vary in size $\left[\mathrm{rom} \frac{3}{8}\right.$ to $\frac{1}{2}$ inch $(0.93-1.25 \mathrm{~cm}$.) in dianeter for ordinary purposes, and from $\frac{3}{4}$ to $\mathrm{I}$ inch $(\mathrm{I} .87-2.50 \mathrm{~cm}$.) when employed to guickly soften and wash out discharges and fecal masses from the rectum or sigmoid. Irrigating pipes when short, 2 to 6 inches $(5-15 \mathrm{~cm}$.), are called rectal, and when long, is to 36 inches $\left(45^{-90} \mathrm{~cm}\right.$.), colontubes. Soft-rubber colon-tubes are employed for giving high colonic irrigation and enemata, and the short, soft, or hard pipes for washing out the rectum. The long tube is convenient, but not necessary for high irrigation, because by elevating the patient's hips anrl having him change his position the solution injected through a short tube can be made to enter all parts of the lowel, colon, and sometimes lower ilemm (Fig. 12.5). Unless contra-indicated, the fluid used for high and low enemata should be warm, and permitted to flow into the gut slowly, to aroid the discomfort and pain which ensue when it is cold or the intestine is quickly distended.

There are two types of irrigators-viz., tubes having an inflow (Fig. I20, A, C, D), and those having both an inflow and an outflow (Fig. I20, B). The single-bore pipe is used when the fluid is to be injected and allowed to remain, and the return-flow irrigator when it is desirable to medicate the bowel, wash out poisons or scybala, and keep the irrigating solution at even temperature. The double-flow ube provides the mechanic, thermic, and solvent action of the Huid without cansing the discomfort and pain from distention incident to the introduction of considerable fluid into the bowel which is allowed to remain until it comes away spontaneously. On the other hanel. when it is desirable to have water or a medicated solution retained the single-bore pipe is preferable. The author prefers the opening to be in the end (Fig. I20, A) rather than in the side (Fig. I20, C) of the tube or irrigator, so that the water may run directly through it and dislodge feces, a fold of nucosa, or a rectal valve which interferes with its passage.

Irrigating pipes of all kinds should be marle in such a way and of such material as to permit their frepuent sterilization.

After experimenting for a number of years with irrigating paraphernalia the athor is convineed that the apparatus described below 
is the mont satisfactory for office and hospital use lecause it is effective, holds enough solution for several irrigations. and the stench from evacuated discharges and feces is prevented from exalping.

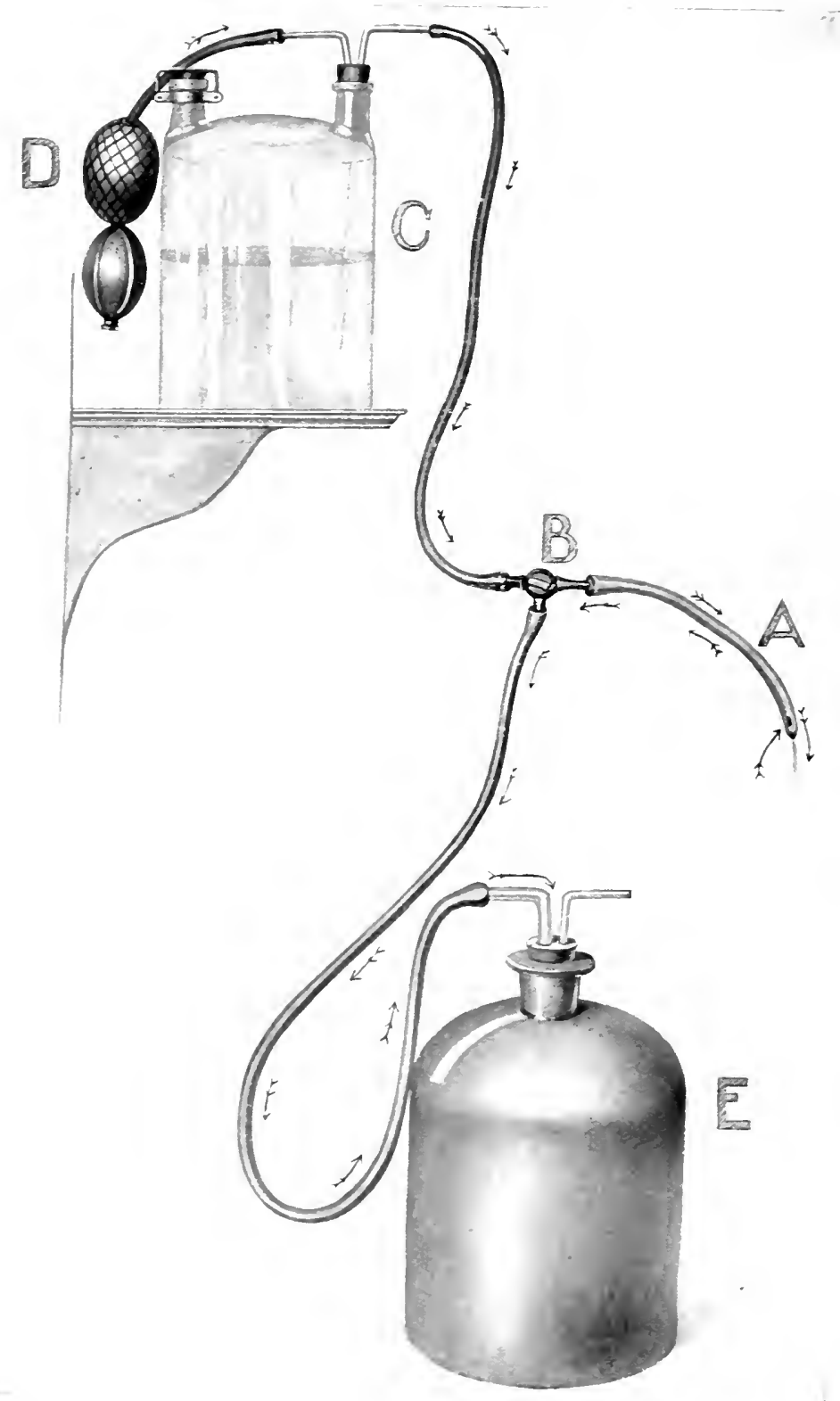

Fir. I25.- - Infaratu- used in auther-office and sanitarium for rectocolonic irrigation.

The apparatu- Fir. 125), which explains iterlf, is employed in the following manner: Introcluce the colon-tube (A) into the bowel and 
turn stop-cock $(B)$ so that the fluid enters the intestine under presiture regulated ley the amount of at which is forced into the lutte. (c) by the rubber hand-bull, (D). By reversing stepecock $(B)$ the bew from the botte (C) is arrested, and the irrigating lluid returns from the lowed and pasoes rownward into botte (E). The contentsol the bottle (E) catn be satisfactorily deolorized ly placing a small amoun of potasium permanganate, charcoal, or ichthyol in the botte prior w the irrigation.

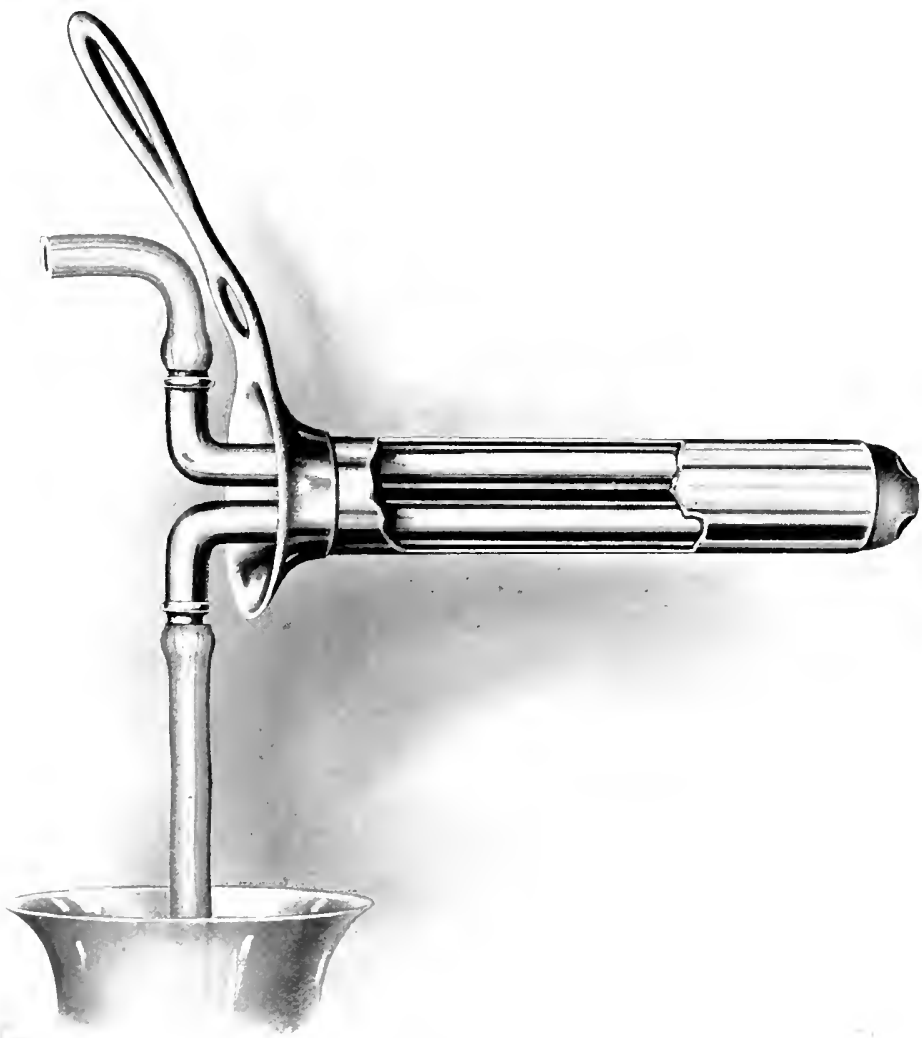

Fig. 1 26.-Gant's double-flow irricating proctoscope.

A low or a high irrigation or lavage can be alministered le altering the pressure, and the lluid be retained for as long or as shert a time as may be desired. This plan of injecting water into all parts of the large bowel is simple, dean, and effective. Amolber satisfactory procedure is to connect one end of the long colon-pipe with a funnel (lig. 110), insert the tube to the desired height, and then pour the Huid or oil inte the funnel, from which it will soon find its way into the colon when 
raised above the patient, while upon his side, back, knees, or in the inverted posture. Still another effective way of introducing oil is to connect the colon-tube with a strong metal syringe (lig. 114), which forces it into the bowel.

Oif should not be employed in the fountain syringe because of its tendency to stick in the tube and rot the rubber.

It is easy to insert a rectal tube, but the introduction of a long or colonic tube is always difficult or impossible (Fig. 127).

The author's technic of passing a colon-tube is as follows: With the patient preferably in the Sims, inverted, or knee-chest postures the pipe, properly warmed and lubricated with vaselin or other stiff lubricant, is grasped by the fingers of the right hand about 2 inches from the end, and gradually inserted into the rectum with a side-to-side or boring motion, while the buttocks are separated with the fingers of the left hand. First it is directed upward and forward until the anal canal and the levator ani muscles have been passed, and thence upward and backward, above the upper rectal valve, into the sigmoid Hexure. After the tube reaches $\mathrm{O}^{\prime}$ Beirne's sphincter its direction cannot be perfectly controlled, and it must be permitted to find its way along the bowel.

The principal anatomic obstructions to introduction of the pipe, named in their order from below, are-viz., the sphincter muscle, folds of mucosa, Houston's valves, O'Beirne's sphincter, and the angulated sigmoid flexure. When the sphincter is irritable and contracts the tube should be continuously pressed against the muscle until it relaxes and permits it to enter the rectum. By allowing the fluid to How through the tube as it passes upward the pipe is stiffened, and this, with the water flowing in, keeps displacing the valves, folds of mucosa, or scybala which may be in the way. When O'Beirne's sphincter spasmodically contracts and blocks the tube the difficulty is orercome by hot fomentations and injection of warm water or oil, which soothe and cause the intestinal musculature to relax.

Force should never be employed when introducing a colon-tube or stiff bougies, because the bowel may be ruptured; and, for the same reason, large amounts of Huid should not be employed at a single sitting except when it is permitted to escape through a return-flow tube.

When it is impossible to insert the long colon-tube in the above manner after introduction of the sigmoidoscope (Fig. I 27), it should be introduced into the lower sigmoid, from which the water can be made to enter all parts of the colon by elevating the hips of the patient and turning him from his left to his right side, irrespective of whether the pipe passes entirely through the sigmoid flexure or not. The accompanying $x$-ray photograph illustrates the futility of attempting to pass a soft tube well into the colon, and it is doubtful if this is ever accomplished (Fig. I27).

Passage of the colon-tube causes but litule discomfort except when it curls up in the rectum or sigmoid llexure, and if it cloes, or the thind or oil does not flow, it should be withdrawn and reintroduced 
until the irrigant passes freely into the bowel. At times it is diffecult to ketermine whether or not the tube has reached the desired height, but by using a little ingenuity one can usually decide the point.

When doubled up (lig. 127) it catses pain in the upper rectum and pelvis, tenesmus, and a tendeney on the part of the bewel to exped it: the solution will not flow, and when slightly withdrawn the tube comes out with a quiver or jerk, and can be felt hy alshominal palpation doubled up when the finger is inserted into the roctum or vaginat, but when the pipe has reached the sigmoid and is molstructed the fluicl runs freely, and when the solution or oil is hot or cold the patient at once feels it entering the colom. Fluid in the rectum is promptly evacuated, but when deposited in the colon it remains several minutes, hours, or orer night.

Except when destined for specific purposes very cold solutions $\left(50^{\circ}\right.$ to $60^{\circ} \mathrm{F}$.) should not be used for irrigating purposes, becaluse they incite a powerful contraction of the intestinal musculature (enterospasm) and are immediately expelled or unduly retained. (In the contrary, warm or hot (100 to $10^{\circ} \mathrm{F}$.) irrigants are desirable because they soothe the bowel and relieve pain and cramps.

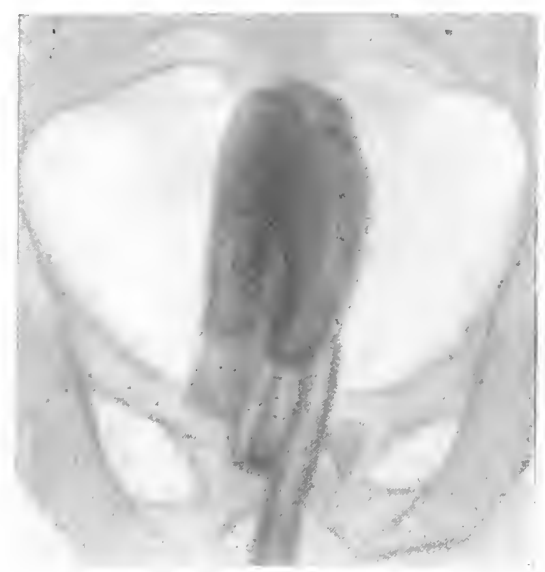

Figr. I 27.-Rarliogram showing how forcible introduction of the colon-lube catuseit to coil upon itself many times, which prevents the solution from passing into the colon above.

When it is desirable to have the irrigations retained for a greater or less time the Strous irrigator is employed, or a large-sized Young's self-retaining anal dilator (Fig. I 20, I)) is inserted to prevent leakage after a sufficient quantity of the fluid has been introduced.

Electrification of the solution is occasionally helpful, in which case a hydriatic tube electrode (Fig. I16) is serviceable.

Following appendicostomy and cecostomy, through-and-through irrigation may lec carried out lyy means of a small catheter, metal, glatss. or hard-rubber irrigator, attached to the conneeting rubber tube of the container by introducing it into the artificial opening and permitting the solution to run into and out of the howel through at proctoscope inserted for the purpose, or it maty be retained and expelleel later, according to indications, after dhe colon has become filled. When the author's appendiceal (Fig. I.52) and enterocolonic irrigators have been introduced at the time of operation they can be useel and the irrigations started at once (Figs. I.5.5, I.56).

When catheters are left in sitn (1iv. r Io) they become soiled and irritable and should be changed from time to time. 


\section{CHAPTER XLII}

\section{OBSTRUCTIVE (MECHANIC, SURGICAL) DIARRHEA}

\section{GENERAI REMIARKS, ETIOLOGY}

General Remarks.-Obstructice represents a type of diarrhea wherein the evacuations are made more frequent or liquid incident to the presence of fecal impactions, foreign bodies, or lesions in or adjacent to the bowel which occlude it so that solid matter is retained, while the watery constituent of the feces is permitted to escape and be eracuated at frequent intervals. From what has been said it may be inferred that this form of loose movements represents one of the manifestations of acute and chronic intestinal obstruction, and that it must necessarily complicate this affection in many cases.

If the frequency and importance of diarrhea as a symptom of intestinal obstruction and other diseases of the intestine was understood by practitioners in general, hundreds of lives would be saved annually, hecause then many lesions which now terminate fatally would be diagnosed, operated upon, and cured.

In this discussion diarrhea and obstipation (constipation) naturally go hand in hand, because, as a rule. patients afflicted with frequent evacuations consequent upon obstruction suffer, first, from constipation: then. costiveness alternating with loose movements, and finally, diarrhea. Owing to this corelation between obstructive diarrhea and constipation the author has made frequent reference to the text, statistics, and illustrations contained in his former work. ${ }^{1}$

Physicians, owing to past and present defective methods of undergraduate teaching, generally hold to the belief that diarrhea is nearly ahwas consequent upon errors in diet, nervous phenomena, digestive disturbances, enteritis, colitis, ptomain-poisoning, affections of the liver, pancreas, and other organs. These affections unquestionably constitute the most frequent factors in diarrhea. but by no means cover all cases, because bowel obstruction is responsible for loose movements in many instances, though one hears little about it in our colleges. medical societies, text-books, or current literature.

The author for many years has done what he could to point out the various types of diarrhea, advise against their medicinal treatment in so far as practicahle, and emphasize the good results obtainable in this class of cases by direct treatment of the bowel and surgical operations.

His interest in this suhject has led his medical friends and former patients to refer to him many sufferers who sought relief from diarrhea. lers: Co.

Gant. Con-tipation and Inte-tinal Obstruction (Ob-tipation), 1010. IV. B. Saun- 
The author has treated many hundreds of individuals for diarhea, and his rather extensive private and clinic statisties indicate that about 20 per cent. Were afflicted with some form of obstruction.

The author operates frepuently for the relief of both obstipation and diarrhea due to intestinal blocking, and he probahly sees a greater percentage of individuals aflicted with obstructive diarthea than the internist or general practitioner, and his large experience has convinced him that the olstructive form of fiartheat prevals in from is to 20 per cent. of the cases, and that the location and character of the blocking can usually be determined when a careful examination is made. The obstructive is almost daily being mistaken and treated for other types of cliarrhea, because, through ignorance or carclessness. the physician does not arrive at a correct diagnosis, and this is surprising, because, with the aid of test-meals, palpation, percussion, bismuth injections, the fluoroscope, $x$-ray photographs, inflatable bag. or sigmoidoscopic examination, the peristaltic waves can be studied and lesions blocking the bowel located with precision in most instances, and the cause of gastrogenic and other diarrheal conditions can be determined through examination of the stomach and intestinal contents, etc.

Obstructions located in the esophagus and stomach seldom induce diarrhea, and when they do, increased frequency of the evacuations results from digestive disturbances or local irritation of the nerves which learl to reflex phenomena and augmentation of peristalsis and glandular secretion.

Obstruction and consequent diarrhea of the small intestine is encountered more frequently and increases in frepuency from above downward; but blocking responsible for diarrhea is located in the colon, sigmoid flexure, or rectum in so per cent. or more of the cases. In the author's experience the obstruction has been encountered most of ten in the rectum, and then, in order of their frequency, the sigmoid, splenic and hepatic flexures, cecum, and transverse colon. It was observed in this class of cases that the ascending and descending colons were rarely blocked, and that the lesions, benign or malignant, causing it were usually located in the above-mentioned or segments of gut which are most frequently subjected to trauma. Splanchnoptosis, coloptosis, adhesions, kinks, angulations, and invagination are the chief etiologic factors in olstruction involving the small lowel, cecum, ascending, descending, and transverse colons; but malignant neoplasms are usually the eause of occlusion of the sigmoid flexure and rectum.

The author has observed both mechanic and other types of diarrhea very much more frepuently in men than in women, and in individuals between twenty and forty years of age more often than in older or younger persons, except calleer patients, whose ages varied from forly to sixty-five.

Numerous types of intestinal olstruction sooner or later lead to frequent or flud evacuations, some of which comstantly block the 
bowel, and the patient suffersall the time, while others intermittently obstruct the fecal current, excite peristalsis, or augment the secretions, and diarrhea is complained of at shorter or longer intervals during the crises.

Sometimes loose movements are indicated by a single mechanic cause, but in others obstipation and diarrhea result from bowel blocking at two or more points, viz., in multiple stricture, angulation, or polypus, and when the above or other lesion is complicated by ptosis, adhesions, extra intestinal pressure, tumor, or fecal impactions located in the rectum and colon.

Physicians often fail to relieve patients under the above circumstances because their examination is superficial and they detect and treat but one obstruction and leave the others undisturbed. The author has several times discovered in the same case obstructing lesions located in the rectum, the sigmoid, colon, and abdomen that were capable of producing mechanic diarrhea. In patients where there are multiple obstructions the diarrhea is usually due to pericolic membranes or athesions, the sequele of appendicitis, typhlitis, colitis, typhoid fever, peritonitis, or suppurative disease in the pelvis or abdomen which binds the bowel to the parietal peritoneum, small intestine, stomach, diaphragm, bladder, tuhes, or uterus.

Exceptionally, lesions causing obstructive diarrhea can be corrected by exercise, massage, electricity, or vibration, but in the majority of instances they cannot be eradicated except by operation, and because of this it has been the author's custom to classify diarrhea as surgical when it is traceable to obstructive or mechanic causes.

It does not follow that because the disease or defect inducing diarrhea is dangerous that the operation necessary for its cure or correction is perilous, for often the lesions responsible for obstinate cases are easy to remedy and with the least disturbance to the patient. As a rule, operations performed for the relief of obstructive diarrhea take but a few moments, are not dangerous, give flattering results, and leave fer sequele, irrespective of whether the block is in the rectum, colon, or abdomen. Naturally, procedures like colonic resection and excision or rectal extirpation and those complicated by abscess are more serious, but they are seldom indicated.

Obstipation prevails when blocking is slight, there is diarrhea alternating with constipation when obstruction is marked, and persistent diarrhea (complicated by impaction) is present when the obstruction is nearly complete.

Etiology.-The frequent and fluid morements incident to acute and chronic intestinal obstruction may be caused in several ways. Sometimes the occlusion is marked and only mushy or liquid feces can get by it; in others, where irritation causes the secretion of mueus in large quantities, and in still others the lesion stimulates peristaltic activity which interferes with digestion and hurries the feces downward to be discharged before absorption can take place or they have been given an opportunity to solidify. Frequently in howel blocking (intestinal stasis), fermentative, putrefactive, and other intestinal bac- 
teria and their toxins are active, and augment intestinal peristalsis. glandular secretion, and the number of daily mosements through their action upon the mucosa, nerves, or circulation.

Another factor which tends to aggravate diarrhea in intestinal obstruction is the nervous state of the patient induced by the thought that he is suffering from a dangerous or incurable disease.

Chronic obstruction is nearly always complicated by ball-lite fecal masses which irritate the bowel and increase the movements. These fecal tumors usually lead to pressure necrosis and stercoral ulcers and exposure of the nerve-filaments, which become irritated and excite peristaltic activity and a greater number of stools. When the rectum is filled with seybala or putty-like masses tluid feces continuously dribble past the impaction and out at the anus. Xow and then irritating gases collect above the obstruction and aggrasate the diarrhea until they have been expelled.

Any of the following mechanic or obstructive lesions may block the bowel and cause diarrhea alone or alternating with ob-tipationviz.: congenital deformities; extra-intestinal pressure; strictures; malisnant and non-malignant neoplasms; foreign bodies; intestinal calculi (enteroliths); fecal impaction (coprostasis); adhesions (including tumefactions, pericolic membranes, and peritoneal and fibrous bands); ansulations, flexures; diverticula and rectocele: pericolitis and perisigmoiditis (pericolic membranes): sacculation (rectocolonic); abnormal or diseased mesentery; ioliulus and kinks; hernia; inzagination, intussusception. rectal procidentia; splanchnoptosis and enteroptosis; paralvitic ilents; dilatation of the colon (congenital and acquired); postoperative sequele: enterospasm; intestinal parasites (aorms); hypertrophy of O'Beirne's sphincter; hypertrophy of the rectal alaes; hypertrophy' of the leiator ani; hypertrophy of the sphincter ani; anterior desiation of the coccy.x: diseases of the rectum and anus (hemorrhoids and fissures) which excite muscular contraction or block the bowel.

Congenital deformities have been encountered in all parts of the small and large intestine, but occur most frequenty in the colon. sigmoid flexure, or rectum. When the gut is abnomally large, narrow. long, kinked. displaced, strictured, or vceluded by membranous partitions, and the deformity prevents the passage of solid feces and leads to fecal impaction, increased frequency and Huidity of the stoots invariably obtains.

Malformations are commonly encountered in the rectum or at the anus, and the one or the other is imperforate, narrowed, or blocked by transverse bands which induce partial or complete obstruction. Diarrhea, however, is most often a sequel of operations performed for the relief of congenital deformities of the colon, rectum (Fig. I 28 ), or anus which have led to peri-intestinal adhesions or stenoses.

Extra-intestinal pressure incilent to abdominal and pelvic tumor: or enlargement and displacement of the uterus (Fig. I20), adnexit. prostate, or other organs occasionally block the bowel and interfere with the morements. 
Foreign bodies (intestinal sand, calculi, etc.), which traumatize, irritate or obstruct the intestine, nsually induce obstinate loose movements.

Fecal impaction (coprostasis) resulting from constipation, chronic intestinal obstruction, enterospasm, or other cause, is frequently com-

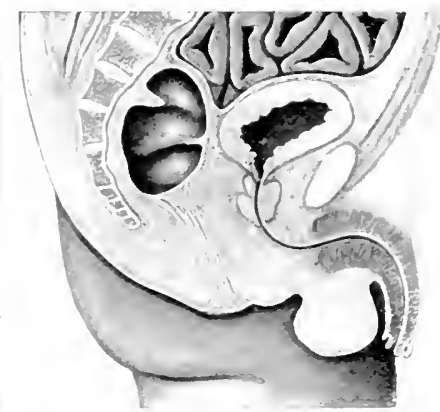

Fig. I 28.-Imperforate anus, the roctum encling in a blind pouch.

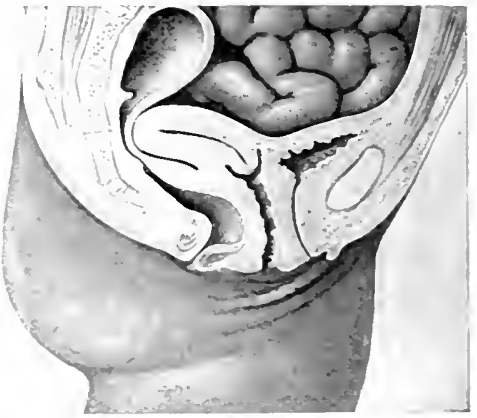

Fig. I 20.-Rectal ob-truction produced by a retroverted uterus.

plicated by diarrhea, because the scybala or latrer firm fecal masses prevent passage of the gas and solid feces, excite peristalsis and the hypersecretion of mucus, augment the pathogenic intestinal bacteria and their toxins, and through disturbance of the circulation lead to the

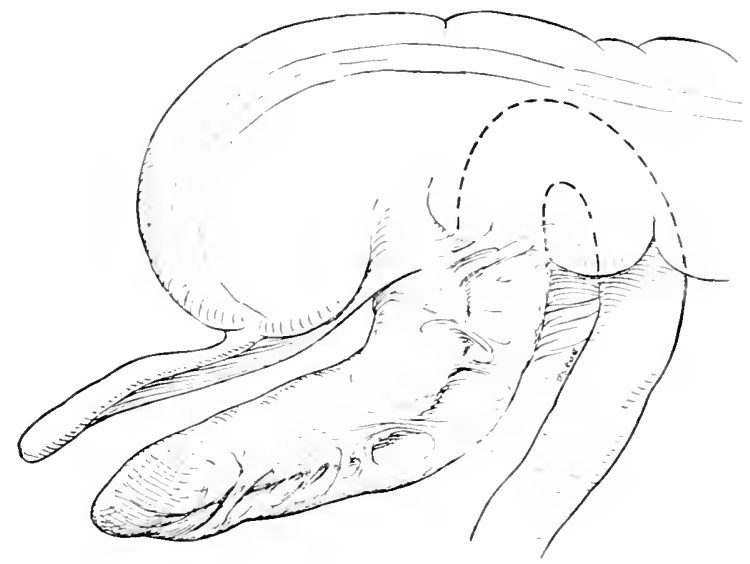

Fig. 130.-Lane's kink at the ileocecal juncture formed by alhesions. (After author's case.)

formation of stercoral ulcers, all of which conditions favor diarrhea alone or alternating with constipation.

Adhesions (Fig. I 30) resulting from operations, colitis, appendicitis, and suppurative or malignant diseases of the intestine or abdominal sroans, frequently cause chronic intestinal obstruction characterized 
by gas accumulation, occasional colic, and constipation followed by (liarrhea.

Strictures and tumors which press upon or constrict the gut or project into and block its caliber are freepently responsible for loose movements, irrespective of the etiology behind them.

Angulations (Fig. I3I), kinks, and twists which olstruct the intestinc (particularly the colon or sigmoid flexure) to a marked degree first induce constipation and later cliarrhea, because they are characterized by coprostasis, intestinal irritability, obstruction and retention of offensive feces, discharges, bacteria, and toxins, all of which tend to augment peristalsis and an increased secretion of mucus. These types

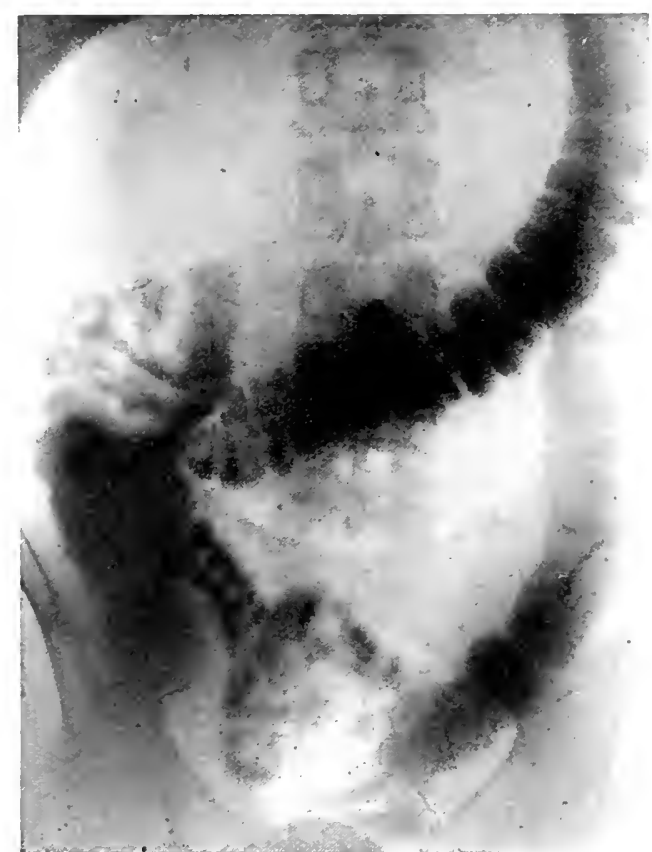

Fig. I3I.-Ptosis and angulation at the hepatic flexure. Yote how the bismuth solution has been projected by the enema into the lower ileum. (Taken by Cole.)

of colonic distortion are usually secondary to a pericolitis or sigmoidit is marked by the formation of pericolic membranes (Jackson's) or adhesions (thread-, hand-like, or fan-shaped adhesions) consequent upon organic changes insicle the bowel, or inflammatory or suppuratire disease within the abdomen or pelivis.

Diverticula and sacculations of the colon (diverticulitis and peridiverticulitis) (rig. I32) and sigmoid flexure and rectoceles which attain considerable size provide a lodging-place for the feces that collect in an amount suffeient to induce an irritative or obstructive diarrhea, or such pockets may become occluded, undergo changes-diverticulitis or peridiverticulitis-which terminate in the formation of an absess and fistula which discharge into the bowel and incite septic diarrhea. 
Pericolitis and perisigmoiditis from whatever cause are invariably accompanied by the formation of adhesions (thread-, band-, fan-, and sheath-like) or a pericolic membrane (Jackson's) (Fig. I33) which

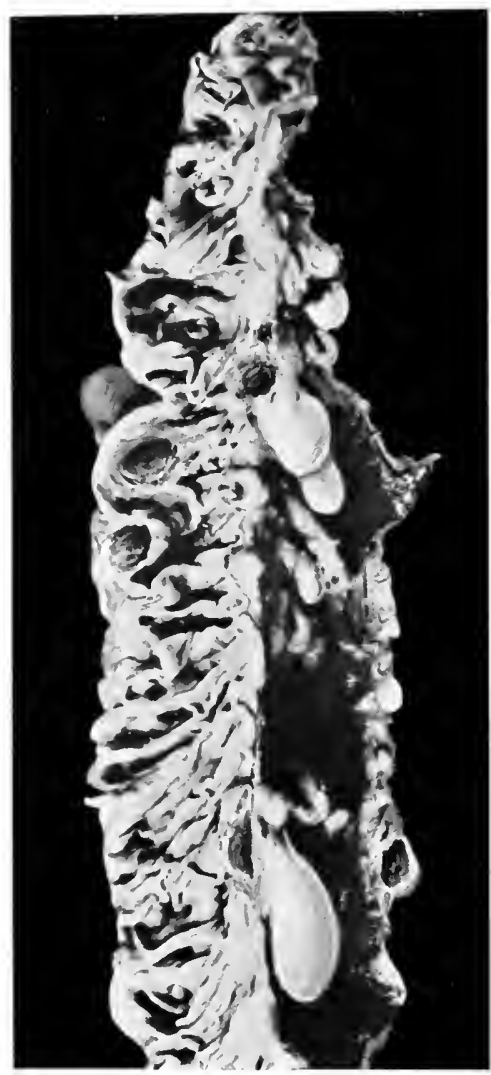

Fig. 132.-Diverticula of the colon and sigmoid flexure. angulate, dislocate, compress or envelop the colon, impair digestion, induce auto-intoxication, fecal impaction, and irritative diarrhea or enterospasm (spastic constipation).

Distortion and disease of the mesentery has been known to cause acute and chronic loose movements by obstructing the

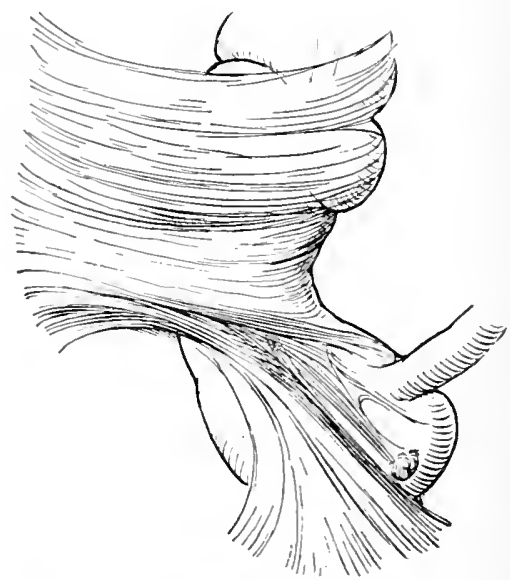

Fig. I33.-Membranous pericolitis. Note the pseudoperitoneal (Jackson's) membrane which binds the ascending colon, cecum, and appendix to the lateral parietal peritoneum.

gut or irritating its nerve mechanism, and chronic incarcerated hermia has in rare instances produced like results.

Invagination, intussusception, and procidentia recti may, when chronic, induce constipation alternating with diarrhea through the retention of toxins, olstruction or irritation caused by them.

Splanchnoptosis and enteroptosis (Fig. I34) are usually complicated by obstipation, but when the colon is compressed by displaced neighboring organs, is markedly ptotic, angulated, and twisted, or is extensively involved by pericolic adhesions or membranes, the patient invariably suffers from intermittent attacks of or continuously from coprostatic diarrhea.

Congenital and acquired dilatation of the colon invariably induce obstipation and stercoral diarrhea.

\footnotetext{
1 Army Med. Museum.
} 
Paralytic ileus frequenty terminates fatally, but when it is partial or the olstruction has been complete, but has been relieved and retained, toxins are discharged in to the lower bowel and toxic diarrhea ensues.

Enterospasm incident to forcign bodies, offensive discharges, and inflammatory, ulecrative, or ohstrueting lesions in the bowel is characterized in the beginning by complete obstipation, and later frecpuent fluid evacuations, which immerliately follow relaxation of the intestinal musculature.

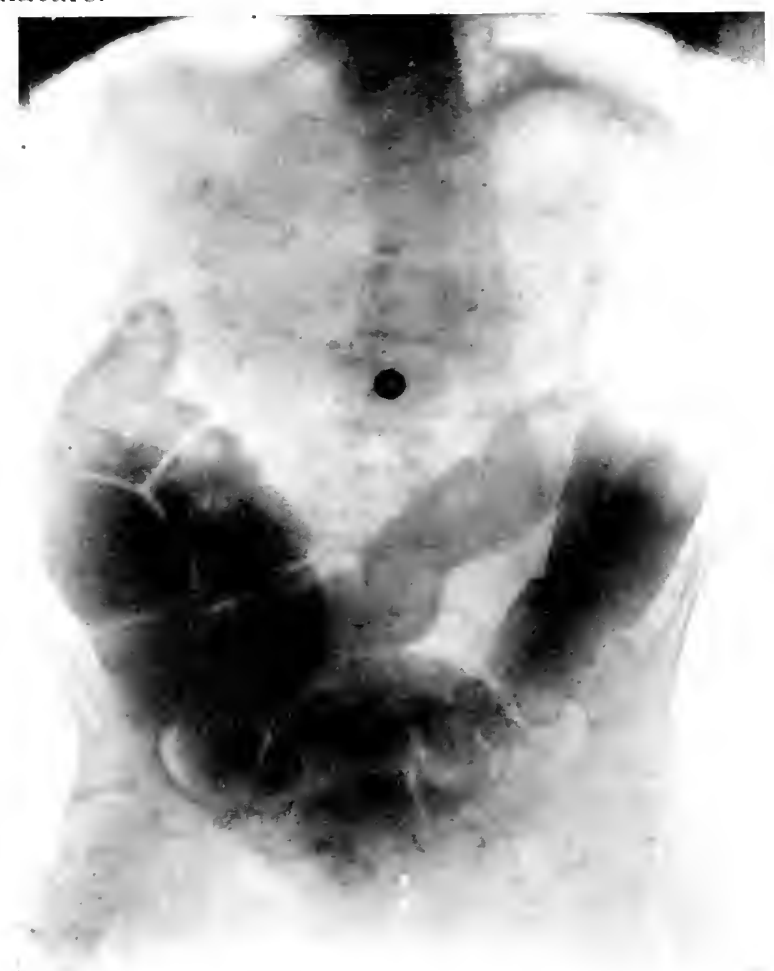

Fig. 134.-Marked ptosis of the transverse colon with redundancy of the sigmoid flexure. (Taken by Cole.)

Postoperative sequele and intestinal iorms which distorted or blocked the bowel have been known to cause constipation, loose movements. or both.

Hypertrophy of O'Beirne's sphincter (rectosigmoidal), the rectal i'alies, lesator ani, and sphincter muscle have one and all been responsible for coprostatic or olstructive diarrhea, and the same can be said of anterior coccygeal deriation.

Rectal diseases-viz., tumors, strictures, hemorrhoids, ulcers, polypi. fissures, procidentia, or fistulc-may augment the frequency and fluidity of the evactations by blocking the bowel or exciting peristalsis or the hypersecretion of mucus. 


\section{CHAPTER ILIII}

\section{OBSTRUCTIVE (MECHANIC, SURGICAL) DIARRHEA (Continued)}

\section{SYMPTOMS}

THE symptoms of obstructive diarrhea depend principally on its course. duration, and the degree of occlusion. When blocking is almost complete, acute constipation ensues; when it is less marked, chronic constipation and diarrhea are induced. The symptoms of the former are more riolent and dangerous than the latter, which derelops slorty and seldom completely obstruct the bowel. In acute obstruction cathartics are ineffectire, and high enemata at first hring away a small amount of feces, and are returned clear thereafter. Persons suffering from acute obstruction frequently have a desire to eracuate the bowel, but pass nothing but mucus. This type of costiveness is most common in childhood, is of sudden onset, and charaeterized by continuous pain in the central abdomen. Lnless complete obstruction is promptly relieved the patient becomes restless. breaks ou in a cold perspiration. hiccups, is nauseated. and at first romits the stomach-contents, then bile, and, finally, fecal matter, has a furred tongue, facial expression of distress. weak, thready pulse, sulnormal temperature. labored respiration, and a distended and tender abdomen. Lsually peristalsis is riolent, and the intestinal morement = may be risible or felt by palpation when the abdominal wall is thin. When the block is not promptly relieved the patient soon dies from exhaustion, toxemia, or septic peritonitis.

Following an attack of acute mechanic obstruction, patients frequently suffer from chronic constipation as a result of the paralyzed condition of the intestine incident to prolonged distention or persistent diarrhea which may prevail where toxins are virulent or abundant.

Chronic mechanic diarrhea or constipation are of common occurrence and encountered more frequently in adults than children. and in women than men.

The srstemic manifestations of chronic obstruction are similar in many ways to those of diarrhea resulting from improper diet, irregular livine. catarrhal and ulcerative colitis, ant digestive ailments, but local clisturbances vary with the nature of the lesion causing the occlusion.

Chronic obstructive constipation or diarrhea may be preceded by peritonitis, appendicitis, typhoid fever, pelvie inflammation, injury, sursical operation. or the formation of adhesions. but most frecquently they 
are caused by one or more lesions of the bowel which impede the fecal current except when caused by a congenital deformity. The patient usually has enjoged good health until the bowel began acting queerly and the stools became too far apart, abnomal in shape, or frecuent and watery. In this type of diarrhea the character of the evacuations and local manifestations depend upon the part of the bowel involved and nature of the obstruction. In one case diarthea is continuous, while in another it is intermittent or alternates with constipation, and there may be periods between the attacks when the bowel acts normally.

Diarrhea and constipation are nore obstinate when the obstructing lesion is in the sigmoid flexure or rectum than in the small intestine or upper colon. When the small gut is blocked the feces, owing to their liquid state, find their way past the obstruction, then become solid, but usually the movements are thin and irregular. When the sigmoid flexure or rectum is involved the evacuations are frefuent, the stools are licjuid or semisolid and irregular in shape, and the patient constantly feels as if there was something in the lower bowel that ought to be expelled (Fig. I35).

The symptomatology of chronic obstruction complicated by diarrhea induced by congenital deformities, extra-intestinal pressure, pericolic bands, adhesions and tumefactions, abnormal mesentery, arolinlus, kinks, angulations, and hernia is similar in many respects. Without previous warning the bowel begins to act irregularly and the stools become further apart; at first a daily stool can be secured by the aid of

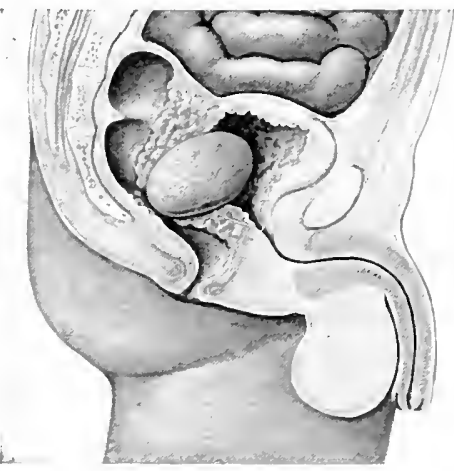

Fir. I35.-Stricture of the rectum produced by a large urinary calculus which ulcerated through the rectoresical septum. (Removed by the author.)

fruit, a special diet, and exercise, but later constipation becomes monte obstinate and mild laxatives are required to obtain a satisfactory movement; and, finally, frequent copious enemata or exceedingly strong cathartics are necessary to secure the coveted evacuation and avoid fecal impaction. When unrelievert, the obstruction increases and obstipation is persistent or alternates with diarrhea, and evidences of auto-intoxication appear. Finally, when the lumen of the gut is almost completely closed, the mucosa is excoriated (stercoral ulcers), the colon above the obstructed point becomes dilated, and diarrhea prevails. because only liquid feces can get by the obstruction, the bowel is irritable, and abnormal peristalsis and glandular activity are easily excited.

Patients who suffer from mechanic chronic constipation or diarrhea sooner or later complain of disturbed digestion, meteorisnt, sensations of weight and fulness along the colon or in the rectum. colic, pulling pains, headache, loss of sleep, nervous phenomena, etc. 
Intestinal stricture (Fig. I 36 ) produce- varying symptons, depending upon the degree of constriction and the part of the bowel involved.

stenosi- of the small intestine due- not occur often. seldom produces obstinate ubstipation. diarrhea, violent peristalsis. intestinal stiffening, or the colicky pains frequently witnessed in persons afflicted with constriction of the colon or rectum. Azain, in occlusion of the small howel. the patient complains of arstric distention, indigestion. nausea, and romiting. owing to backing up of the chrme above the

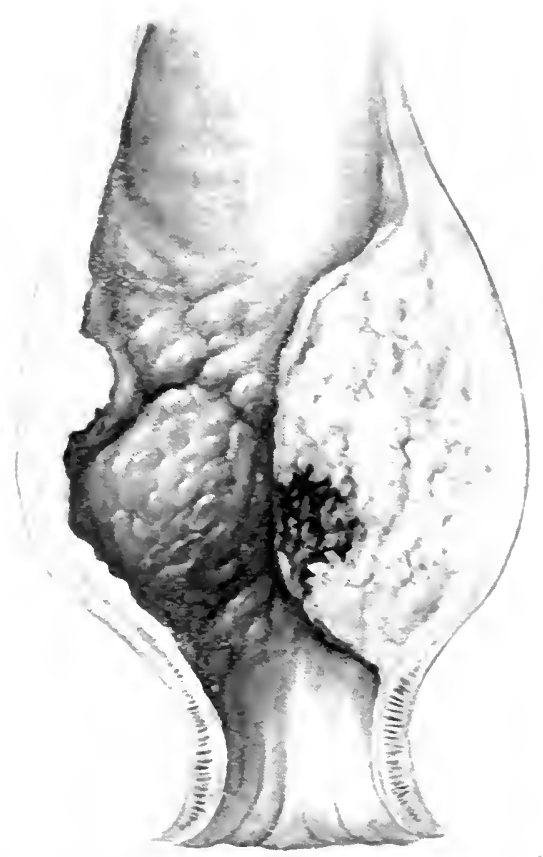

Fic. Isf-Crlindricolled corcinomatous stricture of the rectum-the mass on the rifk shows a charateriatic crater-like wher. Aiter author's case.)

constriction, a condition which som leads to malnutrition and emaciation.

Stricture of the large bowel is fairly common. and is encountered more frequenty in women than men. and oftener in patients orer forty. In the beginning. colonie stricture produces a slight discomfort in the abdomen difficult to locate. Constipation soon begins to play an important rile, and the exacuations become irregular or delared except when stimulated by fruit. Water drinkins. and exercise. Shortly, mild laxative are indicated and after a further time. strong cathartics. puratives alone or in conjunction with enemata of oil or soapsuds. are required to secure daily morements, and. finally, when the con- 
striction becomes tight, constipation alternates with diarrhea or frequent evatuations prevail, and prevention of fecal impaction is difficult.

When once stenosis of the colon or sigmoid tlexure is fully developed the patients not only suffer from ubstinate constipation and diarrheal attacks. but aloo from poor appetite. They aloo complain of irritable stomatel, gas colic, have sallow complexions. blotches on the skin, loso of weight, headaches. nervousness. lasitule. neuralgias. and other manifentations consequent upon copremia or the alsorption of toxins, alsdominal soreness, local tenderness upon presirese sensations of weight and lulness. violent peristalsis (discernible by inspection through the fluoroseope or palpation), intestinal rigility. recurring fecal impation. colitis, mucus, pus. and blood in the stosts, and a sentation as if the howel was lolockerl at a definite point.

Stenoses of the rectum and lower sigmoid flexure are accompanied by the above symptoms and an incessant desire to empty the lowel, straining, hearing-down sensations in the rectum, tenesmus, pain in neighboring organs, the sacrococcygeal region and down the limbs, and tape-or pipestem-shaped stools.

Lsually ulceration is more marked in rectal stricture, the stools foul smelling, more frequent, fluid, and contain an abundance of pus, blorel, and mucus.

In nearly all cases of rectal stenosis the patient suffers from incontinence. owing to destruction of the anal muscle by disease or relasation of the sphincter through overwork. and fluid feces and irritating discharges constantly drilbble through the anus to irritate the skin and cause pruritus.

Enterospasm is of sudden onset and the attacks may last from a few moments to several hours or days and cause partial or complete occlusion, constipation, or diarrhea. At such times the bowel can lee felt through the abdominal wall as a hard-knotted or small ridge-like tube, and the patient suffers from gas distention, cramping, abdominal soreness. rumbling sounds, and olstipation or coprostatic diarrhea.

Intestinal Parasites (Worms). - Suljective and objectivesimptoms, aside from irregular action of the bowel. are entirely absent in certain cases. whereas other patients complain of distresing crawling sensations in the abdomen, cramps, itching of the anus and the nose, digertive disturbances, anorexia alternating with bulimia. li-turbed sleep, extreme nervousnens, and constipated or loose movements.

Benign and malignant neoplasms of the intestine (1.ig. I37) induce constipation alternating with cliarrhea, frefuent straining, and about the same other manifestations as stricture; and, in adklition. cancer patients suffer from cachexia, enlarged glancls, involvement of other organs, a more rapiel loss of flesh, severe pain, and the stors have a characteristic nauseating oflor and eontain pus. blood, mucu-, pigment, and shreds of tisiele.

The symptoms induced by foreign bodies-enteroliths and fecal impactions-are almost iflentical, viz., they are more numerour and distressing when the offending oljects are multiple, large. irregular in 
shape, or lodged at a narrow or angulated portion of the gut, than when single, small, smooth, and loeated in the free intestine.

In the beginning there is constipation alternating with diarrhea, but when the bowel becomes blocked by an impacted mass or stone the eracuations become fluid and escape around it. The stools are offensive and contain considerable mucus, alone or mixed with pus and blood. Impacted fecal masses and foreign bodies situated in the colon and sigmoid flexure induce discomfort in the abdomen. excite frequent and prolonged peristalsis, and interfere greatly with digestion. When situated in the rectum they cause dull aching pressure pain. straining, a constant desire to evacuate the bowel, and sacrococcrgeal pain down the limbs and in neighboring organs, frequent
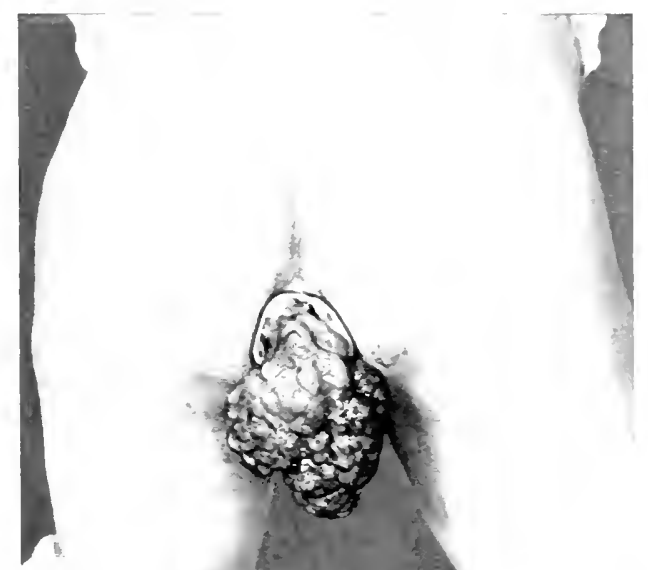

Fig. 137.-Cimple adenoma. larger than a man's fist, removed by operation for the relief of chronic obstructive constipation and diarrhea. (Photograph.) (Author's case.)

micturition, or excite the levator ani and sphincter muscles to contraction.

Diverticula, which become distended and press upon the bowel or obstruct it. cause constipation or diarrhea. and when inflamed induce local pain and symptoms similar to those of appendicitis. Rectocele causes very little trouble except when it serves as a trap in which the feces accumulate. In such cases it induces sensations of weight, fulness, bearing-down pains, and loose moxements or dribbling of fluid feces through the anus.

Splanchnoptosis.-General or aggravated ptosis of special organs reduces the patient to a deplorable state: he is emaciated, suffers from malnutrition, digestive disturbances, loss of appetite, anemia, cold extremities, weak pulse, sinking sensations. dragging pains in the aludomen, a pulsating aorta, tympanites, colic, occipital and temporal headaches, a bulging or pear-shaped abdomen with thin tlabby walls, 
loss of fat and weakened musculature, I)iotl's crises, constipation, which alternates with diarhea, and when the enteroptosis is due to separation of the recti muscles the patient feets as if the aludominal contents were dropping out.

Ptosis of all or any part of the colon is always accompanied by constipation and fecal impaction or coprostatic diarrheas.

Fecal impaction (Fig. 1,3s) canses a still greater displacement of the bowel, pulling pains, hacking up of gases, and favors the formation of adhesions, cansing montural positions of the gut, lessening and imparing its function.

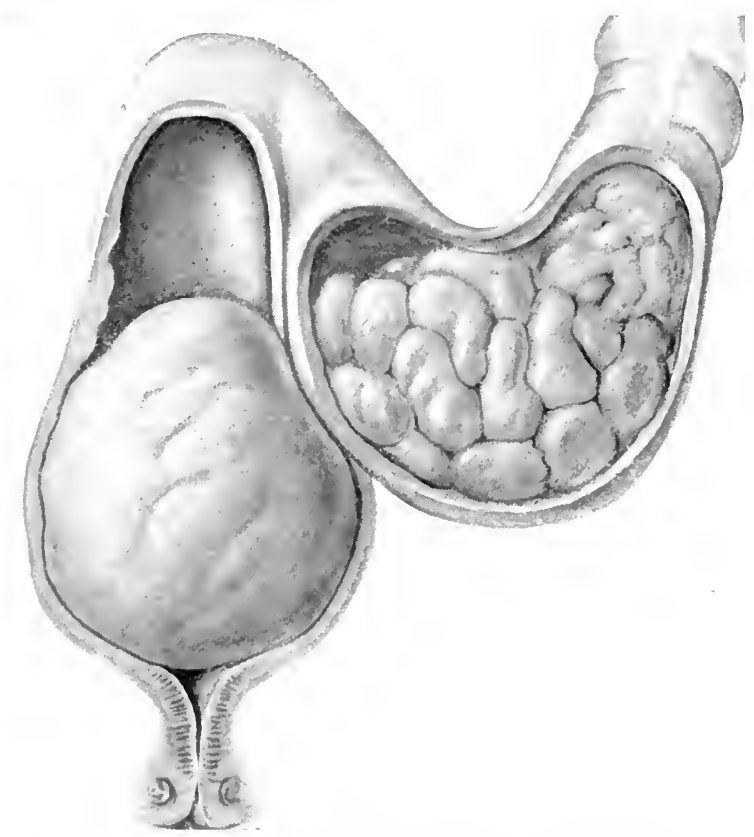

Fig. 138.-Chronic fecal impaction resulting from an angulation in the descending colon and narrow anal canal. The sigmoid flexure is packed with scybala and the rectum filled with a large putty-like mass. (Mfter author's case.)

Ptosis of the Sigmoid Flexure.-The author has treated many patients who suffered from obstructive diarrhea caused by ptosis of the sigmoid. In some cases the accompanying olstipation and coprostatic diarrhea were dependent on massing of the gut in the pelvis, while in others to invagination of the sigmoid into the rectum. The symptoms arising from sigmoid ptosis or invagination are farry clear. The excrement collects in the sigmoid and becomes impateded, causing obstinate constipation alone or alternating with fliarhera, (listention, hulging, tenclerness, sensations of weight, and iragging pains in the left iliac and pelvie regions, uterine and vesical disturbances, occasional discharge of jelly-like or stringy mucus or offensive fluid evacuations, and at sensation of lolocking in the bowel. 
Paralytic Ileus.--This type of obstruction results from complete intestinal ocelusion or extensive operations upon the viscera, the bowel is completely inactive, obstipation is complete, and the condition of the patient beeones grave and he suffers from natusea, meterism, fecal romiting, and a rapid, thready pulse until he is relieved or dies. In some instances the bowel is only partially paralyzed, when the symptoms are less urgent and the patient complains of constipation, diarrhea, gas pains, and the usual manifestations of partial obstruction.

Invagination (Intussusception).-In acute invagination the bowel suddenly becomes blocked and the patielit suffers from acute abdominal pains, distention, rapid pulse, naused and romiting. cold perspiration,

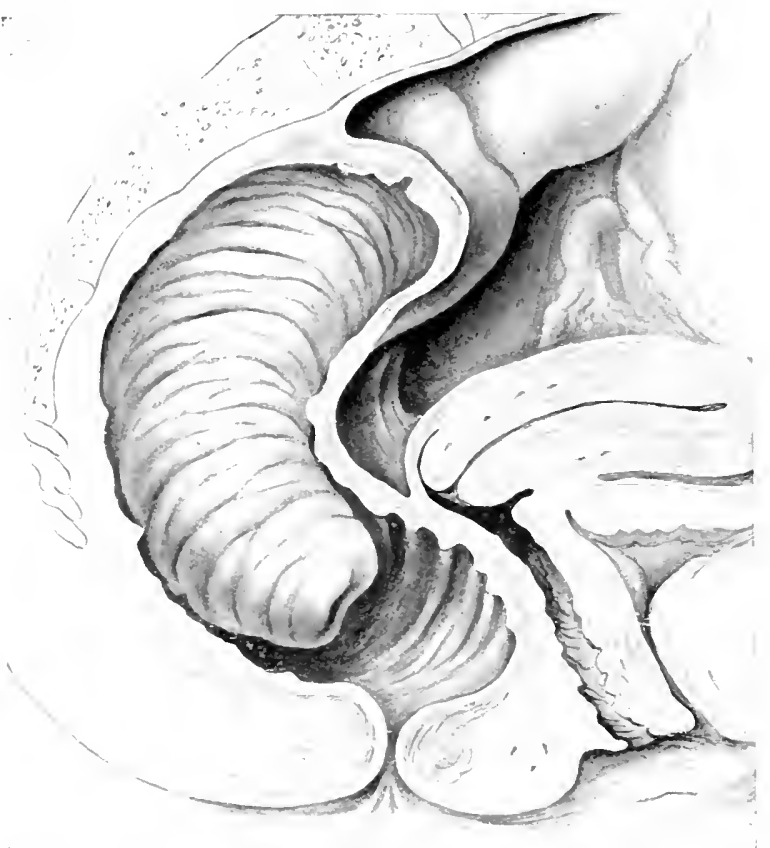

Fig. I39.-Showing chronic invagination of the sismoid flexion into the rectum, as viewed from below through the proctoscope.

partial or complete obstipation, subnormal temperature, and indications of collitpie.

Chronic invagination occasionally takes place at the ileocecal juncture, but the signoid flexure telescopes into the rectum very much more frequently. The manifestations of this condition are similar 10 those of other chronic obstructive lesions, but are modified according 10 the legree in which the gut is incarcerated. Usually the patient suffers from constipation, intermittently or all the time. moderate tympanites, local tenderness and pain, muens in the stools, sensations of weight, fulness, blocking at the obstructed point, or offensive tluid evacuations where fecal impaction prevails. 
When the sigmoid is invaginated into the rectum (Fig. I.39), the patient has an incessant and unrelievable desire to stool, bearingdown pains, feeling as if there was a foreign body in the rectum, strains terrifically at stool, passes more or less mucus, and suffers from persistent constipation or diarrhea, but at other times the stools are normal and the patient is comfortable exeept for the annoyance caused by the accompanying catarrhal inflammation.

The symptoms of congenital and acquired dilatation of the colon resemble those of splanchnoptosis-viz., the feces accumulate in considerable fuantities and the patient complains of gas flistention, indigestion, cardiac disturbances, nervous phenomena, natleca, romiting. and olstipation alternating with diarrhea.

Hypertrophy of O'Beirne's sphincter is accompanied by a fecling of tenderness, weight, and blocking of the gut at the region of the rectosigmoid juncture, backing ap of feces and gas in the colon, and olstipation or diarrhea according to the degree of obstruction catsed by the muscle and the presence of irritating lecal accumulations.

Hypertrophy of the rectal valves is accompanied by the accumulation of leces above them, which forms into scybala or large, firm, or semisolid masses which induce pressure pains, desire for an evacuation, with imperfect and painful defecation. Ordinarily, cathartics are ineffective, and the impacted masses must le remored ly enemata to prevent them completely blocking the rectum and causing fluid evacua(ions.

The manifestations which accompany hypertrophy of the leator ani and sphincter muscles are similar to those just enumerated, with the exception that the blocking is not constant. wing to the fact that the muscles do not remain in a continuous state of contraction, and these patients suffer sererely from sphincteralgia or sacrococesceal pain at long an the muscles remain rigid, but at other imes they are comparattively comfortalyle.

Diseases of the rectum and anus that induce constipation or coprostatic diarrhea in addition to the usual symptoms of chronic lower bowel obstruction may, according to their character, be accompanied by pain in the anal region, pruritus, excoriation of the skin, tenesmus, sphincteralgia, and the discharge of mucus or pus. 


\section{CHAPTER NLIV}

\section{OBSTRUCTIVE (MECHANIC, SURGICAL) DIARRHEA (Continued)}

\section{DIAGNOSIS}

ONE can easily differentiate ordinary from obstructive diarrheas, but it is difficult to distinguish between the rarious intestinal lesions responsible for chronic loose movements and obstipation.

Obstruction rarely takes place in the small bowel, and when it does, diarrhea seldom ensues, because the feces have ample time to become semisolid or firm after passing the block, but obstructive lesions are frequently encountered in the colon, sigmoid flexure, and rectum.

Patients afflicted with chronic mechanic (obstructive) diarrhea usually give a history of having previously suffered from indigestion, irregular evacuations, auto-intoxication, ptomain-poisoning, enteritis, catarrhal or specific colitis, peritonitis, appendicitis, diverticulitis, etc., salpingitis, or suppurative disease within the abdomen, etc., manifestations and diseases which of ten complicate or cause adhesions, pericolic membranes, kinks, twists, stricture or distortions of the colon, and surgical diarrhea.

In such cases, when loose movements prevail or alternate with constipation and recurring fecal impaction, the sufferer complains of abdominal soreness, pain on pressure at a given point, meteorism, one-sided abdominal distention, cramps unrelieved by an evacuation, and intestinal auto-intoxication, one is justified in diagnosing the case as obstructive diarrhea, irrespective of whether or not he can determine the exact nature of the condition responsible for the block.

Palpation, succussion, percussion, auscultation, and distention of the colon with air, gas, or water materially aid in the diagnosis when the patient is made to change his position during the examination. but an opinion should not be given until the abdomen has been examined through the fluoroscope, and radiographs have been made of the colon following the administration of bismuth meals or enemata.

When an obstructing lesion has been located, surgical intervention is indicated, whether its character is known or not.

Congenital deformities of the large intestine are easily located at the anus or in the rectum, and can be seen, felt with the finger, or inspected through the proctoscope. Hirschsprung's disease and megacolon are recognized by the accompanying pot-belly, long-delayed stools, pasty color of the patient, and enormous size of the colon, as revealed by palpation, inflation, fluoroseopic examination, radiographs, and large amount of water it will hold. Malformations in other colonic 
segments may be suspected, but cannot be positively diagnosed except by laparotomy or at autopsy.

Extra-intestinal pressure is sometimes temonstralsle by physical examination, fluoroscoping or $x$-ratying the bowel, and by obtaining a history which would indicate that the colon is being insolved through the extension of diseate or a tumor from neighboring organs or struetures.

Strictures are comparatively easy to diagnose, wing to the previous history, which will show that the patient has suffered from typhoid, ulectative colitis, tuberculosis. syphilis, pelyic or abdominal suppuration, or other affection accompanied by a destruction of the mucosa, thickening of the intestinal tunics, or the formation of arthesions which press upon or constrict the colon.

Their location can be ascertained with the finger or simmidoscope when low, and ly enemata, inflation. the fluoroscope, or radiographs when high.

Rectal strictures produce sensations of fulness, bearing down. incessant desire to stool, and pain. Which are unrelieved by an evacuation.

Benign and malignant tumors present the same manifestarions as stenoses similarly located. Lut, when distinguishable with the finger or through the sigmoidoncope. they are recognizerl by their oxoid or lobulated or pedunculated form. In addition, patients suffering from malignant distase are marherlly cachectic and emaciated and the feces are often typically offensive.

Intestinal calculi are seldom diagnosed except when they are evacuated or felt in the rectum in patients who suffer from gall-stones, and the same can be said of foreing bodies in the absence of a history indicating that the sufferer has swallowed some foreign substance. In a few instances intestinal sand has been accidentally discosered while the feces were being examined for some other condition.

Fecal impaction complicates all forms of chronic intertinal ofs-truction, and scrbala can usually be located above the block. [ noker such ciremmstances fecal can be distingui-hed from other tumors by their mobility, indentability, rapidly increasing size, and tendency to disappear following the administration of castor oil and copious =oapsuds enemata and the evacuation of scybali. Large fecal mases in the lower sigmoid and rectum induce pressure pains. tenesmus. constant dribbling of thuid fecer, and can be felt by digital examination or inspecterl through the proctoscope.

Adhesions are diannsed by getting a history that the patient has been operated upon or suffered from an acute or chronic intlammatory process within or without the bowel, localized rlistention, discomfort. and pulling pains when he changes his proture.

Volvulus (Fig. Ifo), angulations, and kinks thould be -mipected under the sime circumstances because they cannos be differentiated from adhesions except hy radiography, which is not infallible.

Diverticula are usually diseovered hy accident. -ince they to 130 t produce symptoms except when they beeone inflamed, under which 
circumstances they cause manifestations simulating those of appendicitis except that the localized soreness, pain, and muscular rigidity is in the sigmoidal region, which is the segment of gut most of ten the site of diverticula. During acute crises constipation is marked, the temperature is irregular, and a swelling can be seen or felt in the lower left abdominal quadrant, and where perforation ensues the usual symptoms of abscess or peritonitis are present. Sacs in the lower sigmoid and rectum can be detected with the finger or proctuscope by their openings into the bowel through which pus escapes.

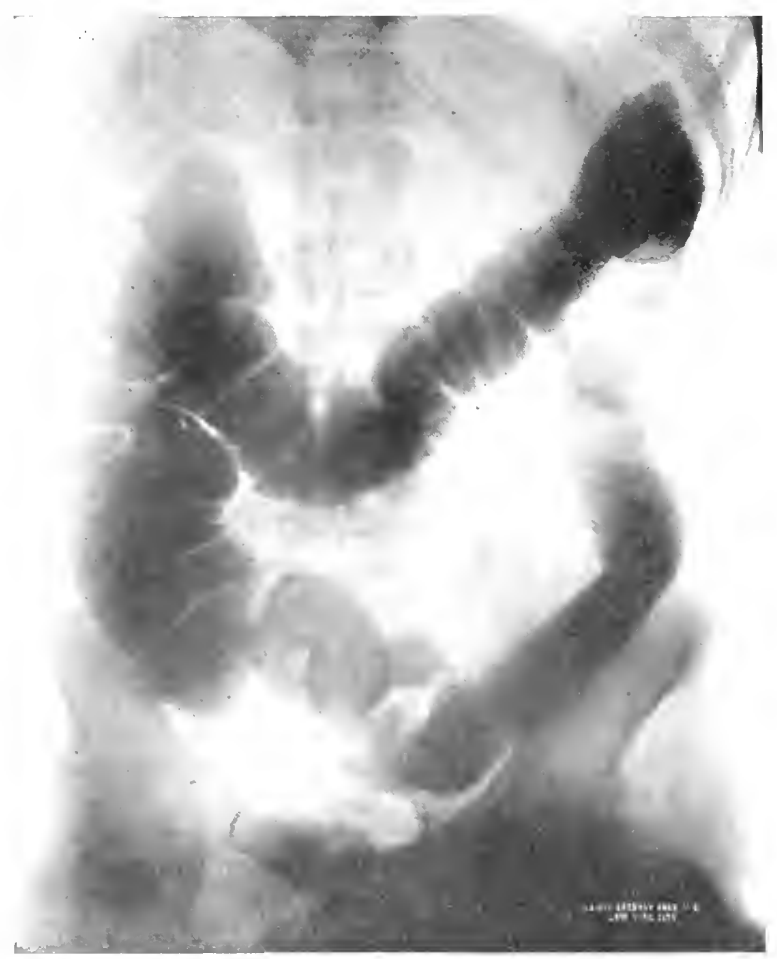

Fig. I 40.--Volvulus complicating a redundant sigmoid flexure. (Taken by Cole.)

Enlarged colonic sacculations, which cannot be liagnosed by radiographs, Huoroscopic examination, or palpation, cannot be positively identified exeept by inspection following celiotomy.

Rectoceles are easily diagnosed owing to the facility with which they can be examined.

Pericolitis and perisigmoiditis are usually caused by inflammatory or obstructive bowel lesions or disease within the ablomen, and it is necessary to get a good history of the case. Pericolitis with adhesions or a pericolic membrane can sometimes be diagnosed by tuoroscopic examination mate while the colon is manipulated with the fingers to determine if it is fixed. Radiographs are helplul in locating the trouble, 
but are not reliable for differentiating pericolitis from other chronic obstructing lesions of the colon.

Internal herniæ and mesenteric disease causing intestinal obstruction may be suspected, but it is impossible to diagnose them without opening the ablomen.

Chronic invagination (intussusception) has leen known to occur at the ileocecal juncture, hut in the vast majority of cases the sigmoid (Fig. 1+1) is involved and projects into the rectum. The latter yede of invagination is easily diagnosed because the displacerl gut can be seen through the proctoseope, which it follows to the ants when the patient strains as it is withdrawn. Patients thus afflicted complain of obstipation, left-sided block, itching, soreness, gas distention, and sily. that water injected into the colon cloes not retum prompty if at all. On the days when invagination does not take place the subject feels comfortable and is not constipated.

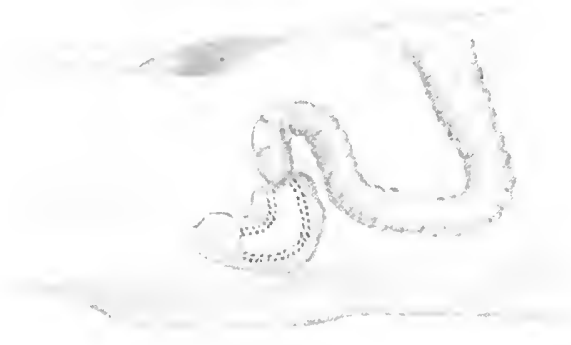

Fig. If I.- Invagination of sigmoid flexure into the rectum.

Splanchnoptosis and enteroptosis are comparatively dats to recognize owing to the patient's emaciated condition, relaxation of his tissucs, constipation, mudk complexion, pulling pains, bulging abdomen, melancholia, inactive mind and muscles, and by palpating and percussing the ablomen, which will reveal that the orwan, or viscera are not in their normal position. When the diagnosis is still doubtiul one should resort to colonic inflation, huoroscopy, radiography, or laparotomy.

Colonic dilatation (Fig. I +2 ) can be differentiated from other chronic obstructions by inflating the bowel with air and delining the position by palpating and percussing the abdomen.

Hirschsprung's disease (congenital colonic dilatation), observerl in young children, is characterized by malnutrition, pot-leelly, olstinate constipation and coprostasis, the stools frepuently being daty, wecks, or months apart. The colon is large, thick, and can le clearly defined by palpation and percussion following its inllation with air, or 
with the fluoroscope and radiographs subsequent to the administration of bismuth meals or enemata.

Enterospasm should be suspected where the intestine is irritable, intlamed, or blocked, and the patient suddenly suffers from complete obstruction. Lsually a semisolid or firm tumor (contracted bowel) can be outlined at the site of the trouble above where gas and feces have collected. When the swelling and symptoms lisappear following the administration of belladonna, applications of hot fomentations

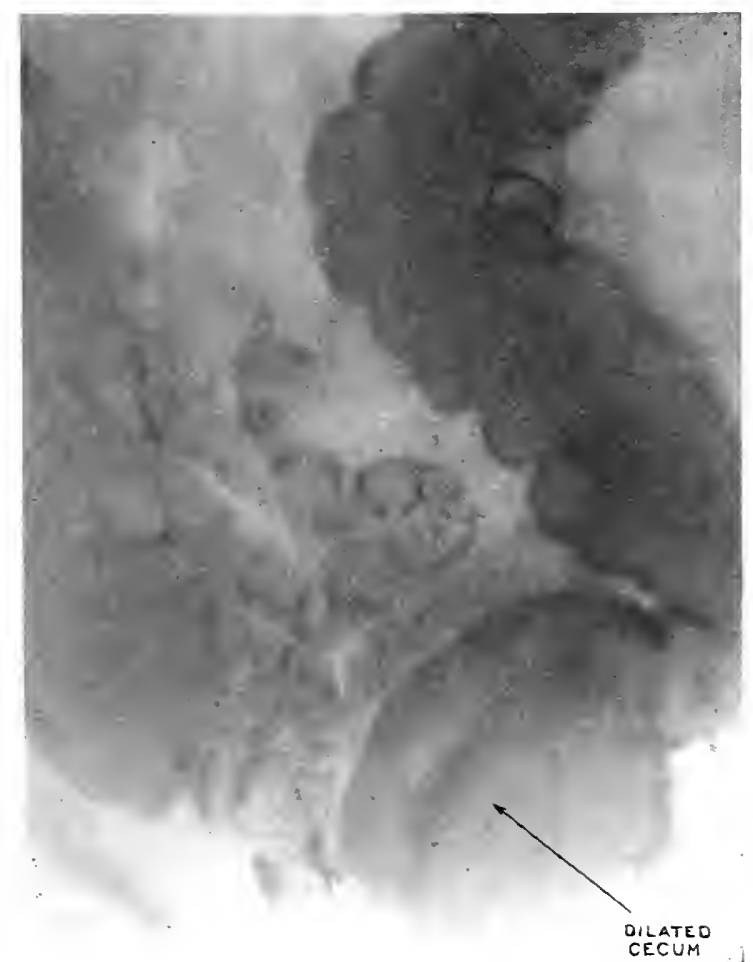

Fig. I 42.-En.rmou-ly ptotic and dilated cecum with enlargement of the ascending colon. Note how the enema projected the bismuth solution for several feet into the small intestine. indicatine ileocecal valse incompetence. (Taken by Cole.)

and copious soapsuds or hot oil injections, a diagnosis of enterospasm is justifiable.

Intestinal parasites seldom cause obstruction, but when they do, the history will show that the patient has previously passed whole or segments of worms. Examination of the stools for pieces of parasites and their ova is indicated when helminths are suspected.

Hypertrophy of O'Beirne's sphincter can be liagnosed by introducing the sigmoidoscope and inflating and defining the size of the rectosimoidal juncture with soft-rubber bougies.

Enlarged rectal valves are easily seen through the proctoscope, and 
when they are rigid and feces can be seen resting upon their upper surface they delay or prevent normal evacuations.

Hypertrophy of the levator ani muscle can be detected by digital examination. In such cases the muscle is greatly increased in size and grasps the finger tightly, about 2 inches above the anus, when introduced into the rectum. The muscle blocks the rectum and leads to coprostasis or fragmentary constipation, heaviness, or dull aching pain in the lower bowel, vesical irritability, and a shutting-off sensation just when the feces are about to be evacuated.

A deformed or fractured coccyx is quickly recognized by the localized pain, finding out if the patient has had a fall, and by inserting the finger into the bowel and grasping the coccygeal lip between the thumb and finger so that it can be manipulated.

Hypertrophy of the sphincter muscle causes constipation, difficult defecation, sphincteralgia, and contraction of the anus, which when stretched causes intense pain.

Hemorrhoids are diagnosed by their bleeding. protrusion, and tumor-like formation; fissure, by its slit-like shape and the sphincteralgia which it induces. 


\section{CHAPTER XLV}

\section{OBSTRUCTIVE (MECHANIC, SURGICAL) DIARRHEA (Concluded)}

\section{TREATMENT (NON-OPERATIVE-PALLIATIVE, SURGICAL)}

THE treatment of mechanic or obstructive diarrhea is non-operative and surgical, but the former only makes the patient more confortable and extends life, while the latter is curative, and should be practised as soon as the patient's consent can be obtained, unless he is unable to withstand operation.

Non-operative Treatment.-The palliative treatment of diarrhea incident to obstructing lesions consists chiefly in (a) regulating the diet so that the bulk of the excreta is diminished and the feces are nonirritating; $(b)$ prescribing antiseptics, astringents, and opiates by mouth to lessen fermentation and putrefaction, attenuate or destroy pathogenic intestinal bacteria (or counteract the effect of their toxins), diminish the evacuations, and relieve pain and cramps; (c) administering castor oil when loose movements are due to coprostasis and frequent colonic medicated irrigation to minimize the effects of auto-intoxication, wash out foul discharges, toxins, and bacteria, dissolve and flush out irritating feces, and heal the inflamed and ulcerated mucosa above and below the obstruction; $(d)$ prescribing nerve, blood, or general tonics to improve the patient's condition; and (e) having the sufferer refrain from worrying, taking violent exercise, remaining in the sun, eating ice-cream or shell-fish, indulging in alcoholic beverages, and consuming articles of diet known to disagree with him.

By following this plan of treatment many patients can be markedly improved and think they are on the road to recovery when such is not the case, because permanent relief is not to be expected until the lesion causing chronic obstruction has been corrected or removed. The author informs the sufferer what he may expect, and lets him decide whether he prefers temporary help, through palliative measures, or a permanent cure, which cannot be accomplished except through operative interference. When the facts have been fully explained, the average patient will insist upon an operation, because he knows from experience that he cannot obtain a cure by less radical measures.

The rearter is referref to the chapters devoted to the surgical treatment of diarrhea for a cletailed description of the operations frefunently employed in the treatment of obstructive or surgical diarrhea. 
Surgical Treatment.-Obstructive diarrhea has a complex etiolegy, and different operations (simple or diffieult) are reepuired to relieve and cure it in a series of cases. Most of the procedures regured for the purpose can be quickly performed, are principally devoid of danger, and bring about surprisingly gool results.

Congenital deformities of the rectum and anus are fairly common, while malformations of the colon and sigmoid are rare, and schlom recognized until it is too late. Palliative measures should be tried in cases of partial obstruction, but where the colon is almost or completely blocked, in consecpuence of abnormalities or sequele incident to an attempt to cure them, surgical intervention is imperative, and the deformity or (listortion should be forthwith corrected or removed by the procerlure inticated.

Congenital narrowing or stricture at the anus is relievalole by forcible divulsion, but division of it and the sphincter, followed by drainage and occasional insertion of the finger, give ruicker and more lasting results.

When the anal aperture or rectum is partially or completely occluded by fibrinomembranous partitions they are gotten rid of hest by seizing and dissecting them free from the bowel with knife or scissors.

Imperforate anus, where the rectum terminates in a blind pouch at or just above the anus, can be corrected by incising the occluding structure at the anal site quickly and without disturbing the sphincter if present. When the culdesac terminates an inch or more above the anus or the lower rectum is strictured, dissections should be carried from below upward, so that the terminal end of the bowel can be freed. brought down, and sutured at the anal site, with preservation of the sphincter, but where this is not feasible, and there is no sphincter, a new anus is established at the most convenient point, which occasionally necessitates removal of the coccyx.

In cases of imperforate anus with a fecal fistula opening into the sasina, bladder, urethra, or surface of the body, the operation must be varied according to indications. Where the rectum ends in the vulva or vagina, the sinus or anus and the sphincter when falscly implanted, are freed through an incision carricel backward and the lower rectum or sphincter are restored to their normal position and a molified procedure is indicated in the presence of surface recto-urethral and rectoresical fistulie.

If the fistulous opening is large and satisfactory evacuations take place, operative interference may be indelinitely postponed, or the deformity or sequelie may he correcterl at once, except in infants and young children, who withstand such operations hadly. In deplorable cases, where as the result of congenital deformities of the colon, rectum, or anus or their sequela, the bowel camnot be replaced and the patient have splincteric control, an artilicial opening should be made above the block.

Extra-intestinal pressure causing diarrhea may be induced by adhesions which constrict the intestine, intra-abdominal tumor, displaced 
organ, or enlarged prostate, under which circumstances adhesions should be divided. Neoplasms should be excised, the uterus restored to its normal position and fixed, or the prostate removed.

Strictures of the small intestine and colon responsible for chronic loose movements require excision, but when this is not feasible, owing to the patient's debilitated condition, complications or number of stenoses, the blocked segment of gut should, according to indications, be excluded by short-circuiting or milateral (Fig. I +3) or bilateral exclusion. Strictures of the lower rectum may be treated by divulsion or division (proctotomy) to increase the bowel lumen, procedures contra-indicated when narrowing is in the upper rectum above the peritoneal attachment. In obstinate and previously operated cases the diseased rectum should be amputated, and the proximal end of the gut sutured to

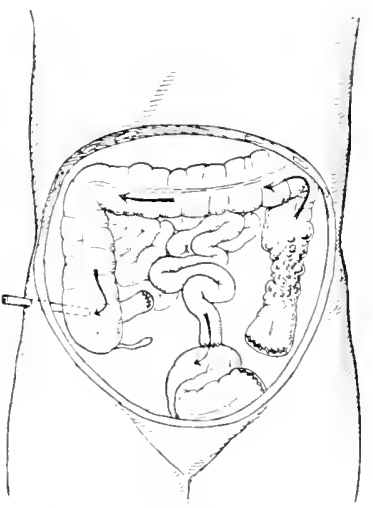

Fig. I+3.-Unilateral exclusion (ileorectostomy) for the relief of inoperable carcinoma of descending colon. Excluded segment of bowel drained by cecostomy. (After author's case.)

tion, may be removed by perineal, a aginal, or sacral (Kraske) proctectomy when located in the lower or middle rectum, and by the combined operation (perineo-abdominal) when situated in the lower sigmoid or rectosigmoid juncture.

Inoperable colonic growths should be excluded because patients prefer this procechure to colostomy, which is imperative in the presence of irremovable rectal cancers.

Polypi when large and numerous should be treated as other tumors of the colon, but when they are single and situated in the rectum they are easily removed by the ligature or clamp-and-cautery operation, linear excision, or by attaching the author's valve clamp to their base and letting them slough off.

Foreign bodies irritating the bowel can occasionaly be dislodged and evacuated through the aid of copious enemata, but when lodged or encysted in the small intestine or colon, enterostomy, colostomy, or, in 
rare instances, resection is necessary to remove them. Foreign bodies in the rectum can be located and removed through the proctoscope if free, but when encysted or they have sharp angles the sphincter should be divulsed to give more room, and the bowel should be protected an they are lrawn downward with foreeps. In rare in-lanees colostomy. is indicated to provide a vent for the feces above them.

Fecal impaction recurs above lesions, causing obstructive diarrhea until they are removed, but when it is incident to atonic eonstipation and a feeal mass of considerable size or seybala form and induce coprostatic diarrhea, relief quickly follows remosal of the feces through the aid of massage and liberal doses of castor oil reinforced by copious high soapsuds, oil, or hydrogen peroxid 25 per cent.) enemata. Impacted masses within the sigmoid and rectum can be broken up through the proetoscope when high, or by the finger when near the anus, after which the rectum can be quickly evacuated by irrisation.

Adhesions viry in form and density and the technic of their removal varies. When obstruction is caused by recent thread-like adhesions or exudates, which constrict or glue the gut to adjacent structures, diarrhea from this source can be relieved by breaking them up with the aid of massage, vibratory treatments, and certain exercises. but when the colon is blocked by organized fibrous band- or sheath-like adhesions operative interference is indicated. To avoid injury to the gut and complications, the involved bowel should be fully exposed, so that exudates and adhesions can be separated or removed by careful dissection or wiping the intestine from side to side, and not by freeing them with the finger within the aldomen. Their possible subsequent re-formation can usually be prevented by suturing or covering tears in the peritoneum. floating the intestine with sterile oil or a normal salt solution before closing the wound, and later prescribing a drug which stimulates peristalsis, and having the patient change his posture frequently to prevent the bowel from remaining too long in the same posture. Where adhesions are numerous, strong, involve a considerable segment of the colon, and cannot be removed or destroyed, resection or exclusion is indicated, and when either of these is not feasible, an artificial anus should be established in the free bowel above.

Pericolic (Jackson's) membranes act similarly to adhesions, but can be eliminated by lisating and dividing their extremities and then peeling them free from the colon. Where this cannot be accomplished, excision, exclusion, or colostomy must be substituted.

Angulations and taitsts (volvulus) responsible for obstructive diarrhea frequently require the same treatment as adherions, since they are causer by them. but when incident to rotation or ptosis of the colon. a distorted segment of bowel should be straightened out. replaced, and anchored in its normal position (igmoidopexy, colopery). In deplorable cases, where the bowel is inseparably adherent to and maned in the pelvis with other viscera, extensive resection, exchusion, or colostomy are unavoidable.

Chronic inagination of the sigmoid (Fig. IfI) has been frequently 
encountered by the author, and where it induced diarrhea alone or alternating with constipation quick and usually permanent relief has,

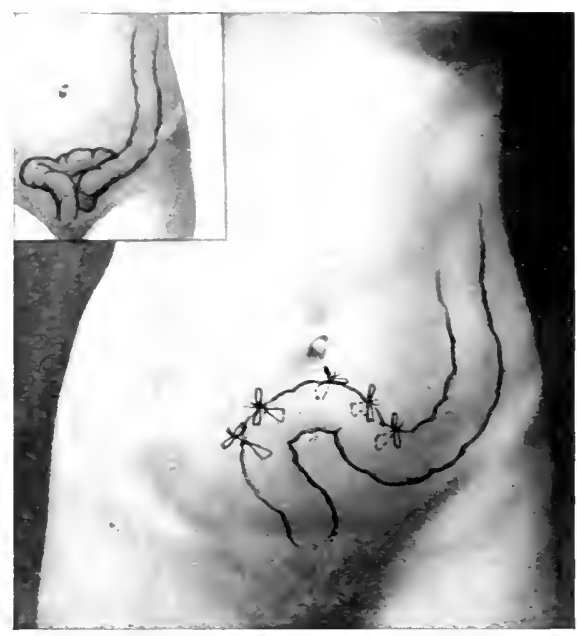

Fig. If4.-Ptosis of the sigmoid flexure is shown in the small drawing. and its replacement and fixation (circular colopexy, in the larger one. (Author's case.)

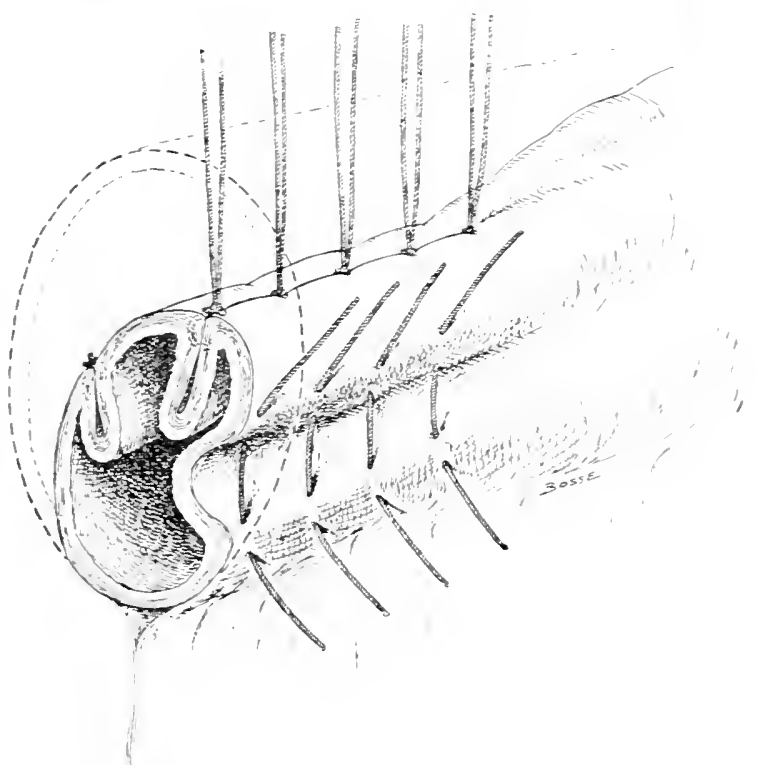

Fig. If - - botted line: indicate the size of the dilated colon. The untied Lembertlike sutures show the way in which the bowel is infolded and the peritoneal surfaces - If the cut are brought in contact. and the tied stitches how the intestine is anchored to the abdominal parietes.

in the majority of instances. followed its withdrawal and fixation to the anterior or posterior abdominal parietes. Where other organs were ptotic they were also anchored where they belonged. 
Congenital and acquired colonic dilatation (Fig. I +5 ) is best controlled by coloplication reinforeed by colopexy, but where the enlarged gut is displaced or completely incapacitated by adhesions, colectomy or, preferably, ileosigmoidostomy should be performed, otherwise a permanent cure cannot be obtained.

Diverticula of the colon and sigmoid that induce diarthea can be eliminated or removed by the ligature, cattery, excision or inversion, and suture when few and small, but when inflamed, large or multiple, as frequently encountered in the sigmoid, the involved segment of gut should be excised. Short-circuiting and colostomy are contra-indicated except in extreme cases or where perforation has occurred resulting in abscess and fistula. Fistulous sinuses should be dissected out, the pus cavity emptied, cleansed and drained, and then the opening in the bowel should be closed.

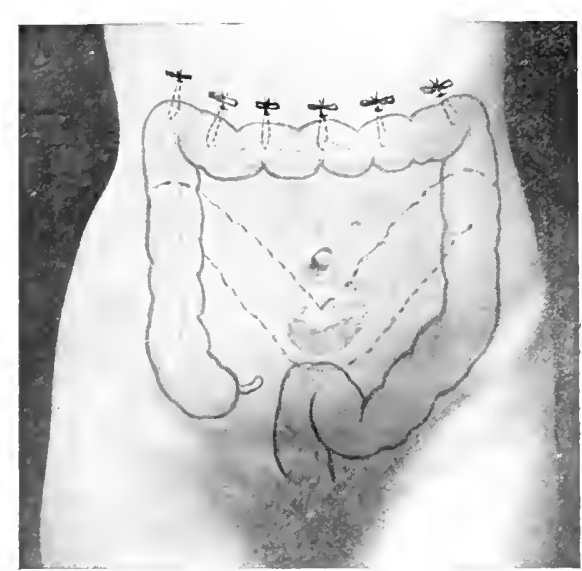

Fig. 1.6.-Dotted lines indicate extensive $\checkmark$-shapert ptosis of the transverse colon; the shaded area the point where the feres col lect in it, and the stitches how the colon is anchored to the abdominal wall (colopexy).

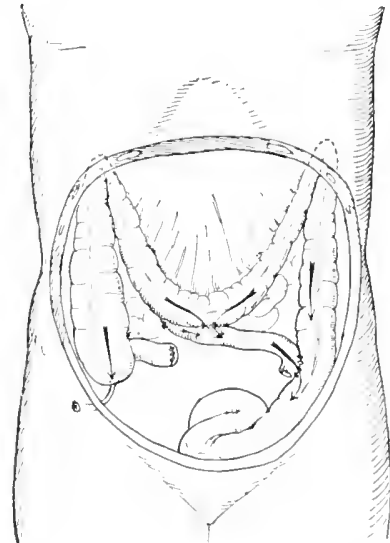

Fig. It7.-Intestinal exilusion (ilensimmoidostomy) for $\mathrm{M}$ shaped fortonic ptosis with angubation of the splenic and hepatic flexures. Cectm and ascending colon dratined by appendicostomy and the transierse colon by ileocolostomy. (After author's calse.)

Splanchnoptosis, enteroptosis, and coloptosis frepuently kad to constipation and coprostatic diarrhea, which continue until the colon and other organs have been returned and fixed in their normal position by colopexy (1ig. If6), sigmoidopexy, splenopexy, hepalopexy. or nephropexy. Where all the large intestine is down, it is necestary to attach it to the alelominal parietes at a number of points to forestatl angulation and sagging, and where it is markerlly displateed and firmly bound down by athesions it is atvisable to exclude it hy joining the lower ileum to the sigmoid flexure or restum (ileosigmonetostomy or rectostomy, Fig. I 47 ). Following restoration of the organs, convalescence can be hastened by having the patient wear at stibable binder and resort to massige, vibratory treatment, electricity, and 
hydrotherapy, to strengthen the bowel and clear up manifestations incident to intestinal auto-intoxication.

For a full description of the author"s colopexy and sigmoidopexy the reader is referred to his work upon "Intestinal Stasis."

Obstruction from parasites (aorms) can usually be relieved by purgation, vermifuges, and medicated irrigations introduced from below or through an appendicustomy, cecostomy- or colustomy opening. In a few instance- worm - blocking the bowel could not be expelled. and it wis necessiry to resect the gut or establish an artificial anus to eliminate them and save the patient's life.

Enterospasm, a- ordinarily encountered, can be speedily relieved by having the patient drink an abundance of hot water, apply hot fomentations to the abdomen, and take several copious high warm enemata, all of which faror relaxation of the intestinal musculature. When relief dues not follow, they should be reinforced by the admin-

GAXTS Valve Clamp Applicator

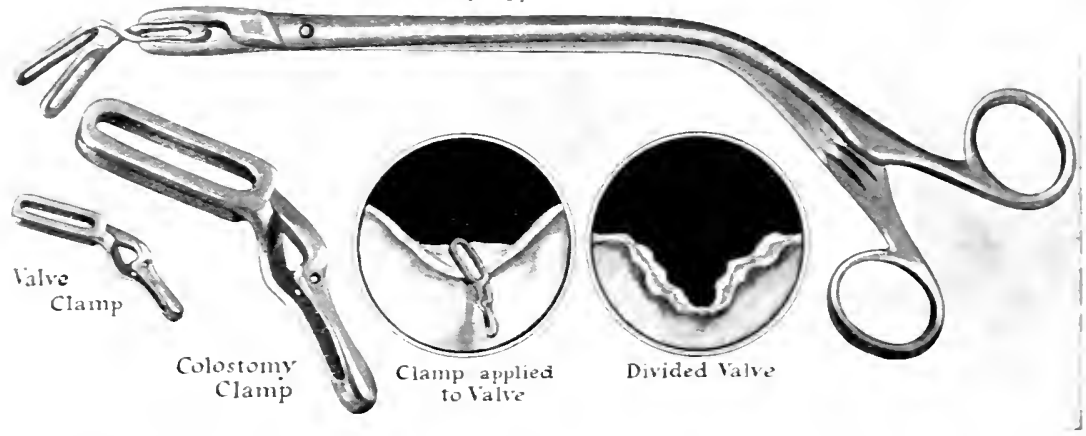

Fir. Ifs.- - Author's valwotomy instruments (clamps and applicator). The drawings show the applied clamp throuch the protoscope and appearance of the ulcerated valve edges aiter it has cut its way out.

i-tration of belladonna. me $\times(0.60)$, administered three or four times daily, until the enterospasm. colic, and constipation or diarrhea have been relieved. Occasionally an opiate is required to relieve suffering. induce sleep. or check frequent movements.

IIypertrophy of $O^{\prime}$ Beirne's sphincter at the rectosigmoid juncture has been known to cause obstructive diarrhea. Ender such circumstances the narrow gut should be distended with an inflating bag, or where the mucosa is inflamed or ulcerated. contration of the gut incident to the lesions can be alleviated or arrested by irrigating the bowel with a hot ichthyol, balsam of Peru, or permanganate (I per cent.) solution of warm high oil enemata contaning bismuth. In a chronic case of the author' - resection became imperative.

IIyertrophied rectal (Itouston's) alies are occasionally" responsible for stercoral diarthea, in which case the division of one or more valves is indicated. Almost universal success has followed the author's valvotomy. The technic of applying his valve clamp can be readily 
understood by a glance at the accompanying illustration (Fig. I f s). The mieldle value usually eatses the obstruction, and division of the others is seldom recpuired.

IIypertrophy of the anal sphincter muscle was formerly treated by forcible divulsion under ether, but experience has tauglit the atuthor that beter and more permanent results are obtained when the muscle is divided under local anesthesia.

Fissure in ano invarially causes sphincteralgia, and usually cannot be cured until the anal muscle has been divulsed or severed. The author prefers the latter becaltse a cure follows more promptly and the

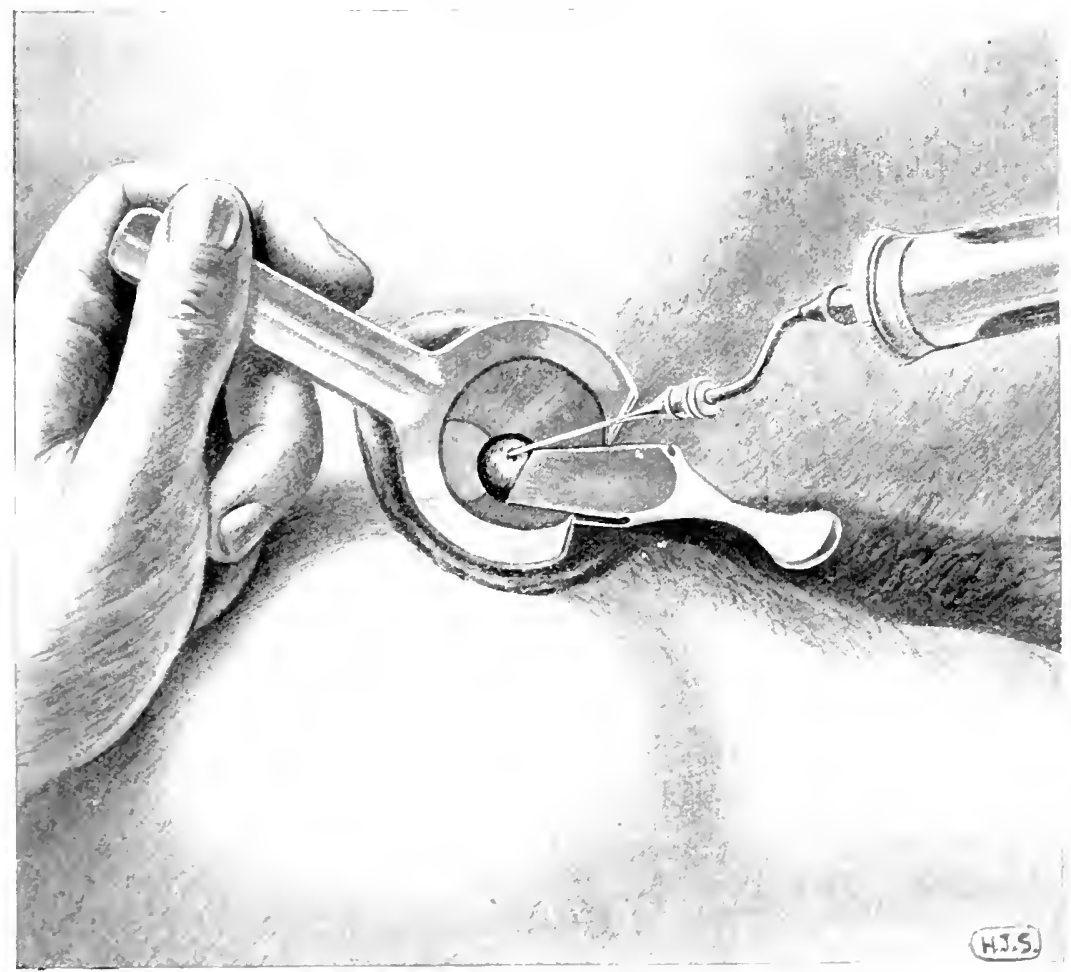

Fig. I $49 .-$ Method of extruding hemorrboids in the author's local ane-thesia "operation.

anal outlet is permanently widened so that defecation is facilitateel. When the patient declines operative interference, one can sometimes heal the rent by keeping the feces soft and making milel (6 per eent.) silver or ichthyol topical applications.

Ilemorthoids seldom induce constipation or diarthea, but when they do, the internal variety should be removed he the damp-and-catery or ligature operation under local anesthesia (Fig. Ifo). Skin-lass can be efuickly gotten rid of ly snipping them off with scissors and suturing or permiting the wounds to heal by granulation, and thrombotic hem- 
orrhoids, by slitting them open, evacuating the clot, and inserting a drain.

Rectal polypi in the lower rectum are easily eliminated by excision, the ligature, or clamp-and-cautery operation, but when situated high they should be removed with a snare, by torsion, or with the author's valve clamp, which, when applied at their base, causes them to drop off through pressure necrosis.

Procidentia recti, moderate in degree, can be corrected by linear cauterization or the removal of segments of the mucosa with the ligature or knife under local anesthesia. In extreme cases the author first remores a segment of the rectum, including the sphincter, and then opens the abdomen, draws the bowel upwarl until taut, and anchors it to the anterior abdominal parietes (sigmoiclopexy).

Coccyoeal deformity, where the bowel projected forward, induced obstinate diarrhea in 3 of the author's cases; in each instance normal evacuations followed excision of the offending segments. 


\section{CHAPTER XLVI}

\section{POSTOPERATIVE DIARRHEA}

IT is only within the present decade that postoperative diarrheas have received any attention, and up to the present only a few good articles have appeared upon this subject, and while these have in a measure cleared up many points concerning this most interesting type of loose movements, the subject still requires much elucidation before it will receive the attention which its importance fleserves.

Errors in diet are often responsible for frefuent loose movements following surgical intervention for the relief of a variety of conditions, particularly those affecting the gastro-intestinal tract; later, after the patient has been on a strict diet, he is permitted to eat whatever he chooses.

\section{ETIOLOGY AND PATHOLOGY}

It is a well-known fatet that a considerable number of patients, laparotomized for various purposes, suffer from constipation and occasionally from coprostasis, where fecal masses assume a putty consistence and size sufficient to obstruct the bowel or form numerous hard irregular or round scybala, which may be scattered along the gut or collected en masse, forming a tumor of considerable proportions. When such accumulations are not promptly dissolved or di-lodged by medication, enemata, the finger, or instruments, they sooner or later produce a stercoral diarrhea that is extremely difficult to correct. This condition may occur shortly following an operation while the patient is on a strictly milk diet, or later when a regular or one of more generous proportions is allowed. particularly in the absence of daily laxatives. and where the attendant, for one reason or another, ties up the patient's bowels for several days. Recent accumulations of the above types may induce diarrhea through obstructing the gut, as would a cancer or other tumor, direct irritation to the bowel, by setting up a localized catarrh, favoring the retention of putrefying material and the multiplication of the normal intestinal pathogenic bacteria and their toxins which lead to increased peristalis, glandular secretion, and cliarrhea owing to their influence upon the local and general mechanism.

In postoperative chronic coprostasis the movements are more persistent, watery, have a very foul odor, may contain pus, blood, and mucus, either alone or admixed, and the patient suffers from insomnia, malaise, dirty complexion, high temperature, furred tongue, abdominal tenderness, and many of the more common manifestations of typhoid fever, for which the condition has frequently been mistaken. Here the diarrhea is incited in the above-mentioned way, together with irritation and trauma to the exposed nerve-endings within the stercoral 
ulcers, which soon form in the vicinity of impacted masses through pressure, necrosis, or infection.

The most exhausting and fatal types of postoperative diarrheas are those which follow gastric operations (gastro-enterostomy, gastric resection, and pyloroplasty), forms frequently encountered in this class of work, but thus far unsatisfactorily accounted for, and are mysterious in that ther arise during the stage of convalescence and often continue in spite of the treatment until the death of the patient a few days later. These conditions are peculiar in that they occur in what are believed favorable cases. where the patient has improved until he is considered out of danger from the operation and is permitted to pass from a restricted to a more liberal diet, following correction of the original condition which was responsible for fermentation, putrefaction, and other disturbances.

In studying this subject it is necessary to take into consideration that the condition for which the operation is performed has, in most instances, previously reduced the patient to a most deplorable state, there is emaciation, imporerished circulation, abnormal secretions and excretions, and a general derangement of metabolism, if it has not already caused a catarrhal or other diseased condition of the gastrointestinal tract.

Surgeons differ as to the cause of the exhausting and frequent evacuations which take place following operation in these cases, and it has been attributed to (a) intoxication dyspepsia incident to putrefactive fermentation: (b) irritative effect of chloroform upon the gastro-intestinal tract: $(c)$ want of neutralization of the gastric secretion (hy drochloric acid) by the bile and pancreatic juices, owing to its being deposited in the intestine below through an artificiai opening: and (d) irritation to the mucosa by the residue of an abundant varied diet following strict abstinence from solid food. In these subjects, owing to the depleted condition of the patient, which minimizes the amount of alkaline fluid secreted and the lack of direct stimulation of biliary and pancreatic secretions which would normally ensue if the gastric acid passed directly from the stomach to the intestine below, and to the fact that after operation the gastric juice does not have to pass through the duodenal reservoir or trap designer to retain the alkaline fluid until a considerable amount is collected, not enough of alkali reaches the small intestine to meet the demands made upon it by the decomposing products within, which, as a result. sets up an irritative diarrhea. !nder this condition. Heile maintains that the alkali, which under normal circumstances would be absorbed by the colon, is lost because it undergoes combustion into alkaline carbonates and is excreted, with the result that aceto-acetic acid, acetone, etc., appear in the urine and the patient derelops enterogenic coma, resembling that of diabetes, which so frequently complicates this rariety of loose movements.

Postoperative gastric diarrhea may follow any operation upon the strmach, but most frequently complicates gastro-enterostomỵ, gastric 
resection, and pyloroplasty. This diarthea occurs more often and is more aggravated when the operation is performed for the relief of cancerous than benign obstruction, when the lesion is at the pylorus than elsewhere, and when a gastro-enterostomy is made with a long intestinal loop than a short one. Dangerous diarrhea rarcly follows gastro-enterostomy performed for the relief of benign affections, particularly when the opening is matle within 12 inclues of the duoflenojejunal angle. This condition is apt to follow operation for whatever purpose when the anastomosis is made low down in the jejunum, hecause the gastric secretions lose the intluence of the bile and pancreatic fluids and imperfect intestinal indigestion naturally ensues.

Auschüz believes that certain individuals show a predisposition to postoperative diarrhea. Decrepit suljects and those sulfering from tubercular lesions independent of bowel trouble are more prone to it. Carle and Fantino attribute the loose movements to chloroform, which callses putrefactive fermentation and gastro-intestinal catarrh, but Auschiitz has disproved this by substituting ether for chloroform. He found that catarrh occurred after one as often as the other, and says that it would be remarkable for the effect of chloroform to manifest itself from the seventh to the tenth day or later, when the diarrhea occurs in these cases. This authority does not believe that gastric postoperative diarrhea is of very common occurrence, as he noticed diarrhea only 30 times in 500 operations, including gastro-enterostomies, resections, and pyloroplasties.

Distressing diarrhea nay occur in connection with peritonitis, sepsis, the formation of abscesses or slonghing of the transierse colon subsequent to gastric or other abdominal operations, and, according to Auschütz, following nutrient enemata when the rectal mucosa is inflamed. Again, a pre-existing intestinal catarrh may be incited to renewed activity by the operative interference and lead to increased frequency of the movements. Consequently, it is important to determine if the bowel is healthy or the patient is already aflicted with diarrhea before deciding upon the more serious gastro-intestinal operations; otherwise, on account of the patient's weakened condition, postoperative diarrhea may ensue and he will soon die from exhaustion.

There is also a variety of loose movements which sometimes follow the establishment of an artificial anus, intestinal cxclusion, or resection made for the purpose of relieving malignant or other forms of intestinal obstruction, or giving rest to a diseased segment of bowel.

After colostomy the frequent evacuations are involuntary, and take place owing to a lack of sphincteric control as a result of increased peristalsis conseduent upon irritation to the exposed nerve-endings in the edges of the yut and wound.

Short-circuiting or exclusion, done to eliminate all or a considerable portion of the colon, makes it necessary to join the proximal end of the ileum to the sigmoid flesure and rectum. After these operations the patient has from five to ten fluid or semisolicl exacuations daty for several weeks, due to the fact that there is no reservoir in which the 
feces may collect and remain until their watery constituents have been absorbed; consequently, they are almost continually poured into the rectum, to be evacuated before they become solid. This type of surgical diarrhea has, in the author's experience, invariably improved without treatment week by week, until at theend of about three months the stools become normal as regards frequency and consistency on account of the ileum acting the part of the colon.

Excision of a short or long piece of the healthy small intestine in dogs is usually followed by a diarrhea which persists permanently or for a consiclerable time, which would indicate that the entire small gut is necessary to the metabolic process, while similar operations upon the colon produce but a slight if any diarrhea, and when this occurs it is probably due to the ensuing trauma. In human beings the operation is usually performed for the relief of serious organic lesions of the gut. Hence it is not surprising that the evacuations should be abnormally frequent after resection, owing to the irritable state of the bowel, nor when several feet of the small intestine or a considerable portion of the colon is excised, because in the former the intestinal juices are prevented from performing their full function, and in the latter because there is no opportunity for the watery contents of the feces to remain in the colon until absorption has taken place. In some instances the diarrheal disturbance is of short duration, the bowel becoming used to the new state of affairs, white in others it becomes chronic. Occasionally, loose movements following resection are due to obstruction resulting from a stricture at the site of anastomosis.

In aggravated cases of acute obstruction, where the bowel has become distended with accumulated irritating gases, putrefactive material, bacteria, and toxins, the patient frequently suffers severely or dies from the absorption of septic material or exhausting diarrhea caused by the effect of the toxic substances from ahove being poured into the healthy bowel below, even though the obstruction has been successfully relieved by operation.

Finally, annoying or dangerous postoperative diarrhea may complicate operations performed for the relief of exophthalmic goiter, certain affections of the liver or pancreas and their ducts, and may follow serious surgical intervention for any purpose in patients who suffer from nephritis, diabetes, or other organic or constitutional disease when the resistance of the paticnt is at a low ebb or is already afflicted with an inflammatory or ulcerative intestinal disease.

\section{SYMPTOMS AND DIAGNOSIS}

The symptomatology of postoperative gastric diarrhea is not very clear, varies considerably in different cases; the suffering is more frequent and persistent in individuals afflicted with cancer than other gastric lesions. The most dangerous manifestation is stagnation of the stomach contents, which may prevail either before or after operation. Ordinarily the patient goes along very well for several days following surgical intervention, and then, while under favorable circum- 
stances-riz., comfortable surroundings, rest in bed. and a controllerl diet-stagnation and diarrhea suddenly supervene, and the latter is diffecult or impossible to control hy antidiarrbeal remedies. In mild cases the stomath condition improves and the diarrhea abates in one or two weeks without greatly exhausting the patient. Postoperative diarrhea is always most severe and often fatal when the frepuent eracuations occur late (tenth to fifteenth day) and following marker stagnation and romiting of offensive blackish material. Some of these patients first beeome delirious, then unconscious, and finally pass into a state resembling diabetic coma.

In this type of cases the evacuations are very frequent. fluid, of a reddish tint, contain shreels of mucus, have a foul odor, and near the end in fatal cases the feces continually dribble through the anus. A case of this kind has been reported by Auschütz where the small intestine, large bowel. and rectum contained numerous deep-seated uleers. while the mucosa of the cecum appeared normal and that of the colon markedly swollen.

\section{TREATMENT}

Following abdominal and operations mentioned, when diarrhea ensues as the result of the patient eating too much. at irregular hours. or indigestible food, the treatment consists in correcting errors of diet. substituting suitable food, and in prescribing an opiate, antiseptic, or astringent to afford temporary relief. In these cases because of an obstruction or other lesion, the patient has, for a considerable time. been left upon a fluid or very restricted diet before and suffers from diarrhea after operation, the latter is usually caused by resuming a regular diet and consuming a greater amount of food than the gastrointestinal tract has been accustomed to taking care of. with the result that indigestion ensues, the gut is irritated, or a catarrhal condition is excited. The treatment is obrious in these cases, and consists in controlling the diet and slowly graduating it up to the normal, and the administration of suitable remedies to control the loose morements during the attack.

In coprostasis the inciting factor of the diarrhea is the impacted fecal masses and hacked-up toxins. Consequently. measures should be instituted which will prevent the undue accumulation of feces, or hasten their removal when they have already collecterl. by atuaching them from abose and below, and to this end castor oil may be prescribed when scybala are present, or liberal doses ( 5 ij to iij-60.0-90.0) of mineral or sweet (ill to soften large putty-like masies and lubricate the intestine. Meanwhile frequent larece (I to 2 quarts-liters) soapsud- enemata, containing ril. $\overline{3}$ ij to iv (60.0-120.0), and turpentine, 5ss to $\mathrm{j}(2.0-4.0)$, should be deposited high up in the coton to help dissolve and dislodge the accumulations, using masige to break them up between the injections. In urgent cases the mechanical breaking up of the mass can be materially hatened by the introluction of a 25 per cent. solution of the peroxid of hydrogen, which has the peculiar power of quickly penetrating fecal masses and rapidly disintegrating 
them so that they may be washed out. Firm mucus-covered fecal tumors in the sigmoid flexure or rectum, upon which water and oil have little effeet, can be quickly broken up with the finger or gouge and eracuated through the proctoscope or sigmoidoscope by continuous irrigation. Following their dislodgment, olive oil, §iv (120.0), and bismuth subnitrate, $\overline{5}$ ss $(\mathbf{1 5 . 0})$, should be injected high into the colon to southe and heal the inflamed or ulcerated mucosa, a therapeutic measure which affords the patient much comfort and diminishes the frequency of the evacuations.

Diarrhea consequent upon gastro-enterostomy, resection, and pyloroplasty is impossible to relieve in aggravated cases where there is profound stagnation, but in the less severe types of loose movements much can be done to palliate the patient's suffering and control the movements providing corrective measures are instituted early. When consequent upon achylia gastrica, acidity should be increased by of trepeated and liberal doses of dilute hydrochloric acid, but when a reverse condition prevals, and there is hyperacidity, bicarbonate of soda, gr. $x$ to $x l(1.3-2.6)$; magnesium, gr. $x$ to $x x(0.60-2.0)$, and lime-water, $5 \mathrm{j}$ to $\mathrm{ij}(4.0-8.0)$; or powder, gr. $\mathrm{x}$ to $\mathrm{xxx}(0.60-2.0)$, should be employed alone or in combination with bismuth, gr. $x$ to xi (0.60-I.3): charcoal, gr. x to $\mathrm{xxx}(0.60-2.0)$; or cerium oxalate, gr. j (0.06), three timesdaily, because of their neutralizing and sedative effect.

When there is marked stagnation with romiting it may not be possible to save the patient, but much can be done for his comfort by washing out the stomach and intestine to free them of their irritating contents, and when this faits to give relief, calomel, strong laxatives. or castor oil may be administered with the object of expelling the offensive material.

Patients afflicted with this stagnant type of diarrhea and some other forms of postoperative diarrhea may first become delirious and then pass into a state resembling diabetic coma, a condition which Heile attributes to the body's loss of alkali. This condition has been successfully treated by him through the actministration of large quantities of alkali, introducing into the body up to $5 \mathrm{v}$ (20.0) of sodium citrate by mouth; a 5 to Io per cent. solution of citric acid and sodium bicarbonate by the rectum; and of sodium bicarbonate up to I per cent. intrarenously, and symptomatically treating the diarrhea. He claims that better results are obtained from this treatment in these cases than in diabetic coma, because the loss of alkali here takes place through the bowel, whereas in the former it is based upon pathologic changes of metaholism.

Surgical Treatment.-In the surgical treatment of postoperative diarrhea following gastro-enterostomy, where the opening has been made low in the small intestine, a second operation may be required and a higher anastomosis made to relieve the condition.

Peritonitis, sepsis, abscess, and sloughing of the transverse colon demand immediate surgical attention and hot abdominal applications, colonic irrigation, and measures to relieve pain and control the more- 
ments are not to be relied upon except for the purpose of relieving the patient's sulfering until the oferation can be performed.

Where diarrlea is induced by the long-continued use of rectal enemata following operations they should be achinistered less frectuently, and the lower bowel shoukd be bathed with a weak boric acid solution or, preferably, with olive oil containing hismuth to reduce the infammation and irritability of the mucosa and relieve tenesmus.

Catarh of the small and large intestine is often a complication of postoperative diartea, having existed prior to, or been caused by, subsecpent gastro-intestinat changes, and requites individual consideration along with the symptomatic treatment of the foose novements.

When diarthea is not improsed by lavage and restricting the diet, an attempt should be made to diminish the frequency of the evacuations by the administration of opium in $\frac{1}{2}-$ gre doses three or four times daily, or laudanum, me to xr (0.60-I.0), may be substituted for it, and bismuth, gr. $\mathrm{x}$ to $\mathrm{xx}(0.60-1.3)$, tannallsin or ichthalbin, gr. $\mathrm{x}$ to $\mathrm{xr}(0.60-$ 1.0), administered every four hours, will be found useful adjuncts, but these reliable remedies usually fail in the most serious or stagnant cases.

To these therapeutic measures should be added bowel irrigation with solutions of ichthyol (1 to a per cent.), horic acid, 2 per cent., permanganate of potash, $\frac{1}{2}$ to 1 per cent., or krameria and sodium hiborate in the folkwing proportions, 10 soothe and heal local inflammatory and ulcerative lesions within the colon and rectum:

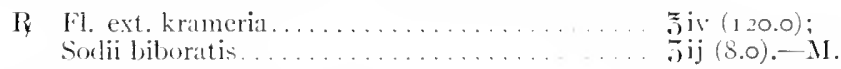

Sig.- One or two tablesponfuls in a cuart of warm water, and inject daily or three times weekly, according to indications.

The frequent movements following the formation of an artificial anus can be somewhat reduced by encouraging the raw edges of the abdominal wound and gut to heal by the application of a 6 per cent. silver nitrate solution, but after healing takes place, where there is incontinence, they are not controllable except by antidiarrheal or constipating remedies. This form of diarrhea is largely unnecessary, and can be avoided by performing colostomy according to the author's method elsewhere described, ${ }^{1}$ which gives the patient a controllalile anus.

Excepting the administration of medicines to quiet peristalsis and solidify the feces, keeping the patient quiet, and having him discard indigestible foods, nothing can be done to control the frepuent huid evacuations following intestinal exclusion and resection. where the ileum has been joined to the sigmoid flexure or rectum. Time, however, will accomplish what therapentic meatures do not, for in these subjects the movements gradually become less frequent and more firm, until normal daily passages occur within from six to fiftecn montlus. The lower ileum gradually assumes the functionating power of the colon, evidence of which has been observed by the atuthor many times. and in two instances the small bowel was found greatly endirged, thickened, and possessed some of the characteristics of the colon.

${ }^{1}$ Gant, Constipation and Intestinal Obstruction, "Diseases of Rectum and Anus." 


\section{CHAPTER NLIII}

\section{MESENTERIC EMBOLISM AND THROMBOSIS (INTESTINAL INFARCTION), DIARRHEA IN}

\section{ETIOLOGY AND PATHOLOGY}

MESEXTERIC embolism or thrombosis and the resulting infarct are encountered most often in individuals having an impaired circulation, such as endocarditis, valvular disease. cirrhosis of the liver, or atheromatous degeneration of the ressels, particularly of the aorta, as a result of lues. The superior or inferior mesenteric ressels may be affected, but in a vast majority of cases it is the former; embolic obstruction occurs in about 3 cases to $\mathrm{I}$ of thrombosis, and men are affected twice as often as women.

In mesenteric rascular obstruction, where a large vessel is involved, the condition of the patient is rendered most serious. There is less danger when a branch is blocked, and the prognosis is still more favorable when a diminutive capillary is occluded. From what has been said it may be inferred that an infarct in one case may be very extensive as regards the mesentery and adjacent segment of the bowel. and insignificant in another, according to the size and number of ressels involved by the obstruction. In rare instances, where interference with the circulation is limited to a small area. possibly a collateral circulation may preserve the part. but usually necrosis of the affected area follows closely upon formation of the emboliom or thrombus. When an artery is blocked, anemia immediately ensues and gangrene quickly follows. hut when a rein is occlucled necrosis may occur more slowly, since the part continues to receive fresh bloot. which early becomes impure, and is extravasated into the surrounding tissue. Except when quichly discorered and remoxed. and this is unusual, a clot in the superior mesenteric artery (or vein or one of its chief branches quickly leads to gangrene and the complete destruction of one or several lengthy segments of the gut, which may include a portion of the small bowel. cecum, or ascending colon, or less frequenty when the inferior mesenteric artery (or vein) or its offshoots are involved, the eolon, sigmoid flexure, or rectum may beeme necrotic and cause the patient's death unless he is immediately operated upon. When distant branches of these resels supplying the muensa become blocked they produce embolic or thrombotic ulcers. according to the capillaries interfered with. These lesions usually result from the clotting of a number of diminutive vescels located in the small or large intestine. but are encountered most often in the jejunum or ileum. 
Thrombosis is prone to occur in individuals who suffer from a chronic catarrhal, tubercular, entamebic, or syphilitic enterocolitis, because of the tendency of the disturbing microbe to enter the capillaries.

Symptoms and Diagnosis.-Intestinal embolism, thrombosis, and infarction may be suspecterl, but are rarely diagnosed, except by opening the abdomen and inspecting the gut and mesentery, and even then have frequently been confused with other bowel lesions. Gebhardt has suggested the following diagnostic rules-viz: (1) There must be a source of embolism; (2) copious intestinal hemorrhages unexplained by organic discase of the bowel or by portal obstruction; (3) a rapid and marked fall of the temperature; (4) more or less colicky aldominal pains; (5) distention of the abdomen and the accumulation of free abdominal Huid; (6) the occurrence of embolism elsewhere, before or simultaneous with obstruction of the mesenteric vessels, and (7) the discovery of a palpable mass (mesenteric hematoma).

The manifestations of mesenteric vascular obstruction usually develop rapidly, though occasionally, in slowly forming thrombi, they may run a subacute or chronic course. The disease is characterized at times 1 y constipation and cliarrhea, the first being intractable in all stages, owing to the obstruction present and the early development of peritonitis, while diarrhea most often occurs in the less acute cases, is due to obstruction, retained irritants, scrbala, toxins, or embolic ulcers.

Blocking of the mesenteric vessels is early characterized by intestinal hemorthages or evidences of obstruction, and serious manifestations rapidly follow each other. Very often there is a sudden fall of temperature, accelerated pulse, focalized tenderness, circumscribed or general peritonitis, colicky pains, nausea, and romiting (which may or may not have fecal characteristics depending on whether or not there is obstruction). Hemorrhages. slight or profuse, always take plate, and the blood may be evacuated immediately or le retained, showing as mekna or dark, blackish-brown, or tarry movements shorty or several days following. Gebhardt maintains that in embolism of the inferior mesenteric bright red blood is evacuated, and, on the other hand, when the superior mesenteric is involvel the movements are dark and tar-like in consistence, but hat offered no convincing reasom as to why this is so. The atthor believes that the character of the blood voided has no relation to the disease of these vessels, but that it depends upon the degree of peristalsis present and the time it is retained, being fresh during or shortly following the hemorrhage and tar-like when it is not evacuated until several hours or dals alterward.

Diarrhea here may be moderate and the stonls composed almost wholly of blood, or they may be very freptuent, watery and exhausting, and contain little if any blood. Again, the loose morements resemble those of acute gastro-enteritis or toxic poisoning and usually the patient suffers considerable abdominal pain, lut occisionally this symptom is absent. Sometines, in ardelition to localized tenderness and pain, a fair-sized tumor resulting from the infarction and swelling can be felt 
in the region of the clot, but such an enlargement is usually mistaken either for a fecal impaction, carcinoma, or invagination. In fatal cases the patient dies from obstruction or peritonitis.

Prognosis.- Where emboli or thrombi block diminutive vessels in the mucosa, nothing more serious than the formation of ulcers, which heal later, occur, but when larger vessels supplying an individual or several segments of the gut are obstructed and collateral circulation to the affected mesentery and bowel is not forthwith established, or the defect corrected by operation, gangrene and obstruction rapidly supervene and cause death within a few hours or, at most, days. A few spontaneous recoveries have been reported.

The treatment of embolic and thrombotic diarrhea is very unsatisfactory, and must necessarily be symptomatic or operative. It is claimed that increased pressure of the portal system is largely responsible for the hemorrhage: consequently, it is advisable to stimulate the heart action with digitalis, strychnin, or nitroglycerin, or the pressure may be relieved by tapping a vein and thereby removing tension. Recently a number of these sufferers have been relieved by resection and anastomosis, or by attaching the affected gut to the skin and forming an artificial opening, the continuity of the gut being re-established later. Most of these operations, however, are fatal, as is shown in the statistics of 47 operated cases collected by Jackson, Porter, and Quimby, wherein the mortality was 92 per cent. 


\section{CHAPTER XLVIII}

\section{FORMULARY}

To meet the needs of the busy practitioner and proxick for exigencies that may arise in diarrheal, inflammatory, and parasitic affections of the gastro-intestinal tract the author has compiled the following suggestions, rules, and prescriptions.

The therapeutic measures herein suggested are more useful in the srmptomatic than in the curative treatment of these affections, ant are intended to carry the patient over sucken crises or until a more complete and rational treatment can be instituted.

Patients afflicted with eliarrhea, intestinal catarrh, or parasitic diseases should $(a)$ dress warmly in winter and coolly in summer; $(b)$ restrict the diet to exclude foods known to disagree with them, refrain from drinking alcoholic ice-cold drinks, strong tea and coffee, or eating ice-cream, articles of diet which leave a large irritating residueraw fruits, shell-fish-and should live chiefly upon milk, soups, eggs. cream, and butter; $(c)$ rest quietly in bet during acute crises and live in the fesh air and indulge in moderate exercise between the attacks; (d) take a nerve and blood tonic when indicated; (e) do everything possible to add to the body fat; $(f)$ avoid excitement, business cares. and mental worries; (g) not employ opiates except when imperative; (h) irrigate the colon three times weekly with a normal saline or one of the medicated solutions given below when the mucosa is highly inflamed or ulcerated, and (i) reinforce the above methods of treatment with astringents, antiseptics, bactericidal, or other medical agents when necessary.

On account of the dissimilarity in which prescriptions of the different authorities quoted have been written, the author has taken the liberty of slightly changing some of them so that all will appear harmonious, but their dosage and method of administration have rarely been altered.

To make the formula more useful in case a prescription is needed in a hurry the author has classified them as far as practicable:

\section{Intestinal Cuturrh Caused by a Cold:}

$\mathrm{P}$ Saloli.

$\bar{j} \mathbf{j}(4.0)$ :

Crensoti

gr. xiv (3.0):

Bismuthi salic rlatis

Pone in capsulas $\mathrm{No} . \mathrm{xx}$.

$5 \mathrm{j}(4.0)-1)$

Sig. - One capsule every three hours. Add opium or phenacetin when pain is troublesome.

(IIill.) 
Formentative Dyspepsia and Diurrha:

IF Creosoti purificatis................. ne xij $(0.75)$;

Alcoholis diluti........................

Ammonii benzoatis ...................

Glycerini purificatis .....................

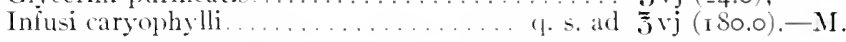

Sig.-Tablespooniul in water two or three times a day between meals.

(Richardson.)

To Check Peristalsis in Acute Enteritis after the Bowel Has Been Completely Emptied:

P Bismuthi subcarbonatis................. gr. $x(0.00)$;

Sodlii bicarbonatis....................... gr. x (0.60);

Tincture opii..................... Iए $\mathrm{x}(0.60)$;

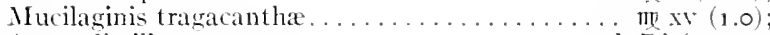

Aque distillatie ..................... s. ad $\tilde{3} \mathrm{j}(30.0)$. - II.

Sig.-Two tablespoonfuls every four hours.

(Nothnagel Clinic.)

Subacute Catarhal Enteritis:

P. Spiritus camphore.

Acicli sulphurici diluti.

$\overline{3} \mathrm{j}(30.0)$

烈

Tincture opii deodorate.................. $\bar{\Xi} \mathbf{j}(30.0)$;

Tincture capsici......................

Spiritus chloroformi ........................

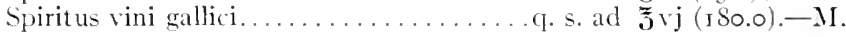

Sig.-One or two teaspoonfuls. well diluted, every three or four hours.

(Scott.)

Acute Intestinal Caturh:

R Bismuthi subnitratis............... 5 iss $(6.0)$;

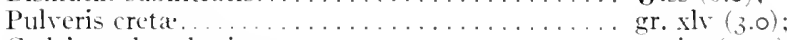

Corleina phosphatis ................... gr. iss $(0.00)$

Elrosaccharis menthe piperita............. gr. lxxv (5.0).-M.

Fiant chartule No.x.

Sig.-One powder three or four times daily.

(Einhorn.)

\section{Coprostatic Diarrhe:}

When fecal masses and scybala collect in the colon, sigmoid flexure, or rectum and cannot be gotten rid of by cathartics and enemata:

R Hydrogenii dioxidi..................... Oss $(250.0)$.

Sig.-Injected in to the bowel will cause their disorganization.

Diarrhea Induced by Caturrhal Coloproctitis:

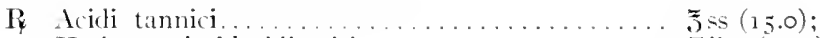

Hydrargyri chloridi mitis.............

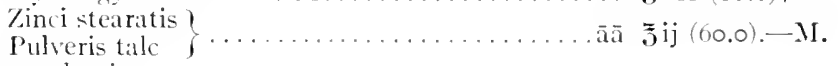

Fiant pulveris.

Sir.--Insufflite the sigmoicl flexure through the sigmoidoscope three times weekly.

This remedy is helpful independently or in combination with remedies otherwise administered.

Rosenoerg's Powder:

R olei thymi...... . . . . . . . .

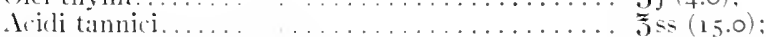

Magnesii carbonatis $\quad \ldots \ldots \ldots \ldots \ldots \ldots \ldots$ J

Sig.-Insufflate the bowel as often as required. 
Diarrhea of Exophthalmic Goiter:

R Listracti dieritalis.

Extracti erentio

gr. iiss (O.I5)

Strychninat sulphatis.

i)

Ferri arseniti.

Lre. $\quad 0.03)$

lone in capsulas No. xxy.

Sir.-One capsule three times daily after meals.

irr. iirs $(0.15),-11$.

(Gint.)

Caturhal Diurrhea with Vausea and Fomiting:

1) Ifydrargy chloridi mitis...............

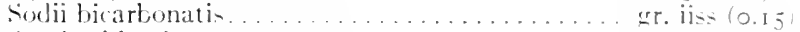

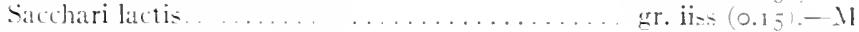

Sig.-Every hour or two on tongue.

G(lnt.)

\section{Mucous Diarrhed:}

R Argenti nitritis

Extracti hyoscymi

gr. ij $(0.12)$;

Fiant pilula $\mathrm{X}$ o. $\mathrm{x}$.

Sig.-One pill three times daily.

gr. $1(0.30)-\mathrm{N}$

$$
\text { (IItre.) }
$$

Diurrhew with Colic:

R Camphora)

Capsici

àa gr. is: (0.00):

Zingiberi-

(tr. ij $(0.12)$ :

Sacchari lactis

arr. Xi (1.0)-M

Fiant pilulie No. xii.

Sig.-one or more pills at two-hour intervals aceording to indications.

Gint.)

Choleriform Diurrhen:

R .Etheri- ate tici.

Olei cajuputi

Tinctura osii

Sig.-Ten or is drojs every two hours on a lump of sugar.

$\overline{5}$ ij $(S .0):$

Tा" XY ( I.O):

$5 \mathrm{i}+1.0 \%-.11$

Beasley.)

Bilious Diurrhen:

P Hydrareyri chloridi mitis..... .......... ur. j 0.00$)$ :

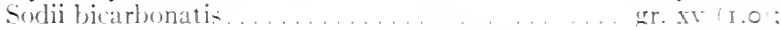

Pulveri-ojii ... ............ ij $10.12 \%-11$

Fiant chartula No. viii.

Sig- One every two or three hours until eight powders are used. follemed by laree doses of bismuth and presin.

Sicitt.

Verious Diarhed:

P Stryhnine sulphatis

dicli -ulphurici aromatici

ar. 510.03

Aquie hamameliclis

il 120.0 :

Fiant solutio.

Sig. - I teasponful in water every three hours.

(imt.)

R Tinctura belladonna

Kalii bromicli

Aquie di-tillatie

$\overline{1}-2.0$

万ilis: 10.0 :

Sig.-I tablesponful three or four time = daily.

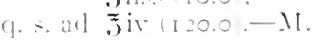

(iunt.) 
Terous Diarhede:

F Liquoris potasii arsenitis ?

Aqua amygdalix amarie

āā $\overline{\mathrm{ij}}(8.0),-\mathrm{M}$.

Sig.-Four alrops three times daily after meals. Increase the dose by a drop every second day until 20 are taken: then reduce the duse in the same way down to $f$ drops. At bedtime administer effervescent bromid salt of Sanduw to encourage sleep.

(Charcot.)

Acute Diurrked:

R Olei ricini

Spiritus chloroformi

gr. $\operatorname{xxiv}(1.50)$;

Morphine hydrochloratis

Pulveris acacie

Syrupi implicis.

Iqua distillatie.

Jiss $(0.0)$;

gr. j (0.06):

5 iiss (10.0);

$\tilde{J}$ SS ( I 5.0$)$;

... $\ldots \ldots \ldots \ldots \ldots \ldots \ldots$

Jैiv (I 20.0).-II.

Sig.-. I dessertspoonful every hour and a half for an adult.

(Ioung.)

Acute Diarrled with Burning and Struining:

1) Tincture opii deodorati .............. nе xij $(0.75)$;

Liquoris potas ri citratis .....................

Sig.-Teasponnful every hour until relieved.

$(G(n !)$.

Diarrhed Accompanicd by Burning in the Bowel:

R Orthoformi

$5 \mathrm{ij}(8.0)$ :

Bismuthi subnitratis

Jis $(45.0)$ :

Olei olira ............................. s. ad Oj (500.0).-MI.

Sig.-Shake well. Warm and inject 6 ounces into the colon after an evacuation.

(Gint.)

Diurrhe Due to Coprostasis or Foreign Bodies:

R Tincture opii .... . . . . . . . . . $\ldots \ldots$

Spiritus ammoniæ $\quad \ldots \ldots \ldots \ldots \ldots \ldots$ mexx $(2.0)$;

spiritus menthe piperita.............. mxए (1.0);

Tincture catechu.....................

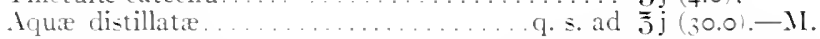

Sig.-. Idminister every three or four hours following a dose of castor oil.

(Leonard II"illiams.)

Diarrheu Wixture:

R Tincturæ lavandulæ compositæ........... Jiv (I 20.0);

Sacchari .... . . . . . . . . . . .

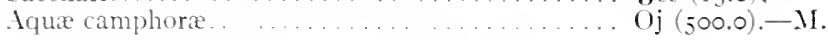

Sig.-. I tablespoonful every three hours, for diarrhea.

(Parrish.)

Diurrhe Due to Indigestion:

R Extracti cannabis indica Huidi . . . . . . . . m (0.06):

Creosoti.... ....................... $\mathrm{g}(0.30)$ :

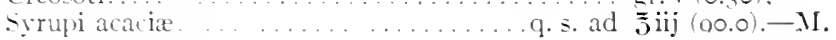

Sir.-. I tearpoonful before meals.

(Germuin Lee.)

Dyspeptic Diurrhed with Nensen or lomiting:

R Cerii oxalatis

IBismuthi subnit ratis

Siritus chloroformi

Liquoris calcis !

Syrupiaria i

Sig.-Desertspooniul in water when necessary.

$(G i n t$. 
Fermentalize Dyspeptic Diarrhea:

If Phenolis liquefati................. gr. $1(0.30)$;

Syrupi alacie

a isis $(45.0) \cdot-\lambda 1$.

Sig.-One teaspoonful before meats.

$($ (illnt.)

Irrilative Diamhed of Adults with Cramps:

I) lulveris rhei.

Sodii bicarbonatis.

Spiritus ammonia a romatici . . . . . . . . . .

Spiritus myristicat

Infusi caryophylli.

Sig.-I hall to a tablespoonful three or four times a day.

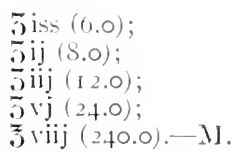

(Shocmaker.)

\section{Tubereular Diurrhea:}

If Tincturie kramerix ................. $5 \mathrm{y}(20.0)$;

Tinctura opii.................... Itw (3.50);

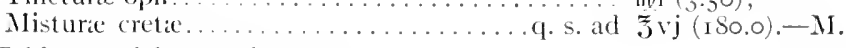

Sig.-Tablespoonful every four hours.

(Gunt.)

R Methylthionina hydrochlorici (Merck)

gr. iiss (O.15);

Sacchari lactis.

gr. xxij (1.50), - - II.

Sig.-In wafer or capsule, one each day.

(Gunt.)

Diarthe lecompaniad by Intestinal Soreness and Burning:

It Acili sulphurici aromatici................

Spiritus chloroformi.....................

Tincturae ofii camphorate.

5 iss $(6.0)$

$\overline{i j}(8.0)$;

Syrupi zinziberis.

吾ij (10.0);

Sig.- Descertsponful in water every two hours.

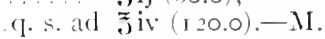

(IIare.)

Serous or II atery Diarrhea:

B Tincture kinu

Tincture gamberis composita...............

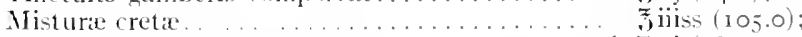

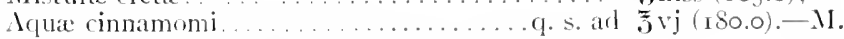

Sig.- Shake and take a tablespoonful every three hours.

$(G(t) t)$

Diurher in Typhoid Fener with Imminen Hemorthage or Peritonilis:

R Argenti nitratis....................... . ij (0.1 2);

Tincturie opii.....................

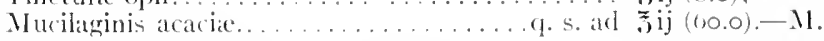

Sig.-I teaspronful three or four times daily.

(II'illiam I'cpper.)

Diurrhea in Typhoid Fear (Lead and Opium Pill):

R Plumbi acetis....................... xij (0.75);

Pulveris opii...........................

Camphora................................ gr. xij $(0.75),-\mathrm{M}$.

Fiant pilulie No. vi.

Sig.--One pill two or three times daily. 
Cholera Drops L'seful in Aeute Diarrhea:

R Olei menthie piperita. gr. xly $(3.0)$

Ncoholi $5 \mathrm{v} \cdot \mathrm{j}(24.0)$

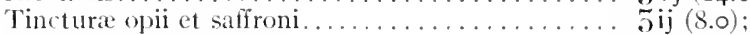

Tinctura ipecacuanha. ............... 5 v

Tinctura valeriance.................. J iss $(45.0) .-\mathrm{M}$.

Sig.-One to two fluidrams as often as required.

(Tiechman.)

\section{Diarrhea or Cholera and Cramps:}

I) Chloralis hydratis................. 5iij (1 2.0);

Morphince sulphatis ................... gr. j (0.06);

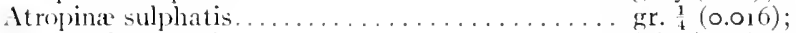

Aqua chloroformi ................. 5 iv $(15.0)$;

Aquat distillata .................... 5 iv (15.0). $-\mathrm{M}$.

Sig.- I dose is 20 minims, repeated every ten minutes as required.

(Bartholow.)

Cholera Mixture:

P. Tincture capsici

Tincturce opii

Tincturce rhei

Spiritus menthe piperita

Spiritus camphora

Sig.--Fifteen to 30 drops in a wineglass of water.

$\bar{a} \bar{a} \cdot-M$.

(Now York Sun.)

Diarrhea in Cholera Asiatiea:

R Tincturie opii................... 6 parts;

Vini ipecacuanha................. \& "

Tincture etheris valeriane.............. I 2

Olei mentha piperita................ I part.-M

Sig.-Fiftexn to 25 drops are given every half-hour.

(Musser and Kelly.)

Syphilitic Diarrhea:

14 Hydrargyri chloricli corrosivi.............. gr. ij (o.1 2);

Potassii iodicli. ..................... $5 \mathrm{5}$ (20.0);

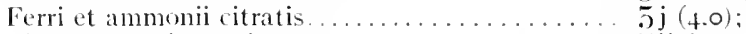

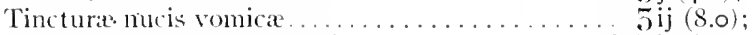

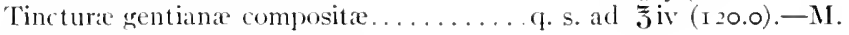

Sig.-Teaspoonful three times daily.

(Gant.)

R Hydrargyri iodidi flavi

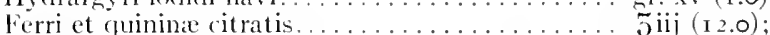

Extracti hyoscyami.................. gr. xij (0.75).-MI.

Fiant pilula No. lx.

Sig.-One pill three times daily.

(Gant.)

P Hydrargyrichloridi corrosivi............... gr. ij (0.12);

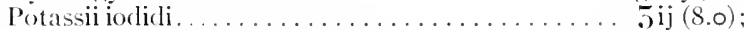

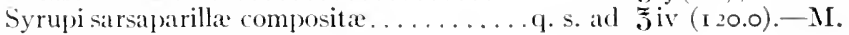

Sig.- Teaspoonful three times daily.

(Gant.)

The above combinations are more effective when their daily administration is reinforced by colonic irrigations, when one of the irrigating solutions given in the formulary is employed. 
Diarrhea and Dysenteric Colitis:

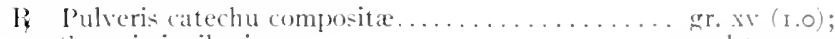

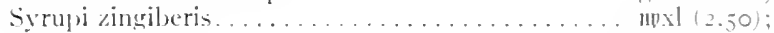

Tincturie op ii . . . . . . . . . . . . . . miv $(0.2+)$;

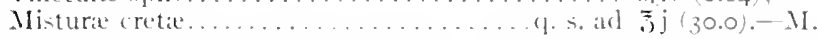

Sig.- I teasponnful to be taken every four hours while diarrlea continues.

(Gint.)

Chronic Dysentery:

R Tincture coto.......................

Tinctura bellarlonnat................ miij (0.18);

Tincture nucis romicie............... miij (o.is);

Tincture krameria...............

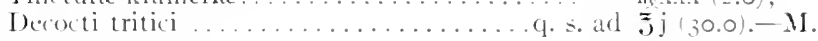

Sig.-Take every four hours.

(Beasley)

Cathartic in Bacillary Dysentery:

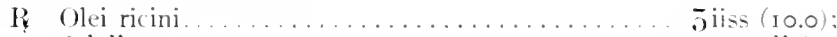

Saloli. . . . . . xxwij (I.75);

Tincture opil deodoratie.................

Pone in capsulat No. xr.

Sig.-One capsule to be taken every two hours.

(Delaficld.)

Entamebic (Dysentery) Diarrhe:

R Pulveris ipecacuanha................ gr. ij (O.12);

Pulveris opii ......................... gr. $\frac{1}{3}(0.02)$;

Hydrargyri chloridi mitis.................. gr. ${ }_{4}^{3}(0.0+5) .-M$.

Pone capsula No. i.

Sig.-One capsule to be taken every two hours.

(Sodré.)

\section{CHRONIC DIARRHEA AND COLITIS}

The following formula are to be relied upon in the treatment of practically all forms of subacute and chronic diarrheas, viz.:

Suhacute and Chronic Diarrhea of Phthisis:

R Irgenti nitratis....................

Syrupi ipecacuanha .............

Miorphine sulphatis . . . . . . . . . . . . . j $(0.065)$ :

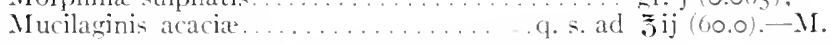

Sig.-. I teaspoonful in water three times a day before meals.

(Shoemaker.)

Chronic Diarrhea. Gastric Caturrh, or Gastro-intestinal Caturrh of Phthisis:

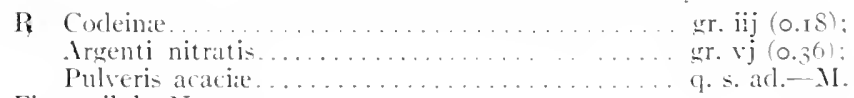

Fiant pilulie No. $x x$

Sig.-Give one pill every two to four hours.

(Shoemaker.)

Chronic Dysentery and Diurrhea:

R Olei ricini.

$5 \sin (2.0)$;

Tincture opii

$\operatorname{limx} 10 \operatorname{xx}(1.30-2.0)$;

Syrupi sarsaparillie or Iqua mentha piperitit

is: $(45.0)$

Pulveris acacie.

(1. S. atl.-M

Sig.-One or two teaspoonfuls three or four times a day.

(Bruntom.) 
Pirsitent Diarrita:

R Pulveris rete umpoitie

5iiis= I 4.0 ;

Bi-muthi abogallatis

Gr. xl 2.00 :

Aqua menthe piperitie.

sig.-Table-poniul every three or fiour hour.

Q. S. ad $\overline{\bar{j}}$ ir I $20.0 .-\mathrm{M}$.

Gunt.)

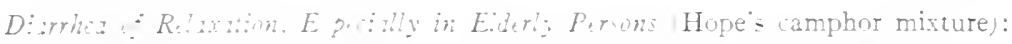

P Acidi nitri i

Tincture of ii

Aquat camphora

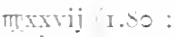

mix I.24:

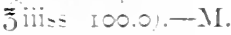

siz- A tablesp wniful every hour or two acurding to symptoms.

IIope.)

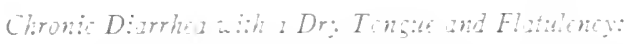

$\mathrm{P}_{\mathbf{2}}$ (O)ei terebinthinat

Olei amyadale expresum

Tincture opii

Mucilarinis acacia

Aque lauro-cerasi

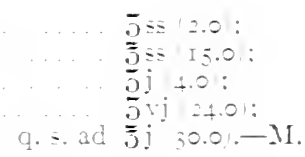

sig.-One teasponiul in water three or iour times daily.

Gunt.)

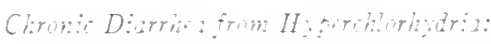

P Acid hydruhlori Codes

simitimonis

Aquat distillatæ

ar. vij to viij $0.36-0.501$

5 iis: 10.0 :

q. S. ad Oij 1000.0.-.11.

Sic - A tumbleriul to be drunk during meals

The ameliorution of smptoms will be manifest in three or four days,

(Lartin.)

$\mathrm{P}$ Tincturee catechu

Bismuthi subsalicylatis

Pulveris crete armatici

Aque, hlorormi

Sir.-Hali a wineclass two or three times a day.

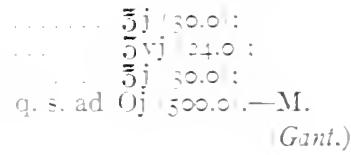

P. Tin ture cepici

A id culphuri a romatici

Tinctura opii

soruri aurantii.

A une camphora

sia-Tu be taken three times a day.

Tाए 0.30 :

$\pi x \quad 0.6:$

ता $0.3:$

$5=2.0:$

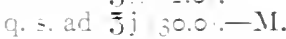

P. Creta preparata

Tinctura catechu

Tincture ofii

Aruat innamomi

Sic-Two tablespornits aiter each stool.

(Beasley.)

Pr Saloli

Bismuthi subnitratis

Misture cretat

sir.-One tablermoniul every two hour.

5 ij 5.0 :

$\overline{3} \div 150$

mlaxx $5.01:$

J) viij $2+0.0 .-\mathrm{M}$.

Fothergill.)

P. Resor ini

Tin ture of

Tin ture cas arillex

Iniusi, ham imil

sig.-Teasminiul every two hours.

5 ij 8.0:

5 iv i 5.0

q. s. ad $\overline{3}$ iij 00.0 -. . II.

Gunt.)

zr. is to iij 0.00-0.IS);

att. ij O.I2 :

git. XY I.0:

$\overline{\bar{J}} \mathrm{ij}(600.0$ - - MI.

( Kinderurzt.) 
Chronic Diurlue from /lyperhhorhydria:

$\mathrm{R}$ Potassii bromidi.

Tinctura opii

Biij (12.0);

Tincture catpici.

万ij (8.0);

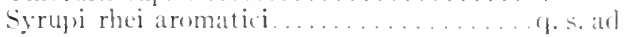

$5 \mathrm{j}(4.0)$;

Sig.-One teasponniul as neerker.

\section{(Bartholow.)}

P. Argenti nitratis

Extracti hyos yami

Extracti opii

Pulveris sycyrhizat

Fiant pilula No. Xx.

Sig.-One pill one hour before each meal.

(rr. Y $(0.30)$;

(rr. x to xxx $(0 .(10-2.0)$;

er. ij tor $(0.12-0.30)$ :

Q. S. -11 .

R Extracti ercotie.

Extracti nucis romicie

Exaracti opii

(ianl.)

Fiant pilule No. xx.

Sig.-One pill every four to six hours.

P. Morphina sulphatis

Bismuthi subnitratis

Fiant chartula No. i.

Sig.-One powder three or four times daily. (In chronic cases.)

(. Honso Clark.)

R Tincture opii camphora !

Bismuthi subnitratis $; \ldots \ldots \ldots \ldots \ldots \ldots$ a $\bar{a}$.joss ( I 5.0 );

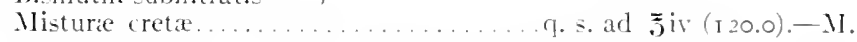

Sig.-Two teaspoonfuls in water every two or three honrs. Shake.

$(K \in m p$.

P. Argenti nitrati:

Resina terebinthina?

Liquoris potasse ,

Pulveris glycyrhiza.

Er. It: $(0.005)$ :

gr. $1=(0.005):$
gr. $V(0.30) .-\mathrm{MI}$.

gr. $v(0.30)$;

ar. \& $(0.100)$. - MI

(Dalosta.)

Fiant pilula Ni. xx.

Sig.-One pill three times daily.

( Kemp.)

$\mathrm{P}$ Pulveris ofii ।

Argenti nitratis

gr. v $(0.30)$

$\bar{u} \bar{a} \bar{D} \mathrm{j}(4.0)$;

q. S.-.I.

Resinx terlyinthina

Liquoris potassie. .

Pulveris enlycrrizize

Fiant pilulie $\mathrm{No}$. Ix.

Sig.-Two or three pills three times a day.

(Thompson.)

\section{HELMINTHIC AND PROTOZOAL COLITIS (PARASITIC DYSENTERY}

Helminthic Caturrh. Diarrhea, or Constipation-Cestodes, Flat (Tonia), Solium (pork), Sagrinata (beef), lata (fish), and Nina (dwarf):

(1) Place patient on a fluid cliet for twenty-four hours.

(2) Clear the bowel thoroughly with a hydragogue cathartic. (Salts or calomel.)

(3) Administer a teniafuge (anthelminthic) such as felix mass (male ferm. $\bar{j}$ ss to ij (2.0-8.0): fluidextract of granatum (pomerranate root). 5 ss to ij (2.0-8.0): pumpkin-seed or pelletierin. gr. v to vij $(0.30-0.45)$. to distodere the parasite.

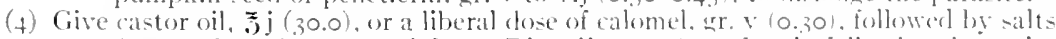
(magnesium citrate or sulphate. $\bar{j} \mathrm{j}$ to $\mathrm{ij}-4.0-8.0$ ), shortly following the teniafuge to cause expulsion of the worm.

(5) When the helminth has been expellert a search should be marle for its head. which. if found, indicates that the treatment has been completely sucressul. In aggravated cases the above vermifuges may be combined to arlvantage. 
When male fern fails to bring the worm, the following combination will be found useful:

R Extracti filicis macis ather............... gtt. Iviij (4.0);

Chloroformi....................... gtt. $\vee$ j $(0.36)$;

Olei ricini

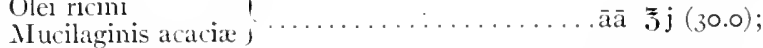

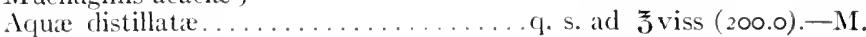

Fiant emulsio.

Sig.-Introduce through a stomach-tube in the morning.

(Cohnheim.)

Nemutodes (Round-and Thead-worms):

Hookworms: These helminths can always be eradicated by the administration of four doses, one hour apart, of beta-naphthol or, preferably, thymol in ${ }_{5} 5$-gr. ( 1.0 ) doses following a two-days' huid diet and cleaning of the bowel with a saline. A second dose of salts should be administered after the last of thymol to wash out the inert or dead worms.

(Gant.)

Lumbricoid Horms: Patients afflicted with Ascaris humbricoides should be advised to destroy their stools and scour their hands after defecation to prevent extension of the infection to others.

Santonin, gr. ij to $\mathrm{v}(0.12-0.30)$, alone or in conjunction with castor oil, is usually effective against these parasites when administered for three successive evenings and followed by a purge, particularly when the bowel has been emptied prior to the first dose.

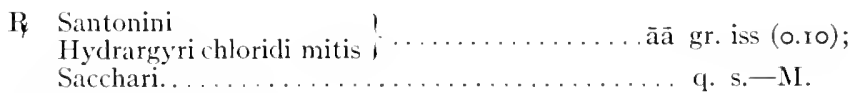

Fiant chartule No, vi.

Sig.-A powder night and morning. $\quad$ (Cohnheim.)

For a detailed discussion of helminthic and protozoal colitis (parasitic dysentery) the reader is referred to Chapters XXXVII and XXXVIII.

\section{ENEMATA AND IRRIGATING SOLUTIONS IN THE TREATMENT OF DIARRHEA DUE TO CATARRHAL OR ULCERATIVE (SPECIFIC) COLITIS}

R Sollii biboratis................. $5 \mathrm{ij}(8.0)$;

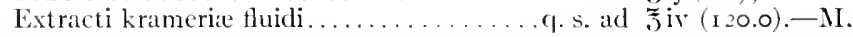

Sig.-Put a tablespoonful in a quart (rooo c.c.) of warm water and irrigate the colon daily until stools are fewer in number, and mucus, pus, and blood disappear.

(Gant.)

This formula acts exceedingly well when the mucosa is inflamed or dotted over with erosions or superficial ulcers.

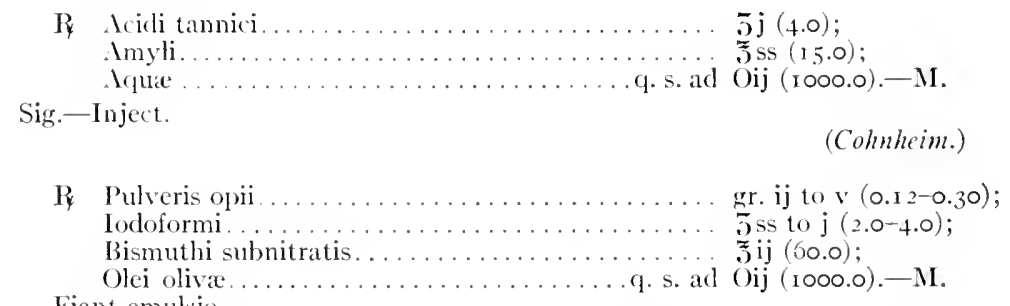

Fiant emulsio.

Sig.- Warm and inject, or pour 4 ounces or more into the colon through sigmoidoscope while the patient is in the inverted posture. Repeat the treatment three times weekly. 
This emulsion quickly relieves intestinal burning and tenesmus, and stimulates healing when the bowel is highly inflamed and sensitive or ulcerated.

Dysenteric Diarrhea (Sublimate Enema):

R Hydrargyri corrosivi .................. ij to iij (0.1 2-0.I 8);

Aqua......................... () j $(500.0)$

Alcoholis................................ .

Sig.-For two injections, one in the morning and one in the evening.

(Bonlmy.)

Enema for Feeble Patients having Dysenteric Chronic Diarrhea:

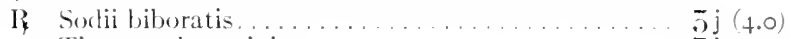

Tinctura benzoini .............

Spiritus camphore...................

Sig.-Inject.

Iqua distillata....................... s. ad Oij (I000.0).-1I.

$(G i n t$.

Wueomembranous Enteritis with Diarrhea:

R Bismuthi subnitratis?

Bismuthi salicylatis

.àa joiiss (10.0);

II ucilaginis cydonii (quince seed)........... Oj (500.0)...II.

Sig.-For rectal injection.

(Revilliod.)

Chronic Colitis:

R Resorcini............................ gr. $v$ to $\mathrm{x}(0.30-0.65)$;

Iquare distillate...................... Oij (1000.0). $-\mathrm{NI}$.

Sig.-L'se for enterocly'sis.

$(G u n t$.

\section{INFANTS AND YOUNG CHIIDREN}

Diarhea of Secondary Sepsis:

Py Bismuthi subcarbonatis............... gr. $\mathrm{x}(0.60)$;

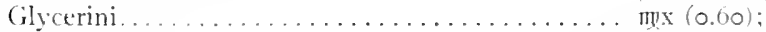

Sodii salicylatis....................... gr. j(0.00):

Aqua distillat $x \ldots \ldots \ldots \ldots \ldots \ldots \ldots \ldots \ldots$ q.

Sig.-Teaspoonful every three or four hours.

$(G(1 n t)$.

Dysenteroid Diarrhea with .I Uueus and Blood in the Stools and Tenesmus:

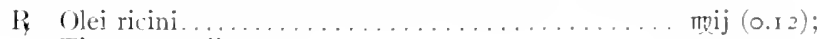

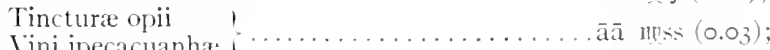

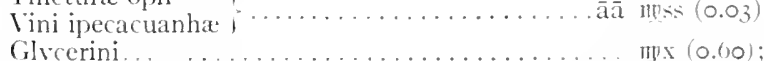

Aquate cinnamomi........................... ad $5 \mathrm{j}(4.0), \mathrm{M}$

Sig.-Teasponful every four hours.

$(G(b) t$.

Diarrhed with Foul-smelling Stools:

R Resorcini.

Tincture rhei aromatica. .............. miij (o.rs);

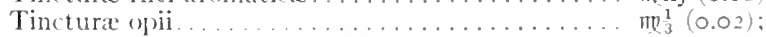

Tinctura cardamomi compositic............ nus $(0.30)$;

Aqua chloroformi ................ mv $(0.30)$ :

Ique mentha piperita................... s. ad $5 \mathrm{j}(4.0) .-\mathrm{M}$.

Sig.-Teaspoonful three times daily. 
Diurrike in ith Fluid Hoitments:

$\mathrm{R}$ spiritu= ammonize aromatici.

$\operatorname{mos}(0.03):$

(ilverini.

miij $0.13:$

- true distillatit

$\operatorname{mix} 0.00$ :

sig.-Teaspouniul every inur hours.

Infuntie Diarthe in ith Greenish Stools accompanied by Abdominal Pain:

R Bionuthi subnitratis

Liquoris calcis

tr. xly 3.0 :

Aqua ieniculi

i. 5 is 10.01 ;

sir.-Teasponful every two hours.

q. $\therefore$ ad 芌ij (00.0).-M.

(.Wod:tied after Grimes.)

\section{DIARRHEA OF OLDER CHILDREN}

The dosage given below must be studied and changed according to the patient's age:

R Bismuthi subcarbonatis spiritus myristice

$5 \leq s$ to iss $(2.0-6.0)$;

spiritus vini callici

$\operatorname{mix}(\mathrm{I} .30)$;

Aqua cinnamomi

i.jij 10.0:

Shake well.

sig-Teaspooniul every two hours.

Binneft.)

P. Siritus camphore

Tincture capsici

spiritus menthæ piperita

Tincture rhei aromatice

$\bar{a} \bar{a}$ ìj 30.0 - M.

sig.-One to 5 drop s in water every two hours

Ruschenberger.)
Pr Spiritus camphore
i. $5=(2.0)$
Epiritus vini gallici
q. S. ad $i \overline{3}$ ij 00.0$)$ - M.

sig.-Twenty drops every two or three hours in cases of prostration irom diarrhea.

Gunt.)

P. Camphor

sterile olive oil

ar. si 0.3 :

$\operatorname{mix} 0.60-\mathrm{M}$.

Sig.-Hypodermically. in cases of collapse as a quick difusible stimulant.

Acule Diarhata and Dusentery Complicated by Secere Pain and Tenesmus:

P. Cocainie hydroihloratis.

Extractie ersutie

gr. j $(0.65)$ :

Extrat tum ofii

ar. j $(0.15)$ :

Thymulis iodidi.

gr. is 10.00$)$ :

() $)$ ei theobromatis

ar. iv (0.24);

ar. cl $(6.0) .-11$

Fiant suppositoria Xi. x.

sig.-One suppository to be used every three hours after prelininiry treatment.

(Gunt.)

P Naphthalini Sacchari lacti-

ar. xij to $\bar{j} \mathrm{j}(0.75-4.0)$ :

Fiant chartulit Nis. xii.

gr. sij to $5=(0.75-2.0),-11$.

sis.-One powder every three hours.

(Starr.) 
Acute Diarrhed and Dyschtery Complicated by Severe Pain and Tinesmus:

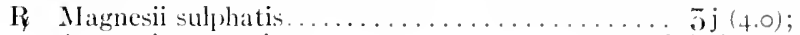

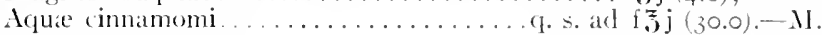

Sig.- Teaspoonful every two hours for a child of one or wo years where saline catharsis is indicated.

I) Extracti hamatoxyli.

Tinctura krameria.

Gilycerini

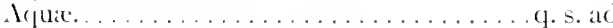

Sig.- Teaspounful three times daily.

R Phenylis salicylatis

Bismuthi subnitratis

Pulveris creta aromatica cum opii

Fiant chartule No. i. gr. iss $(0.00)$

muiiis $(0.20)$ mpxii $(0.75)$;

$5 \mathrm{j}(4.0)-\mathrm{M}$.

(Gint.)

gr. iss $(0.00)$;

gr. iv $(0.24)$;

gr. ss $(0.03)$. - 11 .

Sig.- To be given with 10 drops of glycerin three times daily.

(Gilnt.)

Chronic Intestinal. Diurrhea aith Offensiae Stools; Chronic Dysentery and Colitis:

R Bismuthi subnitratis.

Benzonaphtholi.

gr. v $(0.30)$;

Pulveris ipecacun

gr. j $(0.06)$;

Sacchari albi...

er. $\frac{1}{1}(0.005)$;

Sig.-Of these powders children may be given five daily, one about every three hours. The average dose for children up to one year is 0.05 altogether; $0.25 \mathrm{gm}$. daily; for children of two years $0.1 \mathrm{gm}$. per dose and $0.5 \mathrm{gm}$. daily.

(Zcntrallialt f. d. gesummte Therapie, 28, 1910.)

Note.-Useful after all food elements have been remored.

Acute Colitis and Ileocolitis (Dysentery):

R Bismuthi subnitratis

Tinctura caterhu compositit

Tincturie krameriae

$\bar{a} a \bar{a}$ jij $($ S.o);

Tincture opii camphorate

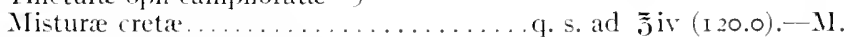

Sig.-Teaspoonful every two hours.

(llater.)

In all varieties of acute and chronic inflammation, and ulcerative lesions accompanied hy diarrhea or mucoid and bloody stools, silver nitrate, potassium permanganate, ichthyol, zinc sulphate, fluidextract of hyclrast is, sodium salicylate, 1 to 2 per cent., are always effective when they are made to reach all parts of the invaded bowel. The strength of irrigating solutions may be doubled in olstinate cases.

When ulceration is extensive and diarrhea is very persistent, in case the stools are exceedingly offensive, they can he succesifully disinfected by flushing the intestine twice daily with a 5 per cent. ich thyol solution of kerosene (lamp oil) or 20 per cent. hydrogen peroxirl solution. 


\section{CHAPTER NLIX}

\section{SURGICAL TREATMENT OF DIARRHEAL, INFLAMMATORY, OBSTRUCTIVE, AND PARASITIC DISEASES OF THE GASTRO-INTESTINAL TRACT}

\section{GENERAI REMIARKS, PREPARATION OF PATIENT}

H.risg elsewhere discussed other measures useful in the treatment of this class of affections, the author will now outline their surgical treatment. Some of the procedures are simple. but other operations are abdominal, and require a shilled surgeon.

Surgical intervention should not be practised in the treatment of diarrheal, catarrhal, and parasitic diseases until after other and less radical therapeutic measures have failed to benefit the patient, except when it is evident that the trouble is incident to acute or chronic intestinal obstruction, in which case an operation is indicated at the first opportunity: Operative interference is also imperative in the presence of intestinal cancer. and in neglected cases where the patient is suffering profoundly from diarrhea, toxemia, exhaustive discharges, and extensive ulcerative lesions of the bowel complicated by mixed infection. On the other hand. operative procedures should not be withheld and the sufferer daily drugged to control diarrhea when it has been demonstrated that the treatment is not curative. because medicines employed for the purpose disturb the stomach. interfere with the secretions and peristalsis, and the patient often becomes addicted to them, and. further, because mans of these sufferers can be quickly and permanently cured by operations that are almost devoid of danger, take but a short time. and keep the patient in hed but a few days. Many phy-icians asert that their patients will not submit to operation. but the author holds that the opposite is true, and that sufferers from either chronic constipation or diarrhea insist upon operative interference as soon as ther are convinced that a correct diagnosis has been made. and that radical treatment will permanently help them, for the are willing to do anything which will reliere them from constant dieting and drugging. The author has had a very extensive experience with the surgical treatment of diarrheal, catarrhal, and parasitic diseases of the stomach and inte-tines, and the results have been almust univer ally good, and in consequence he has no more hesitation in recommending an operation in this class of cases than for other surgical diseases.

Operative measure: are seldom required in the handling of acute inflammatury and ulcerative lesions of the intestine responsible for catarrh or liarrhea, since they can be relieved by the simple neasures outlined elsewhere. 
Where loose movements are incielent to chronic intestinal obstruction a prompt cure ustally follows correction or removal of the lesion, but when catarrh or diarrhea is caused by simple or specific inflammatory or ulcerative lesions a course of postoperative treatment of day's, weeks, or, possibly, months may be necessary to heal them subsequent to intestinal exclusion, appendicostomy, cecostomy, colostony, or resection.

Multiple operations are indicated now and then to relieve individuals suffering from chronic diarrhea, for in one of the author's recent rases the colon was obstructed by an angulation, extensively ulcerated, and the rectum was blocked by a large polyp, all of which were corrected by separate procedures. It is frepuently necessary to do an appendicostomy or cecostomy in connection with resection and other operations performed for the relief of chronic bowel blocking to ruickly overcome the toxemia, heal ulcers or an inflamed mucosa, and hasten the patient's convalescence.

Occasionally one is justified in delaving surgical intervention and giving a prolonged trial to less radical measures where patients have plenty of strength, time, and money, but individuals who work for a living should be operated upon as soon as it has been demonstrated that (liet, medicine, and irrigation (from below), etc., fail to permanently improve them.

Nany types of diarrhea herein discussed are curable with ordinary measures; but loose movements incident to chronic intestinal obstruction, a highly inflamed or ulcerated mucosa, constipation with recurring fecal impaction, irritation or occlusion from parasites, of ten cannot be permanently arrested except by operative procedures.

Before describing individual procedures the author will brietly discuss the method of preparing the patient and other points concerned in the general technic of abdominal and other operations to be discussed.

Preparation of the Patient.-The method of preparing the patient depends upon whether or not the abdomen is to be opened or the rectum operated upon. Except in emergency cases subjects recpuiring laparotomy should remain in the hospital two or more days prior to the operation that they may become accustomed to their surroundings and give the attendant an opportunity to cleanse the bowel. There are many laxatives and cathartics for the purpose, but the author prefers licorice powder, $3 \mathrm{j}$ to $\mathrm{ij}$ (4.0-8.0), a saline, 3 ss (15.0), or when the patient is bilious, calomel and soda, gr. $\frac{1}{2}(0.0,3)$, administered every twenty minutes until five doses have been taken, when it is followed the next morning by salts or a mineral water.

When the intestine is blocked by an olstruction or there is fecal impaction, 1 or 2 ounces of castor oil usually proves effective. Enemata of soapsuds, water, or oil, alone or admixed, are useful for cleansing the colon, sigmoid flexure, and rectum.

Where intestinal resection or rectal extirpation is contemplated more time is required, and several days should be spent in freeing the 
intestine of retained feces and opiates, or astringents should be administered several hours before the operation to arrest peristalsis and the secretions, so that the lower bowel will be empty at the time of operation.

Formerly, the author had the abdomen shaved, thoroughly cleansed, and covered with antiseptic dressings, but recently the latter have been omitted and chief reliance placed on an iodin solution, which is painted over the clean dry skin and then washed off with alcohol just before the operation is begun. In abdominal cases the patient should urinate or be catheterized before he enters the operating room, otherwise the bladder may be opened, as occurred in one of the author's cases.

When the rectum is to be removed or extensively operated upon the patient should be prepared according to the above plan; but when an abscess is to be incised, hemorrhoids removed, the sphincter divulsed or cut. and in other minor procedures, elaborate preparations are unnecessary and should be discarded for an enema, consisting of not more than 6 ounces of water. which will empty and cleanse the rectum. When the author has been bothered with feces rumning over the operative field it has usually occurred in cases where the all-iise intern has administered a strong cathartic the previous night and a copious colonic enema shortly prior to the operation.

Of the operations called for in the surgical treatment of diarrheal, inflammatory, and parasitic diseases of the gastro-intestinal tract, the following are the most useful-viz.:

(I) Cecostomy (Cibson's and Gant's).

(2) Appendicostomy.

(3) Appendicocecostomy.

(4) Appendico-enterocecostomy.

(5) Enterostomy.

(6) Colostomy:

(7) Resection and extirpation (enterectomy, cecectomy, colectomy, sigmoidectomy, and proctectomy).

(s) Sigmoidectomy and proctectomy.

(9) Intestinal exclusion. 


\section{CHAPTER 1 ,}

\section{SURGICAL TREATMENT OF DIARRHEAL, INFLAMMATORY, OBSTRUCTIVE, AND PARASITIC DISEASES OF THE GASTRO-INTESTINAL TRACT (Continued)}

\section{APPENDICOSTOMY, APPENDICOCECOSTOMY, CECOSTOMY \\ HISTORIC NOTE, GENERAL REMARKS}

Historic Note.-Twenty years ago the direct or throngh-andthrough irrigating treatment, now frequently employed for the relief of rliarrhea, catarrbal and infectious colitis, and many other diseases of the colon, was unknown. Mayo Roloson (1893) and Hale White ( 1895$)$ reported cáses of membranous colitis cured by right-sided colostomy and bowel flushing. Cibson (I902) described his valaular cecostomy', and Veir, in the same year, published his method of irrigating the colon through the appendix, a procedure Willy Neyer named appendicostomy. In 1908 the author described his cecostomy which prosides a means of separately or simultaneously irrigating the colon and small iniestine. In 1910² he published a modification of this procedure, describer his enterocolonic irrigator used in connection with it, and at the same time gave the steps and illustrated the technic of his appendicostomy. reporting 105 cases (see accompanying table) of bowel affections treaterl by through-and-through irrigation, since which time he has hard an additional ioo cases.

TABLE SHOWING OPERATIONS ANI AFFECTIOAS FOR WIICH DIRECT BOIIEL TREATHENT WAS EMPLOYED BY THE AUTIOR IN I0.,

IDLLTS. (These operations were performed prior to iolo.)

Operations performed for:

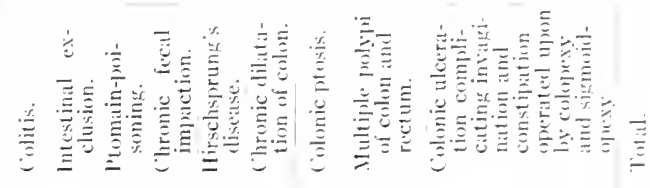

Aprendicostomy

Appendicocerosiomy

Gibson's operation.

. Juthor's cecostomy

Number.

Since Veir, Gibson, and the author outlined the technic of the above operations they have been extensively amplosed in the treat-

1 New York Med. Jour.. August is.

${ }^{2}$ Interstate Med. Jour., vol. xii, No. o. 
ment of colitis and other diseases mentioned below, and many valuable papers by various authorities have been published concerning morlifications in their technic and suggesting new fields of usefulness for them. The author became convinced years ago that ulcerative colitis and other affections responsible for chronic diarrhea required local treatment, and that medicine and dieting were of secondary importance in such cases. In his teaching, books, and articles in current literature he has done his best to emphasize the value of medicated bowel irrigation in the treatment of chronic, inflammatory, ulcerative, and parasitic diseases of the colon, and to point out the value of appendicostomy, cecostomy, colostomy, etc., and through-and-through colonic flushing in cases where the solution cannot be made to reach all parts of the large bowel when introduced from below. A summary of the author's most important contributions along this line are given below:

(1) Report of 3 cases of membranous coloproctitis cured by colostomy and irrigation (Gant, Diseases of the Rectum and Inus, 2d ed., p. 222, 1902).

(2) Tubercular colitis cured by colostomy and irrigation with closure of the artificial anus at end of the third year (Review of Reviews, October, 1900).

(3) Report of 9 cases of dysentery and diarrhea treated by appendicostomy or cecostomy and irrigation (Boston Med. and Surg. Jour., September 6, 1006).

(4) Report of 35 appendicostomies and I 2 cecostomies (New York Med. Jour., August i 5,1908 ).

(5) Surgical Treatment of Diarrhea, with Description of a New Cecostomy which Permits Free Irrigation of the Small and Large Intestines (New York Med. Record, September II, I909).

(6) Some Original Surgical Procedures (which included a description of a new modification of Gant's cecostomy, enterocolonic irrigator, and appendicostomy), an address delivered before the Cleveland, Ohio, Academy of Medicine. November 19, 1900.

(7) Cecostomy and Appendicostomy in the Treatment of Intestinal Affections, with a Report of ${ }^{0} 5$ Cases of Bowel Diseases Treated by the Direct Method (Interstate Med. Jour., vol. xvii, No. 9, 1910).

(8) Constipation and Obstipation (Gant, IgIO).

(9) Analytical and Statistical Study of Bowel Diseases in Infancy and Childhood, etc. (American Medicine, June, IgII).

(10) Intestinal Tuberculosis (The Post-Graduate, June 13, 1913).

(II) Surgical Myxorrhea Coli-M. Membranacea and MI. Colica (Surg., Gyn., and Obstet., December, 1914).

(1 2) Modernized Proctology (N. Y. Med. Jour., January 16, 1915).

General Remarks.-Appendicostomy and cecostomy are called for more often than other operations in the treatment of chronic diarrhea, and are generally practical where the mucosa is extensively involved by a catarrhal. tubercular, entamebic, bacillary, balantidic, helminthic, luetic, membranous, gonorrheal or mixed infection colitis, ptomain-poisoning, chronic intestinal obstruction, colonic polypi, papillomata, and other bowel affections complicated by an inflamed or ulcerated mucosa, profuse discharge of pus, blood, and mucus, or intestinal hemorrhage.

Some idea may be formed of the wide usefulness of appendicostomy and cecostomy by studying the following list of diseases and symptoms, in the treatment of which they have, with varying frequency, been employed-viz., catarrhal and specific inflammatory and ulcerative lesions of the colon, sigmoid and rectum, intestinal cancers, stric- 
tures and tumors, chronic intestinal obstruction incident to angulations, taists, ptosis, extra-intestinal pressure, iniagination, etc.. constipation with recurring coprostasis, intestinal auto-intoxication, myorrhea membranacea and colica, ordinary and pernicious anemia, ptomainpoisoning, helminths, and other intestinal parasites, pain and colic from bowel disturbances, pneumococcic colitis, typhoid fecer, paralytic ileus, septic and tubercular peritonitis, dizerticulitis, simple and membranous pericolitis, epilepsy (complicated by intentinal auto-intoxication), leukocytosis, malnutrition (to facilitate artificial feerlingl, acute and recurring gas distention, defective intestinal flora, ileocecal and colonic intussusception, hemorrhagic colitis, tympanites of lobar pneumonia. appendicitis (where the appendix is infected), gastric ulcer, cancer. and stricture, etc. (where artificial feeding and rest of the organ were indicated), shock (to increase the circulating fluid by the introduction of a saline solution), deficient peristalsis (to augment muscular activity). raise or lower the temperature (by the introduction of hot and cold solutions into the bowel), intestinal fermentation and putrefaction that putrefying food remmants. bacteria, and toxins may be washed sut), general debility (to permit colonic axygen baths, which exhilarate the patient, improve the blood, and forestall collapse during extensive operations).

Appendicostomy and cecostomy have also been emploved as substitutes for gastrostomy. jejunostomy, colostomy. Lane's colonic resection for colonic stasis following resection. intestinal exclusion and fixation of the bowel to the abdominal parietes to prevent tension upon the sutures incident to gas distention, to provide drainage and forestall the backing of discharges and feces when the bowel has been short-circuited, and as a preliminary procedure to resection for intestinal cancer, stricture, and ulceration; and Ver Hoogan. following total cystectomy' (2 cases) ant transplantation of both ureters in to the cecum after its exclusion, performed appendicostomy to insure drainage of the urine, but the patients died within a few hours.

Hollis, Ditmar, Burch, and the author have successfully treated cases of pernicions anemia with appendicostomy or cecostomy and through-and-through irrigation, and it is likely that the benefit derived was due to the flushing out of intestinal toxins elaborated by the anaërobic bacteria peculiar to the colon, for Herter has demonstrated that oxygen is confined to the small bowel.

The author has operated upon many patients suffering from intestinal auto-intoxication, ulceration, or chronic obstruction where anemia was a serious complication, and in every instance the condition was markedly improved by bowel irrigation and drainage subsequent to appendicostomy or cecostomy. In 2 cases he has relieved patients afflicted with epilepsy from recurrences of the attack for periods varying from eight weeks to six months. He has also convinced himself that predigested and other foods emplosed in artificial feeding are absorbed more quickly and in larger amounts when projected into the colon through an appendiceal or cecal opening than when introdueed 
by way of the rectum, and the same may be said of a saline solution employed in large quantities, or the drop method to relieve peritonitis, shock, etc.

Appendicostomy and cecostomy are beneficial in many ways because through their aid one can reach with hot or cold water, saline, or medicated solutions or oils all parts of the diseased and healthy bowel, and therehy dislodge irritating foreign bodies, food, and feces. wash out bacteria, toxins, and foul discharges, heal ulcers, minimize auto-intoxication, diminish or stimulate peristalsis, arrest colic and pain, soothe the inflamed mucosa, nourish debilitated patients, prevent or relieve gas distention and fecal impaction, increase the circulating media, and rapidly diminish the number of evacuations of indiriduals afflicted with chronic diarrhea.

These procedures are also effective in that they improve the psychic state of the patient, who is impressed by the operation, and believes that at last a plan of treatment has been adopted which will lead to his permanent cure.

Irrigation from below would prove as effective as that practised through an artificial opening in the appendix or cecum were it not for the fact that the solution, or tube through which it is to pass, is usually blocked by the anal sphincter, rectal ialies. O'Beirne's sphincter. or a sharp bend in the bowel at the rectosigmoid juncture. Certainly $x$-ray bismuth photographs (see Fig. I 27) demonstrate clearly that a colon-tube seldom if ever passes through the sigmoid flexure. Much of the benefit derived from direct boivel treatment or through-andthrough irrigation is due to the mechanic action of the fluid cleansing the mucosa of irritating discharges, bacteria, toxins, and feces, but the usefulness of the solution can be enhanced by the addition of antiseptic and stimulating medicaments which should be used in greater amounts at the beginning, and then gradually reduced as the ulcers heal and the evacuations diminish in frequency.

In neglected cases complicated by extensive ulceration. repeated hemorrhages, persistent diarrhea, and distressing auto-intoxication, irrigation should be inmediately employed two or three times daily: Under such circumstances the author begins with a solution of silver nitrate, gr. 30 to the quart (2.0-I000.0), after which the bowel is washed out with a normal saline solution to get rid of any excess of silver. The strength of the solution is diminished daily until the diarrhea is greatly improved, when one of the irrigants mentioned below are substituted for the silver. When the stools are very offensive the odor and irritation incident to them can be eradicated by flushing the colon once or, if need be, twice daily with a 5 per cent. ichthyol or 25 per cent. hydrogen peroxid solution.

In cases of moderate diarrhea, which indicates that the bowel is not highly inflamed or extensirely ulcerated, a cure can usually be accomplished by irrigating the colon daily with a + per cent. boric acid, 2 per cent. ichthyol, 3 per cent. balsam of Peru, 2 per cent. permanganate, or 5 per cent. argyrol solution, or the following com- 
bination, which has universally proved satisfactory in the author's hankls:

B Fl. ext. krameria.

Bicarbonate oi sorla. .

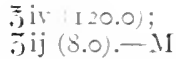

sig.-One or (we tablesponsful to a quart of warm water and irrigate the colon three times weekly.

Salts of quinin (particularly the bisulphate), extensively employed in tropical countries in the irrigating treatment of entamberc and baeillary colitis (dysentery), do not possess specific properties superior to the solutions above mentioned in these or other types of ulcerative colitis, and their beneficial effect is due mainly to the cleansing and healing qualities of the solution. The fuinin should be disolved in dilute sulphuric acid, and enough of the solution added to the water to make a I : Iooo solution, which is employed in the same way ant amounts as other irrigants.

Ilot solutions ( $10^{\circ} \mathrm{F}$.) are soothing. and are indicated when the bowel is sore or irritable, and when the patient complains of pain, cramps, or enterospasm, while cold $\left(65^{\circ} \mathrm{F}\right.$.). except to diminish the temperature, are objectionable, because they cause the intestine to contract and expel the fluid before it has accomplished its purpose, induce cramps, and chill the patient.

Tuttle claims that icc-water irrigation destroys amebre and is a specific for amebic colitis, but the author's experience with ice-water has not been satisfactory in this class of cases. Cold-water irrigation undoubtedly attenuates amebe and relieves the diarrhea, but relapses often occur when cold colonoclysis is suspended. because buried entamebe work to the surface of the nucosa and reinfect it. The author prefers the irrigants already recommended because they are equally if not more effective than ice-water, and because the latter shoch-and chills the patient, causes intense cramps, and is retained but a short time. On the other hand. the warm medicated oils and solution-employed by the author both attenuate or destroy the amebrer other infective agents causing colitis, and through the contained heat wothe the sensitive mucosa and arrest pain and enterospasm. In all form- of ulcerative colitis the strength of the solution and the frequency with which it is employed is regulated by the degree of diarrhea. This class of patients, though apparently cured. should he warned that colitis may recur if they are indiscrete in their manner of living or remain in a community where the infectious forms of diarrhea are endemic. Intestinal stasis from atonic constipation or bowel llocking can be forestalled by appendicostomy or cecostomy and colonedysis, but usually returns when the irrigations are stopped.

Sometimes the bowel is very sensitive and resents the introduction of fluids, in which case, and when cramps and enterospasm are a frequent complication, a few ounces of warm liquid paraffin. mineral, olive, or cotton seed oil should be sulstituted for or alternated with the medicated irrigations. Oil, when introduced into the colon or sig- 
moid from above or below while the patient is in the inverted posture (see Fig. 122), usually remains in the bowel for several hours, particularly when it contains a slight amount of bismuth, because of its soothing and healing effect upon the sensitive mucosa.

As a rule, not more than a quart of the irrigating solution is required, but in aggravated cases, when the bowel is filled with discharges, irritating feces, and débris, it is advisable to employ a halfgallon or more for the first few treatments or until a smaller amount thoroughly cleanses the colon.

Sometimes patients fail to recover following appendicostomy and cecostomy, owing to the fact that the irrigations are always carried out while the patient is in the same posture. The subject should be requested to frequently change his position during the flushings, so that all parts of the diseased mucosa may be reached, otherwise unhealthy areas will remain untouched and relapses will follow.

When through-and-through irrigation has been carefully practised for weeks, and does not completely arrest the diarrhea (which indicates healing of the inflamed and ulcerated mucosa), the stomach and other organs should be examined to see if there is not some other complicating disease or an intestinal obstruction which is interfering with the treatment.

Closure of the Opening.- The appendiceal or cecal vent should not be closed in moderate cases of ulcerative colitis under three months, and in neglected cases (particularly those due to entamebic or tubercular colitis, etc.) it should be left open as a precautionary measure for half a year or longer, even when the patient is apparently cured, because otherwise the ulceration and diarrhea may recur, owing to renewed activity of buried infective agents which are slow in gaining the surface of the mucosa. Individuals who live in tropical conntries where dysenteric diarrhea is endemic are safer when the opening is not closed at all, because they may become infected at any time while there.

Usually an appendiceal opening spontaneously closes following withdrawal of the catheter or the author's appendiceal irrigator and stopping of the irrigation, but when it does not, cauterization of its mucous lining with a silver or copper stick, or an electric or Paquelin cautery tip, facilitates the process. On seven occasions, when these procedures failed, the tissues were infiltrated with a $\frac{1}{8}$ per cent. eucain solution, the appendix was dissected free down to its cecal attachment, ligated, excised, and the wound closed with catgut without entering the peritoneal cavity.

Cecostomy openings are always difficult to close when the cecum has been anchored to the skin, as in Gibson's operation (see Fig. I 54), but when the bowel has been attached to the inner abdominal parietes and a rubber tube is left projecting from it through the integument (Figs. I50, I5I), spontaneous closure of the opening quickly follows withdrawal of the catheter because the wound closes down from above. and blocks the aperture in the intestine. When healing is delayed the mucosa lining the fistula should be cauterized or removed. 
Where less radical measures fail, the abdomen should be opened under local or general anesthesia, the cecum freed from its attachments, and the opening in its wall inverted and buried by infolding Lembert sutures if it is large, or purse-string sutures if it is small. There is less

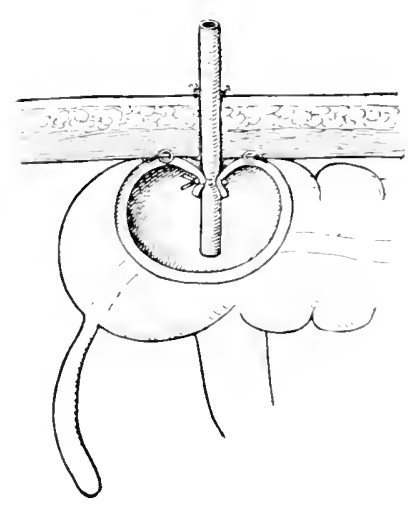

Fig. I50.- Kight way of performing cecostomy. Note that the catheter is carried through the abdominal wall to the cecum and anchored to the inner parietes. After this operation closure of the opening generally follows removal of the catheter and cauterization.

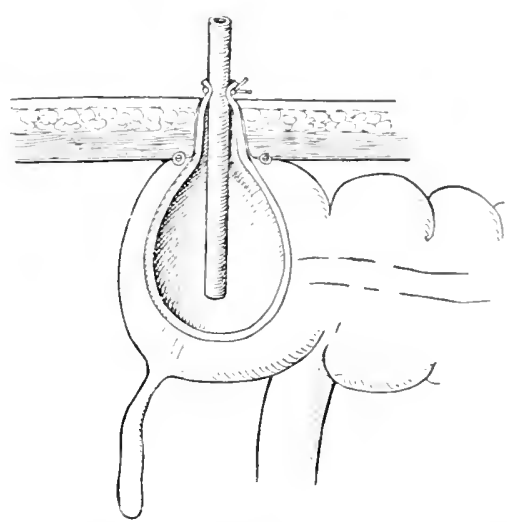

Fin. 15t.-Wrong way of pertorming cecostomy: Note that the cerum is attached to the skin. Closure of the opening after this procedure requires a second and perhaps serious abdominal operation.

danger of leakage and peritonitis after the operation when the cecum is attached to the parietal peritonem before the wound is closed. The author does not know of an instance when the appendiceal or cecal opening has failed to close when handled in the above manner.

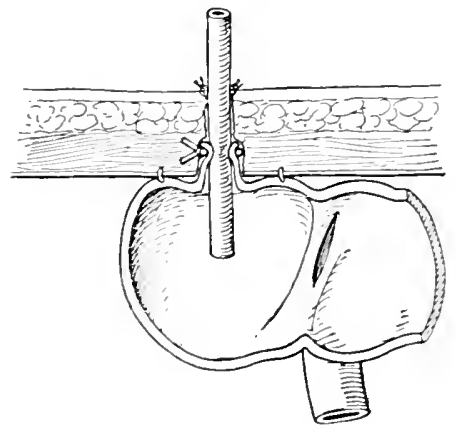

Fig. 152.-Appendicocecostomy showing catheter introduced through the appendiceal stump.

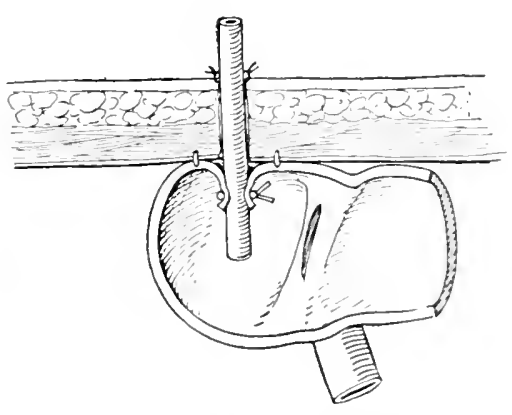

Fir. I 53.- Appendicocecostomy showing catheter introduced through the inverted appendiceal stump.

Appendicocecostomy.- This name is emplosed to indicite an operation wherein a catheter is introduced through the appendiceal stump into the cecum to facilitate throtgh-and-through irrigation, and in cases where appendicostomy proves impractical because the appen- 
dix is diseased, strictured, too short, or otherwise unfit for the purpose. Both appendicostomy and cecostomy should take precedence over appendicocecostomy, which is seldom performed. A few surgeons favor the procedure because it provides a means of direct bowel treatment, and at the same time eliminates the appendix, which might cause future trouble (Figs. 152, 153).

Enterostomy is rarely employed in the treatment of inflammatory, ulcerative, and parasitic affections of the alimentary tract because these types of disease are usually located in the colon, fluid feces almost constantly dribble through the opening to irritate the skin, and a serious operation is required after a cure has been accomplished to close the artificial opening.

Colostomy was occasionally resorted to in the surgical treatment of diarrhea and colitis before the advent of appendicostomy and cecostomy, but since the operation has become obsolete because it is not so effective. Patients strongly object to having bowel movements through their side, and a prolonged and dangerous operation is required to re-establish continuity of the intestine when the treatment has been successful.

Results of Through-and-through Irrigating Treatment Following Appendicostomy and Cecostomy.-The author has treated 200 cases of diarrhea induced by chronic catarrhal, tubercular, bacillary, entamebic, balantidic, syphilitic, helminthic and gonorrheal colitis, intestinal obstruction, or other bowel affections by irrigation subsequent to appendicostomy, cecostomy, appendicocecostomy, appendico-enterocecostomy, and colostomy, alone or in conjunction with other operations, and nearly all patients were improved.

The vast majority were permanently cured, a considerable number had one or more relapses after remaining well for weeks, months, or years, and a few were benefited only for a few days or weeks, and then relapsed into their former deplorable state.

In the most unfarorable cases direct bowel treatment usually improves the manifestations of auto-intoxication, arrests bleeding, diminishes the amount of discharges, increases the number of red bloodcells, deodorizes the stools, decreases the formation of gas, lessens the number of evacuations, and allays cramp, enterospasm, tenesmus, and burning in the rectum, but for one reason or another fails to effect a cure. Perfect results are not to be expected in tubercular colitis complicated by phthisis, dysenteric ulceration, where the mucosa has been extensively destroyed, the patient has a liver abscess or general infection, in stricture, cancer, polyposis, or papillonata of the bowel (unless the stricture or growth has been corrected or removed), when the local is complicated by general or disease in other organs, and when patients indulge freely in alcoholics, dictary incliscretions, violent exercise, worry over business affairs, or fail to carry out the irrigations and treatment as directed.

Convalescence is more rapid when this class of sufferers are placed amid pleasint surroundings, are permitted to eat freely of nourishing 
food which agrees with them, and indulge in moderate exercise except during crises), when general or nerve tonic which lo not up-et the stomatch or intestine are administered to upbuild their general health, and when potassium and mercury are added to the treatment where the diarrhea is secondary to luetic colitis. A complete cure seldom accurs where the bowel is partially blocked hy ptosis, in agination, adhe-ions, a twist or angulation, or extra-intestinal presure, unles the lesion is corrected or extirpated at the time the appendix or cecum in speneel for drainage and irrigation.

Sometimes myxorhea membranacea and colica respond prompty to this method of treatment, and again do not. possibly because the condition is dominated by a nerrous element or a gastrogenic or enterogenic dyspepsia.

Curl, who employed appendicostomy in 22 Isthnian laborers afflicted with entamebic colitis, says that the immediate result. were goud and that a number were permanently cured (others he could not trace). Pettyjohn's 3 patients with amebiasis were dicharged cured and without amele in the stools a few weeks after appendicostomy. In his report on the operation in this form of dyentery he holds that deaths following the operation are not due to $i t$. but is ravagen of the risease. Tuttle, the author. and others have recorded a large number of cases of amebiasis cured by appendicostomy or cecontomy and irrigation, and death has seldom resulted from surgical intervention.

The mortality following appendicostomy and cecostomy is about the same as for the interval operation for appendicitis or colostomy. The actual mortality of the operations of appendicostomy and cecostomy in the author's 200 cases has been 2 per cent. he having bst 2 patients from each of these procedures.

There were 8 or 10 death shortly following the operation. but in some death was due to the disease from which the patient suffered or concurrent ailments: in others to resection. intestinal exclusion. colopexy, etc., where drainage was provided by way of the appendix or cecum, and in still others to the almost moribund -tate of the sufferer at the time of operation.

One patient, upon whom appendicostomy and sigmoldopexy were performed, died from peritonitis caused by an assistant who mi-tosk an aperture at the side for the appendiceal spening and injected the irrigating fluid directly into the peritoneal castity. A seconel died a $=$ a result of sloughing of the cecum about the appendiceal attachment. in conseguence of heary tension upon the appenclix causer hy a fecal impaction and gas retention in the caput coli. ()ne cecostomy terminated fatally from peritonitis caused by the escape of liquid and pus into the abdomen during the operation, and a second pattient. upun whom cecostomy was performerl. died ats a result of a large -lougl in the anterior cecal wall abrout the catheter. apparently catused hy four infolding purse-strings sutures which hat been pliced tox far apart. 
Thus far the author has not had a cleath from his cecostomy, and does not see why the mortality from it should be higher than that of ordinary cecostomy.

The most common complications of appendicostomy are stitchabscesses, sloughing of the organ, tendency of the appendiceal opening to close, retraction of the stump beneath the skin, tension pain when the cecum is distended, ulceration of the mucosa, and discomfort incident to the retained catheter. In one instance a hernia occurred at the side of the appendix, and in another case, where, after a cure, the appendiceal stump was left in situ, the patient had an attack of appendicitis which was relieved by ordinary appendectomy.

Following cecostomy slight infection of the suture line is quite common, owing to soiling of the wound by the feces cluring or subsequent to the operation, but, except in rare instances, the breaking down is confined to the skin, though on two occasions all structures separated down to the transversalis fascia, and two months were required to heal the wound.

Frequently the integument becomes irritated or granulations form around the opening, conditions quickly relieved by a 6 per cent. silver nitrate application.

Occasionally catheters leading to the cecum get soiled or hard, cause considerable discomfort, and require changing.

Much of the annoyance incident to the escape of the fecal discharge can be avoided by clamping the catheter with a cravat clamp and firmly fixing the tube with narrow adhesive strips, which, after being made to surround it, are attached on all sides to the skin.

When the appendiceal or cecal opening tends to remain patent the catheter may be left out on alternate days or all the time, but in either case, when the aperture is inclined to close, it should be dilated with graduated catheters or forceps.

Technic of Cecostomy.-In the original direct or through-andthrough irrigating method of treating catarrhal and infectious colitis causing diarrhea an artificial anus was established - a procedure which conld rightly be called cecostomy. In the light of our present knowledge, however, the operation should be designated as colostomy, since the evacuations take place through the side and the bowel is put at rest, but at present the term "cecostomy" is reserved for operations where a small controllable opening is made in the cecum through which the colon can be irrigated at will-a procedure which does not possess the disgusting features of an artificial anus.

Gibson in $190 \mathrm{I}^{1}$ described his zaluular cecostomy (a great improvement over right-sided colostomy), which has proved useful in the treatment of inflammatory and ulcerative lesions of the colon, sigmoid flexure, and rectum. Briefly described, the steps of the operation are ats follows:

First Step.-The cecum is exposed by a short intermuscular incision. Second Step.-An opening is made in the anterior surface of the 
cecum through the longitudinal band and a medium-ized catheter is inserted.

Third Step.-Two or three tiers of infolding sutures are then introfluced at the sides of the eatheter and tied. which cause- he ceral wall to project inward and form a valve which partially present-subsecpuent leakage (Fig. I5t).

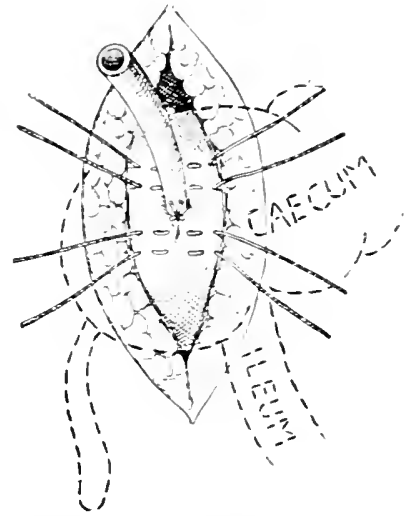

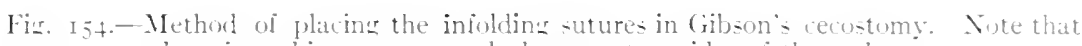
there is nothing to prevent leakate on two -ides of the catheter.

Fourth Step.-The outer sutures, having leeen left lons, are carried through the abtominal wall to close it and to fix the cecum to the inner parietes.

The catheter is left in for a few dass: then it is remosed and reintroduced if further irrigation is required. The operation is fairly successful, the main objection being the leakage which takes place in some cases. 


\section{CHAPTER LI}

\section{SURGICAL TREATMENT OF DIARRHEAL, INFLAMMATORY, OBSTRUCTIVE, AND PARASITIC DISEASES OF THE GASTRO-INTESTINAL TRACT (Continued)}

\section{TECHNIC OF GANT'S ENTEROCECOSTOMY (CECOSTOMY), APPENDI- COSTOMY, APPENDICOCECOSTOMY, AND APPENDICO-ENTERO- CECOSTOMY}

Gant's Cecostomy with an Arrangement for Irrigating the Small Intestine and Colon.- The author will now describe an original cecostomy $y^{1}$ (Fig. I55) which provides a means of separately or simultaneously flushing the small and large intestine, a procedure to which, for want of a better name, he has given the above caption. He at first termed the operation ceco-enterostomy, but this designation proved unsatisfactory because it convered the idea of an artificial opening established by anastomosing the cecum with the small bowel, when, in reality, the communication between the caput coli and ileum is by way of Bauhin's (ileocecal) valve. Taliular ceco-enterostomy and ileocecoenterostomy. also considered, were finally abandoned as confusing in faror of the appellation cecostomy with an arrangement for irrigating the small intestine and colon. which gives one a fair idea of the nature of the operation and what it is intended to accomplish.

This procedure was worked out with the object of providing a method of flushing the colon and small intestine, the author having observed that appendicostomy and ordinary cecostomy, reinforced by irrigation, failed to cure many cases of chronic diarrhea induced by catarrhal and the various types of infectious colitis. The reason for this was that the colon and small bowel were involved to a greater or less extent, and in consequence the solution, when introduced through an artificial opening in the appendis or cecum, failed to reach the diseased mucosa above the ileocecal valve. That such a condition existed was proved by the fact that in many cases a cure followed the author's cecostomy and bowel flushing after appendicostomy or cecostomy and irrigation had been tried without success.

Tubereulosis, intestinal syphilis, entamebic, bacillary, and balantidic colitis are usually confined to the colon, but now and then attack the ileocecal valve and lower ileum, as shown by some of the author's specimens. Inder these circumstances reinfection of the colon after a cure will surely occur unless all diseased areas in the mucosa are

${ }^{1}$ The author first described his " Cecostomy" in a paper read before the Merlical Association of Creater New York, which was published in the New York Med. Jour., August I5, 1008; but the operation harl been performed several times at the Post-Graduate Hospital and in his private sanitarium one, two, and three years before. 
treated and the infecting agents (tubercle bacilli, entitmebre, Batantidium coli, Shisa bacilli, etc.) are destroged. This can only be ace complished through the author's proxedure outlined bedow.

The following briefly described steps of the author's cecostomy can be rearlily grasped by a glance at the aceompanying illustrations.

First Step.-Through a 2-inch intermuscular (or rectus) inci-ion. mate directly oxer the cecum, it and the lowermost part of the iletum

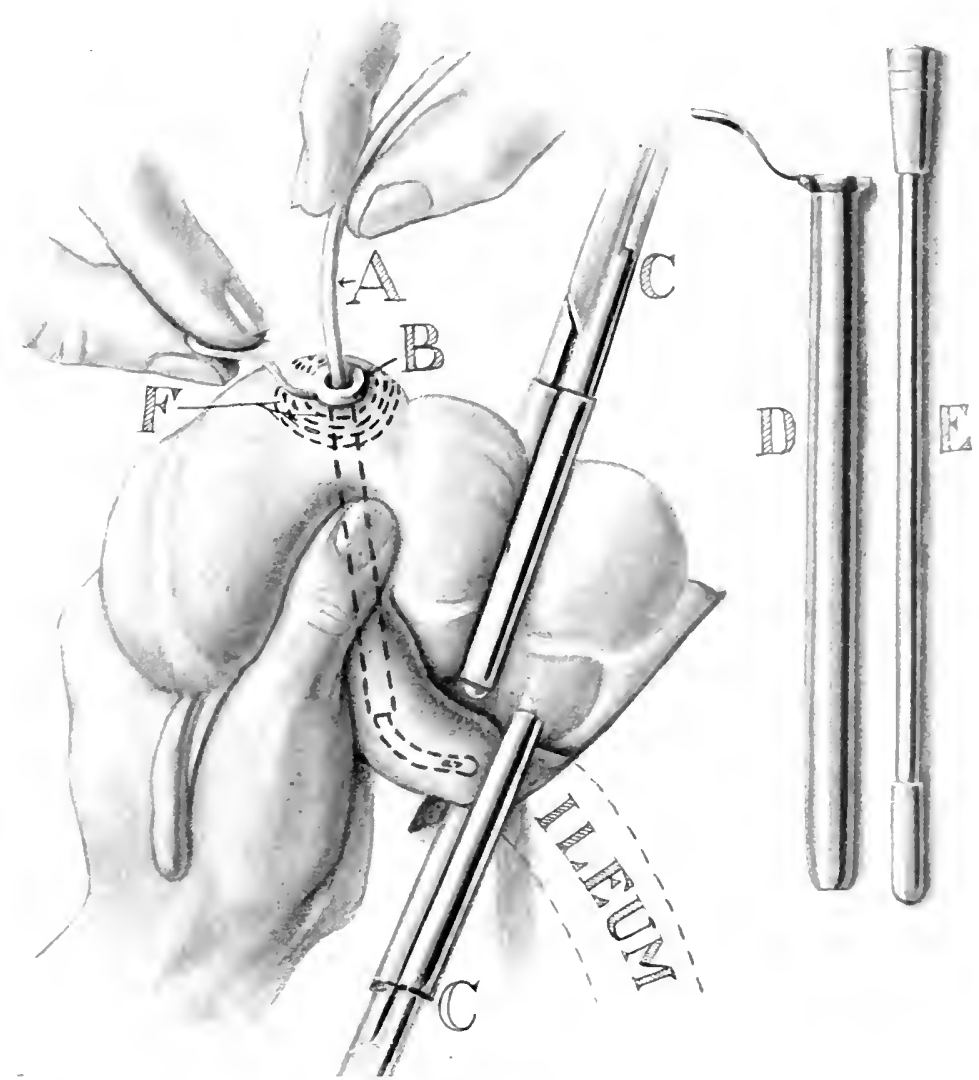

Fig. 155.-First steps in Gant's older cecostomy with an arrangement for irrigating the small intestine: 1. Citheter being introduced through the coum into the -mall lowel: B. atheter guirle in situ through which it passes: ('. C. Intertinal clamps: 1), sirte view of catheter guide: E. obturator from the same; F, purse-string sutures.

are withdrawn and the edges of the wound protected by gatuze handkerchiefs.

Second Step.- The anterior surface of the cecum is scarifierl, after the ascending colon and ileum have been clamped, to prevent soiling of the wound when the lowel is opened (Fig. I55. C).

Third Step.-Two or more linen soromuscular purse-string sutures are placed in the anterior wall of the cecum opposite the ileocecal value (Fig. I55, F) and the bowel is opened inside the suture line. 
Fourth Step.-The gut is grasped at the juncture of the large and small intestine and held in such a way that the ileocecal valve rests between the thumb and fingers of the left hand (Fig. 155). A Gant

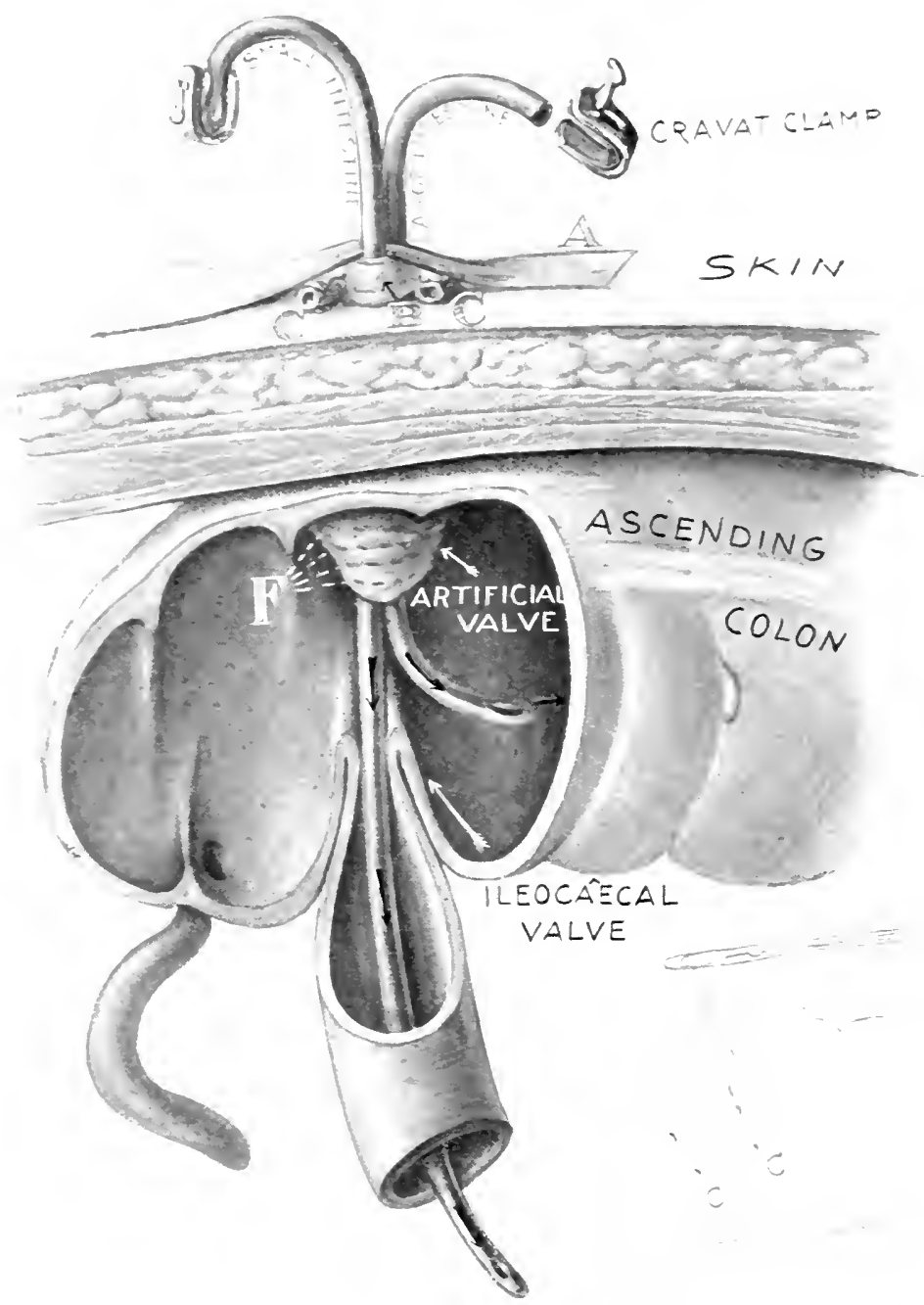

Fir. 156.-Gant = older cecostomy, showing atheters in position through which the small and large intestine can be irrigated ingly or simultaneously. A. A and B. Adhesive -trips which retain the catheters in pusition: $\dot{C}$. C. rubber tubings across which the suspentiry sutures are tied: F. Circular walve formed about the catheters by the infolding purectring sutures. The drawing in lower right-hand corner shows location of cecal wenine and method of introducine the suspensory sutures $(\mathrm{C}$. C).

catheter guide (Fig. I55. B, D, E) is then passed directly across the cecum and through the ileocecal valve into the small intestine, aided in the thumb and fingers. 
Fifth Step.- The obturator is removed from the guide and a catheter is introduced into the small bowel (Fig. I5.5. A) and held there by an assistant until it is anchored to the cecum hy catgut sutures to present its slipping out hefore the operation is funisherl.

Sixth Step.-A short rubber tube 3 inches long is projected into the cecum for an inch or more and anchored beside the one projecting into the small gut (Fig. I56).

Seventh Step.-The infolding purse-string sutures are tied, forming a cone-shaped valve (lig. $156, \mathrm{~F}$ ) about the catheters, to prevent leakage of gas and feces.

Eighth Step.-After removal of the clamps the scarified cecum is anchored to the transversalis fascia, denuded of its peritonem by twe linen suspension sutures, which are passed through the abdominal wall.

Vinth Step.-The suspension sutures are lied across rubber tubing (Fig. 156, C), the wound closecl by the layer method, and the catheters fastened by stitching or by encircling them with an adhesive strip (Fig. I56, B) to hold them together. This is crossed at a right angle with a second piece of plaster placed between the catheters (Fig. 156, A) to prevent their slipping out.

Tenth Step.- The ends of the catheters are closed with cravat clamps (Fig. 156) to prevent leakage, and the operation is completed by applying the dressings about the projecting tubes.

One catheter is left longer than the other, or is identified

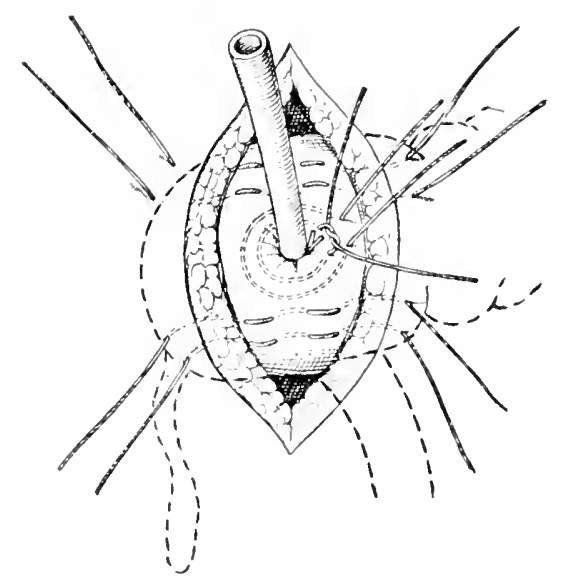

Fig. I $57 .-$ Method of introducing the circular infolding and suspensory sutures in the author's cecostomy. Here a circulatr valve is formed about the catheter which prevents leakage on all sides. in some way, that the intern or nurse may know which is in the large and which in the small intestine when time for irrigation arrives. To avoid danger from infection treatment is not begun before the fifth day, except when diarrhea. hemorrhages, or mixed infection manifestations are alarming.

The catheters may be quickly changed by cutting the attached adhesive strips and withdrawing the one projecting into the cecum. The catheter guide is then passed over the other into the small intestine, where it is retained until the old tube has been removed and a new one introduced. A second piece of catheter is then placed in the cerm and both are prevented from slipping out by adjusting fresh adhesive straps after the manner already described, and replacing the crivit clamps and by the methods shown in the (rawings (Figs. I,56, 1.57).

Before deciding upon the above techuis the writer irrigated the small intestine by passing a glass or silver catheter through the ceal 
opening and into the small gut for each irrigation. Thi=practice was abandoned as impractical, however, because of the difficulty encountered in locating and passing the valve each time, and. further, because the patient could not irrigate himself.

The writer has had no reason to suspect that peristalsis has forced the catheter out of the small intestine except in his first cecostomy, where the tube was soft. cut short, and projected only one inch instead of several inches beyond the ileocecal valie. He feels confident that the catheter remained in the small gut in his other cases because: $(a)$ water was more quickly eracuated when injected through the colonic pipe than when deposited in the small bowel; (b) when a minute quantity of a Io per cent. solution of methylene-blue was injected into the former it appeared in the urine cooner than when introduced into the small gut: (c) the catheter guide could be carried over the tube in the

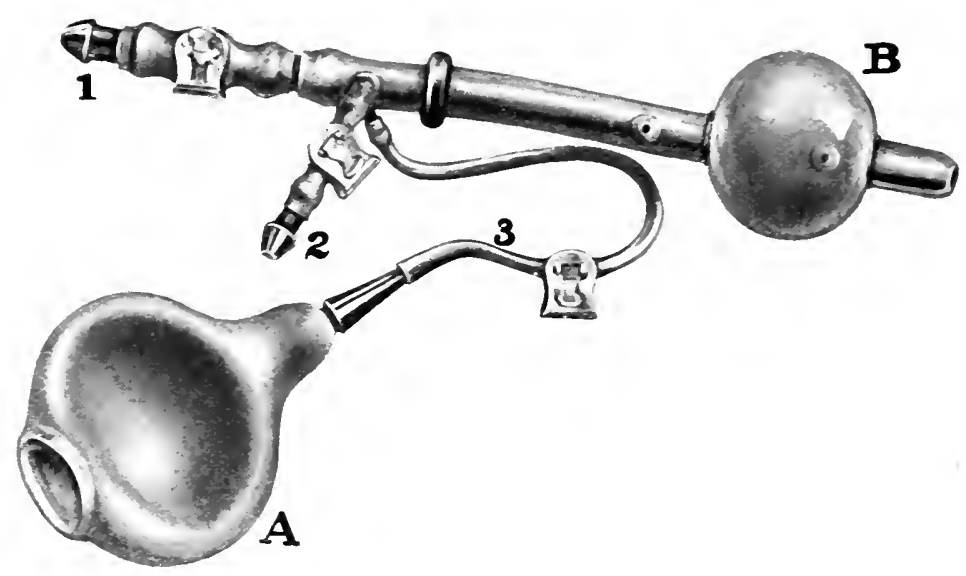

Fig. I 5.-Author's rubber enterocolonic irricator: 1. Bulb used to distend the intating bag: $B$, inflating bag: 1 . tube throurh which the irrigating tuid passes to the small intestine; 2. tube connecting with the cecal opening of the colonic irricator: 3 . inflating tube. The movable hard-rubber ring is used to measure the distance to the ileocecal valve. which varies in different individuals. The variou pipes are closed by cravat clamps.

small intestine, and the latter could be removed and replaced at will; and. further, (d f fuid feces could be withdrawn more often through the pipe in the small intestine than through the colonic catheter.

To aroid posible expulsion of the catheter from the ileum the writer, on different occasions. successfully employed catheters made of silk, silver. glasi, and soft rubber reinforced by an inner tubing of metal or hard rubber, which, owing to their non-flexibility, could not be forced out of the bowel. Only that portion of the pipe projecting into the small bowel was reinforced, as the rubher catheter induced but little irritation hecause of its softness and flexibility and served the desired purpose. 
To facilitate the operation, dispense with soft catheters, and insure the irrigating solution entering and remaining ats long as desired in the small bowel, the atulhor devised an enterocolonic irrigator (Fig. I5s), which he exhibited before the Cleveland Academy of Medicine, November 19, 1909, and the Medical and Chirurgical Factily of Maryland

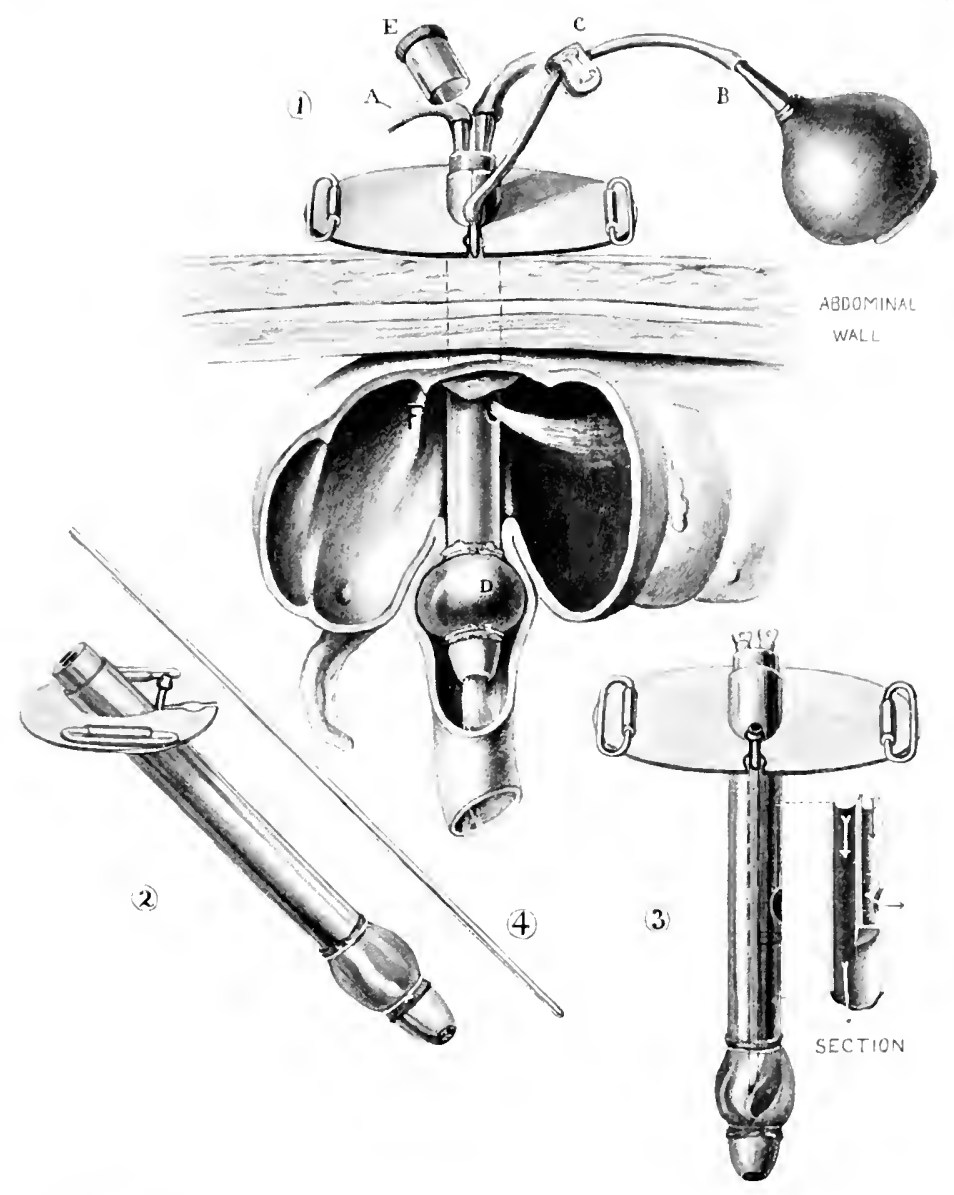

Fig. 159.- Steps showing tiant's newer cecostomy, where the metal enterocolonic irrigator is employed, which provides for irrigat tion of both the small and large intestines: 1, Irrigator in position: 1 , irrigating tubes; $B$, inflating attachment; $C$, cravat clamp for closing same; D, inflating bag distended with air to prevent return of the solution into the colon; $\mathrm{E}$, cover for irrigator: $F$, circular wate formed above the irrigator by purse-string sutures to prevent leakige; '2, side view of Giant's metal enterocolonic irrigator; 3, front and sectional view of same; 1 , rellulovid guide employed in removing and reintroducing the irrigator for cleansing and repairing purposes.

at Baltimore, April 27. 19I0.' The irrigator, which has been suecessfully employed in a considerable number of cases in the treatment of inflammatory and ulcerative lesions of the small intestine and colon,

${ }^{1}$ Interstate Med. Jour., vol. xiji, No. igro. 
or both, is of convenient size, made of hard rubber or metal (Figs. I58, I59), and self-explanatory when studied in connection with the accompanying illustrations.

When the irrigator is in position the inflating bulb (Fig. I59, B) lies in the small intestine at or near the ileocecal valve (Fig. I59, D). When distended it fills the bowel and prevents escape of the solution into the cecum, thereby enabling the attendant accurately to gauge the amount of fluid deposited in the small bowel and to retain it there as long as required. By means of this twin-tube irrigator the small and large intestines can be quickly and scientifically flushed, singly or together, by the physician, nurse, or patient.

The steps in the writer's cecostomy when the irrigator is employed are similar to those already described when catheters are used (see Figs. I 55, I56), except that the Gant catheter guide is unnecessary and the apparatus is retained in position by attached pieces of tape which encircle the body, or by adhesive strips.

Indications for the Author's Cecostomy.-- In a paper read before the Medical Association of Greater New York, April 30, 1908, and since, the author has called attention to the fact that his cecostomy is indicated in the treatment of intestinal parasites, enteritis, enterocolitis, peritonitis, paralytic ileus, intussusception, catarrhal, tuberculous, syphilitic, dysenteric, and gonorrheal colitis, ordinary and pernicious anemia; in the many manifestations dependent upon intestinal auto-intoxication, ptomain-poisoning, diarrhea of aclults and children, intestinal feeding, malnutrition, and following operations upon the mouth, throat, esophagus or stomach, in gastric stricture, ulcer, cancer, and other disturbances where rest of the organ and artificial feeding is required. Again, he has claimed that by means of his cecostomy various intestinal diseases could be investigated to determine the amount and nature of the intestinal juices and discharges, the character of the feces, the action of salines and other cathartics injected directly into the small and large bowels, the marked immediate vasomotor effect following hot and cold enteroclysis, the introduction of bacteria for therapeutic purposes, the injection of a bismuth solution into the intestine for $x$-ray diagnosis, and in the study of many other interesting problems.

In closing the discussion of the above-mentioned paper the author reported several cases successfully treated by his operation, and stated that, while he had not had experience with it in the treatment of cholera and typhoid fever, he believed it was inclicated, and in future would be used in the treatment of these and nearly if not all other non-obstructing diseases of the small and large bowels complicated by diarrhea, hemorrhages, or toxemia.

In his work on "Constipation and Intestinal Obstruction," published in January, I909, the author pointed out the advantage of cecostomy as a means of drainage when the cecum or another part of the colon was excluded. He has also on several oceasions employed his cecostomy when operating for mechanic constipation alone or 
when complicated by colitis, as well as in the palliative treatment of obstipation where the patient declined to have the obstruetion corrected or removed, yet sulfered from deplorable auto-intoxication or recurring impaction.

The author believes his cecostomy to le superior to that of (iilson because, while expally simple in technic, the operation rerpuires no more time, there is less leakage owing to the purse-string infolding leding substituted for his lateral valuukar sutures, both the small and large bowel can be irrigated by the attendant or pationt separately or at the same time, a firmer union is obtained by attaching the corm to the transversalis fascia than to the parietal peritonemm, and the opening heals spontaneously after the catheters are removed.

Comments.- Another methoxl often employed by the writer to prevent leakage in ordinary cecostomy is to bury about an inch of the eatheter in the cecal wall by whipping the gut over it from the opening outward (Fig. I60) with a continuous or interrupted Lembert suture.

To diminish the danger of leakage, peritonitis, and sloughing the opening about the tube, catheter. or irrigator should be made as small as possible, and unnecessary stitching in the cecum should be aroided.

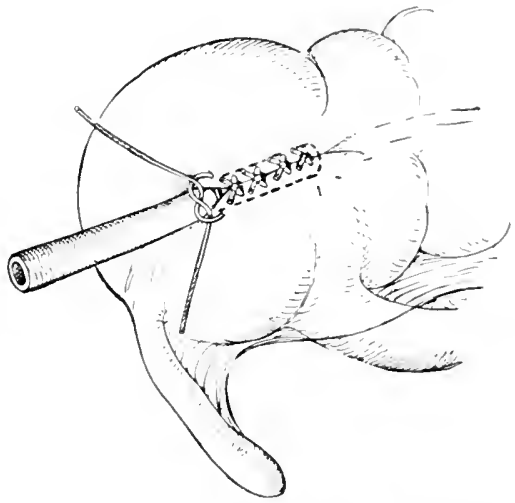

Fig. I60.-Author's method of lurying the catheter in ordinary cecostomy io minimize leakage. The tube can also be placed at a right angle to the cecum.

In a case of the author's a segment of the cecum was caught in the wound when closed, and in conseguence the patient suffered terribly from nausea and romiting until the abdomen was opened and it was releaserl.

During a cecostomy operation the catheter or irrigattor, following its introduction into the cecum or small intestine, should te helel firmly in place until anchored, for in one of the author's cases a carcelest assistant withdrew the ube as the wound was being chesed. necessitating a repetition of the operation.

Soling of the wound can be prevented in a karge measure by damping the ileum and cecum, and not opening the latter motil after the suspension and purse-string sutures have been intrelued. One ran reatily incise the intestinal musculature whth a knife, hut the mucosit recedes upon pressure, and to avoid delay and prevent exersion of the mucous membrane later on the author seizes it with menth-tentherl forceps and removes a small section of it with scissors.

The cecum should never be anchored wh the skin, as in Cibson': operation (see fig. I.5t), because uleeration ensues alout the opening, the feces escape to a greater or less degree, and at serious platstic operat- 
tion is later required to free the bowel and close the opening when the patient recovers. On the other hand, when the caput coli is fixed to the inner abdominal parietes, the fistulous tract leading down to it can be made to close readily by cauterizing its mucosa with silver or a cautery point.

Subsequent to appendicostomy and cecostomy leakage from the catheters can be prevented by snapping a cravat clamp over their ends or sides (Fig. I6I), and the annoyance from their slipping out can be aroided by taking a turn around then with a narrow adhesive strip) and attaching the two ends of the plaster to the skin, then repeating the process, and adjusting the strip so that the ends are fastened to the skin on the other side of the catheter (Fig. I62), and ly passing a safety-pin through the catherer and fastening it to the skin with plaster (Fig. 16.3).

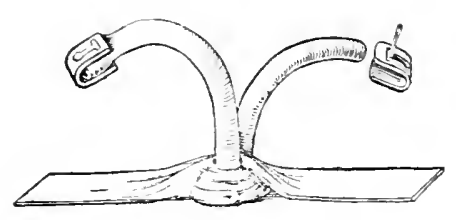

Fin. sor - Method of closing and retaining catheter in place with cravat clamps and adhesive strips following Gant s cecostomy.

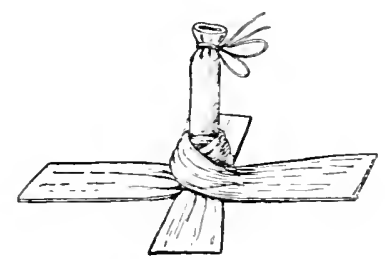

Fig. I62.- Method of closing and retaining catheters in place with a ligature and adhesive strips. (Gant's technic).

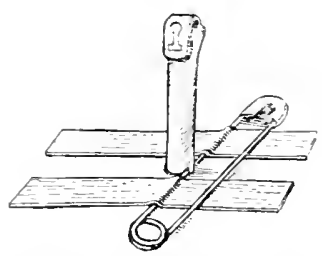

Fig. 163.- Method of closing and retaining catheters in place with safety-pin and adhe-ive strips. (Gant's technic.)

Experience has demonstrated to the author's satisfaction that in the direct treatment of intestinal diseases cecostomy is superior to appendicostomy, and at the June meeting (r909) of the American Proctologic Society he took the position that, with but few exceptions. it should be given the preference. A comparative study of the adiantages of cecostomy and the disadiantages of appendicostomy. as enumerated below. will show why the former should take precedence orer the latter.

Advantages of Cecostomy.-The advantages of this operation. and more especially the author's cecostomy, which provides a means of irrigating both the large and small intestines, are as follows:

(I) Owing to the fact that the cecum lis against the inner abdominal parietes it can be easily anchored without angulating or lwisting the bowel. 
(2) Since the opening is opposite the ileocecal valve a catheter can be introduced into the small bowel for irrigating purfosec or the siphoning of its contents for examination.

(3) The cecal opening can be mate of suitable size.

(4) The circular valve-like projection, formed around the catheter by the infolding purse-string sutures, prevents leakage.

(5) The catheter can be changed without differulty.

(6) Closure of the opening follows withdrawal of the catheter and a few applications of the copper stick or cautery.

(7) Owing to the natural position of the cecum lens tension and pain follows its anchorage to the abelomen than the appendix.

(8) Cecostomy (Cant's) nay be employed in the treatment of lesions located answhere in the intestinal canal, while appendicostomy is limited to those of the colon.

(9) There is no danger of an impaired circulation and scushing.

(10) Therapeutic bacteria and nutriment can be projected clirectly into small intestine or colon following author's enterocecontomy.

Disadvantages of Appendicostomy.- (1) It is more difficult to liring up the appendix than the cecum for anchorage because of its deeper and more uncertain position, and it is frequently bound down hy athesions or a short mesentery.

(2) Anchoring of the appendix causes angulation or twi-tine of the cecum, which, in turn. may induce constipation, discomfort, or painil.

(3) When the recum about the appendiceal base is caught in the wound it incluces nansea and romiting until detached (author"s cante).

(4) When the appendix is small, short. strictured, bound down hy adhesions. blockerl, or otherwise diseased it is useless for irrigating purposes.

(5) Because of the small appendiceal sutlet irrigation is frecpuently difficult and unsatisfactory.

(6) Pain is much greater following appendicostomy than cecostoms owing to the pulling upon the appendix by the loaded cecum. periappendiceal adhesions, or squeezing of the attached mesentery when the wound is closed tightly alout it.

(7) Frepuent dilation or the insertion of a catheter is nectenary 10 keep the appendiceal opening sufficiently large.

(s) Death has followed injection of the irrigating fluid inn the abdomen beside the appendix. where an intern mistoek an winning in the wound for that of the appendix (author's case).

(9) After a cure it is more difficult to chose the apdendiceal than the cecal outlet, and appendectomy may be necesiry.

(Io) Appendicostomy frecpuenty fait berande of the appendix slipping back into the abelemen or retracting sufficiently to malke irrigation almost or quite impositile.

( I ) The appendix has several limes been known to shelgh off owing to tension, its constriction by the sutures, or destruction of its hlendsupply, making excostony imperative. 
(I 2) Appendicostomy is not effective when the disease is located in the small intestine.

(I3) Appendicitis, requiring appendectomy following closure of the appendiceal outlet, has occurred (author's case).

(It) Owing to irritation caused by the catheter or the treatment the appendiceal mucosa may become so inflamed and swollen, ulcerated, or strictured that irrigation must be abandoned.

(I5) According to Reed, the catheter frequently causes the wall of the appendix to perish.

(16) The appendix may be obstructed by a seed, fecalith, or a foreign body.

(17) A short mesentery may prevent bringing of the appendix to the surface for anchorage.

(is) Death has occurred from sloughing of the cecum about the appendiceal attachment on account of tension (author's case).

(19) Prolonged traction upon the meso-appendix has been known to cause frequent and enormous gas distention (author's case).

(20) The appendix may be enormously sacculated and filled with a mucopurulent discharge which renders it unsuitable for appendicostomy.

(2 I) In a case of the author's the organ was larger than the thumb, and cecostomy was substituted for appendicostomy because it was thought that the feces might discharge through the appendix.

(22) In two instances (author's cases) no appendix was found. (In one the deformity was evidently congenital, the cecum at the usual site of the appendiceal attachment being perfectly smooth; in the other an uneven appendiceal stump was presented, appearing as though the appendix might have sloughed at some previous time.)

(23) Strangulation of the appendix at its base may occur from angulation or twisting when tension upon it is great.

(24) A diverticulum at the side of the appendix (author's case) has made appendicostomy unfeasible.

(25) In fat subjects, and where the abdominal muscles are thick, the appendix is often short and cannot be brought out and sutured to the skin.

(26) The appendix may be unsuited because it is congenitally small or deformed.

(27) Sometimes the appendix is located extraperitoneally or is bound down by adhesions, so that it is seriously injured by the dissections and is rendered unfit for irrigating purposes.

(28) Finally, angulation and blocking of the appendix has occurred (author's case) from the stab-wound operation. In this instance the fascia was retracted before the stab-wound was made, and when the wound was closed a sharp bend in the appendix resulted which completely blocked subsequent attempts at irrigation.

Technic of Appendicostomy.--Meir, who originated the operation. opened the appendix, passed a catheter through it into the cecum to see if it was patent, ligated its tip to prevent leakage, and completed 
the operation by suturing it to the skin. Two days later the ligature was removed and the colon was irrigated through the appendix for the first time. Weir failed to anchor the appendiceal base or the cecum to the abdominal parietes, which loft too much tension upon the appendix when the cecum was distended and enough room about it for a hernial protrusion. Dawbarn shortly thereafter modified the procedure by attaching the cecum about the appendiceal base to the parietal peritoneum, and Tuttle (1905) advised at two-stage operation, wherein the appendix was stitched in the wound and later opened for irrigating purposes after healing had taken place, claiming that in this way danger from infection could be minimized. Tuttle also made a practice of ligating the appendiceal artery and stripping the mesentery from the appendix down to the cecum.

Experience has demonstrated to the author's satisfaction that it is not good surgery to stitch an mopened appendix in the wound, since stricture, disease, or blocking by a foreign body may render it useless for irrigating purposes; when infection complicates appendicostomy the trouble is not serious because it is confined to the skin sutures.

Neither does the writer believe in ligating the artery which parallels the appendix (see Fig. I68), having known the organ to slough olf in consequence; he makes a practice of suturing the appendix with its undisturbed mesentery into the wound (see Fig. I67). The author will now give his technic in appendicostomy.

Gant's Appendicostomy.- -Some surgeons, through fear of infection, prefer not to open the appendix during the operation. Having encountered three failures in consequence of this, the author believes this to be a mistake, except when it is obvious that the appendix is unobstructed. In one of these cases the appendix was too small, in another it was strictured, and in the third it was blocked by an encysted grape-seed.

The author amputates the appendiceal tip, probes the appendix, and if its channel is patent immediately introduces his probe-pointed metal or soft rubber appendiceal irrigator (Fig. I64, I); he then knows nothing can interfere with postoperative irrigation. When the appendix is cliseased, strictured, or blocked by a concretion, etc., it is removed and cecostomy substituted for appendicostomy.

It is important that irrigation be started at once in patients suffering from ulcerative colitis who are despondent, greatly delilitated. having many daily movements, losing considerable blood, or sulfering from insomnia, auto-intoxication, or mixed infection.

To meet these conditions the writer has devised a technic for appendicostomy which provides for irrigation both during and following operation (Fig. 164). Since its adoption his pationts have gained very much more rapidly than formerly, when the appenelix was not opened for several days, fluring which time nothing was done to relieve them. Now and then a stitch-abseess has occurred, but no serious complications have arisen during or following operation, and the pres- 
-ure of the irrigator has caused neither pain, atrophy, nor sloughing of the appendix.

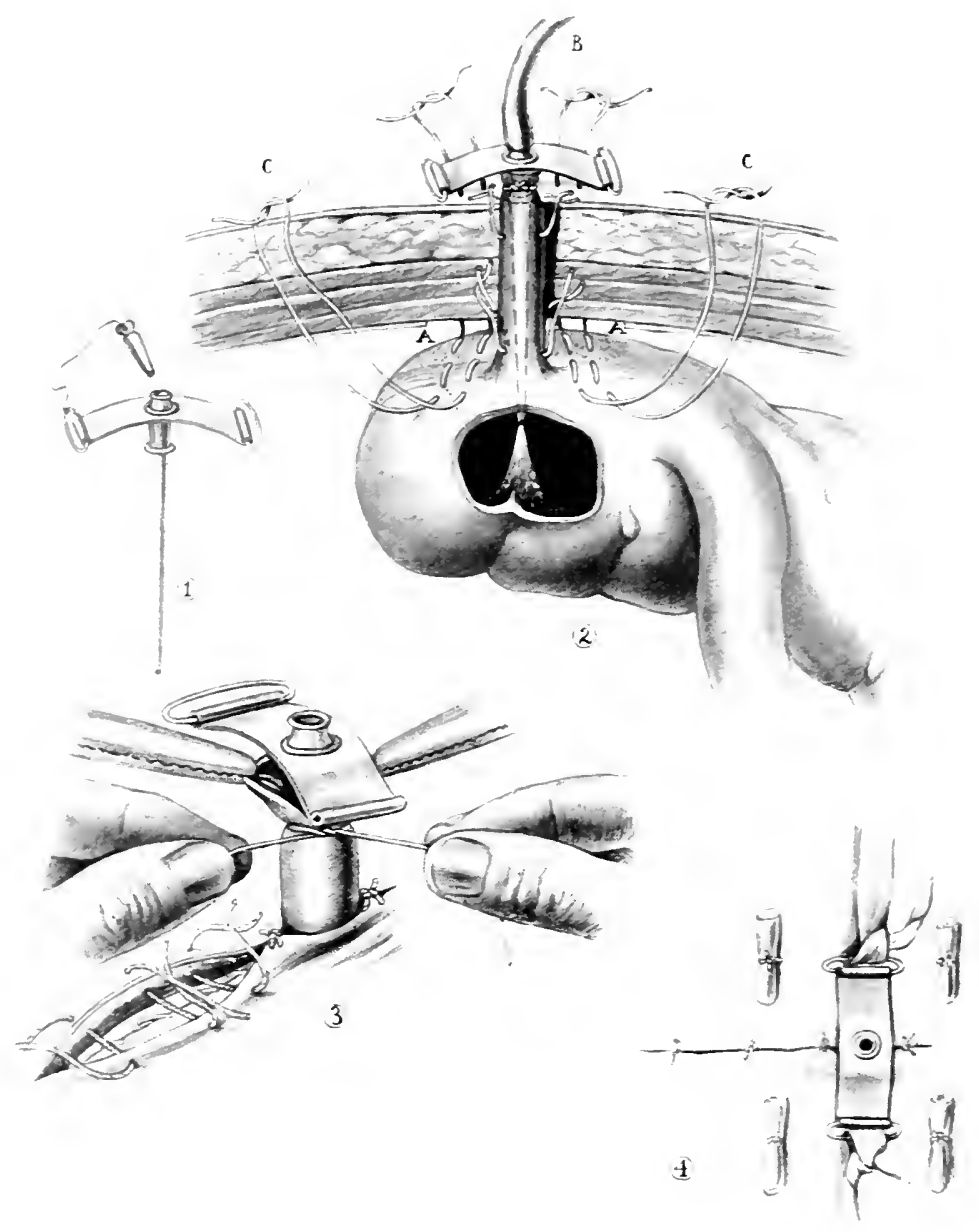

Fir. I 64.- Steps showing Gant's appendicostomy, which provides for immediate irrigation in the direet treatment of buwel diseases: 1. (Gant's appendiceal irrigator: '2. cecum and appendix in position: I. A show peritoneum removed and the rut beine brought in contact with the transersalis fasia: B. tube attached to irrigator; C. C. suspensory sutures which attach the scarilied cecum to the abdominal wall; 3 . method of ligating the appendix about the irrigator and closure of the wound; $t$ show the irrigator in place and the attached pieces of tape which pass around the body and retain it in place when fartened, and the rubber tube across which the suspensory stitches $(C, C)$ are tied.

Briefly described, the steps in appendicostomy as performed by the author are as follows:

First Step.-The appendix is approached through a gridiron incision, and located by tracing the anterior longitudinal band downward, 
when it and the cecum are freed, brought outside, and the wound protected by gauze handkerchiefs.

Second Step.- The cecum is drawn by an assistant to furst one side. and then the other, while the parietal peritonem is removert at the sides of the incision to insure union between the gut and the transversalis fascia.

Third Step.-The appendix is freerl and straightencel l, ligating and dividing adhesions at a safe distance from it, het when the appendix is free the mesentery is not disturbeel.

Fourth Step.- After the cecum has been scarified two seromuscular suspensory sutures are introduced into it, at the sides and near the base of the appendix, each taking three bites in the gut (Fig. If t. (").

Fifth Step.-By means of a long-handled needle the anchoring stitches are carried through the alodominal wall and champer with forceps for identification.

Sixth Step.-Having surrounded the appendix with gauze, a tratetion suture is introduced to steady it white its end is being amputaterl and cauterized.

Seventh Step.-A Gant probe-pointed rubber or silver appendiceal irrigator, closed with a stopper, is introduced, and the appendix is: lisated around it above the projecting rim (Fig. 164.3).

Eighth Step.-The appendix is placed in the lower angle of the wound pointing upward, and anchored by two catgut sutures which pass through the transiersalis fascia.

Ninth Step.- The abdominal layers are then approximated sepalrately, after which the cecal suspensory sutures are tied acrons rubler tubes (Fig. 164, t).

Tenth Step.- The irrigator is prevented from slipping out by the adjustment of adhesive straps or of attached pieces of tape which encircle the body (Fig. I6t, t).

Eleventh Step.- In urgent cases from I to 3 pints of a warm saline solution are immediately injected into the colon, when the irrigator stopper is introduced to prevent leakage.

Tiselfth Step. - The wound is sealed by means of cotton and collodion, and further protected by rubber-covered split-galuze pads. which overlap each other when placed about the appendix.

Thirteenth Step. - The end of the irrigater is surrounded by twisted gatuze strips to prevent pressure upon it when the outer tresings are applied.

The appendiceal irrigators are marle of rubber and silver. and in different sizes and lengths, to meet the individual measurements of the appendix. In a number of (ases, after the irrigator had heen userl wo dilate the appendix and facilitate immediate drainage and irrigation, it was removed and the flushing continued throngh the appendix or at soft catheter, introduced through it into the cecum. In other instances, when the instrument was wet al band, the operation wale performed as above indicated. except that a piece of rubluer tubing was sulstituted for the irrigator. Occasionally lon small a chamnel is 
encountered in an appendix, but if the organ is otherwise suitable for the purpose this difficulty can be overcome by dilatation with graduated catheters, probes, or slender forceps, since the appendix responds readily to stretching.

The appendiceal stump should be left projecting about $\frac{1}{4}$ inch beyond the skin (Fig. 164), so that its opening can easily be seen; otherwise the attendant may inject the irrigant through a mistaken opening beside it, as occurred in one of the author's cases, where death ensued as a result.

Appendico-enterocecostomy.- The author employs this caption to indicate an operation wherein the central part of the appendix is sutured to the skin for irrigating purposes following anastomosis of its distal end with the lower ileum (Fig. I65).

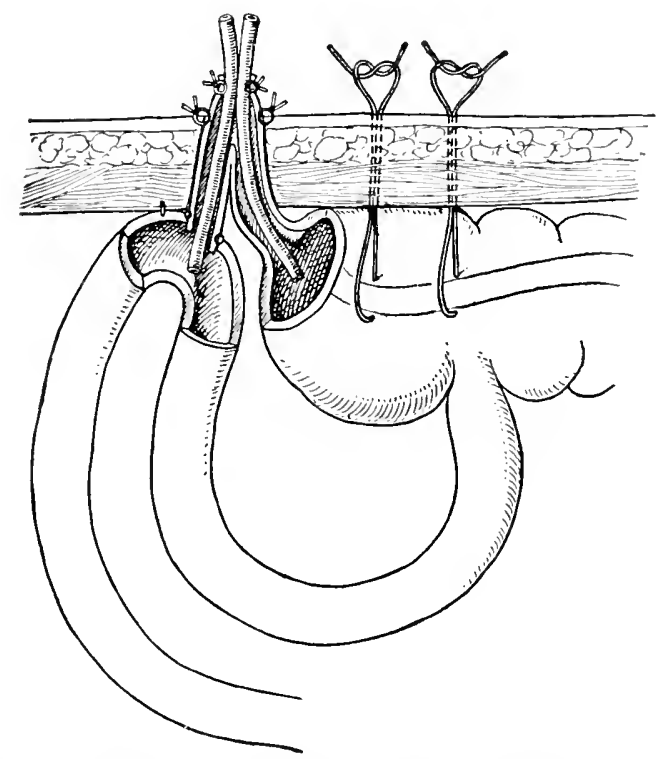

Fig. 165.-Appendico-enterocecostomy. (Author's, technic.)

Keetley (I894) ${ }^{1}$ implanted the opened tip of the appendix into the lower ileum, attached the middle segment of the loop to the skin (appendico-enterocecostomy), and later opened it. so that the lower ileum and colon could be irrigated (Fig. I65). This procedure, however, is in many ways impractical, and surgeons do not think favorably of it. Phillips (I907) performed a similar operation, which he believed to be original.

The author twice attempted the operation without. success, the appendix being too short in one instance and too small in another. On another occasion he succeeded in implanting the end of the appendix in the ileum, suturing its central part into the skin, and later

' British Med. Jour., vol. ii, 1894 . 
opening it (Fig. 165), hut sloughing of the organ enstled at the leend, retraction followed, a fecal fistula formed, and the patient was much more miserable after than before the operation.

Stab-wound Appendicostomy (Fig. 166). - ln 11)07 P'ttyjohn devised his stab-wound operation, performed as follows:

First Step.-The appendix is located through a i-inch incision made over the cecum and retracted toward the merlian line.

Second Step.- After loeating the appendicual attachnent to the cecum with the fingers a vertical stab-womet is mate through the abdominal wall immediately orer this point, while the intestinal lespos are protected with a thick gauze pad.

Third Step.-A pair of narrow forceps is thrust through the opening. and the appendix is drawn upward until the eecum is in contatet with the abdominal wall and anchored in the round by interrupted stitches.

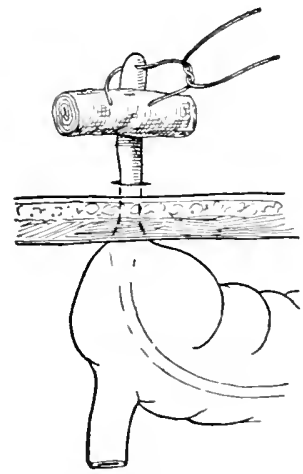

A

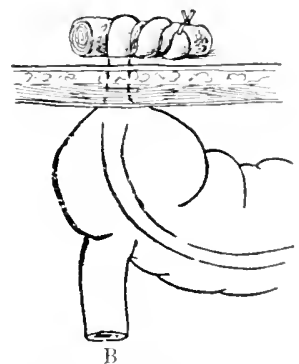

Fig. I66.- Method of attaching gauze (A) and rolling the appendix around it (B) to avoid suturing in stab-wound appendicostomy 1

Fourth Step.-The original wound is closed with three layers of sutures and sealed with collodion.

Fifth Step.-The appendix is then excised and a catheter is inserted to keep it patent.

The author does not believe that this operation posiesses any at vantages over other appendicostomies in the treatment of chronic diarrhea incident to colitis. He has employed the procelure with success on several occasions in conjunction with rescetion, intestinal exclusion, and eolopexy in urgent cases where colonic drainage was indicated, and the patients were so weak that they could endure neither another large incision nor the time reculued for ombinary appendicostomy.

One should know before making the stal,wound that all the abdominal layers are evenly lined up. otherwise failure may result, as sccurred in a case of the author's, where the fascia on one side harl re- 
tracted and calsed a sharp angulation in the appendix when it was sutured to that on the other side of the wound.

In performing appendiestumy one should aroid kinking or twisting of the appendix, which interferes with subseguent irrigation: tension upon it. which causes pain and may lead to cecal sloughing; interferenee with the mesenteric ressels by ligation (Figs. I67, 168) or compression in the wound, as the appendix may thus become gangrenous: suturing the appentix in the wound before it has been opened, since it mix be blocked by a foreign body or stricture; catching the cecum in the wound, which is followed by persistent nausea and romiting; using antinuous sutures for the abdominal layers, for if infection takes plate the wound will open extensively, resulting in prolonged convalescence and a possible hernia: suturing the appendix so that it

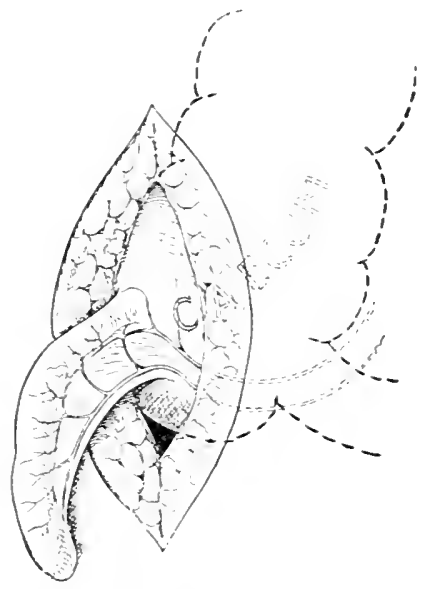

Fig. If-.- Right way of dealing with the mesentery in appendicostomy to prevent sloushing following attachment of the appendis to the skin.

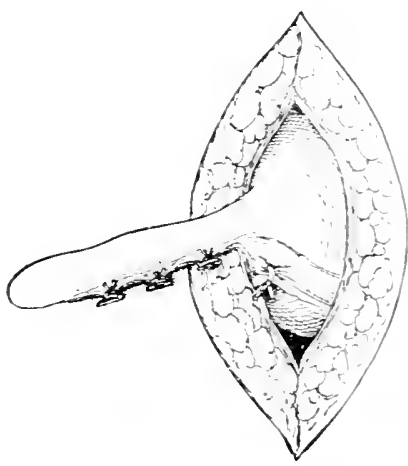

Fig. Ios.- Mrong way of dealing with the mesentery in appendicostony. When the ressels are ligaled and the mesentery adjacent to the appendix is removed. sloughing of the appendix usually ensues.

points lownward, for leakage will ensue; unnecessary injury to the appendix, its blood supply, or intestine to which it may be adherent: permitting adhesions to remain which would give trouble subsequently. Iy pulling upon the organ or cecum: learing the cecum free to make tension upon the appendix when distended with gas of feces: large catheters, which lead to atrophy, ulceration, or sloughing of the appendiceal mucosid.

An appendicostomy properly performed in suitable cases is devoid of danger, brings quick relicf, induces scarcely any pain, is rarely followed by complications or unpleatsant sequelia, and is to be recommended in all rases of chronic diarrhea and ulcerative colitis where cecostomy is for any reason contra-indicated. In the presence of cntametir dysentery the operation should not be performed until after 
the patient has been examined for liver abseess, as a simulaneents operation for this condition may also be neeessary.

Some surgeons scarify the appendix, but a firm union mal be hat without this. It should be kept wrapped in rubber tissue or gatue snteared with vaselin until amputated to prevent it sticking to the dresing and callsing discomfort, and be protected from bandage pressure by arranging the dressing about it in bird's-nest fashion.

In the author's experience no serious complications have followed opening the appendix during the operation, and, as by so doing one knows whether or not the appendix will prove serviceable, he can see no advantage in the tao-stage operation.

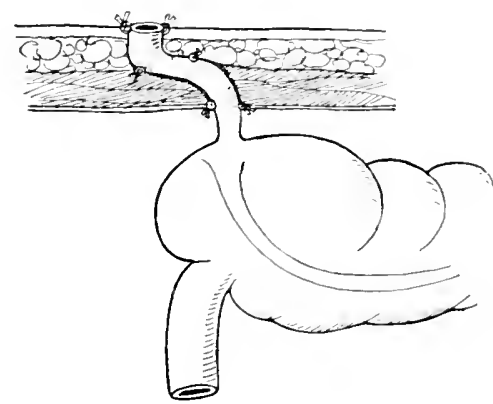

Fir. I60.-Curl's S-shaped method of anchoring the appendix in appendicostomy.

It is advisable to pass the catheter well into the cecum, for in one of the author's cases where a short tube was used ulceration took place below it, a stricture formed, and flushing was greatly interfered with.

When the appendix is bound down extensively by adhesions. clogged, diseased, or otherwise unfit for irrigating purposes. it should be remoxed and an opening made in the cecum.

In the presence of a hepatic abscess or gall-stones the abdominal incision in appendicostomy and cecostomy should be fong enough to permit the operator to perform appendionstomy or cecostomy, as well as remove the stones or operate npon the liver. 


\section{SURGICAL TREATMENT OF DIARRHEAL, INFLAMMATORY, OBSTRUCTIVE, AND PARASITIC DISEASES OF THE GASTRO-INTESTINAL TRACT (Concluded)}

\section{TECHNIC OF ENTEROSTOMY, COLOSTOMY, ENTERECTOMY, CECEC- TOMY, COLECTOMY, SIGMOIDECTOMY, PROCTECTOMY}

Enterostomy.--This operation consists in anchoring the small intestine to the outer abdominal wall or skin and opening it to provide immediate drainage in acute obstruction, or to form an artificial anus in cases of inoperable chronic obstruction of the cecum or small bowel.

The small intestine is rarely fixed and opened, and when it is, it is usually done for the relief of paralytic ileus or an enormously distended bowel filled with gas, feces, and virulent bacteria.

Enterostomy leaves the patient in a deplorable condition because of the almost incessant discharge of fluid feces, which is disgusting to the patient, keeps the skin about the wound in a constant state of irritation, and leads to malnutrition because the chyme is discharged before its nutrient elements have been absorbed.

The colon, on the contrary, is quite frequently opened, either to give vent to the contents of an acutely obstructed bowel for the purpose of forming a permanent artificial anus, or as a preliminary step to excision of the rectum.

Simple enterostomy is a makeshift to carry the patient over a dangerous period in his illness, and the opening is not supposed to be lasting, but when an artificial anus is established it is usually a permanent affair; consequently, the technic of these procedures must necessarily differ.

In enterostomy no attempt is made to give the patient control over the evacuations, the object being to relieve distention and provide a means of irrigation, so that the intestine may be immediately cleansed of contained poisons. On the other hand, when a permanent anus is formed, pains are taken to make it effective, so that the patient will not be annoyed by involuntary movements or protrusion of the gut through the opening.

Simple enterostomy, or a fecal fistula, can be quickly and effectively made by freeing and drawing the segment of bowel situated above the point of obstruction up into the wound and stitching it to the fascia or muscular layers. The stitches should be made to dip into the musculature of the intestine in order to give a firm support. When the colon is opened additional sutures should be introduced at the angles of the incision which will pass beneath the longitudinal band. When 
the manifestations arising from distention are not dangerous the gut should not be opened for a day or two but when the conelition of the patient is critical it should be punctured within a few hours, by which time the peritoneal cavity will have become walled off.

When it is imperative that the bowel should be opened immediately to save life, a loop of the intestine should be brought outsirle the wound, opened, and ligated around a Paul glass tube by means of a seronuscular purse-string suture, or the gut may be divided leetween clamps, and a tube placed in either end and made to connect with a basin under the bed by means of rubber piping. Experience has shown that fecal fistula lieal more frequently and quickly when the intestine is fixed to the deeper abdominal layers than when it is stitched to the shin.

Colostomy.- Now and then it becomes necessary to colostomize patients suffering from chronic mechanic diarrhea induced by a growth. stricture, angulation, adhesion, volvulus, invagination, foreign body, diverticulum, or enteroptosis, etc., after other measures have been tried and proved inefficient.

An artificial anus should never be made to relieve this class of suffurers except as a last resort, because patients seriously object to having the opening in the side, they do not have perfect control over the evacuations, and, further, because a second and more dangerous operation is necessary should they ever desire to have the artificial anus closed. When an inoperable stricture, growth, angulation. etc., causes obstipation, and a new route must be found for the feces, the author prefers entero-anastomosis and exclusion of the involved segment of gut to colostomy, because it relieves the diarrheal state, is not accompanied by the objectionable features of an artificial anus, and does not require a possible secondary operation. An artificial anus may be temporary when made as a preliminary step to excision and resection, or until such time as the condition for the relief of which it was made has been cured; or permanent when the opening is to remain through life.

In temporary colostomy the object sought is to keep the feces away. from the field of operation for a few days before and following the operation and then to close the opening; consequently, it is not necessary to spencl as much time in the formation of a temporary anus as it is in the making of one which is to be permanent.

In permanent colostomy it is of the utmost importance for the patient's comfort to make the opening of a suitable size. and to to the operation in such a way that he may not be botherec with painful eracuations, fecal incontinence, or procidentia.

Practically all surgeons now locate the anus in the left inguinal region, except when the pathologic le-ion necessitating the operation is situated further up, when the outlet is mate in the transverse colon or right inguinal region, accorling to the indications.

Except where there are special reasons for toing otherwise, the aperture should be marle as low down as possible, because here the 
feces are more solid and give less trouble than when the anus is established at or near the cecum.

In recent years Bailey, Weir, Witzel, Tuttle, the author, and others have devised special technics designed to overcome the disgusting features of the old-fashioned artificial ants and give the patient control over the opening, and each operator has met with more or less success.

The technic of the above-named operators has been given elsewhere by the author, ${ }^{1}$ and bere he need not do more than briefly describe his own operation, which has proved eminently satisfactory in more than ioo cases.

The Author's Colostomy.-The sigmoid is approached through a 2-inch incision, which crosses a line extending between the umbilicus

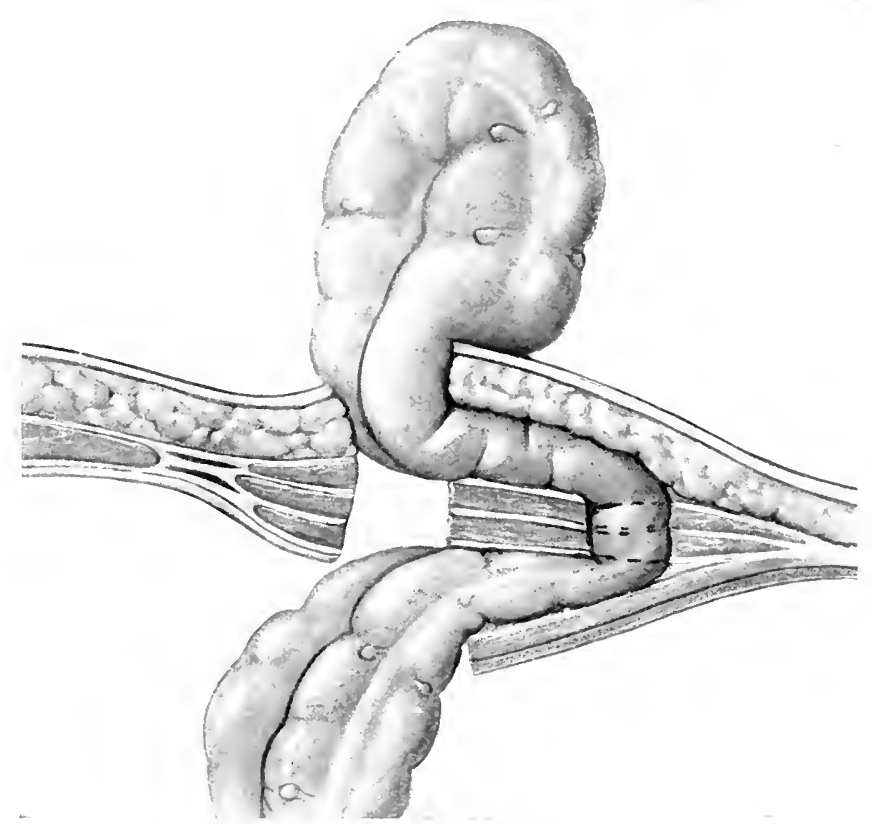

Fig. 170.- Relation of the gut to the muscles, fascia, fat, and skin in Gant's colostomy.

and the anterior spine of the ileum, at the inner border of the oblique muscles; working outward, the transversalis is separated from the internal oblique, with the index- and middle fingers, for about $\mathrm{I}$ to $\mathrm{I}$ ? inches $(2.5-3.8 \mathrm{~cm}$.). The fingers are then forced upward through the two oblique muscles, and finally over the external oblique and inward to the incision, thereby separating the subcutaneous fat from the muscle. A loop of the sigmoid is now hooked up and then made to travel the route taken by the fingers, which makes it pass outward between the internal oblique and the transversalis muscles, and then through and over the external oblique to the incision, where it is

\footnotetext{
${ }^{1}$ Diseases of the Rectum and Inus, $3 \mathrm{~d}$ ed.
} 
sutured after being made taut to avoirl a subsequent procidentia. The angles of the wound are approximated ly two chromicized catgut sutures, which pass through the skin and fascial on one side of the incision and then beneath the longitudinal band of the sigmodid and out through the same structures on the other sirte, where they are tied (Figs. I70, I7I). After the gut has been attached to the kin by a few plain catgut stitches (Fig. I $7 \mathbf{I}$ ), it is cosered with rubler tinste. hubricated with sterile varelin to prevent sticking, and then the outer dressing and binder are applied. Patients have lum little control

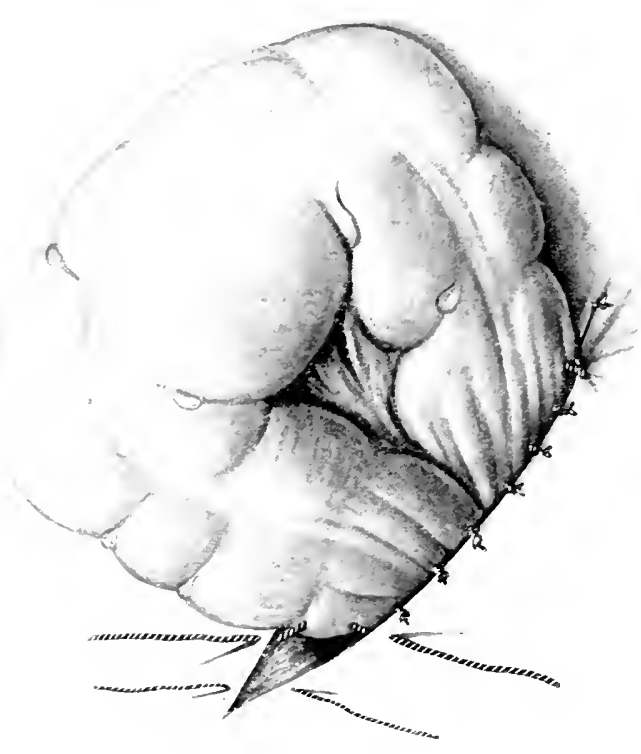

Fig. I, I.- Side view of the sut after it has been brought out of the alodomen, and method of applying the ancular and fixation sutures in Gant"s colostomy.

over an artificial ants for the first few lays, no matter what operation is performed, because the soreness of the wound and the irritalibity of the intestine excite freguent and strong peristalsis accompanied by the involuntary discharge of feces.

Except during attacks of diarrhea the atthor han rarely known a patient to have more than one or two actions daily following his operation. In fact, he has treated many of his colostomized patients for constipation.

A study of the position of the gut, as shown in the accompanying 
illustrations (Figs. I70, I 7 I), will make it easy for the reader to understand why the operation is not followed by incontinence.

This procedure has the advantage over the Bailey, Meir, Mitzel, and Tuttle technics in that but one incision is made instead of two, and, further, because it gives the patient a more perfect control over the morements than do the others. The author's patients rarely complain of the irroluntary escape of gas, and usually do not have an evacuation until they desire, when they stimulate peristalsis by the injection of 2 or 3 ounces (60.0 or 90.0) of warm water into the upper opening.

The bowel is not opened until the third day except when there is a marked distention and suffering; under such circumstances it is punctured at any time after six hours and amputated later. The projecting piece of gut can be quickly and painlessly removed under local anesthesia induced by the injection of a small quantity of a $\frac{1}{4}$ per cent. eucain solution into its mesentery. Cutting of the bowel proper causes no pain and does not require anesthetizing.

The bowel is amputated by a few bold cuts of the scissors about $\frac{1}{4}$ inch from the skin, to allow for retraction. Bleeding points are ligated en masse, and hemorrhage from oozing surfaces is controlled by hot-water compresses, adrenalin, or a solution of iron or the cautery. The raw edges left can be encouraged to heal rapidly by the occasional application of silver nitrate ( 6 per cent.). When the obstruction is located above the sigmoid the steps in the operation must necessarily be modified to meet the indications, but desirable changes in the technic will at once suggest themselves to the experienced surgeon.

ENTERECTOMY, COLECTOMY, CECECTOMY (TYPHLECTOMY)， SIGMOIDECTOMY, AND PROCTECTOMY

Enterectomy, the removal of a fraction of the intestine, is justifiable in acute and chronic obstruction where life is endangered and relicf cannot be given in any other way. In the context the term enterectomy will be limited to partial resections of the small intestine, whereas the corresponding operations upon the large bowel, according to their topography, will be discussed under the headings of Cecectomy or Typhlectomy, Colectomy, Sigmoidectomy, and Proctectomy.

Resection of the small intestine is rarely performed for the relief of chronic diarrhea because lesions responsible for the condition are usually located in the colon, sigmoid flexure, or rectum. In the treatment of this form of diarrhea it quite frequently becomes necessary to remove a small or considerable portion of the large bowel or rectum to free it from a tumor, stricture, foreign body, angulation, twist, diverticulum, arlhesion, stricture, or other obstructing lesions which block passage of the feces.

Operators are often inclined to be conservative as regards the amount of bowel they remove, but this is frequently a mistake. It is far safer to take away a liberal amount of healthy gut than it is to leare even a small portion of diseased intestine, which might lead to 
cutting out of the stitches and peritonitis, the formation of a fecal fistulat or a recursener of the trouble.

A few years ago when making an artificial anus the author lonought outside the ablomen only a sufficient amount of gut to form a relialibe spur ancl provicle a useful opening, but, as a result, many of his colostomized patients suffered from protrusion of the rectum or descending colon through the artificial opening. In his last 200 colostonites he has excised from 31015 inches of the gut to olsiate this complication, and no ill conseduenees have followed.

Experiments upon aninals and lane's colonic excisions and operations upon the large gut have demonstrated that one-thiret of the small intestine can be resected and a large part or all of the colon be excised without appreciable harm, but when as much as one-half or more of the small lowel is remored digestive disturbances arise and death ensues from inanition. Surgeons have on many occasions remoxed from 5 to 2 feet of the small or large bowel, and their patients have recovered and lived in comfort afterward. The following table of cases, collected by Park, ${ }^{1}$ gives many examples where several leet of intestine have been resected:

CASES OF INTESTINAL RESECTION HITH REMOAAL OF MORE TIIAN G FEET \& INCIIES (2O0) (ENTIMETERS) OF INTESTINE.

\begin{tabular}{|c|c|c|}
\hline Operator. & Amount removed. & Result. \\
\hline 1. Koeberle........ & $6 \mathrm{ft} .10 \mathrm{ill} .(205 \mathrm{~cm})$. & Rerovered. \\
\hline 2. Korher........ & $6 \mathrm{ft} . \mathrm{I}$ in. $(208 \mathrm{~cm})$. & Recovered. \\
\hline 3. I)resman......... & $7 \mathrm{fl} .2$ in. $(215 \mathrm{~cm}$.$) .$ & Recovered. \\
\hline 4. Shepherd ........ & $7 \mathrm{fl} .9$ in. $(23+\mathrm{cm}$.$) .$ & Rewered. \\
\hline 5. Kukula ......... & $7 \mathrm{ft} .9$ in. $(237 \mathrm{~cm}$.$) .$ & Recosered. \\
\hline 6. Harris............ & $7 \mathrm{ft} .10 \mathrm{in} .(230 \mathrm{~cm})$. & Recovered. \\
\hline 7. Hayes. ............ & $8 \mathrm{ft} .4_{2}^{1} \mathrm{in} .(248 \mathrm{~cm})$. & Recovered. \\
\hline S. Peck....... & $8 \mathrm{ft} . \quad 5^{\frac{1}{2}} \mathrm{in.}(25 \mathrm{~lm}$.$) .$ & Recovered. \\
\hline 9. Lawers......... & S ft. 0 in. $(205 \mathrm{~cm}$.$) .$ & Recovered. \\
\hline Io. Roswell Park... & S fl. o in. $(205 \mathrm{~cm}$.$) .$ & Recovered. \\
\hline i . Payr......... & o ft. $\frac{1}{3}$ in. (275 (m).). & Recovereel. \\
\hline I 2. Maydl..... & oft. + in. $(2 s+\mathrm{cm}$.$) .$ & I)ied three weeks later of inanition. \\
\hline 13. Fantino....... & $10 \mathrm{ft} .+\mathrm{int} .(310 \mathrm{~cm})$. & Recovered. \\
\hline It. Monprofit..... & $10 \mathrm{ft} .+$ in. $(310(\mathrm{~m})$. & Recovered. \\
\hline 15. Ruggi .......... & $1 \mathrm{lft} \quad(330 \mathrm{~cm})$. & Recovered. \\
\hline 10. Von Eiselsberg... & ir ft. 8 in. $(350$ (n). $)$. & bealh after twenty-five ditys. \\
\hline I7. Obalinski...... & I $2 \mathrm{ft}, 2$ in. $(365 \mathrm{~cm}$.$) .$ & bied. \\
\hline
\end{tabular}

In so far as the colon is concerned, the author's experience warrants the statement that it makes very litule lifference whether a short or long segment or all the colon is removerl, hecatuse the ilemm, to a great extent, takes upon itself the functions of the colon when the latter has been resected or excluded by entero-anastomosis. His experience agrees with that of lane, in that the stools following colonic resection are at first frequent and fluiel, bu gradually the actions become lest frequent, and, finally, normal, mumber and consistence. I neler 
such circumstances the lower ileum, upon examination, was found in 2 of the author's cases to be enlarged and acted as a reservoir in which the feces remained until the watery constituents had been absorbed and the stools became formed and ready for expulsion.

Enterectomy (especially colectomy) is indicated more frequently in men than in women, and has been performed more than twice as frequently in persons between the ages of thirty and sixty than in older or younger individuals.

On account of their great mobility, the small intestine, transverse colon, and sigmoid flexure are more easily resected than the cecum or hepatic and splenic flexures, which are tied down by their mesenteric ligamentous attachments or contracting adhesions, which makes their removal extremely arduous. It is invariably easier to resect a piece of the small bowel than a similar segment of the colon, because the latter is larger, thicker, and more generously surrounded with fat, and is often bound down by adhesions or a short mesocolon.

Except that a lit le more care is necessary in placing the mesenteric sutures following colonic resection, the technic of operations upon the small and large intestine is about the same.

The mortality following colectomy for the relief of cancer is greater than for other types of mechanical obstruction causing obstipation, and the results are not so good.

Patients upon whom a cecectomy, colectomy, or sigmoidectomy is about to be performed should be prepared for the operation by freeing the bowel of accumulated feces (when possible), washing out the stomach, and disinfecting the skin with iodin, etc.

General anesthesia is preferable for resection operations in chronic obstruction from all causes when the condition of the sufferer is good, but if the bowel is completely blocked and vitality is lowered because of enomous distention and absorption of virulent poisons it is frequently advisable to open the abdomen and incise and drain the bowel uncler local or infiltration anesthesia ( $\frac{1}{5}$ per cent. encain solution). When the type and location of the obstruction are known in advance it may be reached, and the intestine resected through an intermuscular incision made directly over the lesion, but when there remains a doubt as to which part of the colon is blocked, a liberal median or Kammerer incision is preferable.

A collapsed large bowel points to small intestinal obstruction, and a highly distended colon to a block in its lowermost segments or the rectum,

When the patient is extremely ill or in a state of collapse from acute obstruction, resection and anastomosis are extremely dangerous, and the patient stands a better chance from the operation and of ultimately obtaining a permanent cure when an immediate vent is provided for the retained feces, gas, and toxins, and the radical operation is postponed until later when the patient's powers of resistance are greater.

In case opening of the bowel is contemplated, it should be brought 
outside and walled off with gauze after it has been freed from exuclates or firm athesions by a gauze wipe or scissors. The distented lumel can then be quickly drained by means of appendienstomy, valutular cecostomy, colostomy, or by simply stitching a part of the colon (to be opened later) above the olstruction to the skin without any attempt at making a spur (simple colostomy). Another popular way of ohtaining quick drainage is to divide the bowet and ligate each pisece of the intestine to one end of a Paull glass-tule, to the other of which is attached a rubber pipe that carries the bewel contents into al vessel beneath the bed. When, for any reason, the surgeon does not with to sever the bowel, it can be opened insiele a purse-string suture, which is then tied around a colon-tube. Some surgeons advorate immerliate incision and irrigation of the gut. While others prefer to empty the bowel by stripping it over a long glass tube similar to the one designed by Moynihan.

Resection of the obstructed segment may be done shortly following drainage if conditions are farorable, on the operation may be deferred for weeks or months, when the opening serves to keep the patient comfortable and the bowel free from fecal accumulations.

As a general rule, obstruction produced by chronic lesions is not complete and a drain is not imperative. Naturally, cecectomy, colectomy, and sigmondectomy are more dangeroun when performed for the relief of acute than chronic obstruction becatse of the distressing manifestations present at the time of operation. In fact, these operations, when done for the retief of chronic mechanic obatruction, are not nearly as dangerous or difficult to perform as surgents of slight experience imagine.

Following cecectomy and colectomy it is often necessary to corer raw surfaces of the aldeminal parietes and bowel with peritonem to aroid the formation of arlhesions or hernia.

The author has resecterl the cecum alone, or including a part of the colon, wice for cecal cancer, once for chronic irreducible ileorecal invagination, wice for otherwise inoperable athesions, once for ateute obstruction and perforation caused by thread-like filorous bands, and once for chronic volvulus (ascending colon) caused ly contrateting athesions which pulled its upper extremity in one direction and the lower in another, with but 2 deaths. In most of thene catess, after a blind end had been formed in the colom, the ilesm was serered, the divided ends closed, and an anastomosis made betwern it and the sigmoid or rectum. Considering the nature of the lesions and the condition of pattents at the time of operation the results aluained from resection in these cases were very satisfartory. Except when arlhowoms are very numerous and firm, or the lowed is greatly distorted, removal of the cecum is not a very difficule prosedure.

(ecectomy (typhtectomy), colectomy, and sigmoiclectmmy are extremely diffecult where the patient is fat, herases of the thickness of the abdomen and the large amount of fat in the mesentery and around

${ }^{1}$ Brit. Med. Jour., May 25, iso5. P. I 139. 
the bowel. Naturally, the most movable portions of the colon are more eas to resect than those which are fixed by the mesocolon.

Bleeding should be completely arrested befort the abdomen is cheed by suturing the raw or torn surface or applying the cautery. adrenalin, iron, or hot water to it until hemorrhage has been controlled.

Postoperative adhesion - may he forestalled by aroiding bruising the viscers and peritunteum. clearing the abdomen of blood. covering denuded surfaces with the serosa, aristol, tallow, or Cargile's membrane. or frequenty changing the position of the patient. and pre-ribing eserin to excite peristalsis and prevent inflamed or raw surfaces of the intestinal loop frum constantly remaining in contact.

Proctectomy.-This procedure is called for in the treatment of inHammatory and ulcerative lesions, strictures, diverticula, benign and

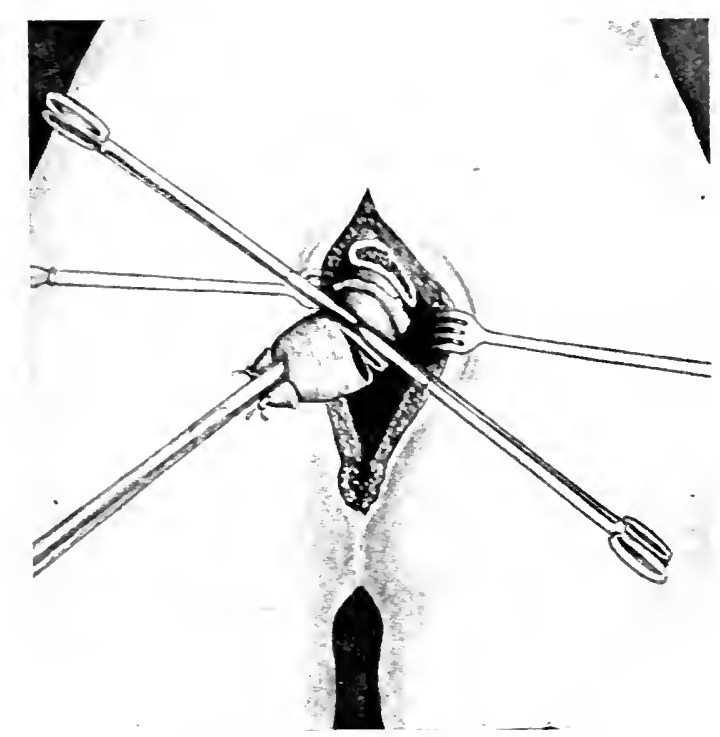

Fir. I-2.-Method of amputating the rectum afier it has been ireed from its attachments in superior procteclumy.

non-maliznant tumors. distortions of the rectum, and other diseases of the rectum causing obstruction or diarrhea which cannot be eliminated by les radical measures.

The author has in many instances cured chronic diarrhea. relieved pain. arresterl offensive discharges. improved digestion. overcome anemic and cachexia, and caused patients to rapidly regain their normal health and weight by removing all or a part of the lower bowel which was blocked, extensively ulcerated, or intensely inflamed and irritables.

Amputation or resection of the rectum is preferable to colostomy in ancirated cane hecause by it the diseased bowel is immediately

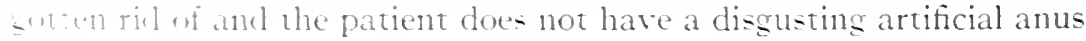


to look after. The rectum, accorcling to indications, may be remorecl by the foltowing methols, viz:

(a) Inferior proctectomy (perincal excision); (b) superior proctectomy (sacral excision); (c) a'asinal proctectomy, and (d) celioproctectomy" (al)dominoperineal proctectomy).

Inferior Proctectomy.-Practically all rectal canerers lexated in the lower 3 inches $(7.62 \mathrm{~cm}$.) of the rectum can le spereflily and safely removed by this procedure, which consists in beginning bedow and fresing the rectum upward sufficiently high to permit the operator to excise the gut at a safe distance alose the growth.

In farorable cases extensive tumors and several inclues of the lawel can be easily removed by perineal excision with little danger to the patient.
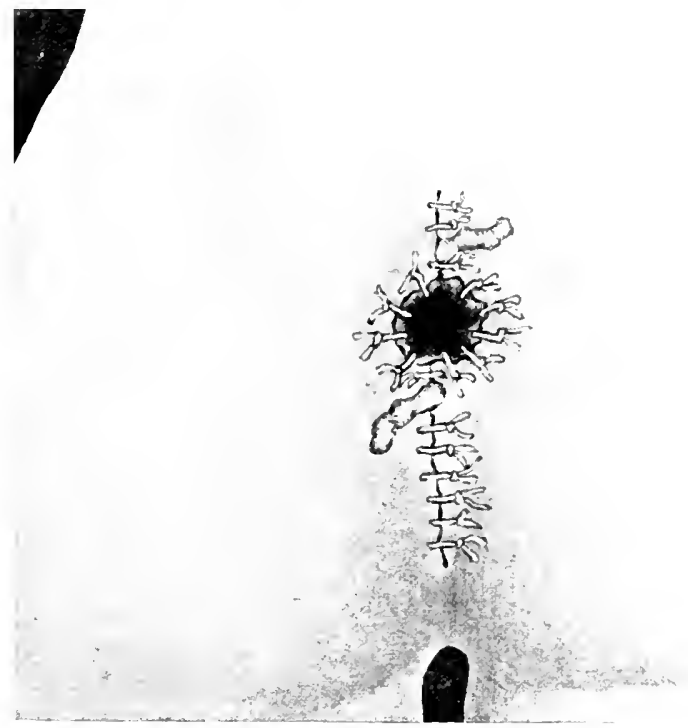

Fig. I 73.-- Aprearance of wound and location of the sacral opening after superior proctectomy where the gut cannot be brought down to the normal site of the anus.

Superior Proctectomy (Sacral Excision).-Aggravated cases al cancer located in the upper third of the rectum or at the rectosigmois juncture cannot always he extirpated by inferior or sersimal proctectomy, and excision of the coceys or lower sarrum (liraske) is neressary to give the surgeon sufficient room in which to isolateand remone the growth (Figs. 172, 17.3).

Cocergeal excision is all that is repuires in mose instances, but removal of the sacrum below the thirel vertelual opening is artrantageous and greatly facilitates the extirpation of high cancers without greatly endangering the patient's life. The formation of al satcral anus is often imperative in this operation, hut the removal of lone weakens petric support. and for these reasons inferior and raginal proctectomies are preferable to kraskes procelure in feasible cases. 
Taginal Proctectomy.-The author invariably removes cancers of the lower, middle, and upper rectum in women by the vaginal route (Figs. I 74. I75), because splitting of the vaginal septum and perineum gives an abundance of room in which to work, frequently avoids destruction of the sphincter muscle, and removal of the bony pelvic support greatly facilitates the operation. On several occasions he has succeeded in removing several inches of the sigmoid flexure with the upper rectum by the vaginal route. When the peritoneal cavity has been opened to free the bowel, it can be easily and quickly clased and drained. In this procedure it is easy to unite the lower and upper rectal segments following extirpation of the tumor, or where

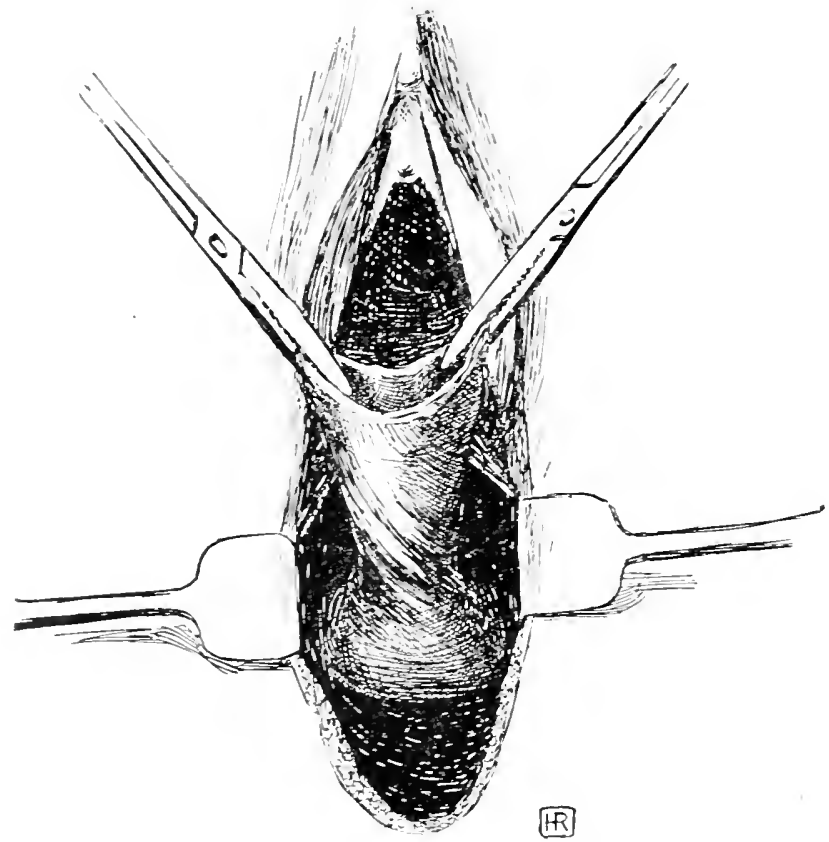

Fig. I 7t.-Proctectomy by the vaginal route, showing Gersuny's twist. (Author's technic.)

the distal end is diseased and must be removed, the proximal end of the bowel can easily be drawn down and sutured to the perianal skin.

Celioproctectomy (Abdominoperineal Excision).-When a growth involves the upper rectum, or it and the lower sigmoid flexure cannot be extirpated by inferior, superior, or aginal proctectomy alone, one is justified in resorting to the combined operation.

In this procedure the abdomen is opened, the cliseased gut is isolated, ligated, and divided, after which the proximal end is stitched in the wound (colostomy) and the distal end, following severance of its peritoneal reflexion, is pushed downward and the serosa is closed above it. The abdominal wound is then closed and the diseased gut is extirpated 
by inferior, superior, or vaginal proctectomy. Patients object to an artificial anus in the side, consequently, when feasible, the sigmoid should be mobilized by sewing its mesenteric attachments, dividing the inferior mesenteric or superior hemorthoidal artery, and incising the peritoneum about the gut, which enables the operator to easily free the upper rectum, push the sigmoid downward, and che the peritoneal cavity. He can then quickly excise the necessary amount of howel by either perincal, sacral, or vaginal excision.

Preseriation of the sphincter muscle is desirable, but unless considerable care is used atl cancerous tissue is not removed, in which case local recurrence will shortly follow. When the anal segment and sphincter are healthy the molilized proximal end of the bowel may be anastomosed with it in situ, pulled down, and sutured to the skin after the mucosa has been removed from the anal canal, or the lower can be invaginated and the upper end of the rectum be drawn through and sutured to it, following which the intact howel is replaced. Many patients have control orer solid fecal matter following the establishment of a sacral anus, and not a few can retain fluid feces when the gut is twisted upon itsolf before being sutured to the skin.

Bleeding is controlled by packing one side of the gut with gauze compresses wrung out of boiling water while the other is being freed. When the gut has been completely isolated and the growth removed between double ligatures placed on either end, ligation of the rectum about a large rubber tube prevents bleeding when it is amputated. Sometimes the autlor sutures the lowel to the skin, cuts it off, and controls hemorrhage by tying the ressels separately as serered.

For a detailed description of inferior, supe-

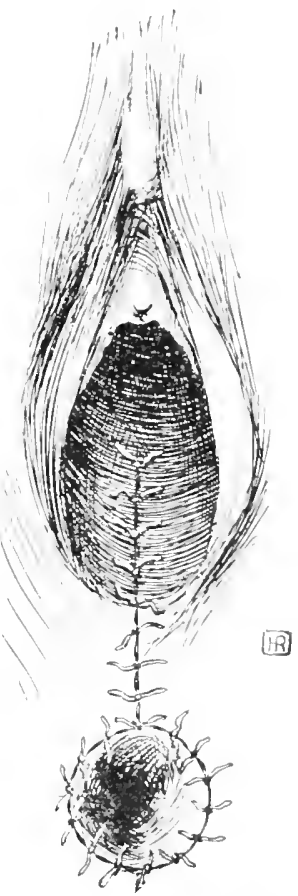

Fig. 175.- Showing the vaginal, perineal, and perianal suture line following proctectomy by the vaginal route. (Author's technic.) rior, and ietginal proctectomy and celioproctectomy the reader is referred to the author's work, Intestinal Stasis ("Constipation and (Obstipation").

Intestinal Exclusion.-This procedure consists in shutting off the fecal current from an intestinal segment to relieve obstruction or sive rest to the mucosa when involved by intlammatory or ulcerative lesions that cause obstipation or loose morements alternating with costiveness or diarrhea. All or a part of the small or large intestine may be exchuded by entero-anastomosis (1ig. 176), closing the bowal on one side of the diseased gut, and uniting the proxinal extremity to the intestine betw unilateral exclusion (Fig. 18); or by boxing in the lesion on both sicles 
and anstomosing the upper end to the healthy bowel below-bilateral exclusion (Fig. I77). The author has resorted to this operation many times as a substitute for colostomy and resection of the intestine in the

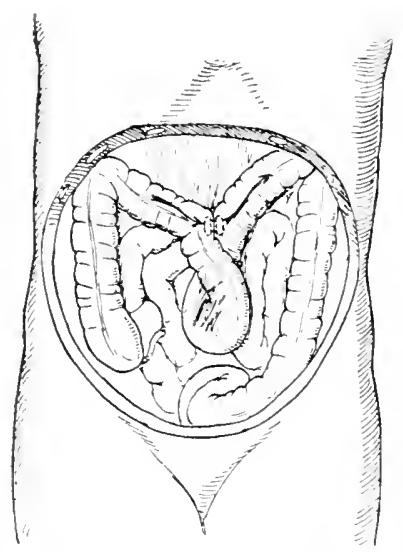

Figr. I -0. - Entero-anastomosis with Murphy button. Performed for the relief of rolvulus and ptosis of the transverse colon. (After author's case.)

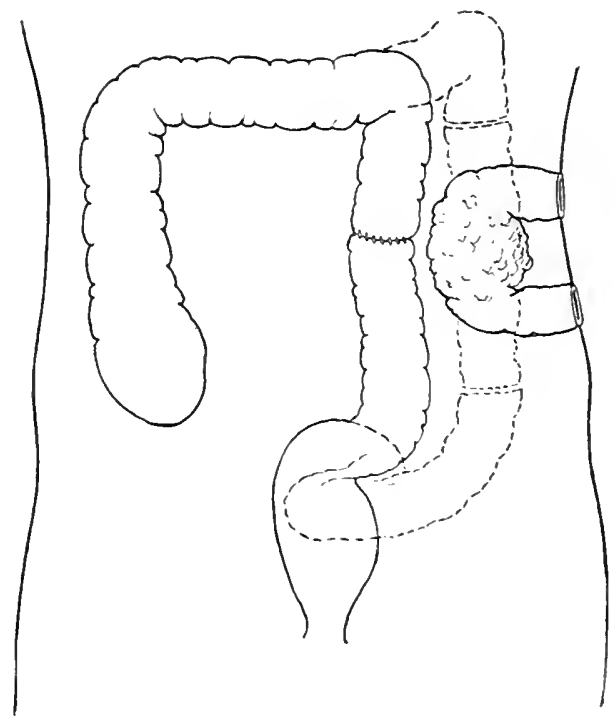

Fig. I7.-Bilateral exclusion for irremorable cancer of the descending colon after the ends of the diseased segment have been brought to the surface for drainage. (Author's case.)

treatment of the various forms of catarrhal and specific colitis, obstructive diarrhea, and intestinal obstruction, and the results have been entirely satisfactory. When it is properly and quickly performed in-

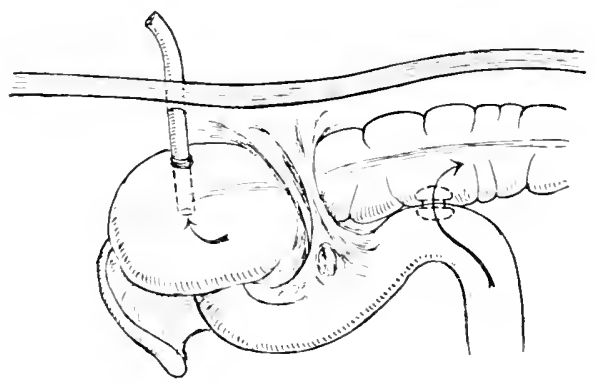

Fig. I 8.-Intestinal exclusion for cecal cancer with adhesions (ileocolostomy) and drainage of the cecum. (Ifter author's case.)

testinal exclusion is not dangerous, brings immediate relief to the patient, and often effects a permanent cure.

In cases where the ileum is joined to the colon, sigmoid, or rectum 
(ileocolostomy, siomoidostomy, or rectostomy) the stools, which are fluirl in the beginning, gradually become fewer, and finally normal within
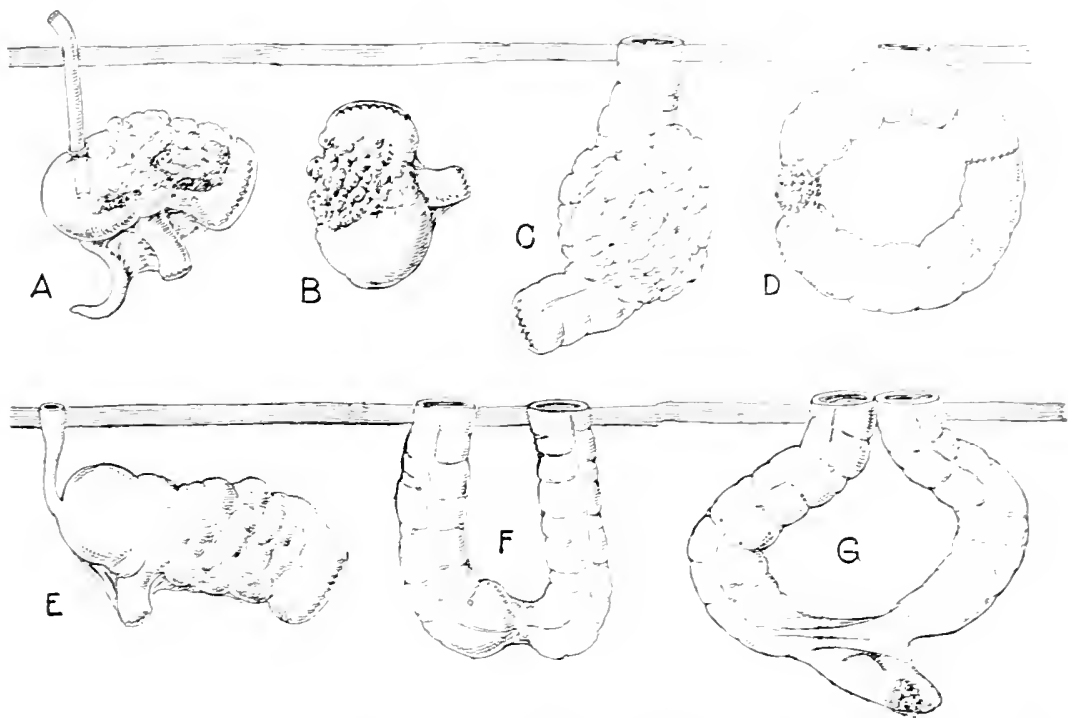

Fig. I; 0.--Author's methods of draining or dealing with the diseased buwel when it cannot be removed iollowing exclusion: A. Cecostomy: B. blind end; ( . I), F, and G. colonic (colostomy: E. appendicostomy.

from six to twelve weeks. The author has in several instance- lemonstrated that the unused bowel does not become u-eless. but func-

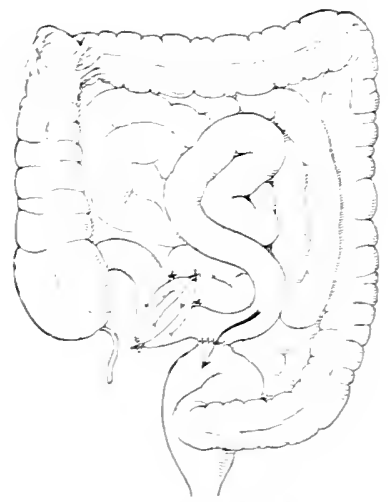

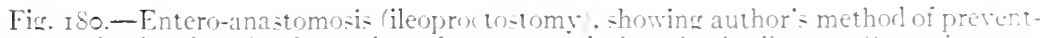
ing recurcitation by the formation of acuteangulation in the ileum. Operation fer formerl for the reliei of olsoructive adhesions situated at the hepatic tlexure. Hiter author' = case.)

tionates normally if healed when continuity of the inte-tine ha- leen re-establi-herl weeks or years after the original operation. 
Regurgitation has been successfully prevented by the author both by narrowing the adjacent gut with a ligature, or by angulating it ${ }^{1}$ with sutures where the large bowel is blocked or ulcerated and the patient shows evidences of intestinal infection or anto-intoxication (Fig. I80). Drainage should be provided for through an appendicostomy or cecostomy after the diseased gut has been excluded.

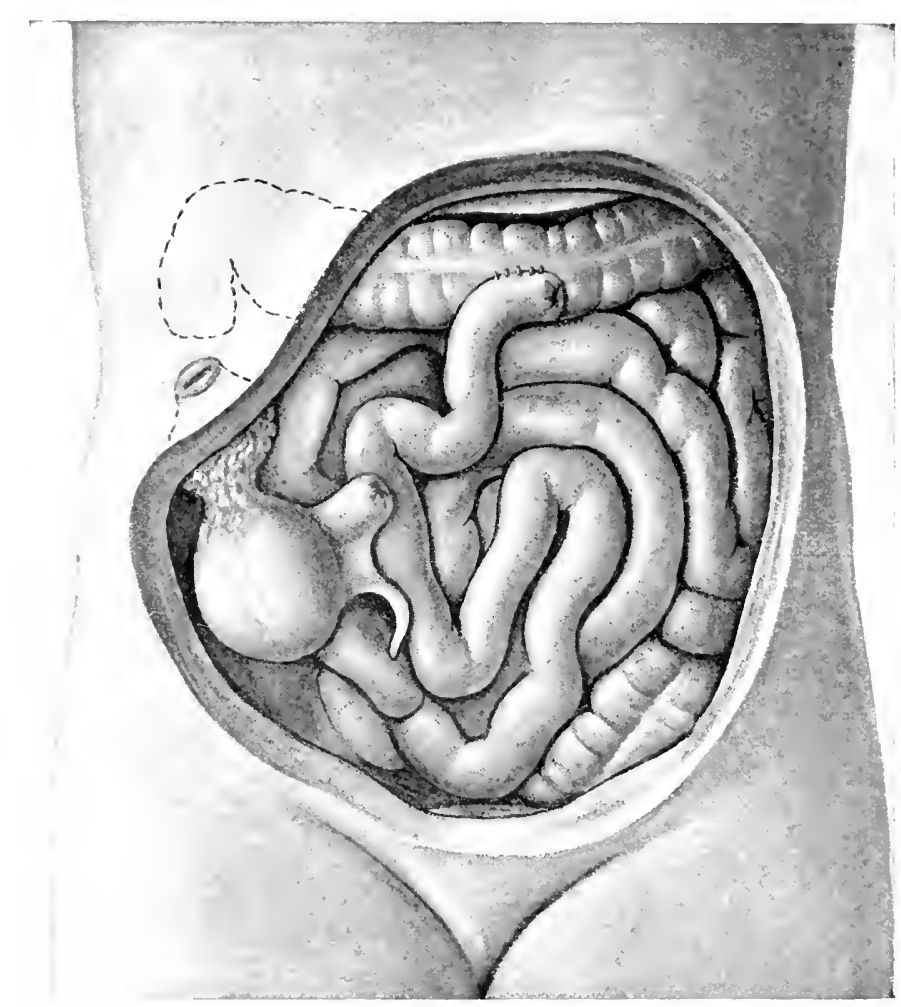

Fig. ISI.-Unilateral exclusion (ileocolostomy) for the relief of a strictured lower ascending colon. The ileum is seen divided and both ends closed, the proximal end being joinerl to the transverse colon. The ascending colon has been divided, and one end closed and the other left open (colostomy) for clrainage.

The technic of intestinal exclusion has been fully discussed in Chapter XXXV, Gant's Intestinal Stasis ("Constipation and Obstipation"), where many cases have been recorded, hence further consideration of the subject is unnecessary here. (1909).

1 This procedure was described in Gant's Constipation and Intestinal Obstruction 


\section{INDEX}

Abscess, hepatic, 364

ischiorectal, in entamebic colitis. 364 perirectal, in entamebic colitis, 364 submucous, in entamebic colitis, 364 tropicat, 364 . See also Hepalic abscess.

Abscesses in entamebic colitis, 36 I in tubercular enteritis, 252 metastatic, in entamelic colitis, $36 \mathrm{I}$

Achytia gistrica, diarrhea in, 123

Acid-poisoning, diarrhea in, 166

Acils in entamebic colitis, 380

Acrodynia, diarrhea in. 07

Aetinomycosis, diarrhea in, ror resembling tubercular enteritis, 259

Acute catarrhal enteritis, is i

Addison"s disease, diarrliea in, $I_{7}$ I

Adenoidism, myxorrhea coli in, $4^{6} 3$

Ige in entamebic colitis, 327 of occurrence in tubercular enteritis, 214

Agelutinating test in bacillary colitis, fro. fI 1

Iroraphobia, diarrhea in, 117

Albuminuria in tubercular enteritis, 257

Aleoholism, diarrhea in. iso treitment. I I

Alkali-poisoning, diarrhea in. 167 treatment, ios

Ameba, detinition of genus. 333

Amebx, chassification of, Calkins', 333

Craig's, 335

Hartmann $\triangle$, 332

and Calkins, points of difference, 33.3

duration of life and resistance, 339

morphology of. $33^{\text {t) }}$

motility of 3.38

non-patherenic, 325

patherenic, 325

reproduction of. 330

Amieba coli aliscovered by loesch, 323

dyenteriet. 324

histolytica, 324

Amyloid degeneration of intestine, differential diagnosis, 108

resembling tubercular enteritis, 259

Amyloidosis, intestinal, diagnosis, 7 I

diarrhea in, 60

etiology, oo

simptoms, jo treatment, 7

Anal fissure, diagnosis, 500 surgical treatment, 517,518

sphincter, hypertrophy of, surgical treatment, $5 \mathrm{i} 7$
Inemia, diarrhea in, 108 dirt-eaters", 427. See also Encinariasis. pernicious, diarrhea in, 100

Ancsthesia, local, in surgrical treatment of tubercular enteritis, 285

Inguillula stercoralis, +30

Inkylostoma duodenale. +27

Inkyfostomiasis, 427. See also Lncinariusis.

Inorectal syphitis, pathology, 302

Anthrax, diarrleca in, of

Intiseptir remedies for entamebic colitis, 380

for tubercular enteritis, $27.3-275$

Inus, intestinal irrigation by, 47

Apjendicerostomy, 5.51

Aprendicitis, catarrhal, diagnosis, i 95

diferent bil diannosis, nos

differentiation from tubercular hyperplastic enteritis, 204

entamebic, in entanebic colitis, 36 I

resembling tubercular enteritis, 245

Alpendicorecostomy, $5+5$

in entamebic colitis. $30 \mathrm{I}$

Ppendico-enterocecostomy, Gant's, 570

Appendicostomy, $5+5$

disadrantalges of , 50,5,560

for diarrheil, 2 I

for syphilitic enteritis. 310

Gant's. technic of $5(1)-5$

in entamebic colitis, 30 I

in tubercular enteritis, $28 S$

mortality from, 5.53

Pettyolin's stab-wound, 57 I-573

results of through-and-through irrigation following, 552

technic of. $56(x)$

Appentix, involvement of in alute entamebic colitis. $3 t^{6}$

in hyperphistic tubercular enteritis, 233

Irsenic-poisoning. diarrhea in, 163

treatment, iot

Arteriosclerosis, diarrheil in, I I 2

Ascariasis. 4,3 I

diagrusis. 4.32

symplons $+31,4.32$

treatment, 43.3

Iscaris lumbricoicles. 4.31

trichinare, $4,3.5$

Istringent remedies in entamebic colitis, in 30 in myorrlea coli, 468

Asylum dyentery, 303, 100, 107, 413. See also Bucillury colilis. 
B.ACILLARI colitis. 303, 400,407, +I3

absorption of toxins in. for

acrelutinating test in, 410.411

and entimebic colitis, differential diagnosis. 400

ascending type. to

blood changes in. +11

ciltarrhal, 402

simptons. 407

caused by Bacillus drsenterie. 30,3

cla--ification of bacilli concerned in. 390

complications. tos

descending type, to

diagnosis. 400

dict regulation in. 4 It

drsenteric. 307

drsenteroid, 307

etiologr, 303

examination of feces in, 409

fulminating type. 404

history. 393

in liver abscess. 406

intestinal stenosis in, 406

mortality after serum therapy, fIS

pathologry. 400

permanent immunity from. is

pseuklomembranous, 405

rest treatment. $+1+$

sequelie. to8

soothing remedies in. + io

symptoms, to-

topical applications in, t to

treatment. irrigating. fis

local. ins

medical +14

prophylactic. +13

serum. + Io

supportive. +13

suruical, +20

vaccine 4 io

ulceratise. 102

dysentery, 303. 400, 407, 413. See also Bucilliry colitis.

Bacillus botulinus in decayed meat. 152 coli in decayed meat. I 52

dysenteria cause of bacillary colitis, 393 table of characteristics. $30 \mathrm{~s}$

enteriticlis in meat-poisoning. $5_{52}$ in milk, I 53

Balantidic colitis $+4^{6}$

diamosis. $+=0$

from infected pork. $+4 \mathrm{~S}$

gengraphic distribution. $44 \mathrm{~S}$

histopatholoery. $45 x-455$

in orangoutangs. $4+$;

postmortem findings in, 451.456

prognosis. +57

symptoms. 455

treatment, 457

ulceration in, 452

Balantidium coli. +40

cultivation ut. +48

in blood-ressels. 454

in teces. +50

morpholery. $4+4$

reproduction of +50
Balantidium minuteum, +46

Beef tapeworm. $+2+$

Bilharzia hamatobia. 438

Biliary insufficiency in gastrogenic diarrhea, 127

Bismuth subcarbonate in Röntgen-ray diagnusis of diarrhea, 52

Blood analyis in tubercular enteritis, $25 \mathrm{~S}$ changes in bacillary colitis, 4 I I

in entamebic colitis. 303

examination in diarrhea, +2

in syphilitic enteritis. 310

in stools. 35

Bothriocephalus latus. +24

Botulism. toxemia from. 152

Bowel perforation in entamebic colitis, 362 treatment, direct, in diarrhea, 20

Burns, diarrhea from. I $6,1 / 3$

Cachexis, diarrhea in, ios

Calkins and Hartmann's dassification of amebu, point - of difference. 333

classification of amebie. 333

Cancer and hyperplastic tubercular enteritis, differential diagnosis. 263 caused by syphilitic enteritis. $30 \mathrm{~S}$

Canned food poisoning, diarrhea in, I 55

Carcinoma and fecal impaction, difierential diagnosis, 105

complicating tubercular enteritis. 254 of stomach, gastrogenic diarrhea from, I 24

Catarrhal enteritis, acute. I $\mathrm{S}$ chronic, iso

Catheter quide, Gant's. 55\%.55S

Cecal involvement in tubercular enteritis, 212

Cecostomy. 545

appendiceal, in tubercular enteritis, 288

closure of opening after. 550

for diarrhea, 2 I

for syphilitic enteritis. 3 I9

Gant $s, 550$

in entamebic colitis. 301

in tubercular enteritis. $2 S S$

mortality from. 553

technic of. 557

through-and-through irrigation following, 552

Celiac disease. of

Celioproctectomy, $5 s_{4}$

control of bleeding in. $5 S_{5}$

preservation of sphincter muscle in. $5 s_{5}$

Ceromonas hominis. $4+4$

Cerebrospinal meningitis, diarıbea in, i 13

Cestodes, +23. See also Tapeworms.

Change of occupation in tubercular enteritis, 260

Clseese-poisoning, diarrhea in. I 53 trrotoxicon in, $15 t$

Chicken-pea poisoning. i $5 \mathrm{~S}$

Children, diseases of enteritis in, 170 older. formula for diarrhea in, 540 roung. formula for diarrhea in, 539

Chilling, diarrhea from, is 
Chilling, diarrhea from, treatment, 119

Chiloron tentatus, $4+0$

Cholera, diagnosis, 82

diarrhea in, Si

prognosis, 83

sporatlic, diagnosis, 85

diarrhea in. st

simptoms. 85

treatment. $s_{5}$

treitment. 83

winter, (liagno-is, 88

etiolog:, 80

mortality. SS

pathology. 87

symptoms. 87

treitment. 88

types 87

Choleriform siarrhea, 84

Cholerine diarrhea, 84

Chronic nephritis, diarrhea in, 62

Ciliates, $4 t^{6}$

Cladorehis watsmi. $4+2$

Classitication of amelyate $3,3,3$

of bacilli concerner in bacillary colitis, 306

of tliarrheas, 21

etiologic, 22, 23

of entamelsic collitis, 357

of entameba. 3.32. See also Amebr. clessificution of.

of tubercular enteritis, 2 ro

Claviceps purpurea. i 58

Clay bolus treatment of chronic enteritis, 208

Clifton Springs pack for chronic enteritis, 209

Climate and entamebic colitis, 320

Clothing, joroper, in tubercular enteritis, 260

Coccidia, 457

Coccidium bigeminum, 457

cuniculi, 457

hominis. 457

Coccygeal deformity. treatment, surgical, $5 \mathrm{r} \dot{8}$

Cochin-China diarrhea, pathology, 03 symptoms. 03

Cochinchinitis, 02

Colel beverages. diarrhea from. 1 io

Colic and enteric catarrh, differentiation, 195

differential diagnosis, 108

entamebic chronic, collar-button ulcers in. 350

in myxorrhea coli. th 5

in syphilitic enteritis. 305

Colica mucosa, $1 \$_{3}, 400$. See also Invorrhea coli.

Colitis, 174, 186, 200. See also Enteritis.

bacillary, 303, t00, to7, 413. See also Bucillary colitis.

balantidie, $44^{\text {ro }}$ See also Baldntidic colitis. chronic, formula for. 535

entamebic, $321,341,356,370,377$. See also Entamebic colitis.

fever in, 104
Colitis, gonorrheal, 458. See also (ionorrhoul colitis.

behmintlaic, +22. See also Helminthic colitis.

localisation, 103

membranous, fifferential diagnosis, sg8

mucoid dejectat in. 194

newroses in, rost

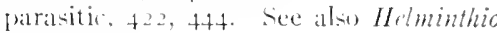
collitis.

protozoil. $4+4$

syphilitic, 204, 30.4, 313. See also Syphilitic cuteritis.

tubercular, $211,219,240,255,260,285$. rea ilso Tuberculur enteritis.

Collar-button ukers in chronic entancbic colitis. 350

Colon, involvement of, in tulecrular enteritis. 2.39

Cohnuc dilatation. diannosis. 50 exclusion in entanebio colitis. 301

intlation for cliagnosis of cliarrhed, 29 rectal tule for, 31

Coloptosis, surmical treatment, jis

(ioloctomy. 552

(iint $\left.s, 5 i^{6}\right)-5,8$

in entamelsi colitis. 301

in tubercular enteritis. 288

technic of, 575

Compensatory diarrhea, 170 treatment, 173

Congenital deformities of large intestine, diarnosis, 504

Constipation, chronic obstructive, symptoms, 400

enteritis in, rso

iratementary, 17,21

in enteritis, is

in belminthic colitis, 422

in mrxorrhea coli, $f^{6}, 5$

in tulverulat enteritis. 243

Contariou- diseases, acute, diarrhea in, 72 , 70

Coprostasis, enteritis in, 180

Coprostati diarrbea, 103

diagnosis, 105

progenosis. 105

rymptomis, 104

treatment. io5

Cravat damps in Gant secostomy. 550 , $5^{6}+4$

Culture of entamelse, 331

cysticerci in measly pork, +24

Dowmsox"s syringe, 473

Dematobia cyanentris, os

I)ialectes, diarrhea in, 1 ;

mellitus, diarrheat in. 03

I) iamosis, differential, of amyloid degeneration of intestine, 108 of alpenticitis. Ios of benign tumors of intestine, 100 of catarrhal enteritis, 107 of collic, ros

of entamebic colitis and bateillary colitis, 409 
Liagnosis. differential. of enteritis from burn. 100

of fecal impaction and carcinoma. 105 of gastrogenic and enterogenic dyspepis. 100

of hyperplastic tubercular enteritis. 202.203

of infectious diseases of intestine. ins of malimant intestinal tumors. Ioo

of neurogenic diarrhea. ios

of pancreatic bowel disturbances. ios

of peritonitis. Ios

of ptomain-poisonine. ro-

of tuberculir and syphilitic enteritis.

$$
305
$$

of typhoid iever. Io

general. of tubercular enteritis. 255

of ascariasis. + iz

of bacillary colitis. 400

of balantidic colitis. +50

of cholera. Sz

of colonic dilatation. 50-

of concenital deformities of intestine. 504 of diarrhea. 24

alba. 04

coprostatic. 105

enterosenic. ist

Hluorociope in. II

gastrotenic. I 26

in eve diseases. it

in liver diseases. 50

in mouth diseases. 55

in naspharyngeal diseases. 55

in pancreatic diseases. 61

in suprarenal diseases. 64

in thyroid diseases. $5-$

neurorenic. Itt

Rintgen rat in. 4 s

of enlarged colonic sacculations. 506

rectal valyes sos

of entamebic colitis. s-o

of enterogenic diarrhea. I it

of enteroperitoneal tuberculosis. 256

oi enteroptosis. 50 -

of enterospasm. $=0$ s

of extra-intestinal pressure. 505

of fecal impaction. 505

of gastrugenic diarrhea. I 26

of conorrheal colitis 450

of hemorrhojis $=50$

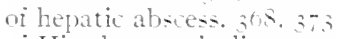

of Hirsthsprung's disease. 50;

of hyperplastic tubercular enteritis. 260 involving peritoneum, 265

of hypertrophy of levator ani. 509

of $0^{\circ}$ Beirne sphincter, $50 \mathrm{~s}$

of sphincter ani. $=00$

of internal herniz. 50 -

of inte-tinal adhesions. 505

afrections. lavage in, +1

amyloidosis. $;$ I

calculi. 505

diverticula. 505

parasites. 50.8

-trictures. 505

tumors. 505
Diagnosis of intestinal volvulus, 505

of mesenteric disease. 507

embolism. 527

of milk-poisoning. 153

of myxurrhea coli. 406

of obstructive diarrhea, 504

oi oxyuriasis. 434

of pericolitis. 500

of perisimmoiditis. 506

of peritoneal tuberculosis, 265

of posteperative diarrhea. 522

of ptomain-poisoning. 159

of rectocele, 506

of schistosomiasis. $4+0$

of splanchnoptosis. 507

of sporadic cholera. 35

oí sprue. 03

of stroneyloidosis. +36

of syphilitic enteritis. 30;

Spirocheta pallida in. so8

test-meals in. Sio

IVasermann reaction in. 308

of teniasi=. +25

of trichuriasis. 436

oi ulcerative tubercular enteritis. 256

of uncinariasis. 4.30

of winter cholera. 53

of vellow fever. - o

Liarrhea adipesa. 6 I

alba. 04

cathartica. II 5

chylosa. of

noiturna. I 2 I

I harrheal disedises surgical treatment 542 . $3+5.536 .5-7$

I)iazo-reaction in tubercular enteritis. $25 \mathrm{~S}$

liet. contrul of. in acute enteritis. 20I

in chronic enteritis. 203.204

in myxurrhea coli. $4^{t}$;

in tubercular enteritis. $=-0$

reculation in bacillary colitis. 4 it

Dietary indis retions. enteritis irom. 1;6

Digital examination in diarrhea. 46

Diphtheria. diarrhea in. $: 5$

Diplnocicus pmeumonite in hemorrhagic enteritis. 1s

Inirt-eater" anemia. 427. See also L'ncinsriasis.

I listoma hamatobium. 4 is

I iverticula, (b)structive diarrhea from, 500 of colon. surcical treatment. 515

I)rus addiction from treatment of diarrhea. 20

I) uodenal involvement in tubercular enteriti=. 212

ulcer. $1: 3$

ard castric ulcer. differentiation. I,35

differentiation irom tubercular enteri-

ti-. $2 \geqslant 0$

in enterogenic diarrhea, 133

treatment, i $; 6$

Duodenitis. localization of. 102

Dysentery, asylum. 30,3, 400, 40, 413. See also Bacillury colitis.

bacillary, 303, 400, 407, 413. See also Bacillary colitis. 
Dysentery, differentiation from tubercular | Entamebic colitis, chronic, destructive enteritis, 260

entamebis, 321, 341, 356, 370, 377. See also Entametie colitis.

helminthic, 422. See also IIetminthic colitis.

protozoal, 444

true, 305

Dyspepiit, enterogenis, enteritis in, I 78

fermentitive, in gitstrogenic diarrhea, 127 gastrogenir and enterogenic, differential diatgmosis, I00

Dyspeptic acid diarmea, I3t

diarrheil, I23, 132. See also Enterogenic diarrleat.

Eczenis, acute, diarrhea in, 67

Einhorn's duodenal tubes, 53

food-carrying cups, 53

obturators, 5.3

stomach bucket, 32

test-beats, 53

El Bicho diarrhea, 90

Electricity for chronic enteritis, 205

Electrode, hydriatic, 473

Embolism, mesenteric, 520

Emetin in entamebic colitis, $3 \mathrm{~S}_{2}$

Emulsions used in intestinal irrigation, 477

Enemal tules, 478

Enemata, 470

amount of fluid used, 472

in acute enteritis, 20?

Entameba, definition of genus, 335

Entamebe, classification of, 332 . See also Imebe, classification of.

culture of, $3.3 \mathrm{I}$

duration of life and resistance, 339

morphology of, $33^{6}$

motility of, 3,38

reproduction by schizogony, 339

by simple division, 330

within cyst, 330

Entamebiasis, 321, 341, 350, 370, 377. See also Entamelic colitis.

Entamebic colitis, $321,341,356,370,377$

abscesses in, $36 \mathrm{I}$

acids in, $3 \mathrm{SO}$

acute, appendiceal involvement in, 346

pathology, $344^{-}-346$

of pre-ulerative stage, 347

arthesions in, 302

age in, 327

and bacillary colitis, differential diagnosis, 400

angulations in, 362

antisentic remedies in, 380

appenelicocecostomy in, $39 \mathrm{I}$

astringent remerlies in, 380

atrophy in, 362

bloot changes in, 363

bowel perforation in, 362

carried by flies, 320

by individuals, 328

caused by Entamoba histolytica, 323 cecostomy in, 39 I

stirge, $35 \mathrm{I}$

Ilarris' ulicers in, 340

pathologer, $34^{\circ}$

of ulcerative statere, 348

stellate whers in, 340

symptoms, 360

undermined ukers in, 350

classes of people infected, 327

classification of, 3.57

colonic exclusion in, 301

colostomy in, 301

complications of, $36 x$

definitions, $32 \mathrm{x}$

diagnosis, 370

effect of climate in, 320

emetin in, 382

entamebic appendicitis in, $36 \mathrm{I}$

enterostomy in, 391

etioloery, 326

examination of lesions at autopsy, 373

of stools in, $37 \mathrm{I}$

fistulie in, 361

from iresh vegetables, 326

gatstro-intestinal risturbances in, $3^{6} 3$

geographic listribution, 330

hemorrhige in, 302

bistory of, $323-320$

in wair, 328

ipecacuanha in, $3 S_{1}$

irrigation in, 383

ischiorestal abscess in, 364

fistulae in, 364

location and rlistribution of lesions, $341-$ 344

metastatic abscesses in, 36 I

occupation in, 327

organisms associated with specific agents of, 322

pathology, $3+1,344-340$

perirectal abscess in, 304

fistulie in, 304

prognosis, $37 t$

racial preclisposition in, 327

rectal complications in, 363

strictures in, 364

ulcers in, 364

relief of tenesmus in. 379

resection and amputation in, 391

salts of fuinin for, 540

sex in, 327

skin affections in, $3^{6}, 2$

small intestine in, 303

stenosis in, 362

submucous abscess in,, 364

fistulae in, 364

surgical procedures in. 30 I

symptoms, $35^{6}, 360$

of algerarated type, 3.58

of diphtheric tye 350

of gangrenous iype. 350

of hatent types. 35 ,

of mild tyox, 3.57

of morleritely severe type, 357

tonics in, 3 so

toprical applications in, $38_{3}, 388$ 
Entamebic colitis, treatment, 376 dietetic, 375

local, 383

irrigants in, 386

merlicial. 379

prophylactic, $3 ; 6$

supportive, 37

surgical. $389-301$

througli-and-through irrigation in. 300

ulcers of, healing tendencies, 355

microscopic appearance, 353

urinary changes in, 303

dysentery, 321, 341, 356, 370, 377. See also Entamebic colitis.

hepatic abscess, 304. See also Itepatic abseess.

Entametba coli, 325 diagram of life cycle, $3+0$

histolytica, 02

cause of entamebic colitis, 323

Schaudinn's demonstration of pathogenicity of, 325

hominis, 325

tetragrena, 325

Enterectomy, $57 \mathrm{~S}-5 \mathrm{~S}_{2}$

technic of, $5 \mathrm{SO}^{-}-5 \mathrm{~S}_{2}$

Enteric catarrh and colic, differentiation, 105

Enteritis, I7+. I 86,200

abdominal examination in, 196

acute catarrhal, is I

macroscopic appearance, $\mathrm{I}_{2}$

microscopic appearance, i $\mathrm{SI}_{\mathrm{I}}$

dietary control in, 20I

enemata in, 202

rest in beel for, 201

treatment, 200 medical, 201

catarrhal, differential diagnosis of, I97

chronic catarrhal, 180

objective symptoms, I9o

intestinal intlation for, 209

irrigation for. 207

missige for, 204

melical treatment, 205

mineral waters for, 205

opotherapy in, 208

organotherapy in. 208

pathology, is $S_{4}$

treatment. 202

by day bolus. 208

by (Clifton Springs pack, 209

dietetic, 203. 204

electric, 205

medical, 205

Rosenberg ilry, $20 \mathrm{~S}$

surgical, 208

vaccines for, $20 \%$

vibration for. 204

constipation in, 1 s.

crouposa enclemira, 183

necrotica, diarrhea in, i 2

diagnosis, Ino

digital examination in, 196

diphtheritica, Is,
Enteritis, dyspeptic, differentiation from tubercular enteritis, 260

enterospasm in, I8S

etiology, 176,177

examination of blood in, 196

of dejecta in. Is;

fever in, i 88

folliculir. Is 3

fermenting stools in, 107

from burns, differential diagnosis, I 99

from chemicals, I $; 0$

from dietary indiscretions, $17 \%$

from intestinal obstruction. I 79

from mechanical irritants, 179

from medicines, 179

Gmelin's reaction in, ISS

green stools in, $10 \bar{i}$

hemorrhagic, Diplococcus pneumonix in, I 82

resemblance to typhoid, ISz

hepatogenic disorders in, i 78

in children's diseases, 179

in constipation, iso

in coprostasis, $\mathbf{I}$ So

in enterogenic dyspepsia, 178

localization of. IOI

membranacea, is 3

mucoid stoels in. ISS

nitusea in. is

nodularis. ist

obstipation in, 188

ocher-colored stools in. 19i

pancreatogenic disorders in, $x_{7} \mathrm{~S}$

pathology, is I

proctosigmoidoscopic examination in, 196

prognosis, 200

putrefying stools in, io

specific bacteria causing, i 75

steatorrhea in, is 8

simptoms, is6

syphilitic, 294. 304. 313. See also $S y h-$ ilitic enteritis.

tubercular, 2I I, 210, 240, 255, 266, 285

See also Tubercular interitis.

vomiting in, is 8

Enterocecostomy for diarrhea, 2I

for syphilitic enteritis, 310

Enterocolitis, I it, IS6, 200. See also Entritis.

syphilitic, 204.304, 313. See also Syphilitic enteritis.

Enteroclysis, t7o. See also Irrigation, inlestinal.

Enterogenic and gastrogenic dyspepsia, differential diagnosis, 199

diarrhea, I 32

liagnosis, 134

svmptoms, 133

treatment, 1,35

disturbances in myxorrhea coli, 462

dyspepsia, enteritis in. 1 ;

Enteroperituneal tubereulosis, diagnosis, $25^{6}$ symptoms. $24 \mathrm{I}$

Enteroptosis, thanosis, 507

obstructive, diarrhea in, 404

surgical treatment, 5 is 
Enterospatsm, diamosis, 508

in cuteritis, I $8 S$

in helminthic colitis, 422

in syphilitic enteritis, 300

obstructive diarrhea in, 495,499

sureical treatment, jo

Enterostomy, 552

in entamebic colitis. $30 \mathrm{I}$

technic oi, $57 t$

Fosinophilic diarrheal, I 2 I

Epilepser, neurogenic diarrhea in, I 43

Ergotismus, $15^{3}$

Erysipelas, diarrhea in, no

Erythema exudativum multiforme, diarrhea in, 67

nodosum, diarrhea in, 67

Examination, abelominal, in enteritis, iof digital, in enteritis. Iot) of rectum in syphilitic enteritis, 3 I I

in diarrheit, 24

in syphilitic enteritis, proctescopic, 3 ro iemoirlosentic. 3 10

of bloot in siarrhea, 42

in enteritis. $10(0)$

in syphilitic enteritis, 310

of feces, 36-40

in bavillary (olitis. 400

in entamelic colitis. 371

in helminthic solitis. $4+3$

in syphilitic enteritis, 300

macroscopic 37

microscosice 30

pathologic signifuance of to

of intestine in syphilitic enteritis, macroscopic, 310

microcopic, 310

of lesions of entamebic colitis at autopsy. 373

of stomach contents in diarrheat, in

of urine in diarrhea $+\mathrm{I}$

in (necritis, $\left.10^{0}\right)$

proctosimmoidosousic, in enteritis, agt rectal, sims' posture for, 25

Exclusion, inte-tinal, for syphilitic enteritis 319

statistics of, 200

of bowel in tuber ular enteritis, bilateral. 280

unfarorable features, 287

unilateril, 280

Exercise in tubercular enteritis, 268

Exophthalmic geiter. diarrhea in, 56,172

Eye diseases, diarrhea in, 54

Fasciolatidocana, $4+2$

Fasciolopsis buskii, $+t^{2}$

iullelwrni, $+4^{2}$

Fecal impaction and carcinoma, differential diagnosis of. 105

diagnosis. 505

surgical treatment, 51,3

Feces, examination oi, $36-40$. See also Stools.

Fever, control of, in tubercular enteritis. 277
Fibrinomentoranous partitions, sureical treitment, $5 \mathrm{I}$

lilaria sanguinis hominis, of

Fish tapeworm, +24

Fish-poisoning, diarrhea in, ist

Fistule in entamelsice whitis, 36 I ischiorectat. 3(1) 4 jerirectal. 30 ) submucous. 314

in tubercular enteritis, 252

Flaredlaten. fth

Hatus in syhilitic enteritis. 306

Flies ats carriers of entamelis colitis, 320

Fluke-worms, +37

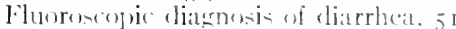

Foreign boxlics, sureriat treatment. 512

Formulae for thronir solitis. 5.35

diarribeat. 5,35

for diarrhea in infant $=5,30$

in olker children, 540

in soung thiletren, 530

for helminthis colitis, 537

Formulary, 526-5+1

Fragmentary comstijation, 21

Fresh air for tuherevlar enteritis. 268 verectables, entamebic oplitis from, 326

functional diarrhea, 1,3s. tiee also Peurogonic diarrh's.

Gint's anal douche 473

appendiceal irrigator, 508,560

ajpendira-enterocestomy, 5, -

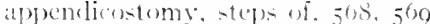
tecthnic of, 5 i)

atheter cuicle, $557,5.58$

c(c) (o) tomy. $\left.55^{6}\right)$

arlvintages of 564

(o)mments

cravat dampin 550,564

indications for. 502

method if burvine watheter in. 563

steps of $555-550$

colostomy. $=-0=-8$

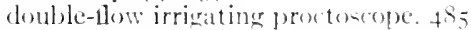

enterocecostumy. 556 . Sete also Gint? crostomy.

enterocolonic irrigator. $560,5(3)$

funnel protuserpe for intertinal irrication. 4 is:

terhnir for pas-ing colom-tulex, q

(iats distention in tulereular cnteritis. $24+4$

(sas-pipe intestine, 301

(iastric dianmolis, stomateh-tuhe for, 33

lavace, I 20

operations, pusperative diarrheal after. 520

uler and dumbenal uleer. differentiation. 135

differentiation from tubercular enteriiis, 250

Gastrodiaphany, 35

Gastrolisolu huminis. 4.2

Gistrugenic and enterogeni dyspepsia, diffurential diacromejs. Joo

diarrhea, 123 
Gastrogenic diarrhea, atonic type, I 24 biliary insufficiency in, 127 deficient motility type. I 24 diannosis. I 26 fermentative dyspepsia in. 127 from carcinoma of stomach, 124 treatment, I $2 S$ palliative, 130 surgical, I 3 I

disturbances in myxorrhea coli, $4^{62}$

Gastro-intestinal disturbances in entamebic colitis, 303

Gastroscopy. 35

Gelatin for intestinal irrication, 480

Genital diseases, diarrhea in. of male, diarrhea in, 06

Genupectoral posture for proctoscopy, it

Glanters, diarrhea in, 97

Glandular tuberculosis, irrigating treatment, 270

Gmelin s reaction in enteritis, $18 S$

Gonorrhea, intestinal. 45 s. See also Gonorrheal colitis.

Gonorrheal colitis, $45 \mathrm{~S}$

diagnosis, +50

in pederasts. $+5 \mathrm{~S}$

pathology $45 \mathrm{~S}$

symptoms. $45 \mathrm{~S}$

treatment,+50

proctitis, +53 . See also Gonormeal colitis.

Gout, diarrhea in. I 3,17 I

Gummata of intestine, pathology, 290

HANes' coal-oil treatment of entamebic colitis. $38-$

Harris ulcers in chronic entamebic colitis. 340

Hartmann's and Calkins' classification of amebe, points of difference, 3.33

classification of anebre, 332

Heat-stroke. diarrhea from. I 20

Helminthiasis, +22. See also II clminthic colitis.

Helminthic colitis, +22 constipation in, +22 enterospasm in, +22 examination of feces in, 443 formulie for. 537

obstipation in, 422

dysentery. +22 . See also II minthic colitis.

Hemorrhage in entamebic colitis, 362

in mesenteric embolism, 527

Hemorrhoirls, diacnosis, 500

surrical treatment. 517

Hepatic abscess, diagnosis, 368,373 entamebic, 30. See also IIcpatic absecss.

histopathology, 366

lisation of, 365

microscopic appearance, 367

number of, 365

prognosis, 37 it

routes of infection, 364

size of. 365
'Hepatic abscess, symptoms. 368

table illustrating site of rupture, 366

treatment, 302

surgical, 302

Hepatogenic disorders in enteritis, $17 \mathrm{~S}$

Heterophyes, $+t^{2}$

Hill-diarrhea, ot

Hirschsprung's disease, diannosis, 507

History taking in diarrhea, 24

Hookworm disease, +27. See also Uncinariasis.

Hot water for intestinal irrigation, 474

Hydriatic electrode. 473

Hỹdrotherapy for chronic enteritis, 205

Hyperacidity diarrhea, 124

Hypersensitive areas, neurogenic diarrhea from. It+

Hypertrophy of anal sphincter, surgical treatment. $5 \mathrm{I}$

of O'Beirne's sphincter, surgical treatment, 516

of rectal valres. surgical treatment, 516

Hypodynamia cordis, diarrhea in, i i 6

Hysteria, neurogenic diarrhea in, Ift

ICE-CRE.U poisoning. diarrhea from. I 53

Ice-water for intestinal irrigation, $4 i t$

Ileac involvement in tubercular enteritis, $2 \mathrm{I} 2$

Ileitis, localization. Io 2

Ileocecal catarrh, diagnosis, 105

Ileocolectomy, statistics oi, 2 So

Immunity, permanent, from bacillary colitis, tis

Imperforate anus, surgical treatment, 5i I

Infants. formula for diarrhea in, 530

Infarction. intestinal, 526. See also Mesenteric cmbolism.

Infectious diseases, acute, diarrhea in, 72 , 79 miscellaneous, diarrhea in, or

Inflammatory diseases, surgical treatment, $5+2,5+5,556,57+$

Inflation of intestine in chronic enteritis, 209

Influenza, diarrhea in, 75

neurogenic liarrhea in, $\mathbf{I}+3$

Inspection of patient in diarrhea, 26

Internal hernia, diagnosis, 507

Intestinal adliesions, diagnosis, 505 surgical treatment. ₹ 13

affections, lavage in diagnosis, t I amvloidosis, oo

auto-intoxication, neurogenic diarrhea in, I +3

calculi, diagnosis, 505

catarrh, non-specinc. I7t. See also $E_{n}$ teritis.

diverticula, diagnosis, 505

exclusion, $5,5-5 \mathrm{SS}$

bilateral. 580

unilateral, $58 s$

gonorrhea, 45 S. See also Gonorrheal colitis.

infarction, 526. See also Mescuteric embolism. 
Intestinal irrigation, 470

myiasis, diarrhea in, 08

neoplasms, obstrurtive diarrhea from, foo

obstruction, enteritis from, 170

from parasites, surgial treatment, 516 parasites, diagnosis, 508

obstrutive diarrhea from, 400

schistosomiltsis, 440

sterosis in bacillary colitis, 400 operative treatment, statisties of, 201

strictures, diagnosis, 505

obstructive diarrhea from, 408

surgical treatment, 512

syphilis, 204, 304, 3r3. See also Syphititic contcritis.

tuberculosis, $211,210,240,255,260,285$. See also Tubercular coleritis.

tumors, diagnosis, 505

surgical treatment, 5 I 2

ulcers, syphilitic, $208-30$ I

volvulus, diagnosis, 505

Intestine, amyloil degeneration of, differential diagnosis, iog

gummata of, pathology, 209

infectious diseases of, differential diagnosis, 108

small, in entamelic colitis, 363

stricture of, pathology, 200-301

tubercular ulcer of, characteristics, 222

Invagination, obstructive diarrhea from, 502

Involuntary bowel moxements in neurogenic diarrhea, $14+4$

Ioxlipin in syphilitic enteritis, 36

Ipecaruanhit in entameloic colitis, 38 s

Irregularities of living, diarrhea from, 114

Irrigants in focal treatment of entamebic colitis, 386 ,

used in intestinal irrigation, 472

Irrigation in chronic enteritis, 207

in entamebic colitis, 383

in myxorrhea coli, tos

intestinal, 470

amount of thid used, 472

of oil userl, 470

apparatus for, $482-487$

by anus, $47 \mathrm{I}$

electrification of irrigant in, 487

emulsions used in, 477

frequency of 472

Giant's funnel proctoscope for, 482

gelatin for, 4 So

hot water for, 477

ire-water for, 474

irrigants lsed in, 473

limitations ot, 473

medical agents useel in, $474^{-477}$

oils userl in, 477

jositions for, 481

technic for, 480

through-and-through, $47 \mathrm{I}$

through artificial opening, 47

Irrigator, Ciant's appendiceal, 568, 560 enterocolonic, $5(00.56)$

Ischiorectal abscess and tubercular enteritis, 245
J.14tson's seat syringe, 470

Jejunal involement in tubercular enteritis, 2 I 2

Jejunitis, localization of, 102

Jelk's formalin-loric solution in treatment of contamclic colitis, 387

KuDNEY diseases, diarrlica in, 62,172

1.1ubLt. intestinislis, 4.4

bardaceous degeneration, 60 . See also Amytoidosis, intestinut.

lathyrismus, 158

liavage, gastric, 120

in diagnosis of intestinal affections, 4I

Learl-peisoning, diarrhea in, ato

leukemia, diarrhea in, roo

levator ani, hypertrophy, diagnosis, 500

lienteric diarrhea, 123,132 . See abo Enterogenie diarthen.

Life-cycle of Entamexbat coli, 340

Liver abscess in bacillary colitis, 406 discases, diarrhea in, 50

Loesch's discovery of Ameda coli, 323

Lumbricoid worms, 431

Mandsutes, diarrhea in, 158

Malaria, diarrhea in, 78,06

Marasmus, diarrhea in, 112

Massage for chromic enteritis, 204

Measles, diarrhea in, 74

Meat-pejsoning, diarrhea from, 150

Mechanic diarrhea, 122, 488, 496, 504, 510. Sice also ()bstructive diurrhere.

Medicinal-chemical poisonine, I03

Melena in syphilitic enteritis, 300

Membranous enteritis, f(o. She also, Myorrhen coli.

Mercurial poisoning, diarrluea in, 165 treatment, io,5 preparations in syphilitic enteritis, 317

Mercury and potassium iondid in sophilitic enteritis, 315

Mesenteric discase, diagnosis, 507

(embolism, 520

diagnosis, 527

etiology, 526

liemorrliage in .527

pithlology, 526

promosis, 528

symptoms, 527

ireatment, 528

thromlosis, 520. Sie also Mesenteric rmbotism.

Methenortobinemia, diarrhea in, 11.3

Migraine, neurogenic diarrhea in, 14t

Milk, Bacillus enteritidis in, 1.5 .7 products, poisoniug from, diarrluea in, I 5.3

Mlilk-poisoning, diagnosis, 153 diarrhea in, $5_{5} 2$

Mineral waters for chronic enteritis, 205

Miscellaneous poisons, chemical, diarrhea from, 108 
Miscellaneous poisons, medicinal, diarrhea from, ios

Mortality in bacillary colitis. 4 is

Mouth diseases, diarrhea in, 54

Mlucus in stools. 39

Muscarin poisoning, diarrhea in, 157

Mushroom poisoning, diarrhea in, I 57

Myiasis, intestinal, diarrlea in, 98

symptoms, oq

treatment, 90

Myxorrlsea coli, is 3,460

colic in, 405

constipation in, $4^{65}$

diannosis, 460

diet in. 407

enterogenic disturbances in, 462

etiology, 460-405

from surgical conditions, 463.464

gastrogenic disturbances in. 402

history of 400

in adenoidism, $4^{6} 3$

in thyroidism, 403

irrigation $\mathrm{in}$, fos

mucoid eracuations in, $4^{65}$

nervous phenomena in, 460

momenclature of, $4^{60}$

pathology, 405

prognosis, 400

symptoms. 465

treatment. 160

ast ringent, foS

surgical, 468

colica, too. See also Myorrhea coli.

membranacea. foo. See also Myorrhea coli.

Nisopharygerl diseases, diarrhea in, 55

Necator americanus, 427

Sematodes. 427

Neosaluarsan $j$ sy splilitic enteritis, 315

Neurasthenia, neurogenic diarrhea in, Iti

Neurogenic diarrhea. I2 I, I is

diamosis, It 4

differential diagnosis, ros

from hypersensitive areas, It

in epilepsy. I +3

in hysteria, ity

in influenza, I 43

in intestinal auto-intoxication, $\mathrm{I}+3$

in migraine, It

in neurasthenia. 144

in tabes dorsalis, $1+3$

involuntary bowel movements in. Ift

reflex disturbances in, I 42

symptoms. 144

Nimht-sweats in tubercular enteritis, troatment, $2-5$

Cocturnal diarrhea, 57, i 35 . See also Veurogenic diarrhed.

Nictotherus africanus, +46

fiaba, $44^{\circ}$

viganteum, $44^{6}$

G'BEIRVE's sphincter, hỹpertrophy of, cliarnovis. 508

obstructive diarrhea from, 405. 503
(') Beirne's sphincter, hypertrophy of, surgical treatment, 510

Obesity, diarrhea in, Ioo

treatment, roo

Obstipation in enteritis. ISS

in helminthic colitis, 422

in iubercular enteritis, 243

Obstructive diarrhea, 21, 485, 496, 504, 510 chronic, symptoms, 496

description, 488

diagnosis, 504

enterospasm from, 409

etiology, 400

from adhesions, to2

from colonic malformation. 493

from congenital reformities. $40 \mathrm{~J}$

from disease of mesentery. 404

from diverticula, 500

from extra-intestinal pressure, 49 I

from fecal impaction. 402

from foreign bodies, 402

from hypert rophy of (0)Beirne's sphincter, 405.503 of rectal values. 503

from intestinal deviation, 493 neoplasms, 409

parasites, 400

stricture, 408

from invagination, 502

from paralytic ileus, 502

from ptosis of sigmoid flexure, 501

from rectal diseases, 405.503 stenosis, 400

from splanchnoptosis. 500

from strictures, 403

from tumors, 40.3

general remarks. $458-490$

in enteroptosis, $49+$

in enterospasm. 405

in paralytic ileus, 405

in pericolitis, 404

in perisigmoiditis, 404

in splanchnoptosis. 494

lesions causing. $49 \mathrm{I}$

location of tso

symptoms, fot

treatment. 5io

non-operative. 5 10

surgical, 5 I I

()cupation in entamebic colitis. 327

(Esophagostoma brumpti, 430

()ils used in intestinal irrigation, 477

()l age, diarrhea in, I2 I, I >0

()psonic index in tubercular enteritis, $25 \mathrm{~S}$

()rganotherapy in chronic enteritis, 208

( )xyuriasis, 433

diagnosis, 434

symptoms, 433

treatment, 4.34

Oxyuris vernicularis, 4.33

P.ilp.itiox in cliarrhear. 27,28

lancreatic bowel clisturbances, differential diagnosis, 100

diseases, diarrliea in, 60 
Pancreatogenic disorders in enteritis, i -8

Paradyentery, 306

larafytic ileus, obstructive diarrhea from, $\$ 05,502$

Parameetsa hominis, 320

Paramecium roli. $44^{6}$

Parasitic colitis, $422,+4.4$. See al lso $/ 1 / 6$ minthic coltitis.

discalses, surgital treatment, $5+2,545$. $55^{0}, 57$.

Patient, preparation of, for sureical treatment, 5.43

Pederasts, enonorrheal colitis in, 458

Pellagra, diarrhea in, 90,158 pethology: 100 simptoms, og trealment, ror

Pemphigus acutus. diarrhea in, 67 vulgaris, liarrhea in, 6-

Peptonitic reat ton in tubercular enteritis. 258

Percussion in cliarrheat. 27

Pericolic membranes, surgical treatment, 513

Pericolitis, diatgnosis, sot

obstructive diarrhea in, 494

Perisignoiditis, diatenosis, 5or, obstructive diarrhea in. 104

Peritoneal tuberculosis. diagnosis, 265 irrigating treatment, 270 symptoms. 248

Peritoneum, involvement of, in tubercular enteritis, 223. 237

Peritonitis, differential rlitgmonis. Ios with perforation in tubestudar enteritis, 251

without perforation in tubercular enteritis, $25 \mathrm{I}$

Perityphlitis antinomyoutica, 102

Pertussis, diarrhea in, 75

Pettyohn's stab-wound appendiontomy. 57.57 .3

Phosphorus-poisoniner. diarrhea in, 160

Physaloptera caucasial. +37 mordens, 4.37

Pin-worms. 4.3 .3

lityriasis rulbra, diarrhea in, 07

I'arge, diarrhea in. es,

Pneumonia, diarrhed in, -6

lork. measly. cysticer i in. 424 tapeworm. +23

Poitions for intestinal irrigation, $48 \mathrm{~s}$

Poitoperative diarrheat, $5 \mathrm{~m}, 520$

(liagnusis. 522

etiulury, $510-522$

fotholiegy, $510-522$

symptoms, 522

treatment. 523

surgialal. 52.4

Postprandial diarrhea, 13 . Sce also Veurogenir diwrhes.

Potassium iodid and meroury in syphiliti enteritis, $3 \mathrm{I} 5$

Potato-poisoning, riarrhea in, $15^{\circ}$

solanin in. 157

symptoms, is 57
Preparation of matient bor surerial treatment, $5+3$

Procidentia reoti, surrical treatment, 518

Prostertomy, 582

inferior, 5it 3

superior, 5 sts

Proctitis, diamenosis, oror gonorrheal. +5s. Sece also Gonorrtat coltitis.

Protomopir examination in rliarrheat. +3

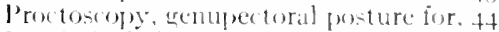

l'rophylaxis in ptomain-poisoninge ror in tubercular enteritis, 207

P'rotozonal onditis. $4+4$ dyenters, +4.4

Pscudodyentery. 305

P'ecurlor lyenteric diarrhea of

Peudo-enteritis, 183

Preudoleutemia, rliarrhea in, i 10

Pseurlomembranous bar illary coliti- 405

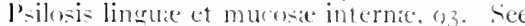
(2) lo Sorwe

Pioats abriess, differentiation from hyperplastic tubercular enteriti- 20,5

P'syhic nervous diarrhea, 1,30)

Ptomain-poisoning, diatono-i-, 150 diarrhea from, i 50

differential diagnosin of. 107

differentiation irom tubercular enteritis, 260

prophylaxis. if

symptoms, 150

(reatment, it)

Pucrueral diarrhea of beneal. of

Purpura, diarrhea in, so

pus in feces. 30

(OCIN. salts of for entameloic colitis, $5+0$

R.1 $1.1 \mathrm{~L}$ predisposition in entametio colitis. 327

Rectal cancers, 1 reatment. sureical. is 2 conmlieations in entamebic colitis. 36,3 diseases. obstrutive diarrhea from. 4o5. 50.3

eximination, Sims posure for. 25 polypi, treatment, sureriat. 5 I -tenois. obstruetive diarrhea from. 400 strictures in entamel)ic (o)litis. 3114 tulse for colonic inflation, 31 ulcers in entameloic colitis, $3^{\text {fit }}$

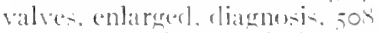

hogertrophied, sureis al treatment. 516 obstrutive diarrheal froms. 503

Rer torele, diagnosi-son

Retorolonic tran-illumination. \&3

Rectum. congenital eleformities of surgial treitment. 5 I I

digital cxamination of. in tubereular enteritis. is

involvement of, in tulerenlarenteritis. 2,30

Reflex di-turbances, rliarrbeat in, 122 in neurogenie diarrheal, $1+2$

Relapsing fever, diarrhea in, si 
Remerlies, symptomatic, for tubercular Spirocheta pallida in syphilitic enterocoenteritis, 276

Resection and amputation in entamebic colitis, 30 I

of bowel in tubercular enteritis, 286

Rest for tubercular enteritis, 268

in bed for acute enteritis, 201

Rhabditis intestinalis, 436

Röntgen ray in diagnosis of diarrhea, is bismutl subcarbonate for, 52

Rosenberg dry treatment for chronic enteritis, 208

Round worms, 427,431

SALMRSIN in syphilitic enteritis, $3 \mathrm{I} 4$

Saluage-poisoning, toxemia from, $I_{5}$

Scarlet fever, liarrhea in, it

Schaudinn's demonstration of pathogenicity of Entamoba histolytica, 325

Schistosoma hicmatobium, 438

japonica, 437

mansoni, +38

Schistosomiasis, 437

etiology, 437

intestinal, $4+0$

treatment, +4I

pathology, 438

prognosis, $4+1$

symptoms, 40

treatment, $4+1$

urinary, +41

Schizogony, reproduction of entameba by, 330

Sclerema neonatorum, diarrhea in, 67

Scurvy, diarrhea in, I 3

Sepsis, diarrhea in, so

treatment, 80

Septedera intestinalis, 4.36

Serum treatment of bacillary colitis, 416 mortality after, $4 \mathrm{~S}$

of tubercular enteritis, $28_{2}$

Sex in entamebic colitis, 327

in tubercular enteritis, 214

Shell-fish poisoning, diarrhea in, 154

Short-circuitine of bowel in tubercular enteritis, 286

Sigmoicl, chronic invagination of, surgical treatment, $5 \mathrm{I} 3$

involvement of, in tubercular enterit is, 239

ptosis of, obstructive diarrhea from, 501

Sigmoilitis, diagnosis, 105

Sirmoicloscopic examination in diarrhea, $+3$

Sims' posture for rectal examination, 25

Sitoplubia, diarrlsea in, is 7

Skin alfections in entanebic colitis, 362 (liarrhea in, 07

treatment, $6 \mathrm{~S}$

Solanin in potato-poisoning, I 57

Soothing remedies for tubercular enteritis, $273-275$

Sphincter ani, hypertrophy, diagnosis, 500 preservation of, in celioproctectomy, 585 
Syphilitic enteritis, 204, 304, 3 I 3 and tubreular enteritis, difierential diannosis. $30 \mathrm{~s}^{\prime}$

appendicostomy for, 310

blood examination in, 310

cancer ratused by. jos

cecostony for, 310

colic in, 305

diagnosis of, 307

Spirmheta pallida in, 308

test-meals in, 310

lliscermann reaction in, 308

differentiation from tubercular enteritis. 250

enterocesolomy for, 310

enterospism in, 300

etiolosy, 200

examination of intestine in, macroscopic, 3 ro

of stools in. 300

flatus in, 306

intestinal exclusion for, 310

ioxlipin in, 316

melena in, 300

mercurial preparations in, 317

mercury and potassium iodid in, 3 I 5

neosaliarsan in, 3 is

pathologr, 207. 302

proctoscopic examination in, 3 io

prognosis, 3 I 1

saluarsan in, $3 \mathrm{I} 4$

siemolibseopic examination in. 3 10

Spirochata pallislat in, $4+6$

simptoms, 304

treatiment, 31,3

medicinal, 31,3

mercurial, by injection, $3 \mathrm{r} 7$

surgical. 318

tuber ulosis caused by, 308

urinalysis in, $3 \mathrm{I}$ I, $3 \mathrm{I}_{2}$

enternculitis. 204. 304. 313. See also Suphititic enteritis.

intestinal ulcers, 20S-30

Syringe, Daridion's, 473

fountain, 475

harel-rubber enema, ti I

Jamison's seat 470

metal piston enemal, 472

T.nBes dorsalis, neurogenic diarthea in, $1+3$

Table of dharacteristics of Bacillus dysenteriat. 30 s

illustrating site of rupture in hepatic abseess, 300

Tienia latil, 424

nana, 425

silvinata, $42+$

solium, 423

Tilpeworm, 423

beef, 424

tish, 424

pork, +23

Technic for intestinal irrigation, 4 So for passing colon-tube. (iant's, +86 of cecostomy, 55t
1 Tenesmus, relief of, in ("ntamelsic (s)litis, 370

Tenialsis, dianmesis, $4^{25}$

simptoms, 425

treitment, 420

Test-meals in cliatemosis of spplilitic enteritis. 310

in riarrheat. 32

Threat-worms, ti.

Thrombuss, menenteris, 526. See also Hesmeric cmbulism.

Through-and-through irriation, +7 following appemalicutumy. 552

(acostomy. 552

in surcial freatment of cntamebic rolitis, 300

in tulbercular enteritic, 285,288

Thyroid diseases, diturrhe'a in, 5i,

lhyroblism, mysorrhea coli in, fos

Tonic remedie in tulserular enteritis, 272

Tonis in entamelis aliti-. 3, so

Topical appliations in batillary colitis, fio in entamelic colitis, 38.3

in tubercular enteritis, so

Toxemia from botuliom, 152

from deatyel meat, 152

from diceided meat, 151

from sallsilge-prisoning. I52

Toxic diarrbea, 150, 193

Toxins, absorption of, in bacillary colitis, for

Transillumination, rectocolonis, tz

Treament, eletric, for chromi enteritis, 205

irrigating, of grandular tubercuksis, 279

of peritoneal tubermbis. 270

of tubercular enteritis. $2-8$

(leep ulecrative, 2-5

librosclerotic, 270

hyperplastic, 270

siuperlicial ulcerative, $2-S$

medical, of rhronic enteritis, 205

of diarrheit, 10,20

of sybilitic enteritis. 313

of tubercular enteritis. 271

merourial, by injetion, in syphilitic enteritis, 317

non-operative of obstruttive diarthea, 510

of acute enteritis, 200

of atscarialsis. tis

of batcillare onlitis, irrisating, +18

latal + is

medicill, tif

prophylartic, 41.3

serum, tro

supportive, +it

slrerical, +20

vature, +10

of bilantili tollti-. 457

of cholera, si

of cliarrlyeat all rat. of

(at hartiois, 110

compensiltory. 17.3

coprestatis ios

conterogente 1 is

from (hilling. i I ) 
Treatment of diarrhea from cold beverages. 'Treatment. operative. of tubercular en$1 \geq 0$ ga-trogenic. I $2 S$

in acute infection diseases. -6

in alcoholism. II

in alkali-poisning. $10 \mathrm{~s}$

in bone diseases. os

in chronic nephritis. 03

in diabetes mellitus. 0 :

in eye diseases. $=1$

in temale cenital diseases. 65

in liver diseases. to

in male aenital diseases. 65

in mercurial poisoning. 165

in mouth diseases. $5 z$

in nasopharyngeal diseases. 56

in obesity. io-

in pancreatic diseases. $6 \mathrm{I}$

in relap-ing iever. si

in sep-is, so

in sin diseases, of

in suprirenal diseases. 64

in throid diseases. 5 -

of entamebic colitis. $3-6$

dietetic. 35

Hane: coal-oil. 5s-

Jelks" formalin-boric solution in. 35 ;

local. is:

irrigant: in. 355

medical. $;-0$

prophylactic. $:-6$

supoortive. $3,-$

surcical. $350-30 \mathrm{I}$

by thrumgh-and-through irrigation. :00

of conorrheal colitis 450

of hepatic absess. 302

surcical. 502

of intestinal amyloidosis. I I

myiasis. 00

shisosomiasis. +41

of me-enteric embolism. 523

of mysorrhea coli. $\rightarrow$ rio

of night-sweat in tubercular enteritis. $2,-5$

of obstructive diarrhea. 510. 5 I I

oi oxruriasis. fit

oi pellagra. ior

of postoperative diarrhea. 523 sursicial. =2.4

of ptomain-poiconing. If I

of shistosomiasis. +? I

rif smoradic cholera. 55

ni strongyloidosis. 4,36

of sphilitic enteritis. 3 I 2

of teniasis. 426

of trichiniasis. $4: 0$

ot trichuriasis. 4 is

oi tropical diarrhea. of

rif tubercular enteritis. irrigating. 280 surgical. 255

ri uncinariasis. $\rightarrow: 0$

oi urinary diarrhea. of

of winter cholera. so

af yellow fever. i

operative of intesinal stenosis, statistic of. 20 I

teritis. author's conclusions in, $201-203$

statistics of, 200

routine. in tubercular enteritis. 266

serum. of tubercular enteritis. 282

surcical, general remarks on, $5+2$

of anal tisure. 5i;

of chronic invagination of sirmoid, 513

of coccyeal deformity. 5 is

of coloptosis. 5 is

of congenital deformitie of rectum. 5 I I

of diarrheal diseases. 542.545 .556 .574

oi diverticula of colon, 5 I 5

of enteroptosis. 515

of enterospasm. 516

of iecal impaction. 513

of nibrinomembranous partitions. 5I I

oíforeign bodies. 512

of hemorrhoids. $51-$

of hypertrophy of anal sphincter. 51;

of $\mathrm{O}^{*}$ Beirne" sphincter. 516

oi rectal valves. 5 I 6

of imperiorate anus. 5 II

of intlammatory disease=. $542,5+5 \cdot 556$. $5-7$

of intestinal adhesions. 513

ob-truction irom parasites, 516

strictures 512

tumors. Fi 2

of mysorthea coli. 4 s

of parasitic diseases. $542.545 \cdot 556.574$

of pericoli membranes. 513

of procidentia recti. sis

oi retal cancers. 5 I 2

polypi. jis

of splanchnoptosis, 515

of syphiliti enteritis. 3is

of tubercular enteritis. local anesthesia

in. 285

of solvulu=. is?

preparation oi patient for. 543

Trematodes. 437

Trichinella spiralis. $\$ 30$

Trichiniasis. +30

simptums. 430

treatment. 430

Trichocephaliasis. 435. See also Trichuriasis.

Trichocephalus dispar. $\$ 35$

hominis. 4,35

trichiuris. $\div: 5$

Trichomonas hominis. 414

Trichostronatlus. types of, 437

Trichuriasis. 435

diamnosis. $4: 36$

symptoms. $+\leqslant 6$

treatment. $\$ 30$

Trichuri- trichiura. 4,35. 4,36

Triodontophorus diminutus. $43 ?$

Tropical abscess. 364. See also IIepatic abscess.

True dyentery. 305

Tubercular colitis. 2II. 210, 240. 255. 266, 285. See also Tubercular cnteritis.

enteritis. 21 I. 210. $240,255.200 .285$ 
Tubercular enteritis, abscesses in, 252 adhesions of bowel in, 252 advanced, mixed infection in, 245 age of oncurrence, $2 \mathrm{I} 4$

albuminuria in, 257

and ischiorectal abscess. $2+5$

and syphilitic enteritis, differential diagno-is, 30 s

antiseptic remedies for, 273-275

appendicostomy in, 288

bilataral exclusion of bowel in, 280

blood analysis in, 258

carcinoma complicating, 254

cecil involvement in, $2 \mathrm{I}_{2}$

cecostomy in, $28 S$

change of occupation in, 269

clasitication, 210

colostomy for, 285

complications, 249

constipation in. $2+3$

controlling fever in. 277

deep ulcerative. irrigating treatment. $2-8$

pathology, 223

diarrhea in, 242,255

diazo-reaction in, $25 \mathrm{~S}$

diet in, $2>0$

differentiation from rluodenal ulcer, 250

from dysentery, 260

from dyspeptic enteritis, 260

from gastric ulcers, 250

from ptomain-poisoniner, 260

from syphilitic enteritis, 259

from typhoid fever. 250

dicital examination of rectum in, 3 I I

duodenal involvement, 212

etiology, 2I3

exclusion of bowel in, bilateral, 286 unfavorable features, 287 unilateral, 286

exercise in, 208

fibrosclerotic, $2.34,248$

irrigating treatment, 279

fistulæ in, 252

loods permitted in, 270

prohibited in, 271

frequency in general tuberculosis, 2 I0

iresh air for. 20,8

gas distention in. $2+5$

general diagnosis, 255

glandular, 2.35

s-mptoms, $2+8$

hyperplastic, 227

age in, 227

and cancer, different iat diagnosis, 263

appearance of intestine in. $23 \mathrm{I}$

bovine bacithos in, 220

diagnosis. 260

difierential diamnosis, 262

diferentiation from appendicitis, 264 from psoal-abaseses, 205

frequency of occurrence, 228

history. 227

involvement of appendix, 233

of enlands, 2.34

of muscular tunic, 232
Tubercular enteritis. hyperpla-tic involvement of pritoneat wat. 233

of sulmucosa, 2,32

() $\hat{\mathrm{f}}-\mathrm{ub})-\mathrm{e}$ rosial, 23.3

irrigating treatment. 270

mucus mentsune in, $2.3 \mathrm{I}$

polyguid erroutlo in, 2,32

symptoms. 2.t'

ilea involvement in, 213

improving exeneral health in, 207 mental condition in. 26$)$.

in miliary tuberculosis, zt'

in tuberculosis of uther wreitns, tilble of. $\left.2{ }^{\prime}\right)$

infrepuency in general tulserculusis, 220

involvenent of apurendix, 2.35

of (a)lon, 2.34)

of peritoneum. 223.2.37

diarnosis, 205

symptoms, 248

of rectum. 230

of siemoint flexure. 230

of stomach, 2,3 s

irriqating treatment, $2-8$

jejunal involvement in. 2I 2

kinks of bowel in. 252

miliary tuberculosis in, 25.3

morle of intection. $21 t$

() $)$-tipation in. 24.3

verative treatment. author's conclusions on, $201-20,3$

opsonic index in. 258

pain and tendernes in. 24.3

peptonitic reartion in, 258

peritonitis with perforation in, $25 \mathrm{I}$

without perforation in. $25 \mathrm{I}$

predisposing culuses. $2 \mathrm{I}_{3}$

primary, 217

table of. 217

proper tothing in. 260

prophylaxis in. $26 \%$

relative ireguency of, 2 I I

resection of bowed in. $28 \%$

resembling ateinomy̧usis, 259

amyloid degeneriation, 250

appendiciti- 245

rest in beel lior. 20,8

rontine treatment. 200

secontlary. 21 ;

serpuelie, 24 ti

serum treatment. 2siz

sexin. 2 It

short-ircuitine of bowel in. 2he

wothiner remedies for. $273-275$

stattistis of operative treatment. 200

stricture, 2f(s)-25I

styptic remerlin - in. $2--$

supernicial ulecative, irrigating treat ment. 2rs

symptomatis remerlic-ior. $2-t)$

through-ind-throuth irriration in. 25 ;. 288

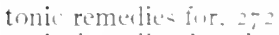

topical applitation-in. 250

treatment. merlinal. $2-1$

surariall, 2ys 
Tubercular enteritis, treatment, surgical, Lncinariasis, prognosis, 430 local anesthesia in, 285 irrigants used, 280 of night-sweits in. 278

tuberculin reaction in. 257

twists of bowel in, 252

ulcerative. diagnosis, 256

symptoms. 240

ulcer of intestine, characteristics, 222

Tuberculin reaction in tubercular enteritis, 257

Tuberculosis, enteroperitoneal, diagnosis, 256 symptoms. $24 \mathrm{x}$

general, frequency in tubercular enteritis. 2 I9

infrequency of tubercular enteritis in. 220

glandular, irrigating treatment, 279

intestinal, $211,210,240,255,266,285$. See also Tubercular cnteritis.

miliary. in tubercular enteritis. 246. 253 of other organs, tubercular enteritis in. table of, 216

peritoneal, diagnosis, 265

irrigating treatment. 279

simptoms, 248

syphilitic enteritis in. 308

Tumors of intestine, malignant, differential diagnosis, I09

benign, differential diagnosis, 199

Typhlitis. diagnosis, I05

Typhoid fever. differential diagnosis of, I97 differentiation from tubercular enteritis, 259

resemblance to hemorrhagic enteritis. $\mathrm{I} 82$

Typhus ferer, diarrbea in. 79, 06

Tyrotoxicon in cheese-poisoning, I5t

LLCERS, uremic, 62

Uncinariasis, 427

diagnosis. 430

pathology +20

symptoms. +29

treatment. +30

Unsegmented worms, 427

Uremic ulcers, 62

Urinalysis in syphilitic enteritis. 3 I I

Urinary changes in entamebic colitis, 363

diarrhea. 60

treatment. 66

schistosomiasis, $44 \mathrm{I}$

Urine, examination of, in diarrhea, $4 \mathrm{I}$

Urticaria, diarrhea in, 67

VACctNe treatment for bacillary colitis, $4 \mathrm{r} 6$

Vaccines for chronic enteritis, 208

Vaginal proctectomy: 584

Vahlkampfia, 3.33

Varicella, diarrhea in. $7 t$

Variola, diarrhea in, 75

Vibration for chronic enteritis, 204

Volvulus, surgical treatment, 5 I 3

WASSERMAx reaction in diagnosis of syphilitic enteritis. 307

Whip-worms. 435

Worms, flat, +23

fluke-, +37

lumbricoid. +3 I

pin-, 433

round, +3 I

tape-, +23

thread-, 433

whip-, 435

X-RAY in diagnosis of diarrhea. See also Röntgen ray.

Yellow fever, diagnosis, So

diarrhea in. 79

prognosis. 80

treatment, $S_{I}$ 




\section{SAUNDERS' BOOKS}

\section{- \\ Skin, Genito-Urinary, \\ Chemistry, Eye, Ear, Nose, and Throat, and Dental}

\section{W. B. SAUNDERS COMPANY}

WEST WASHINGTON SQUARE PHILADELPHIA 9. HENRIETTA STREET, COVENT GARDEN, LONDON

\section{Davis' Accessory Sinuses}

Development and Anatomy of the Nasal Accessory Sinuses in Man. By Warren B. Davis, M. D., Corinna Borden Keen Research Fellow of the Jefferson Medical College, Philadelphia. Octavo of 172 pages, with 57 original illustrations.

Cloth, $\$ 3.50$ net.

\section{ORIGINAL, DISSECTIONS}

This book is based on the study of two hundred and ninety lateral nasal walls, presenting the anatomy and physiology of the nasal accessory sinuses from the sirtieth day of fotal life to aduanced maturity. It represents the original research work and personal dissections of Dr. Davis at the Daniel Baugh Institute of Anatomy of Philadelphia and at the Friedrichshain Krankenhaus of Berlin. It was necessary for Dr, Davis to develop a niw tichic by which the accessory sinus areas could be removed en masse at the time of postmortem examinations, and still permit of reconstruction of the face without marted disfigurement. Ninety-six cases in this series were thus obtained. The tubles of arerages, griving you the age, size of ostia, origin, thickness of septum, and anterior and posterior walls, vertical, lateral, and posterior diameters, and relation to the nasal hoor, form an extremely valuable feature. 


\section{Stelwagon's \\ Diseases of the Skin}

A Treatise on Diseases of the Skin. By Hexry W. STELWAGON, 1I. D., PH. D., Professor of Dermatolog! in the Jefferson Medical College, Philadelphia. Octaro of 1250 pages, with 33 I text-cuts and 33 plates.

Cloth, \$6.00 net; Half Morocco, \$7.50 net.

\section{THE NEW 7th EDITION}

There are two features in Dr. Stelwagon's work that stand out above all the others: The special emphasis given the two practical phases of the subjectdiagnosis and treatment; and the wealth of illustrations. These latter are of real value. They teach you diagnosis as no description can. Many of these illustrations are in colors.

Over 75 pages of the work are devoted to syphilis, giving you the Wassermann test, the salvarsan ("606") treatment, and all the newest adrances. Pellagra, tropical affections, hookworm disease, Oriertal sore, ringworm, impetigo contagiosa -all those diseases being so widely discussed to-day.

George T. Elliot, M. D., Professor of Dermatology, Cornell C'nizersity.

"It is a book that I recommend to my class at Cornell, because for conservative judgment, for accurate observation. and for a thorough appreciation of the essential position of dermatology, I think it holds first place."

\section{Schamberg's $D_{\text {iseases of the }}$ Skin}

\section{and Eruptive Fevers}

Diseases of the Skin and the Eruptive Fevers. By I.AY F. SCHAMBERG, M. D., Professor of Dermatolog: and the Infectious Eruptive Diseases, Philadelphia Polyclinic. Octaro of 573 pages, illustrated. Cloth, $\$ 3.00$ net.

\section{THE NEW (2d) EDITION}

"The acute eruptive fevers constitute a valuable contribution, the statements made emanating from one who has studied these diseases in a practical and thorough manner from the standpont of cutaneous medicine. . . The riews expressed on all topics are conservative, safe to follow, and practical, and are well abreast of the knowledge of the present time, Loth as to general and special pathology", etiology, and treatment."-American Journa.' of Mezical Sizenics. 


\section{Norris' \\ Gonorrhea in Women}

Gonorrhea in Women. By Ciarles C. Norkis, M. I)., Instructor in Gynecology, University of Pennsylvania, with an Introduction by John G. Clark, M. D., Professor of Gynecology, University of Pennsylvania. Large octavo of 520 pages, illustrated. Cluth, \$6.00 nct.

\section{A CLASSIC}

Dr. Norris here presents a work that is destined to take high place among publications on this subject. He has done his work thoroughly. He has searched the important literature very carefully, over 2300 references being utilized. This, coupled with Dr. Norris' long experience, gives his work the stamp of authority. The chapter on serum and vaccine therapy and organotherapy is particularly valuable because it expresses the newest advances. Every phase of the subject is considered.

\section{Pennsylvania Medical Journal}

"Dr. Norris has succeeded in presenting most comprehensively the present knowledge of gonorrhea in women in its many phases. The present status of serum and vaccine therapy is given in detail."

\section{Sharp's}

\section{Ophthalmology for Veterinarians}

Ophthalmology for Veterinarians. By WALTER N. SHARP, M. D., Professor of Ophthalmology in the Indiana Veterinary College. 12 mo of 2 IO pages, illustrated. Cloth, \$2.00 net.

\section{ILLUSTRATED}

This new work covers a much neglected but important field of veterinary practice. Dr. Sharp has presented his subject in a concise, crisp way, so that you can pict: up this book and get to "the point" quickly. Ile first gives you the anatomy of the eye, then examination, followed by the various diseases, including injuries, parasites, errors of refraction, and medicines.

Dr. George H. Glover, Agricultural Experiment Statin, Fort Collins.

"The best book on the subject on the market." 


\section{Barnhill and Wales' Modern Otology}

A Text=Book of Modern Otology. By̆ Johx F. BAkNhill, M. D., Professor of Otology, Laryngology, and Rhinology, and EARNest DE M. Wales, M. D., Associate Professor of Otology, Laryngology, and Rhinology, Indiana University School of Medicine, Indianapolis. Octavo of 598 pages, with 314 original illustrations. Cloth, $\$ 5.50$ net; Half Morocco, s7.00 net.

\section{THE NEW (2d) EDITION}

The authors, in writing this work, kept ever in mind the needs of the physician engaged. in general practice. It represents the results of personal experience as practitioners and teachers, influenced by the instruction given by such authorities as Sheppard, Dundas Grant, Percy Jakins, Jansen, and Alt. Much space is deroted to prophylaxis, diagnosis, and treatment, both medical and surgical. There is a special chapter on the bacteriology of ear affectionsa feature not to be found in any other work on otology. Great pains have been taken with the illustrations, in order to have them as practical and as helpful as possible, and at the same time highly artistic. A large number represent the best work of Mr. H. F. Aitken.

\section{PERSONAL AND PRESS OPINIONS}

Frank Allport, M.D.

Professor of Otology, Northwestern Untersity, Chicago.

"l regard it as one of the best books in the English language on this subject. The pictures are especially good, particularly as they are practically all original and not the old reproduced pictures so frequently seen."

C. C. Stephenson, M. D.

Profissor of Ophthalmology aud Otoligy. College of Physicians and Surgeons, Little Rock Artansas.

"To my mind there is no work on modern otology that can for a moment compare with 'Barnhiil and VIales.".

\section{Journal American Medical Association}

"Its teaching is sound throughout and up to date. The strongest chapters are those on suppuration of the middle ear and the mastoid cells, and the intractarial complications of eat disease." 


\section{DeSchweinitz's Diseases of the Eye}

\section{Seventh Edition}

Diseases of the Eye: A Maxibook of OphThalmic Practice. By G. E. DeScimersitz, M.D., Professor of Ophthalmology in tle University of Pennsylvania, Philadelphia, etc. Handsome octavo of 979 pages, $3^{60}$ text-illustrations, and 7 chromo-lithographic plates. Cloth, $\$ 5.00$ net; Sheep or Half Morocco, $\$ 6.50$ net.

\section{WITH 360 TEXT-ILLUSTRATIONS AND 7 COLORED PLATES}

\section{THE STANDARD AUTHORITY}

Dr. deSchweinitz's book has long been recognized as a standard authority upon eye diseases, the reputation of its author for accuracy of statement placing it far in the front of works on this subject. For this edition Dr. deSchweinitz has subjected his book to a most thorough revision. Nany new subjects have been added, a number in the former edition have been rewritten, and throughout the book reference has been made to vaccine and serum therapy, to the relation of tuberculosis to ocular disease, and to the value of tuberculin as a diagnostic and therapeutic agent.

The text is fully illustrated with black and white cuts and colored plates, and in every way the book maintains its reputation as an authority upon the eye.

\section{PERSONAL AND PRESS OPINIONS}

\section{Samuel Theobald, M.D.}

Clinical Professor of Ophthalmology, Johns Hopkins L 'nireersity, Baltimore.

"It is a work that I have held in high esteem, and is one of the two or three books upon the eye which I have been in the habit of recommending to my students in the Johns IIopkins Medical School."

\section{University of Pennsylvania Medical Bulletin}

"Upon reading through the contents of this book we are impressed ly the remarkalle fulness with which it reflects the notable contributiuns recently made to ophiblimic hiterature. No important subject within its province has been neglected."

\section{Johns Hopkins Hospital Bulletin}

"No single chapter can be selected as the best. They are all the product of a finshed authorship and the work of an exceptional ophthatmologist. The work is certuinly one of the best on ophthalmology extant, and probably the best by an American author." 


\section{GET \\ THE BEST \\ American \\ THE NEW \\ Illustrated Dictionary}

New (7th) Edition-5000 Sold in Two Months

The American Illustrated Medical Dictionary. A new and complete dictionary of the terms used in Medicine, Surgery, Dentistry, Pharmacy, Chemistry, Veterinary Science, Nursing, and kindred branches; with over 100 new and elaborate tables and many illustrations. By W. A. Newhan Dorland, M.D., Editor of "The American Pocket Medical Dictionary." Large octavo, with I IO7 pages, bound in full flexible leather. Price, $\$ 4.50$ net; with thumb index, $\$ 5.00$ net.

\section{KEY TO CAPITALIZATION AND PRONUNCIATION-ALL THE NEW WORDS}

This dictionary is the "new standard." It defines hundreds of the newest terms not defined in any other dictionary-bar none. These terms are live, active words, taken right from modern medical literature.

\section{Howard A. Kelly, M. D.,}

Professor of Gynecologic Surgery, Johns Hopkins Liniversity, Baltimore

"Dr. Dorland's Dictionary is admirable. It is so well gotten up and of such convenient size. No errors have been found in my" use of it."

\section{Theobald's Prevalent Eye Diseases}

Prevalent Diseases of the Eye. By SAmuel Theobald, M. D., Clinical Professor of Ophthalmology and Otology, Johns Hopkins University. Octavo of 550 pages, with 2 I 9 text-cuts and several colored plates. Cloth, $\$ 4.50$ net; Half Morocco, \$6.00 net.

\section{THE PRACTITIONER'S OPHTHALMOLOGY}

With few exceptions all the works on diseases of the eye, although written ostensibly for the general practitioner, are in reality adapted only to the specialist ; but D)r. Theobald in his book has described very clearly and in detail those conditions, the diagnosis and treatment of which come within the province of the genera: practitioner. The therapeutic sugrestions are concise, unequirocal, and specific. It is the one work on the Eye written particularly for the general practitioner.

Charles A. Oliver, M.D.,

Clinical Profissor of Ophthalmology, IToman's Aledical College of Pennsyliania.

"I feel I can conscientiously recommend it, not only to the general physician and medical student, for whom it is primarily written, but also to the experienced ophthalmologist. Most surely Dr. "Theobald has accomplished nis purpose." 


\section{Haab and DeSchweinitz's External Diseases of the Eye}

Atlas and Epitome of External Diseases of the Eye. By DR. O. HAAB, of Zürich. Edited, with additions, by G. E. DeScmwennitz, M. D., Professor of Ophthalmology, University of Pennsylvania. With Ior colored illustrations on 46 lithographic plates and 244 pages of text. Cloth, \$3.00 net. In Saunders' Hand-Atlas Sorics.

\section{THE NEW (3d) EDITION}

Conditions attending diseases of the external eye, which are often so complicated, have probably never been more clearly and comprehensively expounded than in the forelying work, in which the pictorial most happily supplements the verbal description. The price of the book is remarkably low.

The Medical Record, New York

"The work is excellently suited to the student of oplithalmology and to the practising physician. It cannot fail to attain a well-deserved popularity."

\section{Haab and DeSchweinitz's Ophthalmoscopy}

Atlas and Epitome of Ophthalmoscopy and Ophthalmoscopic Diagnosis. By Dr. O. HAAB, of Zürich. Edited, with additions, by G. E. DeScuwernitz, M. D., Professor of Ophthalmology, University of Pennsylvania. With 152 colored lithographic illustrations and 92 pages of text. Cloth, s3.00 net. In Saunders' Hand-Atlas Siries.

\section{THE NEW (2d) EDITION}

The great value of Prof. Ilaab's Atlas of Ophthalmoscopy and Ophthalmoscopic Diagnosis has been fully established and entirely jutified an linglish translation. Not only is the student made acquainted with carefully prepared ophthalmoscopic drawings done into well-executed fithographs of the most imporiant fundus changes, but, in many instances, plates of the microscopic lesions are added. The whole furnishes a manual of the greatest possible service.

The Lancet, London

"We recommend it as a work that should be in the ophthalmic wards or in the library of every hospital into which ophthalmic cases are received." 


\section{Gradle's}

\section{Nose, Pharynx, and Ear}

Diseases of the Nose, Pharynx, and Ear. By Henry Gradle, M.D., late Professor of Ophthalmology and Otology, Northwestern University Medical School, Chicago. Octavo of 547 pages, illustrated, including two full-page plates in colors. Cloth, $\$ 3.50$ net.

\section{INCLUDING TOPOGRAPHIC ANATOMY}

This volume presents diseases of the Nose, Pharynx, and Ear as the author has seen them during an experience of nearly twenty-five years. In it are answered in detail those questions regarding the course and outcome of diseases which cause the less experienced observer the most anxiety in an individual case. Topographic anatomy has been accorded liberal space.

\section{Pennsylvania Medical Journal}

"This is the most practical volume on the nose, pharynx, and ear that has appeared recently. . . . It is exactly what the less experienced observer needs, as it avoids the confusion incident to a categorical statement of everybody's opinion."

\section{Kyle's}

\section{Diseases of Nose and Throat}

Diseases of the Nose and Throat. By D. Braden Krle, M. D., Professor of Laryngology in the Jefferson Medical College, Philadelphia. Octavo, 825 pages; with 25 's illustrations, 28 in colors. Cloth, \$4.50 net; Half Morocco, „c.

\section{JUST OUT-THE NEW (5th) EDITION}

The new (5th) edition of Dr. Kyle's work shows an increase of 100 pages and some to new illustrations. The following new articles have been added: vaccine therapy ; lactic bacteriotherapy; salvarsan in the treatment of syphilis of the upper respiratory tract; sphenopalatine ganglia neuralgia; negative air-pressure in accessory sinus disease; chronic hyperplastic ethmoiditis; and congenital insufficiency of the palate. The tables of differential diagnosis and the prescriptions are striking points of Dr. Kyle's book.

\section{Pennsylvania Medical Journal}

"Dr. Kyle's crisp, terse diction has enabled the inclusion of all needful nose and throat knowledge in this book." 


\section{Ogden on the Urine}

Clinical Examination of Urine and Urinary Diagnosis. A Clinical Guide for the Use of Practitioners and Students of Nerficine and Surgery. By J. Bergen Ogdex, Mi. D., Medical Chemist to the Metropolitan Life Insurance Company, New York. Octavo, 4 IS pages, 54 text illustrations, and a number of colored plates. Cloth, $\$ 3.00$ net.

\section{THE NEW (3d) EDITION}

In this edition the work has been brought absolutely down to the present day, Urinary examinations for purposes of life insurance have been incorporated, because a large number of practitioners are often called upon to make such analyses. Special attention has been paid to diagnosis by the character of the urine, the diagnosis of diseases of the kidneys and urinary passages; an enumeration of the prominent clinical symptoms of each disease; and the peculiarities of the urine in certain general diseases.

\section{The Lancet, London}

"We consider this manual to have been well compiled; and the author's own experience, so clearly stated, renders the volume a useful one both for study and reference."

\section{Pilcher's \\ Practical Cystoscopy}

Practical Cystoscopy. By PAUL M. Plilcher, M. D., Consulting Surgeon to the Eastern Long Island Hospital. Octavo of 398 pages. with 233 illustrations, 29 in colors. Cloth, \$5.50 net.

\section{DIAGNOSIS AND TREATMENT}

Cystoscopy is to-day the most practical manner of diagnosing and treating diseases of the bladder, ureters, kidneys, and prostate. To be properly equipped, therefore, you must have at your instant command the information this book gives you. It explains away all difficulty, telling you why you do not see something when something is there to see, and telling you how to see it. All theory has been uncompromisingly eliminated, devoting every line to practical, neededevery-day facts, telling you how and when to use the cystoscope and cathetertelling you in a way to make jou know. The work is complete in every detail.

Bransford Lewis, M. D., St. Louis Cniversity.

"I am very much pleased with Dr. Pilcher's '1'ractical Cystoscopy." I think it is the best in the English language now." - April $27,1911$. 


\section{Goepp's \\ Dental State Boards}

Dental State Board Questions and Answers.-By R. MAx GoepP, M. D., author "Medical State Board Questions and Answers." Octavo of 428 pages. Cloth, $\$ 2.75$ net.

\section{COMPLETE AND ACCURATE}

This new work is along the same practical lines as Dr. Goepp's successful work on Medical State Boards. The questions included have been gathered from reliable sources, and embrace all those likely to be asked in any State Board examination in any State. They have been arranged and classified in a way that makes for a rapid résumé of every branch of dental practice, and the answers are couched in language unusually explicit-concise, definite, accurate.

The practicing dentist, also, wiil find here a work of great value-a work covering the entire range of dentistry and extremely well adapted for quick reference.

\section{Haab and deSchweinitz's Operative Ophthalmology}

Atlas and Epitome of Operative Ophthalmology. By Dr. O. HAAs, of Zurich. Edited, with additions, by G. E. DESchweinitz, M. D., Professor of Ophthalmology in the University of Pennsylvania. With 30 colored lithographic plates, I 54 text-cuts, and 375 pages of text In Saunders' Hand-Atlas Siries. Cloth, \$3.50 net.

Dr. Haab's Atlas of Operative Ophthalmology will be found as beautiful and as practical as his two former atlases. The work represents the author's thirty years' experience in eye work. The various operative interventions are described with all the precision and clearness that such an experience brings. Recognizing the fact that mere verbal descriptions are frequently insufficient to give a clear idea of operative procedures, Dr. Haab has taken particular care to illustrate plainly the different parts of the operations.

Johns Hopkins Hospital Bulletin

" The descriptions of the various operations are so clear and full that the volume can well hold place with more pretentious text-books." 


\section{Greene and Brooks' Genito-Urinary Diseases}

Diseases of the Genito=Urinary Organs and the Kidney. By Robert H. Greese, M. D., Professor of Genito-Urinary Surgery at Fordham University; and HARlow Brooks, M. D., Assistant Professor of Clinical Medicine, University and Bellerue Hospital Medical School. Octavo of 639 pages, illustrated. Cloth, $\$ 5.00$ net; Half Morocco, s6.jo net.

\section{THE NEW (3d) EDITION}

This new work presents both the medical and surgical sides. Designed as a work of quick reference, it has been written in a clear, condensed style, so that the information can be readily grasped and retained. Kidney discases are very elaborately detailed.

\section{New York Medical Journal}

"As a whole the book is one of the most satisfactory and useful works on genito-urinary diseases now extant, and will undoubtedly be popular among practitioners and students."

\section{Gleason on Nose, Throat, and Ear}

A Irlanual of Diseases of the Nose, Throat, and Ear. By E. Baldwix Glessox, M. D., LI. D., Professor of Otologi-, MedicoChirurgical College, Philadelphia. $12 \mathrm{mo}$ of 590 pages, profusely illustrated. Cloth, \$2.50 net.

\section{JUST OUT-THE NEW (3d) EDITION}

Methods of treatment have been simplified as much as possible, so that in most instances only those methods, drugs, and operations have been advised which have proved beneficial. A valuable feature consists of the collection of formulas.

\section{American Journal of the Medical Sciences}

"For the practitioner who wishes a reliable guide in laryonlogy and otologv there are few books which can be more heartily commended."

American Text=Book of Genito=Urinary Diseases, Syphilis, and Diseases of the Skin. Fdited by I. BoLtox B.AG. .I. D., and IV. A. HARDAWA, M. D. Octaro, I 229 pages, 300 engrarings, 20 colored plates. Cloth, $\$ 7.00$ net. 


\section{Holland's Medical Chemistry and Toxicology}

A Text=Book of Medical Chemistry and Toxicology. By JAMES IV. Hollaxd, M. D., Professor of Medical Chemistry and Toxicology, and Dean, Jefferson Medical College, Philadelphia. Octavo of 675 pages, fully illustrated. Cloth, $\$ 3.00$ net.

\section{THE NEW (3d) EDITION}

Dr. Holland's work is an entirely new one, and is based on his forty years' practical experience in teaching chemistry and medicine. It has been subjected to a thorough revision, and enlarged to the extent of some sixty pages. The additions to be specially noted are those relating to the electronic theory, chemical equilibrium, Kjeldahl's method for determining nitrogen, chemistry of foods and their changes in the body, synthesis of proteins, and the latest improvements in uinary tests. Hore space is given to toxicology than in any other text-book on chemistry.

\section{American Medicine}

"Its statements are clear and terse; its illustrations well chosen; its development logical, systematic, and comparatively easy to follow. . . We heartily commend the work."

\section{Ivy's Applied Anatomy and Oral Surgery for Dental Students}

Applied Anatomy and Oral Surgery for Dental Students. By Robert H. Irr, M.D., D.D.S., Assistant Oral Surgeon to the Philadelphia General Hospital. I 2 mo of 280 pages, illustrated. Cloth, \$1.50 net.

\section{FOR DENTAL STUDENTS}

This work is just what dental students have long wanted-a concise, practical work on applied anatomy and oral surgery, written with their needs solely in mind. No one could be better fitted for this task than Dr. Ivy, who is a graduate in both dentistry and medicine. Having gone through the dental school, he knows precisely the dental student's needs and just how to meet them. His medical training assures you that his a natomy is accurate and his technic modern. The text is well illustrated with pictures that you will find extremely helpful.

H. P. Kuhn, M.D., Hestern Dental College, Känsas City.

"I am delighted with this compact little treatise. It seems to me just to fill the bill." 


\section{American Pocket Dictionary}

New 8th Edition

The Americax Pocker Medcal Dictoxary. Edited by IV. A. NewMax Doklaxo, M. D., Editor "American Illustrated Medical Dictionary:" Containing the pronunciation and definition of the principal words used in medicine and kindred sciences. 67- pages. Flexible leather, with gold edges, SI.OO net; with thumb index, $\$ 1.25$ net.

James W. Holland, M. D.,

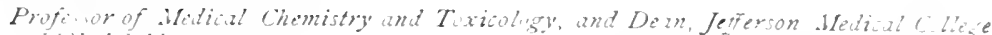
Philidelthiz.

"I am struck at once with armiration at the compat size and aturactive exterior. I can recommend it to our students without reserve.'

\section{Stelwagon's Essentials of Skin}

7th Edition

Fsentals of Diseases of the Skin. Br Hexry IV. StelIVAgox, .I.D., Pн.D., Professor of Dermatolugy in the Jefferson Medical Collegre, Philadelphia. Post-octaro of 29 I pages, with 72 text-illustrations and 8 plates. Cloth, si.oo net. In Saunders' Question-Compend Series.

\section{The Medical News}

"In line with our present knowledge of diseases of the skin.... Continues to ma'n. tain the high standarl of excellence for which thesequeston compenr, have teen noted.

\section{Wolff's Medical Chemistry}

New 7th, Edition

Essextials of Medical Chemistri, Okganic and INorganic. Containing also Questions on Medical Physics, Chemical Physiology, Analytical Processes, Urinalysis, and Toxicology: By LAWRExce WolfF, M. D., Late Demonstrator of Chemistry, Jefferson Medical College. Revised by A. Ferree Mituer, Ph. G., M. D., Formerly Assistant Demonstrator of Physiology, Lniversity of Pennsyliania. Post-octavo of 222 pages. Cloth, SI.00 net. In Saunders' Question-Compend Series.

\section{Bliss' Qualitative Chemical Analysis}

Qcalitative Chemical Axalysis. By A. R. Blin, JR., Ph. G. M. D., Professor of Chemistry and Pharmacy, Bimingham Medical College, Alabama. Octaro of 250 pages. Cloth, $\$ 2.00$ net.

\section{Vecki's Sexual Impotence}

New (4th, Edition

Sextal Impotexce. By Victor G. Vecki, . I. H), Consulting Genito-Urinary Surgeon to Mt. Zion Hospital, San Francincu. I 2 mo of 400 pages. Cloth, \$2.25 net.

\section{Johns Hopkins Hospital Bulletin}

"A scientifie treatise upon an mportant and much n..gi..cicd subj...... The

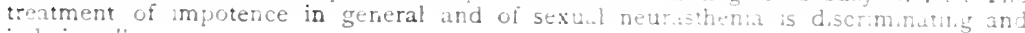
jurlicious. 


\section{Wells' Chemical Pathology secood Wells' Chemical Pathology Exition}

Chemical Pathology. Being a discussion of General Pathology from the Standpoint of the Chemical Prowses Involved. By H. Gideon Wells, Ph. D., M. D., Assistan Professor of Pathology in the University of Chicago. Octavo of 616 pages. Cloth, $\$ 3.25$ net.

Wm. H. Welch, M. D., Professor of Pathology, Johns Hofkins Lnizersity.

"The work fills a real need in the English literature of a very inportant subject, and I shall be glad to recommend it to my students."

\section{Saxe's Urinalysis}

The New (2d) Edition

Examination of the Urine. By G. A. De SAxtos SAxe, M. D., formerly. Instructor in Genito-Urinary Surgery, New York Postgraduate Medical School and Hospital. I 2 mo of 448 pages, fully illustrated. Cloth, SI.75 net.

Francis Carter Wood, M. D.. Adjunct Professor of Clinical Pathology, Columbia Uniiersity.

"It seems to me to be one of the best of the smaller works on this subject; it is, indeed, better than a good many of the larger ones."

\section{deSchweinitz and Randall on the Eye, Ear, Nose, and Throat}

American Text-Book of Diseases of the Eye, Ear, Nose, and Throat. Edited by G. E. DE Schweixitz. M.D., and B. AlexANDER RaNdall, M.D. Imperial octavo, I25I pages, with 766 illustrations, 59 of them in colors. Cloth, \$7.00 net; Half Morocco, $\$ 8.50$ net.

\section{Grünwald and Grayson on the Larynx}

Atlas and Epitome of Diseases of the Larynx. By Dr. L. Grürwald, of Munich. Edited, with additions, by Charles P. Gralsox, M.D., University of Pennsylvania. With Iot colored figures on 44 plates, 25 text-cuts, and 103 pages of text. Cloth, $\$ 2.50$ net. In Samders' Hand-Atlas Series.

\section{Mracek and Stelwagon's Atlas of Skin}

Atlas and Epitome of Diseases of the Skin. By Prof. DR. FrANz MrAcek, of Vienna. Edited, with additions, by HexRY IV. Stelwagox, M.D., Jefferson Medical College. With 77 colored plates, 50 half-tone illustrations, and 280 pages of text. In Samiders' Hand-Atlas Series. Cloth, \$t.00 net. 


\section{de Schweinitz and Holloway on Pulsating Exoph. thalmos}

Pulsating Exophthalios. An analysis of sixty-nine cases not pre. viously analyzed. By (ieorge E. DeSchweinitz, M. D), and I'Homas B. Holloway, M. D. Octavo of I 25 pages. Cloth, $\$ 2.00$ net.

This monograph consists of an analy'sis of sixty-nine cases of this affection not previously analyzed. The therapeutic measures, surgical and otherwise, which have been employed are compared, and an endeavor has been made to determine from these analyses which procedures seem likely to prove of the greatest value. It is the most valuable contribution to ophthalmic literature within recent years.

\section{British Medical Journal}

"The book deals very thoroughly with the whole subject and in it the most complete account of the disease will be found."

\section{Jackson on the Eye}

The New (2d) Edition

A Manual of the Diagnosis and Treatment of Diseases of the Eye. By Edward Jackson, A. M., M. D., Professor of Ophthalmology, University of Colorado. I mo volume of 615 pages, with 184 beautiful illustrations. Cloth, $\$ 2.50$ net.

\section{The Medical Record, New York}

"It is truly an admirable work. . Written in a clear, concise manner, it bears evidence of the author's comprehensive grasp of the subject. The term "multum in parvo" is an appropriate one to apply to this work."

\section{Grant on Face, Mouth, and Jaws}

A Text-Book of the Surgical Principles and Surgical Diseases of the Face, Mouth, and Jaws. For Dental Students. By H. Horace, Grant, A. M., M. D., Professor of Surgery and of Clinical Surgery, Hospital College of Medicine, Louisville. Octavo of 23 I pages, with 68 illustrations. Cloth, $\$ 2.50$ net.

\section{Preiswerk and Warren's Dentistry}

Atlas ane Epitome of Dentistry. By Prof. G. Preiswerk, of Basil. Edited, with additions, by Cieokge W. Wakkex, I.I).S., Professor of Operative Dentistry, Pennsylvania College of Dental Surgery, Philadelphia. With 44 lithographic plates, 152 text-cuts, and $3+3$ pages of text. Cloth, $\$ 3.50$ net. In Samiders' Atlas Serics.

\section{Asher's Chemistry and Toxicology}

Chemistry anu Toxicology for Nurses. By Philip Asiar, Pli. (r., M. D., Dean and Professor of Chemistry, New (irleans College of tharmacy. I 2 mo of 190 pages. Cloth, s1.25 net. 


\section{Wolf's Examination of Urine}

A Laboratory Handbook of Phisiologic Chemistry ano Urine-examination. By Charles G. L. Wolf, M. D., Instructor in Physiologic Chemistry, Cornell University Medical College, New York. I 2 mo volume of 204 pages, fully illustrated. Cloth, \$1.25 net. British Medical Journal

"The methods of examining the urime are very fully lescribed, and there are at the ent of the book some extensive tables drawn up to assist in urinary diagnosis."

\section{Jackson's Essentials of Eye}

Third Revised Edition

Escentials of Refraction and of Diseases of the Eye. By Edward Jackson, A. M., M. D., Emeritus Professor of Diseases of the Eye, Philadelphia Polyclinic. Post-octaro of 261 pages, 82 illustrations. Cloth, Si.OO net. In Sannders' Question-Compend Series. Johns Hopkins Hospital Bulletin

"The entire ground is covered, and the points that most need careful elucidation are made clear and easy."

\section{Gleason's Nose and Throat}

Fourth Edition, Revised

Essentials of Diseases of the Nose and Throat. By E. B. Gleason, S. B., M. D., Clinical Professor of Otology, MedicoChirurgical College, Philadelphia, etc. Post-octavo, 24 I pages, i 12 illustrations. Cloth, \$1.00 net. In Saunders' Question Compends.

\section{The Lancet, London}

"The careful description which is given of the various procedures would be sufficient to enable most people of average intelligence and of slight anatomical knowledge to make a very good attempt at laryngoscopy."

\section{Gleason's Diseases of the Ear}

Third Edition, Revised

Essentials of Diseases of the Ear. By E. B. Gleason, S. B., M. D., Clinical Professor of Otology, Medico-Chirurgical College, Phila., etc. Post-octavo volume of 2 I 4 pages, with 114 illustrations. Cloth, \$i.oo net. In Saunders' Question-Compend Sirie's.

\section{Bristol Medico-Chirurgical Journal}

"We know of no other small work on ear diseases to compare with this, either in freshness of style or completeness of information."

\section{Wilcox on Genito-Urinary and Venereal Diseases}

The New (2d) Edition

Essentials of Genito-Urinary and Venereal Diseases. By' Starling S. Wilcox, M. D., Lecturer on Genito-Urinary Diseases and Syphilology, Starling-Ohio Medical College, Columbus. I 2 mo of 32 I pages, illustrated. Cloth, \$i.oo net. Sannders' Compends.

\section{Stevenson's Photoscopy}

Photoscopy (Skiascopy o: Retinoscopy). By Mark D. StevEnson, M. D., Ophthalmic Surgeon to the Akron City Hospital. 12 mo of 126 pages, illustrated.

Cloth, \$1.25 net

Edward Jackson, M. D., Liniersity of Colorado.

"It is well written and will prove a valuable help. Your treatment of the emergent pencil of rays, and the part falling on the examiner's cye, is decidedly better than anv previous account." 



Return this material to the library from which it was borrowed.

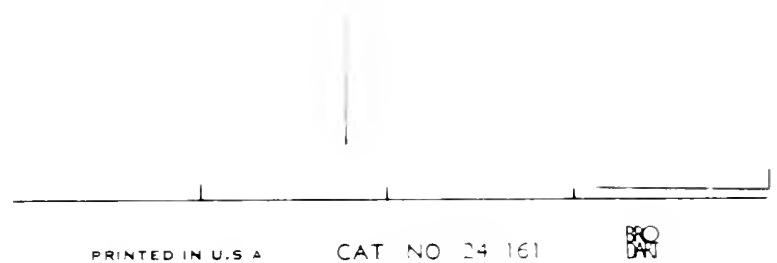

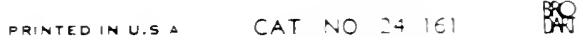



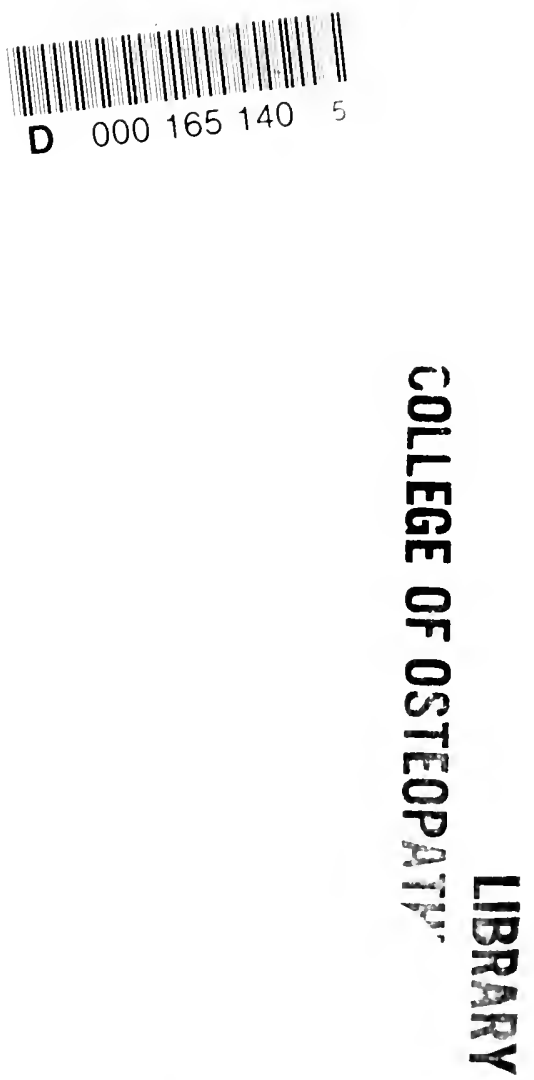

Gant, iamued $G$

Dicrineal, inflarmator, ofstructir.

and rarasitic diseases of the

rastro-intestinal. truct

\section{MEDICAL SCIENCES LIBRARY \\ UNIVERSITY OF CALIFORNIA, IRVINE \\ IRVINE, CALIFORNIA 92664}


